\title{
Youth in India: Situation and Needs 2006-2007, Andhra Pradesh
}

International Institute for Population Sciences (IIPS)

Population Council

Follow this and additional works at: https://knowledgecommons.popcouncil.org/departments_sbsr-pgy

Part of the Demography, Population, and Ecology Commons, Domestic and Intimate Partner Violence Commons, Family, Life Course, and Society Commons, Gender Equity in Education Commons, International Public Health Commons, and the Women's Health Commons How does access to this work benefit you? Let us know!

\section{Recommended Citation}

International Institute for Population Sciences (IIPS) and Population Council. 2010. "Youth in India: Situation and Needs 2006-2007, Andhra Pradesh." Mumbai: IIPS. 
This report is the result of a sub-national study undertaken by the International Institute for Population Sciences, Mumbai and the Population Council, New Delhi, as part of a project to collect information on key transitions experienced by youth in India, including those related to education, work force participation, sexual activity, marriage, health and civic participation; the magnitude and patterns of young people's sexual and reproductive practices before, within and outside of marriage as well as related knowledge, decision-making and attitudes. The project was implemented in six states of India, namely, Andhra Pradesh, Bihar, Jharkhand, Maharashtra, Rajasthan and Tamil Nadu.

\section{For additional copies of this report please contact:}

International Institute for Population Sciences

Govandi Station Road, Deonar

Mumbai 400088

India

Phone: 022-42372400/42372518

email: iipsyouth@rediffmail.com

Website: http://www.iipsindia.org
Population Council

Zone 5-A, Ground Floor

India Habitat Centre

Lodi Road

New Delhi 110003

Phone: 011-2464 2901/02

email: info-india@popcouncil.org

Website: http://www.popcouncil.org/asia/india.html

The International Institute for Population Sciences (IIPS) is a deemed university under administrative control of Ministry of Health and Family Welfare, Government of India. The Institute engages in teaching and research in population sciences, and has been actively involved in building the capacity of Population Research Centres, and other state and central government offices that address population issues in the country and in the Asia-Pacific region. It has a proven record in conducting national- and sub-national-level studies in reproductive health, including the National Family Health Surveys and District Level Household and Facility Survey under the Reproductive and Child Health programme.

The Population Council is an international, non-profit, non-governmental organisation that seeks to improve the well-being and reproductive health of current and future generations around the world and to help achieve a humane, equitable and sustainable balance between people and resources. The Council conducts biomedical, social science and public health research, and helps build research capacities in developing countries.

Copyright @ 2010 International Institute for Population Sciences, Mumbai and Population Council, New Delhi

Suggested citation: International Institute for Population Sciences (IIPS) and Population Council. 2010. Youth in India: Situation and Needs 2006-2007, Andhra Pradesh. Mumbai: IIPS. 


\section{Youth in India: Situation and Needs 2006-2007}

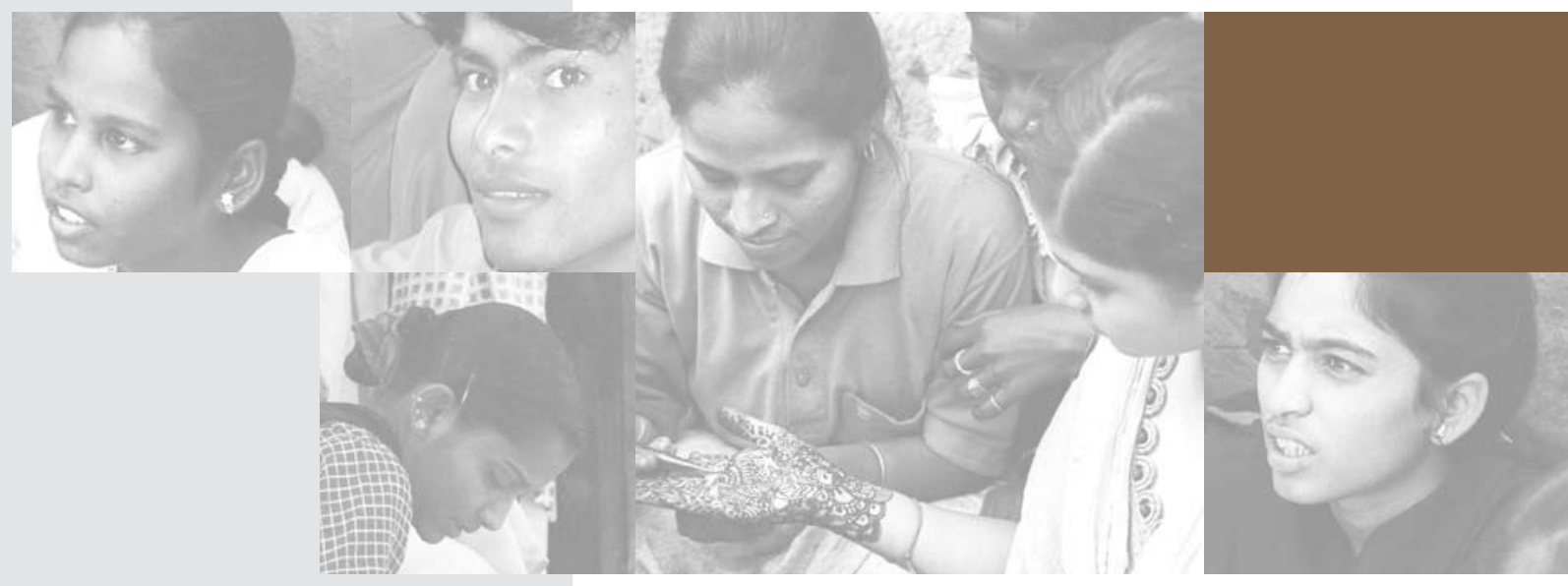

\section{ANDHRA PRADESH}

Usha Ram

S.K. Mohanty

Abhishek Singh

F. Ram

International Institute for Population Sciences, Mumbai

K.G. Santhya

Shireen J. Jejeebhoy

Rajib Acharya

Population Council, New Delhi

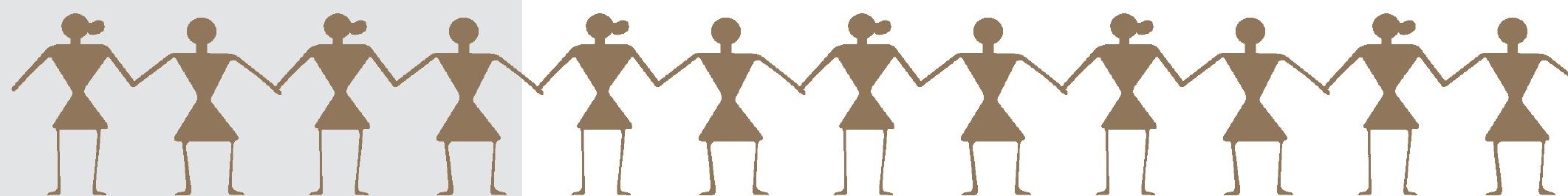




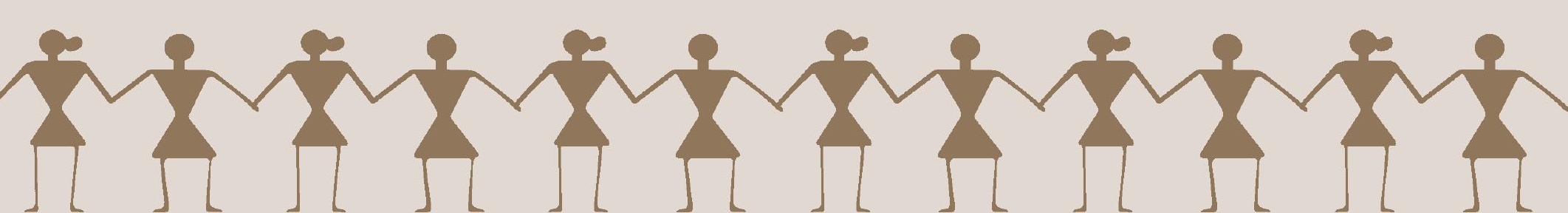




\section{Contents}

Page No.

Tables. viii

Figures xiii

Foreword. xvii

Acknowledgements . ..xix

Executive summary xxii

Chapter 1: Introduction. .1

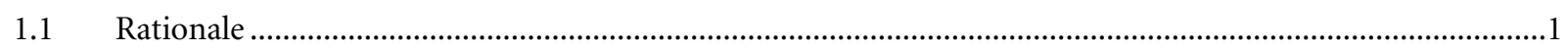

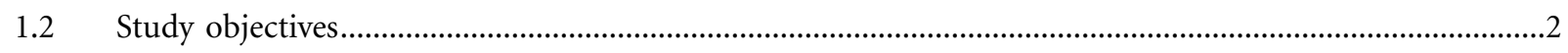

1.3 Andhra Pradesh: Overview of demographic and socio-economic features.................................................2

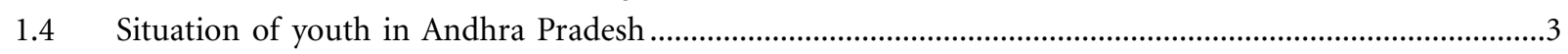

1.5 Youth-related policy and programme environment in Andhra Pradesh ....................................................

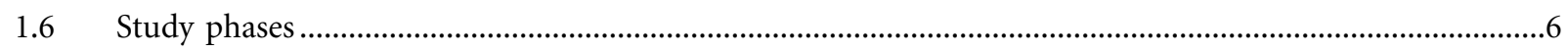

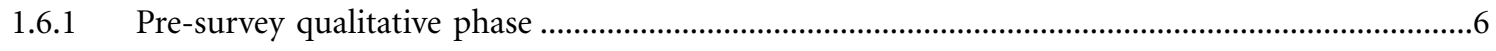

1.6.2 Survey phase .........................................................................................................................

1.6.3 Post-survey qualitative phase ............................................................................................

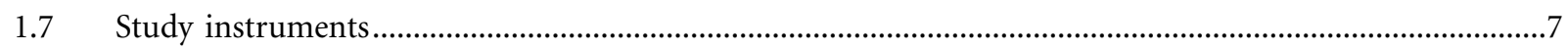

1.7.1 Interview guidelines...........................................................................................................

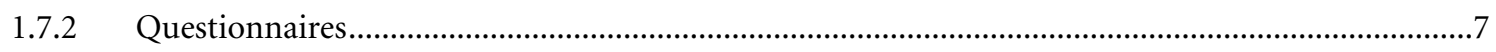

1.8 Study design and sample size estimation for individual interviews ........................................................10

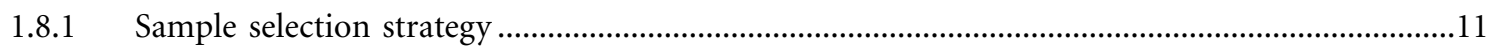

1.8.1.a Selection of households in rural areas ...........................................................................11

1.8.1.b Selection of households in urban areas ........................................................................13

1.8.2 Selection of individual respondents within selected households....................................................15

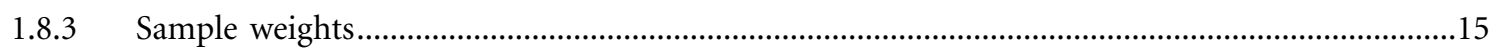

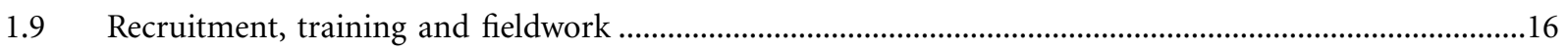

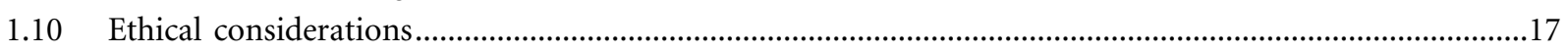

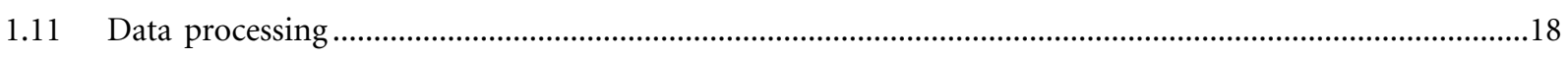

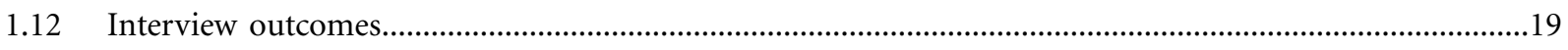

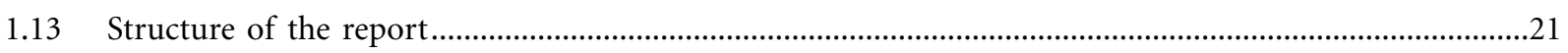

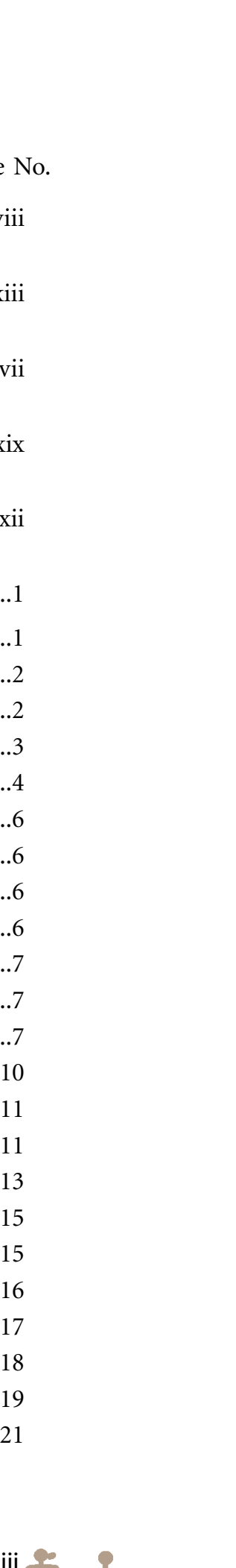


Page No.

Chapter 2: Profile of surveyed communities, households and youth ............................................22

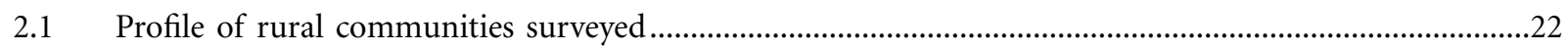

2.2 Profile of the household population: Age-sex distribution ...................................................................25

2.3 Profile of the household population: Marital status.............................................................................27

2.4 Profile of the household population: Educational attainment .............................................................29

2.5 Profile of the household population: Work participation ......................................................................29

2.6 Socio-demographic characteristics of households and heads of households...........................................31

2.7 Profile of the household population: Housing characteristics................................................................34

2.8 Profile of the household population: Ownership of agricultural land ...................................................36

2.9 Profile of the household population: Overall economic status .................................................................36

2.10 Profile of surveyed youth: Background characteristics ...........................................................................38

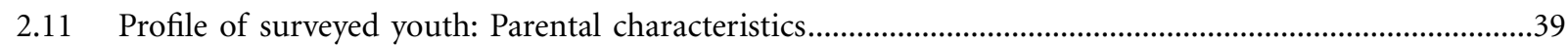

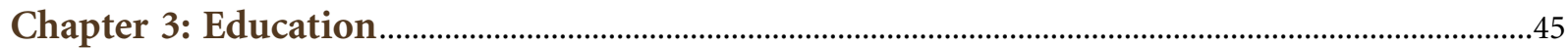

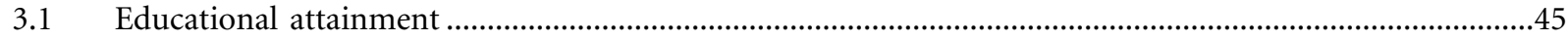

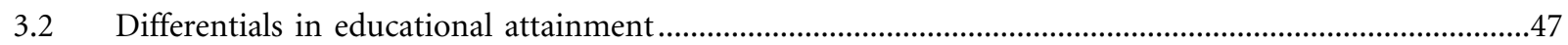

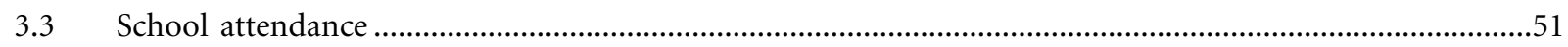

Reasons for school non-attendance or discontinuation ........................................................................52

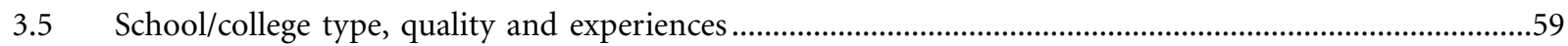

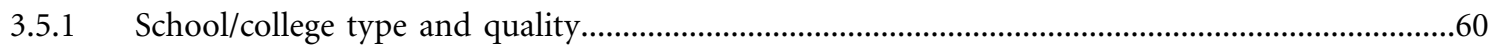

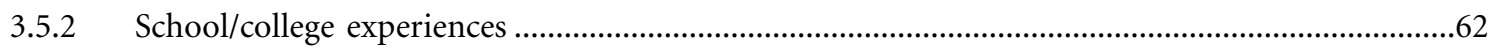

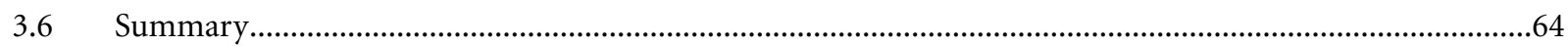

Chapter 4: Economic and non-economic activity.........................................................................66

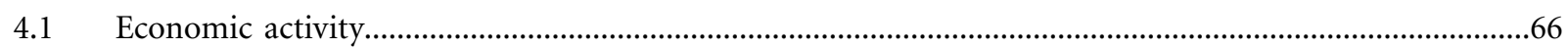

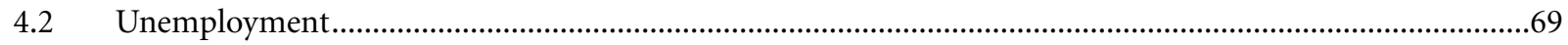

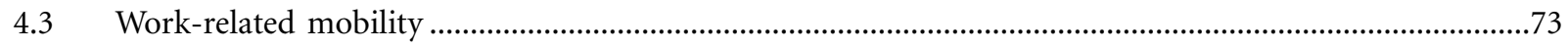

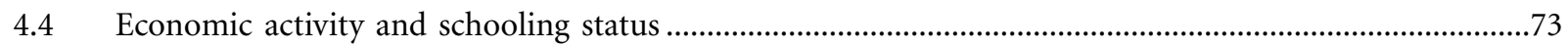

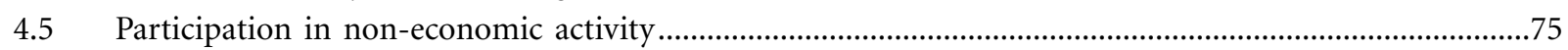

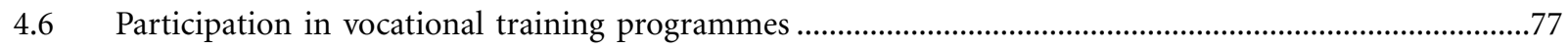

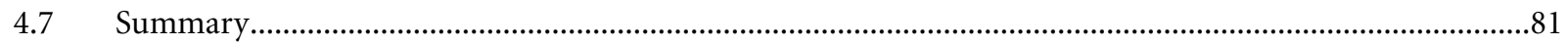

Chapter 5: Media exposure and access to pornographic materials................................................82

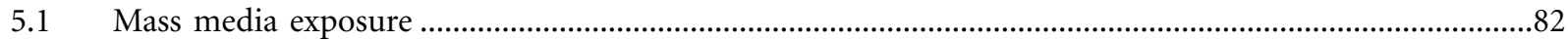

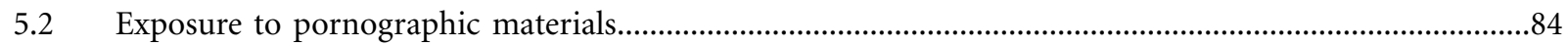

5.3 Youth perceptions about the influence of television and films on youth behaviours .............................87

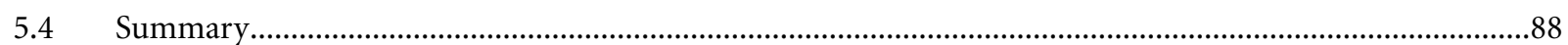


Page No.

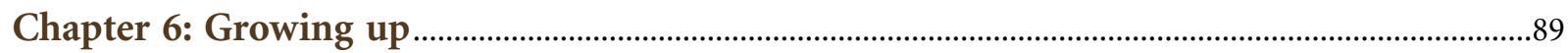

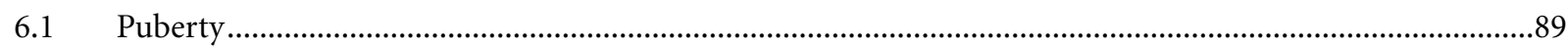

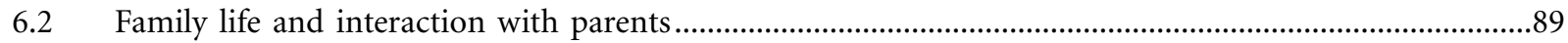

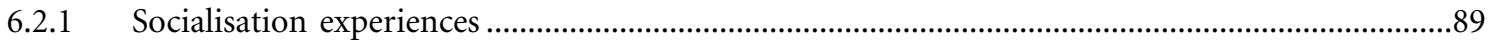

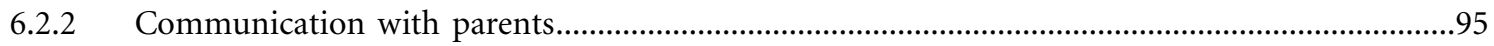

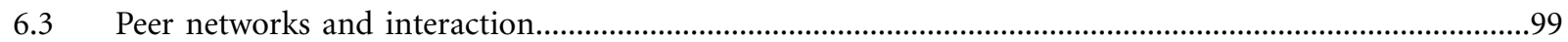

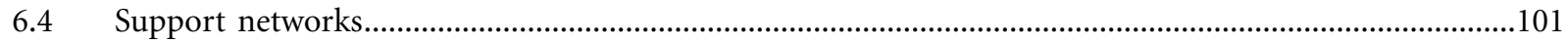

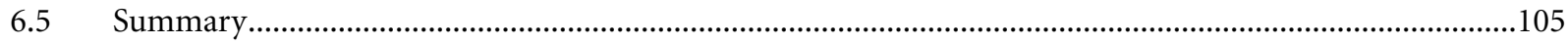

Chapter 7: Agency and gender role attitudes..................................................................................106

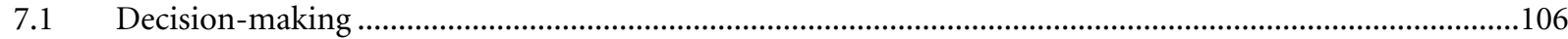

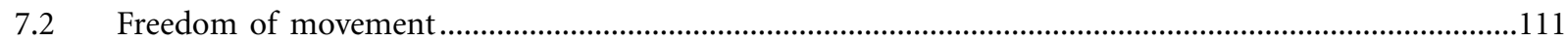

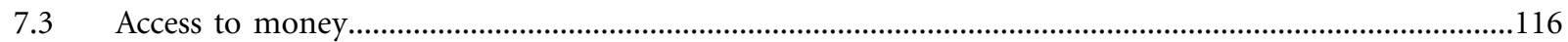

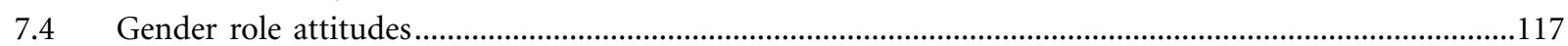

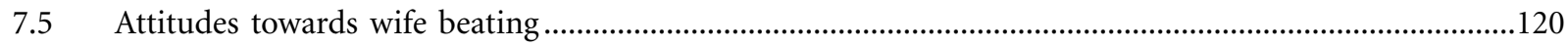

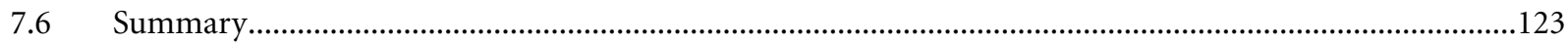

Chapter 8: Awareness of sexual and reproductive health matters..............................................124

8.1 Awareness of sex and pregnancy, contraception, STIs and HIV …........................................................124

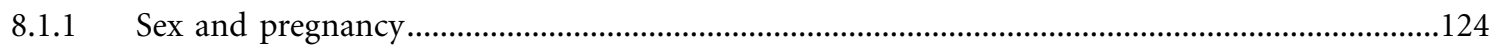

8.1.2 Socio-demographic differentials in awareness of sex- and pregnancy-related matters..............127

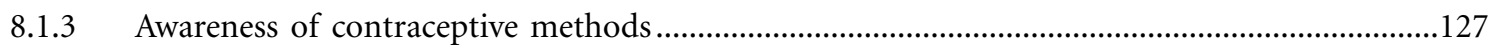

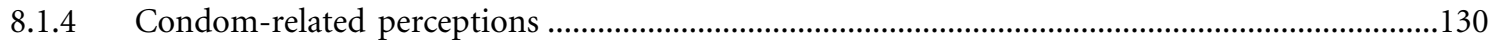

8.1.5 Awareness of contraception prior to marriage ............................................................................133

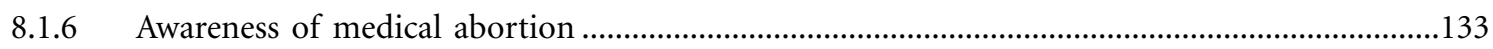

8.1.7 Awareness of sexually transmitted infections (STIs) and HIV/AIDS.........................................133

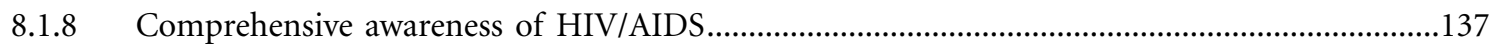

8.2 Knowledge of legal issues related to marriage and abortion......................................................................140

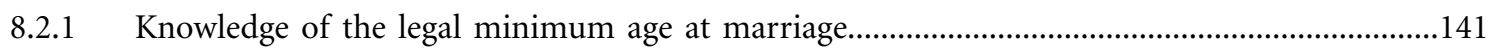

8.2.2 Awareness of the conditions under which abortion is legal ...................................................141

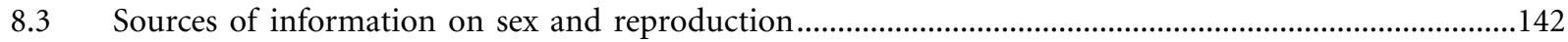

8.3.1 Sources of information on sexual matters ............................................................................

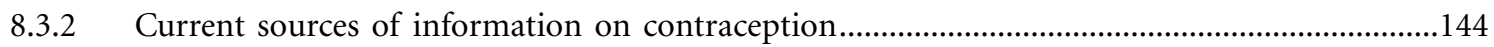

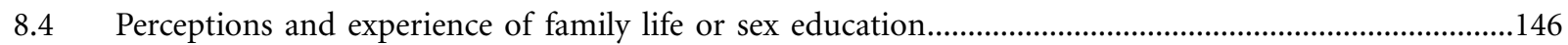

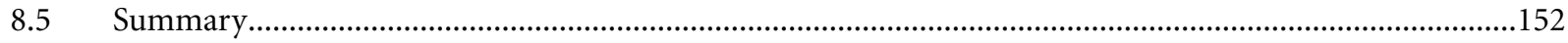


Page No.

\section{Chapter 9: Pre-marital romantic and sexual relationships}

9.1 Development of the questionnaire module on pre-marital romantic and sexual relationships..............153

9.2 Attitudes toward pre-marital physical intimacy and sexual relations .....................................................154

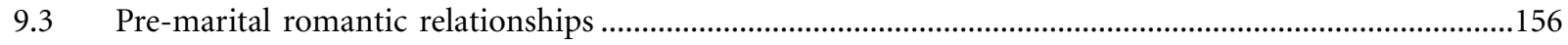

9.3.1 Prevalence of pre-marital romantic relationships ……................................................................156

9.3.2 Characteristics of pre-marital romantic relationships .................................................................158

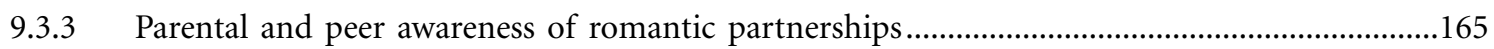

9.3.4 Marriage intentions and duration of pre-marital romantic relationships...................................166

9.3.5 Pre-marital physical intimacy and sex with a romantic partner ...............................................168

9.3.6 Characteristics of sexual experiences within pre-marital romantic relationships.......................168

9.4 Pre-marital sexual experiences within romantic and other relationships..................................................171

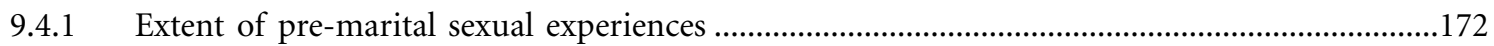

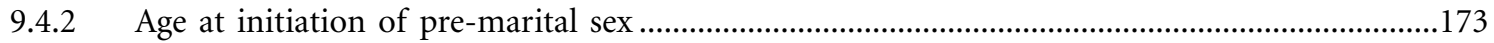

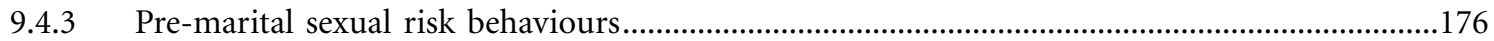

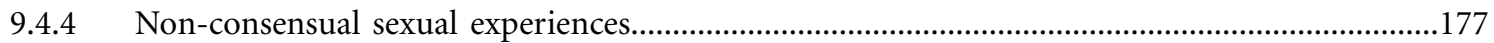

9.5 Triangulation of data on pre-marital sexual experiences among young people.........................................178

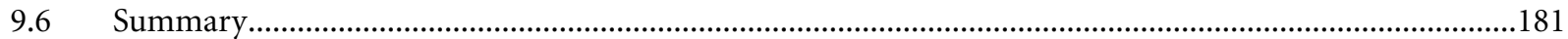

Chapter 10: Transition to marriage and early married life ............................................................182

10.1 Young people's preferences regarding timing and type of marriage ..........................................................182

10.2 Marriage planning and extent of youth involvement ...........................................................................182

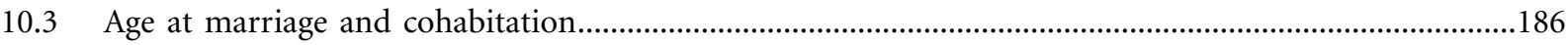

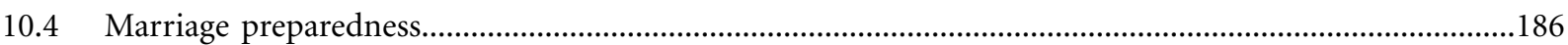

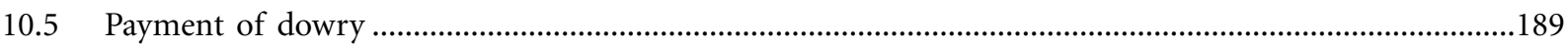

10.6 Early marital experiences: Spousal communication and interaction .......................................................189

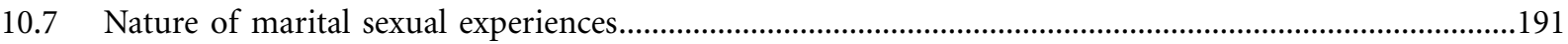

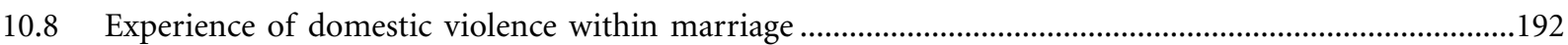

10.9 Extent and nature of extra-marital sexual relations .................................................................................192

10.10 Contraceptive practice within marriage: Lifetime, current and prior to first pregnancy.........................195

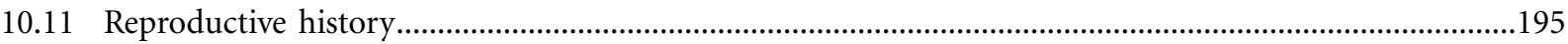

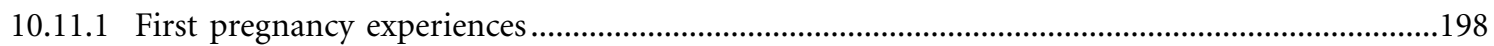

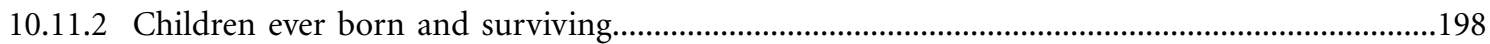

10.11.3 Wantedness of recent pregnancies ......................................................................................198

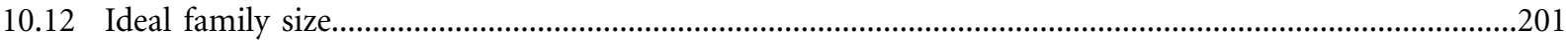

10.13 Summary 


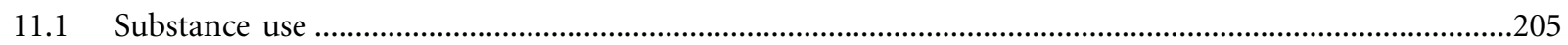

11.2 General and sexual and reproductive health problems ...................................................................206

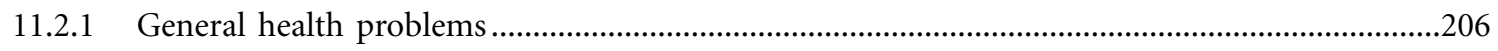

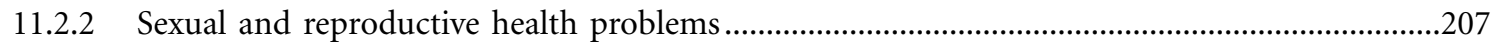

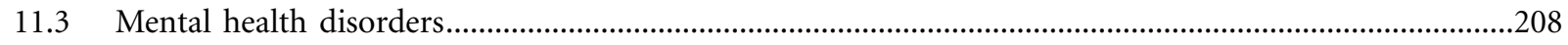

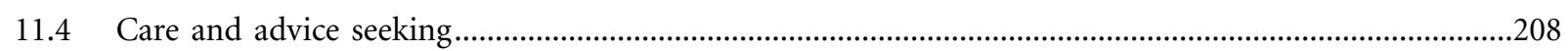

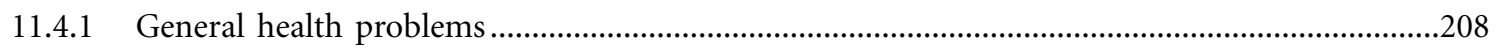

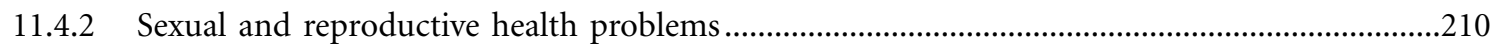

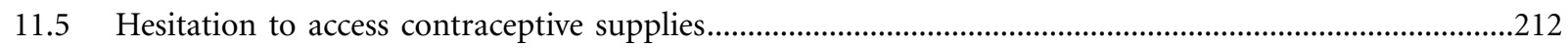

11.6 Attitudes towards pre-marital HIV testing and extent of HIV testing.................................................213

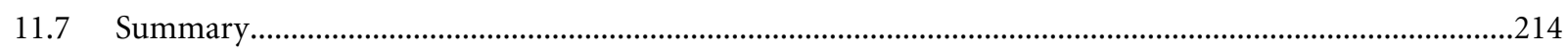

Chapter 12: Participation in civil society and political life ...........................................................215

12.1 Awareness of and participation in government- and NGO-sponsored programmes ............................215

12.2 Participation in community- or panchayat-sponsored programmes ...................................................219

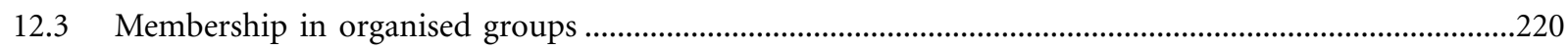

12.4 Perceptions about action taken by panchayats in addressing defiance of social norms .........................220

12.5 Voting behaviour and perceptions of political matters .....................................................................222

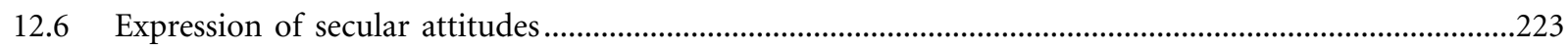

12.7 Physical fights in the village or urban neighbourhood ....................................................................226

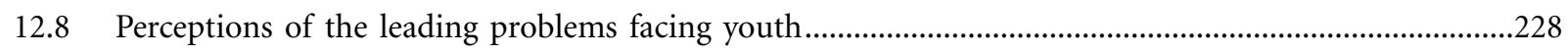

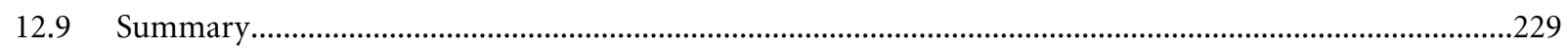

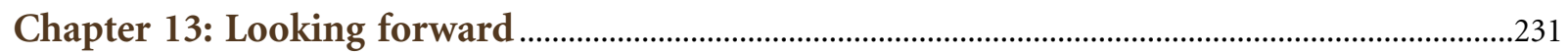

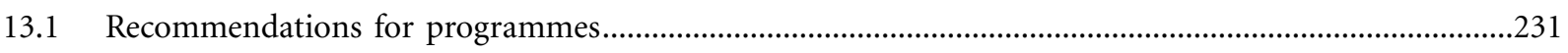

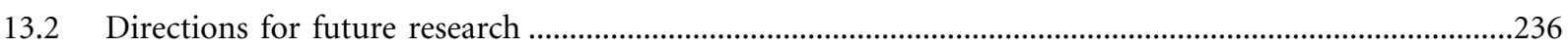

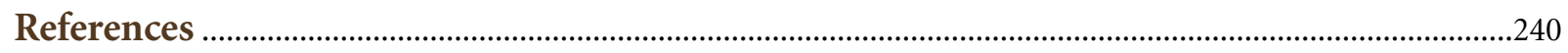

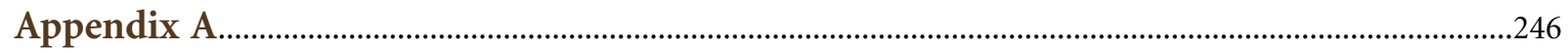

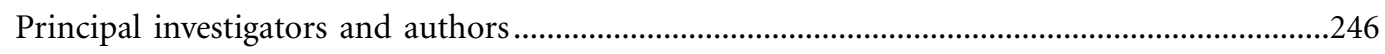

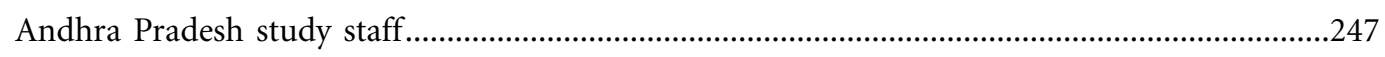

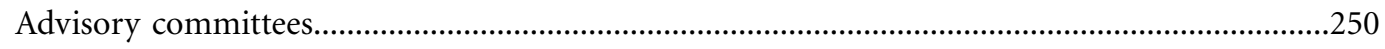

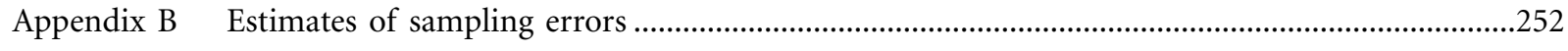

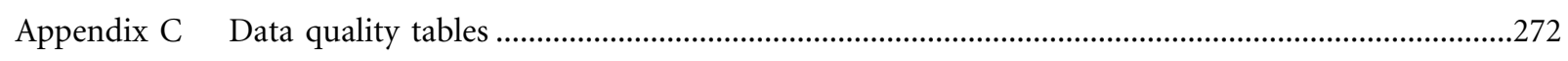




\section{Tables}

Page No.

\section{Chapter 1: Introduction}

Table $1.1 \quad$ Sampling stratification scheme ....................................................................................................

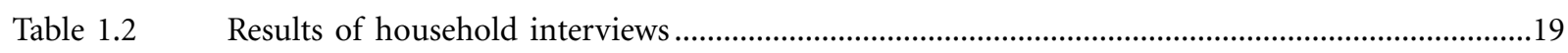

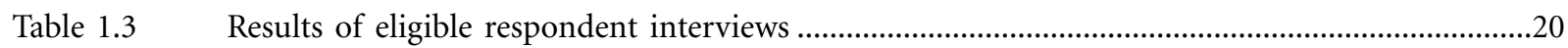

\section{Chapter 2: Profile of surveyed communities, households and youth}

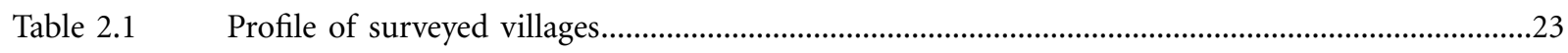

Table 2.2 Proximity of study residents to selected facilities......................................................................24

Table 2.3 Distribution of the surveyed population by age and sex .............................................................25

Table 2.4 Marital status of the surveyed population .....................................................................................28

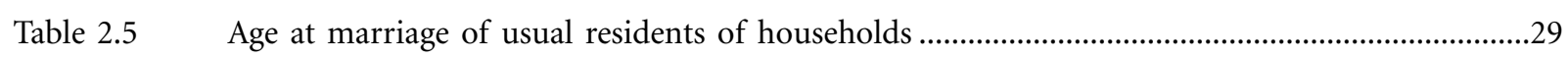

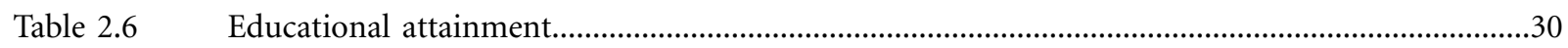

Table $2.7 \quad$ Work participation .......................................................................................................................32

Table 2.8 Socio-demographic characteristics of households and heads of households .................................32

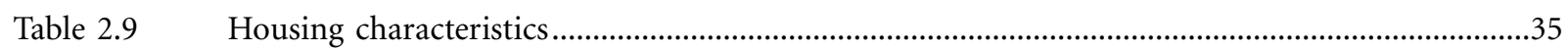

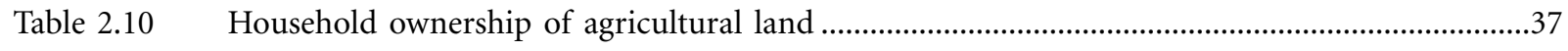

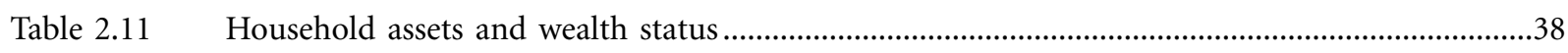

Table $2.12 \quad$ Background characteristics of surveyed youth...................................................................................

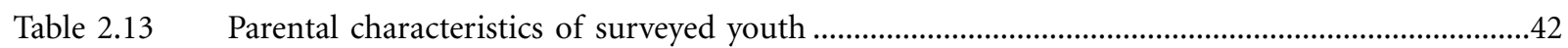

\section{Chapter 3: Education}

Table 3.1 Educational attainment and current educational status ................................................................46

Table 3.2 Educational attainment of young men by selected background characteristics ............................47

Table 3.3 Educational attainment of young women by selected background characteristics.......................49

Table 3.4a Reasons for never attending school ................................................................................................54

Table 3.4b Reasons for school discontinuation by level of education ..........................................................56

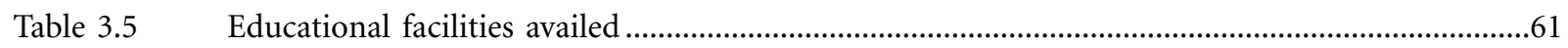

Table $3.6 \quad$ Schooling experiences ..............................................................................................................63 


\section{Chapter 4: Economic and non-economic activity}

Table $4.1 \quad$ Economic activity

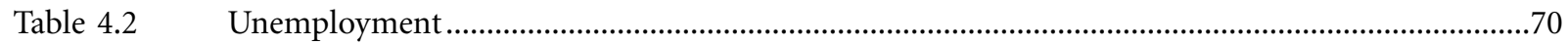

Table 4.3 Unemployment by selected background characteristics.........................................................71

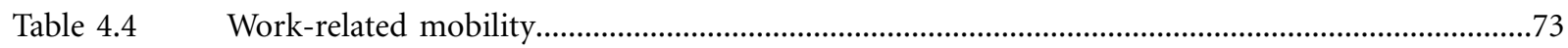

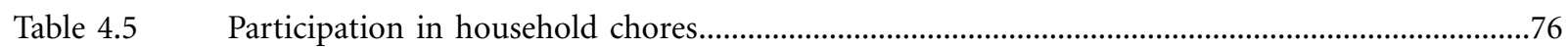

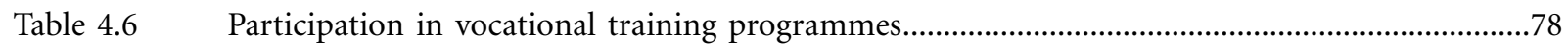

Table $4.7 \quad$ Willingness of youth to participate in vocational training programmes.....................................79

\section{Chapter 5: Media exposure and access to pornographic materials}

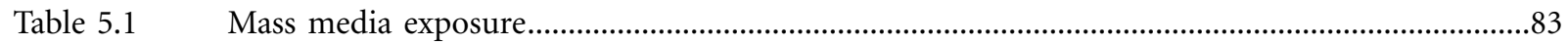

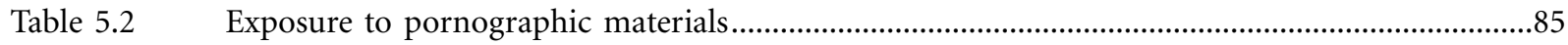

Table 5.3 Perceptions about the influence of television and films on youth behaviours............................87

\section{Chapter 6: Growing up}

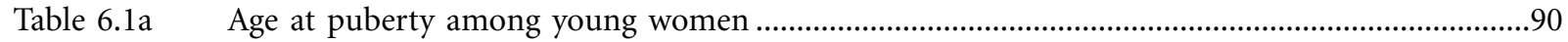

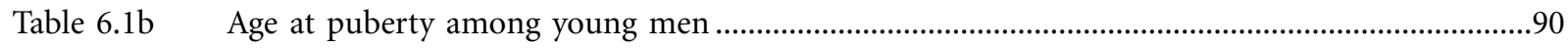

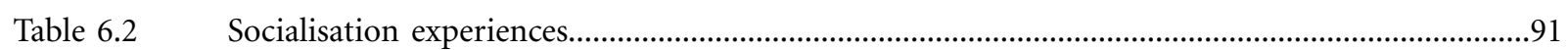

Table 6.3 Perceptions of parental reactions to selected activities ............................................................93

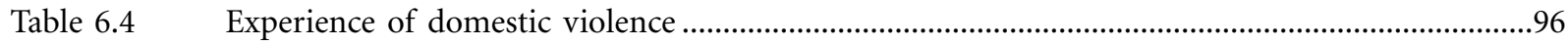

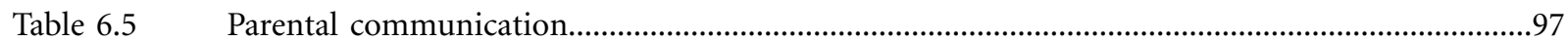

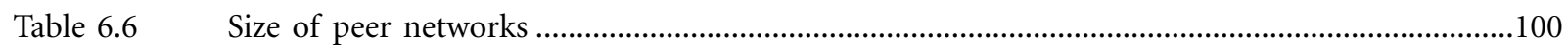

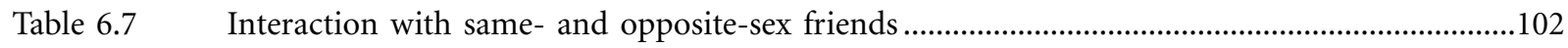

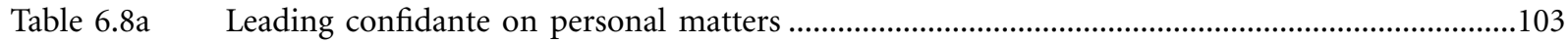

Table $6.8 \mathrm{~b} \quad$ Leading confidante on matters relating to the experience of teasing

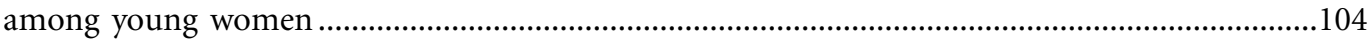

\section{Chapter 7: Agency and gender role attitudes}

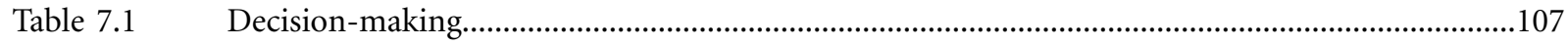

Table 7.2 Decision-making autonomy by selected background characteristics........................................109

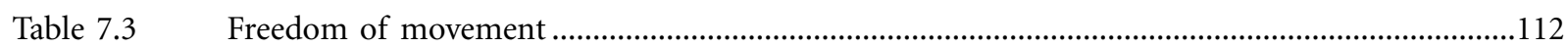

Table 7.4 Freedom of movement by selected background characteristics...............................................114

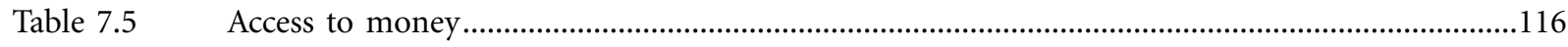

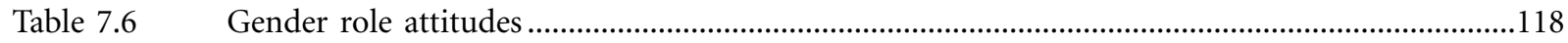

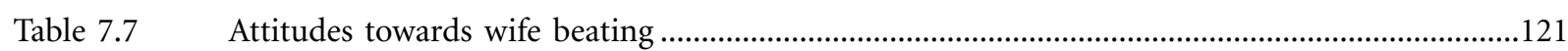




\section{Chapter 8: Awareness of sexual and reproductive health matters}

Table 8.1 Awareness of sex- and pregnancy-related matters.

Table 8.2 Awareness of sex- and pregnancy-related matters by selected

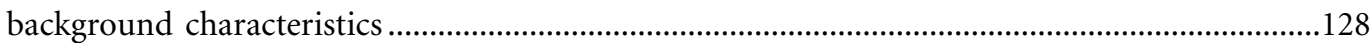

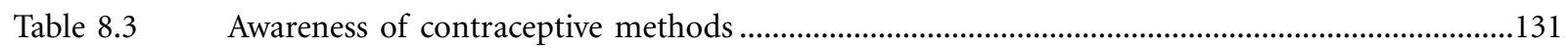

Table 8.4 Perceptions of selected issues related to condom use ...............................................................134

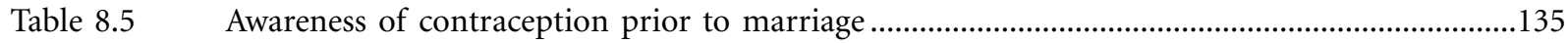

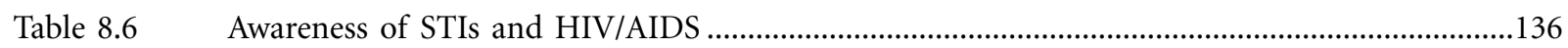

Table 8.7 Comprehensive knowledge of HIV/AIDS by selected background characteristics .....................138

Table $8.8 \quad$ Knowledge of the legal minimum age at marriage ..................................................................142

Table 8.9 Awareness of the conditions under which abortion is legal ...................................................143

Table $8.10 \quad$ Sources of information on sexual matters before marriage.......................................................145

Table $8.11 \quad$ Current sources of information on contraception ...............................................................147

Table $8.12 \quad$ Perceptions about family life or sex education....................................................................148

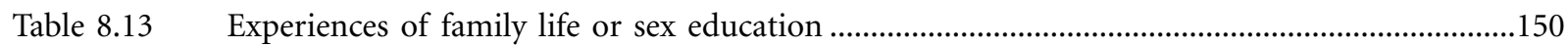

\section{Chapter 9: Pre-marital romantic and sexual relationships}

Table 9.1 Attitudes toward pre-marital physical intimacy and sexual relations ......................................155

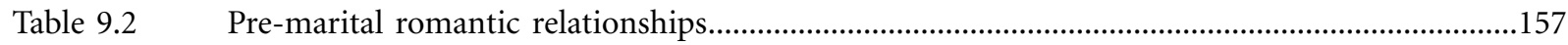

Table 9.3 Prevalence of pre-marital romantic relationships by selected

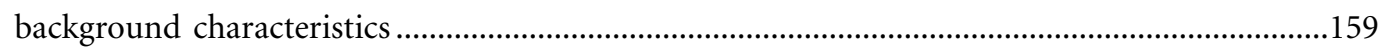

Table 9.4 Characteristics of pre-marital romantic relationships and partners..........................................162

Table 9.5 Meeting places with pre-marital romantic partners.................................................................166

Table 9.6 Peer and parental awareness of first pre-marital romantic relationship...................................167

Table 9.7 Marriage intentions and duration of pre-marital romantic relationships.................................169

Table 9.8 Physical intimacy and sexual experiences in pre-marital romantic relationships......................170

Table 9.9 Characteristics of sexual experiences within pre-marital romantic relationships ......................172

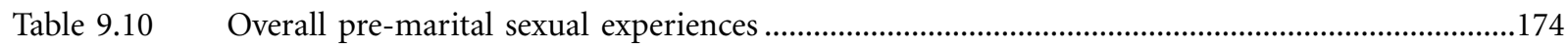

Table 9.11 Overall pre-marital sexual experiences by selected background characteristics.........................175

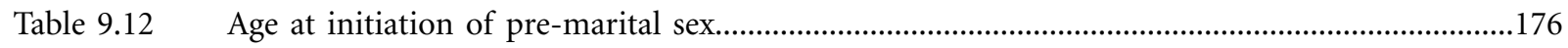

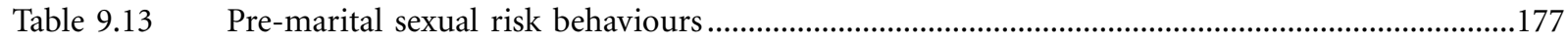

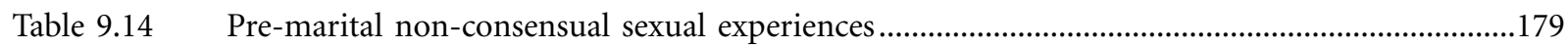

Table 9.15 Levels of pre-marital romantic and sexual experiences by different reporting methods 


\section{Chapter 10: Transition to marriage and early married life}

Table 10.1 Preferences regarding timing and type of marriage................................................................183

Table 10.2 Initiation of discussion on marriage and extent of youth involvement .....................................185

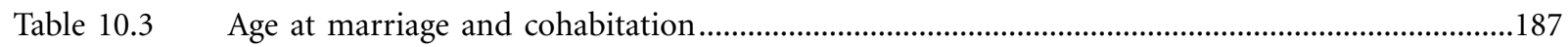

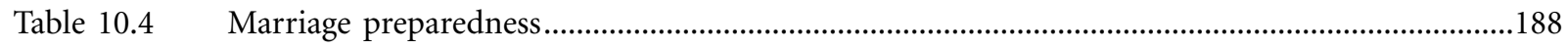

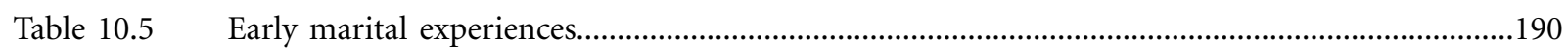

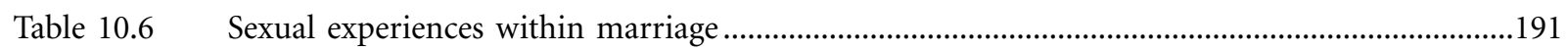

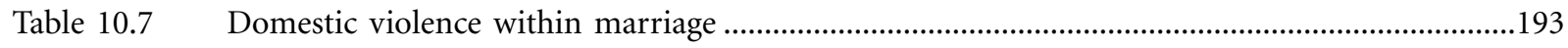

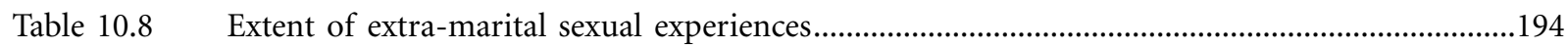

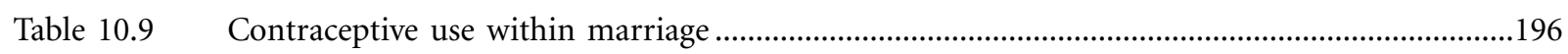

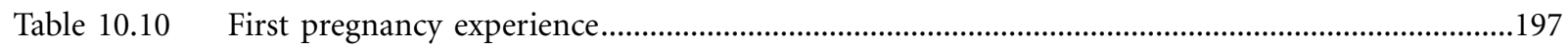

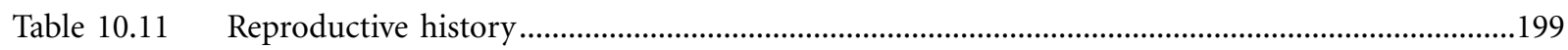

Table 10.12 Children ever born and surviving by selected background characteristics ...............................199

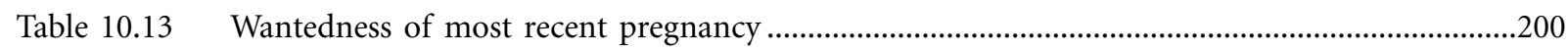

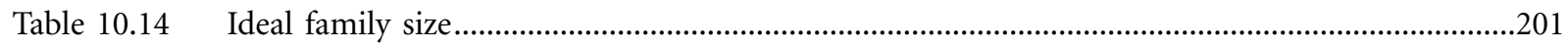

Table 10.15a Married young men's preferences for sons and daughters by selected

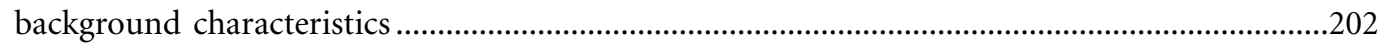

Table 10.15b Married young women's preferences for sons and daughters by selected

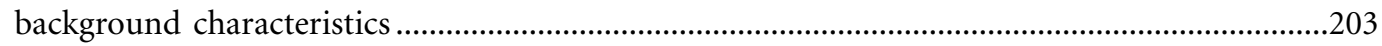

\section{Chapter 11: Health and health seeking behaviour}

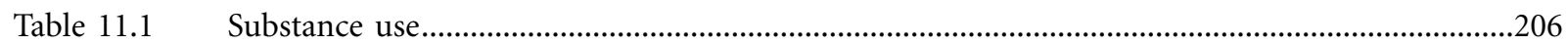

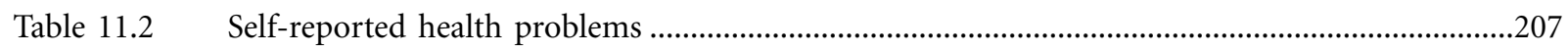

Table 11.3 Reported symptoms or behaviours suggestive of mental health disorders................................209

Table 11.4 Care and advice seeking for reported health problems ...........................................................211

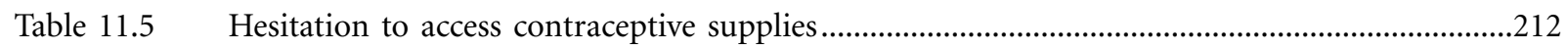

Table 11.6 Attitudes towards pre-marital HIV testing and extent of HIV testing ....................................213

\section{Chapter 12: Participation in civil society and political life}

Table 12.1 Awareness of and participation in government-and NGO-sponsored programmes .................216

Table 12.2 Participation in community-led programmes........................................................................219

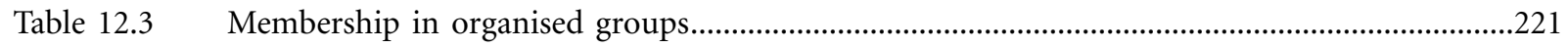

Table 12.4 Perceptions about actions taken by the panchayat in case of defiance of social norms............222

Table 12.5 Voting behaviour of eligible youth and perceptions about political matters............................224

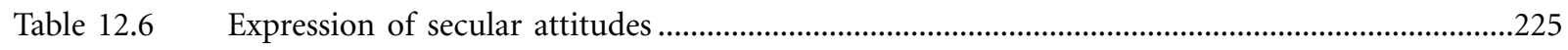

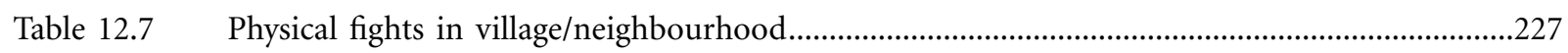

Table $12.8 \quad$ Perceptions about the leading problem facing youth ............................................................228 


\section{Appendix B: Estimates of sampling errors}

Table B.1 List of selected variables for sampling errors, Andhra Pradesh, 2007-08 ................................254

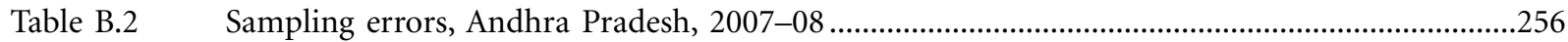

\section{Appendix C: Data quality tables}

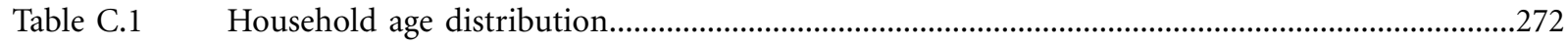

Table C.2 Single-year age distribution of eligible, selected and interviewed young men .........................273

Table C.3 Single-year age distribution of eligible, selected and interviewed young women ......................274

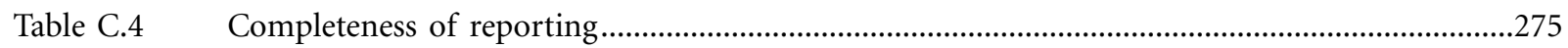




\section{Figures}

Page No.

Figure 3.1 Percentage of youth who were in school at ages 12 and 15, according to residence, Andhra Pradesh, 2007-08.

Figure 3.2a Cumulative percentage of youth who had completed each year of education (Classes1 to 17), Andhra Pradesh (combined), 2007-08

Figure 3.2b Cumulative percentage of youth who had completed each year of education (Classes1 to 17), Andhra Pradesh (urban), 2007-08

Figure 3.2c Cumulative percentage of youth who had completed each year of education (Classes1 to 17), Andhra Pradesh (rural), 2007-08

Figure 3.3a Percentage of married youth who had discontinued schooling by class when discontinued and reasons for discontinuation, Andhra Pradesh 2007-08.

Figure 3.3b Percentage of unmarried youth who had discontinued schooling by class when discontinued and reasons for discontinuation, Andhra Pradesh, 2007-08.

Figure 4.1a Economic activity and schooling status among youth aged 15-24, by age, Andhra Pradesh, 2007-08

Figure 4.1b Economic activity and schooling status among married men aged 15-29 and married women aged 15-24, by age, Andhra Pradesh, 2007-08

Figure 4.1c Economic activity and schooling status among unmarried men and women aged 15-24, by age, Andhra Pradesh, 2007-08.

Figure 4.2 Percentage of youth who participated in domestic chores, according to residence, Andhra Pradesh, 2007-08

Figure 4.3 Percentage of youth who ever attended a vocational training programme and percentage who were interested in participating in such programmes, according to residence, Andhra Pradesh, 2007-08

Figure 5.1 Percentage of youth exposed to television, films, print media and the internet, Andhra Pradesh, 2007-08

Figure 6.1 Percentage of youth reporting gendered socialisation experiences relative to an opposite-sex sibling/cousin, according to residence, Andhra Pradesh, 2007-08

Figure 6.2 Percentage of youth reporting that their father or mother, respectively, would disapprove if they brought same- and opposite-sex friends home, Andhra Pradesh, 2007-08. 
Page No.

Figure 6.3a Percentage of youth who discussed various matters with their father, according to residence, Andhra Pradesh, 2007-08.

Figure $6.3 \mathrm{~b}$ Percentage of youth who discussed various matters with their mother, according to residence, Andhra Pradesh, 2007-08.

Figure 6.4 Percentage of youth reporting at least one opposite-sex friend, according to residence, Andhra Pradesh, 2007-08.

Figure 7.1 Percent distribution of youth by participation in decision-making on selected matters, Andhra Pradesh, 2007-08

Figure 7.2 Percentage of youth allowed to visit selected places within and outside the village/neighbourhood unescorted, Andhra Pradesh, 2007-08.

Figure 7.3 Percentage of youth who expressed egalitarian gender role attitudes on selected issues, Andhra Pradesh, 2007-08

Figure 7.4 Percentage of youth who believed wife beating is justified in selected situations, Andhra Pradesh, 2007-08.

Figure 8.1 Percentage of youth reporting awareness of selected sex- and pregnancy-related matters, according to residence, Andhra Pradesh, 2007-08.

Figure 8.2 Percentage of youth who reported correct specific knowledge of oral pills and condoms, according to residence, Andhra Pradesh, 2007-08.

Figure 8.3 Percent distribution of youth by awareness of medical abortion, according to residence, Andhra Pradesh, 2007-08.

Figure 8.4a Comprehensive knowledge of HIV/AIDS by educational level, Andhra Pradesh, 2007-08 .

Figure $8.4 \mathrm{~b}$ Comprehensive knowledge of HIV/AIDS by wealth quintile, Andhra Pradesh, 2007-08

Figure 8.5 Percentage of youth by awareness of HIV/AIDS, comprehensive knowledge about HIV/AIDS and awareness of STIs, Andhra Pradesh, 2007-08.

Figure 8.6 Percentage of youth who were aware of selected conditions under which abortion is legal, Andhra Pradesh, 2007-08.

Figure 8.7 Percentage of youth who received family life or sex education, according to residence, Andhra Pradesh, 2007-08.

Figure 8.8 Percentage of youth reporting knowledge of selected sexual and reproductive health matters according to whether they had or had not received family life or sex education, Andhra Pradesh, 2007-08.

Figure 9.1 Percentage of youth who had made or received a "proposal" for romantic partnership formation and percentage who had an opposite-sex romantic partner, according to residence, Andhra Pradesh, 2007-08.

Figure 9.2 Percentage of youth reporting experiences of physical intimacy and sex with a pre-marital romantic partner, Andhra Pradesh, 2007-08.....

Figure 9.3 Percentage of youth reporting any pre-marital sexual experiences (in face-to-face interview or sealed envelope), according to residence, Andhra Pradesh, 2007-08 
Figure 10.1 Percentage of youth reporting that their parents had ever sought their opinion on timing of marriage, according to residence, Andhra Pradesh, 2007-08.

Figure 10.2 Percent distribution of married youth by degree of acquaintance with future spouse before marriage, according to residence, Andhra Pradesh, 2007-08.

Figure 10.3 Percentage of married youth who reported receiving or giving dowry, according to residence, Andhra Pradesh, 2007-08.

Figure 10.4 Percentage of married youth who reported spousal communication on selected topics, according to residence, Andhra Pradesh, 2007-08.

Figure 10.5 Percentage of married young women reporting experience of physical violence perpetrated by their husband and percentage of married young men reporting perpetration of physical violence against their wife, according to residence, Andhra Pradesh, 2007-08

Figure 10.6 Percentage of married youth reporting lifetime and current use of contraceptive methods within marriage, Andhra Pradesh, 2007-08.

Figure $11.1 \quad$ Percentage of youth reporting symptoms/behaviours suggestive of mental health disorders in the month preceding the interview, according to residence, Andhra Pradesh, 2007-08.

Figure 12.1 Percentage of youth reporting awareness of and participation in government- and NGO-sponsored programmes in the three years preceding the interview, according to residence, Andhra Pradesh, 2007-08

Figure 12.2 Percentage of youth aged 20 or above who had voted in the last election, according to residence, Andhra Pradesh, 2007-08 


\section{Foreword}

The Government of India is committed to addressing the multiple needs of young people. The Eleventh Five Year Plan, the National Youth Policy, the National Population Policy 2000 and the National Rural Health Mission have all advocated special programmatic attention to addressing this population. National AIDS Control Programme, Reproductive and Child Health Programme and notably the National Adolescent Reproductive and Sexual Health Strategy provide the framework for a range of sexual and reproductive health services to be provided to youth.

Effective implementation of policies and programmes, however, has been difficult because of the lack of evidence on young people's situation and needs. The project Youth in India: Situation and Needs is intended to provide this evidence. Research has been conducted in a total of six states of India-Andhra Pradesh, Bihar, Jharkhand, Maharashtra, Rajasthan and Tamil Nadu. It provides a wealth of evidence on married and unmarried young women and young men from both rural and urban settings of each state. It covers almost every major dimension of youth life: education, work force participation, family life, sexual activity, marriage, health and civic participation. It provides state-level evidence on the magnitude and patterns of sexual and reproductive practices in and outside of marriage as well as related knowledge, decision-making and attitudes. Findings from the study provide important base-line indicators against which the long-term impact of programmes may be measured and will certainly go a long way in guiding policy, programmes and advocacy on youth issues.

This report focuses on findings from Andhra Pradesh and is based on interviews with 8330 youth from all over the state. The report provides an enormous amount of information for the first time at the state level. The information will be useful to policy makers, programme implementers in government and non government sectors, rights activists and researchers alike who are committed to addressing the needs of Andhra Pradesh's young generation. I appreciate the efforts put in by the International Institute for Population Sciences, Population Council and the technical advisory committee who guided the study.

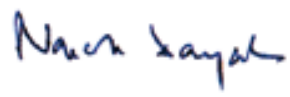

Naresh Dayal

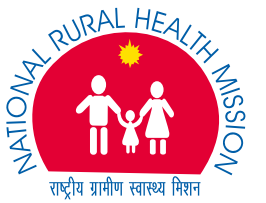




\section{Acknowledgements}

This report from the Youth in India: Situation and Needs study describes the transition to adulthood experienced by young men and women in Andhra Pradesh. It covers multiple dimensions of their situation, ranging from education, work and marriage to sexual and reproductive health and behaviours. Evidence and recommendations contained in this report highlight, moreover, directions for programming and research that will enable youth in Andhra Pradesh to make a successful transition to adulthood.

The Youth in India: Situation and Needs study has benefited immeasurably from the input of many. We are grateful to the Ministry of Health and Family Welfare, Government of India, for granting permission to conduct this study and to the former Secretary, Shri Naresh Dayal, for his support throughout the project, and to the Secretary, Smt. K. Sujatha Rao, for her continuing support. We are also grateful to Shri G.C. Chaturvedi, former Mission Director, National Rural Health Mission, Shri P. K. Pradhan, Additional Secretary and Mission Director, National Rural Health Mission, Smt. S. Jalaja, former Additional Secretary, Shri V. K. Malhotra, Additional Director General, Dr. Rattan Chand, Chief Director, and Shri Rajesh Bhatia, Joint Director, Statistics Division for their support and guidance. We would also like to acknowledge the significant contribution of Smt. S. Jalaja as chair of our Project Advisory Committee; and Shri S.K. Das, Director General, Central Statistical Organisation and former Additional Director General, Ministry of Health and Family Welfare, Government of India for support provided throughout the project.

We would also like to express our thanks to Shri J Satyanarayana, Principal Secretary, Department of Health, Medical and Family Welfare, Government of Andhra Pradesh and Shri A. K. Parida, Principal Secretary, Youth Services and Sports, for facilitating the study in Andhra Pradesh. The smooth functioning of fieldwork was due, in large part, to the support of the state health department, and we would like to acknowledge the contribution of all, including staff at the district headquarters level and primary health centre level. The Office of the Registrar General, New Delhi and the State Census Office, Hyderabad, generously provided the project with information and maps pertaining to the 2001 Census. Their role in enabling the study to implement its sampling design is gratefully acknowledged. We would also like to express our appreciation to the Director, Indian Institute of Health and Family Welfare, Hyderabad and the Director, Osmania University Centre for International Programme, Osmania University, Hyderabad who provided us logistic support in organizing the training programme for the project.

The study received generous financial support from the John D. and Catherine T. MacArthur Foundation and the David and Lucile Packard Foundation; we are grateful for their financial support as well as for useful comments and suggestions provided by Lester Coutinho, Don Lauro and Lana Dakan of the Packard Foundation, and Poonam Muttreja and Dipa Nag Chowdhury of the MacArthur Foundation over the course of the project.

We would like to acknowledge the contribution of the late P. N. Mari Bhat, Director, International Institute for Population Sciences, during 2005-07. His input in the design and implementation of the study and in ensuring the progress of this challenging project contributed immensely to the quality of the study. 
We are grateful to our Project Advisory Committee members for their input, which ensured that our study did indeed address all the key issues that require policy and programme attention. We appreciate their contribution both during meetings of the committee and in various one-on-one discussions during the course of the project.

We acknowledge with gratitude the contribution of members of our Technical Advisory Committee. We were privileged that individuals with a wide range of expertise, from youth health and development to survey and qualitative approaches and ethics in research, agreed to serve as technical committee members. Our technical advisory group—Shalini Bharat, P.M. Kulkarni, Arvind Pandey, Pertti Pelto, T.K. Roy and Leela Visaria-supported the project from conceptualisation to completion. Their guidance at all stages of the study was central in enabling us to confront methodological, ethical and analytical challenges that arose over the course of the study and is gratefully acknowledged. We would like to record our deep appreciation, moreover, to P.M. Kulkarni for giving us so generously of his time in working through problems encountered during the design and implementation phases.

Several specialist group meetings were held over the course of the project that focused on study design, instrument development, qualitative component development and analysis, tabulation planning and report review;; several others who did not attend these meetings provided extensive comments on one or more of these issues. We are grateful to all these specialists_-Dinesh Agarwal, Mallika Alexander, John Cleland, Nimesh Desai, Lalit Deshpande, Sudha Deshpande, Kamla Gupta, M.E. Khan, Sumati Kulkarni, Shiva Kumar, Cynthia Lloyd, S. Niranjan, Sulabha Parasuraman, Vikram Patel and Sunayana Walia-for their valuable contribution. John Cleland provided inputs at almost every phase of this study_design, instrument development and analysis_-and his thought-provoking suggestions are gratefully acknowledged. In particular, we would like to acknowledge our external peer reviewers, P.M. Kulkarni and Sumati Kukarni, for their thoughtful and careful assessments of an earlier draft of this report.

Given that our study probed a number of highly sensitive matters, including young people's sexual and reproductive behaviours, it was an ethical imperative that those in need of information or services would be provided appropriate materials and referrals, respectively. We are grateful to those who provided services to our respondents in need and would like to acknowledge the TARSHI (New Delhi) whose materials (Neeli Rangu Pustakam) were distributed to the young men and women during the survey.

We would like to express our appreciation and gratitude to the International Institute for Population Sciences and the Population Council and all their staff members who helped in small and big ways in making this report possible. T.K. Roy and the late P.N. Mari Bhat as Directors of the International Institute for Population Sciences, and G. Rama Rao and S. Lahiri as officiating Directors, oversaw the activities of the project from the Institute's perspective. Saroj Pachauri, Regional Director, South and East Asia Office, Population Council oversaw the project on behalf of the Population Council. Their insight and supervision are gratefully acknowledged.

A number of research officers, consultants and administrative staff members, both at the International Institute for Population Sciences, Mumbai, and at the Population Council, New Delhi, contributed to the smooth implementation of the study as well as the data management, analysis and report writing phases. We would like to thank those responsible for the administrative aspects of this project. At the International Institute for Population Sciences, we would like to acknowledge M. K. Kulkarni, Registrar and V. Ramakrishnan, Accounts Officer for their support at all stages of the project. We would also like to express our appreciation to the Computer Centre In-Charge, the Library In-Charge, Jeba Kumar, Pranita H. Dalvi, Usha D. Sonawane, Seema Jadhav and Avadesh Kumar who were responsible for the smooth functioning of the project. From the Population Council, we are grateful to Komal Saxena, M.A. Jose, Anil Paul and Ashutosh Mishra who ably managed the administrative, financial and IT aspects of the project. We are grateful to our Senior Research Officers, Research Officers and Assistant Research Officers for their excellent supervision of fieldwork and data management as well as for their support in analysing the data and preparing the tables for this report. Abhishekh Kumar, Lucky Singh, and Prashant Kumar Singh from the International Institute for Population Sciences, and Francis Zavier and Shilpi Rampal from the Population Council, did a painstaking job in ensuring the accuracy of data presented in the report and their contribution is gratefully acknowledged. 
Shanti Menon and Komal Saxena edited and ably managed the preparation of this report; we are grateful to them for their editorial contribution and meticulous attention to detail, which have made the report more readable and precise.

We would also like to acknowledge with thanks the contribution of our team of young and enthusiastic interviewers in eliciting information on difficult topics with sensitivity and skill. They were required to overcome discomfort when asking about intimate experiences and were required to record many disturbing experiences narrated by youth, which they did professionally and empathetically. It was due to their skill and ability to engage with youth in non-judgmental ways that this study was so well received by youth in Andhra Pradesh.

We would also like to record our appreciation of the support and kindness of the people in the villages and urban neighbourhoods in which we conducted our study, and specifically panchayat members and community leaders. While initially sceptical about the study, community members opened their homes to us and acknowledged the importance of this study for the health and development of future generations. Indeed, despite the sensitive issues covered, not a single study community refused our field teams entry. The trust and support of the people are gratefully acknowledged.

Finally, and most importantly, we would like to thank the young women and men from Andhra Pradesh who welcomed us, generously gave their time and shared so many intimate details of their lives with us. We hope that the evidence generated in this report will be useful in influencing the design and content of programmes intended to meet their multiple needs and enable them to make a safe transition to adulthood.

K. G. Santhya

Shireen Jejeebhoy

Rajib Acharya

Population Council, New Delhi
Usha Ram

S. K. Mohanty

Abhishek Singh

F. Ram

International Institute for

Population Sciences, Mumbai 


\section{Executive summary}

The Youth in India: Situation and Needs study (referred to as the Youth Study), implemented by the International Institute for Population Sciences, Mumbai and the Population Council, New Delhi is the first-ever sub-nationally representative study conducted to identify key transitions experienced by married and unmarried youth in India. Young people (aged 10-24) constituted almost 315 million and represented 31\% of the Indian population in 2001. Not only does this cohort represent India's future in the socio-economic and political realms, but its experiences will largely determine India's achievement of its goal of population stabilisation and the extent to which the nation will be able to harness its demographic dividend. While today's youth are healthier, more urbanised and better educated than earlier generations, social and economic vulnerabilities persist. In the course of the transition to adulthood, moreover, young people face significant risks related to sexual and reproductive health, and many lack the knowledge and power to make informed sexual and reproductive choices.

In recognition of the importance of investing in young people, several national policies and programmes formulated since 2000, including the National Population Policy 2000, the National Youth Policy 2003, the Tenth and Eleventh Five-Year Plans, the National Adolescent Reproductive and Sexual Health Strategy and the National Rural Health Mission, have underscored a commitment to addressing the multiple needs of this group in India. Effective implementation of both policies and programmes, however, has been handicapped by the lack of evidence on young people's situation and needs. Currently available evidence is limited, at best, and comes largely from small-scale and unrepresentative studies.

The Youth Study focused on married and unmarried young women and unmarried young men aged 15-24 and, because of the paucity of married young men in the younger ages, married men aged 15-29 in both rural and urban settings. The study collected information pertaining to key transitions experienced by youth, including those related to education, work participation, sexual activity, marriage, health and civic participation; the magnitude and patterns of young people's sexual and reproductive practices within and outside of marriage as well as related knowledge, decision-making and attitudes.

The Youth Study comprised three phases, and included both a survey and qualitative data gathering exercises prior to and after the survey. The study was conducted in a phased manner in six states of India: Andhra Pradesh, Bihar, Jharkhand, Maharashtra, Rajasthan and Tamil Nadu.

This report focuses on findings from the survey conducted in Andhra Pradesh. The survey was undertaken between October 2007 and April 2008. During the survey, 9,121 young people were contacted, of which a total of 8,330 married and unmarried young women and men were successfully interviewed.

\section{Characteristics of the household population}

A total of 32,348 households were selected for the interview. Among these, interviews were successfully completed in 31,123 sample households, and 125,953 individuals, who were usual residents in these households, were enumerated. The age distribution was typical of a population in which fertility has been declining, with relatively smaller proportions in both younger ( $0-9$ years) and older (60+ years) age groups. With regard to the youth population, 
the distribution suggests that, at the time of the survey, $10 \%$ of the population was aged $10-14$ years, $9 \%$ each was aged 15-19 years and $20-24$ years. A total of $18 \%$ of the population was aged 15-24 years. Overall, the sex ratio of the de jure population of the state was 1,018 females per 1,000 males, considerably higher than that observed in the 2001 census (978).

The educational profile of the household population highlights low levels of educational attainment in the state: about two-fifths of the population aged 6 years and above had no formal education. Notably, as many as $47 \%$ of females compared to $29 \%$ of males, and as many as $44 \%$ of the rural population compared to $23 \%$ of the urban population had never been to school. Reaffirming the low levels of educational attainment in the state, findings also indicate that just $10 \%$ of the total population had received 12 or more years of education.

Housing characteristics of the surveyed population suggest that $17 \%$ of all households lived in kachcha houses (constructed from mud, thatch or other low-quality materials), 38\% lived in semi-pucca houses (constructed using a mix of low- and high-quality materials) and $45 \%$ lived in pucca houses (constructed entirely from cement, masonry or other high-quality materials). Some $92 \%$ of households had electricity, including almost all urban households (97\%) and $90 \%$ of rural households. The majority of households (95\%) reported that their main source of drinking water was either piped water, or water obtained from a hand-pump or a covered well. Access to a toilet facility of any kind was reported by about half of all households (47\%).

The distribution of households by wealth quintiles shows that more than two-fifths of urban households were in the wealthiest (fifth) quintile; in contrast, only $12 \%$ of rural households were in this quintile. Likewise, one-fourth of rural households were in the poorest (first) quintile compared to only $6 \%$ of urban households.

\section{Situation of youth}

As mentioned earlier, a total of 8,330 youth were interviewed. Age profiles suggest that young men and women were about equally distributed in the $15-19$ and $20-24$ age groups ( $47-51 \%$ and $49-53 \%$, respectively). The unmarried were, however, younger than the married, and rural youth were somewhat younger than their urban counterparts. Distributions by religion show that $84-85 \%$ of youth were Hindu, $7-10 \%$ were Muslim and $5-8 \%$ were Christian. Caste-wise distributions show that $24-25 \%$ of youth belonged to general castes, $22-23 \%$ to scheduled castes, $6 \%$ to scheduled tribes and $46-49 \%$ to other backward castes. Four in five youth reported that both parents were surviving. For those with just one parent surviving, this parent was more likely to be the mother (13-14\%) than the father $(2-4 \%)$. Finally, $1-2 \%$ reported that neither parent was alive.

\section{Education}

While youth in Andhra Pradesh were better educated than the general population in the state, schooling was far from universal among young people. As many as one in twelve young men and one in five young women had never attended school. Findings show, moreover, that young women in rural areas and married young women in general were particularly disadvantaged; about one-third of married young women and one quarter of women residing in rural areas had never been to school. At the time of the interview, about two-fifths of unmarried youth (and very few married) were still in school or college, and gender differences were negligible.

Not only was school enrolment limited, but school completion rates were also low among young people, particularly young women. For example, among young women, of those who had completed Class 1, only $95 \%$ had completed Class 4 , and completion rates fell below $90 \%$ in Class 5 . Among young men, completion rates fell below $90 \%$ a year later, that is, in Class 6. Declines in school completion were steep among both young men and women at around the time of high school completion, that is, between Classes 9 and 11, suggesting that many youth discontinued their education at high school level. Indeed, just $52 \%$ of young men and $36 \%$ of young women in the state had completed high school. 
Findings suggest moreover that youth were considerably better educated than their parents. More than half of the parents of both young men and women had never been to school.

Leading reasons for never attending school among young men and women were economic (for example, the respondent was required for work on the family farm/business or for outside wage earning work, or the family could not afford school-related expenses), attitude and perception-related (for example, education was unnecessary or the respondent was not interested in schooling) and housework-related reasons (the respondent was required for care of siblings or housework).

Among those who had ever been to school, gender differences in reasons for school discontinuation became more apparent. Leading reasons for school discontinuation among young men were economic and attitude and perception-related, irrespective of the level at which education was discontinued. Far fewer young men cited school-related reasons. Among young women, in contrast, while economic and attitude and perception-related reasons were important, school-related reasons (academic failure, distance to school, poor school quality and infrastructure) were prominent factors underlying discontinuation at all levels; housework responsibilities were also significant, particularly among those who discontinued at primary or middle school levels. Of note are findings that more than one quarter of youth who discontinued their education before completing high school cited academic failure as a reason for discontinuing their education; and that one in five married young women who discontinued their education in Classes 7-9 and one-third of those who discontinued their education in Classes 10-11 reported doing so in order to marry.

As far as type of educational facility youth attended is concerned, while the majority of young men and women attended co-educational facilities at all levels of education, young women were less likely to attend a co-educational facility at higher secondary school and college levels. Typically, more youth who had discontinued their education reported government school attendance than did those who were pursuing their education; private school attendance was reported, moreover, among large proportions who were pursuing a higher secondary or college education or had discontinued their education at this level; while gender differences were relatively mild, youth from urban areas were considerably more likely than their rural counterparts to have attended a private school or college.

Most youth, irrespective of whether they were pursuing their education or not, had access to drinking water and playgrounds. However, for the most part, youth still studying were somewhat more likely, to report the availability of toilets as well as libraries than those who had discontinued their education. The availability of all four amenities was, for the most part, somewhat more likely to be reported by those who were studying at the time of the interview than those who had discontinued their education.

Schooling experiences also differed in some instances between those who had discontinued schooling and those who were studying at the time of the interview. While differences in experience of private tuition were typically narrow, those who had discontinued their education were more likely than those who were continuing their education to perceive the academic workload to be heavy and somewhat less likely to have attended classes regularly. They were considerably less likely, moreover, to have passed the last examination for which they had appeared, suggesting that poor school performance was a significant factor leading to school discontinuation among both young men and women.

\section{Work}

Work profiles suggest that three-quarters of young men and three-fifths of young women had ever engaged in paid or unpaid work. Indeed, almost all married young men and over two-thirds of unmarried young men had done so, compared with over two-thirds and half of married and unmarried young women, respectively. Likewise, more youth in rural than urban areas had ever worked. While the majority of youth were engaged in paid work, considerable proportions of young men and women (26\% each) reported unpaid work on the family farm or business. Economic activity was often initiated at an early age: $37 \%$ and $39 \%$ of young men and women, respectively, reported initiating 
work in childhood or early adolescence (before age 15). The majority of young men (98\% of the married and $67 \%$ of the unmarried) and a substantial proportion of young women ( $51 \%$ and $44 \%$, respectively) had engaged in paid or unpaid work at some point in the 12 months preceding the survey. The majority of young men (85\%) who had worked in the year prior to the interview had done so for the major part (at least six months) of the year. In contrast among young women, $71 \%$ had done so.

Unemployment rates ranged from $7 \%$ among young men to $10 \%$ among young women. Unemployment rates tended to be higher among the unmarried than the married. While rural-urban differences were negligible among young men, young women in urban areas reported considerably higher rates of unemployment than did their rural counterparts. Unemployment was particularly high among the educated and the economically better off than any other group. Youth were clearly interested in acquiring skills that would enable employment generation; $48 \%$ of young men and $63 \%$ of young women reported interest in vocational skills training. However, far fewer-one-third of young men and young women—had attended even one vocational training programme.

\section{Media exposure}

Findings suggest that almost all youth in Andhra Pradesh were exposed to the media, typically television (90\% of all young men and $94 \%$ of all young women), newspapers, magazines or books (94\% of young men and $78 \%$ of young women with five or more years of education) and films either on CD/DVD or at a theatre or video parlour ( $94 \%$ of young men and $77 \%$ of young women). Exposure to the internet was reported by many fewer (22\% of young men and $9 \%$ of young women with five or more years of education). Gender differences were apparent with regard to exposure to the print media, to films and to the internet, with young men considerably more likely than young women to report exposure.

Findings also suggest that almost half of young men and hardly any young women had watched pornographic or "blue" films, and one-third of young men and $4 \%$ of young women had read or looked at pornographic books/magazines. Finally, half of young men (and 7\% of young women) who had accessed the internet reported that they had accessed pornographic materials on the internet. At least half of young men who reported watching pornographic films or reading pornographic materials reported that they accessed these materials sometimes or frequently. Finally, between three-fifths and three-quarters of young men and women acknowledged the influence that media had on youth behaviours.

\section{Socialisation experiences and communication with parents}

Findings reveal a mixed scenario regarding the nature of socialisation experienced by youth. While responses from young men indicate that large proportions of households did discriminate between their sons and daughters in terms of freedom of movement and expectations regarding housework, responses from young women suggest the reverse. While three-quarters of young men reported that they had more freedom to go out than their sisters or female cousins did, many fewer young women, just two-fifths, agreed that they had less freedom to go out than their brothers or male cousins did. Likewise, two-thirds of young men reported that they were expected to do less housework than their sisters or female cousins, and only two-fifths of young women agreed that they were expected to do more housework than their brothers or male cousins.At the same time, far more consistently observed were gender differences in perceptions of parental control: young women were more likely than young men to perceive that their parents would disapprove of social activities in which youth tend to participate that involved members of the opposite sex.

Findings regarding communication with parents on issues relevant to youth—such as school performance, friendships, being teased or bullied, physical maturation, romantic relationships and reproductive processes—reiterate those from other studies, showing that such communication is far from universal. Indeed, sensitive topics such as romantic relationships, reproductive processes and contraception were rarely discussed with either parent. 
That parent-child communication was restricted was also evident from responses to questions probing the most likely confidante on a range of topics from taking a job to boy-girl relationships. While parents were mentioned as leading confidantes on topics such as taking a job, they were rarely cited as leading confidantes on more sensitive matters. Moreover, while young women identified their mother as the most likely confidante on such matters as menstrual problems and the experience of being teased by a boy, young men rarely identified a parent as a leading confidante on matters relating to nocturnal emission or swapnadosh. Indeed, neither young men nor women identified a parent as a leading confidante on boy-girl matters.

Young people's family lives were marked by violence, both experienced and witnessed. Over one in three youth had observed their father beating their mother. Many youth reported being beaten by a parent during adolescence; almost half of young men and almost one-quarter of young women reported such experiences.

\section{Peer networks and interaction}

Growing up was associated with close peer networks. Almost all youth reported having same-sex friends. Young men, however, reported larger networks of friends than did young women. Opposite-sex peer networks were less common but nonetheless reported by one-third of young men and one-seventh of young women. Interaction with friends tended to be restricted to activities such as chatting and studying, although large proportions of young men did report engaging in outside activities such as going out on picnics or to see films and outdoor sports. An important measure of support was derived from these networks, however, with peers reported as the most likely confidante on issues related to boy-girl relationships for both young men and women, and on nocturnal emission for young men.

\section{Agency and gender role attitudes}

Findings highlight young women's limited agency. For example, just one in four young women reported independent decision-making on all three issues explored in the survey, namely, choice of friends, spending one's own money and purchase of clothes for oneself. Likewise, freedom of movement even within the village or neighbourhood was not universal among young women; just four in five young women had the freedom to visit locations within their own village or neighbourhood unescorted. Many fewer, just one in four young women reported freedom to visit at least one place outside their village or neighbourhood unescorted, and one in 10 could visit a health facility unescorted. Access to and control over financial resources tended to be limited among young women; just one in four reported some savings and one in six owned a bank or post office savings account. Of those who owned an account, just three in five operated it themselves.

Within the sub-group of young women, findings indicate that the married were more disadvantaged, in some respects, than the unmarried. For example, compared to the unmarried, married young women were less likely to exercise control over financial resources, and at the same time, more likely to hold unequal gender role attitudes and to justify wife-beating. They were, however, about as likely as the unmarried to report independent decision-making and freedom of movement.

Also notable from the findings is the striking gender divide in most dimensions of young people's agency explored in the survey. Young women were far more disadvantaged than young men. For example, even the least educated young man was more likely than the most educated woman to report independent decision-making on all three issues explored in the survey. Although young women were as likely as young men to have money saved (25\% and $24 \%$, respectively) and own a bank or post office savings account (17\% and 19\%, respectively), they were less likely than young men to operate the account themselves (61\% and $93 \%$, respectively, of those who had an account).

While young men were not as disadvantaged as young women, findings indicate that many young men were also not able to exercise agency in their everyday lives. For example, only 55\% of young men reported independent 
decision-making on all three issues explored in the survey. Similarly, young men's freedom to visit selected localities was far from universal; for example, just over half of unmarried young men (55\%) were allowed to visit a place of entertainment or to attend a programme conducted outside their village or neighbourhood unescorted, and just two-fifths were allowed to visit a health facility unescorted.

Relatively large proportions of youth espoused egalitarian gender role attitudes on such issues as the relative importance attached to educating boys versus girls, the role of the husband as the main decision-maker with regard to spending money, girls' participation in decisions about their own marriage and so on. Even so, it is notable that young men were generally more likely than young women to report unequal gender role attitudes. A somewhat different picture emerged, however, with regard to attitudes to wife-beating. Findings highlight widespread acceptance of violence within marriage among youth, and in this case, young women were more likely than young men to so perceive; $88 \%$ of young women compared to $63 \%$ of young men justified wife beating in at least one situation explored in the survey.

\section{Awareness of sexual and reproductive health matters}

Findings underscore young people's limited awareness of most sexual and reproductive matters, ranging from how pregnancy occurs to contraception, HIV and safe sex practices. For example, just over two-fifths of young men and over three-fifths of young women were aware that a woman can get pregnant at first sex, and just $30 \%$ of young men and $16 \%$ of young women reported awareness of STIs other than HIV.

Even on topics about which young people were generally aware, findings show that in-depth understanding was limited. For example, while $97-98 \%$ of youth reported awareness of at least one modern contraceptive method, in-depth awareness of condoms and oral contraceptives, the non-terminal methods most familiar to youth, was reported by just $80 \%$ and $17 \%$ of young men and $21 \%$ and $15 \%$ of young women, respectively. Likewise, although $94-95 \%$ of youth had heard of HIV/AIDS, just $62 \%$ of young men and $33 \%$ of young women had comprehensive knowledge of HIV/AIDS.

Youth, particularly young women had few sources of information on sex and reproduction. Indeed, half of young women and one-tenth of young men reported that they had never received any information on sexual matters (prior to marriage among the married).

Leading sources of information on sex and reproduction varied considerably between young men and women. Among young men, the leading sources of information reported were friends and neighbours (68\%), followed by the media (47\%). Among young women, leading sources of information were the media (21\%), teachers and schools (18\%) and friends and neighbours (16\%). Notably, just 3-5\% cited a family member. Among the leading current sources of information on contraception among young people who were aware of at least one method were similarly, peers and the media. Teachers and health care providers were relatively infrequently reported as such. Indeed, health care providers were cited as an important source of information on contraception by only a quarter of married youth; they were far less likely to have provided information to the unmarried (9-16\%). Likewise, teachers were cited by just $17-18 \%$ of youth. However considerable proportions of young women (29\%) but hardly any young men cited family members as a leading source of information about contraception. In short, health care providers, teachers and family members - often assumed to be more reliable sources of information than peers and the media—were infrequently and inconsistently cited as sources of information on sensitive topics such as sexual matters and contraception by young people.

Few youth—just one-third of young men and almost one-quarter of young women—had attended family life or sex education programmes either in or outside the school setting. Despite this, youth were overwhelmingly in favour of the provision of family life or sex education to young people; while young men preferred to receive this education from a professional (health care provider or teacher) and peers, young women preferred parents and teachers. Findings suggest, moreover, that youth who had undergone family life or sex education were indeed more knowledgeable about sexual and reproductive matters than those not exposed to this education. 


\section{Pre-marital romantic relations}

Findings confirm that despite strict norms prohibiting pre-marital opposite-sex mixing, opportunities do exist for the formation of pre-marital romantic relationships. Indeed, significant minorities of young men and women have made or received a "proposal" for a romantic relationship (23\%), and noteworthy, if smaller, percentages reported that they had been involved in a romantic partnership (17\% and $11 \%$ of young men and women, respectively). Patterns of pre-marital romantic partnerships suggest that where partnerships occurred, they were initiated at about age 18 among young men and age 16 among young women, and were hidden from parents by many, but were rarely hidden from peers. Notable gender disparities in expectations of a longer-term commitment emerged: young women were more likely than young men to have expected a romantic relationship to lead to marriage. The experiences of the married suggest, moreover, a disconnect between intentions and reality: although almost all (95\%) young women and two-thirds $(68 \%)$ of young men reported the intention to marry their pre-marital partner, fewer- $70 \%$ of young women and $23 \%$ of young men-had done so.

While the majority-95\% of young men and $82 \%$ of young women-had held hands with their romantic partner, fewer-46\%-had engaged in sexual relations with their partner. It is notable that unlike the findings of many previous studies, no gender differences in reports of pre-marital sex with a romantic partner were apparent. Findings suggest, moreover, that the vast majority had engaged in unprotected sex. However, many young men and women who reported contraception at first sex reported that the female partner was involved in the decision. Very few young women who had engaged in sexual relations with a romantic partner reported that their opposite-sex romantic partner had forced them to engage in sex the first time.

\section{Pre-marital sexual experiences in romantic and other relationships}

In total, $16 \%$ of young men and $6 \%$ of young women reported the experience of pre-marital sex within romantic and/or other partnerships. In general, life table estimates reveal that roughly similar proportions of young men and women-3\% and 5\%, respectively-had initiated first sex before age 18; however, larger proportions of youth in rural than urban areas had initiated pre-marital sexual relations in adolescence. Moreover, initiation into pre-marital sexual activity increased as young people transitioned from early into late adolescence and further as they transitioned into young adulthood.

While sex with a romantic partner characterised pre-marital experiences for many of the sexually experienced, findings suggest that young men, but not young women, also engaged in sex in other contexts-mainly with sex workers, married women and casual partners. Many pre-marital sexual experiences reported by youth were risky, for example, one-fifth of young men and $7 \%$ of young women reporting pre-marital sex had engaged in sex with more than one partner. Moreover, consistent condom use was limited-only $17 \%$ and $2 \%$ of sexually experienced young men and women, respectively, reported condom use in all pre-marital encounters.

We acknowledge that youth, especially young women, may not report sexual experience in a survey situation. Hence, the Youth Study supplemented a series of direct questions with an opportunity to report sexual experience in an anonymous format. Surprisingly, findings suggest that a larger percentage of sexually active young men than women reported pre-marital sex only in the anonymous format. In total, direct reporting yielded somewhat higher estimates than anonymous third-party reporting, although the differences were narrow among young women. However, direct questioning supplemented by self-reporting in an anonymous format provided higher estimates of sexual experience than did face-to-face questioning alone or anonymous third-party reporting of peer behaviours.

\section{Transition to marriage and early married life}

Findings indicate that although most youth preferred to marry after age 18, early marriage continues to characterise the lives of many young women and to a certain extent the lives of young men as well. Among young women aged 20-24 years, almost one in five young women (18\%) was married before age 15, 54\% before age 18 and almost 
three in four before age 20. Even though early marriage was less prevalent among young men, one in ten young men aged 20-24 years was married before age 20 .

Almost all youth reported arranged marriages; it is notable, however, that $86-89 \%$ of youth were given an opportunity to participate in the decision on the choice of their spouse. At the same time, reported pre-marital acquaintance was limited. Just a little over one-quarter of young men and one-fifth of young women reported that they had ever had a chance to meet and interact alone with their spouse-to-be prior to marriage. Over two in five married youth reported that they had met their spouse for the first time on the wedding day. Compounding the lack of pre-marital acquaintance was the lack of awareness of what to expect of married life, reported by over two-thirds of young men and over four-fifths of young women.

Despite the existence of laws against the payment of dowry, this practice characterised the marriages of $81 \%$ of young men and $86 \%$ of women. Findings also show that families of urban youth appeared as likely as their rural counterparts to conform to traditional practices, such as payment of dowry.

Reports of marital life suggest that married life was characterised by considerable inter-spousal communication on most topics, yet, just one in eight young men and one in three young women had ever discussed matters relating to contraception with their spouse, clearly undermining married young people's ability to adopt protective actions.

Physical violence and forced sex within marriage were reported by sizeable proportions of youth. For example, a little less than one-quarter of young women reported that they had ever faced physical violence perpetrated by their husband and one-fifth of young men reported perpetrating violence on their wife. Recent physical violence was likewise reported by about one-sixth of young men and about one-fifth of young women. Sexual violence was also reported by significant minorities of youth. One in ten young women reported ever being forced to engage in sex by their husband; one in seven young men reported forcing their wife to engage in sex. Fewer youth (5\%) reported recent sexual violence.

While the Youth Study did not explore extra-marital sexual experiences in detail, the available data indicate that $6 \%$ of young men reported an extra-marital sexual encounter. In contrast, less than $1 \%$ of young women reported an extra-marital sexual encounter.

\section{Contraceptive practice and pregnancy experience}

Contraceptive use at any time within marriage was relatively limited, reported by $10 \%$ of young men and $23 \%$ of young women. Moreover, just $8 \%$ of young men and $21 \%$ of young women reported current use of contraception. Among contraceptive methods typically used, female sterilisation was mostly likely to be reported; indeed, over three-quarters of young men and over four-fifths of young women who were practising contraception at the time of the interview reported that their wife or they had been sterilised. Just 2-3\% of young people practised contraception to delay the first birth. Not surprisingly, pregnancy typically occurred within a year of marriage for over three-fifths of young women and over half of young men who reported that they or their wife had been pregnant at least once. Moreover, substantial proportions of youth reported experiencing unintended pregnancy. For example, among young women who were not pregnant at the time of the interview and among young men whose wife was not pregnant at the time of the interview, $23 \%$ and $15 \%$, respectively, reported that the last pregnancy was mistimed or unwanted.

Circumstances of the first birth suggest that the overwhelming majority of first births were delivered in a health care facility $(72-76 \%)$ and $89-90 \%$ of first birth were delivered by a skilled attendant.

Findings also show that most respondents wanted one child of each sex and just a small minority of young men and women expressed a preference for more sons than daughters. 


\section{Substance use}

Findings show that substantial proportions of young men reported the consumption of tobacco and alcohol; one-fifth of young men reported tobacco consumption and over one-fourth reported alcohol consumption. In contrast, consumption of tobacco products and alcohol was reported by very few young women (less than $2 \%$ ). Drug use was reported by very few young men and not a single young woman.

\section{Health seeking behaviour}

Although youth is a generally healthy period of life, significant minorities reported experiencing general, mental, and sexual and reproductive health problems in the period preceding the interview. For example, $22 \%$ of young men and $33 \%$ of young women had experienced high fever, $7 \%$ and $2 \%$, respectively, had experienced an injury, and $1 \%$ and $11 \%$, respectively, reported experience of symptoms of genital infection. Moreover, $12 \%$ of young women reported menstrual problems; at the same time, $18 \%$ of young men reported anxiety about nocturnal emission. Finally, responses indicative of mental health disorders were reported by $10-11 \%$ of young men and women.

With regard to care seeking for general and sexual and reproductive health problems, patterns varied by type of problem experienced. While the large majority of those who had experienced high fever, for example, had sought care, many fewer had sought care for sexual and reproductive health problems. Of those who had sought treatment, the majority had sought advice or treatment from a private facility or provider, irrespective of the type of problem experienced. It is notable, however, that in the case of anxiety about nocturnal emission, young men rarely sought advice from a health care provider, preferring to do so from peers.

Findings suggest that youth were uncomfortable about seeking sexual and reproductive health services. For example, many youth, including the married, reported that they would indeed hesitate to approach a health care provider or a pharmacy/medical shop for contraceptive supplies.

Finally, although youth were overwhelmingly in favour of pre-marital HIV testing, relatively few had undergone HIV testing. Just 5\% of young men and almost one-quarter of young women had undergone HIV testing. Married young women were far more likely than any other group to have done so; over two-fifths of married young women had undergone HIV testing, most likely as part of antenatal check-ups.

\section{Participation in civil society and political life}

Findings highlight somewhat limited participation of youth in civil society. Although a number of programmes are organised by the government or NGOs at the community level in which youth can participate, familiarity with these programmes was far from universal. Just two-fifths of young men and two-thirds of young women reported familiarity with these programmes and even fewer youth-19\% of young men $22 \%$ of young women-reported participating in such programmes. Considerably more young men (59\%), but fewer young women (17\%) reported that they had participated in community-led activities such as cleanliness drives and the celebration of festivals and national days. Finally, just $10 \%$ of young men compared to $26 \%$ of young women reported membership in organised groups.

Findings suggest that voting behaviour was far from universal. Among those eligible to vote, 62-63\% of young men and women had cast their vote in the most recent election. Also of note is that while most youth (89-90\%) perceived that one could vote freely and without fear and pressure, as many as $60 \%$ of young men and $53 \%$ of young women reported disillusionment with the commitment of political parties to work for change at the community level.

By and large, youth reported secular attitudes; $88-98 \%$ reported that they mixed freely and would eat together with individuals of different religions and castes, and that they would talk to a person who has had an inter-caste marriage. However, only one-third of young men and three-fifths of young women agreed that it was best to tolerate rather than punish someone who had shown disrespect to their religion. 
Considerable proportions of young men and women acknowledged that physical fights among young men as well as among young women did occur in their village or urban neighbourhood. However, few youth-8\% of young men and 3\% of young women-reported that they had been involved in a physical fight in the year preceding the interview.

Youth reported a number of leading problems facing them and gender differences were evident. Among young men, the majority reported difficulty in finding employment and concerns about poverty more generally as leading problems. In contrast, leading problems expressed by young women were poverty and to a lesser extent, lack of amenities and infrastructure, lack of opportunities for education and difficulty in finding employment.

\section{Recommendations for programmes}

Findings presented in the sections above underscore the fact that youth face numerous challenges while making the transition to adulthood. These challenges call for programme interventions at the youth, family and service delivery levels. Key programme recommendations emerging from this study are outlined below.

\section{Address obstacles to school completion}

Youth Study findings highlight that school enrolment was far from universal among young people in the state: indeed, one in twelve young men and one in five young women had never been enrolled in school. School completion rates were also relatively low, particularly among young women; just 52\% of young men and 36\% of young women had completed high school. The state government has articulated its commitment to improving schooling situation in the state, and what is required now, particularly if the state is to achieve the Millennium Development Goal of ensuring universal primary school completion, are actions to implement these commitments. While the achievement of universal primary school completion is a key goal, the importance of high school education in enabling youth to make a successful transition to adulthood underscores the need, at the same time, for efforts to overcome barriers to high school completion. The gender divide and rural-urban divide observed in school enrolment and completion call, moreover, for efforts that target female children and children in rural areas.

A number of factors have been identified in the Youth Study that inhibit school completion; leading among these were economic reasons; attitudes and perceptions; and, among young women, school-related reasons. Multiple activities are needed to address these barriers. Efforts must be made, for example, to address the economic pressures that dissuade parents from enrolling their children in school and from keeping them in school once enrolled. While a number of state government programmes are ongoing that aim to reduce the cost of education, additional inputs, by way of conditional grants that encourage school completion among disadvantaged groups, also need also to be considered. Moreover, there is a need to ensure that ongoing government programmes do indeed reach the most disadvantaged groups. At the same time, activities are needed that raise awareness among parents about the importance of a secondary school education in expanding their children's livelihood opportunities.

Activities must also address school-level barriers, notably, poor infrastructure, quality of education and academic failure, particularly among young women. There is a need to incorporate livelihoods skills building models within the school setting that will not only raise young people's aspirations regarding their education and careers but also provide them opportunities to gain market-driven job skills. There is also a need to focus on providing better training and ensuring accountability for teachers-investments that are likely to improve the quality of the schooling experience for youth.

Findings suggesting that married young men and women remain considerably disadvantaged call for interventions that give the married a second chance to continue their education. Likewise, evidence that rural youth, particularly young women, were more disadvantaged with regard to educational opportunities than their urban counterparts calls for efforts to provide those out of school an opportunity to complete their schooling. 


\section{Strengthen efforts to prevent child labor}

Findings of the Youth Study that almost two in five young men and women had initiated work in childhood or in early adolescence (before age 15) reiterate the recommendation highlighted above regarding the need to provide conditional grants and targeted subsidies to disadvantaged groups, which would encourage parents to opt for schooling over work for their children. At the same time, it is important to vigorously enforce existing laws that prohibit child labour.

\section{Enable opportunities for employment}

Although unemployment rates were relatively low (7-10\%) among youth, findings that unemployment rates were particularly high among the educated suggest a possible disconnect between youth skills and market needs. Indeed, few youth were aware of employment generation programmes and even fewer had availed of these or vocational skills training. It is notable that while considerable proportions of urban youth reported exposure to computer skills, English language skills and so on, rural youth tended to opt for relatively traditional vocational skills and may not have had the opportunity to learn about market needs or develop appropriate skills for which a demand exists. Formal mechanisms need to be developed that enable youth-and particularly rural youth—to acquire skills for which there is an established market demand, and that link eligible youth to market opportunities.

\section{Build upon youth's growing access to the internet}

Findings suggesting that one quarter of young men and one in ten young women with five or more years of education — and considerably more in urban areas—had accessed the internet highlight the role that this medium can play in building youth awareness of the world around them and the opportunities available to them. The development of youth-friendly web-sites in Telugu may be a useful way to convey such information to youth.

\section{Promote agency and gender equitable norms among youth}

Findings highlight the limited agency of young women and the persistence of gender double standards among youth. Young women were particularly disadvantaged in terms of school completion, mobility, opportunities to build peer networks, decision-making authority in matters relating to their own lives and control over resources. And although young women were more likely to express equitable gender role attitudes than young men, they were more likely than young men to express traditional attitudes concerning wife beating. These findings call for attention to promote life skills education programmes for young women, both unmarried and married, that will not only raise their awareness of new ideas and the world around them but also enable them to put information into practice, encourage them to question gender stereotypes, develop self-esteem and strengthen their skills in problem-solving, decision-making, communication and inter-personal relations and negotiation. Safe spaces should be identified in which young women can build social networks and find social support among peers.

Interventions intended to build life skills must also be inclusive of young men. Indeed, findings that inegalitarian gender role attitudes were expressed by many young men, on the one hand, and that considerable numbers of young men were not able to exercise agency in their everyday lives, on the other, call for programmes that build their life skills, promote new concepts of masculinity and femininity and at the same time, promote messages that build egalitarian relations between women and men.

Promoting gender equitable norms and practices requires active engagement with the community. It is essential that programmes for youth work with key community members, such as parents and political and religious leaders in the community, to critically examine prevailing gender norms and forces that perpetuate such norms.

An increasing number of intervention models to build agency and promote egalitarian gender role attitudes among young people have been tested in India. These models could be reviewed and replicated or scaled up as appropriate. 


\section{Provide opportunities for formal saving, especially for young women}

Findings suggest that that just one-quarter of young men and women had some savings and few youth owned a bank or post office account (just 19\% of young men and $17 \%$ of young women). Despite the fact that young women were as likely as young men to report savings and to own a savings account, they were considerably less likely to operate the account independently. Programmes are needed that inculcate a savings orientation among young people, that offer savings products that are attractive and appropriate to the small and erratic savings patterns of young people and that enable young women in particular to overcome obstacles related to owning and controlling savings products.

\section{Promote youth participation in civil society and political processes and reinforce secular attitudes}

Findings have noted that large proportions of youth have exercised their right to vote, that the majority hold secular attitudes with regard to mixing with a person from another caste and religion, and few engage in community-level violence. Nevertheless, not all youth expressed secular attitudes-two thirds of young men and about two-fifths of young women reported that they would endorse violence against someone who showed disrespect to their religion. Moreover, relatively few had taken part in civil society, that is, government- or NGO-sponsored programmes or community-led activities. Programmes are needed—at the school, college and community levels, through national service programmes, sports and other non-formal mechanisms- that encourage civic participation, incorporate value building components and reinforce secular attitudes and values that espouse responsible citizenship.

\section{Strengthen family life or sex education for those in school and out of school}

Youth Study findings provide considerable evidence suggesting that family life or sex education is urgently needed among youth, both for those in school and for those who have discontinued their education. Findings demonstrate a limited understanding of sexual and reproductive matters among young people, including the married. Misconceptions abound on most topics: sex and pregnancy, contraceptive methods including condoms, STIs and HIV/AIDS and the conditions under which abortion is legally available or restricted. Where awareness exists, it is superficial in many cases.

Youth themselves have called for family life or sex education. Findings highlight that large proportions of youth recognised the need for information and education on these issues, and indicated a preference for receiving this education from teachers, health care providers or other experts; many youth also indicated a preference for receiving this education from friends, and large proportions of young women, from parents. However, no more than one in three youth had been exposed to family life or sex education, notwithstanding the School AIDS Education Programme, the Red Ribbon Clubs and special programmes for out-of-school youth. Indeed, substantial proportions of married young women (and some young men) reported entering marriage completely unaware of what marriage entailed. At the same time, several young people had engaged in sexual risk taking.

As mentioned above, a number of state government programmes are ongoing that aim to impart sexual and reproductive health information to young people. What is needed is a strong commitment to ensuring that these programmes do indeed reach young people, both those in school and out-of-school, married and unmarried, and rural and urban. Moreover, there is a need to expand the contents of existing awareness raising programmes to include not just HIV-related information but broader sexual and reproductive topics. These programmes should be designed not only to raise awareness among youth but also to enable young people to correctly understand and assess the risks they face and to adopt appropriate protective actions.

In addition, special attention needs to be paid to the training of trainers. It is important that teachers, health care providers and other experts undergo training that enables them to overcome their reluctance to communicate with youth on sensitive sexual and reproductive matters, dispels their misconceptions on these matters, and enhances their technical knowledge of these issues. Peers and, in the case of young women, parents have also been identified 
as acceptable sources of information and efforts must therefore be made that identify youth leaders and build networks of peer educators. Also needed are efforts to engage parents-providing them with accurate information and working with them to overcome inhibitions about imparting this information to their children, particularly their daughters.

\section{Ensure that the transition to sexual life is safe and wanted}

While for the vast majority of young women sexual activity is initiated within the context of marriage, findings show that small proportions of young men and women had engaged in sex before marriage. As documented in this report, many youth had initiated sexual activities uninformed, reiterating the need for providing family life or sex education to young people. Moreover, the finding that for many youth, pre-marital sexual experiences were unsafe, and for some unwanted, calls for programmes that focus on building sexual and reproductive health awareness among young people, and developing their skills in negotiating safe sex and communicating with their partners on sexual and reproductive health matters. At the same time, programmes must make available appropriate family planning and infection prevention services for both married and unmarried young men and women in a manner acceptable to them.

\section{Intensify efforts to eliminate the practice of early marriage}

Findings indicate that although most youth prefer to marry after age 18, the practice of early marriage is widespread among young women. These findings call for measures that go beyond information campaigns to address the underlying factors-social norms and economic constraints-driving early marriage in the state.

There is a need for a multi-pronged approach to eliminate the practice of early marriage. Strategies are needed that mobilise communities to help parents resist pressures that foster the practice of early marriage; that establish new norms and practices; as well as that initiate campaigns highlighting the adverse consequences of early marriage and how it is a violation of the rights of the child. Community mobilisation efforts must involve youth themselves, their families, as well as influential persons in the community, including religious and political leaders.

Equally important is to ensure greater commitment on the part of law enforcement agencies to enforce existing laws on the minimum age at marriage and the registration of marriages, and to levy penalties on violators. Allowing anonymous reporting, making law enforcement agencies and others aware that the practice of early marriage is not a minor violation, and making the guidelines for penalties clear to enforcement agencies and the wider community are possible steps in this direction.

Efforts to delay marriage also require providing girls with viable alternatives to marriage in the form of accessible and quality schooling and opportunities to build and use livelihood skills. Working with the education sector to make schooling for girls more accessible, and to make classrooms gender-sensitive and responsive to the needs of girls and the concerns of their parents is important. At the same time, it is necessary to provide livelihoods training within and outside the educational system.

Findings that most marriages were arranged with the participation of young people themselves are encouraging. However, the finding that few young people had an opportunity to meet their spouse-to-be prior to the wedding day calls for actions to sensitise parents to the need to enable their children to interact with their prospective spouse prior to the wedding. Parents must also be made aware of the physical and mental health consequences of early marriage and the adverse experiences of many young women (and some young men) who were married early or who were unprepared for marriage.

\section{Support newly-weds to postpone the first pregnancy}

Findings show that the social pressure to bear children as soon as possible following marriage persists. Contraceptives were rarely used to postpone the first pregnancy and many young women experienced their first pregnancy soon 
after marriage. It would appear that numerous forces work against delaying the first pregnancy-young people's lack of awareness of appropriate methods of contraception and access to supplies, their limited skills in countering social expectations and negotiating pregnancy postponement, overwhelming pressure from the family and the community to bear children as soon as possible after marriage, and the lack of attention from health care providers.

Programmes are needed that inform youth about their pregnancy postponement options and enable them to access appropriate contraception. At the same time, providers must be trained and charged with the responsibility of reaching married young women and men-including those who have not yet experienced pregnancy-with information regarding contraception and other reproductive health matters as well as contraceptive supplies. Many married young women lack the freedom of movement to seek health care, underscoring the need for health workers to reach these women—particularly those newly married and first time pregnant—in their homes.

\section{Address power imbalances within marriage}

Findings confirm that within marriage, large proportions reported communication and interaction with their spouse; nevertheless communication on sensitive matters such as contraception was limited for many. In addition, married life was marked by violence for many; for example, notable proportions of young women had suffered physical and sexual violence perpetrated by their husband.

Efforts are needed to encourage couple communication on sensitive issues (contraception, for example), negotiation and conflict management skills early in marriage. Efforts are also needed to inform married young women of their rights so that they have the opportunity to exercise control over their own lives; at the same time, efforts must be made to promote new concepts of masculinity and femininity and egalitarian couple relations among young men and women. Intervention models exist in India that have attempted to address these needs; these should be reviewed and up-scaled as appropriate.

\section{Create a supportive family environment}

Findings highlight the limited interaction and social distance between parents and young people while growing up and the gendered nature of perceptions regarding parental controls on youth behaviours. Efforts must be made to create a supportive environment for young people. While evidence on models that are effective in bridging the distance between parents and children or that enable parents to adopt more gender-egalitarian socialisation practices is not currently available, findings presented in this report call for programmes that address parental inhibitions about discussing sexual matters with their children, encourage greater openness and interaction between parents and children, and enable the adoption of gender-egalitarian child-rearing practices. Programmes that aim to encourage universal education or eliminate child marriage must, likewise, address parental concerns about the potential consequences of keeping daughters in school or delaying their marriage.

\section{Reorient service provision to address the unique needs of unmarried and married young women and men}

Although the RCH Programme has advocated special services for youth, including the unmarried, these services had not reached the youth in our survey. For example, relatively small percentages of young people have ever practised contraception and the method most likely to be adopted was sterilisation. Few had sought care for symptoms of STI or gynaecological problems, and most youth who had sought care for the latter preferred private to public sector facilities. Lack of care seeking and the disconnect between the public health sector and youth underscores the need to sensitise health care providers about the special needs, heterogeneity and vulnerability of unmarried and married young women and men, and to orient them to the need for developing appropriate strategies to reach these diverse groups, including young newlyweds. It raises, at the same time, the need to explore the feasibility of implementing various financing strategies, for example, health insurance, competitive voucher schemes and community financing schemes, which will allow youth to have a wider choice of providers and enhance the possibility of obtaining quality care. 
Programmes must be inclusive of unmarried as well as married young people and recognise their need and right to sexual and reproductive health and related information and services. Counselling and contraceptive services must be made available to all young people, including the unmarried, in a non-threatening, non-judgmental and confidential environment. Indeed, these findings call for the implementation of strategies outlined under the National Rural Health Mission's RCH Programme.

Findings that one in ten young men and women reported symptoms suggestive of mental health disorders suggest that there is a need to establish systems that address young people's mental health. Efforts are needed to screen young people-particularly the married among whom symptoms were more likely to be reported-for mental health disorders when they avail of other primary health services, including, for example, sexual and reproductive health services, and to refer youth with such symptoms to appropriate health facilities and providers.

\section{Directions for future research}

Findings presented in this report provide a broad picture of youth in Andhra Pradesh. At the same time, findings have raised a number of issues that require further investigation, particularly with regard to the determinants and consequences of youth behaviours and practices during the transition to adulthood. While the Youth Study is indeed a rich source of data that will enable investigators to fill many of the information gaps identified, there are several gaps in knowledge that will require additional research.

Youth Study findings highlight the need for further study in terms of formative research that explores in greater depth the factors impeding successful transitions to adulthood, in the areas, for example, of school completion, economic activity, sexual relations, and marriage and parenthood. Research is also needed that explores the role of peers, socialisation practices, young people's access to information and services, and the ways in which these factors may contribute to or impede young people's ability to make successful transitions to adulthood. A general research recommendation is the urgent need for prospective or panel study designs that follow a cohort of adolescents at regular intervals up to age 24 . Prospective study designs would enable researchers to take a life course approach, identify, with compelling data, the factors responsible for healthy transitions to adulthood and point to the ways in which the situation and experiences of youth influence their life course at later ages.

Operations research is also needed. While a number of interventions to address the needs of youth have been initiated in India-for example, addressing the needs of married girls, changing the norms of masculinity and femininity, encouraging education for girls, developing market-oriented vocational skills and providing family life or sex education-few have been rigorously evaluated. Urgently needed, therefore, are carefully designed and rigorously tested intervention models that not only pay attention to the content and delivery of the intervention but also measure its effectiveness and acceptability-in short, that will enable a shift from the implementation of promising to best practices in addressing young people's needs. Ultimately, research is needed that evaluates the reach, effectiveness and feasibility of existing programmes, and that monitors the scaling up of successful interventions in terms of their impact on young people's lives.

In brief, the Youth Study has documented, for the first time, the multi-faceted situation of youth in Andhra Pradesh. The study highlights several positive aspects of young people's lives but also alerts us to the many challenges confronting youth and their ability to make a successful transition to adulthood. It emphasises the heterogeneity of youth, not only in terms of their situation but also with regard to their stated needs and preferred mechanisms to address these needs. Programmes must recognise the heterogeneity of young people, and interventions and delivery mechanisms should be appropriately tailored to meet their needs. Evidence presented here provides not only a blue-print for the programming needs of youth in Andhra Pradesh but also a base-line by which to measure the impact of programmes intended to address youth needs. 


\subsection{Rationale}

The Youth in India: Situation and Needs Study (referred to as the Youth Study) is the first-ever sub-nationally representative study conducted to identify key transitions experienced by married and unmarried youth in India. There is a strong rationale for this study. Young people (aged 10-24) constitute almost 315 million and represent $31 \%$ of the Indian population (Office of the Registrar General and Census Commissioner, 2001a). Numbers are projected to increase and peak at around 358 million in 2011 before stabilising at around 336 million by 2026 (Office of the Registrar General and Census Commissioner, 2006). Not only does this cohort represent India's future in the socio-economic and political realms, but its experiences will largely determine India's achievement of its goal of population stabilisation articulated in the National Population Policy 2000 (MOHFW, 2000) and the extent to which the nation will be able to harness its demographic dividend. In addition, it is clear that the realisation of the Millennium Development Goals (UNDP, 2000) depends, to a considerable extent, upon the situation of young people. While today's youth are healthier, more urbanised and better educated than earlier generations, social vulnerabilities persist and transitions to adulthood are too frequently marked by early entry into the labour force, abrupt and premature exit from school, early marriage and strongly held gender norms. In the course of the transition to adulthood, moreover, young people face significant risks related to sexual and reproductive health, and many lack the knowledge and power to make informed sexual and reproductive choices (Jejeebhoy and Sebastian, 2003).

In recognition of the importance of investing in young people, several national policies formulated since 2000 have underscored a commitment to addressing the multiple needs of this group in India. The National Population Policy 2000 recognised, for the first time, that adolescents constitute an under-served group with special sexual and reproductive health needs, and advocates special programmatic attention to addressing this population (MOHFW, 2000). The National Youth Policy 2003 focuses on the needs of those aged 13-35, but recognises adolescents (aged 13-19) as a special group requiring a different approach from that appropriate for young adults (aged 20-35), and promotes strategies to meet youth needs in areas including education, training and employment, health, recreation and sports, and good citizenship (Ministry of Youth Affairs and Sports, 2003). Also notable is the commitment to addressing the needs of adolescents and young people articulated in the Tenth and Eleventh Five-Year Plans (Planning Commission, 2002; 2006). In addition, the National Adolescent Reproductive and Sexual Health Strategy provides the framework for the adolescent sexual and reproductive health services proposed in the Reproductive and Child Health (RCH) Programme II (MOHFW, 2006). The National Rural Health Mission (2005-12) has incorporated adolescent health services as part of its service guarantees in health sub-centres, primary health centres and schools (MOHFW, 2005).

Effective implementation of both policies and programmes, however, has been handicapped by a lack of evidence on young people's situation and needs. Currently available evidence is limited, at best, and comes largely from small scale and unrepresentative studies. The most recent National Family Health Survey (NFHS-3) obtained, for the first time, valuable data on unmarried young women and men (IIPS and Macro International, 2007a). Even so, the information that it provides on young people's various transitions remains limited and the small sample sizes obtained in most states preclude the possibility of in-depth analysis and of obtaining state-representative estimates of behaviours and practices among different sub-groups of young people. 


\subsection{Study objectives}

The objectives of the Youth Study were to identify key transitions experienced by youth, including those pertaining to education, work force participation, sexual activity, marriage, health and civic participation; provide state level evidence on the magnitude and patterns of young people's sexual and reproductive practices in and outside of marriage as well as related knowledge, decision-making and attitudes; and, finally, identify key factors underlying young people's sexual and reproductive health knowledge, attitudes and life choices. Findings from the study are expected to guide policy, programmes and advocacy on youth issues, enable programmes and policies to recognise the heterogeneity of youth in India, and provide important base-line indicators against which the long-term impact of programmes may be measured.

The Youth Study focused on married and unmarried young women and unmarried young men aged 15-24 and, because of the paucity of married young men in the younger ages, married men aged 15-29 in both rural and urban settings. The study was conducted in a phased manner in six states of India: Andhra Pradesh, Bihar, Jharkhand, Maharashtra, Rajasthan and Tamil Nadu. This report focuses on findings from Andhra Pradesh.

Funding for the Youth Study was provided by the David and Lucile Packard Foundation and the John D. and Catherine T. MacArthur Foundation. The Youth Study was conducted jointly by the International Institute for Population Sciences, Mumbai (IIPS) and the Population Council, New Delhi. The design and implementation of this study were guided by the Project Advisory Committee, headed by the Additional Secretary, Ministry of Health and Family Welfare (MOHFW).

\subsection{Andhra Pradesh: Overview of demographic and socio-economic features}

The state of Andhra Pradesh, situated in the southern part of India, is India's fourth largest state in terms of area, covering a total of 275,069 square kilometers. The state has 23 districts divided into three regions, namely, coastal Andhra, Rayalseema, and Telangana. With a population of 76.2 million in 2001, Andhra Pradesh ranks fifth in terms of total population among states in India (Office of the Registrar General and Census Commissioner, 2001b). The state's population more than doubled between 1961 and 2001 and is projected to have reached 84 million by 2009 (Office of the Registrar General and Census Commissioner, 2006). With 978 females per 1,000 males in 2001, the state registered the fourth highest sex ratio among states in India. Population density in the state was 277 persons per square kilometer in 2001 . The distribution of the population by religion indicates that $89 \%$ of the state's population was Hindu, 9\% was Muslim and 2\% Christians (Office of the Registrar General and Census Commissioner, 2001c). About one-sixth (16\%) of the state's population belonged to scheduled castes and $7 \%$ belonged to scheduled tribes (Office of the Registrar General and Census Commissioner, 2001b).

The state is characterized by a large rural population; just $27 \%$ of the state's population lives in urban areas and the state ranked eleventh in terms of percentage of the population residing in urban areas (Office of the Registrar General and Census Commissioner, 2001b). It has witnessed considerable out-migration. According to the 2001 Census, during the decade 1991-2001, over half a million persons migrated to states outside of Andhra Pradesh (Office of the Registrar General and Census Commissioner, 2001d).

Andhra Pradesh is among the more economically progressive states in the country; in 2006-2007 it accounted for about 7\% to the national Gross Domestic Product (GDP) (Ministry of Statistics and Programme Implementation, 2008). Its per capita income in 2005-06 stood at Rs.26,211, somewhat higher than the national average of Rs.25,716 (Ministry of Finance, 2008). Andhra Pradesh's Gross State Domestic Product (GSDP) at current prices stood at Rs.269,173 crores in 2006-07 (Ministry of Statistics and Programme Implementation, 2008); at constant prices (1993-94), the GSDP increased by $8.7 \%$ between $2004-05$ and 2005-06. Although agriculture was traditionally the main source of income, the primary sector contributed just $26.6 \%$ to the state's economy in 2006-07, compared to $21.6 \%$ by the secondary sector and as much as $51.8 \%$ by the tertiary sector (Planning Department, 
Government of Andhra Pradesh, 2007). Indeed, the large contribution by the tertiary sector reflects the considerable role played by the fields of information technology and biotechnology in the economic development of the state.

About one-sixth of the state's population (15.8\%, based on the Uniform Recall Period consumption distribution) was estimated to live below the poverty line (2004-05), compared to $28 \%$ at national level. Rural-urban differences in the state were wide, with $28 \%$ and $11 \%$ of the urban and rural populations, respectively, living below the poverty line (Planning Commission, 2007).

Unemployment rates are low in Andhra Pradesh. In 2004-05, 1.6\% and 3.9\% of rural and urban males, respectively, were unemployed for a major part of the year, as measured by the usual principal status definition. The corresponding figures for females were $1.1 \%$ and $4.3 \%$, respectively (NSSO, 2006).

Andhra Pradesh lags behind much of India in terms of literacy. Indeed, it ranks twenty-third among Indian states in terms of literacy, with a literacy rate of $61 \%$ in 2001 . Male and female literacy rates in the state, at $50 \%$ for females and $70 \%$ among males, were considerably lower than the national average of $54 \%$ and $75 \%$, respectively (Office of the Registrar General and Census Commissioner, 2001b).

Despite these average education indicators, the state's achievements in the health sector are notable. Life expectancy for females in Andhra Pradesh was higher than the national average during $2002-06$ (66 versus 64 years), life expectancy for males was identical to the national average (63 years) (Office of the Registrar General, 2008a). Likewise, the infant mortality rate was lower than the national average (54 versus 57) in 2005-06. Similarly, fertility levels in Andhra Pradesh are much lower than the national average. Indeed, the fertility rate in the state had reached replacement levels by 2004 (Office of the Registrar General and Census Commissioner, 2006) and the total fertility rate stood at 1.8 in 2005-06, compared to 2.7 for India as a whole. The contraceptive prevalence rate was, correspondingly, much higher than the national average (68\% versus $56 \%$ and just $5 \%$ of women in Andhra Pradesh, compared to $13 \%$ in India as a whole, reported an unmet need for contraception (IIPS and Macro International, 2007a).

As is well known, however, Andhra Pradesh is one of six states of India in which HIV prevalence is highest. During 2005-06, the HIV prevalence rate in the state was reported to be $0.97 \%$, that is, $1.22 \%$ among men and $0.75 \%$ among women, far higher than national rates of $0.22 \%$ and $0.35 \%$, respectively (IIPS and Macro International, $2007 \mathrm{a}$ ).

\subsection{Situation of youth in Andhra Pradesh}

Young people in Andhra Pradesh aged 10-24 constituted a total of 23 million, and accounted for $31 \%$ of the state's population in 2001. The youth population, that is, those aged 15-24 numbered 14.5 million and accounted for about $19 \%$ of the state's population (Office of the Registrar General and Census Commissioner, 2001a). The share of the youth population as a proportion of the total population of Andhra Pradesh is projected to increase to $20 \%$ during 2006-11, before beginning to decline (Office of the Registrar General and Census Commissioner, 2006).

Educational attainment levels among youth in Andhra Pradesh are similar to those in India as a whole, and gender differences in enrolment are relatively narrow. Among young people (aged 10-24), 85\% of men and 71\% of women were literate in 2001 (Office of the Registrar General and Census Commissioner, 2001e). While the state has achieved near universal primary school enrolment (as evident from a gross enrolment ratio of $97 \%$ among children aged 6-11 years), enrolment at secondary school level remains limited. The gross enrolment ratios for 11-14 year-olds and 14-16 year-olds were 72\% and 53\%, respectively. The Gender Parity Index (GPI) at the elementary, secondary and higher secondary levels of education indicates that while learning opportunities are nearly equally available to boys and girls at the elementary level, such opportunities are limited for girls at the secondary and higher secondary level in the state; GPI scores were 0.99 at the elementary level and 0.82 at the secondary and higher secondary level in 2004-05 (Ministry of Human Resource Development, 2007). 
As elsewhere in the country, unemployment rates in Andhra Pradesh were much higher among young people than among the general population discussed above. During 2004-05, unemployment rates among the population aged 15-29, as measured in terms of principal usual status, were $3.4 \%$ and $8.6 \%$ among young men in rural and urban settings, respectively; the corresponding rates for young women were $1.9 \%$ and $10.5 \%$ (NSSO, 2006).

Available evidence on the sexual and reproductive health profile of young people in Andhra Pradesh indicates their vulnerability. Marriage continues to take place before the legal minimum age for both young women and men; as recently as 2005-06, 55\% of women aged 20-24 were married by age 18 and 35\% of men aged 25-29 were married by age 21 . Moreover, $18 \%$ of 15-19 year-old girls had begun childbearing (IIPS and Macro International, 2008).

While it is clear that sexual relations are initiated early and within the context of marriage for large proportions of young men and women in Andhra Pradesh, behavioural surveillance surveys and small-scale studies report that sexual risk taking before or within marriage is not unheard of among young people in the state. For example, the recent National Behavioural Surveillance Survey reports that some 22\% and 3\% of young men and women, respectively, aged 15-24 had engaged in sexual intercourse with a non-regular partner in the 12 months preceding the survey, and of these, just half (49\%) had used condoms consistently with these partners (National Institute of Medical Statistics and NACO, 2008). Likewise, a recent study exploring the pre-marital sexual experiences of married youth in Guntur district reports that 32\% of young men aged 15-29 and 5\% of young women aged 15-24 had experienced pre-marital sex. Moreover, among those married youth who had experienced pre-marital sex, few reported consistent condom use ( $7 \%$ and $2 \%$ of young men and women, respectively) and multiple partner relations were reported by $37 \%$ of young men and $2 \%$ of young women (Santhya, Jejeebhoy and Ghosh, 2008).

Despite the early onset of sexual relations within or before marriage among significant proportions of youth in Andhra Pradesh, available evidence suggests that young people's knowledge of sexual and reproductive health matters tends to be limited. Data from NFHS-3 indicate that only 37\% of young men and even fewer (20\%) of young women had comprehensive knowledge about HIV/AIDS. While $87 \%$ of young men were aware of a source for condoms, just 27\% of young women reported such awareness (IIPS and Macro International, 2007a). Care seeking for symptoms of genital tract infection was, moreover, far from universal. A study of married youth in Guntur district observed that among those reporting symptoms of infection, just 52\% of young women and $64 \%$ of young men sought care promptly, and just $19 \%$ and $25 \%$, respectively, sought care as soon as symptoms were observed (Santhya, Jejeebhoy and Ghosh, 2008).

\subsection{Youth-related policy and programme environment in Andhra Pradesh}

The Andhra Pradesh state government has articulated its commitment to promote and protect the rights of adolescents and youth in a number of policies, including its Population Policy 1997 and its Vision 2020 document. The Population Policy articulates a number of goals to improve the situation of adolescents, particularly girls. In the area of health, it aims to promote family planning among newly-married couples by providing counselling on birth spacing, and raising awareness about such issues as delaying first birth, increasing birth spacing and increasing male responsibility in reproductive behaviour. One of the stated objectives of the Policy is to increase age at marriage of females; the Policy contains a number of recommendations to achieve this objective, including IEC efforts to highlight the adverse effects of early marriage and pregnancy, introduction of the girl child scheme to delay marriage at young ages, involvement of political and community leadership, involvement of women's groups and NGOs in promoting attitudinal changes among young men, parents and communities, and the promotion of education for the girl child through awards and incentives for panchayats/nagarpalikas districts that achieve full enrolment and retention of girl children in schools (Government of Andhra Pradesh, 1997; Santhya and Jejeebhoy, 2007).

The Vision 2020 document of Andhra Pradesh also reaffirms this commitment. In line with the Population Policy, the Vision 2020 also aims to promote family planning among newly-married couples by providing counselling on birth spacing, encouraging male involvement in contraception, and improving maternal health by encouraging adolescents to delay pregnancy until age 21. It seeks to address such health problems of adolescent girls as chronic 
anaemia through the establishment of nutrition programmes for adolescent girls at anganwadi centres. As in the population policy, the document also discusses the need to ensure the enrolment and retention of girls in school, and proposes to expand the number of anganwadi centres to provide crèche services so as to enable older girls to attend schools; provide "Back to School" bridge courses to help girl drop-outs return to school; and equip girls with vocational skills to make them self-reliant. The Vision 2020 document also underscores the need to involve communities in building awareness about the adverse effects of early marriage (Government of Andhra Pradesh, 1999; Santhya and Jejeebhoy, 2007).

A number of programmes have been implemented by the Andhra Pradesh state government to meet the needs of youth. For example, state government initiatives have focused on the expansion of schooling facilities so as to improve physical access to adolescents, especially girls. Over the period 1993 to 2002, the state recorded a $24 \%$ increase in the number of primary schools, a $137 \%$ increase in the number of upper primary schools and a $78 \%$ increase in secondary schools. The state has also paid attention to back-to-school initiatives, including bridge courses (Balika Shikshan Shivir, residential condensed courses), that enable the out of school to return to school and older girls to complete key classes (Centre for Good Governance, n.d.). Under the National Programme for the Education of Girls at Elementary Level, intended to ensure the retention of girls at least up to the completion of elementary school, 3,373 model cluster schools were set up in the state; girls attending these schools were provided vocational skills training as well and teachers were sensitized about the constraints that girls face in attending school. (Planning Department, Government of Andhra Pradesh, 2007).

The Government of Andhra Pradesh has also initiated public-private partnership models to build skills among rural and under-privileged youth under its employment generation programme. In this model, private sector agencies provide training in IT \& ITeS to educated, unemployed youth. The government has recently launched the Rajiv Yuva Shakthi (RYS) Programme, intended to promote self-employment among both educated and uneducated youth in the state. Under this programme, unemployed youth are provided financial assistance to set up small-scale industries as well as service and business sector enterprises. So far 76,038 young people have been supported in establishing self-employment units (Planning Department, Government of Andhra Pradesh, 2007).

Programmes have also been introduced to impart information on sexual and reproductive health to young people. For example, the School AIDS Education Programme has been implemented for students of Class IX and Class X since 2002 (Government of Andhra Pradesh, 2004). As of 2005, the programme has been implemented in over 21,000 secondary schools, that is, nearly $80 \%$ of the schools in the state (MHRD, NACO and UNICEF, 2005). In order to raise awareness among adolescents, the state Education Department has also incorporated a chapter on HIV/AIDS in its biology textbook for students of Class IX and Class X. Similarly, with the support of the Andhra Pradesh State AIDS Control Society (APSACS), the Colleges Talk AIDS Programme has been implemented in 5,000 colleges in the state, covering about 1.2 million students (APSACS, 2005). In addition, the APSACS has formed Red Ribbon Clubs (RRC) in over 15,000 high schools and junior colleges, covering 1.7 million students in classes 9-12. The members of the RRCs are trained to act as change agents to provide correct information to other young people about HIV/AIDS and life skills, notably with regard to coping with peer pressure, promoting healthy life styles and adopting responsible behaviours. It is reported that over $80 \%$ of RRCs formed in the country are located in the state (Planning Department, Government of Andhra Pradesh, 2007). Village AIDS awareness clubs have also been initiated in some districts, starting with youth in junior colleges. And under Chaitanya Kishoram, a scheme implemented by the state Women Development and Child Welfare Department, girls from each district are trained and subsequently impart information on sexual and reproductive health matters to their peers (Government of Andhra Pradesh, 2006).

Programmes that target adolescent girls specifically have also been implemented in the state. For example, the Kishori Shakthi Yojana scheme has been implemented in 376 ICDS blocks in the state. Under this scheme, Balika Mandals, each comprising 25-30 adolescent girls aged 11-18 years, were formed in project villages and activities are carried out to raise these girls' awareness regarding such issues as child marriage, child trafficking, health and hygiene, nutrition, legal rights, home management, child care and so on. So far, 11,340 Balika Mandals 
have been formed, and cover 340,000 adolescent girls in the state (Department of Women Development and Child Welfare, Government of Andhra Pradesh, n.d.). In addition to centrally-sponsored incentive schemes such as the Balika Samridhi Yojana, the state government has launched a number of financial incentive schemes intended to promote girls' education, delay their marriage and prevent discrimination against girls. For example, in order to promote schooling among girls and increase their age at marriage, a pilot scheme, Chaduvula Talli, provides financial incentives to girls belonging to scheduled castes and tribes attending classes 8-10 in all government and government-aided schools in 10 backward districts of the state (Planning Department, Government of Andhra Pradesh, 2007). Another incentive scheme launched in 2005, the Girl Child Protection Scheme, targets economically poor families who have a maximum of two daughters, the youngest of whom is aged 3 years or younger and in which one parent has undergone sterilization (Planning Department, Government of Andhra Pradesh, 2007).

\subsection{Study phases}

The Youth Study comprised three phases and included both a survey and qualitative data gathering exercises.

\subsubsection{Pre-survey qualitative phase}

As the Youth Study was one of the first of its kind in India, precedents did not exist for youth terminologies, particularly with reference to sensitive issues (romantic relationships, sexual experience and so on), youth perceptions or youth willingness to share their experiences with study teams. In order to better understand these matters and to inform the design of the survey instrument, focus group discussions were conducted with married and unmarried young men and women, and key informant interviews conducted with teachers, health care providers, and community and youth leaders, in the first phase of the Youth Study. This phase also offered us an opportunity to explore community reactions to the kinds of issues to be raised by the survey.

In the course of this pre-survey qualitative phase, we also conducted in-depth interviews with parents of youth to collect parental perspectives on young people's situation and needs. In each site, eight categories of parents were selected (mothers and fathers of married and unmarried young men and women, respectively). The discussion focused on the life experiences of the child of interest.

The pre-survey qualitative phase was undertaken during May-July 2005 and covered at least one urban area and one rural area of all five regions of the state. In total, 18 focus group discussions were held with young people; 36 key informant interviews were held with community leaders, health care providers, teachers and youth leaders; and 70 in-depth interviews were held with mothers and fathers.

\subsubsection{Survey phase}

Fieldwork was undertaken between October 2007 and April 2008. A total of 8,330 married and unmarried young men and women were interviewed during this phase.

\subsubsection{Post-survey qualitative phase}

In order to better understand the sexual and reproductive experiences of youth and the factors inhibiting and facilitating safe transitions into these behaviours, in-depth interviews were conducted with consenting survey respondents who reported certain experiences in the course of the survey interview. These experiences included, notably, having an opposite-sex romantic partner; having sexual relations with an opposite-sex romantic partner; experiencing same-sex, forced or exchange sexual relations; and among young men, engaging in relations with sex workers or married women. Among the married, in addition, experiences included exercising choice in spouse selection and practising contraception to delay the first pregnancy. 
At the conclusion of the survey interview, interviewers sought the consent of respondents for an in-depth interview. Those who consented were then approached by a trained investigator who conducted the interview in the form of an unstructured conversation. In-depth interviews therefore took place at around the same time as did the survey. A total of 62 in-depth interviews were completed, 39 from among rural respondents and 23 from among urban respondents. Findings from the survey are presented in this report ${ }^{1}$.

\subsection{Study instruments}

\subsubsection{Interview guidelines}

For the pre-survey qualitative phase, three sets of guidelines were prepared for focus group discussions, key informant interviews and in-depth interviews, respectively. These guidelines were appropriately modified for each youth group (married and unmarried young men and women) and parent group (mothers and fathers of married and unmarried young men and women). As mentioned above, specific guidelines were not prepared for the post-survey in-depth interviews with youth reporting selected behaviours; instead, interviewers were trained to steer the interview to focus on the experience of interest, and obtain information on the circumstances surrounding the experience and the respondent's own perceptions about the experience.

\subsubsection{Questionnaires}

A total of six questionnaires were developed for the study: a community questionnaire; a household questionnaire, administered in each selected household; and four individual questionnaires, one each for married young men, married young women, unmarried young men and unmarried young women. The community questionnaire was administered in each village selected for the survey. This questionnaire collected information on different aspects of village life, including the village population, numbers engaged in agriculture, and the availability of various facilities and infrastructure in and around the village. Team supervisors administered the questionnaire to one or more individuals from each village who were well-informed about the village.

The household questionnaire listed all the usual residents of the selected households and collected basic information on each listed household member, including his or her age, sex, marital status, relationship to the head of the household, education and current activity status. Information was also obtained on the religion and caste of the head of the household as well as on ownership of the residential structure and agricultural land, number of rooms in the residence, and such amenities available as type of toilet facility, main source of lighting, main type of cooking fuel and main source of drinking water. The survey also inquired about ownership of 17 consumer durables. Finally, information was sought on the marriage of any usual resident of the household in the three years preceding the interview as well as the sex and age of the person at the time of marriage.

The development of individual questionnaires was informed by other survey instruments, notably the World Health Organisation core questionnaire for youth surveys (Cleland, 2001) and a recent survey conducted in Pune district on the formation of partnerships among youth (Alexander et al., 2003). Other instruments consulted included surveys of youth conducted in India (Andrew, Patel and Ramakrishna, 2003; IIPS and Population Council, 2002; Sebastian et al. 2003), Pakistan (Sathar et al., 2003), the Philippines (DRDF and UPPI, 2002), Vietnam (Mensch, Anh and Clark, 2000) and sub-Saharan Africa (Guttmacher Institute, 2004a; 2004b; 2004c). Finally, our survey instrument drew upon the questionnaire used in the NFHS-3 (IIPS and Macro International, 2007b).

\footnotetext{
${ }^{I}$ Separate reports, drawn from in-depth interviews with parents and youth, respectively, will discuss parental perspectives on young people's experience of growing up and provide insights on the sexual and reproductive experiences of youth, as well as factors inhibiting and facilitating safe transitions into these behaviours.
} 
The development of individual questionnaires was also informed by insights obtained in the pre-survey qualitative phase. Once the pre-survey qualitative phase was completed in all six states, the data generated were analysed to identify the kinds of issues that would be explored in the survey, ways of presenting sensitive issues, and terminologies to be used that would be comprehensible and acceptable to youth. The survey instruments were finalised after extensive pre-testing in several states.

Individual questionnaires were employed to interview eligible youth who usually resided in selected households. Currently married young men and women aged 15-29 and 15-24, respectively, as well as unmarried young men and women aged 15-24, were eligible for interview. Widowed and divorced individuals were excluded from the survey. Keeping in mind the sensitive nature of the questions, the questionnaire was divided into several sections and arranged in such a way that the most sensitive questions were administered towards the middle of the interview. This strategy of asking a series of non-sensitive questions in the early part of the interview served two purposes: it enabled the interviewer and respondent to build rapport before sensitive questions were posed; it also permitted the investigator to maintain privacy for sensitive questions, as interested bystanders would usually depart while questions in the early sections were posed. The individual questionnaires collected information on the following topics:

Background characteristics: Questions were asked regarding age, education and schooling, quality of school or college attended, work patterns including housework and paid employment, vocational training, short-term migration and characteristics of parents.

Additionally, a Life Event Calendar (LEC), adapted from that used in a nationally representative survey of adolescents and youth in Pakistan (Sathar et al., 2003), was administered to obtain information on education, work, living arrangements, marriage and family building (for married respondents), starting from the age of 12 years. This system of recording life events is considered one of the most effective approaches to minimise recall error.

Media exposure: Respondents were asked whether they were exposed to newspapers, television or the internet, and whether they watched pornographic films or read pornographic magazines. They were also asked about their views on the influence of films and television on their own life as well as young people's lives in general.

Puberty: In order to assess the age at which puberty was experienced, respondents were asked to report their age at key signs of maturation. Young women were questioned about their first menstruation while young men were asked about the onset of voice changes and growth of pubic hair.

Parental interaction/relationship: Detailed questions were asked on the extent of parent-child communication on everyday activities as well as sexual and reproductive issues. Questions were also asked that assessed the extent to which a respondent had witnessed parental violence or been the victim of violence perpetrated by a parent while growing up.

Communication, mobility and decision-making: This section collected information on the person in whom youth were most likely to confide on matters related to getting a job, growing up, boy-girl relationships and personal problems. Detailed questions were also asked on decision-making and, for all groups except married males, mobility.

Gender and self-efficacy: In order to evaluate the respondent's gender role attitudes and level of self-efficacy, questions were asked to probe opinions about a range of gender-related issues, such as, the importance of boys' vis-à-vis girls' education, housework and freedom of movement.

Awareness of sexual and reproductive matters: This section probed young people's awareness about sexual intercourse, pregnancy, contraceptive methods, HIV/AIDS and sexually transmitted infection (STI) as well as the legal minimum age at marriage and conditions under which abortion is legally permitted. This section also probed young people's sources of information on sexual matters and contraception, the extent to which they had obtained formal sex or family life education, and their experiences and perceptions about this education. 
Connectedness and friendship: Questions relating to connectedness and friendship explored respondents' friendship networks among those of the same sex and activities in which they participated with their friends. This was followed in a gradual fashion by questions on interaction with the opposite sex, whether or not the respondent had exchanged a "proposal" of romantic partnership with someone of the opposite sex and whether the respondent had ever met someone of the opposite sex secretly in a number of likely places.

Pre-marital romantic heterosexual relationships: This was a highly sensitive section, conducted only if complete privacy was assured. The section started by probing the pre-marital romantic and sexual experiences of up to five of the respondent's best friends. This technique, known as anonymous third-party reporting (developed by Rossier, 2003), was used to assess the extent to which youth were more likely to report the romantic and sexual relationships of their peers than of themselves. Respondents were then asked about their own experiences of pre-marital romantic partnership and, if reported, detailed questions were asked on the nature of such relationships with the first partner and the last or most recent partner (if more than one partner was reported). Questions were designed to gradually probe sensitive behaviours, for example, starting with whether the respondent had ever held hands with a romantic opposite-sex partner, and continuing with questions on hugging, kissing and finally having sex with the partner. We believe this gradual progression of questions was more culturally appropriate than a single question on pre-marital sex and provided insights into the range of behaviours youth experienced. If sex with a pre-marital romantic partner was reported, a host of questions followed that probed the consensuality of first sex with this partner, condom use, frequency of such relations and experience of pre-marital pregnancy. Questions were also asked about the characteristics of the romantic partner and parental awareness of and reactions to the romantic relationship.

Marriage process: In this section questions covered marriage planning, dowry, the participation of the respondent in decision-making related to marriage and the respondent's feelings about his or her own marriage. This section was administered, suitably modified, to both married and unmarried respondents.

Married life: Married respondents were asked detailed questions on married life. These included the nature of marriage (love or arranged), acquaintance with spouse before marriage and age at cohabitation. Questions about the marital relationship were also covered, including spousal communication and joint decision-making, the nature of the first sexual experience with spouse, experience of forced sex within marriage, inter-spousal violence, pregnancy experiences and outcomes, and contraceptive practice.

Same-sex, paid and forced sexual experiences: This was a second highly sensitive section in which respondents were asked a series of questions on their personal experience of several types of sexual encounters, for example, paid or exchange sex, forced sex perpetrated on the respondent and casual sex. In the case of male respondents, additional questions were asked about sex with a same-sex partner, relations with sex workers and married women (other than their wife for married males) and whether they had ever perpetrated forced sex. All married respondents were also asked about the experience of extra-marital sexual relations. Respondents who reported any of these experiences were probed about their age at their first experience of such a sexual encounter and the extent to which they had used condoms in these encounters.

Attitudes: This section probed respondents' views on pre-marital physical intimacy and wife beating.

Health and health seeking: This section collected information on respondents' experience of common health problems, specifically high fever and injury, as well as symptoms of genital infections in the three months preceding the interview. In addition, respondents were asked whether they had sought treatment for these health issues and, if so, from what source. Respondents' mental health in the last one month was assessed using the 12-item General Health Questionnaire, developed for use in field conditions (Goldberg, 1992).

Substance use and violence: A series of questions were asked about consumption of tobacco products, alcohol or drugs. In each case, questions were asked about use and frequency of use of such substances by family members 
and by the respondents themselves. Additional questions sought respondents' assessments of the frequency with which young people in their neighbourhoods engaged in violence (fights or beatings) and their own participation in such violence.

Programmes and participation: The final section of the questionnaire collected information on programmes available to young people in the village or neighbourhood in which they resided, and the extent to which youth participated in such programmes. In addition, rural respondents were asked about the role of panchayats in decisions affecting young people's lives. All respondents were asked about their participation in community activities, opinions about political processes, secular attitudes and participation in recent elections. Finally, respondents were asked to identify the most important problem facing youth in their village or neighbourhood.

Sealed envelope response: However carefully designed and culturally sensitive the survey questions may have been, the possibility that young people would deliberately withhold information about their sexual experiences in a face-to-face interview could not be discounted. Drawing from other research in the field, an anonymous reporting method was included in our survey to obtain responses to a single question: Have you ever had sex with anyone [for the unmarried]/Did you ever have sex with anyone before marriage [for the married]? Interviewers first explained the technique to respondents, noting in particular its confidential nature. The interviewer then gave each respondent a blank card and asked him or her to simply mark a " $\checkmark$ " or an "X" on the card to indicate that he/she had or had not experienced pre-marital sex. Once marked, the respondent placed the card inside an envelope provided by the interviewer; the envelope was sealed by the respondent and returned to the interviewer. Unique identification numbers linked the individual's questionnaire with his or her responses in the sealed envelope. Envelopes were opened only at the central office at the time of data entry.

Draft tools were extensively reviewed at meetings of the study's Technical Advisory Committee and were then translated into four languages (Hindi, Marathi, Tamil and Telugu), extensively pre-tested and finalised after appropriate modification. Copies of all these instruments are provided in the CD enclosed with this report.

\subsection{Study design and sample size estimation for individual interviews}

The Youth Survey was designed to provide estimates for the state as a whole, as well as for urban and rural areas for each of the four categories of respondents, namely married and unmarried young men and women, separately. The study was not designed to provide estimates at district or sub-district levels.

While arriving at sample size estimates, on the basis of the scarce available evidence, the following assumptions were made:

- $10 \%$ of unmarried young women would report the experience of pre-marital sexual relations;

- Among married men, $20 \%$ would report unsafe sexual relations (multiple partner sex or non-use of condoms, unintended pregnancy or experience of STI symptoms);

- The coefficient of variation was set at $10 \%$ (equivalent to fixing the absolute error at $20 \%$ of the true value and 95\% confidence interval);

- The non-response rate for the individual interviews was assumed to be $25-30 \%$;

- Design effect was assumed to be in the range of 1.5 to 2 .

The chances of finding an unmarried young man were greater than the chances of finding a married young man in a given household, and conversely, the chances of finding a married young woman were greater than the chances of finding an unmarried young woman. As a result, in the case of the male sample, our strategy was to estimate the number of households required to obtain the target number of married young men aged 15-29, that is, the harder to reach group of males. Similarly, in the case of the female sample, the strategy was to identify the total number of households required based on the target number of unmarried young women aged 15-24, again, the harder to reach group of females. 
Following from the assumptions described above, and in consultation with the study's Technical Advisory Committee, the required sample of each sub-group of youth was determined at 1,000 married young men, 1,250 unmarried young men, 1,250 married young women and 1,750 unmarried young women each for urban and rural areas, that is, a total sample size of 5,250 in each area ${ }^{2}$. However, our earlier experience suggested that because of the considerable mobility of youth, there was likely to be a shortfall in achieving these numbers. Hence, in Andhra Pradesh, Bihar and Rajasthan, the sample size in both rural and urban areas was revised to 1,200 married young men, 1,800 unmarried young men, 1,500 married young women and 2,100 unmarried young women, that is, a total sample size of 6,600 in each area. Moreover in Andhra Pradesh, because the ratio of unmarried to married young men in rural areas was smaller than that required by our sampling design, we further inflated the sample of married young men in rural areas to 1,313 in order to obtain a minimum of 1,800 unmarried men. Hence, the targeted sample size for rural Andhra Pradesh was 1,313 married young men, 1,800 unmarried young men, 1,500 married young women and 2,100 unmarried young women, that is, a total sample size of 6,713. In order to achieve the above-mentioned number of individual interviews, an estimated 31,544 households were required to be covered in Andhra Pradesh.

We further determined that a total of 300 primary sampling units (PSUs) — villages in rural areas and Census Enumeration Blocks (CEBs) in urban areas-divided into 150 female PSUs and 150 male PSUs, would be visited in order to conduct interviews in the required number of households. Thus, the average number of household interviews to be conducted in each rural PSU was calculated to be 181 among female PSUs and 69 among male PSUs. Corresponding averages for each urban PSU were 99 and 73, respectively.

\subsubsection{Sample selection strategy}

The study treated rural and urban areas of each state as independent sampling domains and, therefore, drew sample areas independently for each of these two domains. In order to avoid potential risks associated with interviewing both women and men from the same PSU, we decided to conduct interviews in separate PSUs for female and male respondents, that is, interviews with young women in 150 PSUs and young men in the remaining 150 PSUs. These 150 PSUs were further divided equally into rural and urban areas, that is, 75 for rural respondents and 75 for urban respondents. Within each sampling domain, a systematic, multi-stage sampling design was adopted. Sample selection procedures differed somewhat in rural and urban areas, as described below.

\subsection{1.a Selection of households in rural areas}

In rural areas, the 2001 Census list of villages served as the sampling frame for the selection of villages. This list was stratified using four variables, namely, region, village size, proportion of the population belonging to scheduled castes and scheduled tribes, and female literacy. At the first level of stratification, the state of Andhra Pradesh was stratified into six contiguous geographical regions, with districts classified into these regions as follows:

Region I : Srikakulam, Vizianagaram, Vishakhapatnam

Region II : East Godavari, West Godavari, Krishna, Guntur

Region III : Prakasam, Nellore

Region IV : Cuddapah, Kurnool, Anantapur, Chittoor

Region V : Adilabad, Nizamabad, Medak, Rangaredi, Mahbubnagar, Nalgonda

Region VI : Karimnagar, Warangal, Khammam

${ }^{2}$ In estimating the number of households required, the study used the age-sex-marital status distributions observed in rural and urban areas, respectively, in the 2001 Census. The following formula was used to estimate sample size:

$$
\text { Coefficient of Variation } \begin{aligned}
(c v) & =\sqrt{\frac{q}{n p}} \\
n & =\frac{q}{c v^{2} p}
\end{aligned}
$$

In order to obtain the actual number of respondents, the above numbers were multiplied by the design effect and a factor ' $K$ ' ( $1+$ the non-response rate $)$. 
In each region, villages were further stratified by village size and the percentage of the population belonging to scheduled castes or scheduled tribes. Table 1.1 gives detailed information on the stratification scheme in rural areas along with the population in each stratum. The last level of stratification was implicit for all strata, consisting of an ordering of villages within each stratum by level of female literacy, ordered alternatively in increasing and decreasing level of female literacy (obtained from the 2001 Census Village Directory).

The sample in rural areas was selected in two stages. At the first stage of selection, villages were selected systematically from the stratified list arranged as described above, with selection probability proportional to size (PPS). The 150 PSUs thus selected were then ordered by district and taluka codes and numbered from 1 to 150. Odd-numbered PSUs were designated for interviews with young men and even numbered PSUs for interviews with young women. In the case of male PSUs, selected PSUs containing fewer than 75 households were then linked to one or more adjoining villages so that the PSU had approximately 75 households. In the case of female PSUs, selected PSUs containing fewer than 200 households were linked to one or more adjoining villages so that the PSU had approximately 200 households. Those containing more than 300 and fewer than 601 households were segmented into two approximately equal parts, and one was chosen randomly for the survey. In the case of even larger villages, that is, those containing more than 600 households, segments of 150-200 households were made and numbered in a clockwise manner. Two segments were then selected using probability proportional to size.

Table 1.1: Sampling stratification scheme

Details of the stratification used for sampling, Andhra Pradesh (rural), 2007-08

\begin{tabular}{|c|c|c|c|c|}
\hline \multirow{2}{*}{$\begin{array}{l}\text { Stratum } \\
\text { number }\end{array}$} & \multicolumn{3}{|c|}{ Stratification variables } & \multirow{2}{*}{$\begin{array}{c}\text { Total } \\
\text { population }\end{array}$} \\
\hline & Region & $\begin{array}{l}\text { Village size (number of } \\
\text { residential households) }\end{array}$ & $\begin{array}{c}\text { Percent of SC/ST } \\
\text { population }\end{array}$ & \\
\hline 1 & 1 & $\leq 360$ & $\mathrm{NU}$ & $2,044,116$ \\
\hline 2 & 1 & $>360$ & $\leq 10$ & $2,077,716$ \\
\hline 3 & 1 & $>360$ & $>10$ & $2,052,439$ \\
\hline 4 & 2 & $\leq 1400$ & $\leq 26$ & $3,134,426$ \\
\hline 5 & 2 & $\leq 1400$ & $>26$ & $3,242,614$ \\
\hline 6 & 2 & $>1400$ & $\leq 20$ & $3,232,217$ \\
\hline 7 & 2 & $>1400$ & $>20$ & $3,185,578$ \\
\hline 8 & 3 & $\leq 1000$ & $\mathrm{NU}$ & $2,322,693$ \\
\hline 9 & 3 & $>1000$ & $\mathrm{NU}$ & $2,322,928$ \\
\hline 10 & 4 & $\leq 840$ & $\leq 22$ & $2,695,660$ \\
\hline 11 & 4 & $\leq 840$ & $>22$ & $2,525,725$ \\
\hline 12 & 4 & $>840$ & $\leq 18$ & $2,517,360$ \\
\hline 13 & 4 & $>840$ & $>18$ & $2,617,505$ \\
\hline 14 & 5 & $\leq 580$ & $\leq 27$ & $3,428,465$ \\
\hline 15 & 5 & $\leq 580$ & $>27$ & $3,415,277$ \\
\hline 16 & 5 & $>580$ & $\leq 22$ & $3,424,597$ \\
\hline 17 & 5 & $>580$ & $>22$ & $3,307,448$ \\
\hline 18 & 6 & $\leq 600$ & $\mathrm{NU}$ & $2,308,633$ \\
\hline 19 & 6 & $>600$ & $\leq 26$ & $2,571,885$ \\
\hline 20 & 6 & $>600$ & $>26$ & $2,593,438$ \\
\hline Total & NA & NA & NA & $55,020,720$ \\
\hline
\end{tabular}

Note: The level of female literacy (2001 Census) was used for implicit stratification. Villages with less than 50 households in the 2001 census were excluded from the sampling frame. NA: Not applicable. NU: Not used for stratification. SC: Scheduled caste. ST: Scheduled tribe. ${ }^{1} 2001$ Census population 
The rural domain sampling fraction for a particular category, that is, the probability of selecting an eligible respondent of a particular category in rural Andhra Pradesh $\left(f^{R}\right)$, was computed as:

$$
f^{R}=\frac{n^{R}}{N^{R}}
$$

where

$n^{R}=$ number of eligible respondents in a particular category to be interviewed (target number of interviews as described before), and

$N^{R}=$ projected rural population of eligible respondents in the state as of October 1, 2006.

The probability of selecting a PSU from rural Andhra Pradesh $\left(f_{1}^{R}\right)$ was computed as:

$$
f_{1}^{R}=\frac{a \times v_{i}}{\sum v_{i}}
$$

where

$a=$ number of PSUs selected from rural areas for the particular category,

$v_{i}=$ population of the $\mathrm{i}^{\text {th }}$ PSU, and

$\sum v_{i}=$ total rural population of the state.

A complete mapping and household listing operation was carried out in each selected PSU (or in selected segments or linked villages as appropriate). This list of households provided the necessary frame for selecting households at the second stage. Mapping and listing were conducted by teams, each comprising one mapper and one lister. Households to be interviewed were selected with equal probability from the list using systematic sampling.

The probability of selecting a household from a selected rural PSU $\left(f_{2}^{R}\right)$ was calculated as:

$$
f_{2}^{R}=\frac{f^{R}}{f_{1}^{R}}
$$

No replacement for selected households was allowed even if a selected household could not be contacted after several attempts.

All the sampling fractions $\left(f^{R}, f_{1}^{R}, f_{2}^{R}\right)$ described above were computed separately for male and female PSUs on the basis of the target sample of married males and unmarried females, respectively.

Because we expected more unmarried than married males in our age groups, we needed to visit fewer households to obtain the required number of unmarried compared to married males. Likewise, because we expected more married than unmarried females, we needed to visit fewer households to obtain the required number of married compared to unmarried females. Appropriate intervals were computed to operationalise each of these selection processes.

\subsection{1.b Selection of households in urban areas}

In selecting the urban sample, the 2001 Census list of wards (each consisting of several CEBs of 100-200 households) provided the sampling frame. For operational convenience, the Youth Study first determined male PSUs (equivalent to a CEB) and followed this with the selection of female PSUs (another CEB) in CEBs adjacent to male CEBs. As a result, half the total required number of PSUs was first selected.

In urban areas, the 2001 Census list of wards was first ordered by district, and within each district, by level of female literacy. The sample was then selected in three stages. At the first stage of selection, 75 wards were selected systematically with probability proportional to size. At the second stage, within each selected ward, CEBs were 
arranged by their administrative number and one CEB (designated as a male PSU) was selected using probability proportional to size. For each selected male CEB, an adjacent CEB was chosen to represent the female PSU in the same ward.

The urban domain sampling fraction for a particular category, that is, the probability of selecting an eligible respondent of a particular category in urban Andhra Pradesh $\left(f^{U}\right)$, was computed as:

where

$$
f^{U}=\frac{n^{U}}{N^{U}}
$$

$n^{U}=$ number of eligible respondents in a particular category to be interviewed in urban areas (target number of interviews as described before), and

$N^{U}=$ projected urban population of eligible respondents in the state as of October 1, 2006.

The probability of selecting a ward (or section) from urban Andhra Pradesh $\left(f_{1}^{U}\right)$ was computed as:

where

$$
f_{1}^{U}=\frac{a \times w_{i}}{\sum w_{i}}
$$

$a=$ number of wards selected from urban areas for the particular category,

$w_{i}=$ population of $\mathrm{i}^{\text {th }}$ ward, and

$\sum w_{i}=$ total urban population of the state.

The probability of selecting a CEB from a selected ward $\left(f_{2}^{U}\right)$ was computed as:

where

$$
f_{2}^{U}=\frac{c_{i}}{\sum c_{i}}
$$

$c_{i}=$ population of $\mathrm{i}^{\text {th }}$ CEB from a selected ward, and
$\sum c_{i}=$ total population of the selected ward.

A complete mapping and household listing operation was carried out in each selected PSU and the resulting list provided the necessary frame for selecting households at the third stage. Households to be interviewed were selected with equal probability from the list using systematic sampling. In some CEBs the number of households listed was smaller than the minimum expected number of households, and in such cases, a part of an adjacent CEB was listed.

The probability of selecting a household from a selected urban PSU $\left(f_{3}^{U}\right)$ was calculated as:

$$
f_{3}^{U}=\frac{f^{U}}{f_{1}^{U} \times f_{2}^{U}}
$$

As in the case of rural areas, (a) no replacement of selected households was allowed under any circumstances; (b) all sampling fractions $\left(f^{U}, f_{1}^{U}, f_{2}^{U}, f_{3}^{U}\right)$ were computed separately for male and female PSUs on the basis of the target sample of married males and unmarried females, respectively; and (c) appropriate intervals were computed to enable us to select fewer households for the interview of unmarried compared to married males and married compared to unmarried females. 


\subsubsection{Selection of individual respondents within selected households}

In each PSU, households to be interviewed were selected by systematic sampling. The value of the interval (between one selected household and the next) was determined in advance to ensure a self-weighing design. As mentioned earlier, fewer households needed to be selected in order to obtain our sample of unmarried males and married females. Hence, further intervals were computed, using the target sample for unmarried males and married females.

Within each selected household, no more than one married and one unmarried respondent was interviewed, resulting in a maximum of two interviews from any household. In case more than one respondent from a single category was found in the household, one respondent was selected randomly using the Kish table. ${ }^{3}$ No replacement of the respondent thus selected was allowed.

\subsubsection{Sample weights}

In Andhra Pradesh, the sample was weighted at the level of the sampling domain, that is, urban and rural males and females, respectively, making for a total of four sampling domains. In order to consider differential non-response rates in different geographical areas, non-response rates were calculated in smaller sub-domains of 2-3 PSUs within each domain. If $W_{D i}$ is the design weight for the $i^{\text {th }}$ domain $(\mathrm{i}=1 \ldots 4)$ and $R_{H i j}$ is the response rate for households in the $\mathrm{j}^{\text {th }}$ sub-domain within the $\mathrm{i}^{\text {th }}$ domain, then the household weight for the $\mathrm{j}^{\text {th }}$ sub-domain within the $\mathrm{i}^{\text {th }}$ domain $\left(W_{H i j}\right)$ was calculated as follows:

$$
W_{H i j}=\frac{W_{D i}}{R_{H i j}}
$$

where $W_{D i}$ was calculated as the inverse of the probability of selecting an eligible married male in urban and rural male domains, respectively; and similarly, of selecting an eligible unmarried female in urban and rural female domains.

Weights were also calculated for eligible married males and unmarried females, denoted by $W_{E i j}$ and calculated as follows:

$$
W_{E i j}=\frac{W_{D i}}{R_{H i j} \times R_{E i j} \times K_{i j}}
$$

where

$$
\begin{aligned}
R_{E i j}= & \text { response rate for married males or unmarried females in the } \mathrm{j}^{\text {th }} \text { sub-domain within the } \mathrm{i}^{\text {th }} \text { domain, } \\
& \text { and } \\
K_{i j}= & \text { probability that a married male or an unmarried female is selected by the Kish table procedure in } \\
& \text { the } \mathrm{j}^{\text {th }} \text { sub-domain within the } \mathrm{i}^{\text {th }} \text { domain. }
\end{aligned}
$$

The design weight described above was also used in the case of unmarried males and married females in each domain. Also, since the survey did not attempt to interview an unmarried male or a married female in all selected

\footnotetext{
${ }^{3}$ The probability of selection of individuals in rural areas is $\left(f^{R} / K_{i}^{R}\right)$ and in urban areas $\left(f^{U} / K_{i}^{U}\right)$, where $K_{i}^{R}$, and $K_{i}^{U}$ denote the number of individuals of the specified category (married and unmarried males and females, respectively) in the $i^{\text {th }}$ selected household in rural and urban areas, respectively.
} 
households, an additional interval needed to be incorporated in the weight calculation. Hence, weights for eligible unmarried males and married females, denoted by $W_{E i j}$ were calculated using the following equation:

$$
W_{E i j}=\frac{W_{D i}}{R_{H i j} \times R_{E i j} \times K_{i j}} \times I_{i}
$$

where $I_{i}$ is the interval at which selected households were assigned for the interview of a married female (in female PSUs) or an unmarried male (in male PSUs) in the $i^{\text {th }}$ domain.

The weights were then normalised so that the total number of cases was unchanged after weighting. Hence, the normalised weights for households and eligible respondents were:

$$
\begin{aligned}
W_{H i j}^{\prime} & =\frac{\sum n_{i j}}{\sum W_{H i j} \times n_{i j}} \times W_{H i j} \\
W_{E i j}^{\prime} & =\frac{\sum n_{i j}}{\sum W_{E i j} \times n_{i j}} \times W_{E i j}
\end{aligned}
$$

where $n_{i j}$ refers to the number of completed interviews in the $\mathrm{j}^{\text {th }}$ sub-domain within the $\mathrm{i}^{\text {th }}$ domain.

In order to provide estimates for all young males or females (married and unmarried), multiplication factors were computed for married and unmarried males and females (four categories) in urban and rural areas, which, when multiplied with existing individual weights, provided the combined weights for the male and female samples, respectively. For example, the multiplication factor for the male sample $\left(M_{k}^{l}\right)$ was computed as follows:

where

$$
M_{k}^{l}=\frac{\frac{p_{k}^{l}}{p^{l}}}{\frac{s_{k}^{l}}{s^{l}}}
$$

\footnotetext{
$p_{k}^{l}=$ number of eligible male respondents of category $\mathrm{k}$ (married or unmarried) in the $\mathrm{l}^{\text {th }}$ area (urban or rural),

$p^{l}=$ number of eligible male respondents in the $1^{\text {th }}$ area (urban or rural),

$s_{k}^{l} \quad=$ number of completed interviews with male respondents from category $\mathrm{k}$ (married or unmarried) in the $1^{\text {th }}$ area (urban or rural), and

$s^{l} \quad=$ number of completed interviews with male respondents in the $1^{\text {th }}$ area (urban or rural).
}

Similar fractions were computed for the female sample.

\subsection{Recruitment, training and fieldwork}

Some 71 young men and 48 young women underwent interviewer training and 22 underwent training for mapping and house listing exercises. On the basis of their performance, 74 youth were recruited as field investigators. In addition, some 18 individuals were recruited for mapping and house-listing exercises.

Training of interviewers was conducted jointly by principal investigators from IIPS and the Population Council. House-listing staff underwent a four-day training, during which trainees were familiarised with house-listing procedures in both classroom and field situations. Training for field investigators for the main survey lasted three 
weeks. It included lectures and interactive sessions on a range of issues, such as the sexual and reproductive health situation of youth in India, an overview of gender issues, ethical issues in research, violence against women and mental health as well as detailed explanations of sex and contraception. Efforts were also made to enable trainees to overcome their own inhibitions about discussing sexual and reproductive health matters. Trainees were provided opportunities to ask questions via an anonymous drop-box; questions were then answered in the course of training. Trainees were familiarised with each module of the questionnaire, complicated concepts and questions and their underlying rationale. Role-plays and mock interviews were conducted in reference to each module. Towards the end of the training programme, field practice sessions were organised in which trainees were taken to a village and an urban slum setting and asked to conduct interviews. The training team monitored each trainee's progress on a regular basis and selected as interviewers only those trainees who demonstrated full understanding of the questionnaire as well as the ability to ask questions appropriately and record responses accurately.

Interviewers were initially divided into 12 teams, six each to interview young men and women, respectively. Subsequently, because several interviewers found other employment opportunities, remaining interviewers were regrouped into 8 teams, four each to interview young men and women, respectively. Male interviewers interviewed young men and female interviewers interviewed young women. Each team comprised one field editor to take care of field editing, back-checks and quality control of interviews; and one supervisor, responsible for the overall management of fieldwork and team-related logistics as well as assisting in field editing and back-checking. Interviewer and supervisor/editor manuals were prepared, translated into Telugu and provided to each team member as appropriate. These manuals clarified the meaning and appropriate coding of every question in the questionnaire.

Research officers were deputed to oversee fieldwork and ensure that correct survey procedures were followed and data quality maintained. Principal investigators from IIPS and the Population Council made monthly or bi-monthly visits to monitor and supervise data collection operations. Each team filled quality control sheets regularly, giving the team, research officers and coordinators a quick view of the quality of ongoing fieldwork. These control sheets were designed to provide information on response rates in each PSU covered, track sensitive issue reporting and interviewer performance.

\subsection{Ethical considerations}

As this was the first such study in India in which sensitive sexual and reproductive experiences of young people were sought in a survey situation, it was unclear how youth respondents and community members would react. At the same time, it was clear that if youth participated in the interview, its content was likely to prompt questions and problems for which support would be requested. A number of ethical issues arose which influenced the design and implementation of the Youth Study.

First, to address our concern that if interviews with young women and men were conducted in the same PSU, it could lead to teasing, harassment, harm to girls' reputations and even violence, we decided that the study would be undertaken in one set of PSUs for young men and in a completely different set for young women. Likewise, we also ensured that two unmarried brothers or sisters, two married brothers or sisters or two sisters- or brothers-in- law would not be interviewed from the same household in case such a practice caused conflict within the family. Hence, just one individual from any category was selected for interview in each household. In case both a married and an unmarried individual were selected from a particular household, interviews were conducted separately but simultaneously.

Second, youth themselves contributed-albeit indirectly - to the development of the questionnaire. In the course of our pre-survey qualitative phase, youth and key informants informed our study teams of various youth behaviours; youth described the ways in which they referred to various sensitive behaviours. In order to minimise discomfort during questioning, the scenarios and terminologies described by youth themselves were adapted for use in the most sensitive parts of our questionnaire. 
Third, interviewers underwent extensive training in ethical issues. Emphasis was laid on explaining the content of the questionnaire, respondents' right to refuse to participate or answer any question and informed consent. At the same time, we trained interviewers on how to ask sensitive questions-regarding sexual experience, domestic violence and forced sex in particular-in empathetic and non-judgemental ways, and emphasised the importance of offering to refer those in need to appropriate nearby organisations.

Fourth, before entering a PSU, teams were instructed to apprise community leaders of the study and seek their support for its implementation in the community. This step ensured that community support was forthcoming and enabled team members to build rapport within the community easily. We note that despite the sensitive nature of the questions, not a single PSU in Andhra Pradesh refused permission to our teams on the grounds of study content.

Fifth, even though consent was sought from each individual to be interviewed, in the case of unmarried youth aged 15-17, consent was also sought from a parent or guardian.

Sixth, all questionnaires were anonymous and names were never recorded. In order to preserve the confidentiality of the respondent or the parent/guardian, signature on the consent form was optional; however, the interviewer was required to sign that she or he had explained the content of the consent form to the respondent or parent. Consent forms were detached and stored separately from the questionnaires.

Seventh, every effort was made to maintain privacy in the course of the interview. Interviewers were permitted to skip to relatively non-sensitive sections in case the interview was observed by parents or other family members. If possible, particularly in the case of young men, interviews were held outside the home-often in a nearby field-in order to ensure privacy. Each team was trained to assign one interviewer to conduct parallel discussion sessions with bystanders, thereby providing privacy to the interview. This proved particularly useful in the case of interviews with young women. Finally, interviewers were instructed that if privacy could not be ensured, the interview must be terminated without asking sensitive questions. Due to these strategies, few interviews had to be terminated for want of privacy and in no case was a young respondent's privacy breached.

Eighth, the study team realised that this was perhaps one of the first opportunities many youth would have to discuss intimate matters and that respondents might request information on sexual and reproductive issues or seek counselling or treatment for a health problem. In each state, therefore, we approached non-governmental organisations (NGOs) that conduct youth- or health-related activities at the district level and sought their consent for referring any youth in need to their organisation. Many NGOs agreed, and youth (and some adults) in need were later referred to these organisations, along with an indication that the individual had been part of the Youth Study. At the same time, research officers and team members themselves built rapport with public health authorities and referred to their facilities those who preferred to seek public services, again, along with the information that the individual had been part of the Youth Study.

Finally, many youth were in need of information on sexual and reproductive health matters. On occasion, interviewers themselves responded to their questions. In addition, easy to read booklets (for example, the Neeli Rangu Pustakam prepared by TARSHI) were distributed to youth who requested them. In total, some 500 booklets were distributed.

\subsection{Data processing}

All completed questionnaires were sent to the project office at IIPS, Mumbai for editing and data processing. Completed questionnaires were rechecked and further edited in the office for omissions and consistency. Responses to open-ended questions were scrutinised and common responses were provided codes. The edited data were entered using a special software package that was developed using CSPro 3.0. Data were entered twice by different entry operators to minimise entry problems. The raw data were validated and cleaned to remove possible inconsistencies. The analysis of data was carried out using SPSS 14.0. 


\subsection{Interview outcomes}

Table 1.2 provides the outcome of household interviews by type of PSU (male or female) and residence. In all, of the 32,348 households selected for interview, 3\% could not be contacted because the house was vacant or the

Table 1.2: Results of household interviews

Percent distribution of surveyed households by results of interviews, according to residence (unweighted), Andhra Pradesh, 2007-08

\begin{tabular}{|c|c|c|c|c|c|c|}
\hline \multirow[t]{2}{*}{ Results of interviews } & \multicolumn{2}{|c|}{ All PSUs } & \multicolumn{2}{|c|}{ Male PSUs } & \multicolumn{2}{|c|}{ Female PSUs } \\
\hline & Percent & Number & Percent & Number & Percent & Number \\
\hline \multicolumn{7}{|c|}{ Combined } \\
\hline a. Interview completed & 96.2 & 31,123 & 95.6 & 10,484 & 96.5 & 20,639 \\
\hline $\begin{array}{l}\text { b. No respondent or no competent } \\
\text { respondent at home at the time of visit }\end{array}$ & 0.3 & 81 & 0.6 & 66 & 0.1 & 15 \\
\hline $\begin{array}{l}\text { c. Entire household absent for extended } \\
\text { period of time }\end{array}$ & 1.5 & 483 & 2.2 & 241 & 1.1 & 242 \\
\hline d. Refused & 0.2 & 53 & 0.3 & 30 & 0.1 & 23 \\
\hline e. Dwelling vacant/destroyed/not found & 1.2 & 401 & 1.2 & 132 & 1.3 & 269 \\
\hline f. Address not a dwelling & 0.1 & 41 & 0.1 & 7 & 0.2 & 34 \\
\hline g. Other & 0.5 & 166 & 0.1 & 11 & 0.7 & 155 \\
\hline Total households selected & 100.0 & 32,348 & 100.0 & 10,971 & 100.0 & 21,377 \\
\hline Response rate (HRR) & 99.0 & & 99.0 & & 99.1 & \\
\hline \multicolumn{7}{|c|}{ Urban } \\
\hline a. Interview completed & 96.1 & 12,439 & 95.3 & 5,204 & 96.6 & 7,235 \\
\hline $\begin{array}{l}\text { b. No respondent or no competent } \\
\text { respondent at home at the time of visit }\end{array}$ & 0.3 & 43 & 0.7 & 40 & 0.0 & 3 \\
\hline $\begin{array}{l}\text { E. Entire household absent for extended } \\
\text { period of time }\end{array}$ & 1.5 & 196 & 1.9 & 106 & 1.2 & 90 \\
\hline d. Refused & 0.3 & 44 & 0.5 & 25 & 0.3 & 19 \\
\hline e. Dwelling vacant/destroyed/not found & 1.2 & 159 & 1.3 & 73 & 1.1 & 86 \\
\hline f. Address not a dwelling & 0.2 & 25 & 0.1 & 5 & 0.3 & 20 \\
\hline g. Other & 0.3 & 44 & 0.2 & 9 & 0.5 & 35 \\
\hline Total households selected & 100.0 & 12,950 & 100.0 & 5,462 & 100.0 & 7,488 \\
\hline Response rate (HRR) & 99.1 & & 98.6 & & 99.2 & \\
\hline \multicolumn{7}{|c|}{ Rural } \\
\hline a. Interview completed & 96.3 & 18,684 & 95.8 & 5,280 & 96.5 & 13,404 \\
\hline $\begin{array}{l}\text { b. No respondent or no competent } \\
\text { respondent at home at the time of visit }\end{array}$ & 0.2 & 38 & 0.5 & 26 & 0.1 & 12 \\
\hline $\begin{array}{l}\text { c. Entire household absent for extended } \\
\text { period of time }\end{array}$ & 1.5 & 287 & 2.5 & 135 & 1.1 & 152 \\
\hline d. Refused & 0.0 & 9 & 0.1 & 5 & 0.0 & 4 \\
\hline e. Dwelling vacant/destroyed/not found & 1.2 & 242 & 1.1 & 59 & 1.3 & 183 \\
\hline f. Address not a dwelling & 0.1 & 16 & 0.0 & 2 & 0.1 & 14 \\
\hline g. Other & 0.6 & 122 & 0.0 & 2 & 0.9 & 120 \\
\hline Total households selected & 100.0 & 19,398 & 100.0 & 5,509 & 100.0 & 13,889 \\
\hline Response rate (HRR) & 99.2 & & 99.4 & & 99.0 & \\
\hline
\end{tabular}

Note: The household response rate $(H R R)$ was calculated as: $H R R=(a / a+b+d+g)^{*} 100$. PSU: Primary sampling unit. 
Table 1.3: Results of eligible respondent interviews

Percent distribution of eligible respondents by results of interviews, according to residence (unweighted), Andhra Pradesh, 2007-08

\begin{tabular}{|c|c|c|c|c|c|c|c|c|c|c|c|c|}
\hline \multirow[t]{3}{*}{ Results of interviews } & \multicolumn{4}{|c|}{ Combined } & \multicolumn{4}{|c|}{ Urban } & \multicolumn{4}{|c|}{ Rural } \\
\hline & \multicolumn{2}{|c|}{ Married } & \multicolumn{2}{|c|}{ Unmarried } & \multicolumn{2}{|c|}{ Married } & \multicolumn{2}{|c|}{ Unmarried } & \multicolumn{2}{|c|}{ Married } & \multicolumn{2}{|c|}{ Unmarried } \\
\hline & Percent & Number & Percent & Number & Percent & Number & Percent & Number & Percent & Number & Percent & Number \\
\hline \multicolumn{13}{|c|}{ Men (15-24) } \\
\hline a. Interview completed & 89.4 & 1,405 & 90.3 & 2,077 & 92.0 & 648 & 91.7 & 1,132 & 87.2 & 757 & 88.6 & 945 \\
\hline b. Interview partially completed & 0.3 & 5 & 0.1 & 2 & 0.4 & 3 & 0.0 & 0 & 0.2 & 2 & 0.2 & 2 \\
\hline c. Respondent not at home & 8.8 & 138 & 6.8 & 157 & 6.1 & 43 & 4.7 & 58 & 10.9 & 95 & 9.3 & 99 \\
\hline d. Respondent refused & 1.0 & 15 & 1.0 & 22 & 1.1 & 8 & 1.3 & 16 & 0.8 & 7 & 0.6 & 6 \\
\hline e. Respondent's parent refused & 0.1 & 1 & 1.0 & 23 & 0.1 & 1 & 1.5 & 19 & 0.0 & 0 & 0.4 & 4 \\
\hline f. Respondent incapacitated & 0.3 & 4 & 0.8 & 18 & 0.1 & 1 & 0.6 & 8 & 0.3 & 3 & 0.9 & 10 \\
\hline g. No reason given & 0.3 & 4 & 0.1 & 2 & 0.0 & 0 & 0.1 & 1 & 0.5 & 4 & 0.1 & 1 \\
\hline Total selected & 100.0 & 1,572 & 100.0 & 2,301 & 100.0 & 704 & 100.0 & 1,234 & 100.0 & 868 & 100.0 & 1,067 \\
\hline Response rate (IRR) & 89.4 & & 90.3 & & 92.0 & & 91.7 & & 87.2 & & 88.6 & \\
\hline \multicolumn{13}{|c|}{ Women (15-24) } \\
\hline a. Interview completed & 90.5 & 2,330 & 94.2 & 2,518 & 90.7 & 1,037 & 94.0 & 1,089 & 90.3 & 1,293 & 94.4 & 1,429 \\
\hline b. Interview partially completed & 0.5 & 12 & 0.2 & 6 & 0.5 & 6 & 0.3 & 4 & 0.4 & 6 & 0.1 & 2 \\
\hline c. Respondent not at home & 8.1 & 209 & 3.6 & 96 & 7.5 & 86 & 3.9 & 45 & 8.6 & 123 & 3.4 & 51 \\
\hline d. Respondent refused & 0.6 & 16 & 0.4 & 10 & 1.0 & 11 & 0.7 & 8 & 0.3 & 5 & 0.1 & 2 \\
\hline e. Respondent's parent refused & 0.0 & 0 & 0.4 & 10 & 0.0 & 0 & 0.7 & 8 & 0.0 & 0 & 0.1 & 2 \\
\hline f. Respondent incapacitated & 0.1 & 2 & 1.2 & 31 & 0.1 & 1 & 0.4 & 5 & 0.1 & 1 & 1.7 & 26 \\
\hline g. No reason given & 0.2 & 6 & 0.1 & 2 & 0.2 & 2 & 0.0 & 0 & 0.3 & 4 & 0.1 & 2 \\
\hline Total selected & 100.0 & 2,575 & 100.0 & 2,673 & 100.0 & 1,143 & 100.0 & 1,159 & 100.0 & 1,432 & 100.0 & 1,514 \\
\hline Response rate (IRR) & 90.5 & & 94.2 & & 90.7 & & 94.0 & & 90.3 & & 94.4 & \\
\hline
\end{tabular}

Note: The individual response rate (IRR) was calculated as: $I R R=(a / a+b+c+d+e+f+g){ }^{\star} 100$.

entire household was absent over an extended period of time. In total, however, the response to the household questionnaire was high: $96-97 \%$ in male and female PSUs. A total of 12,439 and 18,684 interviews were completed in urban and rural areas, respectively. Response rates in urban and rural areas were identical. We note that hardly any of the selected households in urban and rural areas refused to be interviewed.

Table 1.3 presents similar findings with regard to interviews with eligible respondents. In Andhra Pradesh, 8,330 interviews were completed: 1,405 with married young men, 2,077 with unmarried young men, 2,330 with married young women and 2,518 with unmarried young women. Response rates for individual interviews were in the range of $89-94 \%$. The response rates for young women, both married and unmarried, were slightly higher than those for young men (91-94\% versus $89-90 \%)$. Response rates were slightly higher for young men in urban than rural settings (92\% versus $87-89 \%$ ), but response rates were identical for young women in rural and urban settings. The main reason for non-response was that the respondent was not at home, ranging from 4-8\% among young women to $7-9 \%$ among young men. We attribute the low refusal rates ( $1 \%$ or less) to efforts described earlier that were implemented for ethical reasons, which, at the same time, enabled the development of considerable rapport and trust between study communities and our interview teams. 


\subsection{Structure of the report}

This report is structured as follows. Chapter 2 provides a socio-demographic profile of the surveyed population and respondents, and facilities available to the rural population. Chapters 3,4 and 5 discuss young people's educational attainment patterns, economic and non-economic activity experiences and media exposure, respectively. Chapter 6 discusses growing up issues, including young people's relationships with parents and peers. Chapters 7 and 8 focus, respectively, on young people's autonomy and gender role attitudes, and awareness of sexual and reproductive health matters. Chapter 9 describes the formation of pre-marital romantic relationships and pre-marital sexual experience with romantic and non-romantic partners. Chapter 10 discusses the transition to marriage and experiences in early married life. Chapter 11 presents information on health and health seeking behaviours and substance use. Chapter 12 focuses on civic and political participation and related attitudes. A summary of each chapter (3-12) is provided at its conclusion. Finally, Chapter 13 offers recommendations for programmes and research.

In view of the heterogeneity of youth by sex, marital status and rural-urban residence, in each chapter, tables are presented that describe findings, separately, on the situation of married and unmarried young men and women residing in urban and rural areas, respectively. In order to provide information on all youth in Andhra Pradesh, we provide findings for all young men and women aged 15-24. We excluded married young men aged 25-29) to enable comparison.

All means, medians and percentages indicated in tables have been weighted using normalised weights for the total population. However, in order to show the total number of youth interviewed, unweighted numbers of respondents (Ns) are provided in each table. Because numbers are unweighted and percentages are weighted, we caution readers against deriving numbers based on percentages provided in tables. 


\section{Profile of surveyed communities,} households and youth

This chapter presents a summary of the community-level characteristics of the rural areas surveyed as well as household- and respondent-level profiles of the surveyed population. First, using data drawn from the community questionnaire, it describes the rural communities in which the survey was undertaken in terms of village size, agricultural land holding and access to facilities. Thereafter, drawing on data from the household questionnaire, the chapter profiles surveyed households in terms of socio-demographic and housing characteristics, agricultural land holding and economic status. Comparisons are drawn throughout between the distribution of the population as recorded in the present survey and that reported by the 2001 Census of India (Office of the Registrar General and Census Commissioner, 2001a; 2001b) as well as the most recent NFHS (IIPS and Macro International, 2007a). Finally, we present the socio-demographic characteristics of youth respondents and their parents drawn from individual questionnaires.

\subsection{Profile of rural communities surveyed}

This section provides a profile of the rural PSUs (150 selected villages and 3 link villages) in which the survey was conducted. It should be noted that as sampling of rural PSUs was conducted with the probability of selection proportional to size, the proportion of large villages in our sample is likely to have been greater than the proportion of such villages in Andhra Pradesh as a whole. However, because the selection of villages was made from a list of villages stratified by size, the effect of using the probability proportional to size sampling technique on village size distribution is likely to have been small.

As indicated in Table 2.1, 6\% of the villages surveyed were relatively small in size (less than 1,000 persons, or about 200 households), while $38 \%$ were large villages (5,000 or more persons, or about 1,000 households). The remaining $56 \%$ of the surveyed villages were of medium size (1,000-4,999 persons). The majority of villages surveyed (67\%) contained more than 1,000 hectares of agricultural land. More than half of all agricultural land was irrigated in $71 \%$ of the surveyed villages.

Table 2.2 presents data regarding access to a variety of facilities among the rural population surveyed. Findings show that the median distance to the nearest town was 15 kilometres from the village of residence. Furthermore, $41 \%$ of the rural population had access to all-weather roads in their village; the median distance to the nearest all-weather road was 2 kilometres. Access to banks was limited: just 32\% of the rural population had a bank located in their village. In contrast, four-fifths had a post office located in their village.

Primary schools were available in the village of residence of virtually the entire population (98\%). Middle, secondary and higher secondary schools were progressively less likely to be available within the village of residence $(81 \%, 67 \%$ and $26 \%$ of rural residents, respectively, resided in a village containing these facilities). The median distance to the nearest higher secondary school was 5 kilometres. Colleges and technical institutions were much less accessible; just $11 \%$ and $7 \%$ respectively, of the population had a college and technical institution within the village and median distances to the nearest college and the technical institution were 10 and 18 kilometers, respectively. 
Table 2.1: Profile of surveyed villages

Percent distribution of surveyed villages and residents by village size and agricultural land holding, Andhra Pradesh (rural), 2007-08

\begin{tabular}{|c|c|c|c|c|}
\hline \multirow[t]{2}{*}{ Village characteristics } & \multicolumn{2}{|c|}{ Villages } & \multicolumn{2}{|c|}{ Residents } \\
\hline & Percent & Number & Percent & Number \\
\hline \multicolumn{5}{|c|}{ Current population (no. of persons) } \\
\hline Less than 1,000 & 5.9 & 9 & 4.3 & 3,373 \\
\hline $1,000-4,999$ & 56.2 & 86 & 56.3 & 43,365 \\
\hline $5,000-9,999$ & 21.6 & 33 & 22.3 & 16,345 \\
\hline 10,000 or more & 16.3 & 25 & 17.2 & 11,809 \\
\hline \multicolumn{5}{|c|}{ Size of agricultural land (hectares) } \\
\hline Less than 500 & 11.8 & 18 & 10.4 & 8,804 \\
\hline $500-999$ & 18.3 & 28 & 18.0 & 11,496 \\
\hline $1,000-4,999$ & 49.0 & 75 & 49.7 & 37,892 \\
\hline 5,000 and more & 18.3 & 28 & 19.5 & 14,852 \\
\hline \multicolumn{5}{|c|}{ Proportion of irrigated agricultural land owned } \\
\hline Less than $25 \%$ & 9.8 & 15 & 9.3 & 8,155 \\
\hline $25-49 \%$ & 17.6 & 27 & 18.5 & 12,416 \\
\hline $50-74 \%$ & 20.9 & 32 & 20.6 & 16,383 \\
\hline $75 \%$ or more & 50.3 & 77 & 50.4 & 37,441 \\
\hline Total & 100.0 & 153 & 100.0 & 74,892 \\
\hline
\end{tabular}

Note: All Ns are unweighted. Column totals may not equal 100\% or the total number due to missing cases or "don't know" responses.

As far as health facilities were concerned, anganwadis were available in the village of residence of almost the entire population surveyed (98\%). Nearly three-fifths $(56 \%)$ of the population surveyed had a sub-centre within the village. Primary health centres and community health centres were far less likely to be available within the village; only $20 \%$ and $15 \%$ of the population resided in a village containing a primary health centre and a community health centre, respectively. The median distances to the nearest primary health centre and community health centre were 6 and 8 kilometers, respectively, highlighting that access to government health facilities remains somewhat difficult in rural areas of Andhra Pradesh. Private clinics (including those practising Indian systems of medicine and homoeopathy) were, however, available within the village of residence to almost one-third of the rural population (31\%), and the median distance to such a facility was 7 kilometers. Private hospitals were more distantly located: they were accessible in the village of residence to just $12 \%$ of rural residents and median distance to the nearest private hospital was 10 kilometres.

The availability of civic organisations and entertainment or sports facilities was also assessed. One-third of the population resided in villages containing at least one club or mandal. Almost three-fifths lived in villages containing a community hall (57\%) and 35\% lived in villages containing a playground. Entertainment facilities were less likely to be available; for example, few lived in villages containing a cinema theatre (15\%), a drama theatre (9\%) or a video parlour (7\%) and distances to the nearest such facilities ranged from 8 to 16 kilometres. Not a single village contained a sports club and the median distance to a sports club was 25 kilometers from the village of residence. 
Table 2.2: Proximity of study residents to selected facilities

Percentage of residents covered by the survey by distance from the nearest facility/service, Andhra Pradesh (rural), 2007-08

\begin{tabular}{|c|c|c|c|c|c|c|c|}
\hline \multirow[t]{2}{*}{ Nearest facility/service } & \multicolumn{6}{|c|}{$\%$ of residents } & \multirow{2}{*}{$\begin{array}{c}\text { Median } \\
\text { distance to } \\
\text { nearest facility/ } \\
\text { service }(\mathbf{k m})\end{array}$} \\
\hline & $\begin{array}{l}\text { Within } \\
\text { village }\end{array}$ & $\begin{array}{r}<2 \\
\mathbf{k m}\end{array}$ & $\begin{array}{l}2-5 \\
\mathrm{~km}\end{array}$ & $\begin{array}{l}6-9 \\
\text { km }\end{array}$ & $\begin{array}{c}10-19 \\
\text { km }\end{array}$ & $\begin{array}{c}20 \\
\mathrm{~km} \text { or } \\
\text { more }\end{array}$ & \\
\hline Town & NA & 0.0 & 11.4 & 11.8 & 38.7 & 33.7 & 15.0 \\
\hline District headquarters & NA & 0.0 & 0.0 & 0.7 & 9.5 & 89.1 & 60.0 \\
\hline Railway station & 4.4 & 0.6 & 8.5 & 7.6 & 24.5 & 54.3 & 20.0 \\
\hline Transport service to other places & 27.9 & 1.3 & 19.1 & 16.3 & 21.0 & 14.5 & 6.0 \\
\hline All-weather road & 41.2 & 3.2 & 20.6 & 8.9 & 11.0 & 15.0 & 2.0 \\
\hline Post office & 79.9 & 3.0 & 9.5 & 3.3 & 3.8 & 0.4 & $\mathrm{NC}$ \\
\hline Bank & 31.7 & 0.8 & 31.1 & 19.9 & 13.6 & 2.8 & 4.0 \\
\hline \multicolumn{8}{|l|}{ Educational facilities } \\
\hline Primary school & 98.3 & 0.6 & 1.1 & 0.0 & 0.0 & 0.0 & $\mathrm{NC}$ \\
\hline Middle school & 81.1 & 2.1 & 8.0 & 3.4 & 4.8 & 0.6 & $\mathrm{NC}$ \\
\hline Secondary school & 66.6 & 2.7 & 19.5 & 6.7 & 4.0 & 0.5 & $\mathrm{NC}$ \\
\hline Higher secondary school & 26.0 & 1.2 & 24.3 & 21.8 & 23.5 & 3.2 & 5.0 \\
\hline College & 10.9 & 0.7 & 13.5 & 20.3 & 37.3 & 16.8 & 10.0 \\
\hline Technical school/college & 6.6 & 0.8 & 4.1 & 11.8 & 29.3 & 47.4 & 18.0 \\
\hline Ashram school & 3.1 & 0.0 & 12.3 & 7.9 & 27.6 & 45.8 & 18.0 \\
\hline Madarsa & 5.2 & 0.0 & 5.0 & 7.6 & 21.2 & 53.6 & 27.0 \\
\hline Any of the above & 99.4 & 0.0 & 0.6 & 0.0 & 0.0 & 0.0 & NC \\
\hline \multicolumn{8}{|l|}{ Health facilities } \\
\hline ICDS (anganwadi) & 98.4 & 0.0 & 0.0 & 0.0 & 0.6 & 0.0 & $\mathrm{NC}$ \\
\hline Sub-centre & 55.6 & 0.8 & 20.4 & 12.5 & 8.6 & 2.1 & $\mathrm{NC}$ \\
\hline Primary health centre & 20.4 & 0.0 & 27.2 & 20.8 & 25.1 & 6.5 & 6.0 \\
\hline Community health centre & 15.2 & 0.0 & 21.2 & 18.9 & 24.8 & 18.6 & 8.0 \\
\hline Government dispensary & 9.5 & 0.0 & 15.2 & 17.9 & 31.5 & 25.9 & 12.0 \\
\hline Government hospital & 6.2 & 0.0 & 11.1 & 19.6 & 29.6 & 33.5 & 13.0 \\
\hline Private clinic, including ISMH & 31.4 & 0.6 & 14.7 & 12.0 & 24.7 & 16.5 & 7.0 \\
\hline Private hospital & 12.0 & 0.0 & 14.2 & 19.8 & 29.8 & 23.7 & 10.0 \\
\hline Any of the above & 99.4 & 0.0 & 0.0 & 0.0 & 0.6 & 0.0 & $\mathrm{NC}$ \\
\hline Club/Mandal & 35.2 & NA & NA & NA & NA & NA & NA \\
\hline \multicolumn{8}{|l|}{ Entertainment/sports facilities } \\
\hline Community hall & 57.1 & 0.0 & 10.8 & 11.0 & 12.2 & 7.4 & $\mathrm{NC}$ \\
\hline Playground & 35.3 & 0.0 & 14.1 & 18.3 & 20.6 & 10.9 & 6.0 \\
\hline Sports club & 0.0 & 0.0 & 7.9 & 12.5 & 22.4 & 56.0 & 25.0 \\
\hline Video parlour & 6.7 & 0.0 & 11.4 & 16.7 & 28.6 & 32.5 & 15.0 \\
\hline Cinema theatre & 14.6 & 0.0 & 17.5 & 26.4 & 28.8 & 11.9 & 8.0 \\
\hline Drama theatre & 9.0 & 0.0 & 8.1 & 12.6 & 23.8 & 41.5 & 16.0 \\
\hline Any of the above & 65.2 & 0.0 & 9.9 & 13.3 & 9.0 & 1.8 & $\mathrm{NC}$ \\
\hline
\end{tabular}

Note: ICDS: Integrated Child Development Services. ISMH: Indian Systems of Medicine and Homoeopathy. NA: Not applicable. NC: Median cannot be calculated. 


\subsection{Profile of the household population: Age-sex distribution}

Age and sex distributions play an important role in the study of demographic processes. Details of the age and sex distribution of the de jure population in the survey area are presented in Table 2.3. Corresponding distributions from the 2001 Census are provided to enable comparison.

The age distribution was typical of a population in which fertility has been declining, with relatively smaller proportions in both younger ( $0-9$ years) and older (60+ years) age groups. For example, $17 \%$-of the population was aged 0-9 years, and this proportion was somewhat lower than that reported in the 2001 census (21\%). At the other end of the age spectrum, the population aged $60+$ years represented $9 \%$ of the population, compared to $8 \%$ as reported in the 2001 census. This pattern is observed in both rural and urban areas. Data from consecutive NFHS surveys confirm that the total fertility rate declined from 2.6 in 1992-93 to 2.3 in 1998-99 and further to 1.8 in 2005-06, that is, a 31\% decline in the last 13 years (IIPS and Macro International, 2007a). Sample Registration System data also show a decline in the total fertility rate from 2.4 in 1999 to 1.9 in 2007 (Office of the Registrar General, India, 2003; 2008b).

With regard to the youth population, the distribution of the population suggests that at the time of the survey, $10 \%$ were aged $10-14$ years, and $9 \%$ were aged $15-19$ years and $20-24$ years, respectively. A total of $18 \%$ of the

Table 2.3: Distribution of the surveyed population by age and sex

Percent distribution of the surveyed population by age and sex, according to residence, Andhra Pradesh, 2007-08, and population distribution as reported in the 2001 Census for Andhra Pradesh

\begin{tabular}{|c|c|c|c|c|c|c|}
\hline \multirow[t]{2}{*}{ Age (years) (\%) } & \multicolumn{3}{|c|}{ Youth Study, 2007-08 } & \multicolumn{3}{|c|}{ Census, 2001} \\
\hline & Total & Male & Female & Total & Male & Female \\
\hline \multicolumn{7}{|c|}{ Combined } \\
\hline Below 1 & 1.5 & 1.6 & 1.5 & 1.4 & 1.4 & 1.4 \\
\hline $1-4$ & 6.2 & 6.3 & 6.1 & 7.4 & 7.4 & 7.3 \\
\hline $5-9$ & 9.7 & 9.9 & 9.5 & 11.8 & 11.9 & 11.7 \\
\hline $10-14$ & 10.3 & 10.6 & 10.0 & 11.5 & 11.7 & 11.2 \\
\hline $15-19$ & 8.9 & 8.9 & 9.0 & 9.9 & 10.3 & 9.6 \\
\hline $20-24$ & 9.0 & 8.9 & 9.1 & 9.1 & 9.0 & 9.2 \\
\hline $25-29$ & 9.1 & 8.3 & 9.9 & 8.9 & 8.4 & 9.4 \\
\hline $30-34$ & 7.8 & 7.9 & 7.6 & 7.4 & 7.4 & 7.3 \\
\hline $35-39$ & 8.2 & 8.2 & 8.2 & 7.4 & 7.3 & 7.6 \\
\hline $40-44$ & 6.0 & 6.3 & 5.7 & 5.7 & 6.1 & 5.3 \\
\hline $45-49$ & 5.9 & 6.1 & 5.8 & 5.0 & 5.1 & 4.9 \\
\hline $50-54$ & 4.6 & 4.7 & 4.6 & 4.0 & 4.2 & 3.9 \\
\hline $55-59$ & 3.4 & 3.0 & 3.7 & 2.8 & 2.5 & 3.0 \\
\hline $60-64$ & 3.8 & 3.8 & 3.8 & 3.1 & 2.9 & 3.2 \\
\hline $65-69$ & 2.3 & 2.3 & 2.4 & 1.8 & 1.6 & 2.0 \\
\hline $70-74$ & 1.8 & 1.9 & 1.7 & 1.5 & 1.5 & 1.5 \\
\hline 75 and above & 1.4 & 1.4 & 1.4 & 1.2 & 1.1 & 1.3 \\
\hline Age not stated & 0.0 & 0.0 & 0.0 & 0.2 & 0.2 & 0.2 \\
\hline Number & 125,953 & 63,011 & 62,942 & $76,210,007$ & $38,527,413$ & $37,682,594$ \\
\hline Median age (years) & 27.0 & 26.0 & 27.0 & 23.5 & 23.2 & 23.8 \\
\hline Sex ratio, all ages ${ }^{1}$ & 1,018 & NA & NA & 978 & NA & NA \\
\hline Sex ratio, age $0-6$ years $^{1}$ & 970 & NA & NA & 961 & NA & NA \\
\hline
\end{tabular}

Cont'd on next page... 
Table 2.3: (Cont'd)

\begin{tabular}{|c|c|c|c|c|c|c|}
\hline \multirow[t]{2}{*}{ Age (years) (\%) } & \multicolumn{3}{|c|}{ Youth Study, 2007-08 } & \multicolumn{3}{|c|}{ Census, 2001} \\
\hline & Total & Male & Female & Total & Male & Female \\
\hline \multicolumn{7}{|c|}{ Urban } \\
\hline Below 1 & 1.4 & 1.3 & 1.5 & 1.1 & 1.1 & 1.1 \\
\hline $1-4$ & 6.1 & 6.2 & 6.0 & 6.9 & 7.0 & 6.9 \\
\hline $5-9$ & 9.5 & 9.8 & 9.1 & 10.6 & 10.6 & 10.6 \\
\hline $10-14$ & 9.9 & 10.1 & 9.8 & 11.4 & 11.4 & 11.3 \\
\hline $15-19$ & 9.0 & 8.8 & 9.1 & 11.1 & 11.1 & 11.2 \\
\hline $20-24$ & 10.0 & 9.9 & 10.1 & 10.3 & 10.2 & 10.4 \\
\hline $25-29$ & 10.1 & 9.6 & 10.6 & 9.5 & 9.0 & 10.0 \\
\hline $30-34$ & 8.6 & 8.7 & 8.6 & 7.7 & 7.8 & 7.6 \\
\hline $35-39$ & 8.5 & 8.7 & 8.3 & 7.8 & 7.6 & 7.9 \\
\hline $40-44$ & 6.3 & 6.6 & 6.0 & 5.8 & 6.3 & 5.3 \\
\hline $45-49$ & 5.8 & 5.8 & 5.7 & 5.0 & 5.3 & 4.7 \\
\hline $50-54$ & 4.4 & 4.5 & 4.3 & 3.8 & 4.1 & 3.5 \\
\hline $55-59$ & 3.3 & 3.2 & 3.4 & 2.5 & 2.4 & 2.5 \\
\hline $60-64$ & 3.0 & 3.0 & 3.0 & 2.5 & 2.4 & 2.6 \\
\hline $65-69$ & 1.8 & 1.6 & 2.0 & 1.4 & 1.3 & 1.6 \\
\hline $70-74$ & 1.3 & 1.3 & 1.3 & 1.2 & 1.1 & 1.2 \\
\hline 75 and above & 1.0 & 1.0 & 1.1 & 1.0 & 0.9 & 1.2 \\
\hline Age not stated & 0.0 & 0.0 & 0.0 & 0.3 & 0.3 & 0.2 \\
\hline Number & 51,061 & 25,743 & 25,318 & $20,808,940$ & $10,590,209$ & $10,218,731$ \\
\hline Median age (years) & 26.0 & 26.0 & 26.0 & 23.4 & 23.4 & 23.4 \\
\hline Sex ratio, all ages ${ }^{1}$ & 989 & NA & NA & 965 & NA & NA \\
\hline Sex ratio, age $0-6$ years ${ }^{1}$ & 965 & NA & NA & 955 & NA & NA \\
\hline \multicolumn{7}{|c|}{ Rural } \\
\hline Below 1 & 1.6 & 1.6 & 1.5 & 1.5 & 1.5 & 1.5 \\
\hline $1-4$ & 6.2 & 6.4 & 6.1 & 7.5 & 7.6 & 7.5 \\
\hline $5-9$ & 9.8 & 10.0 & 9.6 & 12.2 & 12.3 & 12.1 \\
\hline $10-14$ & 10.4 & 10.8 & 10.1 & 11.5 & 11.8 & 11.2 \\
\hline $15-19$ & 8.9 & 8.9 & 8.9 & 9.5 & 9.9 & 9.1 \\
\hline $20-24$ & 8.7 & 8.5 & 8.8 & 8.7 & 8.6 & 8.7 \\
\hline $25-29$ & 8.7 & 7.8 & 9.6 & 8.6 & 8.1 & 9.1 \\
\hline $30-34$ & 7.4 & 7.6 & 7.3 & 7.3 & 7.3 & 7.3 \\
\hline $35-39$ & 8.1 & 8.0 & 8.2 & 7.3 & 7.1 & 7.4 \\
\hline $40-44$ & 5.9 & 6.2 & 5.6 & 5.7 & 6.0 & 5.3 \\
\hline $45-49$ & 6.0 & 6.1 & 5.8 & 5.0 & 5.0 & 5.0 \\
\hline $50-54$ & 4.7 & 4.8 & 4.7 & 4.1 & 4.2 & 4.0 \\
\hline $55-59$ & 3.4 & 3.0 & 3.8 & 2.9 & 2.6 & 3.2 \\
\hline $60-64$ & 4.1 & 4.0 & 4.1 & 3.3 & 3.1 & 3.4 \\
\hline $65-69$ & 2.6 & 2.5 & 2.6 & 1.9 & 1.7 & 2.1 \\
\hline $70-74$ & 2.0 & 2.2 & 1.8 & 1.7 & 1.7 & 1.6 \\
\hline 75 and above & 1.5 & 1.5 & 1.6 & 1.3 & 1.2 & 1.3 \\
\hline Age not stated & 0.0 & 0.0 & 0.0 & 0.1 & 0.2 & 0.1 \\
\hline Number & 74,892 & 37,268 & 37,624 & $55,401,067$ & $27,937,204$ & $27,463,863$ \\
\hline Median age (years) & 27.0 & 26.0 & 27.0 & 23.5 & 23.1 & 24.0 \\
\hline Sex ratio, all ages ${ }^{1}$ & 1,029 & NA & NA & 983 & NA & NA \\
\hline Sex ratio, age $0-6$ years $^{1}$ & 971 & NA & NA & 963 & NA & NA \\
\hline
\end{tabular}

Note: All Ns are unweighted. NA: Not applicable. ${ }^{1}$ Sex ratio is defined as the number of females per 1,000 males. 
population was aged 15-24 years, almost identical to that reported in the 2001 Census (19\%) (Office of the Registrar General and Census Commissioner, 2001a).

Overall, the sex ratio of the de jure population of the state was 1,018 females per 1,000 males, considerably higher than that observed in the 2001 census (978). While the urban sex ratio was somewhat higher than that observed in the 2001 census (989 and 965, respectively), the rural sex ratio observed in the Youth Study was considerably higher (1,029 and 983, respectively).

The child sex ratio of the surveyed population was 970 females per 1,000 males aged $0-6$, relatively similar to that reported in the 2001 census (961). The ratios observed in rural and urban areas were, likewise, somewhat similar to that observed in the 2001 census (971 and 963, respectively, in rural areas; 965 and 955, respectively, in urban areas).

\subsection{Profile of the household population: Marital status}

Table 2.4 presents the marital status distribution of the surveyed population, classified by age, residence and sex. A comparison with the marital status distribution as obtained in the 2001 Census (data not shown in tabular form) suggests that proportions never married have increased somewhat in the period 2001-08 (Office of the Registrar General and Census Commissioner, 2001a). The currently married include both those who have married and cohabited with their spouse as well as those for whom cohabitation has not been initiated.

Findings suggest wide gender differences in marriage age distributions, notably between the ages of 15 and 29. Of those aged 15-19 years, just $1.3 \%$ of young men compared to $26 \%$ of young women were currently married. This increased to $28 \%$ and $76 \%$, respectively, for those aged $20-24$ years and further to $72 \%$ and $92 \%$ for those aged 25-29 years. Patterns were similar for both rural and urban areas, but larger percentages of young men were married among those aged 20-29 years in rural than in urban areas and larger percentages of young women were married in each group up to age 25 in rural than in urban areas.

Table 2.4 also provides estimates of the singulate mean age at marriage (SMAM), calculated from the age-specific proportion of never-married individuals obtained in the household survey. As suggested above, the singulate mean age at marriage was considerably higher among the male population compared to the female: 25 and 20 years, respectively. Differences were also observed by rural-urban residence, with the singulate mean age at marriage about two years higher among urban compared to rural males and females. Findings also suggest that women tended to marry men who were an average of five years older than themselves.

In order to assess age at marriage among those married more recently, the Youth Study household questionnaire asked specifically about marriages that had taken place in the three years prior to the interview among the household's usual residents at that time. Table 2.5 suggests that the median age at marriage for those who married in the recent past was 23 years among young men and 18 years among young women. Rural-urban differences were similar to those observed above, that is median ages at marriage were two years higher among urban youth than their rural counterparts. Findings also show that large proportions of both young women and men had married before the legal minimum age at marriage. More than one quarter of young women (28\%) had married before they were aged 18, that is, the legal minimum age at marriage for females. As many as one in five young men had married before they were 21 , the legal minimum age at marriage for males. Rural youth were twice as likely as urban youth to have married before the minimum legal age at marriage: $32 \%$ of rural females compared to $16 \%$ of urban females had married before they were 18 years of age. Among young men, $23 \%$ and $11 \%$ of respondents from rural and urban areas, respectively, had married before they reached 21 years. 
Table 2.4: Marital status of the surveyed population

Percent distribution of the surveyed population aged 6 years and above by marital status and sex, according to residence, Andhra Pradesh, 2007-08

\begin{tabular}{|c|c|c|c|c|c|c|}
\hline \multirow[t]{3}{*}{ Age (years) (\%) } & \multicolumn{6}{|c|}{ Marital status } \\
\hline & \multicolumn{3}{|c|}{ Male } & \multicolumn{3}{|c|}{ Female } \\
\hline & $\begin{array}{c}\text { Never } \\
\text { married }\end{array}$ & $\begin{array}{l}\text { Currently } \\
\text { married }^{1}\end{array}$ & $\begin{array}{l}\text { Separated/ } \\
\text { divorced/ } \\
\text { widowed }\end{array}$ & $\begin{array}{c}\text { Never } \\
\text { married }\end{array}$ & $\begin{array}{l}\text { Currently } \\
\text { married }^{1}\end{array}$ & $\begin{array}{l}\text { Separated/ } \\
\text { divorced/ } \\
\text { widowed }\end{array}$ \\
\hline \multicolumn{7}{|c|}{ Combined } \\
\hline $6-9$ & 99.5 & 0.4 & 0.0 & 99.5 & 0.5 & 0.0 \\
\hline $10-14$ & 99.6 & 0.4 & 0.0 & 99.4 & 0.5 & 0.1 \\
\hline $15-19$ & 98.6 & 1.3 & 0.1 & 73.5 & 26.0 & 0.6 \\
\hline $20-24$ & 71.1 & 28.4 & 0.4 & 21.3 & 75.8 & 2.9 \\
\hline $25-29$ & 26.7 & 72.4 & 0.8 & 4.1 & 91.6 & 4.3 \\
\hline 30 and above & 1.3 & 93.9 & 4.8 & 0.8 & 73.2 & 26.0 \\
\hline Total & 40.3 & 57.2 & 2.5 & 29.7 & 56.6 & 13.8 \\
\hline SMAM $^{2}$ (years) & \multicolumn{3}{|c|}{24.5} & \multicolumn{3}{|c|}{19.6} \\
\hline \multicolumn{7}{|c|}{ Urban } \\
\hline $6-9$ & 99.5 & 0.4 & 0.1 & 99.6 & 0.4 & 0.0 \\
\hline $10-14$ & 99.8 & 0.2 & 0.1 & 99.5 & 0.4 & 0.1 \\
\hline $15-19$ & 99.2 & 0.7 & 0.1 & 82.7 & 16.9 & 0.4 \\
\hline $20-24$ & 83.7 & 16.0 & 0.3 & 34.4 & 63.3 & 2.3 \\
\hline $25-29$ & 40.7 & 58.6 & 0.7 & 7.6 & 89.0 & 3.4 \\
\hline 30 and above & 2.3 & 94.2 & 3.5 & 1.1 & 74.1 & 24.8 \\
\hline Total & 44.0 & 54.2 & 1.8 & 32.4 & 55.0 & 12.6 \\
\hline SMAM $^{2}$ (years) & \multicolumn{3}{|c|}{26.2} & \multicolumn{3}{|c|}{21.0} \\
\hline \multicolumn{7}{|c|}{ Rural } \\
\hline $6-9$ & 99.6 & 0.4 & 0.0 & 99.5 & 0.5 & 0.0 \\
\hline $10-14$ & 99.5 & 0.5 & 0.0 & 99.4 & 0.5 & 0.1 \\
\hline $15-19$ & 98.4 & 1.5 & 0.1 & 70.1 & 29.2 & 0.6 \\
\hline $20-24$ & 65.8 & 33.8 & 0.5 & 16.0 & 80.8 & 3.2 \\
\hline $25-29$ & 20.5 & 78.6 & 0.9 & 2.8 & 92.6 & 4.6 \\
\hline 30 and above & 0.9 & 93.8 & 5.2 & 0.7 & 72.8 & 26.5 \\
\hline Total & 38.9 & 58.3 & 2.8 & 28.7 & 57.1 & 14.1 \\
\hline SMAM $^{2}$ (years) & \multicolumn{3}{|c|}{23.8} & \multicolumn{3}{|c|}{19.1} \\
\hline
\end{tabular}

Note: Row totals may not equal 100\% due to missing cases or "don't know" responses. ${ }^{1}$ Includes both those who are currently married and cohabiting as well as those who have not yet initiated coha]bitation. ${ }^{2}$ SMAM: Singulate mean age at marriage (for those whose first marriage occurred between the ages of 6 and 55 years). 
Table 2.5: Age at marriage of usual residents of households

Age at marriage of usual residents of surveyed households who were married in the three years preceding the interview, according to residence, Andhra Pradesh, 2007-08

\begin{tabular}{|c|c|c|c|}
\hline Age at marriage & Combined & Urban & Rural \\
\hline \multicolumn{4}{|c|}{$\begin{array}{l}\text { Median age at marriage of usual residents married in the } 3 \\
\text { years preceding the interview (years) }\end{array}$} \\
\hline Male & 23.0 & 25.0 & 23.0 \\
\hline Female & 18.0 & 20.0 & 18.0 \\
\hline \multicolumn{4}{|l|}{ Of those married in last 3 years, males married (\%): } \\
\hline Before age 18 & 2.8 & 1.2 & 3.3 \\
\hline Before age 21 & 19.9 & 10.9 & 22.7 \\
\hline $\begin{array}{l}\text { Of those married in last } 3 \text { years, females married } \\
\text { before age } 18(\%)\end{array}$ & 27.6 & 16.4 & 31.5 \\
\hline
\end{tabular}

\subsection{Profile of the household population: Educational attainment}

Table 2.6 shows the percent distribution of the surveyed population aged 6 years and above by educational level and median years of schooling according to sex, age and residence. Findings highlight relatively low levels of educational attainment in the state. For example, about two-fifths of the population aged 6 years and above had no formal education. More females than males (47\% versus $29 \%$ ) fell into this group. Rural-urban differences were also wide: $23 \%$ of the urban population compared to $44 \%$ of the rural population had never been to school. Reaffirming the low levels of educational attainment in the state, findings also indicate that just $10 \%$ of the total population had received 12 or more years of education. Gender and rural-urban differences remained evident: $14 \%$ and $7 \%$ of males and females, and $22 \%$ and $6 \%$ of the urban and rural population, respectively, had reached this level of education. The median years of schooling was 5 years for males and 2 years for females. Rural-urban differences show that years of schooling completed was on average four years higher in the urban compared to the rural population ( 7 and 3 years, respectively).

\subsection{Profile of the household population: Work participation}

Table 2.7 presents the percentage of the surveyed population aged 6 years and above reported to have been working in the seven days prior to interview according to sex and residence. While $54 \%$ of the total population was reported as working, a larger percentage of males than females $(67 \%$ and $41 \%$, respectively) and a larger proportion of the rural than urban population ( $58 \%$ and $43 \%$, respectively) were working. These disparities are attributable to the vast differences in work participation observed among rural and urban females ( $48 \%$ and $21 \%$, respectively). In comparison, percentages of working males were similar in urban and rural areas (64\% and $68 \%$, respectively). A positive association between age and work was observed: 10\% of those aged 10-14 reported working, compared with $39 \%$ of those aged $15-19,60 \%$ in the $20-24$ year age group and continued increases thereafter (reaching $73 \%$ and $71 \%$ among those aged $25-29$ and 30 and above, respectively). This positive association was steeper among males than females. 
Table 2.6: Educational attainment

Percent distribution of the surveyed population aged 6 years and above by educational level and median years of schooling, according to age, sex and residence, Andhra Pradesh, 2007-08

\begin{tabular}{|c|c|c|c|c|c|c|}
\hline \multirow[t]{2}{*}{ Age (years) } & \multicolumn{4}{|c|}{ Completed years of schooling (\%) } & \multirow{2}{*}{$\begin{array}{c}\text { No. of } \\
\text { persons }\end{array}$} & \multirow{2}{*}{$\begin{array}{l}\text { Median } \\
\text { years of } \\
\text { schooling }\end{array}$} \\
\hline & None $^{1}$ & $1-7$ & $8-11$ & $\begin{array}{c}12 \\
\text { and above }\end{array}$ & & \\
\hline \multicolumn{7}{|c|}{ Combined } \\
\hline \multicolumn{7}{|l|}{ Total } \\
\hline $6-9$ & 20.7 & 79.3 & 0.0 & 0.0 & 9,708 & 2.0 \\
\hline $10-14$ & 5.7 & 71.4 & 22.8 & 0.0 & 12,931 & 6.0 \\
\hline $15-19$ & 12.5 & 20.2 & 52.3 & 15.0 & 11,415 & 9.0 \\
\hline $20-24$ & 22.5 & 24.8 & 27.3 & 25.3 & 11,654 & 9.0 \\
\hline $25-29$ & 32.9 & 23.7 & 25.1 & 18.1 & 11,606 & 7.0 \\
\hline 30 and above & 58.2 & 19.9 & 12.6 & 9.2 & 56,544 & $\mathrm{NC}$ \\
\hline Total & 38.4 & 31.8 & 19.3 & 10.4 & 113,861 & 4.0 \\
\hline \multicolumn{7}{|l|}{ Male } \\
\hline $6-9$ & 21.3 & 78.7 & 0.0 & 0.0 & 4,878 & 2.0 \\
\hline $10-14$ & 4.8 & 72.5 & 22.7 & 0.0 & 6,609 & 6.0 \\
\hline $15-19$ & 8.0 & 18.5 & 57.1 & 16.3 & 5,823 & 10.0 \\
\hline $20-24$ & 14.0 & 23.0 & 30.7 & 32.2 & 5,828 & 10.0 \\
\hline $25-29$ & 20.0 & 23.4 & 30.7 & 25.7 & 5,406 & 9.0 \\
\hline 30 and above & 45.0 & 23.4 & 17.4 & 14.0 & 28,267 & 4.0 \\
\hline Total & 29.2 & 33.5 & 23.0 & 14.2 & 56,812 & 5.0 \\
\hline \multicolumn{7}{|l|}{ Female } \\
\hline $6-9$ & 20.1 & 79.8 & 0.0 & 0.0 & 4,830 & 2.0 \\
\hline $10-14$ & 6.6 & 70.3 & 23.1 & 0.0 & 6,322 & 6.0 \\
\hline $15-19$ & 16.8 & 21.8 & 47.6 & 13.7 & 5,592 & 9.0 \\
\hline $20-24$ & 30.8 & 26.4 & 24.0 & 18.8 & 5,826 & 7.0 \\
\hline $25-29$ & 43.6 & 24.0 & 20.6 & 11.8 & 6,200 & 5.0 \\
\hline 30 and above & 71.4 & 16.4 & 7.9 & 4.3 & 28,277 & $\mathrm{NC}$ \\
\hline Total & 47.4 & 30.1 & 15.8 & 6.7 & 57,049 & 2.0 \\
\hline \multicolumn{7}{|c|}{ Urban } \\
\hline \multicolumn{7}{|l|}{ Total } \\
\hline $6-9$ & 19.0 & 80.9 & 0.0 & 0.0 & 3,911 & 2.0 \\
\hline $10-14$ & 3.2 & 73.3 & 23.5 & 0.0 & 5,088 & 6.0 \\
\hline $15-19$ & 7.0 & 14.8 & 55.0 & 23.1 & 4,576 & 10.0 \\
\hline $20-24$ & 11.1 & 19.3 & 28.5 & 41.1 & 5,095 & 10.0 \\
\hline $25-29$ & 17.2 & 18.5 & 29.5 & 34.7 & 5,155 & 10.0 \\
\hline 30 and above & 34.6 & 20.8 & 21.3 & 23.1 & 22,436 & 7.0 \\
\hline Total & 22.6 & 30.6 & 24.8 & 21.9 & 46,264 & 7.0 \\
\hline \multicolumn{7}{|l|}{ Male } \\
\hline $6-9$ & 20.0 & 79.9 & 0.0 & 0.0 & 2,029 & 2.0 \\
\hline $10-14$ & 3.2 & 73.9 & 22.8 & 0.0 & 2,591 & 6.0 \\
\hline $15-19$ & 5.4 & 14.1 & 57.2 & 23.2 & 2,289 & 10.0 \\
\hline $20-24$ & 7.1 & 18.7 & 28.4 & 45.9 & 2,561 & 10.0 \\
\hline $25-29$ & 9.6 & 17.1 & 30.9 & 42.3 & 2,461 & 10.0 \\
\hline 30 and above & 21.9 & 19.9 & 25.4 & 32.5 & 11,360 & 10.0 \\
\hline Total & 15.2 & 30.2 & 26.9 & 27.7 & 23,292 & 9.0 \\
\hline
\end{tabular}


Table 2.6: (Cont'd)

\begin{tabular}{|c|c|c|c|c|c|c|}
\hline \multirow[t]{2}{*}{ Age (years) } & \multicolumn{4}{|c|}{ Completed years of schooling (\%) } & \multirow{2}{*}{$\begin{array}{c}\text { No. of } \\
\text { persons }\end{array}$} & \multirow{2}{*}{$\begin{array}{l}\text { Median } \\
\text { years of } \\
\text { schooling }\end{array}$} \\
\hline & None $^{1}$ & $1-7$ & $8-11$ & $\begin{array}{c}12 \\
\text { and above }\end{array}$ & & \\
\hline \multicolumn{7}{|c|}{ Urban } \\
\hline \multicolumn{7}{|l|}{ Female } \\
\hline $6-9$ & 18.0 & 81.9 & 0.0 & 0.0 & 1,882 & 2.0 \\
\hline $10-14$ & 3.1 & 72.6 & 24.2 & 0.0 & 2,497 & 6.0 \\
\hline $15-19$ & 8.7 & 15.5 & 52.8 & 23.0 & 2,287 & 10.0 \\
\hline $20-24$ & 15.1 & 19.9 & 28.6 & 36.3 & 2,534 & 10.0 \\
\hline $25-29$ & 24.0 & 19.8 & 28.3 & 27.8 & 2,694 & 9.0 \\
\hline 30 and above & 47.6 & 21.6 & 17.2 & 13.5 & 11,076 & 4.0 \\
\hline Total & 30.1 & 31.0 & 22.7 & 16.1 & 22,972 & 6.0 \\
\hline \multicolumn{7}{|c|}{ Rural } \\
\hline \multicolumn{7}{|l|}{ Total } \\
\hline $6-9$ & 21.3 & 78.7 & 0.0 & 0.0 & 5,797 & 2.0 \\
\hline $10-14$ & 6.5 & 70.8 & 22.6 & 0.0 & 7,843 & 6.0 \\
\hline $15-19$ & 14.4 & 22.2 & 51.4 & 12.0 & 6,839 & 9.0 \\
\hline $20-24$ & 27.3 & 27.1 & 26.8 & 18.8 & 6,559 & 7.0 \\
\hline $25-29$ & 39.5 & 25.9 & 23.3 & 11.1 & 6,451 & 5.0 \\
\hline 30 and above & 66.4 & 19.6 & 9.6 & 4.3 & 34,108 & $\mathrm{NC}$ \\
\hline Total & 44.1 & 32.2 & 17.4 & 6.3 & 67,597 & 3.0 \\
\hline \multicolumn{7}{|l|}{ Male } \\
\hline $6-9$ & 21.8 & 78.2 & 0.0 & 0.0 & 2,849 & 2.0 \\
\hline $10-14$ & 5.3 & 72.1 & 22.6 & 0.0 & 4,018 & 6.0 \\
\hline $15-19$ & 9.0 & 20.1 & 57.1 & 13.7 & 3,534 & 9.0 \\
\hline $20-24$ & 16.9 & 24.9 & 31.7 & 26.4 & 3,267 & 9.0 \\
\hline $25-29$ & 24.7 & 26.3 & 30.6 & 18.2 & 2,945 & 7.0 \\
\hline 30 and above & 53.2 & 24.6 & 14.5 & 7.4 & 16,907 & $\mathrm{NC}$ \\
\hline Total & 34.4 & 34.7 & 21.5 & 9.3 & 33,520 & 5.0 \\
\hline \multicolumn{7}{|l|}{ Female } \\
\hline $6-9$ & 20.8 & 79.1 & 0.0 & 0.0 & 2,948 & 2.0 \\
\hline $10-14$ & 7.8 & 69.5 & 22.7 & 0.0 & 3,825 & 6.0 \\
\hline $15-19$ & 19.7 & 24.1 & 45.8 & 10.4 & 3,305 & 9.0 \\
\hline $20-24$ & 37.1 & 29.1 & 22.1 & 11.7 & 3,292 & 5.0 \\
\hline $25-29$ & 51.2 & 25.6 & 17.5 & 5.6 & 3,506 & $\mathrm{NC}$ \\
\hline 30 and above & 79.4 & 14.6 & 4.7 & 1.2 & 17,201 & $\mathrm{NC}$ \\
\hline Total & 53.4 & 29.8 & 13.4 & 3.4 & 34,077 & NC \\
\hline
\end{tabular}

Note: All Ns are unweighted. Row totals may not equal 100\% due to missing cases or "don't know" responses. NC: Median cannot be calculated as more than $50 \%$ had no formal education. ${ }^{1}$ Includes non-literate and literate with no formal schooling.

\subsection{Socio-demographic characteristics of households and heads of households}

Table 2.8 presents selected characteristics pertaining to households and their heads, according to residence, for all households as well as for those containing youth eligible for interview (that is, all young women aged 15-24 years, unmarried young men aged 15-24 years and married young men aged 15-29 years). 
Table 2.7: Work participation

Percentage of the surveyed population aged 6 years and above by work participation, according to age, sex and residence, Andhra Pradesh, 2007-08

\begin{tabular}{|l|c|c|c|c|c|c|c|c|c|}
\multirow{2}{*}{ Age (years) (\%) } & \multicolumn{3}{|c|}{ Combined } & \multicolumn{3}{c|}{ Urban } & \multicolumn{3}{c|}{ Rural } \\
\cline { 2 - 12 } & Total & Male & Female & Total & Male & Female & Total & Male & Female \\
\hline $6-9$ & 1.0 & 0.8 & 1.2 & 0.5 & 0.6 & 0.4 & 1.2 & 0.9 & 1.5 \\
$10-14$ & 10.2 & 9.7 & 10.7 & 4.0 & 5.2 & 2.8 & 12.3 & 11.2 & 13.4 \\
$15-19$ & 38.9 & 45.0 & 32.9 & 23.0 & 33.3 & 13.0 & 44.7 & 49.3 & 40.2 \\
$20-24$ & 59.6 & 76.8 & 43.2 & 44.7 & 67.8 & 21.8 & 65.7 & 80.6 & 51.8 \\
$25-29$ & 72.6 & 94.8 & 54.3 & 59.0 & 92.1 & 28.9 & 78.3 & 95.9 & 64.3 \\
30 and above & 70.6 & 89.1 & 52.2 & 58.2 & 88.2 & 27.5 & 74.9 & 89.4 & 60.5 \\
Total & $\mathbf{5 3 . 7}$ & $\mathbf{6 7 . 0}$ & $\mathbf{4 0 . 7}$ & $\mathbf{4 2 . 5}$ & $\mathbf{6 4 . 2}$ & $\mathbf{2 0 . 7}$ & $\mathbf{5 7 . 7}$ & $\mathbf{6 8 . 1}$ & $\mathbf{4 7 . 7}$ \\
\hline
\end{tabular}

Note: Work participation is defined as reported work activity in the seven days prior to interview.

Findings suggest that heads of all households and households with eligible youth were overwhelmingly male and half (50\%) were aged 45 years and above. Heads of all surveyed urban households were somewhat younger than heads of all surveyed rural households: for example, the age of the head of household was 45 years or more among $45 \%$ of urban households compared to $52 \%$ of rural households. Among households that contained youth eligible for interview in the Youth Study, no differences were observed (the head of household was aged 45 years or more among half of both urban and rural households).

Table 2.8: Socio-demographic characteristics of households and heads of households

Percent distribution of all surveyed households and households containing youth eligible for interview by selected socio-demographic characteristics of heads of households, household size and type of family, according to residence, Andhra Pradesh, 2007-08

\begin{tabular}{|c|c|c|c|c|c|c|}
\hline \multirow{2}{*}{$\begin{array}{l}\text { Socio-demographic } \\
\text { characteristics (\%) }\end{array}$} & \multicolumn{2}{|c|}{ Combined } & \multicolumn{2}{|c|}{ Urban } & \multicolumn{2}{|c|}{ Rural } \\
\hline & $\begin{array}{c}\text { All } \\
\text { households }\end{array}$ & $\begin{array}{l}\text { Households } \\
\text { with youth }\end{array}$ & $\begin{array}{c}\text { All } \\
\text { households }\end{array}$ & $\begin{array}{l}\text { Households } \\
\text { with youth }\end{array}$ & $\begin{array}{c}\text { All } \\
\text { households }\end{array}$ & $\begin{array}{l}\text { Households } \\
\text { with youth }\end{array}$ \\
\hline $\begin{array}{l}\text { Sex of household head } \\
\text { Male }\end{array}$ & 86.9 & 89.9 & 87.3 & 88.0 & 86.7 & 90.6 \\
\hline Female & 13.1 & 10.1 & 12.7 & 12.0 & 13.3 & 9.4 \\
\hline $\begin{array}{l}\text { Current age of househo } \\
\text { (years) }\end{array}$ & & & & & & \\
\hline Below 25 & 2.7 & 7.3 & 2.8 & 6.5 & 2.7 & 7.6 \\
\hline $25-34$ & 19.7 & 24.6 & 21.9 & 24.4 & 19.0 & 24.7 \\
\hline $35-44$ & 27.7 & 18.5 & 30.4 & 19.7 & 26.8 & 18.1 \\
\hline $45-54$ & 23.2 & 29.6 & 23.1 & 31.5 & 23.2 & 29.0 \\
\hline 55 and above & 26.6 & 19.9 & 21.8 & 17.8 & 28.4 & 20.7 \\
\hline Religion of household $h$ & 867 & 850 & 83. & 811 & 880 & 864 \\
\hline Muslim & 6.9 & 8.1 & 10.7 & 12.5 & 5.5 & 6.5 \\
\hline Christian & 6.3 & 6.8 & 6.1 & 6.3 & 6.4 & 7.0 \\
\hline Buddhist/Neo-Buddhist & 0.0 & 0.0 & 0.0 & 0.0 & 0.0 & 0.0 \\
\hline Other $^{1}$ & 0.1 & 0.0 & 0.2 & 0.1 & 0.0 & 0.0 \\
\hline
\end{tabular}

Cont'd on next page... 


\begin{tabular}{|c|c|c|c|c|c|c|}
\hline \multirow{2}{*}{$\begin{array}{l}\text { Socio-demographic } \\
\text { characteristics (\%) }\end{array}$} & \multicolumn{2}{|c|}{ Combined } & \multicolumn{2}{|c|}{ Urban } & \multicolumn{2}{|c|}{ Rural } \\
\hline & $\begin{array}{c}\text { All } \\
\text { households }\end{array}$ & $\begin{array}{l}\text { Households } \\
\text { with youth }\end{array}$ & $\begin{array}{c}\text { All } \\
\text { households }\end{array}$ & $\begin{array}{l}\text { Households } \\
\text { with youth }\end{array}$ & $\begin{array}{c}\text { All } \\
\text { households }\end{array}$ & $\begin{array}{l}\text { Households } \\
\text { with youth }\end{array}$ \\
\hline Caste/tribe of household head & & & & & & \\
\hline SC & 21.6 & 22.3 & 17.1 & 18.7 & 23.2 & 23.6 \\
\hline ST & 6.1 & 6.3 & 2.3 & 2.8 & 7.4 & 7.5 \\
\hline OBC & 45.3 & 47.3 & 45.6 & 47.1 & 45.3 & 47.4 \\
\hline General $^{2}$ & 26.8 & 23.9 & 34.9 & 31.2 & 23.9 & 21.3 \\
\hline Caste/tribe unknown & 0.2 & 0.2 & 0.1 & 0.1 & 0.2 & 0.2 \\
\hline \multicolumn{7}{|l|}{$\begin{array}{l}\text { Schooling of household head } \\
\text { (years) }\end{array}$} \\
\hline None $^{3}$ & 47.6 & 46.1 & 26.0 & 28.9 & 55.2 & 52.3 \\
\hline $1-7$ & 23.8 & 26.4 & 20.8 & 24.2 & 24.9 & 27.2 \\
\hline $8-10$ & 15.5 & 16.5 & 22.8 & 24.5 & 12.8 & 13.5 \\
\hline $11-12$ & 5.2 & 4.9 & 9.8 & 8.2 & 3.5 & 3.6 \\
\hline Above 12 & 7.8 & 5.9 & 20.4 & 13.8 & 3.4 & 3.1 \\
\hline \multicolumn{7}{|l|}{$\begin{array}{l}\text { Current work status } \\
\text { of household head }\end{array}$} \\
\hline Working & 87.4 & 90.8 & 85.0 & 86.9 & 88.3 & 92.2 \\
\hline Not working & 12.5 & 9.1 & 14.9 & 13.0 & 11.6 & 7.7 \\
\hline \multicolumn{7}{|l|}{$\begin{array}{l}\text { Number of members in the } \\
\text { household }\end{array}$} \\
\hline 1 & 4.7 & 0.2 & 3.4 & 0.2 & 5.1 & 0.1 \\
\hline 2 & 14.9 & 6.1 & 12.6 & 7.2 & 15.6 & 5.7 \\
\hline 3 & 17.5 & 16.2 & 18.3 & 16.1 & 17.2 & 16.2 \\
\hline 4 & 28.8 & 30.1 & 32.5 & 31.6 & 27.5 & 29.6 \\
\hline 5 & 17.5 & 21.9 & 17.9 & 21.7 & 17.3 & 22.0 \\
\hline 6 & 9.0 & 11.9 & 8.2 & 11.3 & 9.2 & 12.1 \\
\hline 7 or more & 7.7 & 13.7 & 7.1 & 11.9 & 7.9 & 14.4 \\
\hline Mean household size & 4.1 & 4.8 & 4.1 & 4.6 & 4.0 & 4.8 \\
\hline \multicolumn{7}{|l|}{ Type of family } \\
\hline Nuclear & 67.9 & 55.0 & 70.3 & 61.4 & 67.0 & 52.6 \\
\hline Non-nuclear & 32.1 & 45.0 & 29.7 & 38.6 & 33.0 & 47.4 \\
\hline $\begin{array}{l}\text { Households with at least one } \\
\text { literate member aged } 18 \text { and } \\
\text { above }\end{array}$ & 72.8 & 85.9 & 88.5 & 93.4 & 67.2 & 83.2 \\
\hline Number of households & 31,123 & 9,920 & 12,439 & 4,070 & 18,684 & 5,850 \\
\hline
\end{tabular}

Note: All Ns are unweighted. Column totals may not equal 100\% due to missing cases or "don't know" responses. OBC: Other backward caste. SC: Scheduled caste. ST: Scheduled tribe. ${ }^{1}$ Includes Sikh, Jain, Jewish, Parsi/Zoroastrian and no specified religion. ${ }^{2}$ Includes all those not belonging to SC, ST or OBC. ${ }^{3}$ Includes non-literate and literate with no formal schooling. ${ }^{4}$ Defined as reported work activity in the seven days prior to interview.

Distributions by religion suggest that $87 \%$ of all household heads were Hindu, $7 \%$ were Muslim, $6 \%$ were Christian and just $0.1 \%$ belonged to other religions. Distributions were by and large similar among households containing youth eligible for interview in the Youth Study. Rural-urban differences were, however evident. The urban population consisted of a somewhat larger proportion of Muslims than did the rural population ( $11 \%$ and $6 \%$, respectively among all households surveyed, for example). As far as caste was concerned, the largest group of all household heads belonged to other backward castes (45\%), followed by general castes $(27 \%)$ and scheduled castes $(22 \%)$. 
Scheduled tribes comprised just $6 \%$ of all household heads. This distribution closely resembles that obtained in the NFHS-3 (other backward castes, 49\%; general castes, 27\%; scheduled castes, 18\% and scheduled tribes, 7\%). Rural-urban differences indicate that households belonging to scheduled castes were more likely to reside in rural than urban areas (23\% and $17 \%$, respectively) and those belonging to general castes more likely to reside in urban than rural areas (35\% and $24 \%$, respectively).

Educational attainment levels suggest that $48 \%$ of all heads of households had no schooling and almost one-quarter had 1-7 years of schooling (24\%). Distributions were similar among households containing youth eligible for interview in the Youth Study. Just as educational distributions differed for the general population, here too, heads of households in urban areas were better educated than their rural counterparts. The vast majority of all heads of households reported working in the last seven days (87\%), with negligible rural-urban differences (85-88\%).

Households contained an average of 4.1 members. This number was slightly higher (4.8) among those containing youth eligible for interview in the Youth Study. Rural-urban differences were negligible. As far as family type was concerned, $68 \%$ of all households consisted of a nuclear family, with little rural-urban difference (67-70\%). However, among households containing youth eligible for interview in the Youth Study, many fewer (55\%) were nuclear, ranging from $53 \%$ in rural areas to $61 \%$ in urban areas.

Finally, 73\% of all households contained at least one literate member aged 18 and above, a percentage that was considerably higher $(86 \%)$ in households containing eligible youth. Rural-urban differences were wide: $89 \%$ and $67 \%$ of urban and rural households, respectively, of the total population surveyed contained at least one literate member aged 18 and above, as did 93\% and 83\%, respectively, of those containing eligible youth.

\subsection{Profile of the household population: Housing characteristics}

Table 2.9 provides information on ownership of residence, housing quality, access to basic amenities and indicators of crowding. Information was obtained from responses to the household questionnaire and, in the case of housing type, interviewer observations. Information is presented by rural-urban residence separately for all surveyed households and households containing youth eligible for the Youth Study.

The characteristics of both types of households are basically similar. A little less than four-fifth of households, irrespective of whether or not they contained youth, owned the structure in which they resided. Considerably more rural than urban households, however, reported owning their residence ( $88 \%$ and $51 \%$, respectively). Overall, interviewers observed that $17 \%$ of all households lived in kachcha houses (constructed from mud, thatch or other low-quality materials), 38\% lived in semi-pucca houses (constructed using a mix of low- and high-quality materials) and 45\% lived in pucca houses (constructed entirely from cement, masonry or other high-quality materials). Rural households were much less likely than urban households to reside in pucca structures (38\% versus $65 \%$ ).

Most residential structures contained 2-3 rooms (58\%); urban households were somewhat more likely to report 4 or more rooms than rural households $(20 \%$ and $10 \%$ respectively). The mean number of persons per room was 2.3 for all households and 2.6 for those containing eligible youth. The mean number of persons per room was somewhat larger among rural than urban households (2.3 and 2.0, respectively).

Respondents were asked about their household's main source of lighting and drinking water. In addition, information was gathered on toilet facilities typically accessed and cooking fuel generally used. As Table 2.9 shows, $92 \%$ of households had electricity, including almost all urban households (97\%) and 90\% of rural households. This compares with $88 \%$ for Andhra Pradesh as a whole as assessed in NFHS-3 (IIPS and Macro International, 2007a). The majority of households (95\%) reported that their main source of drinking water was either piped water, water obtained from a hand-pump or a covered well (while not entirely comparable, $94 \%$ of households reported that they had access to an improved source of drinking water, defined to include piped water, tube-well or borehole, protected well or spring, rainwater or bottled water in NFHS-3 (IIPS and Macro International, 2007a). These facilities were 
Table 2.9: Housing characteristics

Percent distribution of all surveyed households and households containing youth eligible for interview by selected housing characteristics, according to residence, Andhra Pradesh, 2007-08

\begin{tabular}{|c|c|c|c|c|c|c|}
\hline \multirow{2}{*}{$\begin{array}{l}\text { Housing } \\
\text { characteristics (\%) }\end{array}$} & \multicolumn{2}{|c|}{ Combined } & \multicolumn{2}{|c|}{ Urban } & \multicolumn{2}{|c|}{ Rural } \\
\hline & $\begin{array}{l}\text { All } \\
\text { households }\end{array}$ & $\begin{array}{l}\text { Households } \\
\text { with youth }\end{array}$ & $\begin{array}{l}\text { All } \\
\text { households }\end{array}$ & $\begin{array}{c}\text { Households } \\
\text { with youth }\end{array}$ & $\begin{array}{c}\text { All } \\
\text { households }\end{array}$ & $\begin{array}{l}\text { Households } \\
\text { with youth }\end{array}$ \\
\hline Ownership of residence & & & & & & \\
\hline Yes & 78.3 & 79.1 & 50.8 & 52.7 & 88.0 & 88.8 \\
\hline No & 21.7 & 20.9 & 49.2 & 47.3 & 12.0 & 11.2 \\
\hline Type of house & & & & & & \\
\hline Kachcha & 16.8 & 16.0 & 6.5 & 7.4 & 20.5 & 19.1 \\
\hline Semi-pucca & 37.8 & 38.1 & 28.0 & 30.2 & 41.3 & 41.0 \\
\hline Pucca & 45.4 & 45.9 & 65.4 & 62.4 & 38.2 & 39.9 \\
\hline Number of rooms in the house ${ }^{1}$ & & & & & & \\
\hline 1 & 29.3 & 27.1 & 22.6 & 22.7 & 31.7 & 28.7 \\
\hline $2-3$ & 58.0 & 59.0 & 57.7 & 59.1 & 58.1 & 58.9 \\
\hline $4-5$ & 10.8 & 11.9 & 16.9 & 15.5 & 8.6 & 10.6 \\
\hline 6 or more & 1.9 & 2.0 & 2.8 & 2.7 & 1.6 & 1.7 \\
\hline $\begin{array}{l}\text { Average number of persons } \\
\text { per room }\end{array}$ & & & & & & \\
\hline Up to 2 & 72.0 & 64.6 & 77.7 & 72.2 & 70.0 & 61.9 \\
\hline $3-4$ & 20.3 & 25.7 & 16.5 & 20.8 & 21.7 & 27.5 \\
\hline $5-6$ & 6.5 & 7.7 & 4.9 & 5.6 & 7.0 & 8.5 \\
\hline More than 6 & 1.2 & 1.9 & 0.8 & 1.3 & 1.3 & 2.2 \\
\hline $\begin{array}{l}\text { Mean number of persons per room } \\
\text { Source of lighting }\end{array}$ & 2.3 & 2.6 & 2.0 & 2.3 & 2.3 & 2.7 \\
\hline Electricity & 91.6 & 94.3 & 97.2 & 97.4 & 89.6 & 93.2 \\
\hline Kerosene & 8.3 & 5.6 & 2.7 & 2.6 & 10.3 & 6.7 \\
\hline Other lighting sources ${ }^{2}$ & 0.1 & 0.0 & 0.1 & 0.0 & 0.1 & 0.0 \\
\hline Source of drinking water & & & & & & \\
\hline $\begin{array}{l}\text { Own piped water/ } \\
\text { hand-pump/covered well }\end{array}$ & 33.1 & 33.3 & 56.7 & 53.6 & 24.7 & 25.9 \\
\hline $\begin{array}{l}\text { Public piped water/ } \\
\text { hand-pump/covered well }\end{array}$ & 61.4 & 61.1 & 40.3 & 43.4 & 68.8 & 67.6 \\
\hline Own open well & 0.6 & 0.7 & 0.0 & 0.0 & 0.8 & 0.9 \\
\hline Public open well & 2.1 & 2.0 & 0.1 & 0.2 & 2.8 & 2.7 \\
\hline Surface water ${ }^{3}$ & 1.3 & 1.2 & 0.0 & 0.0 & 1.8 & 1.7 \\
\hline Other water sources ${ }^{4}$ & 1.5 & 1.6 & 2.7 & 2.7 & 1.1 & 1.2 \\
\hline Toilet facility & & & & & & \\
\hline Own flush toilet & 35.5 & 35.4 & 64.1 & 63.0 & 25.3 & 25.4 \\
\hline Shared flush toilet & 5.2 & 5.4 & 10.9 & 10.8 & 3.2 & 3.4 \\
\hline Own pit toilet & 5.6 & 5.8 & 10.1 & 10.0 & 4.0 & 4.2 \\
\hline Shared pit toilet & 1.0 & 1.0 & 2.4 & 2.3 & 0.5 & 0.5 \\
\hline Other toilet facility ${ }^{5}$ & 0.1 & 0.0 & 0.0 & 0.0 & 0.1 & 0.1 \\
\hline No toilet facility & 52.7 & 52.4 & 12.5 & 14.0 & 67.0 & 66.5 \\
\hline
\end{tabular}

Cont'd on next page... 
Table 2.9: (Cont'd)

\begin{tabular}{|c|c|c|c|c|c|c|}
\hline \multirow{2}{*}{$\begin{array}{l}\text { Housing } \\
\text { characteristics (\%) }\end{array}$} & \multicolumn{2}{|c|}{ Combined } & \multicolumn{2}{|c|}{ Urban } & \multicolumn{2}{|c|}{ Rural } \\
\hline & $\begin{array}{c}\text { All } \\
\text { households }\end{array}$ & $\begin{array}{l}\text { Households } \\
\text { with youth }\end{array}$ & $\begin{array}{c}\text { All } \\
\text { households }\end{array}$ & $\begin{array}{l}\text { Households } \\
\text { with youth }\end{array}$ & $\begin{array}{c}\text { All } \\
\text { households }\end{array}$ & $\begin{array}{l}\text { Households } \\
\text { with youth }\end{array}$ \\
\hline $\begin{array}{l}\text { Main type of fuel } \\
\text { used for cooking }\end{array}$ & & & & & & \\
\hline Liquid petroleum gas & 27.2 & 25.8 & 66.9 & 63.2 & 13.1 & 12.2 \\
\hline Bio-gas & 0.4 & 0.4 & 0.4 & 0.3 & 0.4 & 0.5 \\
\hline Kerosene & 2.6 & 2.8 & 7.9 & 8.7 & 0.8 & 0.6 \\
\hline $\begin{array}{l}\text { Wood/crop residue/ } \\
\text { dung cakes/coal/charcoal }\end{array}$ & 69.5 & 70.7 & 24.4 & 27.4 & 85.6 & 86.6 \\
\hline Other types of fuel ${ }^{6}$ & 0.2 & 0.2 & 0.3 & 0.4 & 0.1 & 0.1 \\
\hline Number of households & 31,123 & 9,920 & 12,439 & 4,070 & 18,684 & 5,850 \\
\hline
\end{tabular}

Note: All Ns are unweighted. Column totals may not equal $100 \%$ due to missing cases or "don't know" responses. ${ }^{1}$ Excludes toilets/bathrooms but includes kitchen. ${ }^{2}$ Includes oil, gas, etc. ${ }^{3}$ Includes water of a spring, river, stream, pond, lake or dam. ${ }^{4}$ Includes rain water and tanker truck. ${ }^{5}$ Includes twin pit/composting and dry toilets. ${ }^{6}$ Includes electricity, straw, shrubs and grass.

reported as self-owned for almost one-third of households, and as public or shared facilities for the remainder. While rural-urban differences in access to these safe sources of drinking water were negligible (94-97\%), it was clear that urban households were more likely to report that the facility was owned by the household than were rural households (57\% and $25 \%$, respectively).

Access to a toilet facility of any kind was reported by less than half of all households (47\%, compared to $42 \%$ as assessed in NFHS-3; IIPS and Macro International, 2007a): these included owned or shared flush toilets (35\% and 5\%, respectively); owned pit toilets $(6 \%)$ and shared pit toilets (1\%). Large rural-urban differences were observed: $67 \%$ of rural households, compared to $13 \%$ of urban households had no access to toilet facilities.

Finally, the main source of cooking fuel was coal, charcoal, wood, crop residue or dung cakes, reported by $70 \%$ of all households (compared to 66\% as assessed in NFHS-3; IIPS and Macro International, 2007a), and 86\% of all rural households compared to $24 \%$ of urban households. Liquid petroleum gas was used, in contrast, by $27 \%$ of all households (compared to $29 \%$ as assessed in NFHS-3; IIPS and Macro International, 2007a), ranging from 13\% in rural areas to $67 \%$ in urban areas. Patterns of access to these facilities in households containing youth eligible for interview in the Youth Study were similar to those observed for all households, described above.

\subsection{Profile of the household population: Ownership of agricultural land}

Table 2.10 presents information on ownership of agricultural land for households in both rural and urban areas (irrigated and non-irrigated). Most households, irrespective of whether or not they contained youth eligible for interview, owned no land (65-67\%) or only marginal holdings (21-22\%). A larger proportion of urban than rural households were landless (92\% and 59\%, respectively). Just 7\% owned between 2.51 and 5 acres of land, and $4 \%$ owned more than 5 acres of land. Moreover, land holdings were, by and large, not irrigated. Even in rural areas, only $38 \%$ of all households owned some irrigated land.

\subsection{Profile of the household population: Overall economic status}

Household economic status was measured using a wealth index, composed of household asset data on ownership of selected durable goods, including means of transportation, as well as data on access to a number of amenities. The wealth index was constructed by allocating the following scores to a household's reported assets or amenities: 
Table 2.10: Household ownership of agricultural land

Percent distribution of all surveyed households and households containing youth eligible for interview by ownership of agricultural land, according to residence, Andhra Pradesh, 2007-08

\begin{tabular}{|c|c|c|c|c|c|c|}
\hline \multirow{2}{*}{ Land holding (\%) } & \multicolumn{2}{|c|}{ Combined } & \multicolumn{2}{|c|}{ Urban } & \multicolumn{2}{|c|}{ Rural } \\
\hline & $\begin{array}{c}\text { All } \\
\text { households }\end{array}$ & $\begin{array}{l}\text { Households } \\
\text { with youth }\end{array}$ & $\begin{array}{c}\text { All } \\
\text { households }\end{array}$ & $\begin{array}{l}\text { Households } \\
\text { with youth }\end{array}$ & $\begin{array}{c}\text { All } \\
\text { households }\end{array}$ & $\begin{array}{l}\text { Households } \\
\text { with youth }\end{array}$ \\
\hline Land holding (in acres) & & & & & & \\
\hline Landless & 67.4 & 65.1 & 92.3 & 91.3 & 58.6 & 55.6 \\
\hline Marginal $(\leq 2.50)$ & 21.0 & 22.3 & 3.3 & 4.0 & 27.3 & 29.0 \\
\hline Small (2.51-5.00) & 7.3 & 8.1 & 2.2 & 2.7 & 9.2 & 10.1 \\
\hline Medium (5.01-10.00) & 2.6 & 2.7 & 1.1 & 0.9 & 3.1 & 3.3 \\
\hline Large $(>10.00)$ & 1.2 & 1.3 & 0.6 & 0.7 & 1.4 & 1.6 \\
\hline Own any irrigated land & 29.8 & 32.1 & 6.5 & 7.4 & 38.0 & 41.1 \\
\hline Number of households & 31,123 & 9,920 & 12,439 & 4,070 & 18,684 & 5,850 \\
\hline
\end{tabular}

Note: All Ns are unweighted. Column totals may not equal 100\% due to missing cases or "don't know" responses.

Type of house: 2 for pucca; 1 for semi-pucca; 0 for kachcha

Agricultural land owned: 4 for more than 10 acres; 3 for 5.1-10.0 acres; 2 for 2.6-5.0 acres; 1 for less than 2.6 acres, or if the household owns some land but does not know how much; 0 for no land

Irrigated land owned: 1 for any irrigated land; 0 for no land

Access to toilet facility: 4 for own flush toilet; 2 for shared flush toilet or own pit toilet; 1 for shared pit toilet or other types of toilet; 0 for no toilet facility

Cooking fuel used: 2 for liquid petroleum gas, electricity or bio-gas; 1 for kerosene, wood, crop residue, dung cakes, coal or charcoal; 0 for other types of cooking fuel, for example, straw, shrubs or grass

Access to drinking water facility: 4 for own piped water, hand-pump or covered well; 3 for own open well; 2 for public or shared piped water, hand-pump or covered well; 1 for public or shared open well; 0 for other sources of drinking water, for example, surface water, tanker/truck or rainwater

Access to electricity: 3 for electricity; 0 for no electricity

Ownership of household assets: 4 for car or truck; 3 each for motorcycle or scooter, refrigerator, computer/laptop, telephone (landline or mobile), colour television; 2 each for bicycle, electric fan, radio or transistor, black and white television, sewing machine, water pump, animal-drawn cart; 1 for watch or clock; 0 for each of the above items that the household does not possess.

Index scores, so constructed, ranged from 0 to 54 . Households were then ranked according to the index score. This ranked sample was divided into quintiles-i.e., five groups, each containing an equal number of households-with the first quintile representing households of the lowest (poorest) wealth status and the fifth quintile representing households with the highest (wealthiest) status. In the Youth Study, the wealth quintiles were developed at the state level on the basis of the weighted sample for the whole state.

Findings are presented in Table 2.11. As far as ownership of household assets was concerned, the items most likely to be owned by all households were a watch or clock (80\%) and an electric fan (78\%). Other items owned by one-fifth or more of all households included a bicycle (41\%), a telephone (32\%), a colour television set $(30 \%)$, and a black and white television set $(23 \%)$. In addition, $15 \%$ reported ownership of a motorcycle/scooter. Wide rural-urban differences were observed, with rural households considerably less likely than urban households to report ownership of most items. For example, while 56\% of urban households owned a colour television set, just $21 \%$ of rural households did; and while $58 \%$ of all urban households owned a telephone, just $22 \%$ of rural households did. A small proportion of households did not own a single item ( $9 \%$ of all households and $5 \%$ of those containing eligible youth); rural households were more likely than urban households to report so (12\% and $7 \%$ of all households and those containing youth, respectively, did not own a single item in rural areas, compared to $2 \%$ each in urban areas). 
Table 2.11: Household assets and wealth status

Percentage of all surveyed households and households containing youth eligible for interview owning selected household assets and percent distribution of households by wealth quintile, according to residence, Andhra Pradesh, 2007-08

\begin{tabular}{|c|c|c|c|c|c|c|}
\hline \multirow{2}{*}{$\begin{array}{l}\text { Housing } \\
\text { characteristics (\%) }\end{array}$} & \multicolumn{2}{|c|}{ Combined } & \multicolumn{2}{|c|}{ Urban } & \multicolumn{2}{|c|}{ Rural } \\
\hline & $\begin{array}{c}\text { All } \\
\text { households }\end{array}$ & $\begin{array}{l}\text { Households } \\
\text { with youth }\end{array}$ & $\begin{array}{c}\text { All } \\
\text { households }\end{array}$ & $\begin{array}{c}\text { Households } \\
\text { with youth }\end{array}$ & $\begin{array}{c}\text { All } \\
\text { households }\end{array}$ & $\begin{array}{l}\text { Households } \\
\text { with youth }\end{array}$ \\
\hline \multicolumn{7}{|l|}{ Assets owned } \\
\hline Watch/clock & 80.4 & 85.0 & 92.0 & 93.3 & 76.3 & 81.9 \\
\hline Electric fan & 78.3 & 82.0 & 93.5 & 93.6 & 72.8 & 77.8 \\
\hline Bicycle & 41.1 & 49.1 & 44.0 & 50.3 & 40.1 & 48.7 \\
\hline Radio and/or transistor & 9.7 & 10.3 & 14.6 & 13.6 & 8.0 & 9.0 \\
\hline Colour television & 30.0 & 32.3 & 55.7 & 54.1 & 20.9 & 24.3 \\
\hline $\mathrm{B} / \mathrm{W}$ television & 23.1 & 26.7 & 20.3 & 22.1 & 24.2 & 28.4 \\
\hline Telephone (landline/mobile) & 31.6 & 35.5 & 57.5 & 57.9 & 22.4 & 27.4 \\
\hline Refrigerator & 9.0 & 7.7 & 25.0 & 20.3 & 3.3 & 3.0 \\
\hline Motorcycle/scooter & 14.8 & 15.0 & 31.5 & 28.6 & 8.9 & 10.0 \\
\hline Sewing machine & 9.6 & 10.8 & 17.4 & 16.9 & 6.9 & 8.6 \\
\hline Animal-drawn cart & 3.4 & 4.5 & 0.3 & 0.3 & 4.5 & 6.0 \\
\hline Water pump & 13.2 & 13.3 & 20.5 & 18.9 & 10.6 & 11.3 \\
\hline Personal computer/laptop & 1.9 & 1.9 & 6.5 & 6.1 & 0.2 & 0.4 \\
\hline Car/truck & 1.1 & 0.8 & 3.4 & 2.4 & 0.3 & 0.2 \\
\hline Tractor & 0.6 & 0.6 & 0.3 & 0.3 & 0.7 & 0.7 \\
\hline Thresher & 0.1 & 0.1 & 0.2 & 0.2 & 0.1 & 0.1 \\
\hline None of the above & 9.3 & 5.1 & 2.2 & 1.5 & 11.9 & 6.5 \\
\hline \multicolumn{7}{|l|}{ Wealth quintile } \\
\hline First & 20.0 & 14.4 & 6.2 & 5.6 & 24.9 & 17.5 \\
\hline Second & 20.0 & 20.0 & 9.0 & 8.8 & 23.9 & 24.1 \\
\hline Third & 20.0 & 22.0 & 14.2 & 14.9 & 22.1 & 24.5 \\
\hline Fourth & 20.0 & 23.5 & 27.4 & 30.4 & 17.4 & 21.0 \\
\hline Fifth & 20.0 & 20.1 & 43.3 & 40.3 & 11.7 & 12.8 \\
\hline Number of households & 31,123 & 9,920 & 12,439 & 4,070 & 18,684 & 5,850 \\
\hline
\end{tabular}

Note: All Ns are unweighted.

The distribution of households by wealth quintiles shows that more than two-fifths of urban households were in the wealthiest (fifth) quintile; in contrast, only $12 \%$ of all rural households were in this quintile. Likewise, $25 \%$ of rural households were in the poorest (first) quintile of the index compared to only $6 \%$ of all urban households.

\subsection{Profile of surveyed youth: Background characteristics}

A total of 8,330 youth were interviewed. Table 2.12 presents the background characteristics of surveyed youth. Age profiles suggest that young men and women were about equally distributed in the 15-19 and 20-24 age groups ( $47-51 \%$ and $49-53 \%$, respectively). The unmarried were, however, younger than the married; while about one-third of married young women were aged 15-19 (31\%), almost four-fifths (78\%) of unmarried young women fell into these ages. In contrast, few married young men were aged 15-19 (1.2\%), and the majority was aged 25-29 (70\%); unmarried young men were more evenly divided, with $57 \%$ and $43 \%$ aged $15-19$ and $20-24$, respectively. Rural-urban differences suggest that rural youth were somewhat younger than their urban counterparts. 
The distribution of youth by religion and caste was fairly similar to that observed in the household population: $84-85 \%$ of youth were Hindu, $7-10 \%$ were Muslim and $5-8 \%$ were Christian. While no differences were evident by marital status, rural-urban differences suggest that youth in urban areas were somewhat less likely than their rural counterparts to be Hindu $(80-81 \%$ versus $86 \%)$ and conversely, more likely to be Muslim $(12-14 \%$ versus $5-8 \%)$.

Caste-wise distributions were similar among young men and women, with $24-25 \%$ of youth falling into general castes, $22-23 \%$ into scheduled castes, $6 \%$ into scheduled tribes and $46-49 \%$ into other backward castes. Distributions by marital status show that more unmarried than married youth belonged to general castes (27\% versus $20-21 \%$ ). Rural-urban differences were also evident; urban youth were more likely than rural youth to belong to general castes $(30-31 \%$ versus $21-22 \%)$; conversely young men in urban areas were less likely to belong to scheduled castes $(16 \%$ versus $25 \%)$.

Educational distributions suggest that youth were better educated than the population at large. In total, $8 \%$ of young men and $21 \%$ of young women had no formal education (compared to $29 \%$ and $47 \%$, respectively, of the total population described in Table 2.6) and $23 \%$ of young men and $14 \%$ of young women had 12 or more years of education (compared to $14 \%$ and $7 \%$ of the total population). As can be seen from the above, gender differences were evident, with young women more likely than young men to be concentrated among the uneducated or poorly educated. This difference is attributable to differences observed primarily among the married. Differences were also evident by marital status and rural-urban residence. Among married youth for example, $21 \%$ of young men and $31 \%$ of young women had no formal education, and $14 \%$ and $7 \%$, respectively had attained 12 or more years of education. The unmarried were typically better educated than the married $-5 \%$ of young men and $7 \%$ of young women had no formal education and $26 \%$ and $24 \%$, respectively, had more than 12 years of education. Urban youth were generally better educated than rural youth: for example, $5 \%$ of young men and $10 \%$ of young women in urban areas had no formal education compared to $9 \%$ and $25 \%$ of rural youth, respectively. Similarly, $32 \%$ and $28 \%$ of urban young men and women had completed 12 or more years of education compared to $19 \%$ and $9 \%$ of rural youth, respectively.

Gender differences were wide with regard to work status: $73 \%$ of young men compared to $47 \%$ of young women had ever worked in paid or unpaid activities in the 12 months preceding the interview. Married youth were typically more likely to be engaged in work activities than the unmarried: while almost all married young men had worked in the year preceding the interview, $67 \%$ of unmarried young men had done so, and among young women, correspondingly, $51 \%$ and $44 \%$ reported so. Also evident was that more rural than urban youth were engaged in work activities and that gender differences were narrower in rural than urban areas: $76 \%$ of young men and $57 \%$ of young women in rural areas were engaged in work activites compared to $65 \%$ and $25 \%$, respectively, in urban areas.

Household economic status distributions, as measured by wealth quintiles, suggest that young men were somewhat less likely than young women to belong to households in the poorer (first and second) quintiles (30\% versus $36 \%$ ), and somewhat more likely than young women to belong to households in the wealthier (fourth and fifth) quintiles (49\% versus $42 \%$ ). Likewise, the married were more likely than the unmarried to belong to households in the poorer quintiles (34-41\% versus $28-30 \%)$ and conversely, less likely to fall into households in the wealthier quintiles (35-43\% versus $49-51 \%)$. Rural-urban differences were wide, with rural youth more likely than their urban counterparts to belong to households in the poorer quintiles; conversely, more urban than rural youth belonged to households in the wealthiest quintiles.

\subsection{Profile of surveyed youth: Parental characteristics}

The Youth Study inquired about the characteristics of respondents' parents, including their survival status, education and occupation. Four in five respondents reported that both parents were surviving (Table 2.13). Fewer married than unmarried youth reported that both parents were surviving ( $71-77 \%$ and $84-85 \%$, respectively). 
Table 2.12: Background characteristics of surveyed youth

Percent distribution of surveyed youth by selected background characteristics, according to residence, Andhra Pradesh, 2007-08

\begin{tabular}{|c|c|c|c|c|c|c|c|c|c|c|c|c|}
\hline \multirow[t]{2}{*}{$\begin{array}{l}\text { Background } \\
\text { characteristics }\end{array}$} & \multicolumn{2}{|c|}{ 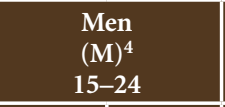 } & \multicolumn{2}{|c|}{$\begin{array}{l}\text { Women } \\
(W)^{4} \\
15-24 \\
\end{array}$} & \multicolumn{2}{|c|}{$\begin{array}{c}\text { Married } \\
\text { men }(\mathrm{MM})^{4} \\
15-29 \\
\end{array}$} & \multicolumn{2}{|c|}{$\begin{array}{c}\text { Married } \\
\text { women }(\mathrm{MW})^{4} \\
15-24\end{array}$} & \multicolumn{2}{|c|}{$\begin{array}{c}\text { Unmarried } \\
\text { men }(U M)^{4} \\
15-24\end{array}$} & \multicolumn{2}{|c|}{$\begin{array}{c}\text { Unmarried } \\
\text { women }(\mathrm{UW})^{4} \\
15-24 \\
\end{array}$} \\
\hline & Percent & Number & Percent & Number & Percent & Number & Percent & Number & Percent & Number & Percent & Number \\
\hline \multicolumn{13}{|c|}{ Combined } \\
\hline Age (years) & & & & & & & & & & & & \\
\hline $15-19$ & 47.2 & 1,165 & 50.9 & 2,603 & 1.2 & 14 & 30.6 & 661 & 57.3 & 1,151 & 78.2 & 1,942 \\
\hline $20-24$ & 52.8 & 1,314 & 49.1 & 2,245 & 29.2 & 388 & 69.4 & 1,669 & 42.7 & 926 & 21.8 & 576 \\
\hline $25-29$ & NA & NA & NA & NA & 69.6 & 1,003 & NA & NA & NA & NA & NA & NA \\
\hline \multicolumn{13}{|l|}{ Religion } \\
\hline Hindu & 84.9 & 2,075 & 84.3 & 4,051 & 86.4 & 1,202 & 84.7 & 1,947 & 84.7 & 1,735 & 84.2 & 2,104 \\
\hline Muslim & 9.8 & 276 & 7.1 & 386 & 7.4 & 123 & 6.3 & 175 & 10.4 & 241 & 7.9 & 211 \\
\hline Christian & 5.3 & 128 & 8.4 & 405 & 6.2 & 80 & 8.9 & 206 & 4.9 & 101 & 7.8 & 199 \\
\hline Other ${ }^{1}$ & 0.0 & 0 & 0.0 & 3 & 0.0 & 0 & 0.0 & 0 & 0.0 & 0 & 0.1 & 3 \\
\hline \multicolumn{13}{|l|}{ Caste } \\
\hline $\mathrm{SC}$ & 22.7 & 506 & 21.9 & 1,085 & 24.4 & 308 & 21.5 & 519 & 21.8 & 408 & 22.5 & 566 \\
\hline ST & 6.3 & 133 & 6.0 & 248 & 8.1 & 100 & 7.1 & 136 & 5.1 & 94 & 4.8 & 112 \\
\hline $\mathrm{OBC}$ & 45.9 & 1,163 & 48.5 & 2,319 & 47.2 & 674 & 50.6 & 1,164 & 45.6 & 967 & 46.0 & 1,155 \\
\hline General $^{2}$ & 24.7 & 670 & 23.5 & 1,195 & 20.4 & 323 & 20.8 & 510 & 27.1 & 601 & 26.7 & 685 \\
\hline No caste/do not know & 0.4 & 7 & 0.0 & 1 & 0.0 & 0 & 0.0 & 1 & 0.5 & 7 & 0.0 & 0 \\
\hline \multicolumn{13}{|l|}{ Educational level (years) } \\
\hline None $^{3}$ & 7.8 & 166 & 20.8 & 809 & 21.1 & 262 & 31.3 & 635 & 5.1 & 94 & 7.2 & 174 \\
\hline $1-7$ & 23.1 & 537 & 28.4 & 1,255 & 33.0 & 448 & 36.2 & 805 & 18.8 & 375 & 18.4 & 450 \\
\hline $8-11$ & 46.4 & 1,129 & 36.4 & 1,929 & 31.5 & 460 & 25.6 & 672 & 50.3 & 1,003 & 50.7 & 1,257 \\
\hline 12 and above & 22.7 & 647 & 14.4 & 855 & 14.4 & 235 & 6.9 & 218 & 25.8 & 605 & 23.8 & 637 \\
\hline \multicolumn{13}{|c|}{ Worked in last 12 months } \\
\hline Yes & 72.6 & 1,729 & 47.4 & 2,051 & 98.4 & 1,388 & 50.5 & 989 & 66.6 & 1,333 & 44.1 & 1,062 \\
\hline No & 27.4 & 749 & 52.6 & 2,797 & 1.4 & 15 & 49.5 & 1,341 & 33.3 & 743 & 55.9 & 1,456 \\
\hline \multicolumn{13}{|l|}{ Wealth quintile } \\
\hline First & 11.3 & 219 & 16.4 & 687 & 12.4 & 143 & 19.3 & 377 & 9.9 & 161 & 13.0 & 310 \\
\hline Second & 18.5 & 375 & 19.9 & 857 & 21.2 & 247 & 22.1 & 447 & 18.3 & 303 & 17.4 & 410 \\
\hline Third & 21.6 & 480 & 22.1 & 1,012 & 23.5 & 297 & 23.6 & 520 & 20.8 & 381 & 20.3 & 492 \\
\hline Fourth & 26.1 & 680 & 22.0 & 1,165 & 25.6 & 400 & 20.3 & 549 & 26.4 & 573 & 23.9 & 616 \\
\hline Fifth & 22.5 & 725 & 19.6 & 1,127 & 17.2 & 318 & 14.7 & 437 & 24.6 & 659 & 25.3 & 690 \\
\hline Total & 100.0 & 2,479 & 100.0 & 4,848 & 100.0 & 1,405 & 100.0 & 2,330 & 100.0 & 2,077 & 100.0 & 2,518 \\
\hline \multicolumn{13}{|c|}{ Urban } \\
\hline \multicolumn{13}{|l|}{ Age (years) } \\
\hline $15-19$ & 44.6 & 583 & 47.6 & 975 & 0.3 & 3 & 22.5 & 234 & 50.9 & 580 & 68.0 & 741 \\
\hline $20-24$ & 55.4 & 706 & 52.4 & 1,151 & 24.2 & 154 & 77.5 & 803 & 49.1 & 552 & 32.0 & 348 \\
\hline $25-29$ & NA & NA & NA & NA & 75.5 & 491 & NA & NA & NA & NA & NA & NA \\
\hline \multicolumn{13}{|l|}{ Religion } \\
\hline Hindu & 81.1 & 1,046 & 79.6 & 1,700 & 84.0 & 544 & 81.1 & 843 & 81.0 & 917 & 78.4 & 857 \\
\hline Muslim & 14.0 & 180 & 12.0 & 248 & 11.4 & 75 & 10.5 & 107 & 14.3 & 160 & 13.2 & 141 \\
\hline Christian & 4.8 & 63 & 8.2 & 175 & 4.6 & 29 & 8.4 & 87 & 4.8 & 55 & 8.0 & 88 \\
\hline Other $^{1}$ & 0.0 & 0 & 0.1 & 3 & 0.0 & 0 & 0.0 & 0 & 0.0 & 0 & 0.3 & 3 \\
\hline \multicolumn{13}{|l|}{ Caste } \\
\hline SC & 16.3 & 208 & 22.4 & 484 & 16.3 & 106 & 22.9 & 244 & 16.2 & 181 & 22.0 & 240 \\
\hline ST & 3.4 & 42 & 2.2 & 48 & 4.6 & 30 & 3.2 & 34 & 3.2 & 35 & 1.3 & 14 \\
\hline OBC & 48.7 & 627 & 45.2 & 971 & 50.7 & 327 & 49.5 & 508 & 47.9 & 543 & 41.7 & 463 \\
\hline General $^{2}$ & 31.4 & 411 & 30.3 & 623 & 28.4 & 185 & 24.4 & 251 & 32.5 & 372 & 35.0 & 372 \\
\hline No caste/do not know & 0.1 & 1 & 0.0 & 0 & 0.0 & 0 & 0.0 & 0 & 0.2 & 1 & 0.0 & 0 \\
\hline
\end{tabular}


Table 2.12: (Cont'd)

\begin{tabular}{|c|c|c|c|c|c|c|c|c|c|c|c|c|}
\hline \multirow[t]{2}{*}{$\begin{array}{l}\text { Background } \\
\text { characteristics }\end{array}$} & \multicolumn{2}{|c|}{$\begin{array}{c}\text { Men } \\
(\mathrm{M})^{4} \\
15-24\end{array}$} & \multicolumn{2}{|c|}{$\begin{array}{l}\text { Women } \\
\qquad(W)^{4} \\
15-24\end{array}$} & \multicolumn{2}{|c|}{$\begin{array}{c}\text { Married } \\
\text { men }(\mathrm{MM})^{4} \\
15-29\end{array}$} & \multicolumn{2}{|c|}{$\begin{array}{c}\text { Married } \\
\text { women }(\mathrm{MW})^{4} \\
15-24\end{array}$} & \multicolumn{2}{|c|}{$\begin{array}{c}\text { Unmarried } \\
\text { men }(\mathrm{UM})^{4} \\
15-24\end{array}$} & \multicolumn{2}{|c|}{$\begin{array}{c}\text { Unmarried } \\
\text { women }(\mathrm{UW})^{4} \\
15-24\end{array}$} \\
\hline & Percent & Number & Percent & Number & Percent & Number & Percent & Number & Percent & Number & Percent & Number \\
\hline \multicolumn{13}{|c|}{ Urban } \\
\hline Educational level (years) & & & & & & & & & & & & \\
\hline None $^{3}$ & 4.8 & 61 & 10.0 & 228 & 13.7 & 89 & 17.2 & 182 & 3.3 & 38 & 4.2 & 46 \\
\hline $1-7$ & 19.4 & 248 & 20.9 & 456 & 29.7 & 192 & 30.5 & 314 & 16.7 & 188 & 13.1 & 142 \\
\hline $8-11$ & 43.5 & 561 & 40.9 & 865 & 35.0 & 227 & 36.6 & 379 & 44.6 & 505 & 44.6 & 486 \\
\hline 12 and above & 32.3 & 419 & 28.1 & 577 & 21.6 & 140 & 15.6 & 162 & 35.4 & 401 & 38.2 & 415 \\
\hline Worked in last 12 months & & & & & & & & & & & & \\
\hline Yes & 65.0 & 831 & 24.5 & 518 & 99.7 & 645 & 23.1 & 238 & 59.9 & 674 & 25.6 & 280 \\
\hline No & 35.0 & 458 & 75.5 & 1,608 & 0.3 & 2 & 76.9 & 799 & 40.1 & 458 & 74.4 & 809 \\
\hline \multicolumn{13}{|l|}{ Wealth quintile } \\
\hline First & 4.0 & 51 & 6.8 & 152 & 5.6 & 36 & 8.9 & 93 & 3.5 & 39 & 5.2 & 59 \\
\hline Second & 8.2 & 108 & 9.0 & 196 & 10.5 & 67 & 11.8 & 122 & 7.4 & 86 & 6.7 & 74 \\
\hline Third & 14.4 & 188 & 15.7 & 338 & 15.4 & 100 & 18.8 & 196 & 13.3 & 153 & 13.1 & 142 \\
\hline Fourth & 30.2 & 388 & 30.2 & 644 & 34.3 & 223 & 31.9 & 330 & 29.8 & 338 & 28.7 & 314 \\
\hline Fifth & 43.2 & 554 & 38.3 & 796 & 34.3 & 222 & 28.5 & 296 & 46.0 & 516 & 46.3 & 500 \\
\hline Total & 100.0 & 1,289 & 100.0 & 2,126 & 100.0 & 648 & 100.0 & 1,037 & 100.0 & 1,132 & 100.0 & 1,089 \\
\hline \multicolumn{13}{|c|}{ Rural } \\
\hline Age (years) & & & & & & & & & & & & \\
\hline $15-19$ & 48.2 & 582 & 52.3 & 1,628 & 1.5 & 11 & 33.0 & 427 & 60.1 & 571 & 84.0 & 1,201 \\
\hline $20-24$ & 51.8 & 608 & 47.7 & 1,094 & 30.7 & 234 & 67.0 & 866 & 39.9 & 374 & 16.0 & 228 \\
\hline $25-29$ & NA & NA & NA & NA & 67.9 & 512 & NA & NA & NA & NA & NA & NA \\
\hline \multicolumn{13}{|l|}{ Religion } \\
\hline Hindu & 86.3 & 1,029 & 86.3 & 2,351 & 87.1 & 658 & 85.7 & 1,104 & 86.3 & 818 & 87.4 & 1,247 \\
\hline Muslim & 8.2 & 96 & 5.0 & 138 & 6.3 & 48 & 5.1 & 68 & 8.8 & 81 & 4.8 & 70 \\
\hline Christian & 5.5 & 65 & 8.5 & 230 & 6.6 & 51 & 9.0 & 119 & 4.9 & 46 & 7.7 & 111 \\
\hline Other ${ }^{1}$ & 0.0 & 0 & 0.0 & 0 & 0.0 & 0 & 0.0 & 0 & 0.0 & 0 & 0.0 & 0 \\
\hline \multicolumn{13}{|l|}{ Caste } \\
\hline SC & 25.2 & 298 & 21.7 & 601 & 26.6 & 202 & 21.1 & 275 & 24.2 & 227 & 22.8 & 326 \\
\hline ST & 7.4 & 91 & 7.6 & 200 & 9.1 & 70 & 8.2 & 102 & 5.9 & 59 & 6.8 & 98 \\
\hline $\mathrm{OBC}$ & 44.9 & 536 & 50.0 & 1,348 & 46.2 & 347 & 50.9 & 656 & 44.6 & 424 & 48.4 & 692 \\
\hline General $^{2}$ & 22.0 & 259 & 20.6 & 572 & 18.1 & 138 & 19.8 & 259 & 24.7 & 229 & 22.0 & 313 \\
\hline No caste/do not know & 0.6 & 6 & 0.0 & 1 & 0.0 & 0 & 0.1 & 1 & 0.7 & 6 & 0.0 & 0 \\
\hline \multicolumn{13}{|l|}{ Educational level (years) } \\
\hline None $^{3}$ & 8.9 & 105 & 25.3 & 581 & 23.1 & 173 & 35.3 & 453 & 5.9 & 56 & 8.9 & 128 \\
\hline $1-7$ & 24.5 & 289 & 31.6 & 799 & 34.0 & 256 & 37.8 & 491 & 19.7 & 187 & 21.4 & 308 \\
\hline $8-11$ & 47.5 & 568 & 34.4 & 1,064 & 30.6 & 233 & 22.4 & 293 & 52.7 & 498 & 54.1 & 771 \\
\hline 12 and above & 19.0 & 228 & 8.6 & 278 & 12.3 & 95 & 4.4 & 56 & 21.6 & 204 & 15.6 & 222 \\
\hline Worked in last 12 months & & & & & & & & & & & & \\
\hline Yes & 75.5 & 898 & 57.0 & 1,533 & 98.2 & 743 & 58.4 & 751 & 69.6 & 659 & 54.5 & 782 \\
\hline No & 24.3 & 291 & 43.0 & 1,189 & 1.7 & 13 & 41.6 & 542 & 30.3 & 285 & 45.5 & 647 \\
\hline \multicolumn{13}{|l|}{ Wealth quintile } \\
\hline First & 14.2 & 168 & 20.5 & 535 & 14.4 & 107 & 22.3 & 284 & 12.8 & 122 & 17.5 & 251 \\
\hline Second & 22.5 & 267 & 24.5 & 661 & 24.2 & 180 & 25.2 & 325 & 23.0 & 217 & 23.4 & 336 \\
\hline Third & 24.5 & 292 & 24.7 & 674 & 25.8 & 197 & 24.9 & 324 & 24.0 & 228 & 24.4 & 350 \\
\hline Fourth & 24.5 & 292 & 18.5 & 521 & 23.2 & 177 & 17.0 & 219 & 24.9 & 235 & 21.1 & 302 \\
\hline Fifth & 14.3 & 171 & 11.7 & 331 & 12.5 & 96 & 10.6 & 141 & 15.3 & 143 & 13.6 & 190 \\
\hline Total & 100.0 & 1,190 & 100.0 & 2,722 & 100.0 & 757 & 100.0 & 1,293 & 100.0 & 945 & 100.0 & 1,429 \\
\hline
\end{tabular}

Note: All Ns are unweighted. Column totals may not equal 100\% due to missing cases or "don't know" responses. NA: Not applicable. OBC: Other backward caste. SC: Scheduled caste. ST: Scheduled tribe. ${ }^{1}$ Includes Sikh, Jain, Jewish, Parsi/Zoroastrian and no specified religion. ${ }^{2}$ Includes all those not belonging to SC, ST or OBC. ${ }^{3}$ Includes non-literate and literate with no formal schooling. ${ }^{4}$ These abbreviations have been used in subsequent tables in this report. 
Table 2.13: Parental characteristics of surveyed youth

Percent distribution of surveyed youth by selected parental characteristics, according to residence, Andhra Pradesh, 2007-08

\begin{tabular}{|c|c|c|c|c|c|c|}
\hline Parental characteristics (\%) & $\underset{15-24}{M}$ & $\begin{array}{c}W \\
15-24\end{array}$ & $\begin{array}{c}\text { MM } \\
15-29\end{array}$ & $\begin{array}{c}\text { MW } \\
15-24\end{array}$ & $\begin{array}{c}\text { UM } \\
15-24\end{array}$ & $\begin{array}{c}\text { UW } \\
15-24\end{array}$ \\
\hline \multicolumn{7}{|c|}{ Combined } \\
\hline \multicolumn{7}{|l|}{ Survival status } \\
\hline Both parents dead & 1.2 & 2.2 & 3.6 & 3.0 & 1.1 & 1.3 \\
\hline Only father alive & 2.2 & 3.9 & 4.8 & 5.1 & 2.0 & 2.5 \\
\hline Only mother alive & 13.1 & 13.5 & 20.9 & 15.4 & 12.8 & 10.8 \\
\hline Both parents alive & 83.5 & 80.4 & 70.6 & 76.6 & 84.1 & 85.4 \\
\hline \multicolumn{7}{|l|}{ Educational attainment level } \\
\hline Median years of schooling of father & $\mathrm{NC}$ & NC & NC & $\mathrm{NC}$ & $\mathrm{NC}$ & 5.0 \\
\hline Median years of schooling of mother & $\mathrm{NC}$ & $\mathrm{NC}$ & $\mathrm{NC}$ & $\mathrm{NC}$ & $\mathrm{NC}$ & $\mathrm{NC}$ \\
\hline \multicolumn{7}{|l|}{ Current/last occupational status of father } \\
\hline Cultivator & 18.4 & 23.8 & 20.9 & 28.3 & 18.2 & 18.4 \\
\hline Agricultural labourer & 30.5 & 31.0 & 38.0 & 34.2 & 27.6 & 27.5 \\
\hline Administrative/executive/managerial/clerical & 6.0 & 6.8 & 3.4 & 4.0 & 6.9 & 10.2 \\
\hline Business & 4.6 & 4.1 & 3.8 & 3.0 & 5.2 & 5.4 \\
\hline Skilled manual/machinery & 18.6 & 16.8 & 13.2 & 14.4 & 19.7 & 19.6 \\
\hline Unskilled non-agricultural labourer & 21.1 & 16.5 & 19.8 & 15.2 & 21.7 & 17.7 \\
\hline Other & 0.3 & 0.5 & 0.2 & 0.4 & 0.3 & 0.7 \\
\hline Never worked & 0.3 & 0.4 & 0.6 & 0.5 & 0.3 & 0.3 \\
\hline \multicolumn{7}{|l|}{ Current/last occupational status of mother } \\
\hline Cultivator & 11.7 & 16.0 & 13.8 & 18.8 & 11.0 & 12.6 \\
\hline Agricultural labourer & 28.2 & 33.6 & 32.1 & 38.2 & 25.7 & 28.6 \\
\hline Administrative/executive/managerial/clerical & 1.0 & 1.4 & 0.4 & 0.7 & 1.2 & 2.3 \\
\hline Business & 1.3 & 1.1 & 1.0 & 0.8 & 1.3 & 1.4 \\
\hline Skilled manual/machinery & 4.4 & 5.1 & 2.7 & 3.9 & 4.8 & 6.6 \\
\hline Unskilled non-agricultural labourer & 11.5 & 9.6 & 11.0 & 9.4 & 11.4 & 9.6 \\
\hline Other & 0.1 & 0.1 & 0.0 & 0.1 & 0.1 & 0.2 \\
\hline Housewife/never worked & 41.8 & 33.0 & 39.0 & 27.9 & 44.5 & 38.7 \\
\hline Number of respondents & 2,479 & 4,848 & 1,405 & 2,330 & 2,077 & 2,518 \\
\hline \multicolumn{7}{|c|}{ Urban } \\
\hline \multicolumn{7}{|l|}{ Survival status } \\
\hline Both parents dead & 1.6 & 1.9 & 5.6 & 3.2 & 1.1 & 0.8 \\
\hline Only father alive & 2.7 & 2.5 & 5.6 & 4.2 & 2.4 & 1.2 \\
\hline Only mother alive & 14.0 & 14.2 & 22.9 & 17.5 & 13.2 & 11.5 \\
\hline Both parents alive & 81.7 & 81.4 & 66.0 & 75.1 & 83.4 & 86.6 \\
\hline \multicolumn{7}{|l|}{ Educational attainment level } \\
\hline Median years of schooling of father & 5.4 & 6.0 & $\mathrm{NC}$ & $\mathrm{NC}$ & 7.0 & 8.0 \\
\hline Median years of schooling of mother & $\mathrm{NC}$ & $\mathrm{NC}$ & $\mathrm{NC}$ & NC & $\mathrm{NC}$ & 5.0 \\
\hline \multicolumn{7}{|l|}{ Current/last occupational status of father } \\
\hline Cultivator & 6.1 & 9.0 & 11.4 & 15.6 & 5.4 & 3.6 \\
\hline Agricultural labourer & 5.4 & 9.9 & 13.1 & 15.4 & 4.6 & 5.5 \\
\hline Administrative/executive/managerial/clerical & 15.5 & 14.7 & 9.5 & 8.4 & 16.7 & 19.8 \\
\hline
\end{tabular}

Cont'd on next page... 
Table 2.13: (Cont'd)

\begin{tabular}{|c|c|c|c|c|c|c|}
\hline Parental characteristics (\%) & $\begin{array}{c}M \\
15-24\end{array}$ & $\begin{array}{c}\text { W } \\
15-24\end{array}$ & $\begin{array}{c}\text { MM } \\
15-29\end{array}$ & $\begin{array}{c}\text { MW } \\
\text { 15-24 }\end{array}$ & $\begin{array}{c}\text { UM } \\
15-24\end{array}$ & $\begin{array}{c}\text { UW } \\
\text { 15-24 }\end{array}$ \\
\hline \multicolumn{7}{|c|}{ Urban } \\
\hline Business & 8.2 & 8.4 & 7.2 & 7.2 & 8.7 & 9.2 \\
\hline Skilled manual/machinery & 33.2 & 28.1 & 24.5 & 25.0 & 33.5 & 30.6 \\
\hline Unskilled non-agricultural labourer & 30.4 & 28.0 & 32.7 & 26.5 & 29.8 & 29.2 \\
\hline Other & 0.3 & 1.0 & 0.7 & 0.8 & 0.2 & 1.4 \\
\hline Never worked & 0.7 & 0.6 & 0.7 & 0.8 & 0.8 & 0.6 \\
\hline \multicolumn{7}{|l|}{ Current/last occupational status of mother } \\
\hline Cultivator & 2.1 & 3.7 & 5.6 & 7.4 & 1.7 & 0.7 \\
\hline Agricultural labourer & 3.1 & 8.6 & 7.5 & 15.2 & 2.9 & 3.3 \\
\hline Administrative/executive/managerial/clerical & 2.5 & 3.0 & 1.3 & 0.8 & 2.7 & 4.7 \\
\hline Business & 1.6 & 2.0 & 1.3 & 2.1 & 1.3 & 2.0 \\
\hline Skilled manual/machinery & 7.6 & 8.5 & 4.3 & 7.4 & 7.8 & 9.5 \\
\hline Unskilled non-agricultural labourer & 15.0 & 15.2 & 16.1 & 16.2 & 14.1 & 14.3 \\
\hline Other & 0.1 & 0.3 & 0.0 & 0.4 & 0.2 & 0.3 \\
\hline Housewife/never worked & 67.8 & 58.4 & 63.9 & 50.3 & 69.4 & 65.1 \\
\hline Number of respondents & 1,289 & 2,126 & 648 & 1,037 & 1,132 & 1,089 \\
\hline \multicolumn{7}{|c|}{ Rural } \\
\hline \multicolumn{7}{|l|}{ Survival status } \\
\hline Both parents dead & 1.1 & 2.4 & 3.1 & 2.9 & 1.0 & 1.5 \\
\hline Only father alive & 2.0 & 4.5 & 4.6 & 5.3 & 1.9 & 3.2 \\
\hline Only mother alive & 12.7 & 13.2 & 20.4 & 14.8 & 12.6 & 10.5 \\
\hline Both parents alive & 84.2 & 79.9 & 71.9 & 77.0 & 84.4 & 84.8 \\
\hline \multicolumn{7}{|l|}{ Educational attainment level } \\
\hline Median years of schooling of father & $\mathrm{NC}$ & NC & $\mathrm{NC}$ & $\mathrm{NC}$ & $\mathrm{NC}$ & $\mathrm{NC}$ \\
\hline Median years of schooling of mother & $\mathrm{NC}$ & NC & $\mathrm{NC}$ & $\mathrm{NC}$ & $\mathrm{NC}$ & $\mathrm{NC}$ \\
\hline \multicolumn{7}{|l|}{ Current/last occupational status of father } \\
\hline Cultivator & 23.3 & 30.0 & 23.5 & 32.0 & 23.7 & 26.8 \\
\hline Agricultural labourer & 40.4 & 39.8 & 45.0 & 39.6 & 37.8 & 40.0 \\
\hline Administrative/executive/managerial/clerical & 2.3 & 3.5 & 1.7 & 2.7 & 2.7 & 4.8 \\
\hline Business & 3.1 & 2.3 & 2.8 & 1.7 & 3.5 & 3.2 \\
\hline Skilled manual/machinery & 12.9 & 12.1 & 10.0 & 11.3 & 13.6 & 13.4 \\
\hline Unskilled non-agricultural labourer & 17.5 & 11.6 & 16.2 & 11.9 & 18.2 & 11.3 \\
\hline Other & 0.3 & 0.3 & 0.1 & 0.3 & 0.3 & 0.2 \\
\hline Never worked & 0.1 & 0.3 & 0.6 & 0.4 & 0.1 & 0.1 \\
\hline \multicolumn{7}{|l|}{ Current/last occupational status of mother } \\
\hline Cultivator & 15.4 & 21.1 & 16.1 & 22.2 & 15.1 & 19.4 \\
\hline Agricultural labourer & 38.1 & 44.1 & 38.9 & 44.9 & 35.7 & 42.9 \\
\hline Administrative/executive/managerial/clerical & 0.4 & 0.8 & 0.1 & 0.6 & 0.6 & 1.0 \\
\hline Business & 1.2 & 0.7 & 0.9 & 0.4 & 1.2 & 1.1 \\
\hline Skilled manual/machinery & 3.1 & 3.7 & 2.3 & 2.8 & 3.5 & 5.0 \\
\hline Unskilled non-agricultural labourer & 10.2 & 7.2 & 9.7 & 7.4 & 10.2 & 6.9 \\
\hline Other & 0.1 & 0.0 & 0.0 & 0.0 & 0.1 & 1.1 \\
\hline Housewife/never worked & 31.6 & 22.3 & 32.1 & 21.4 & 33.6 & 23.7 \\
\hline Number of respondents & 1,190 & 2,722 & 757 & 1,293 & 945 & 1,429 \\
\hline
\end{tabular}

Note: All Ns are unweighted. Column totals may not equal 100\% due to missing cases or "don't know" responses. NC: Not calculated, as more than $50 \%$ had no formal education. 
The finding that married young men were less likely than both married young women and unmarried youth to report that both parents were living is likely a function of the fact that married young men in our sample were older than other youth. Rural-urban differences were generally narrow. For those with just one parent surviving, this parent was more likely to be the mother $(13-14 \%)$ than the father $(2-4 \%)$. Finally, $1-2 \%$ of youth reported that neither parent was alive.

Parents' educational attainment was considerably lower than that of youth respondents. Indeed, the parents of more than half of both young men and women had never been to school. The only exception was fathers of unmarried young women, who typically had 5 years of education. Rural-urban differences in educational attainment were wide as far as father's educational attainment levels were concerned; fathers of over half of rural youth had never been to school and fathers of urban young men and women had 5 and 6 years of schooling, respectively (7-8 years among fathers of the unmarried youth). Other rural-urban differences were not observed.

The Youth Study also inquired about the current or last main occupation of respondents' parents. Distributions of occupational status suggest that fathers of $18 \%$ and $24 \%$ of young men and women, respectively, were working on their own farms, compared to mothers of $12 \%$ and $16 \%$, respectively, of young men and women. In contrast, $31 \%$ of fathers and $28-34 \%$ of mothers were agricultural labourers; $17-19 \%$ of fathers and $4-5 \%$ of mothers were engaged in skilled manual occupations; and $17-21 \%$ of fathers and $10-12 \%$ of mothers were unskilled non-agricultural labourers. Few parents reported administrative, executive, managerial or clerical occupations (6-7\% of fathers and $1 \%$ of mothers); few, likewise, reported that they were doing their own business ( $4-5 \%$ of fathers and $1 \%$ of mothers). Finally, mothers of $42 \%$ and $33 \%$ of young men and women, respectively, were housewives; just a handful of fathers (less than 1\%) had never worked. Differences by marital status were evident. Fathers of married youth were more likely to have been engaged in agricultural activities and less likely to have been engaged in skilled manual or administrative, executive, managerial or clerical occupations than were fathers of unmarried youth. Mothers of the married were similarly more likely than mothers of the unmarried to have been engaged in agricultural activities, and conversely, mothers of the unmarried were more likely to be housewives. Rural-urban differences were evident. While rural parents were largely cultivators and agricultural labourers, urban parents were more likely than their rural counterparts to be concentrated in skilled manual occupations and unskilled non-agricultural activities. Additionally, urban fathers were more likely than rural fathers to be engaged in administrative, managerial or clerical occupations, and urban mothers were more likely than rural mothers to be engaged in housework. 


\section{Education}

Young people in India are spending more of their adolescent years acquiring an education than ever before. Educational attainment levels have increased, the percentage that has never been to school has declined and gender differences in educational attainment levels have diminished (Office of the Registrar General and Census Commissioner, 2001a). This does not mean, however, that schooling is universal or that gender differences are no longer a concern. Attainment of primary school education is still far from universal, especially among girls. Differences by caste, religion, region and poverty levels persist; and the quality of education varies widely for different sub-groups of youth. This chapter examines the schooling experiences of youth in terms of educational attainment, reasons for school non-attendance or discontinuation, quality of schools and colleges attended and schooling experiences.

\subsection{Educational attainment}

The Youth Study obtained information on whether the respondent had ever been to school and, if so, the number of years of schooling successfully completed. Current schooling status was also assessed and a Life Event Calendar inquired about the schooling status of all respondents from the age of 12. Findings are presented in Table 3.1.

Findings highlight that schooling was far from universal among young people, particularly among young women in Andhra Pradesh: $8 \%$ of young men and $21 \%$ of young women had never been to school. Wide differences by marital status were evident: $5 \%$ of unmarried young men compared to $21 \%$ of married young men had no formal education. The corresponding difference among young women was much wider: $7 \%$ of unmarried young women compared to $31 \%$ of married young women had never been to school. As expected, a larger percentage of rural than urban youth-particularly young women - had never attended school; indeed, one quarter of young women in rural areas, compared to $10 \%$ of those in urban areas had never been to school.

Educational attainment levels of young people also reconfirm differences by sex, marital status and rural-urban residence. Young men, on average, had 2 more years of schooling than young women (10 versus 8); however, gender differences were not observed among the unmarried, among whom both young men and women had attained 10 years of schooling. Differences by marital status were evident, with unmarried young men attaining 3 more years of education than their married counterparts (10 versus 7 ); and unmarried young women attaining 5 more years of education than their married counterparts (10 versus 5). Rural-urban differences suggest that urban young men typically had 1 more year of schooling than their rural counterparts (10 versus 9$)$; the corresponding difference among young women was 3 years (10 versus 7$)$.

Similar patterns were evident in terms of the proportion who had completed high school (Class 10). Young men were more likely than young women to have completed high school (52\% versus $36 \%$ ). However, this gender difference was observed only among the married; about equal proportions of unmarried young men and women had completed high school (56-57\%). Married youth were considerably less likely to have completed high school than the unmarried (31\% and 57\%, respectively, among young men; $21 \%$ and $56 \%$, respectively, among young women). We note that disparities by marital status may be wider than what is reflected here because the unmarried were younger and more likely to be pursuing their education at the time of the interview. Finally, rural youth were considerably less likely to have completed high school than urban youth (49\% and 59\%, respectively, among young men; $29 \%$ and $53 \%$ among young women, respectively). 
At the time of the interview, 33\% of young men compared to $19 \%$ of young women were in school or college. These gender differences were strongly influenced by marital status distributions of youth. Indeed, gender differences disappeared when the married and the unmarried were considered separately: $40 \%$ and $41 \%$ of unmarried young men and women were still studying, compared to just $1-2 \%$ of married youth. Among the unmarried, in addition, rural-urban differences were pronounced, with urban youth considerably more likely to be studying at the time of

Table 3.1: Educational attainment and current educational status

Percent distribution of youth by years of schooling successfully completed, median years of schooling and percentage currently in school, according to residence, Andhra Pradesh, 2007-08

\begin{tabular}{|c|c|c|c|c|c|c|}
\hline Schooling status (\%) & $\begin{array}{c}M \\
15-24\end{array}$ & $\begin{array}{c}\text { W } \\
15-24\end{array}$ & $\begin{array}{c}\text { MM } \\
15-29\end{array}$ & $\begin{array}{c}\text { MW } \\
15-24\end{array}$ & $\begin{array}{c}\text { UM } \\
15-24\end{array}$ & $\begin{array}{c}\text { UW } \\
\text { 15-24 }\end{array}$ \\
\hline \multicolumn{7}{|c|}{ Combined } \\
\hline \multicolumn{7}{|l|}{ Completed years of schooling } \\
\hline None $^{1}$ & 7.8 & 20.8 & 21.1 & 31.3 & 5.1 & 7.2 \\
\hline $1-4$ & 6.2 & 8.3 & 11.0 & 11.1 & 4.3 & 4.6 \\
\hline $5-7$ & 16.9 & 20.2 & 22.1 & 25.1 & 14.5 & 13.7 \\
\hline $8-9$ & 17.5 & 14.8 & 14.7 & 11.8 & 18.7 & 18.9 \\
\hline $10-11$ & 28.9 & 21.5 & 16.8 & 13.8 & 31.6 & 31.8 \\
\hline 12 and above & 22.7 & 14.4 & 14.4 & 6.9 & 25.8 & 23.7 \\
\hline Median years of schooling & 10.0 & 8.0 & 7.0 & 5.0 & 10.0 & 10.0 \\
\hline Currently in school/college & 33.1 & 18.8 & 0.8 & 1.7 & 40.3 & 40.9 \\
\hline Number of respondents & 2,479 & 4,848 & 1,405 & 2,330 & 2,077 & 2,518 \\
\hline \multicolumn{7}{|c|}{ Urban } \\
\hline \multicolumn{7}{|l|}{ Completed years of schooling } \\
\hline None $^{1}$ & 4.8 & 10.0 & 13.7 & 17.1 & 3.3 & 4.2 \\
\hline $1-4$ & 4.1 & 4.8 & 10.5 & 7.2 & 3.0 & 3.0 \\
\hline $5-7$ & 15.3 & 16.0 & 19.3 & 23.2 & 13.8 & 10.2 \\
\hline $8-9$ & 17.0 & 16.6 & 15.0 & 16.8 & 17.3 & 16.5 \\
\hline $10-11$ & 26.4 & 24.4 & 19.9 & 20.0 & 27.3 & 28.0 \\
\hline 12 and above & 32.3 & 28.1 & 21.6 & 15.6 & 35.3 & 38.1 \\
\hline Median years of schooling & 10.0 & 10.0 & 9.0 & 8.0 & 10.0 & 10.0 \\
\hline Currently in school/college & 39.7 & 31.2 & 0.7 & 3.6 & 45.4 & 53.5 \\
\hline Number of respondents & 1,289 & 2,126 & 648 & 1,037 & 1,132 & 1,089 \\
\hline \multicolumn{7}{|c|}{ Rural } \\
\hline \multicolumn{7}{|l|}{ Completed years of schooling } \\
\hline None $^{1}$ & 8.9 & 25.3 & 23.1 & 35.3 & 5.9 & 8.9 \\
\hline $1-4$ & 6.9 & 9.7 & 11.2 & 12.2 & 4.8 & 5.6 \\
\hline $5-7$ & 17.6 & 21.9 & 22.8 & 25.7 & 14.9 & 15.8 \\
\hline $8-9$ & 17.7 & 14.1 & 14.7 & 10.4 & 19.3 & 20.3 \\
\hline $10-11$ & 29.8 & 20.3 & 15.9 & 12.1 & 33.4 & 33.9 \\
\hline 12 and above & 19.0 & 8.6 & 12.3 & 4.4 & 21.6 & 15.6 \\
\hline Median years of schooling & 9.0 & 7.0 & 7.0 & 5.0 & 10.0 & 9.0 \\
\hline Currently in school/college & 30.5 & 13.5 & 0.7 & 1.2 & 38.2 & 33.8 \\
\hline Number of respondents & 1,190 & 2,722 & 757 & 1,293 & 945 & 1,429 \\
\hline
\end{tabular}

Note: All Ns are unweighted. Column totals may not equal 100\% due to missing cases or "don't know" responses. ${ }^{1}$ Includes non-literate and literate with no formal schooling. 
the interview than their rural counterparts. Differences were particularly wide among young women; for example, $45 \%$ and $38 \%$ of unmarried young men in urban and rural areas respectively, were pursuing their education at the time of the interview, compared to $54 \%$ and $34 \%$ respectively, of unmarried young women.

\subsection{Differentials in educational attainment}

Differentials in educational levels of young men and women, measured with respect to completed years of schooling, are presented in Tables 3.2 and 3.3, respectively. Although findings show that the younger cohort (15-19 years) was, in general, better educated than the older cohort (20-24 years), this pattern is not consistently observed when the married and unmarried and those in rural and urban settings are considered separately. For example, the older cohort was more likely than the younger cohort to have completed Class 10 among both married and unmarried young men and women. Age differences by rural-urban residence suggest that in urban settings, about equal proportions of both cohorts had completed Class 10; among rural youth, in contrast, 15-19 year olds were considerably more likely to have done so, suggesting perhaps that the thrust on school completion in rural areas has been relatively recent.

Differences by religion among young men, shown in Tables 3.2, indicate that Hindu youth were typically better educated than both Muslim youth and those belonging to other religions. For example, 54\% of Hindu young men had completed at least 10 years of education, compared to $44 \%$ of Muslim young men and $33 \%$ of young men belonging to other religions. Among young women, although differentials were narrower, the same pattern held;

Table 3.2: Educational attainment of young men by selected background characteristics

Percent distribution of young men by educational level, according to selected background characteristics and residence, Andhra Pradesh, 2007-08

\begin{tabular}{|c|c|c|c|c|c|c|c|c|c|c|c|c|}
\hline \multirow{3}{*}{$\begin{array}{l}\text { Background } \\
\text { characteristics (\%) }\end{array}$} & \multicolumn{4}{|c|}{ M, 15-24 } & \multicolumn{4}{|c|}{ MM, 15-29 } & \multicolumn{4}{|c|}{ UM, 15-24 } \\
\hline & \multicolumn{12}{|c|}{ Completed years of schooling } \\
\hline & None $^{1}$ & $1-7$ & 8-9 & $10+$ & None $^{1}$ & $1-7$ & 8-9 & $10+$ & None $^{1}$ & $1-7$ & 8-9 & $10+$ \\
\hline \multicolumn{13}{|c|}{ Combined } \\
\hline \multicolumn{13}{|l|}{ Age (years) } \\
\hline $15-19$ & 5.2 & 17.2 & 22.1 & 55.6 & * & * & * & * & 4.8 & 17.1 & 22.3 & 55.8 \\
\hline $20-24$ & 10.1 & 28.4 & 13.5 & 48.0 & 18.8 & 42.7 & 12.4 & 26.1 & 5.6 & 21.0 & 14.0 & 59.4 \\
\hline $25-29$ & NA & NA & NA & NA & 21.9 & 29.3 & 15.8 & 33.0 & NA & NA & NA & NA \\
\hline \multicolumn{13}{|l|}{ Religion } \\
\hline Hindu & 7.5 & 21.1 & 17.7 & 53.7 & 21.2 & 31.7 & 14.9 & 32.2 & 4.8 & 16.5 & 18.8 & 59.9 \\
\hline Muslim & 6.5 & 35.0 & 14.6 & 43.9 & 17.3 & 33.7 & 15.4 & 33.7 & 6.0 & 33.2 & 16.6 & 44.2 \\
\hline Other ${ }^{2}$ & 14.4 & 31.8 & 20.5 & 33.3 & 24.1 & 50.6 & 12.6 & 12.6 & 9.7 & 28.2 & 21.4 & 40.8 \\
\hline \multicolumn{13}{|l|}{ Caste } \\
\hline SC & 9.5 & 22.3 & 21.1 & 47.2 & 24.2 & 34.7 & 16.3 & 24.8 & 6.0 & 17.1 & 22.6 & 54.3 \\
\hline ST & 25.0 & 26.9 & 9.0 & 39.1 & 37.4 & 33.9 & 5.2 & 23.5 & 21.0 & 22.9 & 9.5 & 46.7 \\
\hline OBC & 6.7 & 24.6 & 17.3 & 51.4 & 21.5 & 34.7 & 14.2 & 29.6 & 4.0 & 19.2 & 19.1 & 57.6 \\
\hline General $^{3}$ & 4.1 & 19.5 & 16.7 & 59.7 & 9.8 & 26.9 & 18.2 & 45.1 & 3.4 & 17.8 & 16.7 & 62.1 \\
\hline \multicolumn{13}{|l|}{ Wealth quintile } \\
\hline First & 19.1 & 35.3 & 12.4 & 33.2 & 37.1 & 37.7 & 10.3 & 14.9 & 17.0 & 28.6 & 14.6 & 39.8 \\
\hline Second & 12.7 & 30.9 & 15.6 & 40.8 & 35.6 & 40.3 & 9.7 & 14.4 & 7.1 & 27.2 & 16.9 & 48.8 \\
\hline Third & 6.8 & 26.8 & 19.6 & 46.9 & 19.9 & 37.8 & 18.1 & 24.2 & 4.6 & 21.5 & 20.6 & 53.2 \\
\hline Fourth & 5.0 & 21.1 & 19.8 & 54.0 & 11.1 & 32.2 & 18.1 & 38.6 & 3.3 & 18.2 & 20.4 & 58.0 \\
\hline Fifth & 2.1 & 9.2 & 17.1 & 71.6 & 7.9 & 15.7 & 14.5 & 62.0 & 1.2 & 6.8 & 18.2 & 73.8 \\
\hline Total & 7.8 & 23.1 & 17.5 & 51.6 & 21.1 & 33.0 & 14.7 & 31.1 & 5.1 & 18.8 & 18.7 & $\mathbf{5 7 . 4}$ \\
\hline
\end{tabular}

Cont'd on next page... 
Table 3.2: (Cont'd)

\begin{tabular}{|c|c|c|c|c|c|c|c|c|c|c|c|c|}
\hline \multirow{3}{*}{$\begin{array}{l}\text { Background } \\
\text { characteristics (\%) }\end{array}$} & \multicolumn{4}{|c|}{ M, 15-24 } & \multicolumn{4}{|c|}{ MM, 15-29 } & \multicolumn{4}{|c|}{ UM, 15-24 } \\
\hline & \multicolumn{12}{|c|}{ Completed years of schooling } \\
\hline & None $^{1}$ & $1-7$ & 8-9 & $10+$ & None $^{1}$ & $1-7$ & $8-9$ & $10+$ & None $^{1}$ & $1-7$ & $8-9$ & $10+$ \\
\hline \multicolumn{13}{|c|}{ Urban } \\
\hline \multicolumn{13}{|l|}{ Age (years) } \\
\hline $15-19$ & 4.4 & 14.9 & 21.6 & 59.0 & * & * & * & * & 4.4 & 15.0 & 21.5 & 59.2 \\
\hline $20-24$ & 5.1 & 23.1 & 13.3 & 58.5 & 15.1 & 38.4 & 15.1 & 31.5 & 2.3 & 18.4 & 12.9 & 66.3 \\
\hline $25-29$ & NA & NA & NA & NA & 13.4 & 26.8 & 15.2 & 44.6 & NA & NA & NA & NA \\
\hline \multicolumn{13}{|l|}{ Religion } \\
\hline Hindu & 3.8 & 16.3 & 17.1 & 62.8 & 11.7 & 28.0 & 15.6 & 44.7 & 2.5 & 13.5 & 17.1 & 66.9 \\
\hline Muslim & 9.1 & 36.4 & 14.1 & 40.4 & 22.9 & 34.3 & 14.3 & 28.6 & 7.8 & 33.3 & 15.6 & 43.3 \\
\hline Other $^{2}$ & 8.8 & 23.5 & 23.5 & 44.1 & $(28.6)$ & $(42.9)$ & $(7.1)$ & $(21.4)$ & 6.5 & 19.4 & 25.8 & 48.4 \\
\hline \multicolumn{13}{|l|}{ Caste } \\
\hline SC & 6.0 & 18.1 & 24.1 & 51.7 & 22.0 & 32.0 & 18.0 & 28.0 & 2.9 & 16.7 & 24.5 & 55.9 \\
\hline ST & $(20.8)$ & $(20.8)$ & $(8.3)$ & $(50.0)$ & $(21.4)$ & $(50.0)$ & $(0.0)$ & $(28.6)$ & $(10.0)$ & $(25.0)$ & $(10.0)$ & $(55.0)$ \\
\hline OBC & 4.4 & 20.7 & 18.4 & 56.6 & 12.3 & 29.0 & 16.8 & 41.9 & 3.3 & 17.5 & 18.2 & 61.1 \\
\hline General $^{3}$ & 3.6 & 17.5 & 12.6 & 66.4 & 10.3 & 26.4 & 12.6 & 50.6 & 2.5 & 14.7 & 13.2 & 69.6 \\
\hline \multicolumn{13}{|l|}{ Wealth quintile } \\
\hline First & 17.9 & 46.4 & 21.4 & 14.3 & $(44.4)$ & $(38.9)$ & $(11.1)$ & (5.6) & $(14.3)$ & $(47.6)$ & $(23.8)$ & (14.3) \\
\hline Second & 15.3 & 42.4 & 16.9 & 25.4 & 29.0 & 48.4 & 12.9 & 9.7 & 8.7 & 41.3 & 17.4 & 32.6 \\
\hline Third & 9.8 & 29.4 & 11.8 & 49.0 & 19.1 & 34.0 & 17.0 & 29.8 & 7.1 & 27.4 & 13.1 & 52.4 \\
\hline Fourth & 3.8 & 22.1 & 21.1 & 53.1 & 12.4 & 31.4 & 17.1 & 39.0 & 3.2 & 19.7 & 21.3 & 55.9 \\
\hline Fifth & 0.7 & 7.2 & 15.7 & 76.4 & 2.9 & 18.1 & 14.3 & 64.8 & 0.7 & 5.5 & 15.5 & 78.4 \\
\hline Total & 4.8 & 19.4 & 17.0 & 58.7 & 13.7 & 29.7 & 15.0 & 41.5 & 3.3 & 16.7 & 17.3 & 62.7 \\
\hline \multicolumn{13}{|c|}{ Rural } \\
\hline \multicolumn{13}{|l|}{ Age (years) } \\
\hline $15-19$ & 5.4 & 18.0 & 22.2 & 54.4 & * & * & * & * & 4.9 & 18.0 & 22.4 & 54.7 \\
\hline $20-24$ & 12.2 & 30.6 & 13.5 & 43.7 & 19.6 & 43.5 & 11.9 & 25.0 & 7.5 & 22.2 & 14.6 & 55.8 \\
\hline $25-29$ & NA & NA & NA & NA & 24.5 & 30.0 & 16.1 & 29.4 & NA & NA & NA & NA \\
\hline \multicolumn{13}{|l|}{ Religion } \\
\hline Hindu & 8.9 & 23.0 & 17.8 & 50.3 & 23.7 & 32.7 & 14.7 & 28.8 & 5.8 & 17.7 & 19.5 & 57.0 \\
\hline Muslim & 4.8 & 34.0 & 15.0 & 46.3 & $(14.3)$ & $(32.9)$ & $(15.7)$ & $(37.1)$ & 4.8 & 32.5 & 17.5 & 45.2 \\
\hline Other ${ }^{2}$ & 16.3 & 34.7 & 19.4 & 29.6 & 23.6 & 51.4 & 13.9 & 11.1 & (11.1) & (31.9) & (19.4) & $(37.5)$ \\
\hline \multicolumn{13}{|l|}{ Caste } \\
\hline SC & 10.4 & 23.3 & 20.5 & 45.8 & 24.7 & 34.9 & 16.1 & 24.3 & 6.9 & 17.4 & 22.0 & 53.7 \\
\hline ST & 26.3 & 27.8 & 9.0 & 36.8 & 39.0 & 32.0 & 6.0 & 23.0 & 23.3 & 23.3 & 9.3 & 44.2 \\
\hline OBC & 7.6 & 26.3 & 16.9 & 49.3 & 24.3 & 36.5 & 13.4 & 25.8 & 4.3 & 20.0 & 19.7 & 56.0 \\
\hline General $^{3}$ & 4.5 & 20.5 & 19.2 & 55.8 & 9.5 & 27.1 & 20.6 & 42.7 & 3.9 & 19.6 & 18.7 & 57.8 \\
\hline \multicolumn{13}{|l|}{ Wealth quintile } \\
\hline First & 19.1 & 34.0 & 11.7 & 35.2 & 36.7 & 37.3 & 10.1 & 15.8 & 16.8 & 26.6 & 13.6 & 42.9 \\
\hline Second & 12.1 & 29.2 & 15.6 & 43.1 & 36.5 & 39.1 & 9.4 & 15.0 & 6.9 & 25.2 & 16.8 & 51.1 \\
\hline Third & 6.1 & 26.1 & 21.1 & 46.6 & 20.1 & 38.0 & 18.7 & 23.2 & 4.0 & 20.1 & 22.4 & 53.4 \\
\hline Fourth & 5.7 & 20.4 & 19.3 & 54.6 & 10.6 & 32.3 & 18.5 & 38.6 & 3.3 & 17.5 & 20.0 & 59.2 \\
\hline Fifth & 4.2 & 11.6 & 18.5 & 65.6 & 11.7 & 13.9 & 14.6 & 59.9 & 2.3 & 8.6 & 21.7 & 67.4 \\
\hline Total & 8.9 & 24.5 & 17.7 & 48.9 & 23.1 & 34.0 & 14.7 & 28.2 & 5.9 & 19.7 & 19.3 & 55.1 \\
\hline
\end{tabular}

Note: Row totals may not equal 100\% due to missing cases or "don't know" responses. ( ) Based on 25-49 unweighted cases. ${ }^{*}$ Percentage not shown, based on fewer than 25 unweighted cases. NA: Not applicable. OBC: Other backward caste. SC: Scheduled caste. ST: Scheduled tribe. ${ }^{1}$ Includes non-literate and literate with no formal schooling. ${ }^{2}$ Includes Christian, Buddhist, Neo-Buddhist, Sikh, Jain, Jewish, Parsi/Zoroastrian and no specified religion. ${ }^{3}$ Includes all those not belonging to SC, ST or OBC. 
$37 \%$ of Hindu women, compared to $34 \%$ of Muslim women and $29 \%$ of women belonging to other religions had completed 10 or more years of education. Patterns were by and large similar for both the unmarried and the married and those residing in rural and urban areas.

Caste differences were more consistent, with those belonging to general castes considerably more likely than others to have completed 10 or more years of schooling among both young men and young women, irrespective of marital status and rural-urban residence. Those belonging to scheduled tribes were typically least likely to have attained this level of education.

Finally, a positive association was consistently observed between the economic status of young people's households, measured in wealth quintiles, and young people's educational attainment levels. For example, among young men, $33 \%$ of those from households in the poorest (first) quintile had completed 10 or more years of schooling, compared to $72 \%$ of those from households in the wealthiest (fifth) quintile. Among young women however, an even stronger positive association was observed: just $14 \%$ of those from households in the poorest quintile (first) had completed 10 or more years of education. This percentage increased to $66 \%$ among those belonging to the wealthiest quintile (fifth). Patterns were similar for both the unmarried and the married and those residing in rural and urban areas.

Table 3.3: Educational attainment of young women by selected background characteristics

Percent distribution of young women by educational level, according to selected background characteristics and residence, Andhra Pradesh, 2007-08

\begin{tabular}{|c|c|c|c|c|c|c|c|c|c|c|c|c|}
\hline \multirow{3}{*}{$\begin{array}{l}\text { Background } \\
\text { characteristics (\%) }\end{array}$} & \multicolumn{4}{|c|}{ W, 15-24 } & \multicolumn{4}{|c|}{ MW, 15-24 } & \multicolumn{4}{|c|}{ UW, 15-24 } \\
\hline & \multicolumn{12}{|c|}{ Completed years of schooling } \\
\hline & None ${ }^{1}$ & $1-7$ & $8-9$ & $10+$ & None $^{1}$ & $1-7$ & $8-9$ & $10+$ & None $^{1}$ & $1-7$ & $8-9$ & $10+$ \\
\hline \multicolumn{13}{|c|}{ Combined } \\
\hline Age (years) & & & & & & & & & & & & \\
\hline $15-19$ & 15.2 & 26.9 & 18.6 & 39.3 & 29.9 & 40.2 & 12.8 & 17.1 & 7.7 & 20.2 & 21.6 & 50.5 \\
\hline $20-24$ & 26.6 & 30.0 & 11.0 & 32.4 & 31.8 & 34.4 & 11.4 & 22.3 & 5.6 & 11.8 & 9.1 & 73.4 \\
\hline \multicolumn{13}{|l|}{ Religion } \\
\hline Hindu & 21.1 & 27.2 & 14.9 & 36.8 & 31.9 & 34.8 & 11.7 & 21.6 & 7.0 & 17.4 & 19.2 & 56.5 \\
\hline Muslim & 20.7 & 32.9 & 12.5 & 33.8 & 33.3 & 40.1 & 9.5 & 17.0 & 7.6 & 25.8 & 15.7 & 51.0 \\
\hline Other ${ }^{2}$ & 18.0 & 36.8 & 16.1 & 29.1 & 24.2 & 46.4 & 14.2 & 15.2 & 9.4 & 22.3 & 18.8 & 49.5 \\
\hline \multicolumn{13}{|l|}{ Caste } \\
\hline SC & 21.4 & 32.6 & 15.6 & 30.4 & 30.5 & 41.7 & 11.4 & 16.4 & 9.9 & 21.3 & 21.0 & 47.8 \\
\hline ST & 41.8 & 24.3 & 11.0 & 22.9 & 55.2 & 26.7 & 6.7 & 11.5 & 15.7 & 19.0 & 19.0 & 46.3 \\
\hline OBC & 21.8 & 29.8 & 15.3 & 33.1 & 31.7 & 36.3 & 12.2 & 19.8 & 7.7 & 20.7 & 19.6 & 52.0 \\
\hline General $^{3}$ & 12.6 & 22.7 & 14.3 & 50.4 & 22.5 & 33.6 & 13.0 & 30.9 & 2.5 & 11.9 & 15.8 & 69.8 \\
\hline \multicolumn{13}{|l|}{ Wealth quintile } \\
\hline First & 43.3 & 32.9 & 9.7 & 14.2 & 53.3 & 34.0 & 6.7 & 6.0 & 23.7 & 30.4 & 15.5 & 30.4 \\
\hline Second & 27.2 & 37.0 & 15.6 & 20.2 & 37.1 & 40.8 & 11.4 & 10.6 & 10.3 & 30.4 & 23.1 & 36.3 \\
\hline Third & 21.6 & 32.1 & 15.7 & 30.5 & 31.7 & 38.6 & 12.4 & 17.3 & 6.3 & 21.9 & 20.7 & 51.1 \\
\hline Fourth & 11.7 & 27.5 & 15.7 & 45.1 & 19.2 & 40.3 & 12.2 & 28.3 & 3.5 & 13.3 & 19.5 & 63.7 \\
\hline Fifth & 4.5 & 13.1 & 16.4 & 66.0 & 9.6 & 22.2 & 17.5 & 50.6 & 0.8 & 6.1 & 15.7 & 77.4 \\
\hline Total & 20.8 & 28.4 & 14.8 & 35.9 & 31.3 & 36.2 & 11.8 & 20.7 & 7.2 & 18.4 & 18.9 & 55.5 \\
\hline
\end{tabular}

Cont'd on next page... 
Table 3.3 (Cont'd)

\begin{tabular}{|c|c|c|c|c|c|c|c|c|c|c|c|c|}
\hline \multirow{3}{*}{$\begin{array}{l}\text { Background } \\
\text { characteristics (\%) }\end{array}$} & \multicolumn{4}{|c|}{ W, 15-24 } & \multicolumn{4}{|c|}{ MW, 15-24 } & \multicolumn{4}{|c|}{ UW, 15-24 } \\
\hline & \multicolumn{12}{|c|}{ Completed years of schooling } \\
\hline & None $^{1}$ & $1-7$ & 8-9 & $10+$ & None $^{1}$ & $1-7$ & 8-9 & $10+$ & None $^{1}$ & $1-7$ & $8-9$ & $10+$ \\
\hline \multicolumn{13}{|c|}{ Urban } \\
\hline \multicolumn{13}{|l|}{ Age (years) } \\
\hline $15-19$ & 8.4 & 19.2 & 19.2 & 53.2 & 23.7 & 35.6 & 13.6 & 27.1 & 4.4 & 14.9 & 20.7 & 60.1 \\
\hline $20-24$ & 11.6 & 22.3 & 14.2 & 51.9 & 15.5 & 29.0 & 17.4 & 38.1 & 4.1 & 9.3 & 7.6 & 79.0 \\
\hline \multicolumn{13}{|l|}{ Religion } \\
\hline Hindu & 10.2 & 19.4 & 16.6 & 53.9 & 17.6 & 28.2 & 17.1 & 37.1 & 3.8 & 12.1 & 16.3 & 67.9 \\
\hline Muslim & 10.5 & 25.0 & 16.9 & 47.7 & 16.7 & 37.0 & 13.0 & 33.3 & 5.9 & 17.6 & 19.3 & 57.1 \\
\hline Other ${ }^{2}$ & 8.3 & 29.2 & 15.8 & 46.7 & 13.3 & 44.4 & 17.8 & 24.4 & 5.3 & 15.8 & 14.5 & 64.5 \\
\hline \multicolumn{13}{|l|}{ Caste } \\
\hline SC & 13.7 & 27.7 & 17.1 & 41.4 & 21.5 & 36.4 & 15.7 & 26.4 & 7.0 & 20.6 & 18.6 & 53.8 \\
\hline ST & $(25.8)$ & $(22.6)$ & $(16.1)$ & $(35.5)$ & $(31.3)$ & $(25.0)$ & (18.8) & $(25.0)$ & * & * & * & * \\
\hline OBC & 11.1 & 21.3 & 17.6 & 50.0 & 18.8 & 30.0 & 16.9 & 34.2 & 3.7 & 12.9 & 17.9 & 65.4 \\
\hline General $^{3}$ & 4.6 & 15.0 & 14.5 & 65.8 & 9.3 & 26.4 & 16.3 & 48.1 & 2.2 & 8.5 & 13.6 & 75.7 \\
\hline \multicolumn{13}{|l|}{ Wealth quintile } \\
\hline First & 35.4 & 38.4 & 11.1 & 15.2 & 43.8 & 37.5 & 10.4 & 8.3 & 23.9 & 39.1 & 10.9 & 26.1 \\
\hline Second & 24.0 & 37.2 & 12.4 & 26.4 & 28.6 & 38.1 & 11.1 & 22.2 & 17.7 & 37.1 & 14.5 & 30.6 \\
\hline Third & 16.1 & 31.3 & 21.4 & 31.3 & 23.2 & 34.3 & 21.2 & 21.2 & 6.7 & 28.3 & 22.5 & 42.5 \\
\hline Fourth & 7.2 & 21.3 & 18.8 & 52.8 & 11.9 & 35.1 & 17.9 & 35.1 & 3.1 & 8.8 & 19.5 & 68.7 \\
\hline Fifth & 2.0 & 9.3 & 14.9 & 73.8 & 5.4 & 17.4 & 17.4 & 59.7 & 0.2 & 5.0 & 13.8 & 81.0 \\
\hline Total & 10.0 & 20.9 & 16.6 & 52.5 & 17.1 & 30.5 & 16.8 & 35.6 & 4.2 & 13.1 & 16.5 & 66.2 \\
\hline \multicolumn{13}{|c|}{ Rural } \\
\hline \multicolumn{13}{|l|}{ Age (years) } \\
\hline $15-19$ & 17.8 & 29.9 & 18.4 & 34.0 & 31.3 & 41.1 & 12.5 & 15.2 & 9.2 & 22.7 & 22.1 & 46.0 \\
\hline $20-24$ & 33.6 & 33.5 & 9.5 & 23.5 & 37.4 & 36.3 & 9.3 & 17.0 & 7.4 & 14.7 & 10.5 & 67.4 \\
\hline \multicolumn{13}{|l|}{ Religion } \\
\hline Hindu & 25.3 & 30.3 & 14.2 & 30.2 & 35.8 & 36.6 & 10.2 & 17.4 & 8.5 & 20.1 & 20.6 & 50.7 \\
\hline Muslim & 31.0 & 40.9 & 8.2 & 19.9 & 43.0 & 41.9 & 7.5 & 7.5 & 10.3 & 38.5 & 10.3 & 41.0 \\
\hline Other ${ }^{2}$ & 22.0 & 39.7 & 16.3 & 22.0 & 27.1 & 47.0 & 13.3 & 12.7 & 12.0 & 25.6 & 21.6 & 40.8 \\
\hline \multicolumn{13}{|l|}{ Caste } \\
\hline SC & 24.7 & 34.8 & 15.0 & 25.6 & 33.7 & 43.4 & 10.0 & 12.9 & 11.2 & 21.8 & 22.3 & 44.7 \\
\hline ST & 43.9 & 24.0 & 10.3 & 21.8 & 58.4 & 26.8 & 4.7 & 10.1 & 14.8 & 19.4 & 20.4 & 45.4 \\
\hline OBC & 25.9 & 33.1 & 14.4 & 26.6 & 35.4 & 38.0 & 10.9 & 15.7 & 9.6 & 24.5 & 20.4 & 45.4 \\
\hline General $^{3}$ & 17.3 & 27.4 & 14.2 & 41.1 & 27.2 & 36.1 & 11.8 & 24.9 & 2.8 & 14.7 & 17.8 & 64.7 \\
\hline \multicolumn{13}{|l|}{ Wealth quintile } \\
\hline First & 44.4 & 32.0 & 9.6 & 14.0 & 54.3 & 33.5 & 6.5 & 5.7 & 23.8 & 28.8 & 16.0 & 31.3 \\
\hline Second & 27.7 & 36.9 & 16.1 & 19.2 & 38.3 & 41.2 & 11.5 & 9.0 & 9.0 & 29.4 & 24.4 & 37.1 \\
\hline Third & 23.2 & 32.2 & 14.2 & 30.3 & 33.3 & 39.6 & 10.7 & 16.4 & 6.1 & 19.9 & 20.4 & 53.6 \\
\hline Fourth & 14.8 & 31.8 & 13.6 & 39.8 & 22.9 & 43.1 & 9.2 & 24.8 & 4.1 & 16.5 & 19.7 & 59.7 \\
\hline Fifth & 8.0 & 18.2 & 18.5 & 55.4 & 13.0 & 26.0 & 17.7 & 43.2 & 1.8 & 8.3 & 19.3 & 70.6 \\
\hline Total & 25.3 & 31.6 & 14.1 & 29.0 & 35.3 & 37.8 & 10.4 & 16.5 & 8.9 & 21.4 & 20.3 & 49.5 \\
\hline
\end{tabular}

Note: Row totals may not equal 100\% due to missing cases or "don't know" responses. ( ) Based on 25-49 unweighted cases. ${ }^{*}$ Percentage not shown, based on fewer than 25 unweighted cases. OBC: Other backward caste. SC: Scheduled caste. ST: Scheduled tribe. ${ }^{1}$ Includes non-literate and literate with no formal schooling. ${ }^{2}$ Includes Christian, Buddhist, Neo-Buddhist, Sikh, Jain, Jewish, Parsi/Zoroastrian and no specified religion. ${ }^{3}$ Includes all those not belonging to SC, ST or OBC. 


\subsection{School attendance}

Figure 3.1 presents schooling status at ages 12 and 15, representing periods before and after puberty was attained for many. Findings suggest limited school attendance among young people in Andhra Pradesh: $81 \%$ and $63 \%$ of young men and women, respectively, were in school at age 12 and far fewer- $66 \%$ and $46 \%$ - at age 15 . Married youth and rural youth were far less likely than their unmarried and urban counterparts to be in school at ages 12 or 15 .

Findings also imply different rates of retention in school between ages 12 and 15 between young men and women as well as between the married and the unmarried. For example, while $61 \%$ of married young men were in school at age 12, just $43 \%$ were still in school at age 15 . Correspondingly, among married young women, $47 \%$ and $28 \%$ were in school at ages 12 and 15 . Among the unmarried, there were, in contrast, considerably higher rates of retention: $86 \%$ and $83 \%$ of unmarried young men and women, respectively, were in school at age 12, and $73 \%$ and $69 \%$, respectively, remained in school at age 15 .

Figures 3.2 a-c show graphically the cumulative percentages of youth (all youth who had completed at least one year of schooling) who had completed each year of education from Class 2 to Class 17, using life table techniques. Findings show that youth who had completed at least one year of schooling by and large remained in school up to Class 4 (95-96\% had completed Class 4). Declines in school completion and gender difference in school completion became more evident thereafter. For example, among young women, completion rates fell to $90 \%$ in Class 5 and $80 \%$ in Class 6 . Among young men, completion rates fell to $88 \%$ and $81 \%$ in Classes 6 and 7 , respectively. Considerable differences in school completion were evident by marital status. For example, completion rates fell below $90 \%$ as early as in Class 5 among married youth; in contrast among unmarried youth, completion rates fell below $90 \%$ in Class 7 among both young men and women. Rural-urban differences suggest that among those in urban areas, completion rates fell below $90 \%$ in Class 7 among young men and Class 6 among young women. Completion rates fell below 90\% a year earlier among young men and women in rural areas.

Two notable subsequent declines in completion rates were observed. The first set of declines occurred between Classes 9 and 11, where declines exceeded $10 \%$ a year (Figure 3.2a). Declines were steep at around the time of high school completion: For example, while $73 \%$ of young men and $61 \%$ of young women had completed Class $9,62 \%$ and $50 \%$, respectively, had completed Class 10, and percentages fell further to $47 \%$ and 33\%, respectively by Class 11 . Findings suggest therefore that many youth may have discontinued their education because they did not pass Class 10, and many who passed Class 10 opted not to pursue further education. The second decline occurred between Classes 15 and 16, typically associated with the transition to postgraduate degree courses: $28 \%$ and $17 \%$ of young men and women, respectively, had completed Class 15, and just 13\% and 8\%, respectively, completed Class 16 .
Figure 3.1: Percentage of youth who were in school at ages 12 and 15, according to residence, Andhra Pradesh, 2007-08
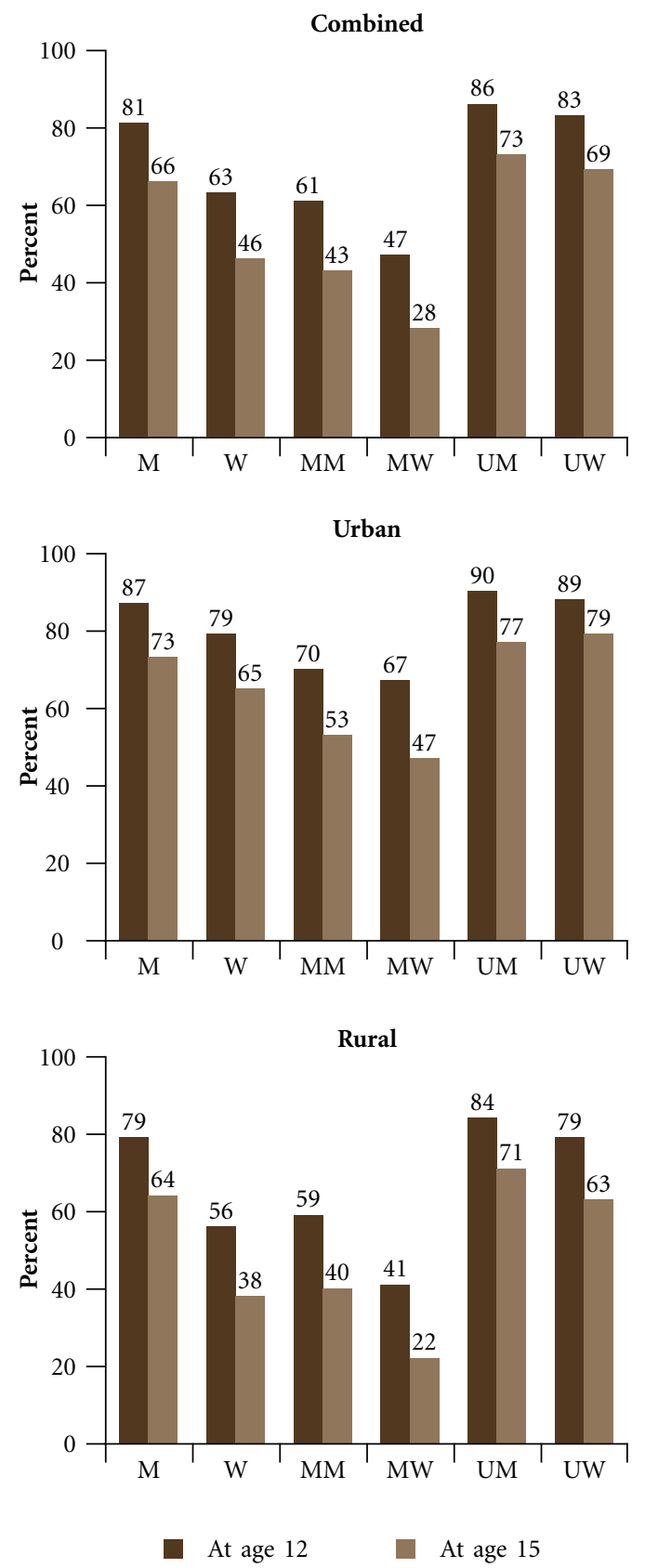
Marital status differences were evident, with steep declines occurring earlier among the married than the unmarried. For example, declines in the range of 9-14\% per year were observed among both married young men and women between Classes 5 and 8. In comparison, the unmarried recorded declines of just 4-6\% per year during this period. Declines between Classes 9 and 11 were, in contrast, similarly steep among the married and the unmarried (11-18\% per year). As a result, among the married, just 39\% and 30\% of young men and women, respectively, had completed Class 10 and $18 \%$ and 10\%, respectively, had completed Class 12 . In contrast, among the unmarried, as many as $67-69 \%$ and $46-47 \%$ had completed Classes 10 and 12, respectively. These marital status differences persisted in percentages of youth completing each class thereafter as well.

Rural and urban patterns of school completion (Figures 3.2b and 3.2c) diverged from an early stage of education, particularly among young women, and then widened. For example, while $89 \%$ of young women in urban areas had competed Class 6 , just $75 \%$ of their rural counterparts had done so; $63 \%$ compared to $43 \%$ had completed Class 10. Among young men differences were typically milder and diverged from a later stage. Nevertheless, while $67 \%$ and $50 \%$ of urban young men had completed Classes 10 and 12, respectively, just 59\% and 36\% of their rural counterparts had completed these classes. Notably, the female disadvantage in school completion was considerably more noticeable among rural than urban youth.

\subsection{Reasons for school non-attendance or discontinuation}

The Youth Study inquired about reasons for never going to school from all those who so reported, and reasons for discontinuing school from all those who had not completed Class 12. Responses are provided in Table 3.4a for those who had never gone to school and have been grouped into five categories: economic reasons (work on the family farm or business, wage earning work, family poverty, i.e., the family could not afford to keep the respondent in school); housework-related reasons (required for care of siblings or housework); attitude or perception-related reasons (unsafe to send children to school, education not considered necessary, respondent's lack of interest); school-related reasons (school located too far away, appropriate transport not available, poor school quality and infrastructure, poor quality of teaching); and health-related reasons (health problems of respondent, illness or death of a family member).

Figure 3.2a: Cumulative percentage of youth who had completed each year of education (Classes 1 to 17), Andhra Pradesh (combined), 2007-08

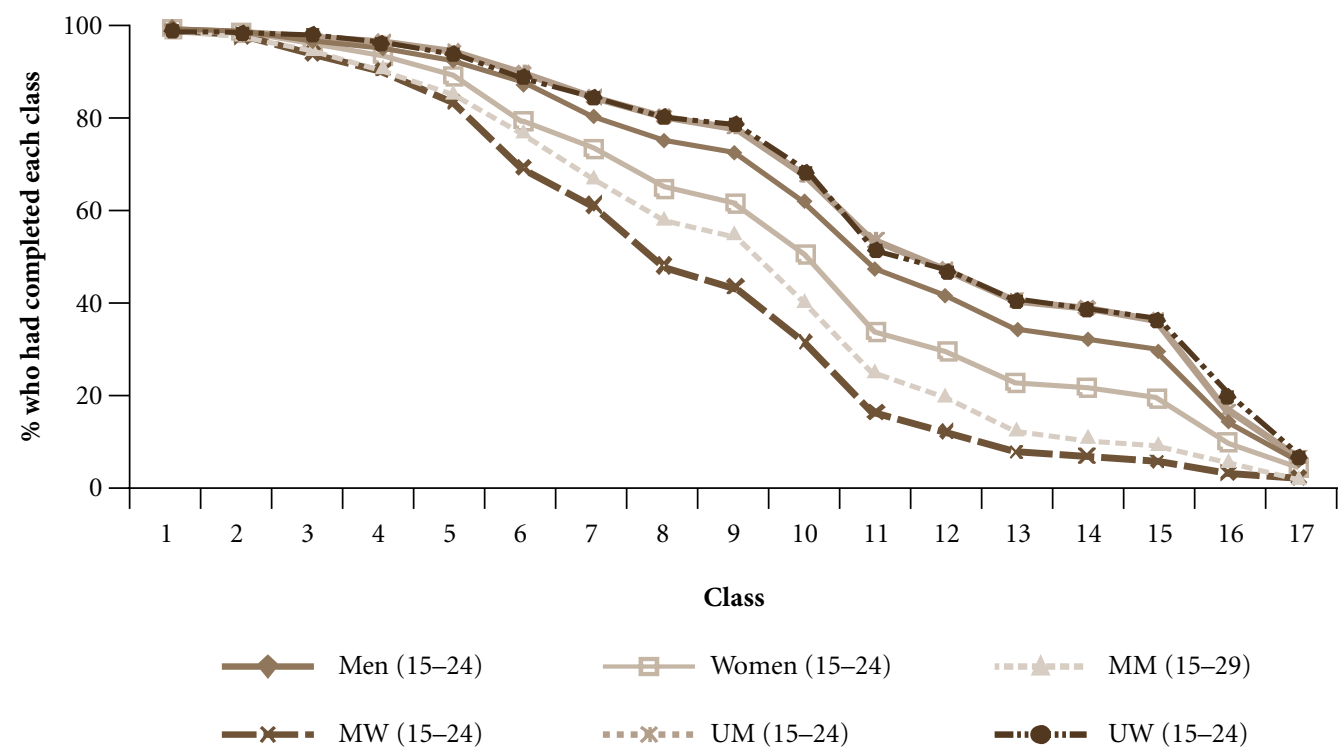


Figure 3.2b: Cumulative percentage of youth who had completed each year of education (Classes 1 to 17), Andhra Pradesh (urban), 2007-08

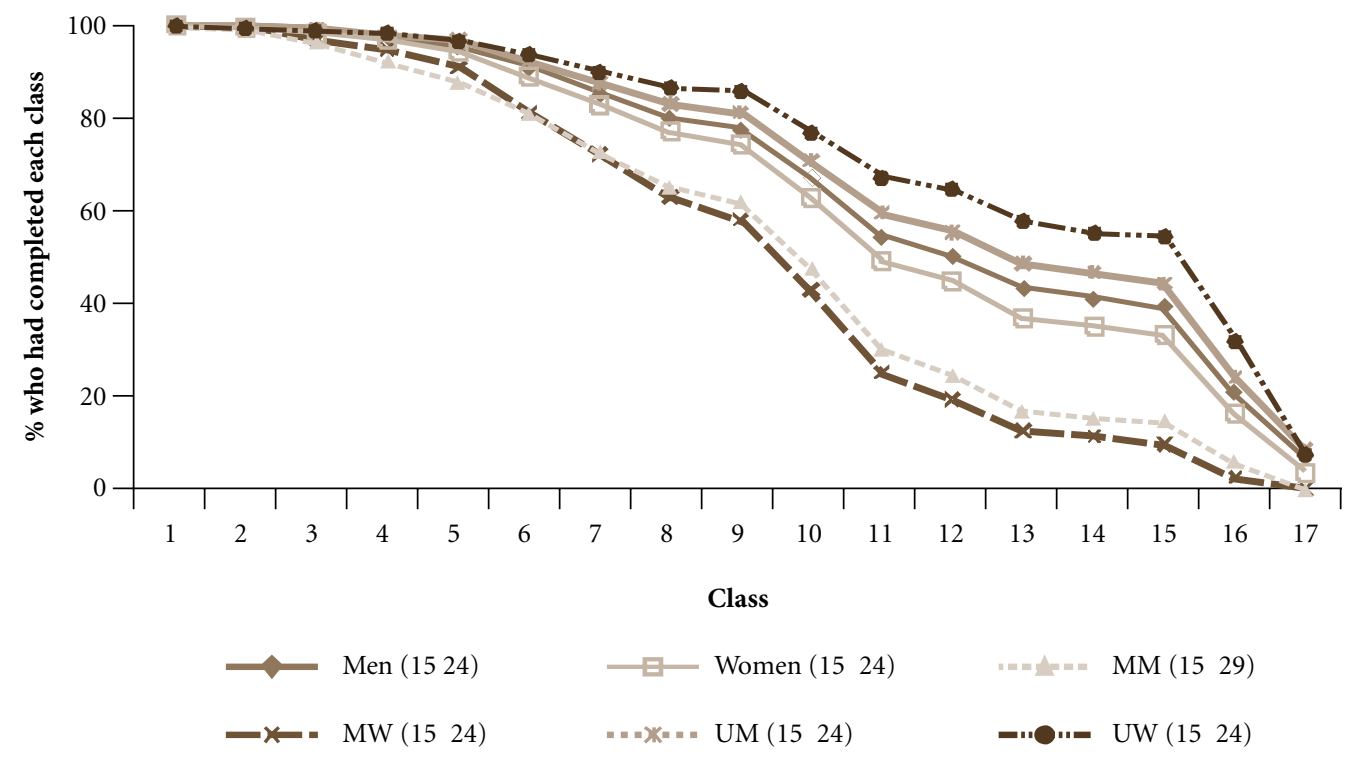

Figure 3.2c: Cumulative percentage of youth who had completed each year of education (Classes 1 to 17), Andhra Pradesh (rural), 2007-08

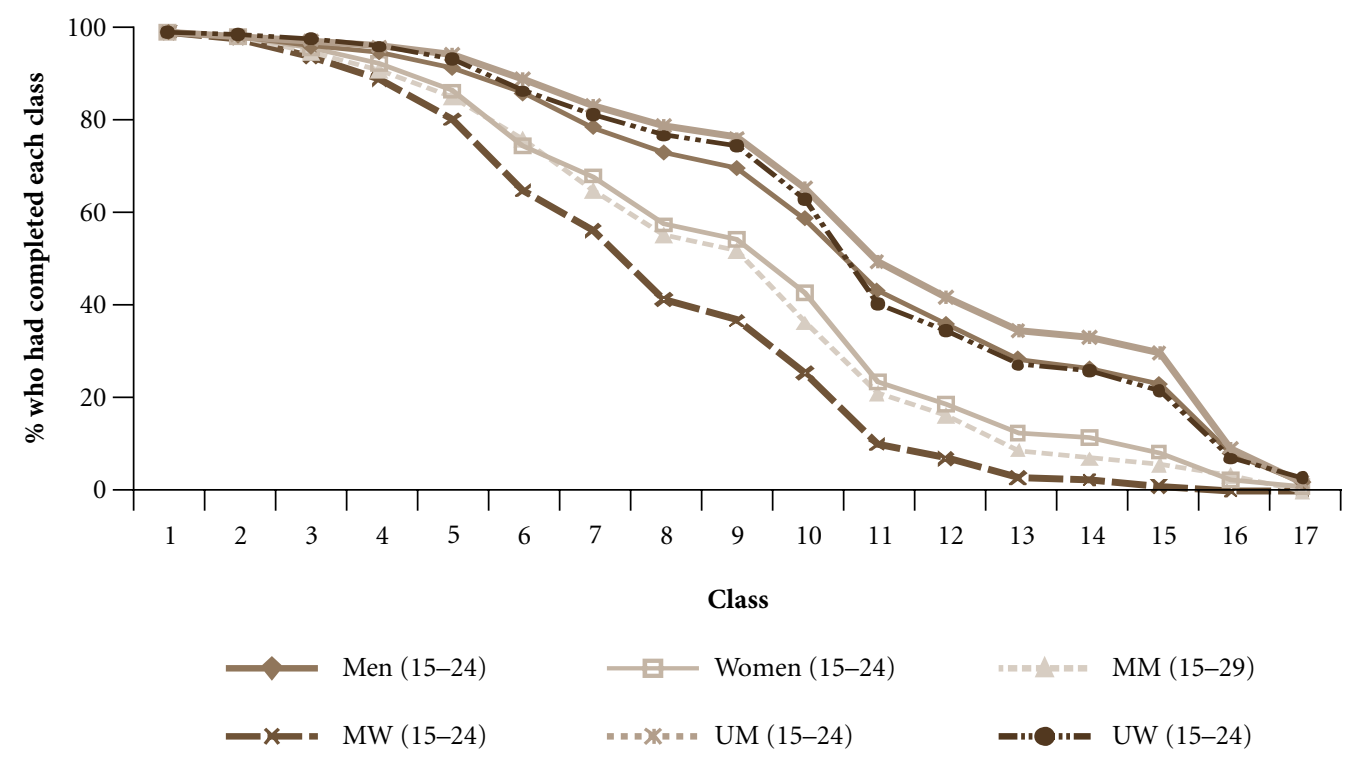

Findings suggest that key reasons for never going to school were economic, cited by 57\% of young men and 53\% of young women. Specifically, over two-fifths of young men and almost one-quarter of young women reported that they were required for wage earning activities; $23 \%$ and $29 \%$ of young men and women, respectively, reported that their families could not afford to send them to school; and 10-11\% youth reported that they were required to work on the family farm or business. Housework-related factors were reported by larger percentages of young 
Table 3.4a: Reasons for never attending school

Percentage of youth who never attended school by reasons for never attending school, according to residence, Andhra Pradesh, 2007-08

\begin{tabular}{|c|c|c|c|c|c|c|}
\hline Reasons (\%) & $\begin{array}{c}M \\
15-24\end{array}$ & $\begin{array}{c}\text { W } \\
15-24\end{array}$ & $\begin{array}{c}\text { MM } \\
15-29\end{array}$ & $\begin{array}{c}\text { MW } \\
15-24\end{array}$ & $\begin{array}{c}\text { UM } \\
15-24\end{array}$ & $\begin{array}{c}\text { UW } \\
\text { 15-24 }\end{array}$ \\
\hline \multicolumn{7}{|c|}{ Combined } \\
\hline \multicolumn{7}{|l|}{ Economic reasons } \\
\hline Required for work on farm/family business & 9.8 & 10.6 & 18.6 & 11.6 & 5.7 & 6.1 \\
\hline Required for outside work for payment in cash/kind & 40.2 & 23.5 & 40.9 & 23.8 & 36.4 & 21.7 \\
\hline Family could not afford it (cost too much) & 23.1 & 29.1 & 22.0 & 27.6 & 24.5 & 36.7 \\
\hline At least one economic reason & 57.4 & 52.8 & 61.8 & 52.3 & 53.3 & 56.1 \\
\hline Housework-related reasons & 37.4 & 47.1 & 40.9 & 48.4 & 37.4 & 38.9 \\
\hline \multicolumn{7}{|l|}{ Parental or youth attitudes and perceptions } \\
\hline Not safe to send girls/boys to school & 0.5 & 4.5 & 0.7 & 4.8 & 0.0 & 2.8 \\
\hline Education not considered necessary & 16.9 & 14.5 & 13.2 & 15.0 & 14.0 & 11.7 \\
\hline Respondent not interested in studies & 35.9 & 28.6 & 33.8 & 27.9 & 38.7 & 32.8 \\
\hline At least one attitude-/perception-related reason & 49.2 & 43.6 & 44.6 & 43.5 & 45.8 & 44.4 \\
\hline \multicolumn{7}{|l|}{ School-related reasons } \\
\hline School too far away/transport not available & 0.5 & 8.7 & 2.0 & 9.2 & 0.0 & 5.6 \\
\hline Poor quality of school facilities, teaching or education & 2.6 & 4.5 & 2.7 & 4.4 & 1.9 & 4.4 \\
\hline At least one school-related reason & 3.6 & 12.5 & 4.7 & 13.2 & 1.9 & 8.9 \\
\hline Health-related reasons & 8.7 & 10.9 & 6.1 & 10.6 & 11.2 & 13.3 \\
\hline Number who never attended school & 166 & 807 & 262 & 634 & 94 & 173 \\
\hline \multicolumn{7}{|c|}{ Urban } \\
\hline \multicolumn{7}{|l|}{ Economic reasons } \\
\hline Required for work on farm/family business & 2.9 & 2.8 & 4.8 & 3.3 & $(4.8)$ & $(0.0)$ \\
\hline Required for outside work for payment in cash/kind & 41.2 & 14.1 & 38.1 & 13.5 & $(38.1)$ & $(16.2)$ \\
\hline Family could not afford it (cost too much) & 24.2 & 35.2 & 23.8 & 30.3 & $(23.8)$ & $(51.4)$ \\
\hline At least one economic reason & 52.9 & 46.5 & 50.0 & 42.2 & $(47.6)$ & $(59.5)$ \\
\hline Housework-related reasons & 29.4 & 44.8 & 38.1 & 45.6 & $(28.6)$ & $(43.2)$ \\
\hline \multicolumn{7}{|l|}{ Parental or youth attitudes and perceptions } \\
\hline Not safe to send girls/boys to school & 2.9 & 5.6 & 2.4 & 6.7 & $(0.0)$ & $(2.7)$ \\
\hline Education not considered necessary & 12.1 & 13.4 & 9.5 & 15.6 & $(4.8)$ & $(5.4)$ \\
\hline Respondent not interested in studies & 47.1 & 31.7 & 40.5 & 30.0 & $(52.4)$ & $(35.1)$ \\
\hline At least one attitude-/perception-related reason & 55.9 & 46.9 & 50.0 & 48.9 & $(52.4)$ & $(40.5)$ \\
\hline \multicolumn{7}{|l|}{ School-related reasons } \\
\hline School too far away/transport not available & 0.0 & 4.9 & 0.0 & 3.3 & $(0.0)$ & $(10.8)$ \\
\hline Poor quality of school facilities, teaching or education & 2.9 & 4.2 & 2.4 & 3.3 & $(4.5)$ & $(5.4)$ \\
\hline At least one school-related reason & 2.9 & 9.8 & 4.8 & 6.7 & $(4.5)$ & $(\mathbf{1 8 . 4})$ \\
\hline Health-related reasons & 5.9 & 7.7 & 7.1 & 7.8 & $(4.8)$ & $(8.1)$ \\
\hline Number who never attended school & 61 & 227 & 89 & 182 & 38 & 45 \\
\hline
\end{tabular}




\begin{tabular}{|c|c|c|c|c|c|c|}
\hline Reasons (\%) & $\begin{array}{c}\text { M } \\
15-24\end{array}$ & $\begin{array}{c}\text { W } \\
15-24\end{array}$ & $\begin{array}{c}\text { MM } \\
15-29\end{array}$ & $\begin{array}{c}\text { MW } \\
\text { 15-24 }\end{array}$ & $\begin{array}{c}\text { UM } \\
15-24\end{array}$ & $\begin{array}{c}\text { UW } \\
15-24\end{array}$ \\
\hline \multicolumn{7}{|c|}{ Rural } \\
\hline \multicolumn{7}{|l|}{ Economic reasons } \\
\hline Required for work on farm/family business & 11.8 & 12.1 & 20.9 & 12.7 & 7.0 & 7.7 \\
\hline Required for outside work for payment in cash/kind & 39.8 & 25.0 & 41.3 & 25.3 & 36.0 & 23.8 \\
\hline Family could not afford it (cost too much) & 22.5 & 28.0 & 21.7 & 27.2 & 24.7 & 33.3 \\
\hline At least one economic reason & 58.8 & 53.9 & 63.4 & 53.7 & 54.7 & 55.2 \\
\hline Housework-related reasons & 39.1 & 47.4 & 40.9 & 48.9 & 39.5 & 37.8 \\
\hline \multicolumn{7}{|l|}{ Parental or youth attitudes and perceptions } \\
\hline Not safe to send girls/boys to school & 0.0 & 4.3 & 0.4 & 4.6 & 0.0 & 2.8 \\
\hline Education not considered necessary & 17.5 & 14.6 & 13.8 & 14.9 & 16.3 & 13.3 \\
\hline Respondent not interested in studies & 33.5 & 28.2 & 32.7 & 27.5 & 35.3 & 31.5 \\
\hline At least one attitude-/perception-related reason & 47.8 & 43.1 & 43.7 & 42.7 & 44.2 & 45.5 \\
\hline \multicolumn{7}{|l|}{ School-related reasons } \\
\hline School too far away/transport not available & 0.6 & 9.4 & 2.4 & 10.0 & 0.0 & 4.2 \\
\hline Poor quality of school facilities, teaching or education & 3.1 & 4.5 & 2.8 & 4.6 & 2.3 & 3.5 \\
\hline At least one school-related reason & 3.7 & 13.1 & 5.1 & 14.0 & 2.3 & 7.0 \\
\hline Health-related reasons & 9.3 & 11.5 & 6.3 & 11.0 & 12.8 & 14.7 \\
\hline Number who never attended school & 105 & 580 & 173 & 452 & 56 & 128 \\
\hline
\end{tabular}

Note: All Ns are unweighted. Column totals may exceed 100\% due to multiple responses. ( ) Based on 25-49 unweighted cases.

women than men ( $47 \%$ and $37 \%$, respectively). Attitude or perception-related reasons for never attending school were also reported by large percentages of youth ( $49 \%$ and $44 \%$ of young men and women, respectively); the leading attitude or perception-related reason expressed was lack of interest in studies, reported by $36 \%$ of young men and $29 \%$ of young women. School-related reasons were cited by few youth, just $4 \%$ of young men and 13\% of young women. Finally, a small but notable percentage of respondents-9-11\%-cited health-related reasons (mostly the sickness or death of a family member) for never attending school. Differences by marital status were generally mild. However, more married than unmarried young men reported economic reasons for never attending school (62\% versus 53\%), and more married than unmarried young women reported housework related reasons (48\% versus $39 \%)$ for never attending school.

Rural-urban differences suggest young men and women in urban settings were less likely than those in rural settings to report economic reasons (53\% and 59\%, respectively, among young men; $47 \%$ and $54 \%$, respectively, among young women). Young men in urban areas, moreover, were more likely to report attitude or perceptions-related reasons (56\% versus $48 \%$ ), particularly lack of interest in studies ( $47 \%$ versus $34 \%$ ), and less likely to report housework related reasons (29\% versus $39 \%)$ than their rural counterparts.

Table 3.4b reports findings for youth who had discontinued their education before completing Class 12. In addition to the five sets of reasons included above, an additional category, early transition into adult roles, has been included, containing such reasons as marriage and employment. Reasons are presented separately for those who had discontinued school before completing middle school (Class 7), high school (Class 10) and higher secondary education (Class 12), respectively. As evident from Figures 3.3a and 3.3b, reasons varied considerably by level at which education was discontinued, as well as sex and marital status of the respondent. 


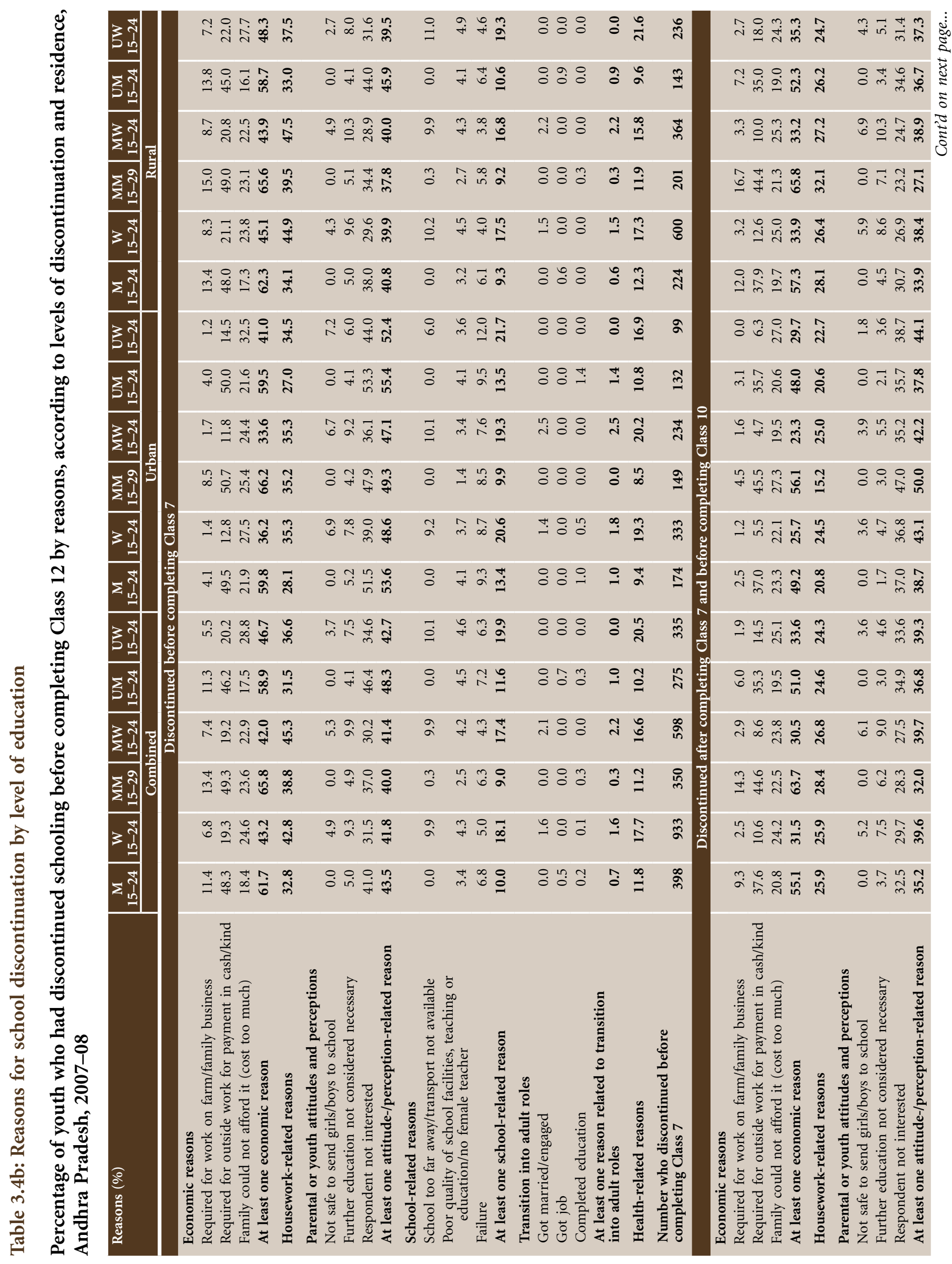




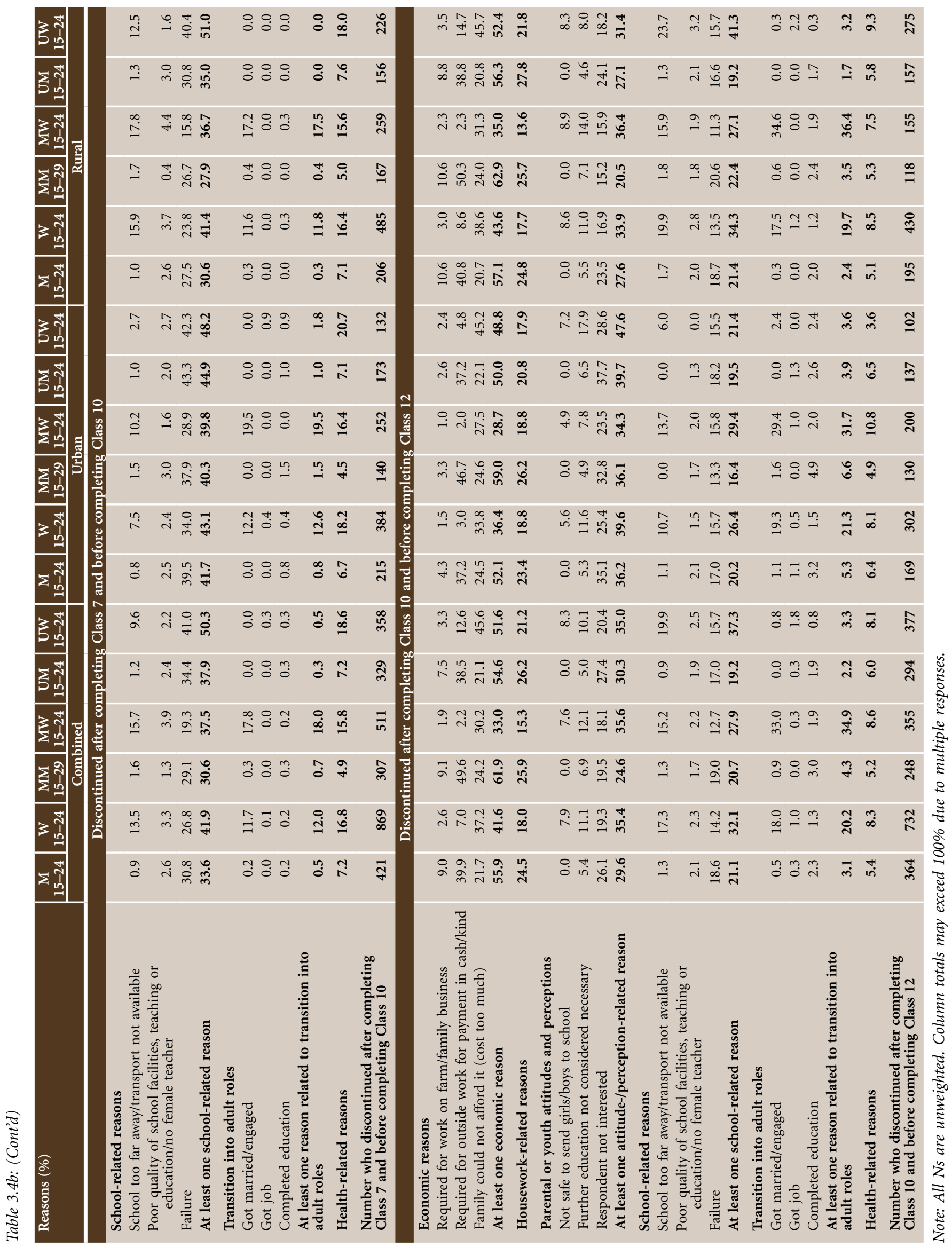


Among those who had completed just 1-6 years of schooling, three main sets of reasons for school discontinuation were cited by both young men and women: economic considerations, attitude or perception-related factors, and housework-related factors. Gender differences were evident. Among young men, for example, economic considerations $(62 \%)$ and attitude or perception-related reasons (44\%) dominated; fewer young men cited housework-related reasons (33\%). Among young women, each of the three key reasons was cited by about or more than two in five (42-43\%). School-related reasons were reported by relatively few young men and women (10\% and $18 \%$, respectively). Similar patterns were observed among the married and the unmarried, except that married young men were somewhat more likely than their unmarried counterparts to report economic reasons (66\% versus 59\%) and housework related reasons (39\% versus 32\%), and conversely less likely to report attitude or perception-related reasons (40\% versus 48\%); married young women were somewhat more likely than their unmarried counterparts to report housework-related reasons (45\% versus 37\%). Although reasons reported by rural and urban youth were somewhat similar, some notable differences were evident: urban youth were considerably more likely than the rural to cite attitude or perception-related reasons (54\% versus $41 \%$ among young men; $49 \%$ versus $40 \%$ among young women), and conversely, less likely to cite housework related reasons (28\% versus 34\% among young men; $35 \%$ versus $45 \%$ among young women); urban young women were also less likely than their rural counterparts to cite economic reasons (36\% versus $45 \%)$.

Among those who had completed Classes 7-9, gender differences in leading reasons reported for school discontinuation persisted. Among young women, leading reasons for discontinuation at this level were school-related, expressed by $42 \%$ of all young women; other reasons were attitude or perception-related (40\%), economic (32\%) and housework-related (26\%). Among young men, economic reasons continued to dominate, reported by over half $(55 \%)$, and were followed by attitude or perception-related and school-related reasons (34-35\%) and housework-related reasons $(26 \%)$. While reported by relatively few youth, considerably more young women than men reported a health-related reason $(17 \%$ versus $7 \%)$. Findings also show that transitions into adult roles were rarely expressed as a reason for discontinuation at this level of education by young men but by more than one in ten young women ( $1 \%$ versus $12 \%$ ). The leading sets of reasons indicated by married and unmarried youth as also urban and rural youth were by and large similar; with some notable exceptions. Married young men were more likely than unmarried young men to report economic (64\% versus 51\%); married young women were considerably less likely than their unmarried counterparts to report school-related reasons (38\% versus 50\%). In addition, as many as $18 \%$ of married young women reported marriage as a reason for school discontinuation. In addition, more rural than urban youth reported economic reasons. More urban than rural young men reported school-related reasons, notably academic failure, as a reason for school discontinuation; while corresponding rural-urban differences were not observed among young women $(41-43 \%)$, it is notable that considerably more rural than urban young women reported long distances as a reason for school discontinuation (16\% versus $8 \%$ ) and fewer rural than urban young women reported academic failure as a reason (24\% versus $34 \%)$.

Among those who had discontinued their education after completing Classes 10 or 11, leading sets of reasons cited for discontinuation were similar. Leading reasons for both young men and women were economic (56\% and $42 \%$, respectively), attitude or perception-related (30\% and 35\%, respectively), school-related ( $21 \%$ and $32 \%$, respectively), and housework-related (25\% and 18\%, respectively). For considerable proportions-one in five-of young women, moreover, marriage was cited as the reason for school discontinuation. Of note is the finding that academic failure was cited as a reason for discontinuing education by fewer youth who discontinued their education at this level (19\% of young men and $14 \%$ of young women) than those who discontinued their education in Classes $7-9$ ( $31 \%$ of young men and $27 \%$ of young women).

Differences by marital status were mild among young men; among young women, however, the married were less likely than the unmarried to cite economic (33\% versus 52\%) and school-related reasons (28\% versus 37\%). Also notable is that a sizeable proportion of married young women-35\%—reported discontinuing their education in order to marry. Rural-urban differences were evident in a few instances. For example, more rural than urban young women reported economic (44\% versus 36\%) and school-related reasons (34\% versus 26\%) and more urban than rural youth reported attitude or perception-related reasons (36\% versus 28\% among young men; $40 \%$ versus $34 \%$ 
Figure 3.3a: Percentage of married youth who had discontinued schooling by class when discontinued and reasons for discontinuation, Andhra Pradesh 2007-08

Married men

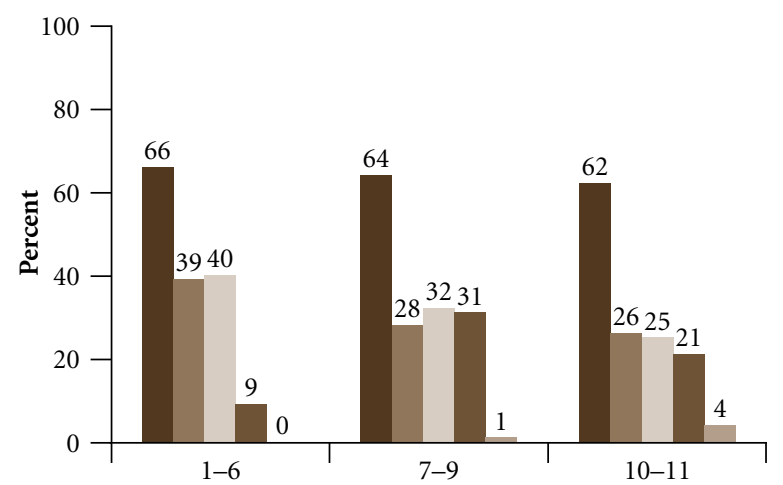

Married women

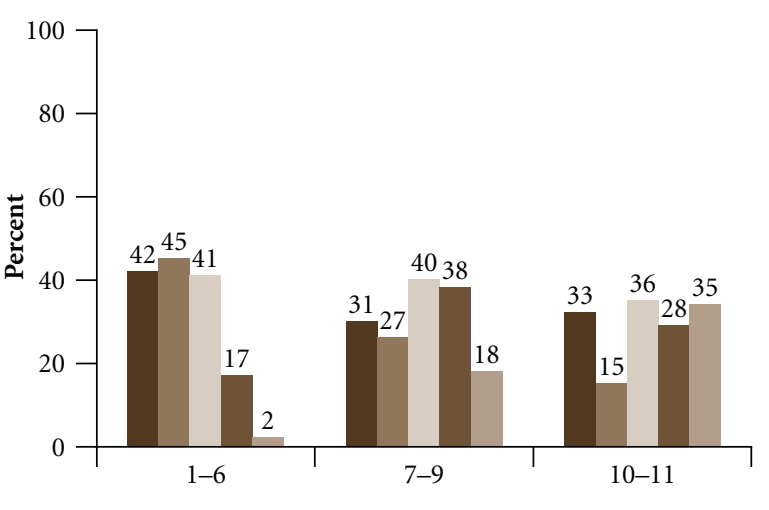

Class at discontinuation

At least one economic reason

At least one attitude-/perception-related reason

At least one reason related to transition into adult roles
At least one housework-related reason

At least one school-related reason

Figure 3.3b: Percentage of unmarried youth who had discontinued schooling by class when discontinued and reasons for discontinuation, Andhra Pradesh, 2007-08

Unmarried men

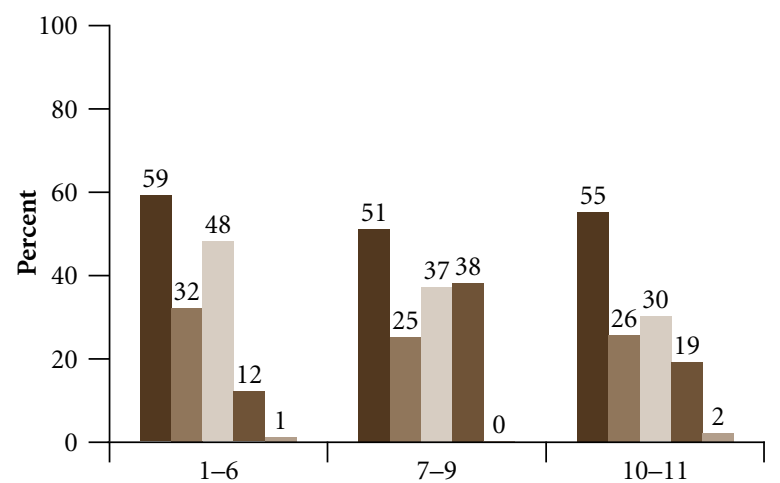

Unmarried women

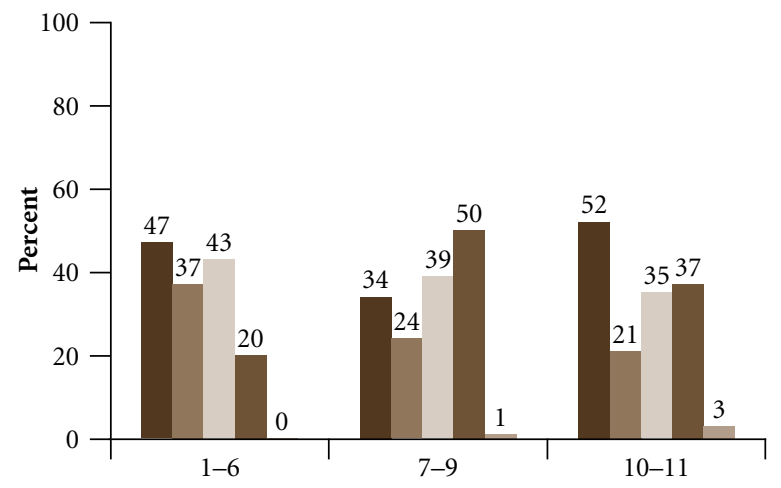

Class at discontinuation

At least one economic reason

- At least one attitude-/perception-related reason

- At least one reason related to transition into adult roles
At least one housework-related reason

- At least one school-related reason

among young women). Finally, among married young women, more of those from rural than urban areas reported marriage as a reason for discontinuing their education (35\% versus $29 \%$ ).

\subsection{School/college type, quality and experiences}

All respondents were asked about the kind of school or college they last attended or were attending at the time of the interview, and the facilities available in that school or college. Respondents were also asked about their experiences: 
whether they attended classes regularly, their attitudes towards education and their performance in that school or college. Tables 3.5 and 3.6 present findings on type and quality of educational institutions most recently attended and schooling experiences, respectively; as school quality and experiences are unlikely to be different for the married and unmarried, information is provided by sex and rural-urban residence of respondents only. Moreover, because school/college quality and experiences may have differed for those who were still in school or college at the time of the interview and those who had discontinued their education, findings are presented separately by level of education attained, namely, for those currently in high school or higher secondary school or college (few youth were in primary or middle school at the time of interview) and those who had discontinued their education at primary or middle levels, high school level or before completing Class 12.

\subsubsection{School/college type and quality}

Findings presented in Table 3.5 show that the majority of youth irrespective of sex, level of education attained and current schooling status attended co-educational facilities. Typically, percentages studying in a co-educational facility declined with level of education attained. Gender differences were evident, particularly among those currently studying in higher secondary school or college; young men were considerably more likely than young women to attend a co-educational facility (90\% versus $75 \%)$. Young men in urban areas were considerably more likely than young women to report co-educational school attendance, both at high school (95\% versus 88\%) and higher secondary school and college ( $88 \%$ versus $71 \%$ ); among rural youth, this difference was only evident among those at secondary or higher levels of education (90\% versus $80 \%$ ). Among those who had discontinued their education, a generally similar pattern prevailed.

Attendance at government versus private educational facilities also varied. The majority of youth who had discontinued their education tended to have attended a government school or college; even so, percentages attending a government school or college declined with level at which education was discontinued (92-93\%, 84-88\% and $57-62 \%$ at primary/middle, high and higher secondary and higher levels, respectively). Among those continuing their education, percentages attending a government facility revealed a similar pattern: attendance declined from $69-72 \%$ among those at high school level to $40-41 \%$ among those at higher secondary or higher levels. More youth studying at higher secondary or higher levels were attending a private institution than those studying at high school level; 47-54\% versus 26-28\%. Irrespective of current schooling status gender differences were typically mild. Rural-urban differences indicate that more urban than rural youth attended a private educational institution, particularly among those still studying at the time of the interview; for example, among those studying at high school levels, 55\% and 54\% of young men and women in urban areas compared to $17 \%$ and $10 \%$, respectively, in rural areas were attending a private educational institution. The only exception was that among young women who discontinued their education at higher secondary or higher levels, more of those from rural than urban settings had attended a private educational facility (37\% versus $23 \%)$.

School quality was assessed by questions on the availability of drinking water, toilets, playgrounds and library facilities. Findings from Table 3.5 show vast differences between those pursuing their education at the time of the interview and those who had discontinued their education; differences were also observed by level of education attained and by type of amenity considered. Among those pursuing their education at the time of the interview, well over $90 \%$ of youth, irrespective of sex or rural-urban residence, had access to drinking water. Between $75 \%$ and $94 \%$ had access to playgrounds, and between $74 \%$ and $96 \%$ had access to toilet facilities. Library facilities were less likely to be available, and differences emerged by level of education attained-while 29-32\% of those in high school had access to library facilities, $63-71 \%$ of those in higher secondary school or college did. By and large, more urban than rural youth reported toilet facilities (88-91\% versus 68-82\% among those in high school; 95-99\% versus 82-93\% among those at higher secondary and higher levels). At higher secondary levels, more urban than rural youth reported the availability of a library (76-79\% compared to 56-61\%). In contrast, fewer urban than rural youth attending high school reported the availability of a playground $(78-88 \%$ and $87-97 \%$, respectively). 


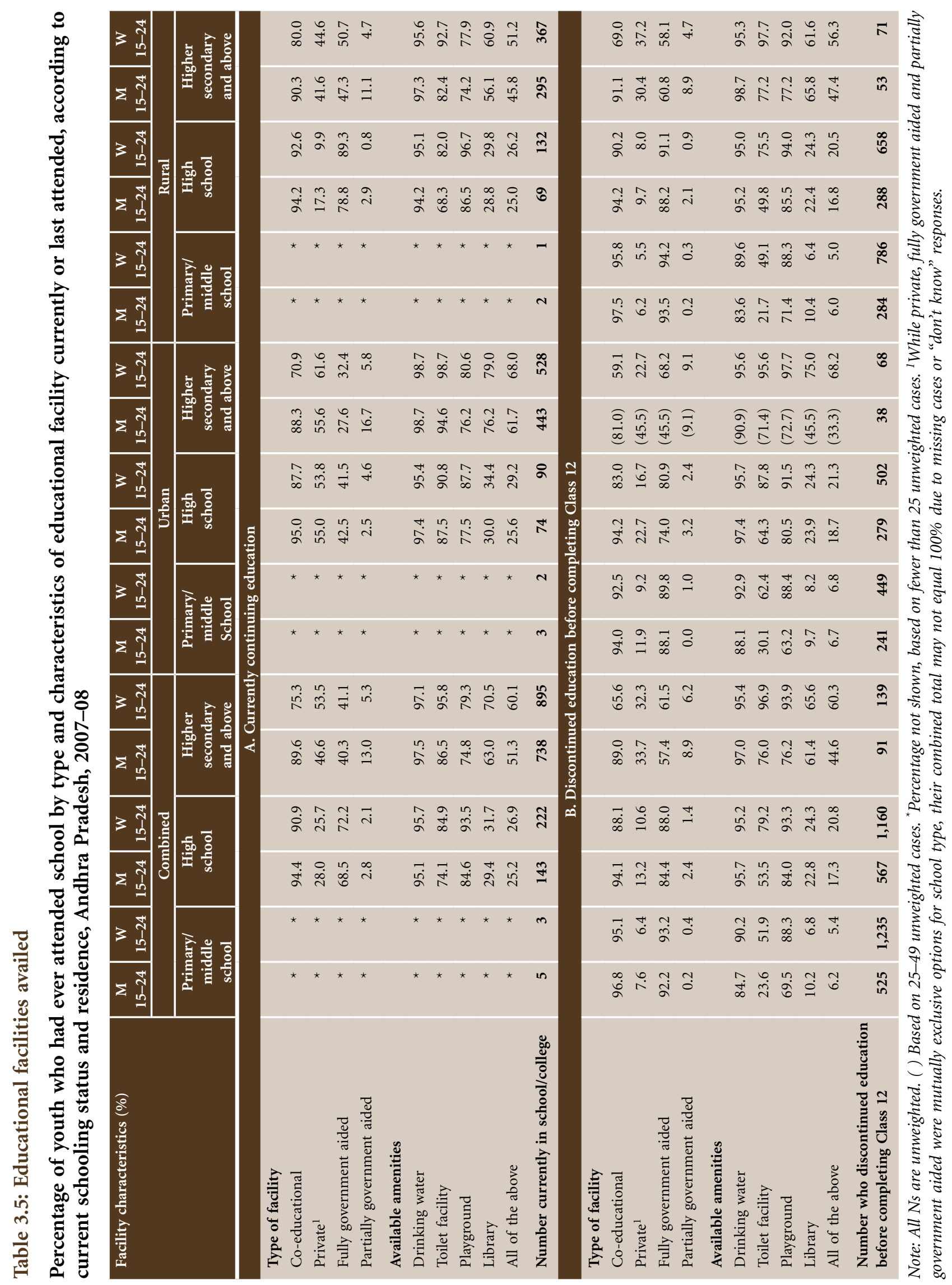


The situation was somewhat different among youth who had discontinued their education. Drinking water was available at schools/colleges attended by over $80 \%$ of youth, irrespective of the level at which they had discontinued their education, and irrespective of sex and rural-urban residence. Likewise, $70 \%$ or more youth, irrespective of the level at which they had discontinued their education, reported that a playground was available in the school/college they attended. Access to toilet facilities was more likely to be reported by young women than men, and by those who had discontinued their education at progressively higher levels. For example, 24\% of young men, compared to $52 \%$ of young women who had discontinued their education at primary or middle levels reported that toilet facilities were available; considerably larger proportions of young men and women who had discontinued at high school (54\% and 79\%, respectively) or higher secondary levels (76\% and 97\%, respectively) reported the availability of toilets. With regard to the availability of library facilities, similarly, access increased with level at which education was discontinued $(10 \%, 23 \%$ and $61 \%$, respectively, at each of the three levels of education among young men who had discontinued their education; and 7\%, 24\% and 66\%, respectively, among young women). Rural-urban differences were evident in some instances; for example, availability of toilet facilities were more likely to be reported by urban than rural youth, particularly at primary or middle level and high school levels.

The availability of all four amenities_-drinking water, playgrounds, toilets and libraries_increased systematically with level of schooling attained among all youth, irrespective of whether or not they had discontinued their education. Among those still in school, all four amenities were available to $25 \%$ and $27 \%$ of young men and women, respectively, in high school and $51 \%$ and $60 \%$ of young men and women, respectively, at higher levels. Among those who had discontinued their education, the availability of all four amenities increased from 5-6\% among those who had discontinued their education at primary or middle school levels, to 17-21\% among those who had discontinued at high school, and 45-60\% among those who had discontinued at higher levels. Gender differences were relatively narrow, however, more young women than men women who were currently studying at higher secondary or college levels or had discontinued their education at these levels reported the availability of all four amenities. Rural-urban differences were also narrow, except that urban youth attending higher secondary schools and colleges were more likely than their rural counterparts to have access to all four amenities. Also narrow were differences in the availability of all four amenities reported by those who were studying at the time of the interview than those who had discontinued their education; in most instances, those pursuing their education were, however, somewhat more likely than those who had discontinued their education to report so.

\subsubsection{School/college experiences}

Table 3.6 presents young people's schooling experiences, namely, whether or not they attended class regularly, took private tuition, considered the academic workload to be heavy and had passed the last examination for which they had appeared. Among those still in school or college, almost all youth (95\% or more) reported that they attended classes regularly, almost all youth reported that they had passed the last examination for which they had appeared (96-99\%) and relatively few youth (10-19\%) reported feeling that the academic workload was heavy. In contrast, $42 \%$ and $36 \%$ of young men and women in high school and considerably fewer, $20 \%$ and $14 \%$, respectively, of those at higher secondary or higher levels, reported that they had taken private tuition. Rural-urban differences were notably narrow on most indicators; however, among those in high school, urban youth were somewhat more likely than their rural counterparts to have attended coaching classes. Additionally, among those in high school young men in rural areas were somewhat more likely than those in urban areas to feel that the academic workload was heavy.

Among youth who had discontinued their education, regular attendance was reported by $70 \%$ or more youth, irrespective of sex, rural-urban residence and level at which education was discontinued. As far as private tuition was concerned, few $(10-11 \%)$ of those who discontinued at primary or middle school levels had taken private tuition, compared to $27-33 \%$ and $15-22 \%$ of those who discontinued at high school or higher levels, respectively. Between $28 \%$ and $44 \%$ of young men and somewhat fewer young women-22\% to $27 \%$ - perceived the academic workload to be heavy. Finally, percentages who passed the last examination for which they had appeared declined systematically as level of education increased: $79 \%, 65 \%$ and $24 \%$ of young men, and $88 \%, 70 \%$ and $28 \%$ of young women who discontinued at primary/middle school, high school, or higher secondary or higher levels, respectively. 


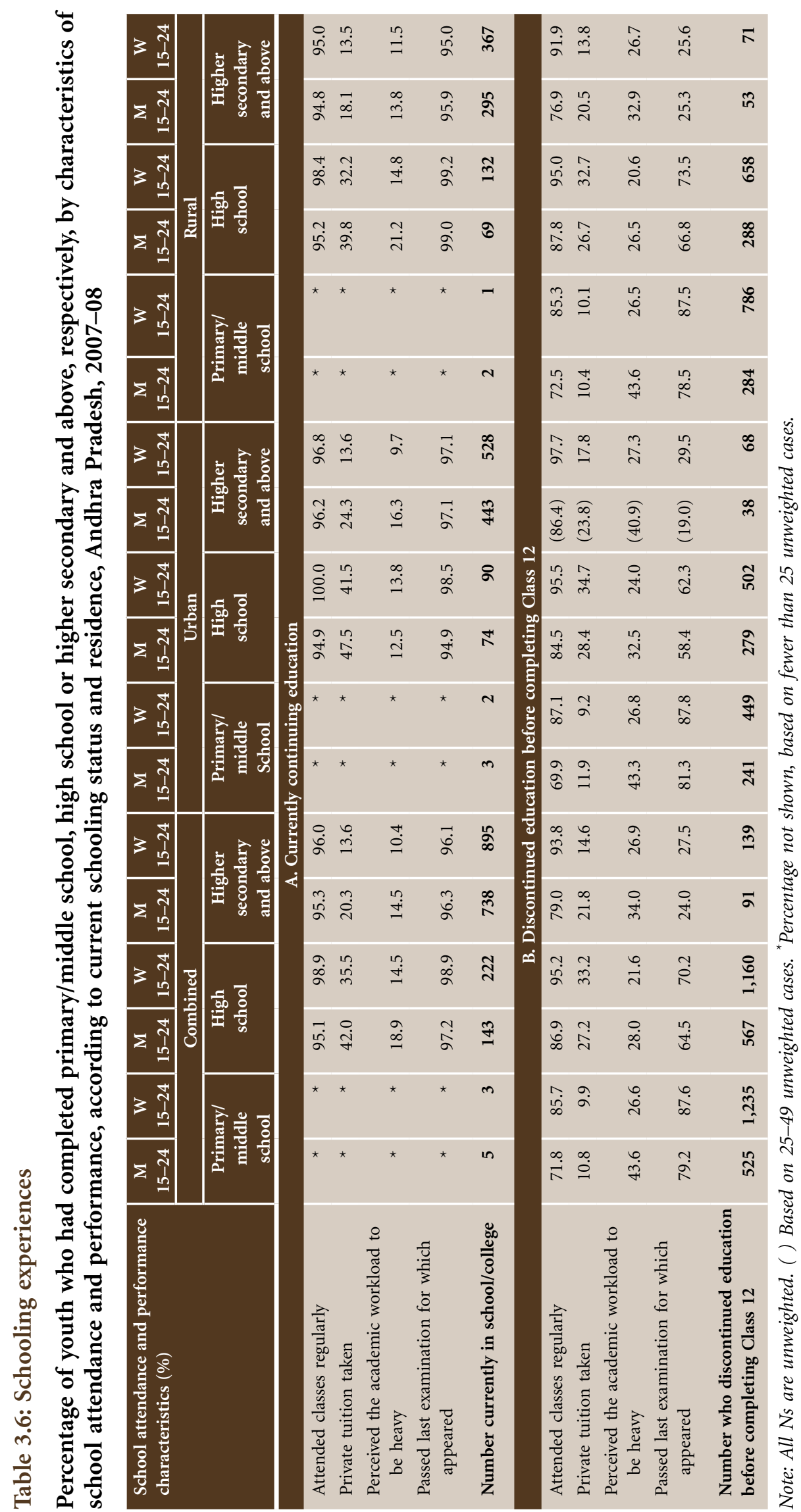


Rural-urban differences were narrow, however, fewer urban than rural youth who had discontinued their education at high school level had passed the last examination for which they had appeared and more urban than rural youth who discontinued their education at higher secondary and above level had attended classes regularly.

Schooling experiences were different among those who had discontinued schooling and those who were studying at the time of the interview. For example, youth who were pursuing their education at the time of the interview were less likely than those who had discontinued their education to report feelin $\mathrm{g}$ that the academic workload was heavy and somewhat more likely to have attended classes regularly. A much wider difference was observed in reporting of school performance: while almost all youth who were still in school reported passing the last examination for which they had appeared (96-99\%), only $65 \%$ and $70 \%$ of young men and women, respectively, who had discontinued their education at high school level, and even fewer $(24 \%$ and $28 \%$, respectively) of those who had discontinued at higher levels, so reported. Clearly, academic failure was an important factor precipitating school discontinuation for both young men and young women.

\subsection{Summary}

While youth in Andhra Pradesh were better educated than the population at large in the state, schooling was far from universal among young people. As many as one in twelve young men and one in five young women had never attended school. Findings show, moreover, that young women in rural areas and married young women in general were particularly disadvantaged; about one-third of married young women and one quarter of women residing in rural areas had never been to school. At the time of the interview, about two-fifths of unmarried youth (and very few married) were still in school or college, and gender differences were negligible.

Not only was school enrolment limited, but school completion rates were also low among young people, particularly among young women. For example, among young women, of those who had completed Class 1, only 95\% had completed Class 4, and completion rates fell below $90 \%$ in Class 5 . Among young men, completion rates fell below $90 \%$ a year later, that is, in Class 6. Declines in school completion were steep, among both young men and women at around the time of high school completion, that is, between Classes 9 and 11, suggesting that many youth discontinued their education at high school level. Indeed, just 52\% of young men and $36 \%$ of young women in the state had completed high school.

Leading reasons for never attending school among young men and women were economic (for example, the respondent was required for work on the family farm/business or for outside wage earning work, or the family could not afford school-related expenses), attitude and perception-related (for example, education was unnecessary or the respondent was not interested in schooling) and housework-related reasons (the respondent was required for care of siblings or housework).

Among those who had ever been to school, gender differences in reasons for school discontinuation became more apparent. Leading reasons for school discontinuation among young men were economic and attitude and perception-related, irrespective of level at which education was discontinued. Far fewer young men cited school-related reasons. Among young women, in contrast, while economic and attitude and perception-related reasons were important, school-related reasons (academic failure, distance to school, poor school quality and infrastructure) were prominent factors underlying discontinuation at all levels; housework responsibilities were also significant, particularly among those who discontinued at primary or middle school levels. Of note are findings that more than one quarter of youth who discontinued their education before completing high school cited academic failure as a reason for discontinuing their education; and that one in five married young women who discontinued their education in Classes 7-9 and one-third of those who discontinued their education in Classes 10-11 reported doing so in order to marry.

As far as type of educational facility youth attended is concerned, while the majority of young men and women attended co-educational facilities at all levels of education, young women were less likely to attend a co-educational 
facility at higher secondary school and college levels. Typically, more youth who had discontinued their education reported government school attendance than did those who were pursuing their education; private school attendance was reported, moreover, among large proportions who were pursuing a higher secondary or college education or had discontinued their education at this level; while gender differences were relatively mild, youth from urban areas were considerably more likely than their rural counterparts to have attended a private school or college.

Most youth, irrespective of whether they were pursuing their education or not, had access to drinking water and playgrounds. However, for the most part, youth still studying were somewhat more likely, to report the availability of toilets as well as libraries than those who had discontinued their education. The availability of all four amenities was, for the most part, somewhat more likely to be reported by those who were studying at the time of the interview than those who had discontinued their education. Gender and rural-urban differences were, by and large, narrow.

Schooling experiences differed in some instances between those who had discontinued schooling and those who were studying at the time of the interview. While differences in experience of private tuition were typically narrow, those who had discontinued their education were more likely than those who were still in school to perceive the academic workload to be heavy and somewhat less likely to have attended classes regularly. They were considerably less likely, moreover, to have passed the last examination for which they had appeared, suggesting that poor school performance was a significant factor leading to school discontinuation among both young men and women. 


\section{Economic and non-economic} activity

The period between the ages of 15 and 29 marks, for many young people, entry into the labour market and economic independence, acquisition of professional and technical skills and new living arrangements. Economic uncertainty, however, dominates the lives of many youth. According to International Labour Organisation (ILO) estimates, although youth (aged 15-24) comprise around $25 \%$ of the world's working-age population, they constitute around $44 \%$ of the unemployed (ILO, 2006). The unemployment rate among youth has also been identified as one of the key indicators for monitoring the progress towards achieving the UN Millennium Development Goals (UNDP, 2000). For many young people, this period also marks the discontinuation of education and increasing acceptance of domestic responsibilities. This chapter explores the economic activity of young people, their work-related mobility, their participation in non-economic activities (domestic work) and their vocational skill-building experiences and preferences.

\subsection{Economic activity}

During the survey, a number of questions were asked to assess the economic activity and occupational status of youth. Youth were asked whether they had ever worked, either for or without remuneration. They were also asked whether they had worked in the 12 months preceding the interview, whether they were seeking employment, the type of work in which they engaged, and the number of months during which they had worked or sought work in the year preceding the interview.

Work profiles varied widely, as shown in Table 4.1. In total, three-fourths of young men and three-fifths of young women reported that they had been engaged in paid or unpaid work at some point in their lives. They were, moreover, more likely to have been engaged in paid than in unpaid work: $71 \%$ and $26 \%$, respectively, among young men, $53 \%$ and $26 \%$, respectively, among young women. Marital status differences indicate that the married were more likely than the unmarried to have engaged in paid or unpaid work. Differences were wide, particularly among young men. Almost all married young men compared to $71 \%$ of unmarried young men reported ever having worked; among young women, correspondingly, $70 \%$ and $48 \%$, respectively, had ever worked. Rural-urban differences suggest, moreover, that more rural than urban youth had ever worked-79\% versus $68 \%$ of young men and $70 \%$ versus $37 \%$ of young women.

For many, work was initiated in childhood or early adolescence, that is, by age $15 ; 37 \%$ of young men and $39 \%$ of young women reported that they had initiated either paid or unpaid work before age 15. More married than unmarried and more rural than urban youth had initiated economic activity from an early age, irrespective of sex.

Table 4.1 also presents the percentages of youth reporting that they had worked any time in the 12 months prior to the interview. We note that the measure of work in the year prior to the interview covers a wide range of experiences that go beyond what is typically considered an employment rate (for example, as per the usual principal status definition, employment is defined as those who worked for the major part of the year preceding the interview as a fraction of those in the labour force, that is, those who had worked or had sought work for the major part of the year). Included in our measure of work are youth who worked for any length of time during the year as a proportion of all youth irrespective of whether they had worked or sought work in the year preceding the interview. 
Table 4.1: Economic activity

Percentage of youth who ever worked and who worked in the last 12 months, and percent distribution of youth by duration of work and main occupation in the last 12 months, according to residence, Andhra Pradesh, 2007-08

\begin{tabular}{|c|c|c|c|c|c|c|}
\hline Economic activity (\%) & $\begin{array}{c}\mathrm{M} \\
15-24\end{array}$ & $\begin{array}{c}\text { W } \\
15-24\end{array}$ & $\begin{array}{c}\text { MM } \\
15-29\end{array}$ & $\begin{array}{c}\text { MW } \\
15-24\end{array}$ & $\begin{array}{c}\text { UM } \\
15-24\end{array}$ & $\begin{array}{c}\text { UW } \\
\text { 15-24 }\end{array}$ \\
\hline \multicolumn{7}{|c|}{ Combined } \\
\hline \multicolumn{7}{|l|}{ Ever worked } \\
\hline Paid work & 70.9 & 53.1 & 98.4 & 62.6 & 64.5 & 41.1 \\
\hline Unpaid work & 25.5 & 26.0 & 32.7 & 31.2 & 23.1 & 19.7 \\
\hline Either paid or unpaid work & 76.0 & 60.4 & 99.4 & 70.1 & 70.7 & 48.4 \\
\hline Started working before age 15 & 37.2 & 38.6 & 53.9 & 50.6 & 31.7 & 23.4 \\
\hline \multicolumn{7}{|l|}{ Ever worked in last 12 months } \\
\hline Paid work & 67.8 & 41.2 & 97.3 & 44.5 & 60.9 & 37.5 \\
\hline Unpaid work & 13.1 & 16.5 & 11.5 & 17.6 & 13.1 & 15.5 \\
\hline Either paid or unpaid work & 72.6 & 47.4 & 98.4 & 50.5 & 66.6 & 44.1 \\
\hline Number of respondents & 2,479 & 4,848 & 1,405 & 2,330 & 2,077 & 2,518 \\
\hline \multicolumn{7}{|l|}{ Duration of paid work in last 12 months } \\
\hline Most of the year ( 6 months or more) & 84.6 & 71.4 & 96.1 & 72.7 & 80.9 & 69.4 \\
\hline Part of the year ( $3-5$ months) & 7.8 & 16.4 & 2.9 & 16.9 & 9.2 & 15.8 \\
\hline Rarely (less than 3 months) & 7.5 & 11.8 & 1.0 & 9.8 & 10.0 & 14.8 \\
\hline \multicolumn{7}{|l|}{ Main occupation (paid work) } \\
\hline Cultivator & 3.7 & 2.2 & 7.0 & 2.7 & 2.6 & 1.3 \\
\hline Agricultural labourer & 29.4 & 61.8 & 30.5 & 68.7 & 27.3 & 52.3 \\
\hline Administrative/executive/managerial/clerical & 7.3 & 7.0 & 6.1 & 3.6 & 8.6 & 12.0 \\
\hline Business & 4.0 & 0.7 & 4.5 & 0.8 & 4.1 & 0.4 \\
\hline Skilled manual/machinery & 31.9 & 17.3 & 30.7 & 15.3 & 33.4 & 20.2 \\
\hline Unskilled non-agricultural labourer & 23.0 & 10.9 & 21.1 & 9.0 & 23.2 & 13.3 \\
\hline Other & 0.5 & 0.2 & 0.3 & 0.0 & 0.6 & 0.4 \\
\hline Number engaged in paid work in last 12 months & 1,612 & 1,774 & 1,375 & 872 & 1,218 & 902 \\
\hline \multicolumn{7}{|c|}{ Urban } \\
\hline \multicolumn{7}{|l|}{ Ever worked } \\
\hline Paid work & 63.0 & 31.7 & 99.7 & 39.6 & 57.6 & 25.3 \\
\hline Unpaid work & 19.7 & 10.8 & 24.8 & 13.7 & 17.9 & 8.5 \\
\hline Either paid or unpaid work & 68.1 & 36.8 & 100.0 & 45.4 & 63.4 & 29.7 \\
\hline Started working before age 15 & 26.0 & 15.0 & 42.7 & 24.0 & 22.3 & 7.7 \\
\hline \multicolumn{7}{|l|}{ Ever worked in last 12 months } \\
\hline Paid work & 60.5 & 21.1 & 99.3 & 20.6 & 54.8 & 21.6 \\
\hline Unpaid work & 8.8 & 5.7 & 5.2 & 5.0 & 9.2 & 6.4 \\
\hline Either paid or unpaid work & 65.0 & 24.5 & 99.7 & 23.1 & 59.9 & 25.6 \\
\hline Number of respondents & 1,289 & 2,126 & 648 & 1,037 & 1,132 & 1,089 \\
\hline
\end{tabular}


Table 4.1: (Cont'd)

\begin{tabular}{|c|c|c|c|c|c|c|}
\hline Economic activity (\%) & $\begin{array}{c}\mathrm{M} \\
15-24\end{array}$ & $\begin{array}{c}\text { W } \\
15-24\end{array}$ & $\begin{array}{c}\text { MM } \\
15-29\end{array}$ & $\begin{array}{c}\text { MW } \\
15-24\end{array}$ & $\begin{array}{c}\text { UM } \\
15-24\end{array}$ & $\begin{array}{c}\text { UW } \\
\text { 15-24 }\end{array}$ \\
\hline \multicolumn{7}{|c|}{ Urban } \\
\hline \multicolumn{7}{|l|}{ Duration of paid work in last $\mathbf{1 2}$ months } \\
\hline Most of the year ( 6 months or more) & 89.2 & 71.2 & 99.0 & 67.9 & 86.1 & 73.0 \\
\hline Part of the year ( $3-5$ months) & 5.9 & 15.6 & 1.0 & 18.3 & 7.5 & 13.3 \\
\hline Rarely (less than 3 months) & 4.9 & 11.9 & 0.0 & 10.1 & 6.4 & 13.8 \\
\hline \multicolumn{7}{|l|}{ Main occupation (paid work) } \\
\hline Cultivator & 0.5 & 0.0 & 0.0 & 0.0 & 0.3 & 0.0 \\
\hline Agricultural labourer & 1.2 & 4.6 & 3.0 & 8.5 & 0.9 & 2.0 \\
\hline Administrative/executive/managerial/clerical & 14.7 & 22.8 & 14.2 & 14.2 & 15.9 & 29.6 \\
\hline Business & 5.1 & 2.3 & 7.0 & 2.8 & 4.6 & 2.0 \\
\hline Skilled manual/machinery & 43.5 & 33.1 & 47.7 & 43.4 & 42.6 & 25.0 \\
\hline Unskilled non-agricultural labourer & 32.9 & 36.4 & 27.5 & 31.1 & 33.3 & 40.3 \\
\hline Other & 1.9 & 0.7 & 0.7 & 0.0 & 2.0 & 1.0 \\
\hline Number engaged in paid work in last 12 months & 772 & 446 & 642 & 211 & 616 & 235 \\
\hline \multicolumn{7}{|c|}{ Rural } \\
\hline \multicolumn{7}{|l|}{ Ever worked } \\
\hline Paid work & 74.0 & 62.0 & 98.1 & 69.3 & 67.4 & 50.0 \\
\hline Unpaid work & 27.7 & 32.4 & 35.0 & 36.2 & 25.3 & 26.0 \\
\hline Either paid or unpaid work & 79.2 & 70.3 & 99.2 & 77.3 & 73.9 & 58.9 \\
\hline Started working before age 15 & 41.6 & 48.5 & 57.0 & 58.3 & 35.8 & 32.3 \\
\hline \multicolumn{7}{|l|}{ Ever worked in last 12 months } \\
\hline Paid work & 70.7 & 49.6 & 96.8 & 51.5 & 63.5 & 46.4 \\
\hline Unpaid work & 14.8 & 21.0 & 13.2 & 21.3 & 14.8 & 20.6 \\
\hline Either paid or unpaid work & 75.5 & 57.0 & 98.2 & 58.4 & 69.6 & 54.5 \\
\hline Number of respondents & 1,190 & 2,722 & 757 & 1,293 & 945 & 1,429 \\
\hline \multicolumn{7}{|l|}{ Duration of paid work in last 12 months } \\
\hline Most of the year ( 6 months or more) & 83.1 & 71.5 & 95.4 & 73.2 & 78.9 & 68.3 \\
\hline Part of the year ( $3-5$ months) & 8.5 & 16.6 & 3.3 & 16.7 & 9.8 & 16.6 \\
\hline Rarely (less than 3 months) & 8.4 & 11.8 & 1.3 & 9.8 & 11.3 & 15.1 \\
\hline \multicolumn{7}{|l|}{ Main occupation (paid work) } \\
\hline Cultivator & 4.9 & 2.5 & 8.9 & 3.0 & 3.5 & 1.6 \\
\hline Agricultural labourer & 38.9 & 72.0 & 38.3 & 75.5 & 37.2 & 65.5 \\
\hline Administrative/executive/managerial/clerical & 4.8 & 4.1 & 3.8 & 2.4 & 5.9 & 7.5 \\
\hline Business & 3.6 & 0.4 & 3.8 & 0.6 & 3.9 & 0.0 \\
\hline Skilled manual/machinery & 27.9 & 14.5 & 25.9 & 12.0 & 29.9 & 19.0 \\
\hline Unskilled non-agricultural labourer & 19.6 & 6.3 & 19.3 & 6.5 & 19.4 & 6.1 \\
\hline Other & 0.2 & 0.1 & 0.1 & 0.0 & 0.0 & 0.3 \\
\hline Number engaged in paid work in last 12 months & 840 & 1,328 & 733 & 661 & 602 & 667 \\
\hline
\end{tabular}

Note: All Ns are unweighted. Column totals may not equal 100\% due to missing cases or "don't know" responses. 
Percentages of youth who had worked in the last 12 months largely mirrored lifetime economic activity for young men. Among young women, this was true for the unmarried. Considerably fewer married young women reported economic activity in the last 12 months compared to lifetime economic activity (51\% compared to $70 \%$ ), a finding that may be attributed to conflict with childbearing and childrearing activities, on the one hand, and the tendency of married young women to be secluded from outside work, on the other.

Findings also suggest that among young men and women who worked for remuneration in the year prior to the interview, most young men (85\%) and somewhat fewer young women (71\%) worked for at least six months of the year. Marital status differences were not wide among young women, but more married than unmarried young men reported working for most of the year, and this pattern prevailed in both urban and rural settings. Differences by rural-urban residence were narrow, with similar proportions of youth in urban and rural areas reporting working for most of the year $(83 \%$ and $89 \%$ among young men in rural and urban areas, respectively; $72 \%$ and $71 \%$, respectively, among young women).

Occupational distributions of those engaged in remunerated work in the 12 months preceding the interview differed widely between young men and women. Young men were far more likely than young women to be engaged in skilled manual labour (32\% versus $17 \%$ ) and unskilled non-agricultural labour (23\% versus $11 \%)$ and far less likely to be employed as agricultural labourers (29\% versus $62 \%)$.

Occupational distributions also differed widely among youth in rural and urban areas. Leading occupations for young men in rural areas were agricultural labour (39\%), skilled manual labour (28\%) and unskilled non-agricultural labour (20\%). In contrast, young men in urban areas were most likely to report skilled manual labour (44\%), unskilled non-agricultural labour (33\%) and administrative, executive, managerial and clerical occupations (15\%). While percentages differed, the three leading occupations reported by young women in rural and urban areas, respectively, resembled those of young men. For example, in rural areas, as many as $72 \%$ of working young women were engaged in agricultural labour, while skilled manual labour and unskilled non-agricultural labour occupied small proportions (15\% and 6\%, respectively). In urban areas, in contrast, $36 \%, 33 \%$ and $23 \%$ of working women reported engaging in unskilled non-agricultural labour, skilled manual labour and administrative, executive, managerial and clerical occupations, respectively.

Differences by marital status were not wide among young men, irrespective of rural-urban residence. Among young women, however, differences were notable and these varied by rural-urban residence. For example, in rural areas, the married were more likely to be engaged in agricultural labour than the unmarried ( $76 \%$ and $66 \%$, respectively) and somewhat less likely to have engaged in skilled manual labour (12\% versus $19 \%)$. Differences in urban areas were wider: the unmarried were considerably more likely than the married to have engaged in administrative, executive, managerial or clerical occupations ( $30 \%$ versus $14 \%$ ), somewhat more likely to have engaged in unskilled non-agricultural labour ( $40 \%$ versus $31 \%$ ) and considerably less likely to have worked as skilled manual labourers ( $25 \%$ versus $43 \%)$.

\subsection{Unemployment}

To measure unemployment rates among respondents, the Youth Study assessed (a) whether youth had worked in the 12 months preceding the interview and if so, the number of months worked; and (b) whether youth were seeking work and if so, the number of months during which they had been searching for work. Table 4.2 presents unemployment rates, defined as those seeking employment for the major part of the year preceding the interview as a fraction of those in the labour force. Labour force refers to those who were working or seeking work for the major part of the year. It does not, therefore, include those exclusively studying, those who may have worked for a short period in the year preceding the interview, or those who had sought work for a short period in the year preceding the interview.

Measured in this way, the percentage of unemployed youth was 7\% among young men and $10 \%$ among young women, rates somewhat similar to those observed by the National Sample Survey (NSS) (NSSO, 2006) among youth using the principal usual status definition. We note, however, that rates obtained in the Youth Study are not 
quite comparable to the NSS, not only because questions were not identical, but also because of differences in the frequency with which information was obtained and corresponding differences in the recall period (quarterly in the NSS as compared to a 12-month recall period in the Youth Study) and differences in the household member eligible to provide information on youth unemployment (any household member in the NSS compared to the individual herself or himself in the Youth Study).

Findings suggest that unemployment rates among young men were similar, irrespective of rural-urban residence ( $7 \%$ and $6 \%$, respectively). They were, in contrast, wide among young women: unemployment rates ranged from $7 \%$ among young women in rural areas to $23 \%$ among those in urban areas.

\section{Table 4.2: Unemployment}

Percentage of youth in the labour force who were unemployed, according to residence, Andhra Pradesh, 2007-08

\begin{tabular}{|c|c|c|c|c|c|c|}
\hline Unemployment $(\%)^{1}$ & $\underset{15-24}{M}$ & $\begin{array}{c}\text { W } \\
15-24\end{array}$ & $\begin{array}{c}\text { MM } \\
15-29\end{array}$ & $\begin{array}{c}\text { MW } \\
15-24\end{array}$ & $\begin{array}{c}\text { UM } \\
15-24\end{array}$ & $\begin{array}{c}\text { UW } \\
\text { 15-24 }\end{array}$ \\
\hline \multicolumn{7}{|c|}{ Combined } \\
\hline Unemployed & 6.8 & 10.1 & 1.1 & 7.7 & 9.1 & 13.5 \\
\hline Number in labour force & 1,551 & 1,553 & 1372 & 758 & 1,162 & 795 \\
\hline \multicolumn{7}{|c|}{ Urban } \\
\hline Unemployed & 6.3 & 23.3 & 0.7 & 27.1 & 7.8 & 20.5 \\
\hline Number in labour force & 752 & 451 & 642 & 209 & 595 & 242 \\
\hline \multicolumn{7}{|c|}{ Rural } \\
\hline Unemployed & 6.9 & 7.2 & 1.2 & 5.0 & 9.6 & 11.2 \\
\hline Number in labour force & 799 & 1,102 & 730 & 549 & 567 & 553 \\
\hline
\end{tabular}

Note: All Ns are unweighted. ${ }^{1}$ Unemployment rate: Youth who were seeking work for the major part of the year preceding the interview as a proportion of those in the labour force (namely, those who were employed and/or seeking work for the major part of the year).

Differences by marital status indicate that unmarried young men were more likely than married young men to be unemployed (9\% versus 1\%) and the differences were apparent in both rural and urban areas. Among young women too, unemployment rates among the unmarried exceeded those of the married (14\% versus $8 \%$ ), but rural-urban patterns differed, with unemployment rates higher among the married than the unmarried in urban areas (27\% versus $21 \%)$ and lower than the unmarried in rural areas (5\% versus $11 \%)$.

Table 4.3 describes socio-economic differentials in reported unemployment among young men and women. Unemployment rates appeared to be somewhat similar among younger (aged 15-19) and older (aged 20-24) young men $(10 \%$ versus $5 \%)$ and women $(8 \%$ versus $12 \%)$. Age differences were, however, notable among unmarried young women and young women in urban areas; among them, unemployment rates were higher among women aged 20-24 than those aged 15-19. Differences by religion were, for the most part, narrow. However, among young men, unemployment rates were lower among Hindus and Muslims than those from other religions and this difference was particularly notable among unmarried young men. Caste-wise differences showed that among young women, those belonging to general castes experienced higher unemployment rates than did other young women. Among young men, unemployment rates were similar. Differences by education were, in contrast, wide and consistent and suggest the relative dearth of opportunities for the educated. Unemployment rates were just $1-2 \%$ among those with no education or 1-7 years of education; in contrast, young men and women who had completed Class 12 reported exceptionally high rates of unemployment (23\% and $46 \%$, respectively), a finding reiterated in other studies (Chandrasekhar, Ghosh and Roychowdhury, 2006; NSSO, 2006). Differences by household economic status, measured in wealth quintiles, similarly suggest that unemployment rates increased with economic status; more sharply among young women than men.

Patterns of unemployment observed were by and large similar among both unmarried and married youth. Patterns by rural-urban residence were, for the most part, also similar to those observed for young men and women in general. 
Table 4.3: Unemployment by selected background characteristics

Percentage of youth in the labour force who were unemployed by selected background characteristics, according to residence, Andhra Pradesh, 2007-08

\begin{tabular}{|c|c|c|c|c|c|c|}
\hline Background characteristics (\%) & $\begin{array}{c}\mathrm{M} \\
15-24\end{array}$ & $\begin{array}{c}\text { W } \\
15-24\end{array}$ & $\begin{array}{c}\text { MM } \\
15-29\end{array}$ & $\begin{array}{c}\text { MW } \\
\text { 15-24 }\end{array}$ & $\begin{array}{c}\text { UM } \\
15-24\end{array}$ & $\begin{array}{c}\text { UW } \\
\text { 15-24 }\end{array}$ \\
\hline \multicolumn{7}{|c|}{ Combined } \\
\hline \multicolumn{7}{|l|}{ Age (years) } \\
\hline $15-19$ & 10.2 & 8.2 & * & 5.6 & 10.3 & 9.8 \\
\hline $20-24$ & 5.0 & 11.8 & 0.3 & 8.6 & 8.1 & 24.3 \\
\hline $25-29$ & NA & NA & 1.3 & NA & NA & NA \\
\hline \multicolumn{7}{|l|}{ Religion } \\
\hline Hindu & 6.4 & 9.9 & 0.9 & 7.6 & 8.8 & 13.5 \\
\hline Muslim & 7.7 & 11.5 & 1.0 & $(6.3)$ & 8.6 & 15.3 \\
\hline Other $^{1}$ & 10.0 & 11.0 & 3.8 & 9.7 & 16.1 & 12.0 \\
\hline \multicolumn{7}{|l|}{ Caste } \\
\hline SC & 8.3 & 10.4 & 2.2 & 6.9 & 10.9 & 14.9 \\
\hline ST & 4.5 & 10.0 & 0.0 & 8.2 & 7.6 & 15.8 \\
\hline $\mathrm{OBC}$ & 6.9 & 8.4 & 0.9 & 7.7 & 9.8 & 9.4 \\
\hline General $^{2}$ & 4.9 & 15.3 & 0.7 & 9.4 & 5.8 & 22.3 \\
\hline \multicolumn{7}{|l|}{ Educational level (years) } \\
\hline None $^{3}$ & 1.1 & 0.9 & 0.0 & 1.1 & 2.0 & 0.0 \\
\hline $1-7$ & 0.6 & 1.5 & 0.2 & 1.5 & 0.8 & 1.1 \\
\hline $8-11$ & 7.6 & 19.6 & 1.2 & 24.1 & 9.2 & 16.4 \\
\hline 12 and above & 22.9 & 45.8 & 4.6 & 57.8 & 26.6 & 40.8 \\
\hline \multicolumn{7}{|l|}{ Wealth quintile } \\
\hline First & 4.4 & 5.4 & 0.6 & 4.3 & 6.6 & 7.6 \\
\hline Second & 5.0 & 6.8 & 0.4 & 5.9 & 6.2 & 8.5 \\
\hline Third & 7.3 & 8.0 & 0.9 & 3.8 & 10.0 & 14.9 \\
\hline Fourth & 7.4 & 15.1 & 1.1 & 11.3 & 10.1 & 20.0 \\
\hline Fifth & 9.0 & 22.0 & 2.5 & 23.1 & 11.5 & 19.8 \\
\hline Total & 6.8 & 10.1 & 1.1 & 7.7 & 9.1 & 13.5 \\
\hline \multicolumn{7}{|c|}{ Urban } \\
\hline \multicolumn{7}{|l|}{ Age (years) } \\
\hline $15-19$ & 5.6 & 12.3 & * & $(12.5)$ & 5.6 & 12.1 \\
\hline $20-24$ & 6.5 & 28.6 & 0.0 & 29.7 & 8.9 & 28.4 \\
\hline $25-29$ & NA & NA & 0.9 & NA & NA & NA \\
\hline \multicolumn{7}{|l|}{ Religion } \\
\hline Hindu & 6.7 & 23.8 & 0.8 & 26.4 & 8.5 & 21.2 \\
\hline Muslim & 3.0 & (19.4) & 0.0 & * & 3.6 & $(15.4)$ \\
\hline Other $^{1}$ & (9.5) & (23.1) & $(0.0)$ & * & (11.8) & * \\
\hline \multicolumn{7}{|l|}{ Caste } \\
\hline SC & 7.4 & 24.4 & 0.0 & 25.9 & 11.1 & 24.5 \\
\hline ST & (6.7) & * & $(0.0)$ & * & * & * \\
\hline OBC & 6.1 & 18.5 & 0.7 & 21.4 & 7.7 & 14.8 \\
\hline General $^{2}$ & 5.9 & 30.6 & 0.0 & $(42.1)$ & 7.1 & 26.3 \\
\hline
\end{tabular}


Table 4.3: (Cont'd)

\begin{tabular}{|c|c|c|c|c|c|c|}
\hline Background characteristics (\%) & $\begin{array}{c}\mathrm{M} \\
15-24\end{array}$ & $\begin{array}{c}\text { W } \\
15-24\end{array}$ & $\begin{array}{c}\text { MM } \\
15-29\end{array}$ & $\begin{array}{c}\text { MW } \\
15-24\end{array}$ & $\begin{array}{c}\text { UM } \\
15-24\end{array}$ & $\begin{array}{c}\text { UW } \\
15-24\end{array}$ \\
\hline \multicolumn{7}{|c|}{ Urban } \\
\hline \multicolumn{7}{|l|}{ Educational level (years) } \\
\hline None $^{3}$ & 0.0 & 0.0 & 0.0 & $(0.0)$ & $(0.0)$ & * \\
\hline $1-7$ & 0.8 & 2.6 & 1.1 & 3.4 & 1.0 & 2.0 \\
\hline $8-11$ & 3.6 & 31.3 & 0.0 & 43.8 & 5.1 & 19.1 \\
\hline 12 and above & 20.7 & 42.7 & 0.0 & $(60.9)$ & 23.8 & 36.0 \\
\hline \multicolumn{7}{|l|}{ Wealth quintile } \\
\hline First & $(8.0)$ & $(6.5)$ & $(0.0)$ & $(7.7)$ & $(11.1)$ & * \\
\hline Second & 4.3 & 12.8 & 0.0 & $(18.8)$ & 5.7 & $(9.1)$ \\
\hline Third & 4.3 & 18.5 & 0.0 & $(25.0)$ & 6.0 & $(11.8)$ \\
\hline Fourth & 5.5 & 26.4 & 1.9 & 26.7 & 7.4 & 26.6 \\
\hline Fifth & 7.8 & 33.3 & 0.0 & 42.9 & 9.3 & 27.0 \\
\hline Total & 6.3 & 23.3 & 0.7 & 27.1 & 7.8 & 20.5 \\
\hline \multicolumn{7}{|c|}{ Rural } \\
\hline \multicolumn{7}{|l|}{ Age (years) } \\
\hline $15-19$ & 11.5 & 7.4 & * & 4.7 & 11.6 & 9.1 \\
\hline $20-24$ & 4.5 & 7.1 & 0.3 & 5.2 & 7.7 & 21.1 \\
\hline $25-29$ & NA & NA & 1.4 & NA & NA & NA \\
\hline \multicolumn{7}{|l|}{ Religion } \\
\hline Hindu & 6.4 & 7.1 & 1.0 & 5.1 & 8.9 & 11.1 \\
\hline Muslim & 10.7 & $(7.0)$ & (1.5) & * & 12.0 & (12.5) \\
\hline Other $^{1}$ & (11.4) & 8.6 & $(4.5)$ & $(7.8)$ & (17.8) & 10.8 \\
\hline \multicolumn{7}{|l|}{ Caste } \\
\hline SC & 8.5 & 7.1 & 2.6 & 4.0 & 11.0 & 12.3 \\
\hline ST & 4.2 & 8.7 & 0.0 & 6.5 & (7.4) & (15.4) \\
\hline OBC & 7.3 & 6.4 & 1.0 & 5.6 & 10.7 & 7.9 \\
\hline General $^{2}$ & 4.4 & 9.6 & 1.0 & 3.1 & 5.2 & 19.6 \\
\hline \multicolumn{7}{|l|}{ Educational level (years) } \\
\hline None $^{3}$ & 1.3 & 1.0 & 0.0 & 1.1 & 2.6 & 0.0 \\
\hline $1-7$ & 0.5 & 1.3 & 0.0 & 1.7 & 0.8 & 0.9 \\
\hline $8-11$ & 8.8 & 16.7 & 1.2 & 17.8 & 10.8 & 15.8 \\
\hline 12 and above & 24.2 & 50.0 & 7.0 & * & 29.0 & 47.5 \\
\hline \multicolumn{7}{|l|}{ Wealth quintile } \\
\hline First & 4.0 & 5.4 & 0.7 & 4.5 & 6.0 & 7.2 \\
\hline Second & 5.1 & 6.2 & 0.4 & 4.9 & 6.3 & 8.4 \\
\hline Third & 7.9 & 6.3 & 1.1 & 1.6 & 10.9 & 15.6 \\
\hline Fourth & 8.4 & 10.1 & 0.8 & 7.5 & 11.7 & 15.5 \\
\hline Fifth & 10.1 & 12.9 & 4.4 & $(14.3)$ & 13.8 & $(11.5)$ \\
\hline Total & 6.9 & 7.2 & 1.2 & 5.0 & 9.6 & 11.2 \\
\hline
\end{tabular}

Note: ( ) Based on 25-49 unweighted cases. ${ }^{*}$ Percentage not shown, based on fewer than 25 unweighted cases. NA: Not applicable. OBC: Other backward caste. SC: Scheduled caste. ST: Scheduled tribe. ${ }^{1}$ Includes Christian, Buddhist, Neo-Buddhist, Sikh, Jain, Jewish, Parsi/Zoroastrian and no specified religion. ${ }^{2}$ Includes all those not belonging to SC, ST or OBC. ${ }^{3}$ Includes non-literate and literate with no formal schooling. 


\subsection{Work-related mobility}

Among young men who had ever worked, almost one-quarter reported the experience of work-related mobility, as shown in Table 4.4. Fewer young women, in contrast, had lived away from home for work-related reasons (8\%). Gender differences may be attributed to the finding observed earlier that men were more likely than women to be engaged in such activities as non-agricultural labour and skilled manual labour, which entail mobility; they may also be attributed to the greater restrictions placed on the independent movement of young women than young men. Differences by marital status and rural-urban residence were mild. A large proportion of young men (62\%) and women $(42 \%)$ who reported work-related mobility had remained outside their home village or neighbourhood for three months or longer.

\subsection{Economic activity and schooling status}

While the period of transition to adulthood is marked by discontinuation of schooling and entry into the labour market for many young people, some combine schooling and work and others are neither in school nor working. Data collected through the Life Event Calendar component of the Youth Study provided an opportunity to explore the pattern of these events (that is, studying, working, both studying and working, and neither studying nor working) in young people's lives from the age of 12, and these are presented in Figures 4.1 a-c. We note that Figures 4.1a and $4.1 \mathrm{~b}$ convey the situation both prior to and following marriage for married youth.

Patterns varied widely by sex and marital status of the respondent. A comparison of the two panels of Figure 4.1a shows, first, that the proportion of youth reporting school attendance declined steadily across all groups as young

Table 4.4: Work-related mobility

Percentage of youth who had ever lived outside their home village/area for work, according to residence, Andhra Pradesh, 2007-08

\begin{tabular}{|c|c|c|c|c|c|c|}
\hline Mobility characteristics (\%) & $\begin{array}{c}\mathrm{M} \\
15-24\end{array}$ & $\begin{array}{c}\text { W } \\
15-24\end{array}$ & $\begin{array}{c}\text { MM } \\
15-29\end{array}$ & $\begin{array}{c}\text { MW } \\
15-24\end{array}$ & $\begin{array}{c}\text { UM } \\
15-24\end{array}$ & $\begin{array}{c}\text { UW } \\
15-24\end{array}$ \\
\hline \multicolumn{7}{|c|}{ Combined } \\
\hline \multicolumn{7}{|l|}{ Work-related mobility } \\
\hline Ever stayed outside village/area for work & 23.0 & 7.5 & 26.0 & 7.4 & 22.1 & 7.7 \\
\hline Number ever worked & 1,813 & 2,630 & 1,398 & 1,463 & 1,414 & 1,167 \\
\hline Stayed outside village/area for 3 months or longer & 62.2 & 41.6 & 68.9 & 38.0 & 61.9 & 47.9 \\
\hline Number ever stayed out of home village/area for work & 409 & 185 & 378 & 97 & 305 & 88 \\
\hline \multicolumn{7}{|c|}{ Urban } \\
\hline \multicolumn{7}{|l|}{ Work-related mobility } \\
\hline Ever stayed outside village/area for work & 22.5 & 4.6 & 30.1 & 4.2 & 21.3 & 5.2 \\
\hline Number ever worked & 871 & 795 & 647 & 470 & 714 & 325 \\
\hline Stayed outside village/area for 3 months or longer & 63.0 & $(66.7)$ & 69.2 & * & 63.1 & * \\
\hline Number ever stayed out of home village/area for work & 194 & 36 & 194 & 19 & 150 & 17 \\
\hline \multicolumn{7}{|c|}{ Rural } \\
\hline Work-related mobility & & & & & & \\
\hline Ever stayed outside village/area for work & 23.2 & 8.1 & 24.9 & 8.0 & 22.5 & 8.4 \\
\hline Number ever worked & 942 & 1,835 & 751 & 993 & 700 & 842 \\
\hline Stayed outside village/area for 3 months or longer & 62.0 & 38.5 & 68.8 & 36.6 & 61.5 & 42.5 \\
\hline Number ever stayed out of home village/area for work & 215 & 149 & 184 & 78 & 155 & 71 \\
\hline
\end{tabular}

Note: All Ns are unweighted. ( ) Based on 25-49 unweighted cases. ${ }^{*}$ Percentage not shown, based on fewer than 25 unweighted cases. 
Figure 4.1a: Economic activity and schooling status among youth aged 15-24, by age, Andhra Pradesh, 2007-08
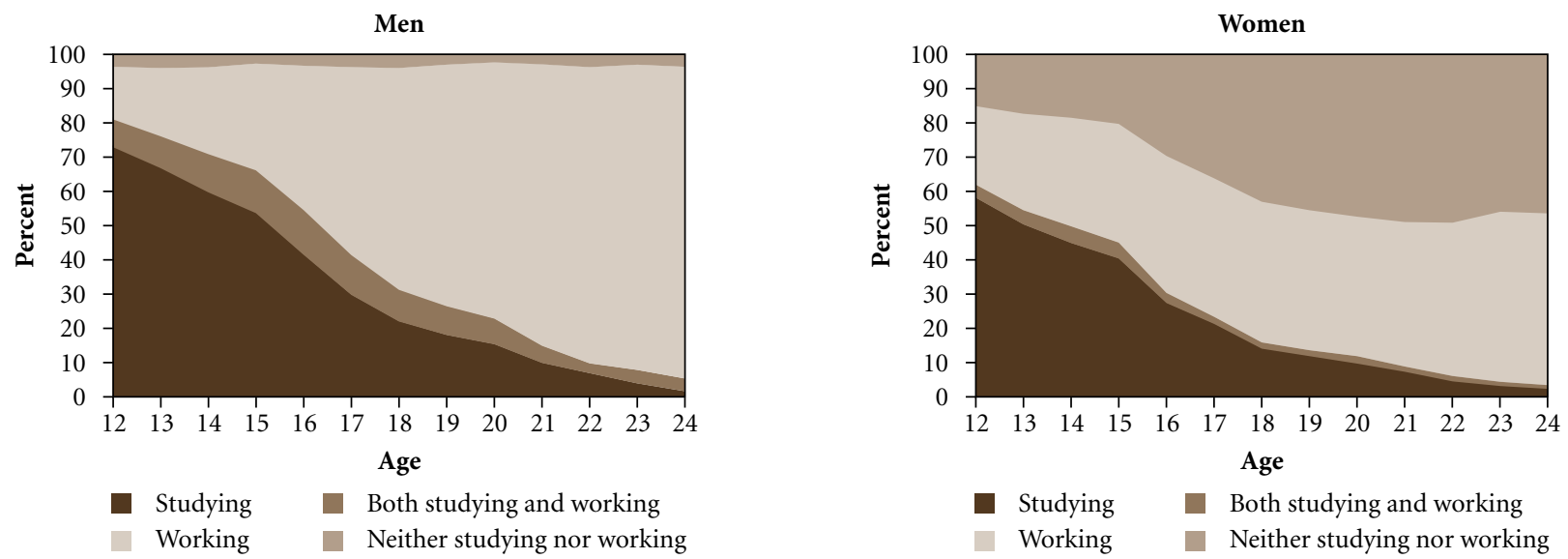

Note: For married youth, the figure conveys the situation prior to and following marriage.

Figure 4.1b: Economic activity and schooling status among married men aged 15-29 and married women aged 15-24, by age, Andhra Pradesh, 2007-08
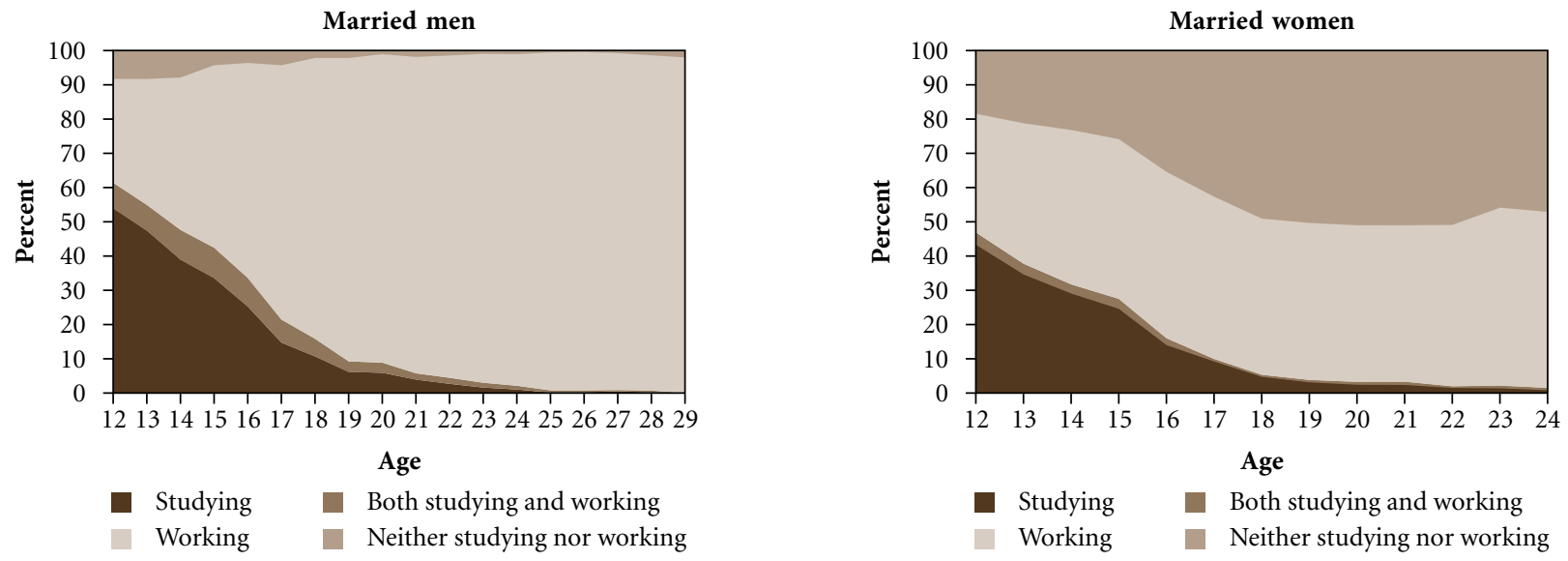

Note: For married youth, the figure conveys the situation prior to and following marriage.

Figure 4.1c: Economic activity and schooling status among unmarried men and women aged 15-24, by age, Andhra Pradesh, 2007-08
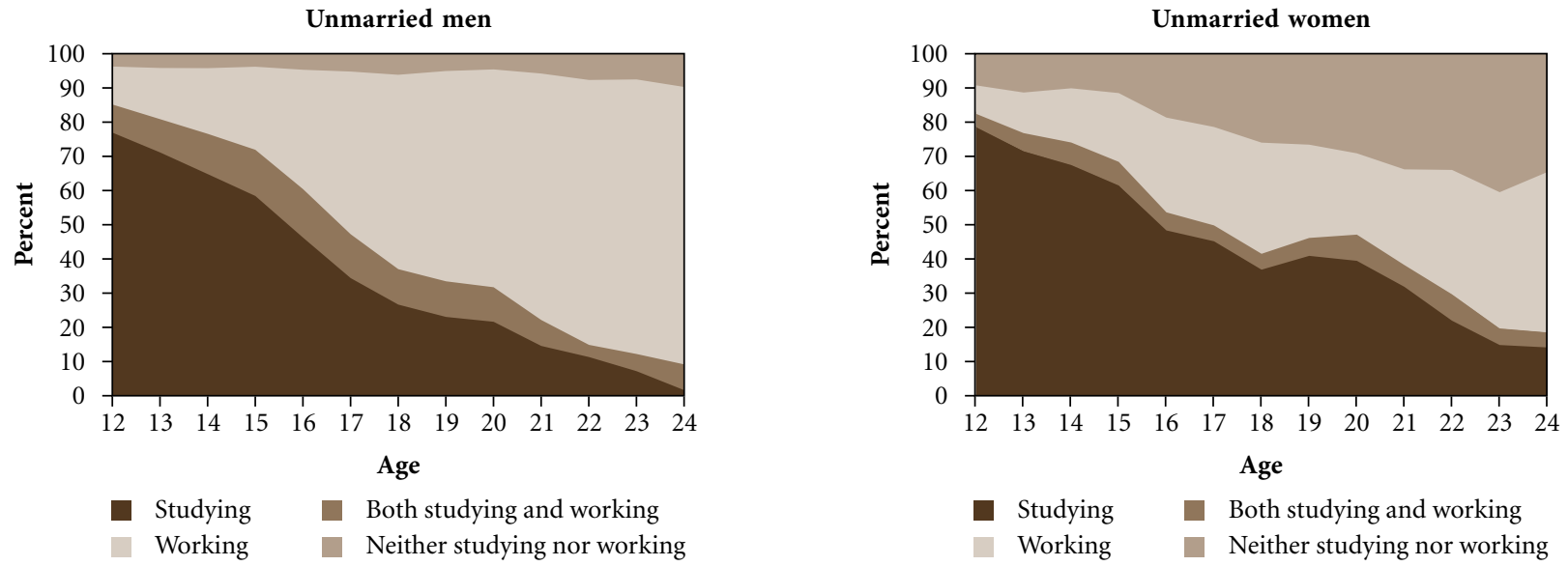
people transitioned out of childhood or early adolescence into late adolescence and young adulthood. For example, while $81 \%$ of young men and $63 \%$ of young women were in school (a small minority of these were also working) at age 12, the percentage who remained in school at age 15 fell to $66 \%$ for young men and $46 \%$ for young women. Second, very few youth (4-13\% of young men; $1-5 \%$ of young women) reported having combined studying and working at any age. Third, exit from school was accompanied by a rise in work participation over the ages for both young men and women; however, the increase was steeper among young men than among young women. Among young men, work participation increased consistently from age 12 onwards; among young women, in contrast, work participation increased between ages 12 and 16 and then remained more or less steady up to age 24. Findings also suggest that larger percentages were working than in school by age 17 among young men and 16 among young women. Finally, significant proportions of young women but not young men were neither in school nor working from age 12 onwards. Among young men, small proportions (less than 5\%) were neither working nor in school at any age. On the contrary among young women, there was a steady increase in proportion of women who were neither studying nor working by age. At age 12,14\% of young women were neither working nor in school; percentages increased to $20 \%$ at age 15 and $47 \%$ at age 20 .

Figures $4.1 \mathrm{~b}$ and $4.1 \mathrm{c}$ suggest that patterns differed between married and unmarried youth. For one, the married were less likely than the unmarried to be in school at each age. For example, $62 \%$ and $47 \%$ of married young men and women, respectively, and $86 \%$ and $82 \%$ of the unmarried, respectively, were in school (a small minority of these were also working) at age 12, and the percentages of those who remained in school fell thereafter. At age 20, for example, only $9 \%$ of married young men and $4 \%$ of married young women were pursuing their education compared to $32 \%$ and $48 \%$ of unmarried young men and women, respectively. Second, the unmarried were more likely than the married to combine studying and working at any age. Third, while slightly larger percentages of married than unmarried young men were neither in school nor working in early adolescence, a reverse pattern was observed once young men transitioned out of adolescence to young adulthood. Among young women, more married than unmarried were neither in school nor working from age 12. The percentage of those neither in school nor working increased steadily till age 18 and thereafter plateaued among married young women; among unmarried, it continued to increase even at later ages. Finally, more youth were working than in school at a younger age among the married than the unmarried. Indeed, by ages 13 and 14, respectively, more married young men and women were working than in school; this crossover occurred at considerably older ages among the unmarried (17 and 22 among young men and women, respectively).

\subsection{Participation in non-economic activity}

The Youth Study also inquired about the extent to which young men and women participated in domestic chores. All youth were asked whether and how frequently they were engaged in activities such as housework (cooking, cleaning, child/sibling care), shopping for groceries for the family and tasks such as collecting firewood or fetching water, and paying electricity or phone bills (as appropriate for urban and rural areas). Findings, reported in Table 4.5 and Figure 4.2, highlight the gendered nature of young people's participation in domestic chores. They show that the large majority of young women (83\%), compared with just $8 \%$ of young men were often engaged in housework. It is notable, nonetheless, that two in three young men reported "sometimes" participating in housework. In contrast, $94 \%$ of young men compared with $75 \%$ of young women reported sometimes or often shopping for groceries. Tasks such as collecting firewood or fetching water, and paying electricity or phone bills were conducted by both young men and women. Larger proportions of young men than women reported participating in these tasks-95\% and $74 \%$, respectively_-suggesting that young women were more likely than young men to be engaged in work inside the home, and less likely to be engaged in tasks that violated norms restricting their mobility outside the home.

In terms of differences in household work participation by marital status, patterns varied by type of activity and sex of the respondent. For example, among young women, the unmarried were about as likely as the married to engage in housework and shopping but less likely than the married to engage in other tasks; among young men, differences by marital status were modest. These patterns were evident, by and large, in both rural and urban areas. 
Table 4.5: Participation in household chores

Percent distribution of youth by extent of participation in various household chores, according to residence, Andhra Pradesh, 2007-08

\begin{tabular}{|c|c|c|c|c|c|c|}
\hline Types of chores (\%) & $\begin{array}{c}\text { M } \\
15-24\end{array}$ & $\begin{array}{c}\text { W } \\
15-24\end{array}$ & $\begin{array}{c}\text { MM } \\
15-29\end{array}$ & $\begin{array}{c}\text { MW } \\
15-24\end{array}$ & $\begin{array}{c}\text { UM } \\
15-24\end{array}$ & $\begin{array}{c}\text { UW } \\
15-24\end{array}$ \\
\hline \multicolumn{7}{|c|}{ Combined } \\
\hline \multicolumn{7}{|l|}{ Housework $^{1}$} \\
\hline Never & 24.2 & 1.3 & 25.0 & 0.0 & 24.5 & 3.1 \\
\hline Sometimes & 67.6 & 15.7 & 65.3 & 2.0 & 67.9 & 33.6 \\
\hline Often & 8.1 & 82.9 & 9.8 & 98.0 & 7.6 & 63.3 \\
\hline \multicolumn{7}{|l|}{ Shopping } \\
\hline Never & 6.0 & 24.7 & 5.2 & 23.0 & 5.8 & 27.0 \\
\hline Sometimes & 79.5 & 52.7 & 75.2 & 49.2 & 80.5 & 57.1 \\
\hline Often & 14.6 & 22.6 & 19.6 & 27.8 & 13.8 & 15.9 \\
\hline \multicolumn{7}{|l|}{ Other tasks ${ }^{2}$} \\
\hline Never & 5.4 & 25.7 & 3.2 & 19.8 & 5.9 & 32.6 \\
\hline Sometimes & 75.6 & 27.4 & 73.0 & 23.3 & 75.4 & 32.7 \\
\hline Often & 19.0 & 47.0 & 23.8 & 56.9 & 18.7 & 34.7 \\
\hline Number of respondents & 2,479 & 4,848 & 1,405 & 2,330 & 2,077 & 2,518 \\
\hline \multicolumn{7}{|c|}{ Urban } \\
\hline \multicolumn{7}{|l|}{ Housework $^{1}$} \\
\hline Never & 31.6 & 2.5 & 27.1 & 0.0 & 32.6 & 4.6 \\
\hline Sometimes & 65.0 & 26.2 & 69.9 & 3.4 & 64.0 & 44.8 \\
\hline Often & 3.4 & 71.2 & 2.9 & 96.6 & 3.3 & 50.6 \\
\hline \multicolumn{7}{|l|}{ Shopping } \\
\hline Never & 5.5 & 22.8 & 3.3 & 19.2 & 5.7 & 25.7 \\
\hline Sometimes & 81.8 & 59.0 & 77.2 & 55.8 & 82.7 & 61.6 \\
\hline Often & 12.6 & 18.2 & 19.5 & 25.0 & 11.6 & 12.7 \\
\hline \multicolumn{7}{|l|}{ Other tasks ${ }^{2}$} \\
\hline Never & 7.9 & 44.9 & 3.6 & 39.6 & 8.6 & 49.3 \\
\hline Sometimes & 81.4 & 28.8 & 79.7 & 24.6 & 81.5 & 32.2 \\
\hline Often & 10.6 & 26.2 & 16.7 & 35.8 & 10.0 & 18.5 \\
\hline Number of respondents & 1,289 & 2,126 & 648 & 1,037 & 1,132 & 1,089 \\
\hline \multicolumn{7}{|c|}{ Rural } \\
\hline \multicolumn{7}{|l|}{ Housework $^{1}$} \\
\hline Never & 21.4 & 0.8 & 24.4 & 0.0 & 20.9 & 2.2 \\
\hline Sometimes & 68.6 & 11.3 & 64.0 & 1.6 & 69.6 & 27.3 \\
\hline Often & 10.0 & 87.8 & 11.6 & 98.4 & 9.5 & 70.5 \\
\hline \multicolumn{7}{|l|}{ Shopping } \\
\hline Never & 6.1 & 25.5 & 5.7 & 24.1 & 5.9 & 27.7 \\
\hline Sometimes & 78.5 & 50.1 & 74.6 & 47.3 & 79.4 & 54.5 \\
\hline Often & 15.3 & 24.5 & 19.7 & 28.6 & 14.7 & 17.8 \\
\hline \multicolumn{7}{|l|}{ Other tasks ${ }^{2}$} \\
\hline Never & 4.4 & 17.6 & 3.1 & 14.1 & 4.8 & 23.2 \\
\hline Sometimes & 73.4 & 26.7 & 71.2 & 22.9 & 72.7 & 33.0 \\
\hline Often & 22.2 & 55.7 & 25.7 & 63.0 & 22.5 & 43.8 \\
\hline Number of respondents & 1,190 & 2,722 & 757 & 1,293 & 945 & 1,429 \\
\hline
\end{tabular}

Note: All Ns are unweighted. Column totals may not equal 100\% due to missing cases or "don't know" responses. ${ }^{1}$ Includes cooking, cleaning, etc. ${ }^{2}$ Respondents were given examples of other tasks such as collecting firewood, fetching water, grazing livestock, paying bills, etc. 
Frequency of engaging in domestic activities varied. Young women were more likely than young men to engage in all three tasks on a regular basis. Differences by marital status suggest that larger proportions of married than unmarried young women reported engaging in all three tasks on a regular basis. Likewise, married young men were more likely than the unmarried to report shopping and engaging in such tasks as collecting firewood or fetching water, and paying bills on a regular basis.

Rural-urban differences were moderate but suggest that urban youth were considerably less likely than their rural counterparts to report engaging in such tasks as collecting firewood or fetching water, and paying bills on a regular basis. Differences were particularly evident for young women upon whom much of the responsibility for collecting firewood and water rests in rural areas. Moreover, rural young women were more likely than urban young women to have engaged in housework and shopping on a regular basis.

\subsection{Participation in vocational training programmes}

A number of vocational training opportunities are available to youth through government, non-government and private organisations. Our survey inquired whether respondents had attended any such programmes, and the kinds of programmes they would like to attend, if offered. Findings, presented in Table 4.6 and Figure 4.3, indicate that about one-third of young men and women $(31 \%$ and $30 \%$, respectively) had ever attended a vocational training programme. The unmarried were somewhat more likely to have received training than the married, particularly among young women. Similarly, urban youth were far more likely to have received training than their rural counterparts.

The kind of training received varied widely by sex of the respondent and rural-urban residence. Among young men, leading training programmes reported were focused on computer skills $(43 \%)$, and to a lesser extent, auto mechanics or electrical work (19\%), driving (17\%) and plumbing or masonry (11\%). Key training received by young women was quite different: $56 \%$ reported training in tailoring, $37 \%$ in handicrafts, painting, embroidery or cooking and $25 \%$ in computer skills. The unmarried were more likely to report training in new technologies than the married. For example, $49 \%$ of unmarried young men compared to $15 \%$ of married young men reported computer training; corresponding figures for young women were $36 \%$ and $11 \%$, respectively. Conversely, larger proportions of married than unmarried youth reported training in-driving (30\% versus $13 \%)$ and plumbing or masonry ( $20 \%$ versus $8 \%$ ), and more married than unmarried young women received training in tailoring (69\% versus $44 \%)$ and handicrafts, painting, embroidery or cooking ( $40 \%$ versus $33 \%$ ). Finally, training received by rural youth was considerably more likely than that obtained by urban youth to fall into more traditional activities.
Figure 4.2: Percentage of youth who participated in domestic chores, according to residence, Andhra Pradesh, 2007-08
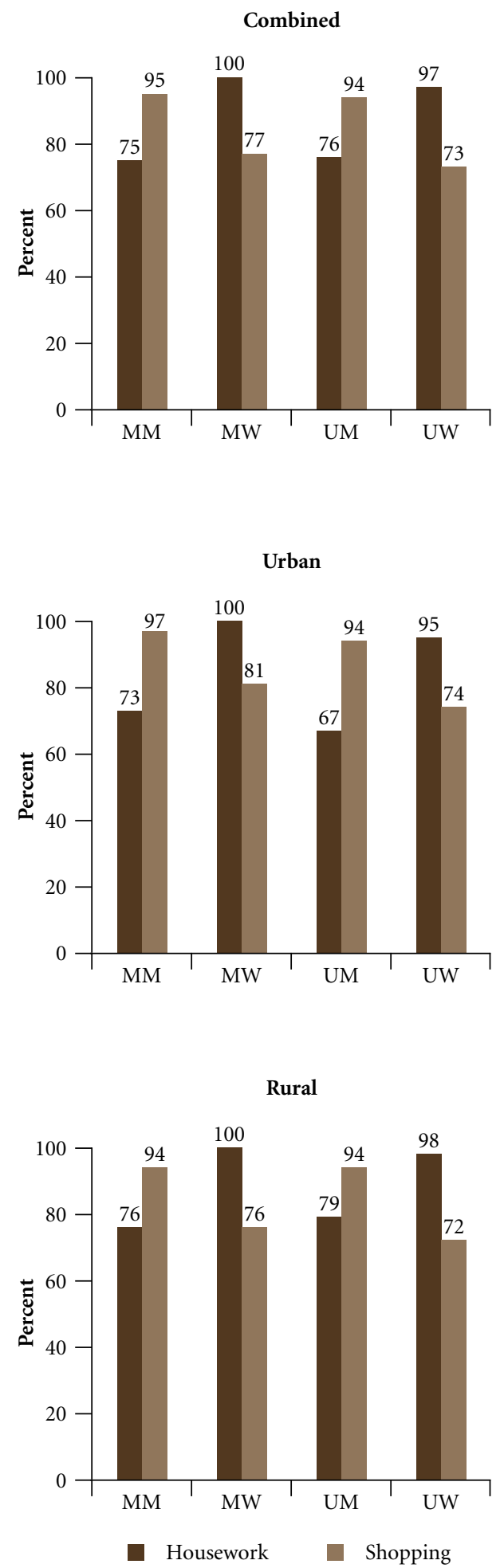
Table 4.6: Participation in vocational training programmes

Percentage of youth who ever attended a vocational training programme and type of programme attended, according to residence, Andhra Pradesh, 2007-08

\begin{tabular}{|c|c|c|c|c|c|c|}
\hline Programmes/courses attended (\%) & $\begin{array}{c}\mathrm{M} \\
15-24\end{array}$ & $\begin{array}{c}\text { W } \\
15-24\end{array}$ & $\begin{array}{c}\text { MM } \\
15-29\end{array}$ & $\begin{array}{c}\text { MW } \\
15-24\end{array}$ & $\begin{array}{c}\text { UM } \\
15-24\end{array}$ & $\begin{array}{c}\text { UW } \\
15-24\end{array}$ \\
\hline \multicolumn{7}{|c|}{ Combined } \\
\hline Ever attended a vocational training programme & 31.0 & 30.1 & 27.6 & 24.8 & 32.0 & 36.4 \\
\hline Number of respondents & 2,479 & 4,848 & 1,405 & 2,330 & 2,077 & 2,518 \\
\hline \multicolumn{7}{|l|}{ Types of programmes/courses attended } \\
\hline Tailoring & 3.3 & 55.7 & 8.0 & 69.1 & 2.4 & 44.4 \\
\hline Auto mechanic/electrical work & 18.6 & 0.0 & 21.2 & 0.0 & 17.6 & 0.0 \\
\hline Driving & 16.7 & 0.2 & 29.6 & 0.0 & 13.3 & 0.3 \\
\hline Plumbing/masonry & 10.7 & 0.1 & 20.1 & 0.0 & 8.1 & 0.0 \\
\hline Poultry/goat farm & 0.0 & 0.0 & 0.8 & 0.0 & 0.0 & 0.0 \\
\hline Beauty parlour/salon & 1.4 & 1.4 & 1.8 & 1.7 & 1.0 & 1.1 \\
\hline Nurse's aid & 1.3 & 3.0 & 0.8 & 2.6 & 1.5 & 3.4 \\
\hline Computer training & 43.3 & 24.5 & 15.2 & 11.2 & 49.3 & 35.7 \\
\hline English language/typing/shorthand & 6.6 & 7.3 & 3.9 & 4.0 & 7.2 & 9.9 \\
\hline Handicrafts/painting/embroidery/cooking & 7.6 & 36.7 & 7.0 & 40.2 & 7.5 & 33.4 \\
\hline Number ever attended any vocational training & 861 & 1,626 & 437 & 677 & 737 & 949 \\
\hline \multicolumn{7}{|c|}{ Urban } \\
\hline Ever attended a vocational training programme & 42.4 & 44.8 & 39.9 & 39.4 & 41.8 & 49.1 \\
\hline Number of respondents & 1,289 & 2,126 & 648 & 1,037 & 1,132 & 1,089 \\
\hline \multicolumn{7}{|l|}{ Types of programmes/courses attended } \\
\hline Tailoring & 1.7 & 41.8 & 4.1 & 58.5 & 1.1 & 31.0 \\
\hline Auto mechanic/electrical work & 16.0 & 0.0 & 20.3 & 0.0 & 15.1 & 0.0 \\
\hline Driving & 11.3 & 0.5 & 20.3 & 0.0 & 7.9 & 0.7 \\
\hline Plumbing/masonry & 7.7 & 0.2 & 18.0 & 0.0 & 5.3 & 0.0 \\
\hline Poultry/goat farm & 0.0 & 0.0 & 0.0 & 0.0 & 0.0 & 0.0 \\
\hline Beauty parlour/salon & 1.0 & 2.5 & 0.8 & 3.4 & 1.1 & 1.8 \\
\hline Nurse's aid & 1.0 & 2.5 & 1.6 & 2.4 & 1.1 & 2.5 \\
\hline Computer training & 53.8 & 39.5 & 26.8 & 21.7 & 60.2 & 51.0 \\
\hline English language/typing/shorthand & 10.7 & 11.7 & 7.3 & 6.3 & 11.7 & 15.2 \\
\hline Handicrafts/painting/embroidery/cooking & 7.4 & 36.2 & 10.7 & 45.2 & 7.2 & 30.5 \\
\hline Number ever attended any vocational training & 546 & 942 & 256 & 408 & 474 & 534 \\
\hline \multicolumn{7}{|c|}{ Rural } \\
\hline Ever attended a vocational training programme & 26.5 & 23.9 & 24.1 & 20.6 & 27.8 & 29.1 \\
\hline Number of respondents & 1,190 & 2,722 & 757 & 1,293 & 945 & 1,429 \\
\hline \multicolumn{7}{|l|}{ Types of programmes/courses attended } \\
\hline Tailoring & 4.4 & 66.6 & 10.2 & 74.8 & 3.2 & 57.1 \\
\hline Auto mechanic/electrical work & 20.3 & 0.0 & 21.9 & 0.0 & 19.2 & 0.0 \\
\hline Driving & 20.1 & 0.0 & 34.0 & 0.0 & 16.9 & 0.0 \\
\hline Plumbing/masonry & 12.6 & 0.0 & 21.1 & 0.0 & 10.2 & 0.0 \\
\hline Poultry/goat farm & 0.0 & 0.0 & 1.1 & 0.0 & 0.0 & 0.0 \\
\hline Beauty parlour/salon & 1.7 & 0.5 & 2.3 & 0.8 & 1.2 & 0.2 \\
\hline Nurse's aid & 1.5 & 3.6 & 0.8 & 2.7 & 1.7 & 4.5 \\
\hline Computer training & 36.6 & 12.6 & 9.8 & 5.4 & 42.2 & 21.1 \\
\hline English language/typing/shorthand & 4.2 & 3.8 & 2.6 & 2.7 & 4.2 & 5.1 \\
\hline Handicrafts/painting/embroidery/cooking & 7.6 & 36.9 & 5.3 & 37.6 & 8.0 & 36.2 \\
\hline Number ever attended any vocational training & 315 & 684 & 181 & 269 & 263 & 415 \\
\hline
\end{tabular}

Note: All Ns are unweighted. Column totals may exceed 100\% due to multiple responses. 
Figure 4.3: Percentage of youth who ever attended a vocational training programme and percentage who were interested in participating in such programmes, according to residence, Andhra Pradesh, 2007-08

Combined

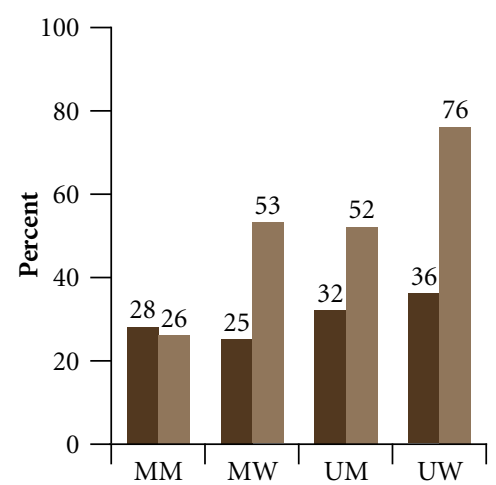

Urban

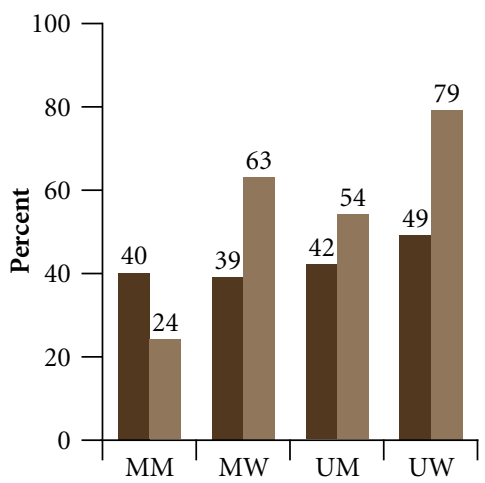

Rural

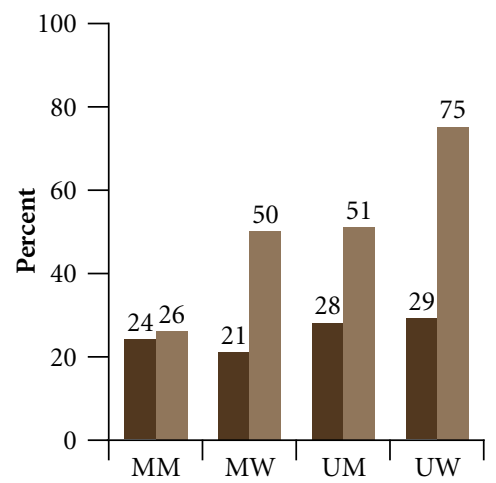

Ever attended a vocational training programme

Interested in participating in a vocational training programme

For example, rural young women were far more likely than their urban counterparts to have received training in tailoring (67\% versus $42 \%)$ and, conversely, less likely to have been trained in computer skills ( $13 \%$ versus $40 \%$ ).

Large proportions of youth-48\% of young men and $63 \%$ of young women-reported interest in attending vocational training programmes, as shown in Table 4.7. Although more unmarried than married youth expressed interest in attending vocational training programmes, it is notable that one quarter of married men (26\%) and

Table 4.7: Willingness of youth to participate in vocational training programmes

Percentage of youth interested in participating in vocational training programmes and type of programme they were interested in participating in, according to residence, Andhra Pradesh, 2007-08

\begin{tabular}{|c|c|c|c|c|c|c|}
\hline Programmes/courses (\%) & $\begin{array}{c}\text { M } \\
15-24\end{array}$ & $\begin{array}{c}\text { W } \\
15-24\end{array}$ & $\begin{array}{c}\text { MM } \\
15-29\end{array}$ & $\begin{array}{l}\text { MW } \\
15-24\end{array}$ & $\begin{array}{c}\text { UM } \\
15-24\end{array}$ & $\begin{array}{l}\text { UW } \\
15-24\end{array}$ \\
\hline \multicolumn{7}{|c|}{ Combined } \\
\hline $\begin{array}{l}\text { Interested in participating in a vocational } \\
\text { training programme }\end{array}$ & 47.6 & 63.2 & 25.8 & 53.1 & 51.9 & 76.4 \\
\hline Number of respondents & 2,479 & 4,848 & 1,405 & 2,330 & 2,077 & 2,518 \\
\hline \multicolumn{7}{|l|}{$\begin{array}{l}\text { Types of programmes in which youth } \\
\text { wished to participate }\end{array}$} \\
\hline Tailoring & 1.3 & 63.6 & 1.7 & 78.0 & 1.4 & 50.8 \\
\hline Auto mechanic/electric work & 18.8 & 0.1 & 34.6 & 0.0 & 16.2 & 0.1 \\
\hline Driving & 23.1 & 0.3 & 43.3 & 0.0 & 20.1 & 0.5 \\
\hline Plumbing/masonry & 4.3 & 0.1 & 12.4 & 0.1 & 3.3 & 0.0 \\
\hline Poultry/goat farm & 0.3 & 0.0 & 3.6 & 0.0 & 0.1 & 0.0 \\
\hline Beauty parlour/salon & 0.4 & 3.3 & 0.8 & 2.8 & 0.4 & 3.6 \\
\hline Nurse's aid & 0.3 & 2.4 & 0.3 & 1.3 & 0.3 & 3.4 \\
\hline Computer training & 62.5 & 31.7 & 23.6 & 10.9 & 68.5 & 50.3 \\
\hline English language/typing/shorthand & 23.9 & 10.9 & 6.1 & 3.0 & 26.9 & 18.0 \\
\hline Handicrafts/painting/embroidery/cooking & 3.3 & 43.9 & 7.2 & 50.8 & 2.8 & 37.6 \\
\hline $\begin{array}{l}\text { Number interested in participating in a vocational } \\
\text { training programme }\end{array}$ & 1,205 & 3,238 & 350 & 1,303 & 1,093 & 1,935 \\
\hline
\end{tabular}


Table 4.7: (Cont'd)

\begin{tabular}{|c|c|c|c|c|c|c|}
\hline Programmes/courses (\%) & $\begin{array}{c}M \\
15-24\end{array}$ & $\begin{array}{c}\text { W } \\
15-24\end{array}$ & $\begin{array}{c}\text { MM } \\
15-29\end{array}$ & $\begin{array}{l}\text { MW } \\
15-24\end{array}$ & $\begin{array}{c}\text { UM } \\
15-24\end{array}$ & $\begin{array}{l}\text { UW } \\
15-24\end{array}$ \\
\hline \multicolumn{7}{|c|}{ Urban } \\
\hline $\begin{array}{l}\text { Interested in participating in a vocational } \\
\text { training programme }\end{array}$ & 50.3 & 71.7 & 24.1 & 62.7 & 54.0 & 79.1 \\
\hline Number of respondents & 1,289 & 2,126 & 648 & 1,037 & 1,132 & 1,089 \\
\hline \multicolumn{7}{|l|}{$\begin{array}{l}\text { Types of programmes in which youth } \\
\text { wished to participate }\end{array}$} \\
\hline Tailoring & 0.3 & 48.6 & 2.7 & 69.6 & 0.3 & 35.0 \\
\hline Auto mechanic/electric work & 13.0 & 0.1 & 20.5 & 0.0 & 11.7 & 0.0 \\
\hline Driving & 15.8 & 0.7 & 28.8 & 0.0 & 15.0 & 1.1 \\
\hline Plumbing/masonry & 3.7 & 0.0 & 8.1 & 0.0 & 3.2 & 0.0 \\
\hline Poultry/goat farm & 0.0 & 0.1 & 2.7 & 0.0 & 0.0 & 0.0 \\
\hline Beauty parlour/salon & 0.3 & 5.5 & 0.0 & 4.9 & 0.3 & 5.8 \\
\hline Nurse's aid & 0.3 & 1.5 & 1.4 & 1.8 & 0.3 & 1.1 \\
\hline Computer training & 70.1 & 47.5 & 35.1 & 21.0 & 73.0 & 64.5 \\
\hline English language/typing/shorthand & 24.5 & 16.6 & 10.8 & 6.4 & 26.1 & 23.4 \\
\hline Handicrafts/painting/embroidery/cooking & 3.1 & 39.0 & 8.1 & 50.8 & 2.6 & 31.5 \\
\hline $\begin{array}{l}\text { Number interested in participating in a vocational } \\
\text { training programme }\end{array}$ & 650 & 1,511 & 154 & 650 & 612 & 861 \\
\hline \multicolumn{7}{|c|}{ Rural } \\
\hline $\begin{array}{l}\text { Interested in participating in a vocational } \\
\text { training programme }\end{array}$ & 46.7 & 59.6 & 26.3 & 50.4 & 50.9 & 74.9 \\
\hline Number of respondents & 1,190 & 2,722 & 757 & 1,293 & 945 & 1,429 \\
\hline \multicolumn{7}{|l|}{$\begin{array}{l}\text { Types of programmes in which youth } \\
\text { wished to participate }\end{array}$} \\
\hline Tailoring & 1.8 & 71.1 & 1.7 & 81.1 & 1.9 & 60.2 \\
\hline Auto mechanic/electric work & 21.3 & 0.1 & 38.3 & 0.0 & 18.3 & 0.2 \\
\hline Driving & 26.2 & 0.0 & 46.9 & 0.0 & 22.7 & 0.1 \\
\hline Plumbing/masonry & 4.5 & 0.1 & 13.8 & 0.1 & 3.4 & 0.0 \\
\hline Poultry/goat farm & 0.4 & 0.0 & 3.8 & 0.0 & 0.1 & 0.0 \\
\hline Beauty parlour/salon & 0.6 & 2.1 & 1.0 & 2.1 & 0.4 & 2.2 \\
\hline Nurse's aid & 0.2 & 2.8 & 0.0 & 1.1 & 0.3 & 4.7 \\
\hline Computer training & 59.3 & 23.7 & 20.7 & 7.2 & 66.3 & 41.7 \\
\hline English language/typing/shorthand & 23.6 & 8.0 & 5.2 & 1.8 & 27.3 & 14.9 \\
\hline Handicrafts/painting/embroidery/cooking & 3.3 & 46.3 & 6.9 & 50.8 & 3.0 & 41.3 \\
\hline $\begin{array}{l}\text { Number interested in participating in a vocational } \\
\text { training programme }\end{array}$ & 555 & 1,727 & 196 & 653 & 481 & 1,074 \\
\hline
\end{tabular}

Note: All Ns are unweighted. Column totals may exceed 100\% due to multiple responses.

half of married women (53\%) were interested in developing vocational skills. Rural-urban differences in interest in attending vocational training programmes were muted among young men. Among young women, those in urban areas were more likely to express interest than their rural counterparts; even so, three-fifths of rural young women did express such an interest. Skills in which youth wished to be trained virtually mirrored the patterns revealed above. The majority of young women continued to wish to be trained in areas such as tailoring and handicrafts, although substantial proportions-particularly those in urban areas-reported a preference for computer training. Young men's preferences, in contrast, were focused on computer training, English language, typing and shorthand skills, auto mechanics or electrical work and driving. 


\subsection{Summary}

Work profiles suggest that three-quarters of young men and three-fifths of young women had at some time engaged in paid or unpaid work. Indeed, almost all married young men and over two-thirds of unmarried young men had done so, compared with over two-thirds and half of married and unmarried young women, respectively. Likewise, more youth in rural than urban areas had ever worked. While the majority of youth were engaged in paid work, considerable proportions of young men and women ( $26 \%$ each) reported unpaid work on the family farm or business. Economic activity was often initiated at an early age: $37 \%$ and $39 \%$ of young men and women, respectively, reported initiating work in childhood or early adolescence (before age 15). Data on work participation in the 12 months prior to the interview indicate that the majority of young men ( $98 \%$ of married and $67 \%$ of unmarried) and a substantial proportion of young women ( $51 \%$ and $44 \%$, respectively) had engaged in paid or unpaid work at some point in the 12 months preceding the survey. The majority of young men (85\%) who worked in the year prior to the interview had done so for the major part (at least six months) of the year. In contrast among young women, $71 \%$ had done so.

Occupational profiles among those working for wages differed widely among youth in rural and urban areas. Among young men in rural areas, leading occupations were agricultural labour, skilled manual labour and unskilled non-agricultural labour, together reported by $86 \%$. Those in urban areas were also likely to report skilled manual labour and unskilled non-agricultural labour, but in addition, many reported administrative, executive, managerial and clerical occupations; almost all men (91\%) reported these occupations. While percentages differed, leading occupations reported by young women resembled those of young men, although considerably more young women than men in rural areas were engaged in agricultural labour (72\% versus $39 \%)$.

Unemployment rates ranged from $7 \%$ among young men to $10 \%$ among young women. Unemployment tended to be higher among the unmarried than the married. While rural-urban differences were negligible among young men, young women in urban areas reported considerably higher rates of unemployment than did their rural counterparts. Unemployment was particularly high among the educated and the economically better off.

Youth were interested in acquiring skills that would enable employment generation; $48 \%$ of young men and $63 \%$ of young women expressed interest in vocational skills training. However, far fewer-just one-third each of young men and women — had attended at least one vocational training programme. 


\section{Media exposure and access to} pornographic materials

Media may play an important role in shaping the attitudes and behaviors of youth. Youth gain access to new information through a variety of sources, including print and visual media and, increasingly, the internet. Many are also exposed to pornography through these channels. The Youth Study probed young people's exposure to various media sources, the extent of their exposure to pornographic materials by way of books/ magazines, films and the internet, and their perceptions about the influence of television and films on youth behaviors.

\subsection{Mass media exposure}

The survey asked a number of questions regarding youth exposure to mass media. These included whether and how frequently young people read newspapers, magazines or books, watched films or television programmes other than movies, and accessed the internet. Questions regarding exposure to print media and the internet were asked only among those who had attained at least five years of education, as this was considered a pre-requisite for basic literacy and, thus, understanding of such materials. Youth were asked to rate the frequency of their exposure to each medium according to the categories "never," "sometimes" and "often". If any young person did not respond in this format but rather, in terms of days per week, three or more exposures per week were classified as "often" and less frequent exposure as "sometimes".

Findings are presented in Table 5.1 and Figure 5.1. They suggest that youth were exposed to a variety of media. While almost all young men and women reported exposure to television (90\% and 94\%, respectively), young men were more likely than young women to report exposure to other media. For example, $94 \%$ of young men, compared to $77 \%$ of young women were exposed to films either on CD/DVD or at a theatre or video parlour and of those who had completed five or more years of education, $94 \%$ of young men and $78 \%$ of young women were exposed to print materials (newspapers, magazines or books). Frequency of exposure to television and print media varied by sex of the respondent; while young women were more likely than young men to report frequent exposure to television ( $45 \%$ versus $27 \%$ ), a reverse pattern was apparent with regard to frequent exposure to print media (17\% versus $38 \%$ ). Exposure to the internet was reported by
Figure 5.1: Percentage of youth exposed to television, films, print media and the internet, Andhra Pradesh, 2007-08
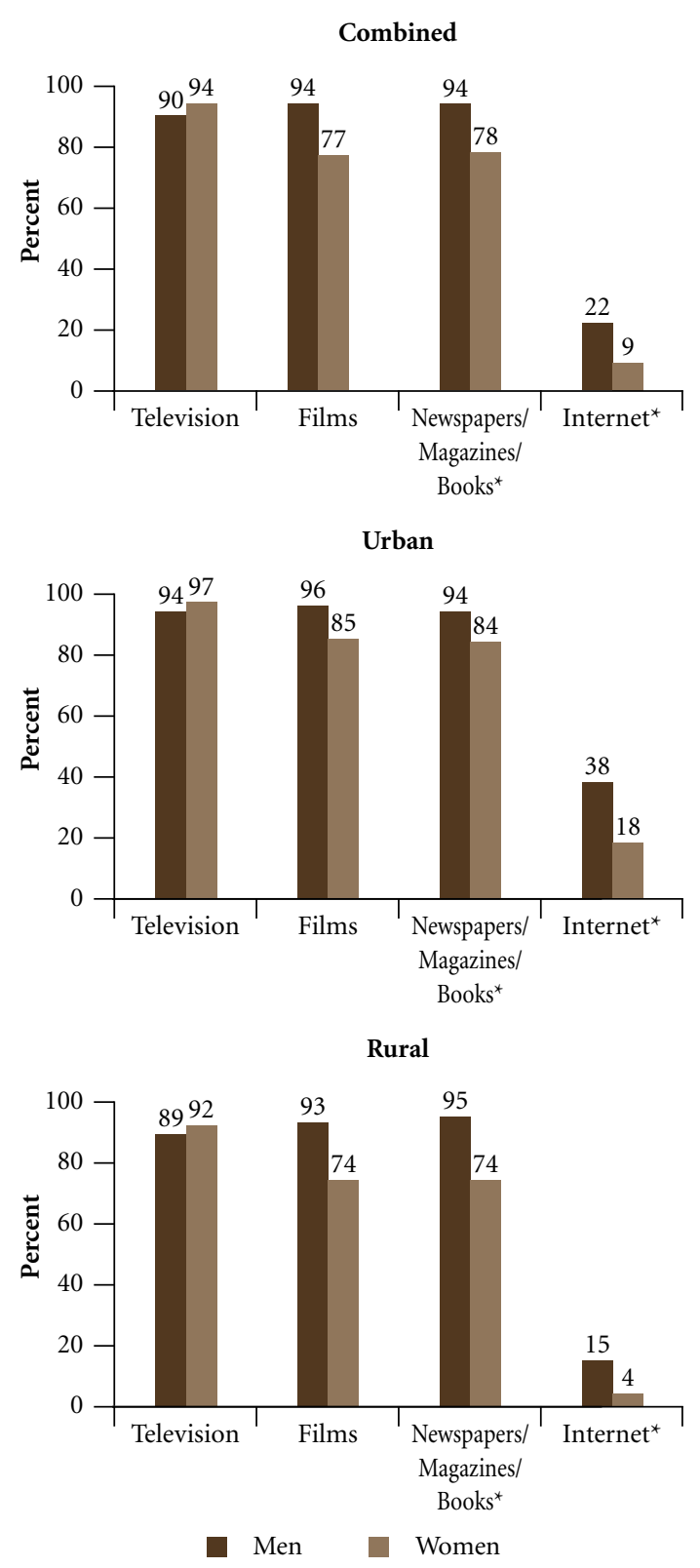

Note: ${ }^{*}$ Question asked only of respondents who had completed five or more years of education. 
Table 5.1: Mass media exposure

Percent distribution of youth exposed to various mass media by frequency of exposure, according to residence, Andhra Pradesh, 2007-08

\begin{tabular}{|c|c|c|c|c|c|c|}
\hline Exposure indicators (\%) & $\begin{array}{c}\text { M } \\
15-24\end{array}$ & $\begin{array}{c}\text { W } \\
15-24\end{array}$ & $\begin{array}{c}\text { MM } \\
15-29\end{array}$ & $\begin{array}{c}\text { MW } \\
15-24\end{array}$ & $\begin{array}{c}\text { UM } \\
15-24\end{array}$ & $\begin{array}{c}\text { UW } \\
15-24\end{array}$ \\
\hline \multicolumn{7}{|c|}{ Combined } \\
\hline \multicolumn{7}{|l|}{ Frequency of watching television } \\
\hline Never & 9.7 & 6.4 & 12.7 & 8.5 & 8.9 & 3.6 \\
\hline Sometimes & 63.7 & 48.9 & 63.5 & 50.1 & 63.5 & 47.7 \\
\hline Often & 26.6 & 44.7 & 23.8 & 41.3 & 27.5 & 48.7 \\
\hline \multicolumn{7}{|l|}{ Frequency of watching films } \\
\hline Never & 6.0 & 22.8 & 7.5 & 24.9 & 5.5 & 20.3 \\
\hline Sometimes & 87.1 & 72.8 & 86.8 & 71.2 & 87.7 & 74.7 \\
\hline Often & 6.8 & 4.4 & 5.6 & 3.9 & 6.8 & 5.0 \\
\hline Number of respondents & 2,479 & 4,848 & 1,405 & 2,330 & 2,077 & 2,518 \\
\hline \multicolumn{7}{|l|}{$\begin{array}{l}\text { Frequency of reading newspapers/magazines/ } \\
\text { books }^{1}\end{array}$} \\
\hline Never & 5.4 & 22.3 & 7.9 & 31.8 & 5.0 & 14.2 \\
\hline Sometimes & 56.6 & 60.3 & 59.4 & 59.9 & 55.3 & 60.9 \\
\hline Often & 37.7 & 17.2 & 32.6 & 8.0 & 39.5 & 24.8 \\
\hline \multicolumn{7}{|l|}{ Frequency of accessing the internet ${ }^{1}$} \\
\hline Never & 77.5 & 91.0 & 90.2 & 96.3 & 75.1 & 86.7 \\
\hline Sometimes & 19.9 & 7.6 & 7.9 & 3.1 & 21.9 & 11.2 \\
\hline Often & 2.2 & 1.3 & 1.5 & 0.4 & 2.6 & 2.0 \\
\hline Number with 5 or more years of education & 2,180 & 3,696 & 993 & 1,464 & 1,904 & 2,232 \\
\hline \multicolumn{7}{|c|}{ Urban } \\
\hline \multicolumn{7}{|l|}{ Frequency of watching television } \\
\hline Never & 5.7 & 2.7 & 9.1 & 3.8 & 4.9 & 1.9 \\
\hline Sometimes & 60.3 & 42.2 & 59.6 & 42.2 & 60.5 & 42.4 \\
\hline Often & 33.9 & 55.0 & 31.3 & 54.0 & 34.4 & 55.7 \\
\hline \multicolumn{7}{|l|}{ Frequency of watching films } \\
\hline Never & 4.4 & 15.3 & 4.9 & 15.2 & 4.4 & 15.3 \\
\hline Sometimes & 88.0 & 79.5 & 90.2 & 79.2 & 87.8 & 79.8 \\
\hline Often & 7.6 & 5.1 & 4.9 & 5.3 & 7.8 & 4.8 \\
\hline Number of respondents & 1,289 & 2,126 & 648 & 1,037 & 1,132 & 1,089 \\
\hline \multicolumn{7}{|l|}{$\begin{array}{l}\text { Frequency of reading newspapers/magazines/ } \\
\text { books }^{1}\end{array}$} \\
\hline Never & 6.1 & 15.8 & 6.4 & 25.7 & 5.6 & 9.2 \\
\hline Sometimes & 54.4 & 56.8 & 59.2 & 61.2 & 54.3 & 53.9 \\
\hline Often & 39.3 & 27.2 & 34.3 & 12.6 & 39.9 & 36.7 \\
\hline \multicolumn{7}{|l|}{ Frequency of accessing the internet ${ }^{1}$} \\
\hline Never & 62.1 & 82.2 & 79.3 & 92.2 & 59.4 & 75.6 \\
\hline Sometimes & 32.0 & 14.6 & 16.8 & 6.0 & 34.3 & 20.3 \\
\hline Often & 5.8 & 2.9 & 3.9 & 1.3 & 6.1 & 4.0 \\
\hline Number with 5 or more years of education & 1,177 & 1,793 & 493 & 781 & 1,061 & 1,012 \\
\hline
\end{tabular}


Table 5.1: (Cont'd)

\begin{tabular}{|c|c|c|c|c|c|c|}
\hline Exposure indicators (\%) & $\begin{array}{c}\text { M } \\
15-24\end{array}$ & $\begin{array}{c}\text { W } \\
15-24\end{array}$ & $\begin{array}{c}\text { MM } \\
15-29\end{array}$ & $\begin{array}{c}\text { MW } \\
15-24\end{array}$ & $\begin{array}{c}\text { UM } \\
15-24\end{array}$ & $\begin{array}{c}\text { UW } \\
\text { 15-24 }\end{array}$ \\
\hline \multicolumn{7}{|c|}{ Rural } \\
\hline Frequency of watching television & & & & & & \\
\hline Never & 11.2 & 7.9 & 13.7 & 9.9 & 10.7 & 4.5 \\
\hline Sometimes & 64.9 & 51.8 & 64.6 & 52.4 & 64.7 & 50.7 \\
\hline Often & 23.8 & 40.4 & 21.8 & 37.6 & 24.6 & 44.8 \\
\hline \multicolumn{7}{|l|}{ Frequency of watching films } \\
\hline Never & 6.6 & 25.9 & 8.2 & 27.7 & 6.0 & 23.0 \\
\hline Sometimes & 86.8 & 70.0 & 85.8 & 68.8 & 87.6 & 71.9 \\
\hline Often & 6.4 & 4.1 & 5.7 & 3.5 & 6.4 & 5.1 \\
\hline Number of respondents & 1,190 & 2,722 & 757 & 1,293 & 945 & 1,429 \\
\hline \multicolumn{7}{|l|}{$\begin{array}{l}\text { Frequency of reading newspapers/magazines/ } \\
\text { books }^{1}\end{array}$} \\
\hline Never & 5.2 & 25.8 & 8.2 & 34.4 & 4.7 & 17.2 \\
\hline Sometimes & 57.6 & 62.3 & 59.5 & 59.5 & 55.8 & 65.2 \\
\hline Often & 36.9 & 11.8 & 32.0 & 6.0 & 39.3 & 17.5 \\
\hline \multicolumn{7}{|l|}{ Frequency of accessing the internet ${ }^{1}$} \\
\hline Never & 84.0 & 95.8 & 93.6 & 98.0 & 82.4 & 93.6 \\
\hline Sometimes & 14.7 & 3.7 & 5.0 & 1.9 & 16.3 & 5.7 \\
\hline Often & 0.7 & 0.3 & 0.7 & 0.0 & 0.9 & 0.7 \\
\hline Number with 5 or more years of education & 1,003 & 1,903 & 500 & 683 & 843 & 1,220 \\
\hline
\end{tabular}

Note: All Ns are unweighted. Column totals may not equal 100\% due to missing cases or "don't know" responses. ${ }^{1}$ Question asked only of respondents who had completed five or more years of education.

relatively few youth and gender differences were wide: more than one in five young men (22\%) and one-tenth of young women $(9 \%)$ had accessed the internet.

Differences were observed by marital status in the case of exposure to print materials and the internet. Unmarried youth were consistently more likely to be exposed to the internet than the married and more unmarried than married young women reported exposure to the print media, irrespective of rural-urban residence. Findings, moreover, suggest that unmarried youth were more likely than married youth to report frequent exposure to television and print media. Differences by rural-urban residence were also evident. Urban youth were considerably more likely to report that they accessed the internet than their rural counterparts: while $38 \%$ of young men and $18 \%$ of young women in urban settings accessed the internet, just $15 \%$ and $4 \%$, respectively, of rural youth had done so. Other differences were apparent only among young women; indeed, considerably more young women in urban than rural areas reported access to print materials and films. Frequency of exposure to mass media varied somewhat by rural-urban residence; urban youth were more likely than their rural counterparts to report frequent exposure to television and the internet and urban young women, in addition, to report frequent exposure to print media.

\subsection{Exposure to pornographic materials}

Youth were asked whether they were exposed to pornographic materials by way of films, books and magazines, and the internet (for those who accessed the internet). Table 5.2 reports that $47 \%$ of young men compared to $1 \%$ of young women had watched "blue" or pornographic films. Differences by marital status were negligible for the overall population; however, more married than unmarried young men were exposed to pornographic films in urban settings (63\% versus 55\%). Differences by rural-urban residence were negligible for young women, but among young men, those in urban areas were more likely than their rural counterparts to have ever watched pornographic films (56\% versus $44 \%)$. 
Table 5.2: Exposure to pornographic materials

Percentage of youth exposed to different pornographic materials, according to residence, Andhra Pradesh, 2007-08

\begin{tabular}{|c|c|c|c|c|c|c|}
\hline Exposure indicators (\%) & $\underset{15-24}{M}$ & $\begin{array}{c}\text { W } \\
15-24\end{array}$ & $\begin{array}{c}\text { MM } \\
15-29\end{array}$ & $\begin{array}{c}\text { MW } \\
15-24\end{array}$ & $\begin{array}{c}\text { UM } \\
15-24\end{array}$ & $\begin{array}{c}\text { UW } \\
\text { 15-24 }\end{array}$ \\
\hline \multicolumn{7}{|c|}{ Combined } \\
\hline Ever watched a "blue"/pornographic film & 47.2 & 1.0 & 47.1 & 1.2 & 46.6 & 0.7 \\
\hline Number of respondents & 2,479 & 4,848 & 1,405 & 2,330 & 2,077 & 2,518 \\
\hline \multicolumn{7}{|l|}{ Frequency of watching "blue"/pornographic films } \\
\hline Rarely & 52.2 & 17.3 & 61.3 & $(0.0)$ & 50.7 & * \\
\hline Sometimes & 46.3 & 76.9 & 38.1 & $(96.6)$ & 47.3 & * \\
\hline Often & 1.4 & 3.8 & 0.6 & $(3.4)$ & 1.8 & * \\
\hline \multicolumn{7}{|l|}{$\begin{array}{l}\text { Person accompanying when watching "blue"/ } \\
\text { pornographic films }\end{array}$} \\
\hline Alone & 2.7 & 11.8 & 3.0 & $(6.9)$ & 2.9 & * \\
\hline $\operatorname{Peer}(\mathrm{s})$ & 93.5 & 15.7 & 92.9 & $(0.0)$ & 93.2 & * \\
\hline Other(s) & 3.8 & 7.8 & 4.1 & (93.1) & 3.9 & * \\
\hline $\begin{array}{l}\text { Ever forced by anyone to watch "blue"/ } \\
\text { pornographic films }\end{array}$ & 15.2 & 12.0 & 12.4 & $(10.3)$ & 15.4 & * \\
\hline $\begin{array}{l}\text { Number who ever watched "blue"/ } \\
\text { pornographic films }\end{array}$ & 1,243 & 56 & 735 & 37 & 1,028 & 19 \\
\hline Ever read/looked at pornographic books/magazines & 31.9 & 3.8 & 25.4 & 3.0 & 32.8 & 4.9 \\
\hline Number of respondents & 2,479 & 4,848 & 1,405 & 2,330 & 2,077 & 2,518 \\
\hline \multicolumn{7}{|l|}{$\begin{array}{l}\text { Frequency of reading/looking at pornographic } \\
\text { books/magazines }\end{array}$} \\
\hline Rarely & 41.4 & 56.7 & 44.0 & 57.1 & 42.2 & 56.0 \\
\hline Sometimes & 57.6 & 42.2 & 55.2 & 41.4 & 56.7 & 42.4 \\
\hline Often & 1.0 & 0.5 & 0.8 & 1.4 & 1.0 & 0.8 \\
\hline $\begin{array}{l}\text { Number who ever read/looked at pornographic } \\
\text { books/magazines }\end{array}$ & 833 & 205 & 403 & 79 & 715 & 126 \\
\hline Ever accessed pornographic materials on the internet & 50.4 & 7.2 & 53.9 & 6.4 & 51.0 & 7.5 \\
\hline Number who ever accessed the internet & 599 & 389 & 131 & 69 & 570 & 320 \\
\hline \multicolumn{7}{|c|}{ Urban } \\
\hline Ever watched a "blue"/pornographic film & 56.2 & 1.4 & 63.4 & 2.3 & 55.0 & 0.7 \\
\hline Number of respondents & 1,289 & 2,126 & 648 & 1,037 & 1,132 & 1,089 \\
\hline Frequency of watching "blue"/pornographic films & & & & & & \\
\hline Rarely & 50.9 & $(9.5)$ & 64.4 & $(0.0)$ & 49.9 & * \\
\hline Sometimes & 48.6 & $(85.7)$ & 35.1 & $(100.0)$ & 49.6 & * \\
\hline Often & 0.5 & $(0.0)$ & 0.5 & $(0.0)$ & 0.6 & * \\
\hline \multicolumn{7}{|l|}{$\begin{array}{l}\text { Person accompanying when watching "blue"/ } \\
\text { pornographic films }\end{array}$} \\
\hline Alone & 2.8 & $(5.0)$ & 5.2 & $(8.3)$ & 2.9 & * \\
\hline Peer(s) & 94.5 & $(10.0)$ & 91.8 & $(0.0)$ & 94.5 & * \\
\hline Other(s) & 2.8 & $(5.0)$ & 3.1 & $(91.7)$ & 2.6 & * \\
\hline $\begin{array}{l}\text { Ever forced by anyone to watch "blue"/ } \\
\text { pornographic films }\end{array}$ & 11.8 & $(19.0)$ & 8.8 & $(16.7)$ & 12.4 & * \\
\hline $\begin{array}{l}\text { Number who ever watched "blue"/ } \\
\text { pornographic films }\end{array}$ & 723 & 33 & 410 & 25 & 622 & 8 \\
\hline
\end{tabular}


Table 5.2: (Cont'd)

\begin{tabular}{|c|c|c|c|c|c|c|}
\hline Exposure indicators (\%) & $\underset{15-24}{M}$ & $\begin{array}{c}W \\
15-24\end{array}$ & $\begin{array}{c}\text { MM } \\
15-29\end{array}$ & $\begin{array}{c}\text { MW } \\
15-24\end{array}$ & $\begin{array}{c}\text { UM } \\
15-24\end{array}$ & $\begin{array}{c}\text { UW } \\
15-24\end{array}$ \\
\hline \multicolumn{7}{|c|}{ Urban } \\
\hline Ever read/looked at pornographic books/magazines & 37.4 & 4.7 & 36.3 & 4.2 & 37.6 & 5.1 \\
\hline Number of respondents & 1,289 & 2,126 & 648 & 1,037 & 1,132 & 1,089 \\
\hline \multicolumn{7}{|l|}{$\begin{array}{l}\text { Frequency of reading/looking at pornographic } \\
\text { books/magazines }\end{array}$} \\
\hline Rarely & 44.3 & 54.4 & 54.5 & $(52.2)$ & 44.3 & 55.3 \\
\hline Sometimes & 54.5 & 42.6 & 45.5 & $(43.5)$ & 54.9 & 40.4 \\
\hline Often & 1.1 & 1.5 & 0.0 & $(4.3)$ & 0.8 & 2.1 \\
\hline $\begin{array}{l}\text { Number who ever read/looked at pornographic } \\
\text { books/magazines }\end{array}$ & 478 & 101 & 232 & 44 & 423 & 57 \\
\hline Ever accessed pornographic materials on the internet & 55.6 & 6.5 & 60.4 & 10.3 & 56.9 & 5.8 \\
\hline Number who ever accessed the internet & 445 & 299 & 103 & 56 & 427 & 243 \\
\hline \multicolumn{7}{|c|}{ Rural } \\
\hline Ever watched a "blue"/pornographic film & 43.6 & 0.8 & 42.5 & 0.9 & 42.9 & 0.7 \\
\hline Number of respondents & 1,190 & 2,722 & 757 & 1,293 & 945 & 1,429 \\
\hline Frequency of watching "blue"/pornographic films & & & & & & \\
\hline Rarely & 52.8 & * & 60.0 & * & 51.2 & * \\
\hline Sometimes & 45.2 & * & 39.3 & * & 46.1 & * \\
\hline Often & 1.8 & * & 0.6 & * & 2.4 & * \\
\hline \multicolumn{7}{|l|}{$\begin{array}{l}\text { Person accompanying when watching "blue"/ } \\
\text { pornographic films }\end{array}$} \\
\hline Alone & 2.8 & * & 2.1 & * & 2.9 & * \\
\hline Peer(s) & 92.9 & * & 93.4 & * & 92.4 & * \\
\hline Other(s) & 4.3 & * & 4.5 & * & 4.7 & * \\
\hline $\begin{array}{l}\text { Ever forced by anyone to watch "blue"/ } \\
\text { pornographic films }\end{array}$ & 16.9 & * & 13.9 & * & 17.1 & * \\
\hline $\begin{array}{l}\text { Number who ever watched "blue"/ } \\
\text { pornographic films }\end{array}$ & 520 & 23 & 325 & 12 & 406 & 11 \\
\hline Ever read/looked at pornographic books/magazines & 29.7 & 3.5 & 22.4 & 2.7 & 30.8 & 4.8 \\
\hline Number of respondents & 1,190 & 2,722 & 757 & 1,293 & 945 & 1,429 \\
\hline \multicolumn{7}{|l|}{$\begin{array}{l}\text { Frequency of reading/looking at pornographic } \\
\text { books/magazines }\end{array}$} \\
\hline Rarely & 40.0 & 58.0 & 39.1 & $(59.6)$ & 41.3 & 56.4 \\
\hline Sometimes & 59.1 & 42.0 & 59.7 & $(40.4)$ & 57.5 & 43.6 \\
\hline Often & 0.9 & 0.0 & 1.2 & $(0.0)$ & 1.1 & 0.0 \\
\hline $\begin{array}{l}\text { Number who ever read/looked at pornographic } \\
\text { books/magazines }\end{array}$ & 355 & 104 & 171 & 35 & 292 & 69 \\
\hline Ever accessed pornographic materials on the internet & 45.1 & 8.8 & $(46.3)$ & * & 44.6 & 11.5 \\
\hline Number who ever accessed the internet & 154 & 90 & 28 & 13 & 143 & 77 \\
\hline
\end{tabular}

Note: All Ns are unweighted. Column totals may not equal 100\% due to missing cases or "don't know" responses. ( ) Based on 25-49 unweighted cases. ${ }^{*}$ Percentage not shown, based on fewer than 25 unweighted cases. 
Among young men who had ever watched a pornographic film, about half (48\%) reported having viewed such films sometimes or frequently (see Table 5.2). Marital status differences were evident with more unmarried than married young men reporting having watched such films sometimes or often (49\% versus 39\%). Rural-urban differences were negligible.

For the most part, young men had watched films together with friends. A small minority (15\%) reported that they had been pressured or forced, at least once, to do so.

Exposure to pornographic books and magazines was reported by 32\% of young men and $4 \%$ of young women. While marital status and rural-urban differences were negligible among young women, somewhat more unmarried than married young men (33\% versus 25\%) and more young men from urban than rural areas (37\% versus $30 \%)$ reported exposure to pornographic books and magazines. Three-fifths of young men and two-fifths of young women who reported reading or looking at pornographic books or magazines did so sometimes or often.

Of those exposed to the internet, half of young men (50\%) had accessed pornographic materials on the internet; this compared with $7 \%$ of young women. Differences by marital status were negligible. Differences by rural-urban residence were negligible among young women, however, considerably more young men in urban than rural areas reported having accessed pornographic materials on the internet (56\% and $45 \%$, respectively).

\subsection{Youth perceptions about the influence of television and films on youth behaviours}

The survey also questioned youth about their perceptions of the influence of television and films on youth behaviours. Specifically, they were asked whether they believed that television and films influenced the way in which their friends dressed, whether violence on television and in films could make youth aggressive and whether they had ever felt like having sex after watching certain films. Table 5.3 suggests that two-thirds of young men and women

Table 5.3: Perceptions about the influence of television and films on youth behaviours

Percentage of youth reporting perceptions regarding the influence of television and films on youth behaviours, according to residence, Andhra Pradesh, 2007-08

\begin{tabular}{|c|c|c|c|c|c|c|}
\hline $\begin{array}{l}\text { Perceptions about the influence of } \\
\text { television and films (\%) }\end{array}$ & $\begin{array}{c}M \\
15-24\end{array}$ & $\begin{array}{c}W \\
15-24\end{array}$ & $\begin{array}{c}\text { MM } \\
15-29\end{array}$ & $\begin{array}{l}\text { MW } \\
15-24\end{array}$ & $\begin{array}{l}\text { UM } \\
15-24\end{array}$ & $\begin{array}{l}\text { UW } \\
15-24\end{array}$ \\
\hline \multicolumn{7}{|c|}{ Combined } \\
\hline TV/films influence the way friends dress & 68.3 & 67.8 & 60.4 & 65.4 & 68.7 & 71.0 \\
\hline Violence on TV and in films can make youth aggressive & 60.3 & 75.3 & 58.4 & 74.2 & 61.2 & 76.7 \\
\hline Certain films make respondent want to have sex & 34.3 & 11.4 & 29.7 & 18.0 & 34.8 & 2.6 \\
\hline Number of respondents & 2,479 & 4,848 & 1,405 & 2,330 & 2,077 & 2,518 \\
\hline \multicolumn{7}{|c|}{ Urban } \\
\hline TV/films influence the way friends dress & 70.4 & 71.7 & 59.8 & 70.1 & 70.8 & 73.0 \\
\hline Violence on TV and in films can make youth aggressive & 65.6 & 79.4 & 61.3 & 81.5 & 65.8 & 77.6 \\
\hline Certain films make respondent want to have sex & 35.6 & 9.6 & 37.1 & 19.0 & 34.8 & 1.8 \\
\hline Number of respondents & 1,289 & 2,126 & 648 & 1,037 & 1,132 & 1,089 \\
\hline \multicolumn{7}{|c|}{ Rural } \\
\hline TV/films influence the way friends dress & 67.5 & 66.2 & 60.5 & 64.0 & 67.7 & 69.8 \\
\hline Violence on TV and in films can make youth aggressive & 58.3 & 73.6 & 57.6 & 72.1 & 59.2 & 76.1 \\
\hline Certain films make respondent want to have sex & 33.9 & 12.2 & 27.5 & 17.7 & 34.8 & 3.1 \\
\hline Number of respondents & 1,190 & 2,722 & 757 & 1,293 & 945 & 1,429 \\
\hline
\end{tabular}

Note: All Ns are unweighted. 
(68\% each) believed that television and films influenced the way their friends dressed. Three-fifths of young men and three-quarters of young women believed that violence on television and in films could make youth aggressive. One-third $(34 \%)$ of young men and one-tenth $(11 \%)$ of young women reported that watching certain films had made them desire sex. Clearly, considerable proportions of youth felt that television and films influenced their friends', and in the case of young men their own, behaviours.

Differences in reported perceptions by marital status were narrow, although unmarried youth were somewhat more likely than others to acknowledge the influence of television and films on the way youth dress. Married young women, moreover, were more likely than unmarried young women to report that watching certain films had made them desire sex. Differences by rural-urban residence were, likewise, narrow, although urban youth were somewhat more likely than rural youth to report that violence on television and in films could make youth aggressive (66\% versus $58 \%$ among urban and rural young men; $79 \%$ versus $74 \%$, respectively, among young women) and urban young women were more likely than their rural counterparts to report that TV/films influenced the way their friends dressed $(72 \%$ versus $66 \%)$.

\subsection{Summary}

Findings suggest that almost all youth in Andhra Pradesh were exposed to the media, typically television ( $90 \%$ of all young men and $94 \%$ of all young women), newspapers, magazines or books ( $94 \%$ of young men and $78 \%$ of young women with five or more years of education) and films either on CD/DVD or at a theatre or video parlour ( $94 \%$ of young men and $77 \%$ of young women). Exposure to the internet was reported by many fewer ( $22 \%$ of young men and $9 \%$ of young women with five or more years of education). Gender differences were apparent with regard to exposure to the print media, to films and to the internet, with young men considerably more likely than young women to report exposure.

Findings also suggest that almost half of young men and hardly any young women had watched pornographic or "blue" films, and one-third of young men and $4 \%$ of young women had read or looked at pornographic books and magazines. Finally, half of young men (and 7\% of young women) who had accessed the internet reported that they had accessed pornographic materials on the internet. At least half of young men who reported watching pornographic films or reading pornographic materials reported that they accessed these materials sometimes or frequently. Finally, between three-fifths and three-quarters of young men and women acknowledged the influence that media have on youth behaviours. 


\section{Growing up}

This chapter focuses on such experiences as puberty as well as youth interaction with parents and peers while growing up. Globally, studies have suggested a declining age at puberty for young men and women and stress that this, along with rising age at marriage, provides a longer window in which young people can make same- and opposite-sex friends (National Research Council and Institute of Medicine, 2005). Several studies have highlighted the importance of close parental interaction for the healthy development of young people (Laird et al., 2003; Marta, 1997; Sroufe, 1991). Others note that young people's interaction with parents is particularly limited when it comes to discussion of sensitive issues, for example, girl-boy relations or sexual and reproductive matters (Alexander et al., 2006a; 2006b; Lambert and Wood, 2005; Mehra, Savithri and Coutinho, 2002). In addition, a few studies have shown that the peer group is, for many youth, a central source of both information and support, but at the same time, a source of misinformation and pressure to adopt risky behaviours (Bhuiya et al., 2003; Sachdev, 1998; Ul Haque and Faizunnisa, 2003).

The Youth Study included several questions relating to each of these issues. This chapter begins by describing the age at which young people experienced signs of puberty. It then explores aspects of their family life and interaction with parents on various matters of importance to youth. It also addresses peer networks and interaction, specifically, the size of the same- and opposite-sex peer network and peer activities in which respondents engaged. Finally, the chapter discusses young people's access to support networks for discussing personal matters.

\subsection{Puberty}

In order to examine age at which puberty occurs among young men and women, the Youth Study included questions on age at menarche for young women and age at which voice change and growth of pubic hair were noticed for young men. Table 6.1a shows that mean age at menarche was 12.9 years for young women, irrespective of rural-urban residence.

Voice change and appearance of pubic hair for young men occurred 2 to 2.5 years later than did menarche for young women. Table $6.1 \mathrm{~b}$ shows that the average age at which young men reported voice change was 15.1 years, and growth of pubic hair was 15.7 years, with those in rural and urban areas reporting similar mean age at which these changes occurred.

\subsection{Family life and interaction with parents}

The Youth Study explored a variety of issues that capture the nature of family life, and youth interaction with parents in particular. Married respondents were specifically asked to recall the period before marriage.

\subsubsection{Socialisation experiences}

Table 6.2 presents findings on the socialisation experiences of youth during their teenage years as compared with siblings, or cousins of the opposite sex if the respondent did not have an opposite-sex sibling. Wide gender differences were evident in relation to respondents' freedom to go out as well as housework expectations. For example, three-quarters of young men (75\%) reported that they had more freedom to go out than their sisters or female 
Table 6.1a: Age at puberty among young women

Percent distribution of young women aged 15-24 by age at puberty, according to residence, Andhra Pradesh, 2007-08

\begin{tabular}{|c|c|c|c|}
\hline Puberty indicators (\%) & Combined & Urban & Rural \\
\hline Age at menarche (years) & & & \\
\hline Below 12 & 2.8 & 4.1 & 2.3 \\
\hline 12 & 31.7 & 33.3 & 31.1 \\
\hline 13 & 45.5 & 41.2 & 47.3 \\
\hline 14 & 12.6 & 14.3 & 11.9 \\
\hline 15 and above & 6.0 & 6.4 & 5.8 \\
\hline Not yet menstruated & 0.3 & 0.2 & 0.4 \\
\hline Mean age at menarche (years) ${ }^{1}$ & 12.9 & 12.9 & 12.9 \\
\hline Number of respondents & 4,848 & 2,126 & 2,722 \\
\hline
\end{tabular}

Note: All Ns are unweighted. Column totals may not equal 100\% due to missing cases or "don't know" responses. ${ }^{1}$ Excludes those who had not menstruated at the time of the interview.

Table 6.1b: Age at puberty among young men

Percent distribution of young men aged 15-24 by age at puberty, according to residence, Andhra Pradesh, 2007-08

\begin{tabular}{|c|c|c|c|}
\hline Puberty indicators (\%) & Combined & Urban & Rural \\
\hline \multicolumn{4}{|l|}{ Age at which voice change noticed (years) } \\
\hline Below 14 & 10.0 & 9.1 & 10.4 \\
\hline 14 & 15.3 & 16.1 & 15.0 \\
\hline 15 & 29.1 & 30.6 & 28.6 \\
\hline 16 & 17.1 & 20.3 & 15.8 \\
\hline 17 and above & 9.6 & 8.9 & 9.8 \\
\hline No voice change yet & 3.6 & 2.4 & 4.0 \\
\hline Did not notice/don't remember & 15.3 & 12.6 & 16.4 \\
\hline Mean age at voice change (years) ${ }^{1}$ & 15.1 & 15.1 & 15.0 \\
\hline \multicolumn{4}{|l|}{ Age at which pubic hair noticed (years) } \\
\hline Below 14 & 1.6 & 1.3 & 1.7 \\
\hline 14 & 10.5 & 10.6 & 10.4 \\
\hline 15 & 32.7 & 36.5 & 31.2 \\
\hline 16 & 25.5 & 28.5 & 24.3 \\
\hline 17 and above & 18.3 & 15.2 & 19.6 \\
\hline No pubic hair yet & 3.7 & 2.8 & 4.1 \\
\hline Did not notice/don't remember & 7.7 & 5.1 & 8.8 \\
\hline Mean age at which pubic hair noticed ${ }^{1}$ & 15.7 & 15.6 & 15.7 \\
\hline Number of respondents & 2,479 & 1,289 & 1,190 \\
\hline
\end{tabular}

Note: All Ns are unweighted. Column totals may not equal 100\% due to missing cases or "don't know" responses. ${ }^{1}$ Excludes those who had not noticed voice changelappearance of pubic hair at the time of the interview or did not remember age at the time of voice changelappearance of pubic hair.

cousins did; only two-fifths (40\%) of young women, in contrast, agreed that they had less freedom to go out than their brothers or male cousins did. Likewise, two-thirds of young men (66\%) reported that they were expected to do less housework than their sisters or female cousins, and only two-fifths of young women (41\%) agreed that they were expected to do more housework than their brothers or male cousins. In other words, young men and women perceived their socialization experiences somewhat differently, with young men far more likely than young women to acknowledge that their parents discriminated between their sons and daughters. Even so, it is notable that many 
households in Andhra Pradesh did not discriminate between their sons and daughters in terms of freedom of movement and expectations regarding housework ( $23 \%-32 \%$ by young men's assessment and $57 \%-58 \%$ by young women's assessment).

Differences by marital status and rural-urban residence were muted among young men. Among young women, in contrast, differences were observed. For example, the unmarried, particularly those in urban settings, were more likely than the married to report gender egalitarian socialisation experiences. Likewise, young women in urban areas were more likely than their rural counterparts to report gender egalitarian socialisation experiences (see Figure 6.1). These findings may be explained by the likelihood that rural respondents and those who married early belonged to families adhering more closely to traditional gender norms than the families of other young women.

\section{Table 6.2: Socialisation experiences}

Percent distribution of youth by degree of mobility and housework responsibilities relative to an opposite-sex sibling/cousin, according to residence, Andhra Pradesh, 2007-08

\begin{tabular}{|c|c|c|c|c|c|c|}
\hline Socialisation experiences (\%) & $\begin{array}{c}M \\
15-24\end{array}$ & $\begin{array}{c}\text { W } \\
15-24\end{array}$ & $\begin{array}{c}\text { MM } \\
15-29\end{array}$ & $\begin{array}{c}\text { MW } \\
15-24\end{array}$ & $\begin{array}{c}\text { UM } \\
15-24\end{array}$ & $\begin{array}{c}\text { UW } \\
15-24\end{array}$ \\
\hline \multicolumn{7}{|c|}{ Combined } \\
\hline \multicolumn{7}{|c|}{$\begin{array}{l}\text { Respondent had less freedom }(\mathrm{W}) / \text { more freedom }(\mathrm{M}) \text { to } \\
\text { roam/go out than opposite-sex sibling or cousin }\end{array}$} \\
\hline Yes & 75.4 & 40.1 & 75.2 & 44.0 & 76.6 & 35.1 \\
\hline No & 22.7 & 57.9 & 22.8 & 54.1 & 21.7 & 62.7 \\
\hline \multicolumn{7}{|c|}{$\begin{array}{l}\text { Respondent was expected to do more housework }(\mathrm{W}) / \text { less } \\
\text { housework (M) than opposite-sex sibling or cousin }\end{array}$} \\
\hline Yes & 65.9 & 41.3 & 65.1 & 45.7 & 67.0 & 35.7 \\
\hline No & 32.3 & 56.9 & 33.1 & 52.5 & 31.4 & 62.3 \\
\hline Number of respondents & 2,479 & 4,848 & 1,405 & 2,330 & 2,077 & 2,518 \\
\hline \multicolumn{7}{|c|}{ Urban } \\
\hline \multicolumn{7}{|c|}{$\begin{array}{l}\text { Respondent had less freedom }(\mathrm{W}) / \text { more freedom }(\mathrm{M}) \text { to } \\
\text { roam/go out than opposite-sex sibling or cousin }\end{array}$} \\
\hline Yes & 79.6 & 35.0 & 82.1 & 41.7 & 79.7 & 29.6 \\
\hline No & 18.9 & 63.5 & 16.3 & 56.8 & 18.7 & 69.0 \\
\hline \multicolumn{7}{|c|}{$\begin{array}{l}\text { Respondent was expected to do more housework (W)/less } \\
\text { housework (M) than opposite-sex sibling or cousin }\end{array}$} \\
\hline Yes & 68.7 & 32.7 & 70.0 & 39.3 & 69.4 & 27.4 \\
\hline No & 29.9 & 65.9 & 28.3 & 59.2 & 29.2 & 71.4 \\
\hline Number of respondents & 1,289 & 2,126 & 648 & 1,037 & 1,132 & 1,089 \\
\hline \multicolumn{7}{|c|}{ Rural } \\
\hline \multicolumn{7}{|c|}{$\begin{array}{l}\text { Respondent had less freedom }(\mathrm{W}) / \text { more freedom }(\mathrm{M}) \text { to } \\
\text { roam/go out than opposite-sex sibling or cousin }\end{array}$} \\
\hline Yes & 73.8 & 42.2 & 73.2 & 44.7 & 75.1 & 38.2 \\
\hline No & 24.2 & 55.5 & 24.7 & 53.3 & 22.9 & 59.2 \\
\hline \multicolumn{7}{|c|}{$\begin{array}{l}\text { Respondent was expected to do more housework }(\mathrm{W}) / \text { less } \\
\text { housework (M) than opposite-sex sibling or cousin }\end{array}$} \\
\hline Yes & 64.9 & 44.9 & 63.7 & 47.6 & 65.9 & 40.5 \\
\hline No & 33.3 & 53.1 & 34.5 & 50.6 & 32.4 & 57.2 \\
\hline Number of respondents & 1,190 & 2,722 & 757 & 1,293 & 945 & 1,429 \\
\hline
\end{tabular}

Note: All Ns are unweighted. Column totals may not equal 100\% due to "unsure" responses. For married respondents, questions referred to the period prior to marriage. 
Parental attitudes towards youth friendships and social activities were probed by asking young men and women about whether their mother and father, respectively, would disapprove if they engaged in a series of activities, ranging from bringing a same-sex friend to their home to having a love marriage. Married youth were asked to respond according to their experience prior to marriage. Findings, presented in Table 6.3, suggest considerable variation in youth perceptions by activity. What is clearly noticeable is that parents were most likely to be perceived to disapprove of love marriages for their children. This was reported by $67-69 \%$ of young men and $93 \%$ of young women. Also noticeable is that youth perceived their parents to be far more likely to disapprove of activities conducted with members of the opposite sex than those conducted with same-sex individuals (see Figure 6.2). For example, just $5-7 \%$ of young men and $2-5 \%$ of young women reported that their mother or father would be angry if they brought same-sex friends to their home. In contrast, fathers and mothers of $44-61 \%$ of young men and fathers and mothers of $70-85 \%$ of young women were perceived to disapprove of activities such as bringing an opposite-sex friend to their home, talking to a person of the opposite sex who did not belong to the family and going to a mela or film with someone of the opposite sex. As evident from the findings discussed above, young women were far more likely than young men to perceive that their mother or father would disapprove of love marriages and activities conducted with members of the opposite sex.

Also evident from Table 6.3 is that even though interactions with same-sex friends were less likely to meet with disapproval, about $19-20 \%$ of young men and $17-23 \%$ of young women reported expecting parental disapproval if they went out with same-sex friends to a mela or film. Likewise, as many as $28 \%$ of young men and $48-59 \%$ of young women reported expecting parental disapproval if they joined a club or mandal. By and large, differences in perceived reactions of fathers versus mothers were negligible, although somewhat larger proportions of young women perceived disapproval from their father than their mother of activities such as talking to a person of the opposite sex who did not belong to the family, going to a mela or film with same-sex friends and joining a club or mandal. For instance, $59 \%$ and $48 \%$ of young women reported expecting disapproval from their father and mother, respectively, if they joined a club or mandal.

Differences by marital status were narrow, except that married young women were somewhat more likely than the unmarried to report that their mother would disapprove of activities such as bringing an opposite-sex friend to their home, talking to a person of the opposite sex who did not belong to the family and going to a mela or film with someone of the opposite sex. Differences by rural-urban residence were mild; even so, it was rural youth who were somewhat more likely than their urban counterparts to report parental disapproval.
Figure 6.1: Percentage of youth reporting gendered socialisation experiences relative to an opposite-sex sibling/cousin, according to residence, Andhra Pradesh, 2007-08
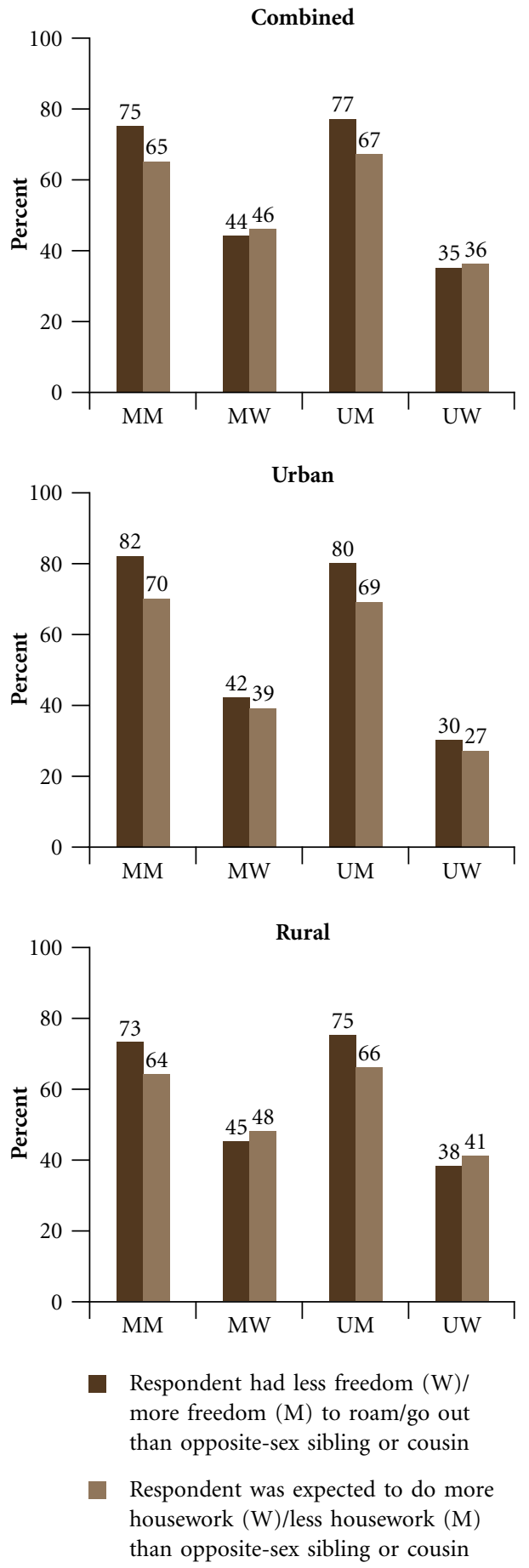

Note: For married respondents, questions referred to the period prior to marriage. 
Table 6.3: Perceptions of parental reactions to selected activities

Percentage of youth who perceived that their parents would disapprove of their engaging in selected activities, according to residence, Andhra Pradesh, 2007-08

\begin{tabular}{|c|c|c|c|c|c|c|}
\hline \multirow[t]{2}{*}{ Perceptions of parental reactions (\%) } & $\begin{array}{c}M \\
15-24\end{array}$ & $\begin{array}{c}W \\
15-24\end{array}$ & $\begin{array}{c}\text { MM } \\
15-29\end{array}$ & $\begin{array}{c}\text { MW } \\
15-24\end{array}$ & $\begin{array}{c}\text { UM } \\
15-24\end{array}$ & $\begin{array}{c}\text { UW } \\
15-24\end{array}$ \\
\hline & \multicolumn{6}{|c|}{ Father } \\
\hline \multicolumn{7}{|c|}{ Combined } \\
\hline \multicolumn{7}{|l|}{ Father would disapprove if respondent: } \\
\hline Brought same-sex friends home & 6.8 & 4.7 & 6.3 & 5.6 & 6.7 & 3.6 \\
\hline Brought opposite-sex friends home & 59.6 & 84.6 & 62.3 & 86.0 & 59.7 & 83.0 \\
\hline $\begin{array}{l}\text { Talked to a person of the opposite sex from outside } \\
\text { the home }\end{array}$ & 46.5 & 77.2 & 52.1 & 79.2 & 45.9 & 74.7 \\
\hline Went to a mela/film with same-sex friends & 19.2 & 23.2 & 18.9 & 23.8 & 18.4 & 22.5 \\
\hline Went to a mela/film with opposite-sex friends & 53.9 & 81.6 & 55.7 & 83.2 & 53.8 & 79.7 \\
\hline Joined a club or mandal & 28.1 & 58.9 & 27.2 & 59.6 & 29.0 & 58.2 \\
\hline Had a love marriage & 68.5 & 93.2 & 69.4 & 93.9 & 68.7 & 92.3 \\
\hline Found a job & NA & 9.7 & NA & 10.9 & NA & 8.3 \\
\hline Number of respondents ${ }^{1}$ & 2,115 & 4,096 & 1,045 & 1,885 & 1,787 & 2,211 \\
\hline \multicolumn{7}{|c|}{ Urban } \\
\hline \multicolumn{7}{|l|}{ Father would disapprove if respondent: } \\
\hline Brought same-sex friends home & 6.7 & 3.8 & 6.4 & 4.8 & 6.6 & 3.1 \\
\hline Brought opposite-sex friends home & 57.8 & 81.4 & 65.0 & 85.6 & 57.2 & 78.4 \\
\hline $\begin{array}{l}\text { Talked to a person of the opposite sex from outside } \\
\text { the home }\end{array}$ & 43.5 & 75.5 & 53.9 & 80.3 & 43.3 & 71.9 \\
\hline Went to a mela/film with same-sex friends & 15.6 & 21.1 & 15.1 & 21.9 & 15.0 & 20.7 \\
\hline Went to a mela/film with opposite-sex friends & 50.0 & 77.7 & 50.7 & 82.9 & 50.5 & 73.8 \\
\hline Joined a club or mandal & 28.2 & 58.9 & 26.5 & 60.8 & 29.0 & 57.6 \\
\hline Had a love marriage & 69.9 & 92.2 & 75.0 & 93.8 & 70.1 & 91.1 \\
\hline Found a job & NA & 9.3 & NA & 10.3 & NA & 8.5 \\
\hline Number of respondents ${ }^{1}$ & 1,090 & 1,777 & 464 & 822 & 972 & 955 \\
\hline \multicolumn{7}{|c|}{ Rural } \\
\hline Father would disapprove if respondent: & & & & & & \\
\hline Brought same-sex friends home & 6.8 & 5.0 & 6.3 & 5.8 & 6.7 & 3.8 \\
\hline Brought opposite-sex friends home & 60.4 & 85.9 & 61.6 & 86.1 & 60.8 & 85.6 \\
\hline $\begin{array}{l}\text { Talked to a person of the opposite sex from outside } \\
\text { the home }\end{array}$ & 47.6 & 77.9 & 51.6 & 78.9 & 47.1 & 76.3 \\
\hline Went to a mela/film with same-sex friends & 20.7 & 24.0 & 19.8 & 24.3 & 19.9 & 23.6 \\
\hline Went to a mela/film with opposite-sex friends & 55.4 & 83.2 & 57.0 & 83.3 & 55.2 & 83.0 \\
\hline Joined a club or mandal & 28.2 & 58.9 & 27.4 & 59.3 & 29.0 & 58.4 \\
\hline Had a love marriage & 67.9 & 93.6 & 67.9 & 93.9 & 68.2 & 92.9 \\
\hline Found a job & NA & 9.8 & NA & 11.0 & NA & 8.1 \\
\hline Number of respondents ${ }^{1}$ & 1,025 & 2,319 & 581 & 1,063 & 815 & 1,256 \\
\hline
\end{tabular}

Cont'd on next page... 
Table 6.3: (Cont'd)

\begin{tabular}{|c|c|c|c|c|c|c|}
\hline \multirow[t]{2}{*}{ Perceptions of parental reactions (\%) } & $\begin{array}{c}\mathrm{M} \\
15-24\end{array}$ & $\begin{array}{c}\text { W } \\
15-24\end{array}$ & $\begin{array}{c}\text { MM } \\
15-29\end{array}$ & $\begin{array}{c}\text { MW } \\
15-24\end{array}$ & $\begin{array}{c}\text { UM } \\
15-24\end{array}$ & $\begin{array}{c}\text { UW } \\
15-24\end{array}$ \\
\hline & \multicolumn{6}{|c|}{ Mother } \\
\hline \multicolumn{7}{|c|}{ Combined } \\
\hline \multicolumn{7}{|l|}{ Mother would disapprove if respondent: } \\
\hline Brought same-sex friends home & 5.4 & 1.6 & 5.8 & 2.0 & 5.1 & 1.3 \\
\hline Brought opposite-sex friends home & 60.5 & 80.7 & 60.0 & 83.5 & 60.9 & 77.1 \\
\hline $\begin{array}{l}\text { Talked to a person of the opposite sex from outside } \\
\text { the home }\end{array}$ & 44.0 & 69.7 & 44.5 & 72.9 & 44.2 & 65.7 \\
\hline Went to a mela/film with same-sex friends & 19.7 & 16.9 & 18.1 & 17.5 & 19.8 & 16.1 \\
\hline Went to a mela/film with opposite-sex friends & 51.3 & 77.6 & 48.6 & 80.5 & 51.5 & 73.9 \\
\hline Joined a club or mandal & 28.2 & 48.4 & 25.8 & 47.9 & 28.4 & 48.9 \\
\hline Had a love marriage & 67.3 & 93.0 & 67.7 & 93.8 & 66.8 & 92.0 \\
\hline Found a job & NA & 4.7 & NA & 5.8 & NA & 3.4 \\
\hline Number of respondents ${ }^{1}$ & 2,389 & 4,575 & 1,274 & 2,147 & 2,012 & 2,428 \\
\hline \multicolumn{7}{|c|}{ Urban } \\
\hline \multicolumn{7}{|l|}{ Mother would disapprove if respondent: } \\
\hline Brought same-sex friends home & 5.2 & 1.4 & 6.3 & 1.9 & 4.8 & 1.0 \\
\hline Brought opposite-sex friends home & 57.4 & 77.3 & 60.5 & 82.5 & 58.3 & 73.3 \\
\hline $\begin{array}{l}\text { Talked to a person of the opposite sex from outside } \\
\text { the home }\end{array}$ & 41.6 & 67.5 & 45.6 & 72.6 & 40.8 & 63.7 \\
\hline Went to a mela/film with same-sex friends & 15.1 & 16.4 & 15.4 & 17.7 & 14.4 & 15.4 \\
\hline Went to a mela/film with opposite-sex friends & 46.2 & 74.2 & 44.6 & 79.6 & 46.8 & 70.0 \\
\hline Joined a club or mandal & 29.0 & 50.5 & 24.0 & 50.7 & 29.7 & 50.3 \\
\hline Had a love marriage & 67.3 & 92.0 & 71.2 & 94.7 & 67.2 & 90.1 \\
\hline Found a job & NA & 5.3 & NA & 6.6 & NA & 4.4 \\
\hline Number of respondents ${ }^{1}$ & 1,236 & 2,026 & 575 & 960 & 1,095 & 1,066 \\
\hline \multicolumn{7}{|c|}{ Rural } \\
\hline Mother would disapprove if respondent: & & & & & & \\
\hline Brought same-sex friends home & 5.5 & 1.7 & 5.7 & 1.9 & 5.3 & 1.4 \\
\hline Brought opposite-sex friends home & 61.7 & 82.1 & 59.8 & 83.8 & 62.0 & 79.4 \\
\hline $\begin{array}{l}\text { Talked to a person of the opposite sex from outside } \\
\text { the home }\end{array}$ & 45.0 & 70.6 & 44.2 & 73.0 & 45.7 & 66.8 \\
\hline Went to a mela/film with same-sex friends & 21.6 & 17.1 & 18.9 & 17.5 & 22.1 & 16.5 \\
\hline Went to a mela/film with opposite-sex friends & 53.2 & 79.1 & 49.7 & 80.8 & 53.5 & 76.3 \\
\hline Joined a club or mandal & 27.9 & 47.5 & 26.3 & 47.0 & 27.8 & 48.1 \\
\hline Had a love marriage & 67.3 & 93.4 & 66.9 & 93.7 & 66.6 & 93.1 \\
\hline Found a job & NA & 4.5 & NA & 5.6 & NA & 2.8 \\
\hline Number of respondents ${ }^{1}$ & 1,153 & 2,549 & 699 & 1,187 & 917 & 1,362 \\
\hline
\end{tabular}

Note: All Ns are unweighted. NA: Not applicable. For married respondents, questions referred to the period prior to marriage. ${ }^{1}$ Includes only those respondents reporting that their father or mother, respectively, was alive at the time of the interview. 
Figure 6.2: Percentage of youth reporting that their father or mother, respectively, would disapprove if they brought same- and opposite-sex friends home, Andhra Pradesh, 2007-08

Father would disapprove

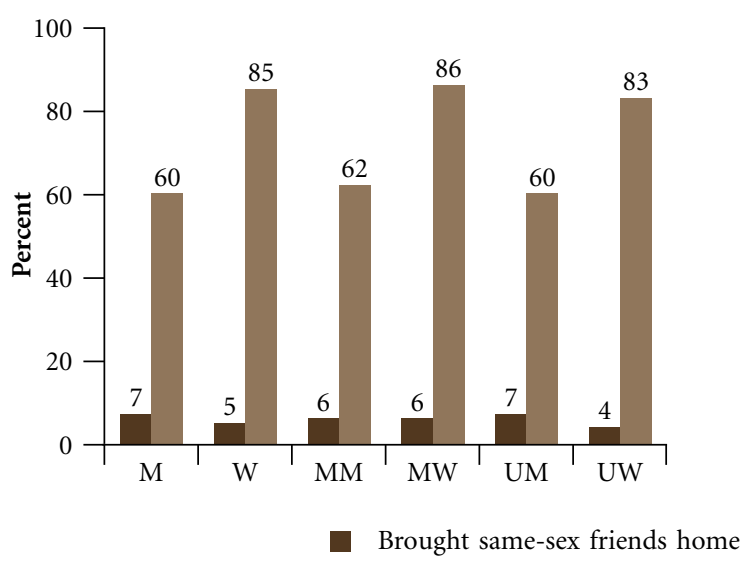

Mother would disapprove

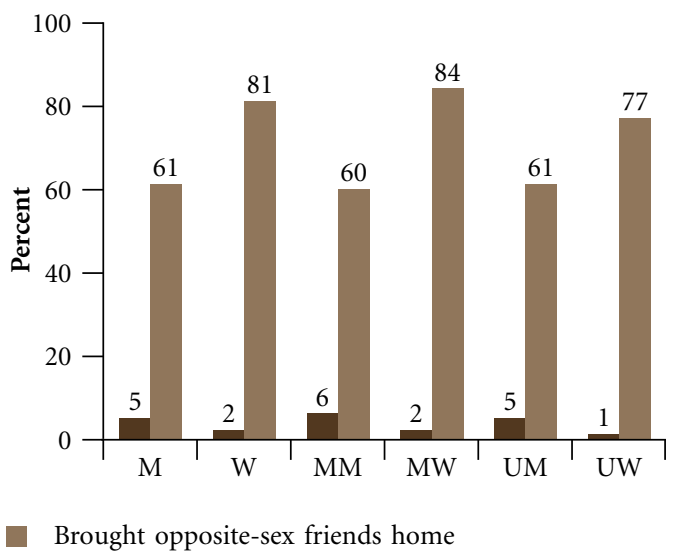

Note: For married respondents, questions referred to the period prior to marriage. Percentages were calculated only of those respondents reporting that their father or mother, respectively, was alive at the time of the interview.

Youth were also asked about the extent to which family life was characterised by quarrels and domestic violence between parents, and whether they had witnessed their father beating their mother or vice versa. Findings are reported in Table 6.4. They suggest that about half of young men and two-thirds of young women with both parents living acknowledged that they had ever witnessed quarrels between their parents. About $2-3 \%$ reported that they had witnessed their mother beating their father. Considerably larger proportions-over one-third-reported ever witnessing their father beating their mother. Differences by marital status indicate that more married than unmarried youth reported witnessing their father beating their mother (43\% versus 35\% among young men and $40 \%$ versus $32 \%$ among young women). Rural-urban differences were muted among young men; but among young women, those in rural areas were considerably more likely than the urban to have witnessed their father beating their mother (39\% versus $28 \%)$.

Youth were also asked whether one or both parents had ever beaten them since the age of 12. Findings, shown in Table 6.4, suggest that sizeable proportions of youth with at least one parent alive at the time of the interview reported being beaten by a parent at any time since the age of 12 . Gender differences were evident, with young men twice as likely as young women to have experienced beatings ( $48 \%$ compared to $23 \%$ ). No clear differences were observed by marital status or rural-urban residence.

\subsubsection{Communication with parents}

Information regarding communication with parents on issues relevant to youth-such as school performance, friendships, romantic relationships, being teased or bullied, physical maturation, reproductive processes and contraception-was elicited from all respondents reporting that their mother or father was alive at the time of the interview. Findings, presented in Table 6.5 and Figures $6.3 \mathrm{a}-\mathrm{b}$, reveal that communication on any topic was far from universal. In general, among all groups but particularly young men, sensitive topics—such as romantic relationships, reproduction and contraception-were rarely discussed with either parent. 
Table 6.4: Experience of domestic violence

Percentage of youth reporting violence between parents and being beaten by parents, according to residence, Andhra Pradesh, 2007-08

\begin{tabular}{|c|c|c|c|c|c|c|}
\hline Experiences of domestic violence (\%) & $\begin{array}{c}M \\
15-24\end{array}$ & $\begin{array}{c}\text { W } \\
15-24\end{array}$ & $\begin{array}{c}\text { MM } \\
15-29\end{array}$ & $\begin{array}{l}\text { MW } \\
15-24\end{array}$ & $\begin{array}{c}\text { UM } \\
15-24\end{array}$ & $\begin{array}{l}\text { UW } \\
15-24\end{array}$ \\
\hline \multicolumn{7}{|c|}{ Combined } \\
\hline Parents ever fought & 48.5 & 65.3 & 52.7 & 66.7 & 47.1 & 63.6 \\
\hline Mother ever beat father & 3.2 & 2.0 & 4.1 & 2.1 & 3.1 & 1.8 \\
\hline Father ever beat mother & 36.6 & 35.8 & 42.8 & 39.7 & 34.6 & 31.5 \\
\hline Number with both parents alive & 2,057 & 3,926 & 973 & 1,774 & 1,744 & 2,152 \\
\hline $\begin{array}{l}\text { Respondent beaten by father and/or mother } \\
\text { since age } 12\end{array}$ & 48.2 & 22.5 & 50.6 & 21.8 & 47.6 & 23.3 \\
\hline Number with at least one parent alive & 2,447 & 4,745 & 1,346 & 2,258 & 2,055 & 2,487 \\
\hline \multicolumn{7}{|c|}{ Urban } \\
\hline Parents ever fought & 47.9 & 62.0 & 56.9 & 66.5 & 46.2 & 58.8 \\
\hline Mother ever beat father & 2.4 & 1.5 & 3.0 & 1.5 & 2.1 & 1.5 \\
\hline Father ever beat mother & 35.7 & 27.7 & 44.1 & 32.0 & 33.8 & 24.7 \\
\hline Number with both parents alive & 1,056 & 1,720 & 427 & 779 & 947 & 941 \\
\hline $\begin{array}{l}\text { Respondent beaten by father and/or mother } \\
\text { since age } 12\end{array}$ & 46.0 & 25.6 & 50.2 & 25.0 & 44.6 & 26.1 \\
\hline Number with at least one parent alive & 1,270 & 2,083 & 612 & 1,003 & 1,120 & 1,080 \\
\hline \multicolumn{7}{|c|}{ Rural } \\
\hline Parents ever fought & 48.7 & 66.6 & 51.6 & 66.8 & 47.5 & 66.4 \\
\hline Mother ever beat father & 3.5 & 2.1 & 4.4 & 2.2 & 3.4 & 1.9 \\
\hline Father ever beat mother & 36.9 & 39.3 & 42.5 & 41.9 & 35.0 & 35.5 \\
\hline Number with both parents alive & 1,001 & 2,206 & 546 & 995 & 797 & 1,211 \\
\hline $\begin{array}{l}\text { Respondent beaten by father and/or mother } \\
\text { since age } 12\end{array}$ & 49.0 & 21.2 & 50.7 & 20.8 & 48.9 & 21.7 \\
\hline Number with at least one parent alive & 1,177 & 2,662 & 734 & 1,255 & 935 & 1,407 \\
\hline
\end{tabular}

Note: All Ns are unweighted. Domestic violence refers exclusively to physical violence.

Topics most likely to be discussed with fathers were schooling and friendships: about two-thirds of young men and women reported discussing schooling (67\% and 63\%, respectively) and half reported discussing friendships ( $49 \%$ and $52 \%$, respectively) with their father. About one-fifth of young men and two-fifths of young women reported discussing being teased/bullied with their father. While one-tenth of young men discussed adolescent body changes, one percent or less reported discussing such topics as romantic relationships, reproduction and contraception with their father. Among young women, very few- $5 \%$ or less_-reported having discussed any topic aside from schooling, friendships and being teased/ bullied with their father.

As far as discussion with mothers was concerned, young women were considerably more likely than young men to have discussed all seven topics with their mother. Topics that young men and women were most likely to have discussed with their mother were identical to those discussed with their father, described above: most youth reported discussing schooling ( $62 \%$ of young men and $68 \%$ of young women) and friendships ( $57 \%$ and $84 \%$, respectively) 
Table 6.5: Parental communication

Percentage of youth who discussed selected matters with parents, according to residence, Andhra Pradesh, 2007-08

\begin{tabular}{|c|c|c|c|c|c|c|c|c|c|c|c|c|}
\hline \multirow[t]{2}{*}{$\begin{array}{l}\text { Issues } \\
\text { discussed (\%) }\end{array}$} & $\begin{array}{c}\mathrm{M} \\
15-24 \\
\end{array}$ & $\begin{array}{c}\text { W } \\
15-24 \\
\end{array}$ & $\begin{array}{c}\text { MM } \\
15-29 \\
\end{array}$ & $\begin{array}{c}\text { MW } \\
15-24 \\
\end{array}$ & $\begin{array}{c}\text { UM } \\
15-24 \\
\end{array}$ & $\begin{array}{c}\text { UW } \\
15-24 \\
\end{array}$ & $\begin{array}{c}M \\
15-24 \\
\end{array}$ & $\begin{array}{c}\mathrm{W} \\
15-24 \\
\end{array}$ & $\begin{array}{c}\text { MM } \\
15-29 \\
\end{array}$ & $\begin{array}{c}\text { MW } \\
15-24 \\
\end{array}$ & $\begin{array}{c}\text { UM } \\
15-24 \\
\end{array}$ & $\begin{array}{c}\text { UW } \\
15-24 \\
\end{array}$ \\
\hline & \multicolumn{6}{|c|}{ Father } & \multicolumn{6}{|c|}{ Mother } \\
\hline \multicolumn{13}{|c|}{ Combined } \\
\hline School performance & 66.5 & 62.6 & 49.1 & 52.7 & 70.9 & 74.5 & 62.3 & 68.1 & 45.8 & 57.9 & 66.2 & 80.6 \\
\hline Friendships & 49.4 & 51.7 & 36.2 & 46.1 & 53.1 & 58.3 & 56.5 & 84.2 & 43.4 & 79.6 & 59.2 & 89.8 \\
\hline Romantic relationships & 1.4 & 0.2 & 1.4 & 0.1 & 1.3 & 0.3 & 0.9 & 4.6 & 0.7 & 6.1 & 1.0 & 2.7 \\
\hline Being teased/bullied & 18.6 & 42.1 & 19.0 & 41.0 & 18.8 & 43.2 & 17.7 & 62.6 & 17.3 & 58.0 & 17.1 & 68.4 \\
\hline Adolescent body changes & 11.0 & 4.7 & 10.8 & 4.8 & 10.8 & 4.5 & 12.1 & 83.3 & 11.4 & 81.8 & 11.6 & 85.2 \\
\hline Reproductive processes & 0.3 & 2.7 & 0.8 & 4.7 & 0.2 & 0.2 & 1.3 & 11.5 & 1.7 & 16.9 & 1.2 & 4.7 \\
\hline Contraception & 0.1 & 3.4 & 0.1 & 6.2 & 0.2 & 0.1 & 0.2 & 12.4 & 0.5 & 18.6 & 0.1 & 4.6 \\
\hline Number of respondents ${ }^{1}$ & 2,115 & 4,096 & 1,045 & 1,885 & 1,787 & 2,211 & 2,389 & 4,575 & 1,274 & 2,147 & 2,012 & 2,428 \\
\hline \multicolumn{13}{|c|}{ Urban } \\
\hline School performance & 74.7 & 73.8 & 57.1 & 65.1 & 77.3 & 80.1 & 67.0 & 81.0 & 52.4 & 73.3 & 69.8 & 86.9 \\
\hline Friendships & 56.0 & 59.1 & 42.0 & 52.3 & 58.4 & 64.2 & 60.3 & 89.9 & 50.7 & 86.2 & 62.7 & 92.7 \\
\hline Romantic relationships & 1.3 & 0.2 & 0.9 & 0.0 & 1.3 & 0.4 & 1.3 & 4.2 & 1.5 & 5.3 & 1.1 & 3.4 \\
\hline Being teased/bullied & 19.8 & 43.4 & 18.2 & 38.0 & 19.8 & 47.3 & 18.2 & 68.6 & 18.0 & 64.4 & 17.7 & 71.8 \\
\hline Adolescent body changes & 11.6 & 3.7 & 10.5 & 2.9 & 11.1 & 4.4 & 11.8 & 83.7 & 10.3 & 80.7 & 11.0 & 86.1 \\
\hline Reproductive processes & 0.7 & 1.2 & 0.5 & 2.4 & 0.6 & 0.3 & 2.4 & 9.5 & 3.3 & 14.6 & 1.8 & 5.6 \\
\hline Contraception & 0.3 & 1.2 & 0.5 & 2.9 & 0.4 & 0.1 & 0.3 & 10.1 & 0.7 & 16.0 & 0.3 & 5.4 \\
\hline Number of respondents ${ }^{1}$ & 1,090 & 1,777 & 464 & 822 & 972 & 955 & 1,236 & 2,026 & 575 & 960 & 1,095 & 1,066 \\
\hline \multicolumn{13}{|c|}{ Rural } \\
\hline School performance & 63.3 & 58.0 & 47.0 & 49.3 & 68.2 & 71.4 & 60.5 & 62.5 & 44.1 & 53.3 & 64.7 & 77.0 \\
\hline Friendships & 46.8 & 48.6 & 34.7 & 44.4 & 50.7 & 55.0 & 55.0 & 81.7 & 41.5 & 77.6 & 57.7 & 88.0 \\
\hline Romantic relationships & 1.4 & 0.1 & 1.5 & 0.1 & 1.4 & 0.2 & 0.8 & 4.7 & 0.6 & 6.3 & 0.9 & 2.4 \\
\hline Being teased/bullied & 18.2 & 41.5 & 19.3 & 41.9 & 18.3 & 40.9 & 17.4 & 60.1 & 17.2 & 56.1 & 16.9 & 66.3 \\
\hline Adolescent body changes & 10.8 & 5.0 & 10.9 & 5.3 & 10.7 & 4.6 & 12.2 & 83.1 & 11.7 & 82.1 & 11.8 & 84.7 \\
\hline Reproductive processes & 0.2 & 3.4 & 0.8 & 5.4 & 0.0 & 0.2 & 0.9 & 12.3 & 1.3 & 17.6 & 0.9 & 4.1 \\
\hline Contraception & 0.1 & 4.3 & 0.0 & 7.1 & 0.2 & 0.1 & 0.1 & 13.4 & 0.5 & 19.3 & 0.0 & 4.2 \\
\hline Number of respondents ${ }^{1}$ & 1,025 & 2,319 & 581 & 1,063 & 815 & 1,256 & 1,153 & 2,549 & 699 & 1,187 & 917 & 1,362 \\
\hline
\end{tabular}

Note: All Ns are unweighted. For married respondents questions referred to the period prior to marriage. ${ }^{1}$ Includes only those respondents reporting that their father or mother, respectively, was alive at the time of the interview.

with their mother. On such topics as adolescent body changes and being teased, far more young women than men reported discussion with their mother. For example, while $83 \%$ of young women had discussed adolescent body changes and $63 \%$ had discussed being bullied or teased with their mother, only $12-18 \%$ of young men had done so. Few youth reported discussing such topics as romantic relationships, reproduction and contraception with their mother and gender differences were mild: only $5-12 \%$ of young women and one percent or less of young men had discussed these with their mother.

Findings suggest that while young men were about as likely to discuss each issue with their mother as their father, with the exception of friendship, young women were far more likely to discuss all matters with their mother than with their father. 
Figure 6.3a: Percentage of youth who discussed various matters with their father, according to residence, Andhra Pradesh, 2007-08
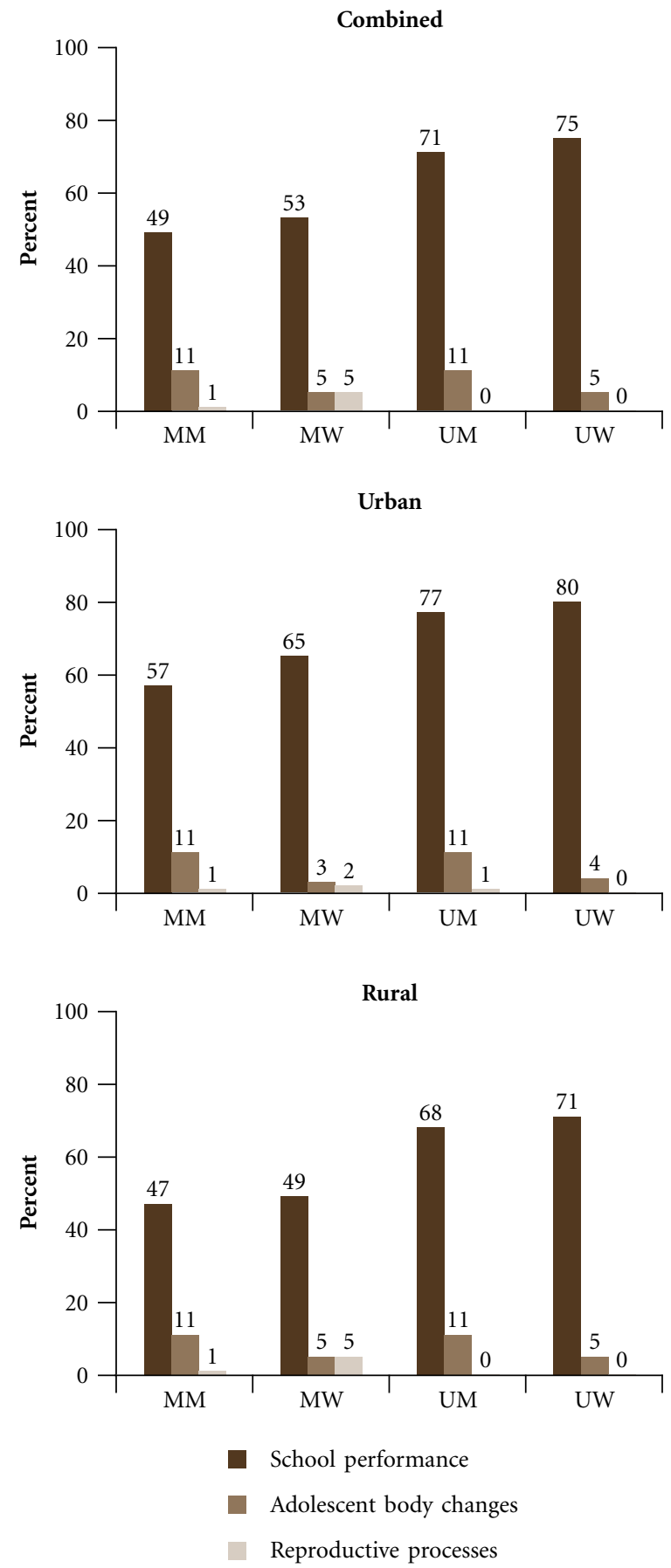

Note: For married respondents, questions referred to the period prior to marriage. Percentages were calculated only of those respondents reporting that their father was alive at the time of the interview.
Figure 6.3b: Percentage of youth who discussed various matters with their mother, according to residence, Andhra Pradesh, 2007-08
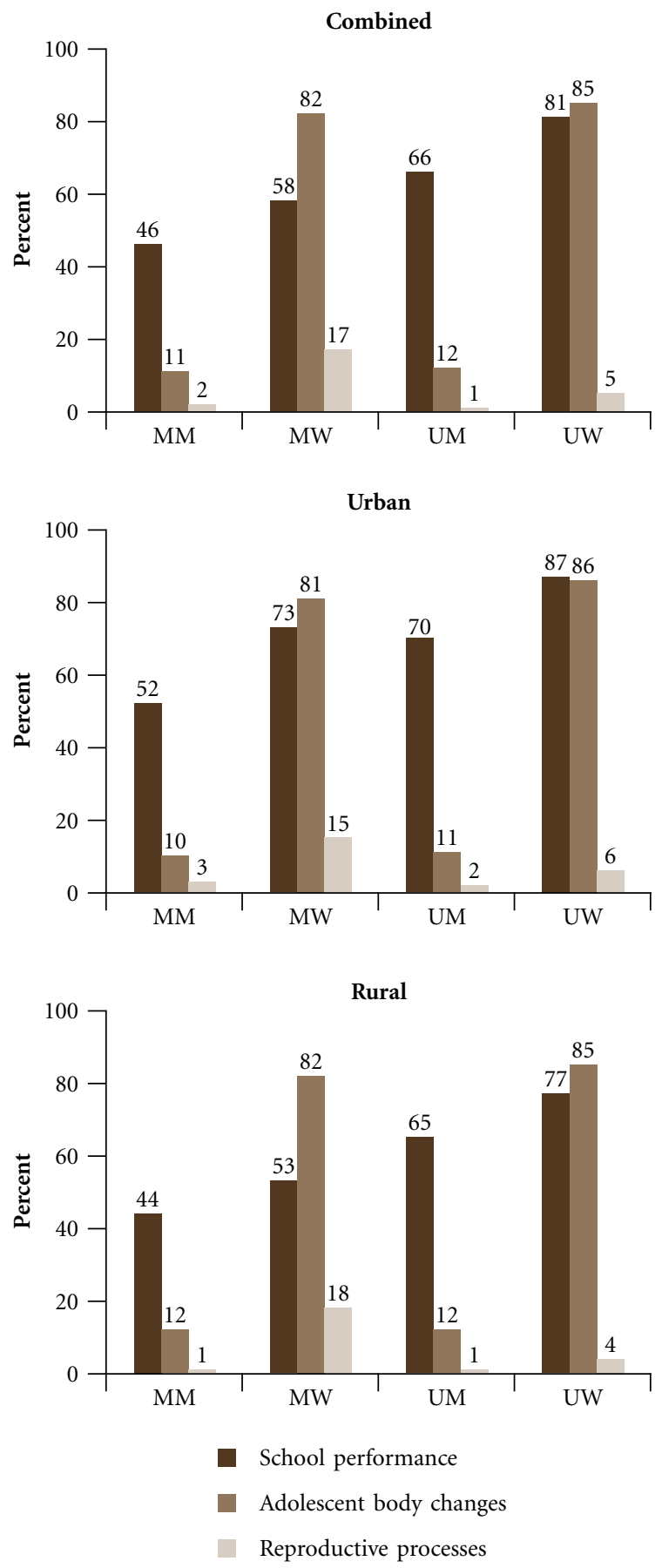

Note: For married respondents, questions referred to the period prior to marriage. Percentages were calculated only of those respondents reporting that their mother was alive at the time of the interview. 
Differences by marital status suggest that unmarried youth were more likely than the married to have discussed such topics as school performance and friendships with their father or mother. Additionally, unmarried young women were more likely than married women to have discussed being teased/bullied with their mother. In contrast, married women were more likely than unmarried women to share such sensitive topics as reproduction and contraception with their parents, particularly their mother. Differences by rural-urban residence suggest greater openness between a parent and youth in urban areas compared to those in rural areas on non-sensitive topics-schooling and friendships. Additionally, urban young women were more likely than their rural counterparts to have discussed being teased/ bullied with their mother.

\subsection{Peer networks and interaction}

In order to assess the size of peer networks and the nature of peer interaction, the Youth Study asked young people about the number of same-sex friends they had, whether they had opposite-sex friends and the kinds of activities in which they engaged with their same- and opposite-sex friends. Married respondents were asked to recall the situation prior to marriage.

Table 6.6 reports findings on the size of peer networks. Same-sex peer networks of young men were typically wider than those of young women. The proportion of youth reporting five or more same-sex friends ranged from $58 \%$ among young men to $25 \%$ among young women, and the median number of same-sex friends reported by young men exceeded that reported by young women by two (5 versus 3 ). Unmarried young men reported more same-sex friends - a median of one additional friend-than their married counterparts; however, among young women, no differences were evident by marital status. In terms of rural-urban differences, the median number of same-sex friends reported by young men in urban settings (6) exceeded, by one, the median number reported by those in rural settings (5). In the case of young women, however, no differences were observed (all reported a median number of three friends).

Opposite-sex peer networks were reported by sizeable proportions of young people and gender differences were apparent. Young men were twice as likely as young women to report having at least one opposite-sex friend (31\% compared to $15 \%)$. The unmarried were, likewise, more likely than the married to report an opposite-sex friend $-33 \%$ and $22 \%$, respectively, among young men, and $23 \%$ and $10 \%$, respectively, among young women (see Figure 6.4). Finally, rural young men and women were considerably less likely than their urban counterparts to report an opposite-sex friend.

Table 6.7 reports the nature of interaction with same- and opposite-sex friends. Again, the married were asked to recall the situation prior to marriage. Respondents were asked if they went on picnics or to films with their peers, studied together, spent time chatting, engaged in sporting activities or drank or gambled with their friends.

The activity in which almost all young people engaged was chatting with their same-sex friends. Also, over $70 \%$ of young men and women reported studying together with their same-sex friends. Significant gender differences were evident in other forms of interaction: young men were considerably more likely than young women to report going on picnics or to films (92\% versus $61 \%$ ) or engaging in sports or outdoor games ( $84 \%$ versus $63 \%$ ) with same-sex friends. It is notable that one in four young men (26\%) reported drinking or gambling with their same-sex friends; very few young women so reported $(1 \%)$.

Among young men and women, the unmarried were more likely than the married to report studying with their same-sex friends (77\% versus 51\% among young men and $85 \%$ versus 59\% among young women), presumably a function of the higher levels of school attainment of the unmarried, on the one hand, and the relatively curtailed adolescent experience of the married, on the other. Additionally among young men, the unmarried were more likely to report engaging in sports ( $88 \%$ versus $65 \%$ ), and conversely, the married were more likely than the unmarried to report drinking or gambling with their same-sex friends (47\% versus $23 \%$ ). The patterns remained by and large similar in both urban and rural settings. Rural-urban differences were generally mild, with some exceptions. For example, urban young men were somewhat more likely than their rural counterparts to report drinking or gambling 
Table 6.6: Size of peer networks

Percent distribution of youth by number of same- and opposite-sex friends, according to residence, Andhra Pradesh, 2007-08

\begin{tabular}{|c|c|c|c|c|c|c|}
\hline Number of friends (\%) & $\underset{15-24}{M}$ & $\begin{array}{c}\text { W } \\
15-24\end{array}$ & $\begin{array}{c}\text { MM } \\
15-29\end{array}$ & $\begin{array}{l}\text { MW } \\
15-24\end{array}$ & $\begin{array}{l}\text { UM } \\
15-24\end{array}$ & $\begin{array}{l}\text { UW } \\
15-24\end{array}$ \\
\hline \multicolumn{7}{|c|}{ Combined } \\
\hline \multicolumn{7}{|l|}{ Number of same-sex friends } \\
\hline None & 0.8 & 2.1 & 1.4 & 3.0 & 0.6 & 0.9 \\
\hline 1 & 3.6 & 13.4 & 6.3 & 14.5 & 2.8 & 12.1 \\
\hline 2 & 14.6 & 28.7 & 17.7 & 30.6 & 13.1 & 26.3 \\
\hline 3 & 12.9 & 19.1 & 14.5 & 19.3 & 13.1 & 18.8 \\
\hline 4 & 10.1 & 11.8 & 11.5 & 11.5 & 9.8 & 12.0 \\
\hline 5 or more & 57.9 & 24.9 & 48.6 & 21.1 & 60.5 & 29.8 \\
\hline Median number of same-sex friends & 5.0 & 3.0 & 4.0 & 3.0 & 5.0 & 3.0 \\
\hline At least one opposite-sex friend (\%) & 31.4 & 15.3 & 21.9 & 9.7 & 33.1 & 22.6 \\
\hline Number of respondents & 2,479 & 4,848 & 1,405 & 2,330 & 2,077 & 2,518 \\
\hline \multicolumn{7}{|c|}{ Urban } \\
\hline \multicolumn{7}{|l|}{ Number of same-sex friends } \\
\hline None & 0.3 & 1.6 & 0.3 & 2.7 & 0.2 & 0.9 \\
\hline 1 & 2.5 & 12.1 & 3.9 & 12.0 & 2.2 & 12.1 \\
\hline 2 & 10.9 & 26.2 & 14.7 & 26.7 & 10.1 & 25.9 \\
\hline 3 & 12.0 & 20.8 & 12.4 & 22.5 & 12.7 & 19.3 \\
\hline 4 & 9.5 & 12.6 & 11.4 & 13.5 & 9.2 & 11.8 \\
\hline 5 or more & 64.7 & 26.8 & 57.2 & 22.7 & 65.6 & 30.1 \\
\hline Median number of same-sex friends & 6.0 & 3.0 & 5.0 & 3.0 & 6.0 & 3.0 \\
\hline At least one opposite-sex friend (\%) & 37.2 & 21.5 & 24.8 & 12.0 & 38.1 & 29.0 \\
\hline Number of respondents & 1,289 & 2,126 & 648 & 1,037 & 1,132 & 1,089 \\
\hline \multicolumn{7}{|c|}{ Rural } \\
\hline \multicolumn{7}{|l|}{ Number of same-sex friends } \\
\hline None & 1.1 & 2.3 & 1.6 & 3.1 & 0.8 & 0.9 \\
\hline 1 & 4.1 & 14.0 & 7.0 & 15.2 & 3.1 & 12.2 \\
\hline 2 & 16.1 & 29.8 & 18.5 & 31.8 & 14.5 & 26.5 \\
\hline 3 & 13.3 & 18.4 & 15.1 & 18.3 & 13.3 & 18.5 \\
\hline 4 & 10.3 & 11.4 & 11.6 & 11.0 & 10.0 & 12.2 \\
\hline 5 or more & 55.2 & 24.1 & 46.2 & 20.7 & 58.4 & 29.6 \\
\hline Median number of same-sex friends & 5.0 & 3.0 & 4.0 & 2.0 & 5.0 & 3.0 \\
\hline At least one opposite-sex friend (\%) & 29.1 & 12.7 & 21.1 & 8.9 & 31.0 & 18.9 \\
\hline Number of respondents & 1,190 & 2,722 & 757 & 1,293 & 945 & 1,429 \\
\hline
\end{tabular}

Note: All Ns are unweighted. For married respondents, questions referred to the period prior to marriage. 
Figure 6.4: Percentage of youth reporting at least one opposite-sex friend, according to residence, Andhra Pradesh, 2007-08
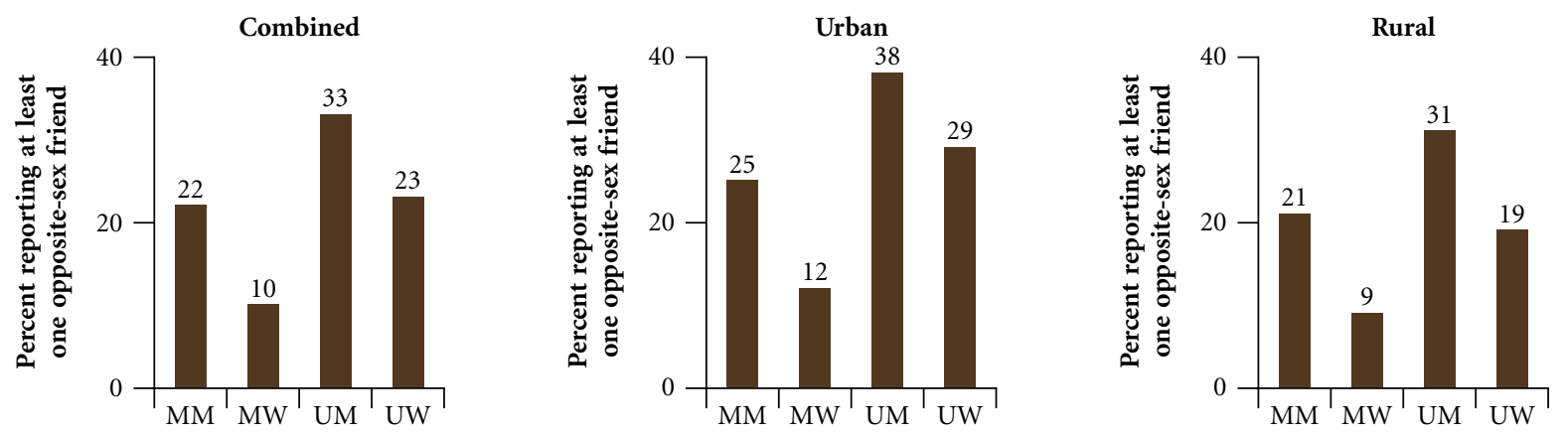

Note: For married respondents, questions referred to the period prior to marriage.

with their same-sex friends (32\% versus 24\%). Among young women, those in urban areas were more likely than their rural counterparts to report going on picnics or to films (68\% versus 58\%) and studying ( $83 \%$ versus $66 \%$ ) with their same-sex friends.

The range of activities in which youth engaged with their opposite-sex peers was much narrower. As shown in Panel B of Table 6.7, the only activity in which about as many youth engaged with their opposite-sex friends as their same-sex friends was chatting and gossiping, mentioned by $95-96 \%$ of youth reporting opposite-sex friends. Differences by gender in activities in which youth engaged with their opposite-sex friends were relatively narrow, except that as in the case of interactions with same-sex friends, young men were considerably more likely than young women to report that they went out on picnics or to films (59\% versus $33 \%$ ) with their opposite-sex friends. Differences by marital status in activities conducted with opposite-sex friends were apparent in only a couple of activities. For example, more unmarried than married young men reported studying with their opposite-sex friends; more married than unmarried young women reported engaging in sports with their opposite-sex friends. Finally, rural-urban differences were typically mild, except that urban youth were more likely than rural youth to have gone out on picnics or to films $(63 \%$ and $40 \%$ among urban young men and women, respectively, compared with $57 \%$ and $29 \%$ among the rural).

\subsection{Support networks}

The Youth Study also asked respondents about the individual with whom they would most likely discuss a range of personal matters, namely, taking a job, menstrual problems (females) and nocturnal emission or swapnadosh (males), and boy-girl relationships. All those aged 20 and above were asked to think back to the time they were aged 15-18 while responding to these questions.

Findings, reported in Table 6.8a, indicate that the person with whom youth would most likely discuss personal matters varied considerably by sex of the respondent and type of topic. Young women tended to consider their mother or father as their leading confidante on matters relating to taking a job (30-31\%). Mothers were, additionally, cited as the leading confidante on matters relating to menstrual problems (81\%). Matters pertaining to boy-girl relationships, however, were most likely to be confided in peers (57\%). Young men, in contrast, were less likely to consider a parent as a key confidante, except on the non-sensitive issue of taking a job (on which $28-31 \%$ cited their mother or father). Parents were rarely cited as key confidantes ( $1 \%$ or less) by young men on such issues as nocturnal emission or swapnadosh, and boy-girl relationships, for both of which most young men reported peers as their leading confidante ( $79 \%$ and $93 \%$, respectively). Differences by marital status were negligible among young men but notable among young women. Among young women, the unmarried were more likely than the married to 
Table 6.7: Interaction with same- and opposite-sex friends

Percentage of youth reporting interaction with same- and opposite-sex friends by types of activities, according to residence, Andhra Pradesh, 2007-08

\begin{tabular}{|c|c|c|c|c|c|c|}
\hline Activities (\%) & $\begin{array}{c}\mathrm{M} \\
15-24\end{array}$ & $\begin{array}{c}\mathrm{W} \\
15-24\end{array}$ & $\begin{array}{c}\text { MM } \\
15-29\end{array}$ & $\begin{array}{c}\text { MW } \\
15-24\end{array}$ & $\begin{array}{c}\text { UM } \\
15-24\end{array}$ & $\begin{array}{c}\text { UW } \\
15-24\end{array}$ \\
\hline \multicolumn{7}{|c|}{ A. Activities with same-sex friends } \\
\hline \multicolumn{7}{|c|}{ Combined } \\
\hline Going on picnics/to see films & 91.5 & 60.9 & 90.1 & 59.2 & 92.1 & 62.6 \\
\hline Studying together & 73.2 & 70.5 & 51.2 & 59.1 & 77.3 & 85.0 \\
\hline Spending time chatting/gossiping & 99.6 & 99.5 & 99.6 & 99.4 & 99.5 & 99.5 \\
\hline Playing sports & 84.2 & 62.8 & 65.4 & 60.7 & 87.8 & 65.5 \\
\hline Drinking or gambling & 26.4 & 1.3 & 47.3 & 1.2 & 22.7 & 1.4 \\
\hline Number with at least one same-sex friend & 2,464 & 4,758 & 1,391 & 2,263 & 2,068 & 2,495 \\
\hline \multicolumn{7}{|c|}{ Urban } \\
\hline Going on picnics/to see films & 93.9 & 68.4 & 93.4 & 65.6 & 94.0 & 70.5 \\
\hline Studying together & 75.0 & 82.6 & 55.6 & 74.2 & 78.2 & 89.2 \\
\hline Spending time chatting/gossiping & 99.6 & 99.6 & 99.7 & 99.6 & 99.5 & 99.7 \\
\hline Playing sports & 84.7 & 64.0 & 65.7 & 62.3 & 87.0 & 65.4 \\
\hline Drinking or gambling & 31.7 & 1.4 & 59.3 & 1.4 & 28.3 & 1.3 \\
\hline Number with at least one same-sex friend & 1,286 & 2,090 & 646 & 1,010 & 1,130 & 1,080 \\
\hline \multicolumn{7}{|c|}{ Rural } \\
\hline Going on picnics/to see films & 90.6 & 57.7 & 89.2 & 57.4 & 91.2 & 58.2 \\
\hline Studying together & 72.5 & 65.5 & 50.0 & 54.7 & 77.0 & 82.7 \\
\hline Spending time chatting/gossiping & 99.6 & 99.4 & 99.6 & 99.4 & 99.5 & 99.4 \\
\hline Playing sports & 84.0 & 62.3 & 65.3 & 60.2 & 88.2 & 65.6 \\
\hline Drinking or gambling & 24.3 & 1.2 & 44.0 & 1.1 & 20.2 & 1.4 \\
\hline Number with at least one same-sex friend & 1,178 & 2,668 & 745 & 1,253 & 938 & 1,415 \\
\hline \multicolumn{7}{|c|}{ B. Activities with opposite-sex friends } \\
\hline \multicolumn{7}{|c|}{ Combined } \\
\hline Going on picnics/to see films & 58.9 & 33.2 & 62.2 & 29.9 & 58.1 & 34.7 \\
\hline Studying together & 67.4 & 70.0 & 46.9 & 67.6 & 70.9 & 71.3 \\
\hline Spending time chatting/gossiping & 95.8 & 95.4 & 93.5 & 98.2 & 95.5 & 93.8 \\
\hline Playing sports & 27.0 & 30.7 & 24.4 & 37.8 & 27.0 & 26.7 \\
\hline Drinking or gambling & 1.1 & 1.9 & 2.3 & 2.7 & 1.0 & 1.4 \\
\hline Number with at least one opposite-sex friend & 829 & 831 & 317 & 243 & 726 & 588 \\
\hline \multicolumn{7}{|c|}{ Urban } \\
\hline Going on picnics/to see films & 63.0 & 39.7 & 72.0 & 42.2 & 62.1 & 39.0 \\
\hline Studying together & 66.8 & 70.8 & 56.0 & 68.8 & 69.2 & 71.3 \\
\hline Spending time chatting/gossiping & 96.6 & 96.4 & 96.0 & 100.0 & 96.3 & 95.5 \\
\hline Playing sports & 27.9 & 29.6 & 24.0 & 39.7 & 28.8 & 26.1 \\
\hline Drinking or gambling & 0.8 & 1.6 & 1.3 & 3.2 & 0.8 & 0.8 \\
\hline Number with at least one opposite-sex friend & 481 & 444 & 157 & 126 & 433 & 318 \\
\hline \multicolumn{7}{|c|}{ Rural } \\
\hline Going on picnics/to see films & 56.8 & 28.6 & 59.1 & 25.5 & 56.0 & 30.9 \\
\hline Studying together & 67.7 & 69.6 & 44.0 & 67.1 & 71.9 & 71.4 \\
\hline Spending time chatting/gossiping & 95.4 & 94.7 & 92.7 & 97.5 & 95.1 & 92.4 \\
\hline Playing sports & 26.5 & 31.3 & 24.1 & 37.0 & 25.9 & 27.0 \\
\hline Drinking or gambling & 1.3 & 2.1 & 2.6 & 2.5 & 1.3 & 2.0 \\
\hline Number with at least one opposite-sex friend & 348 & 387 & 160 & 117 & 293 & 270 \\
\hline
\end{tabular}

Note: All Ns are unweighted. For married respondents, questions referred to the period prior to marriage. 


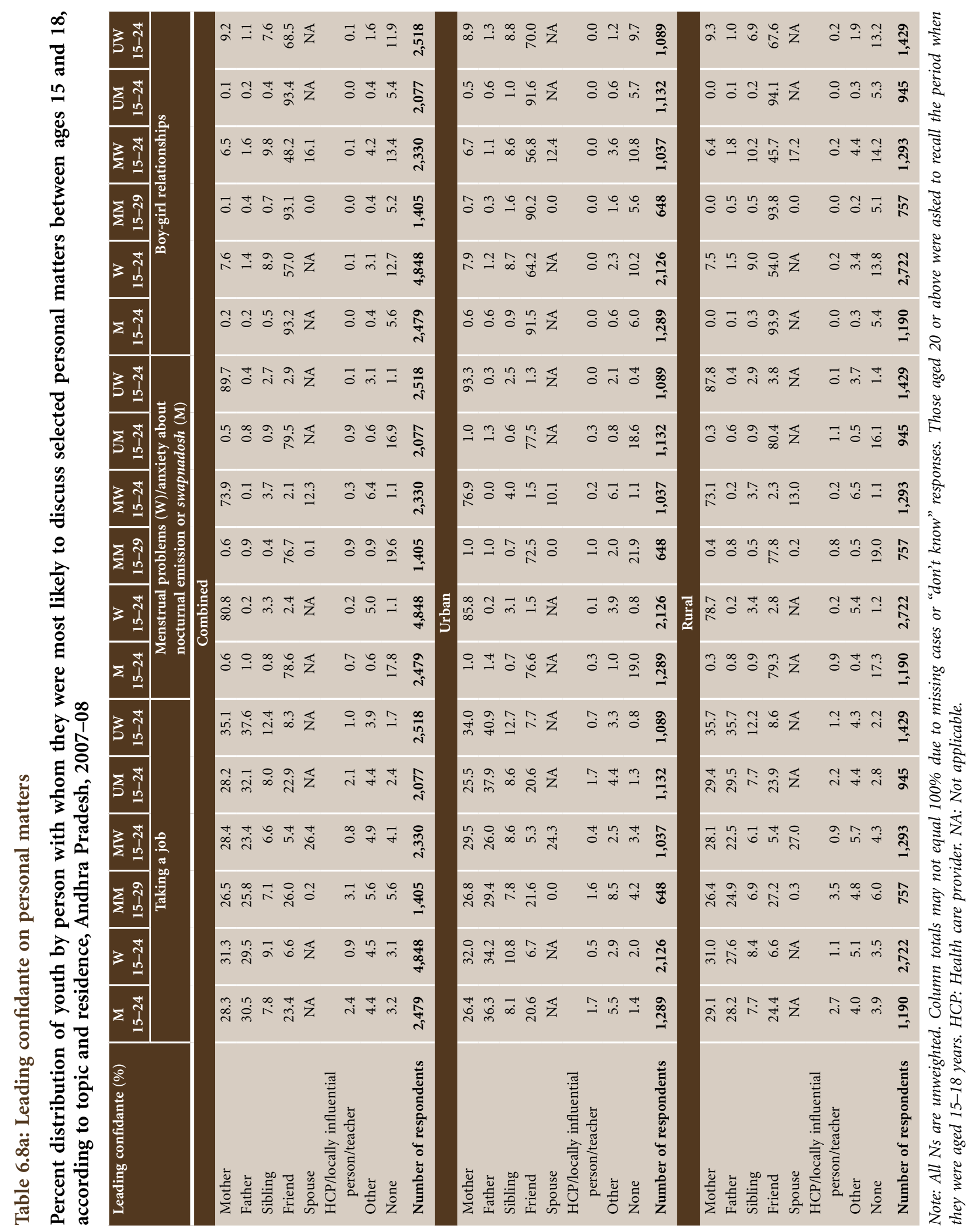


report a parent as a confidante on matters related to taking a job, the mother as the leading confidante on menstrual problems, and a friend as a confidante on boy-girl relationship issues. Of note is that while about $12-26 \%$ of married young women reported their spouse as a leading confidante on all three topics, hardly any married young men reported so, reflecting the fact that many more young women than men were married at ages 15-18, the ages about which these questions were posed. Patterns in rural and urban settings remained fairly similar; urban youth were slightly more likely than their rural counterparts to report their father as a confidante on matters related to taking a job and young women in urban areas were somewhat more likely than their rural counterparts to report their mother as a leading confidante on menstrual problems.

Notably, significant minorities of youth reported that they would not confide in anyone on some of these topics; for example, almost one-fifth of young men reported that they would not confide in anyone on nocturnal emission and likewise, $13 \%$ of young women would not confide in anyone on boy-girl relationships.

The Youth Study also asked young women about the individual in whom they were most likely to confide if they were teased by a boy. Findings, reported in Table $6.8 \mathrm{~b}$, show that one-third reported that they would confide in their mother compared to just $13 \%$ who reported that they would confide in their father. About one-fifth reported that they would confide in a friend and $13 \%$ in a sibling. Differences by marital status suggest that the unmarried were more likely than the married to confide in a parent ( $52 \%$ versus $44 \%)$ or a peer (25\% versus $15 \%)$. One-fifth of married young women reported that they would confide in their husband. Rural-urban differences were negligible.

Table 6.8b: Leading confidante on matters relating to the experience of teasing among young women

Percent distribution of young women by person with whom they were most likely to discuss being teased by a boy, according to residence, Andhra Pradesh, 2007-08

\begin{tabular}{|c|c|c|c|c|c|c|c|c|c|}
\hline \multirow[t]{2}{*}{ Leading confidante (\%) } & $\begin{array}{c}\mathrm{W} \\
15-24\end{array}$ & $\begin{array}{c}\text { MW } \\
15-24\end{array}$ & $\begin{array}{c}\text { UW } \\
15-24\end{array}$ & $\begin{array}{c}\text { W } \\
15-24\end{array}$ & $\begin{array}{c}\text { MW } \\
15-24\end{array}$ & $\begin{array}{c}\text { UW } \\
15-24\end{array}$ & $\begin{array}{c}\mathrm{W} \\
15-24\end{array}$ & $\begin{array}{c}\text { MW } \\
15-24\end{array}$ & $\begin{array}{c}\text { UW } \\
15-24\end{array}$ \\
\hline & \multicolumn{3}{|c|}{ Combined } & \multicolumn{3}{|c|}{ Urban } & \multicolumn{3}{|c|}{ Rural } \\
\hline Mother & 34.1 & 31.5 & 37.4 & 34.3 & 30.7 & 37.3 & 34.0 & 31.9 & 37.5 \\
\hline Father & 13.3 & 12.7 & 14.3 & 12.3 & 10.9 & 13.5 & 13.8 & 13.2 & 14.7 \\
\hline Sibling & 12.9 & 10.9 & 15.5 & 16.6 & 14.3 & 18.6 & 11.4 & 10.0 & 13.7 \\
\hline Friend & 19.1 & 14.7 & 25.0 & 21.5 & 18.3 & 24.1 & 18.1 & 13.6 & 25.5 \\
\hline Spouse & NA & 20.1 & NA & NA & 17.4 & NA & NA & 20.8 & NA \\
\hline $\begin{array}{l}\mathrm{HCP} / \text { locally influential } \\
\text { person/teacher }\end{array}$ & 1.4 & 1.2 & 1.8 & 1.5 & 1.0 & 1.9 & 1.4 & 1.3 & 1.7 \\
\hline Other person & 3.9 & 4.4 & 3.3 & 2.7 & 3.4 & 2.0 & 4.4 & 4.7 & 4.0 \\
\hline None & 3.7 & 4.4 & 2.8 & 3.2 & 3.8 & 2.6 & 4.0 & 4.6 & 2.9 \\
\hline Number of respondents & 4,848 & 2,330 & 2,518 & 2,126 & 1,037 & 1,089 & 2,722 & 1,293 & 1,429 \\
\hline
\end{tabular}

Note: All Ns are unweighted. Column totals may not equal 100\% due to missing cases or "don't know" responses. Those aged 20 or above were asked to recall the period when they were aged 15-18 years. HCP: Health care provider. NA: Not applicable. 


\subsection{Summary}

Youth Study findings reveal a mixed scenario regarding the nature of socialisation experienced by young people. While responses from young men indicate that large proportions of households did discriminate between their sons and daughters in terms of freedom of movement and expectations regarding housework, responses from young women suggest the reverse. While three-quarters of young men reported that they had more freedom to go out than their sisters or female cousins did, many fewer young women, just two-fifths, agreed that they had less freedom to go out than their brothers or male cousins did. Likewise, two-thirds of young men reported that they were expected to do less housework than their sisters or female cousins, and only two-fifths of young women agreed that they were expected to do more housework than their brothers or male cousins. At the same time, far more consistently observed were gender differences in perceptions of parental control: young women were more likely than young men to perceive that their parents would disapprove of social activities in which youth tend to participate that involved members of the opposite sex.

Findings regarding communication with parents on issues relevant to youth—such as school performance, friendships, being teased or bullied, physical maturation, romantic relationships and reproductive processes-reiterate those from other studies, showing that such communication is far from universal. Indeed, sensitive topics-such as romantic relationships, reproduction and contraception among all youth-were rarely discussed with either parent.

That parent-child communication was restricted was also evident from responses to questions probing the most likely confidante on a range of topics from taking a job to boy-girl relationships. While parents were mentioned as leading confidantes on topics such as taking a job, they were rarely cited as leading confidantes on more sensitive matters. Moreover, while young women identified their mother as the most likely confidante on such matters as menstrual problems and experience of teasing, young men rarely identified a parent as a leading confidante on matters relating to nocturnal emission or swapnadosh. Indeed, neither young men nor women identified a parent as a leading confidante on boy-girl matters.

Young people's family lives were marked by violence, both experienced and witnessed. Over one in three youth had observed their father beating their mother. Many young people reported experiencing a beating by a parent during adolescence; almost half of young men and almost one-quarter of young women reported such experiences.

In contrast, growing up was associated with close peer networks. Almost all youth reported having some same-sex friends. Young men, however, reported larger networks of friends than did young women. Opposite-sex peer networks were less common but nonetheless reported by nearly one-third of young men and one-seventh of young women. Interactions with friends tended to be restricted to activities such as chatting and studying, although young men did report engaging in activities such as going out on picnics or to films and playing games and outdoor sports. Indeed, findings suggest that youth derived an important measure of support from their peer networks on personal matters: friends were by far the leading confidante on boy-girl relationships for both young men and women, and on nocturnal emission for young men. 


\section{Agency and gender role attitudes}

Evidence on agency among youth, although sparse, suggests that in traditional settings such as India, young women and even some young men have limited agency in terms of decision-making on matters affecting their own lives, freedom of movement and access to resources. Gender role attitudes, similarly, tend to be traditional, assigning greater value to young men than young women (Alexander et al., 2006a; 2006b; Ram et al., 2006; Santhya, Jejeebhoy and Ghosh, 2008; Sebastian, Grant and Mensch, 2005). This chapter discusses Youth Study findings on agency and gender role attitudes.

\subsection{Decision-making}

In order to assess young people's involvement in decision-making, the Youth Study asked all respondents about their involvement in decisions related to three specific matters: choice of friends, spending one's own money and buying clothes for oneself. If youth reported that they were involved in decision-making on any issue, they were asked whether they made the decision entirely on their own or jointly with other family members.

Findings, presented in Table 7.1 and Figure 7.1, reveal that irrespective of sex, youth were overwhelmingly likely to choose their friends on their own (92\%). Differences by marital status and rural-urban residence were also narrow.

Fewer youth were involved in making decisions on spending their own money than on choice of friends, and this was particularly evident among young women. For example, while $63 \%$ of young men reported that they made decisions on their own about spending money, only $48 \%$ of young women so reported. Findings further suggest that a significant minority of young men $(8 \%)$ and women $(15 \%)$ reported that it was other family members who made decisions on spending money, without their involvement. As shown in Figure 7.1, marital status differences were mild among young women, but notable among young men among whom the married were more likely than the unmarried ( $77 \%$ versus $61 \%)$ to make this decision independently. Rural-urban differences were notable, particularly among young women; urban youth were more likely than rural youth to make independent decisions related to spending their own money.

With regard to decisions about the purchase of clothes for themselves, while $74 \%$ of young men decided on their own about purchasing clothes, only $34 \%$ of young women did so. One in seven young women reported that they did not have any say in decisions to buy clothes for themselves, and that it was other family members who made this decision for them, compared to $6 \%$ of young men.

Differences by marital status were observed among young men but not among young women; married young men were considerably more likely than unmarried young men to make decisions related to buying clothes on their own ( $86 \%$ versus $72 \%)$. Finally, rural-urban differences were muted among young men. Among young women, however, more urban than rural women made independent decisions relating to the purchase of clothes (41\% versus $32 \%)$.

In order to assess the extent to which youth had independent decision-making authority on all three matters, Table 7.2 presents the percentage of youth who reported that they independently made decisions on choice of 
Table 7.1: Decision-making

Percent distribution of youth by participation in decision-making on selected matters, according to residence, Andhra Pradesh, 2007-08

\begin{tabular}{|c|c|c|c|c|c|c|}
\hline Participation in decision-making (\%) & $\begin{array}{c}M \\
15-24\end{array}$ & $\begin{array}{c}\text { W } \\
15-24\end{array}$ & $\begin{array}{c}\text { MM } \\
15-29\end{array}$ & $\begin{array}{c}\text { MW } \\
15-24\end{array}$ & $\begin{array}{c}\text { UM } \\
15-24\end{array}$ & $\begin{array}{c}\text { UW } \\
15-24\end{array}$ \\
\hline \multicolumn{7}{|c|}{ A. Choice of friends } \\
\hline \multicolumn{7}{|c|}{ Combined } \\
\hline Respondent only & 92.2 & 92.0 & 94.7 & 90.8 & 92.3 & 93.4 \\
\hline Jointly with others & 5.6 & 4.7 & 4.1 & 5.3 & 5.3 & 3.9 \\
\hline Others only & 2.2 & 3.4 & 1.2 & 3.9 & 2.4 & 2.8 \\
\hline Number of respondents & 2,479 & 4,848 & 1,405 & 2,330 & 2,077 & 2,518 \\
\hline \multicolumn{7}{|c|}{ Urban } \\
\hline Respondent only & 94.6 & 94.3 & 97.7 & 94.1 & 94.6 & 94.4 \\
\hline Jointly with others & 4.1 & 3.1 & 2.3 & 3.2 & 3.8 & 3.0 \\
\hline Others only & 1.3 & 2.6 & 0.0 & 2.7 & 1.6 & 2.6 \\
\hline Number of respondents & 1,289 & 2,126 & 648 & 1,037 & 1,132 & 1,089 \\
\hline \multicolumn{7}{|c|}{ Rural } \\
\hline Respondent only & 91.3 & 91.0 & 93.9 & 89.9 & 91.4 & 92.8 \\
\hline Jointly with others & 6.2 & 5.3 & 4.6 & 5.9 & 5.9 & 4.3 \\
\hline Others only & 2.6 & 3.7 & 1.5 & 4.2 & 2.8 & 2.9 \\
\hline Number of respondents & 1,190 & 2,722 & 757 & 1,293 & 945 & 1,429 \\
\hline \multicolumn{7}{|c|}{ B. Spending money } \\
\hline \multicolumn{7}{|c|}{ Combined } \\
\hline Respondent only & 63.2 & 48.0 & 77.0 & 46.2 & 61.1 & 50.1 \\
\hline Jointly with others & 28.5 & 36.7 & 19.9 & 39.2 & 29.6 & 33.6 \\
\hline Others only & 8.3 & 15.3 & 3.1 & 14.6 & 9.3 & 16.3 \\
\hline Number of respondents & 2,479 & 4,848 & 1,405 & 2,330 & 2,077 & 2,518 \\
\hline \multicolumn{7}{|c|}{ Urban } \\
\hline Respondent only & 67.3 & 55.5 & 83.4 & 51.8 & 65.6 & 58.4 \\
\hline Jointly with others & 25.5 & 31.4 & 14.3 & 36.0 & 26.5 & 27.7 \\
\hline Others only & 7.2 & 13.1 & 2.3 & 12.2 & 7.9 & 13.9 \\
\hline Number of respondents & 1,289 & 2,126 & 648 & 1,037 & 1,132 & 1,089 \\
\hline \multicolumn{7}{|c|}{ Rural } \\
\hline Respondent only & 61.6 & 44.9 & 75.2 & 44.5 & 59.2 & 45.4 \\
\hline Jointly with others & 29.7 & 38.9 & 21.4 & 40.2 & 31.0 & 36.9 \\
\hline Others only & 8.7 & 16.2 & 3.5 & 15.3 & 9.9 & 17.7 \\
\hline Number of respondents & 1,190 & 2,722 & 757 & 1,293 & 945 & 1,429 \\
\hline
\end{tabular}


Table 7.1: (Cont'd)

\begin{tabular}{|c|c|c|c|c|c|c|}
\hline Participation in decision-making (\%) & $\begin{array}{c}\text { M } \\
15-24\end{array}$ & $\begin{array}{c}\text { W } \\
15-24\end{array}$ & $\begin{array}{c}\text { MM } \\
15-29\end{array}$ & $\begin{array}{c}\text { MW } \\
15-24\end{array}$ & $\begin{array}{c}\text { UM } \\
15-24\end{array}$ & $\begin{array}{c}\text { UW } \\
\text { 15-24 }\end{array}$ \\
\hline \multicolumn{7}{|c|}{ C. Buying clothes for oneself } \\
\hline \multicolumn{7}{|c|}{ Combined } \\
\hline Respondent only & 74.0 & 34.4 & 86.3 & 34.6 & 72.0 & 33.8 \\
\hline Jointly with others & 19.6 & 50.7 & 11.4 & 51.1 & 20.6 & 50.4 \\
\hline Others only & 6.4 & 14.9 & 2.3 & 14.3 & 7.4 & 15.8 \\
\hline Number of respondents & 2,479 & 4,848 & 1,405 & 2,330 & 2,077 & 2,518 \\
\hline \multicolumn{7}{|c|}{ Urban } \\
\hline Respondent only & 75.5 & 41.4 & 90.5 & 39.2 & 73.2 & 43.1 \\
\hline Jointly with others & 17.9 & 48.7 & 7.2 & 50.7 & 19.5 & 47.1 \\
\hline Others only & 6.7 & 9.9 & 2.3 & 10.1 & 7.3 & 9.8 \\
\hline Number of respondents & 1,289 & 2,126 & 648 & 1,037 & 1,132 & 1,089 \\
\hline \multicolumn{7}{|c|}{ Rural } \\
\hline Respondent only & 73.5 & 31.5 & 85.2 & 33.2 & 71.5 & 28.6 \\
\hline Jointly with others & 20.2 & 51.6 & 12.5 & 51.2 & 21.1 & 52.3 \\
\hline Others only & 6.3 & 16.9 & 2.4 & 15.6 & 7.4 & 19.1 \\
\hline Number of respondents & 1,190 & 2,722 & 757 & 1,293 & 945 & 1,429 \\
\hline
\end{tabular}

Note: All Ns are unweighted. Column totals may not equal 100\% due to missing cases or "don't know" responses.

Figure 7.1: Percent distribution of youth by participation in decision-making on selected matters, Andhra Pradesh, 2007-08
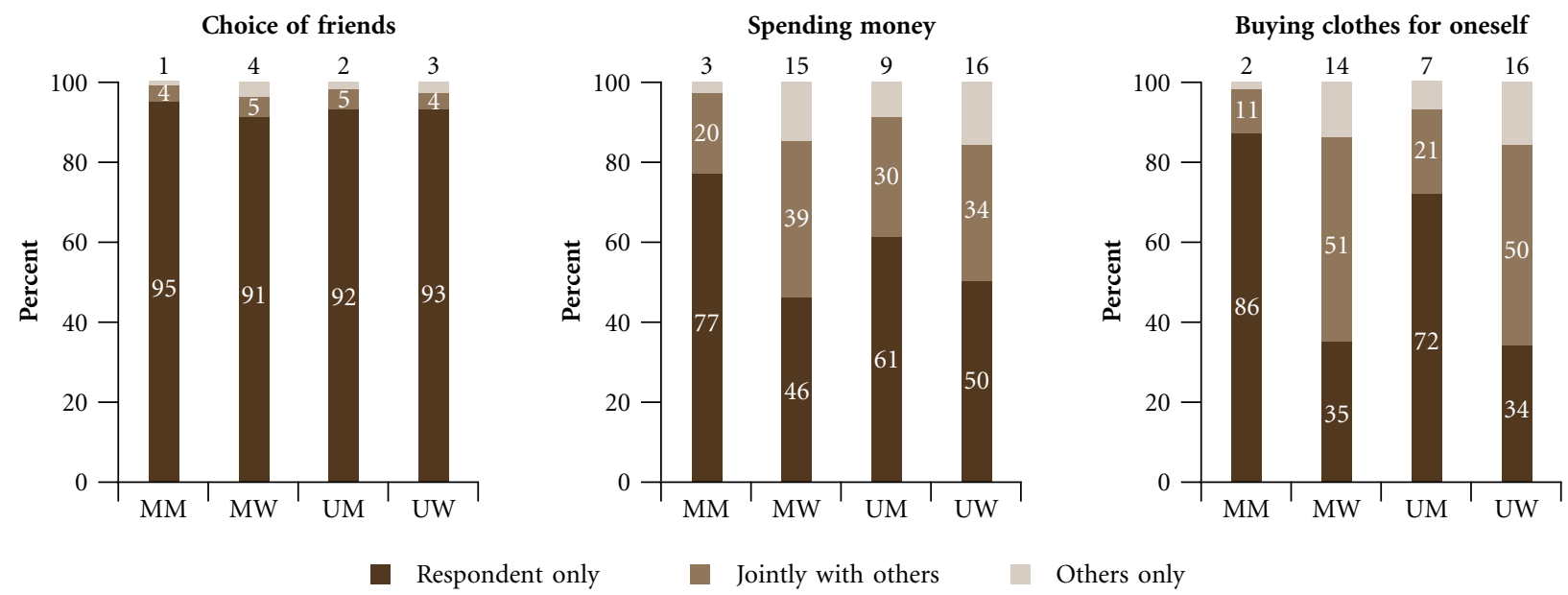

Note: Percentages may not equal 100.0 because of rounding.

friends, spending money and purchase of clothes. In total, 55\% of young men compared to $26 \%$ of young women reported independent decision-making on all three issues. While differences by marital status were muted among young women, among young men, the married were considerably more likely to report independent decision-making on all three matters than the unmarried ( $72 \%$ versus $52 \%)$. Rural-urban differences indicate that urban youth, particularly young women, were more likely to report independent decision-making on all three matters than rural youth (59\% versus 53\% among young men and $33 \%$ versus $24 \%$ among young women). 
Table 7.2 also presents independent decision-making on all three matters by selected background characteristics. Findings reveal that independent decision-making was indeed higher among older than younger respondents, irrespective of sex, marital status or rural-urban residence; differences were most pronounced among the unmarried. Differences by religion were muted for the overall and rural samples; however in urban areas, young men belonging to other religions were less likely to report independent decision-making on all three matters than Hindu and Muslim young men (50\% versus 59-61\%) and Muslim young women were less likely to report independent decision-making than women belonging to Hindu and other religions (27\% versus $34-36 \%)$. Caste-wise differences were negligible, however, among young women, those from general castes were more likely and those from scheduled tribes less likely than others to report independent decision-making.

Table 7.2: Decision-making autonomy by selected background characteristics

Percentage of youth who independently made decisions on choice of friends, spending money and buying clothes for themselves by selected background characteristics, according to residence, Andhra Pradesh, 2007-08

\begin{tabular}{|c|c|c|c|c|c|c|}
\hline Background characteristics (\%) & $\begin{array}{c}\mathrm{M} \\
15-24\end{array}$ & $\begin{array}{c}\text { W } \\
15-24\end{array}$ & $\begin{array}{c}\text { MM } \\
15-29\end{array}$ & $\begin{array}{c}\text { MW } \\
15-24\end{array}$ & $\begin{array}{c}\text { UM } \\
15-24\end{array}$ & $\begin{array}{c}\text { UW } \\
\text { 15-24 }\end{array}$ \\
\hline \multicolumn{7}{|c|}{ Combined } \\
\hline $\begin{array}{l}\text { Age (years) } \\
15-19 \\
20-24 \\
25-29\end{array}$ & $\begin{array}{r}45.9 \\
62.8 \\
\text { NA }\end{array}$ & $\begin{array}{r}21.9 \\
30.9 \\
\text { NA }\end{array}$ & $\begin{array}{r}* \\
64.6 \\
75.3\end{array}$ & $\begin{array}{r}18.9 \\
28.9 \\
\text { NA }\end{array}$ & $\begin{array}{r}45.5 \\
61.8 \\
\text { NA }\end{array}$ & $\begin{array}{r}23.2 \\
38.8 \\
\text { NA }\end{array}$ \\
\hline $\begin{array}{l}\text { Religion } \\
\text { Hindu } \\
\text { Muslim } \\
\text { Other }^{1}\end{array}$ & $\begin{array}{l}54.9 \\
55.5 \\
52.6\end{array}$ & $\begin{array}{l}26.6 \\
25.4 \\
23.6\end{array}$ & $\begin{array}{l}72.2 \\
75.0 \\
66.7\end{array}$ & $\begin{array}{l}26.4 \\
28.4 \\
19.0\end{array}$ & $\begin{array}{l}52.2 \\
55.8 \\
48.0\end{array}$ & $\begin{array}{l}26.7 \\
22.3 \\
29.9\end{array}$ \\
\hline $\begin{array}{l}\text { Caste } \\
\text { SC } \\
\text { ST } \\
\text { OBC } \\
\text { General }^{2}\end{array}$ & $\begin{array}{l}57.5 \\
60.5 \\
53.7 \\
54.0\end{array}$ & $\begin{array}{l}24.3 \\
21.2 \\
25.6 \\
30.9\end{array}$ & $\begin{array}{l}71.4 \\
75.7 \\
71.3 \\
73.4\end{array}$ & $\begin{array}{l}22.8 \\
19.4 \\
25.6 \\
31.8\end{array}$ & $\begin{array}{l}54.4 \\
51.4 \\
51.5 \\
53.5\end{array}$ & $\begin{array}{l}25.9 \\
24.8 \\
25.4 \\
29.7\end{array}$ \\
\hline $\begin{array}{l}\text { Educational level (years) } \\
\text { None } \\
1-7 \\
8-11 \\
12 \text { and above }\end{array}$ & $\begin{array}{l}50.8 \\
56.1 \\
53.4 \\
57.8\end{array}$ & $\begin{array}{l}20.9 \\
22.0 \\
26.6 \\
41.7\end{array}$ & $\begin{array}{l}70.9 \\
70.0 \\
71.8 \\
79.2\end{array}$ & $\begin{array}{l}22.4 \\
23.7 \\
28.6 \\
42.2\end{array}$ & $\begin{array}{l}42.1 \\
51.5 \\
51.6 \\
56.6\end{array}$ & $\begin{array}{l}12.2 \\
17.7 \\
25.1 \\
41.1\end{array}$ \\
\hline $\begin{array}{l}\text { Worked in last } 12 \text { months } \\
\text { Yes } \\
\text { No }\end{array}$ & $\begin{array}{l}57.1 \\
48.8\end{array}$ & $\begin{array}{l}22.5 \\
29.8\end{array}$ & $\begin{array}{r}71.7 \\
*\end{array}$ & $\begin{array}{l}21.5 \\
30.3\end{array}$ & $\begin{array}{l}54.6 \\
48.0\end{array}$ & $\begin{array}{l}23.8 \\
28.9\end{array}$ \\
\hline $\begin{array}{l}\text { Wealth quintile } \\
\text { First } \\
\text { Second } \\
\text { Third } \\
\text { Fourth } \\
\text { Fifth }\end{array}$ & $\begin{array}{l}48.8 \\
52.1 \\
57.2 \\
55.6 \\
57.0\end{array}$ & $\begin{array}{l}21.8 \\
22.0 \\
23.9 \\
27.0 \\
36.1\end{array}$ & $\begin{array}{l}62.3 \\
72.7 \\
75.2 \\
73.1 \\
73.0\end{array}$ & $\begin{array}{l}22.7 \\
23.2 \\
24.6 \\
25.5 \\
36.3\end{array}$ & $\begin{array}{l}45.4 \\
47.6 \\
54.4 \\
53.7 \\
55.7\end{array}$ & $\begin{array}{l}19.8 \\
20.0 \\
22.9 \\
28.6 \\
35.7\end{array}$ \\
\hline Total & 54.8 & 26.3 & 72.1 & 25.8 & 52.4 & 26.6 \\
\hline \multicolumn{7}{|c|}{ Urban } \\
\hline $\begin{array}{l}\text { Age (years) } \\
15-19 \\
20-24 \\
25-29\end{array}$ & $\begin{array}{r}46.3 \\
69.2 \\
\text { NA }\end{array}$ & $\begin{array}{r}29.2 \\
36.6 \\
\text { NA }\end{array}$ & $\begin{array}{r}\star \\
75.3 \\
81.8\end{array}$ & $\begin{array}{r}26.3 \\
32.4 \\
\text { NA }\end{array}$ & $\begin{array}{r}46.1 \\
67.4 \\
\text { NA }\end{array}$ & $\begin{array}{r}29.9 \\
44.7 \\
\text { NA }\end{array}$ \\
\hline
\end{tabular}

Cont'd on next page... 
Table 7.2: (Cont'd)

\begin{tabular}{|c|c|c|c|c|c|c|}
\hline Background characteristics (\%) & $\begin{array}{c}M \\
15-24\end{array}$ & $\begin{array}{c}\text { W } \\
15-24\end{array}$ & $\begin{array}{c}\text { MM } \\
15-29\end{array}$ & $\begin{array}{c}\text { MW } \\
15-24\end{array}$ & $\begin{array}{c}\text { UM } \\
15-24\end{array}$ & $\begin{array}{c}\text { UW } \\
15-24\end{array}$ \\
\hline \multicolumn{7}{|c|}{ Urban } \\
\hline $\begin{array}{l}\text { Religion } \\
\text { Hindu } \\
\text { Muslim } \\
\text { Other }^{1}\end{array}$ & $\begin{array}{l}59.3 \\
60.6 \\
50.0\end{array}$ & $\begin{array}{l}33.6 \\
27.3 \\
35.8\end{array}$ & $\begin{array}{r}80.2 \\
82.9 \\
(71.4)\end{array}$ & $\begin{array}{l}31.3 \\
29.1 \\
31.8\end{array}$ & $\begin{array}{l}56.9 \\
59.3 \\
43.3\end{array}$ & $\begin{array}{l}35.6 \\
25.8 \\
39.5\end{array}$ \\
\hline $\begin{array}{l}\text { Caste } \\
\text { SC } \\
\text { ST } \\
\text { OBC } \\
\text { General }^{2}\end{array}$ & $\begin{array}{r}60.0 \\
(62.5) \\
60.8 \\
55.4\end{array}$ & $\begin{array}{r}33.2 \\
(36.7) \\
31.3 \\
35.3\end{array}$ & $\begin{array}{r}82.4 \\
(78.6) \\
81.9 \\
76.7\end{array}$ & $\begin{array}{r}33.1 \\
(29.4) \\
28.8 \\
34.1\end{array}$ & $\begin{array}{r}54.9 \\
(65.0) \\
58.3 \\
53.9\end{array}$ & $\begin{array}{r}33.5 \\
* \\
33.7 \\
36.2\end{array}$ \\
\hline $\begin{array}{l}\text { Educational level (years) } \\
\text { None }^{3} \\
1-7 \\
8-11 \\
12 \text { and above }\end{array}$ & $\begin{array}{l}58.8 \\
66.4 \\
53.1 \\
62.7\end{array}$ & $\begin{array}{l}23.8 \\
23.7 \\
30.3 \\
47.3\end{array}$ & $\begin{array}{l}76.2 \\
84.6 \\
78.5 \\
80.3\end{array}$ & $\begin{array}{l}27.5 \\
24.2 \\
31.6 \\
47.6\end{array}$ & $\begin{array}{r}(57.1) \\
61.3 \\
50.7 \\
62.2\end{array}$ & $\begin{array}{r}(13.2) \\
23.3 \\
29.5 \\
47.0\end{array}$ \\
\hline $\begin{array}{l}\text { Worked in last } 12 \text { months } \\
\text { Yes } \\
\text { No }\end{array}$ & $\begin{array}{l}64.7 \\
48.2\end{array}$ & $\begin{array}{l}31.6 \\
33.5\end{array}$ & $\begin{array}{r}80.0 \\
*\end{array}$ & $\begin{array}{l}29.8 \\
31.5\end{array}$ & $\begin{array}{l}62.2 \\
48.2\end{array}$ & $\begin{array}{l}33.0 \\
35.2\end{array}$ \\
\hline $\begin{array}{l}\text { Wealth quintile } \\
\text { First } \\
\text { Second } \\
\text { Third } \\
\text { Fourth } \\
\text { Fifth }\end{array}$ & $\begin{array}{l}64.3 \\
54.2 \\
61.8 \\
61.3 \\
57.0\end{array}$ & $\begin{array}{l}31.6 \\
27.7 \\
25.3 \\
30.1 \\
40.1\end{array}$ & $\begin{array}{r}(76.5) \\
68.8 \\
87.2 \\
85.7 \\
75.2\end{array}$ & $\begin{array}{l}32.6 \\
27.4 \\
23.2 \\
28.6 \\
40.0\end{array}$ & $\begin{array}{r}(57.1) \\
53.2 \\
54.8 \\
58.7 \\
56.2\end{array}$ & $\begin{array}{l}29.8 \\
27.9 \\
27.7 \\
31.4 \\
40.0\end{array}$ \\
\hline \multicolumn{7}{|c|}{ Rural } \\
\hline $\begin{array}{l}\text { Age (years) } \\
15-19 \\
20-24 \\
25-29\end{array}$ & $\begin{array}{r}45.7 \\
60.2 \\
\text { NA }\end{array}$ & $\begin{array}{r}19.1 \\
28.3 \\
\text { NA }\end{array}$ & $\begin{array}{r}* \\
62.3 \\
73.2\end{array}$ & $\begin{array}{r}17.5 \\
27.7 \\
\text { NA }\end{array}$ & $\begin{array}{r}45.2 \\
58.8 \\
\text { NA }\end{array}$ & $\begin{array}{r}20.2 \\
32.2 \\
\text { NA }\end{array}$ \\
\hline $\begin{array}{l}\text { Religion } \\
\text { Hindu } \\
\text { Muslim } \\
\text { Other }^{1}\end{array}$ & $\begin{array}{l}53.3 \\
52.4 \\
53.5\end{array}$ & $\begin{array}{l}23.9 \\
24.0 \\
18.6\end{array}$ & $\begin{array}{r}70.1 \\
(71.0) \\
65.8\end{array}$ & $\begin{array}{l}25.0 \\
28.0 \\
15.7\end{array}$ & $\begin{array}{r}50.4 \\
53.5 \\
(50.0)\end{array}$ & $\begin{array}{l}22.2 \\
17.7 \\
24.0\end{array}$ \\
\hline $\begin{array}{l}\text { Caste } \\
\text { SC } \\
\text { ST } \\
\text { OBC } \\
\text { General }^{2}\end{array}$ & $\begin{array}{l}56.8 \\
60.2 \\
50.6 \\
53.0\end{array}$ & $\begin{array}{l}20.5 \\
19.5 \\
23.5 \\
28.1\end{array}$ & $\begin{array}{l}69.5 \\
75.2 \\
68.0 \\
72.0\end{array}$ & $\begin{array}{l}19.7 \\
18.2 \\
24.6 \\
31.1\end{array}$ & $\begin{array}{l}54.2 \\
48.2 \\
48.4 \\
53.2\end{array}$ & $\begin{array}{l}21.8 \\
22.0 \\
21.4 \\
24.0\end{array}$ \\
\hline $\begin{array}{l}\text { Educational level (years) } \\
\text { None }^{3} \\
1-7 \\
8-11 \\
12 \text { and above }\end{array}$ & $\begin{array}{l}49.1 \\
53.0 \\
53.5 \\
54.7\end{array}$ & $\begin{array}{l}20.4 \\
21.6 \\
24.8 \\
33.9\end{array}$ & $\begin{array}{l}70.5 \\
66.5 \\
69.6 \\
78.7\end{array}$ & $\begin{array}{l}21.6 \\
23.6 \\
27.4 \\
35.9\end{array}$ & $\begin{array}{l}38.8 \\
48.1 \\
51.9 \\
52.7\end{array}$ & $\begin{array}{l}11.9 \\
15.7 \\
23.0 \\
33.1\end{array}$ \\
\hline $\begin{array}{l}\text { Worked in last } 12 \text { months } \\
\text { Yes } \\
\text { No }\end{array}$ & $\begin{array}{l}54.5 \\
49.1\end{array}$ & $\begin{array}{l}20.8 \\
27.0\end{array}$ & $\begin{array}{r}69.4 \\
*\end{array}$ & $\begin{array}{l}20.6 \\
29.6\end{array}$ & $\begin{array}{l}51.6 \\
47.9\end{array}$ & $\begin{array}{l}21.3 \\
23.1\end{array}$ \\
\hline $\begin{array}{l}\text { Wealth quintile } \\
\text { First } \\
\text { Second } \\
\text { Third } \\
\text { Fourth } \\
\text { Fifth }\end{array}$ & $\begin{array}{l}47.1 \\
51.7 \\
56.1 \\
52.8 \\
57.0\end{array}$ & $\begin{array}{l}20.5 \\
21.3 \\
23.6 \\
25.0 \\
30.7\end{array}$ & $\begin{array}{l}61.1 \\
72.9 \\
73.1 \\
67.5 \\
71.5\end{array}$ & $\begin{array}{l}21.6 \\
22.7 \\
24.9 \\
23.9 \\
33.3\end{array}$ & $\begin{array}{l}44.0 \\
46.8 \\
54.3 \\
51.1 \\
55.2\end{array}$ & $\begin{array}{l}18.1 \\
18.8 \\
21.2 \\
26.5 \\
27.5\end{array}$ \\
\hline Total & 53.2 & 23.5 & 69.9 & 24.3 & 50.6 & 22.1 \\
\hline
\end{tabular}

Note: ${ }^{*}$ Percentage not shown, based on fewer than 25 unweighted cases. ( ) Based on 25-49 unweighted cases. NA: Not applicable. OBC: Other backward caste. SC: Scheduled caste. ST: Scheduled tribe. ${ }^{1}$ Includes Christian, Buddhist, Neo-Buddhist, Sikh, Jain, Jewish, Parsi/Zoroastrian and no specified religion. ${ }^{2}$ Includes all those not belonging to SC, ST or OBC. ${ }^{3}$ Includes non-literate and literate with no formal schooling. 
Independent decision-making increased consistently with level of education among young women but not so among young men, and this pattern was observed irrespective of marital status and rural-urban residence. Among young women, for example, $42 \%$ of those with 12 or more years of education decided independently on all three issues, compared with just $21 \%$ of non-literate young women or those without any formal education. The differences were much wider in urban than in rural settings ( $47 \%$ and $24 \%$ in urban settings compared to $34 \%$ and $20 \%$ in rural settings). Among young men, however, differences in percentages who decided independently on all three issues between those with no formal education and those with 12 or more years of education were typically narrow; even so, when the married and unmarried were considered separately, those with 12 or more years of education were more likely to have decided independently on all three issues than those without any formal education. Interestingly, even the least educated young men were more likely than the most educated young women to report independent decision-making on all three matters.

Associations between economic activity status and independent decision-making differed among young men and women. Among young men, those who had worked in the last 12 months tended to be more likely than others to make decisions independently; however, among young women, a reverse pattern was apparent. The pattern remained by and large similar among the married and the unmarried.

Associations between the economic status of households and independent decision-making were inconsistent among young men. Among young women, however, those belonging to the wealthiest (fifth) quintile were more likely than those belonging to other quintiles to have made independent decisions on all three matters. As in the case of education, even young men from households in the poorest (first) quintile were typically more likely to report independent decision-making than young women in the wealthiest quintile.

\subsection{Freedom of movement}

Freedom of movement was assessed only for all young women and unmarried young men because married young men generally have unrestricted mobility. Mobility was measured by a number of questions relating to whether the respondent was permitted to visit places within and outside the village (rural) or neighbourhood (urban) unescorted, only if accompanied by someone else, or was not permitted to visit the place at all. Places within the village or neighbourhood included a shop/market, the home of a friend/relative and a community programme. Places outside the village or neighbourhood included the home of a relative or friend, movie theatre, video parlour or other place of entertainment and a community programme. Finally, all respondents were asked if they could go to a health facility unescorted if required. Table 7.3 and Figure 7.2 report findings relating to mobility.

Findings confirm that freedom of movement even within the village or neighbourhood was not universal, although the mobility of young women, both married and unmarried, was more restricted than that of unmarried young men. For example, findings suggest that $80 \%$ of young women could go unescorted to a shop or market within the village or neighbourhood compared with $89 \%$ of unmarried young men. Freedom to attend programmes within the village or neighbourhood was more restricted, particularly among young women. Only $9 \%$ of young women compared with $61 \%$ of unmarried young men were allowed to attend community programmes within the village or neighbourhood unescorted. While three-quarters of young women were allowed to attend such programmes, if accompanied, as many as one in seven were not allowed to attend such programmes under any circumstances.

Freedom to visit places outside the village or neighbourhood unescorted was even more restricted than mobility within the village. Of the three sites, freedom to visit a place of entertainment or to attend a programme was more curtailed than freedom to visit a friend or relative residing outside the village or neighbourhood. Young women's mobility was particularly limited: for example, just $22 \%$ were permitted to visit a friend or relative outside the village unescorted and just 3-4\% were permitted to visit a place of entertainment or to attend a programme conducted outside the village or neighbourhood unescorted. While $85 \%$ and $70 \%$ of young women were allowed to visit a place of entertainment or attend a programme, respectively, if accompanied, as many as $27 \%$ were not allowed to 
attend a programme and about $12 \%$ were not allowed to visit a place of entertainment outside the home village or neighbourhood under any circumstances. While young men's mobility was not curtailed to the same extent as young women's, it was far from universal: findings show that just over half of unmarried young men were allowed to visit a place of entertainment or to attend a programme conducted outside their village or neighbourhood unescorted, and just $71 \%$ were allowed to visit a friend or relative residing outside the village or neighbourhood unescorted.

Table 7.3: Freedom of movement

Percent distribution of youth by extent of freedom to visit selected locations within or outside the village/neighbourhood, according to residence, Andhra Pradesh, 2007-08

\begin{tabular}{|c|c|c|c|c|c|c|c|c|c|c|c|c|}
\hline \multirow[t]{2}{*}{ Mobility indicators (\%) } & $\begin{array}{c}\text { W } \\
15-24\end{array}$ & $\begin{array}{c}\text { MW } \\
15-24\end{array}$ & $\begin{array}{l}\text { UW } \\
15-24\end{array}$ & $\begin{array}{l}\text { UM } \\
15-24\end{array}$ & $\begin{array}{c}\text { W } \\
15-24\end{array}$ & $\begin{array}{c}\text { MW } \\
15-24\end{array}$ & $\begin{array}{l}\text { UW } \\
15-24\end{array}$ & $\begin{array}{c}\text { UM } \\
15-24\end{array}$ & $\begin{array}{c}\text { W } \\
15-24\end{array}$ & $\begin{array}{c}\text { MW } \\
15-24\end{array}$ & $\begin{array}{l}\text { UW } \\
15-24\end{array}$ & $\begin{array}{c}\text { UM } \\
15-24\end{array}$ \\
\hline & \multicolumn{4}{|c|}{ Combined } & \multicolumn{4}{|c|}{ Urban } & \multicolumn{4}{|c|}{ Rural } \\
\hline \multicolumn{13}{|l|}{ Permitted to: } \\
\hline $\begin{array}{l}\text { Visit shop/market withir } \\
\text { neighbourhood }\end{array}$ & & & & & & & & & & & & \\
\hline Alone & 79.6 & 78.2 & 81.3 & 88.8 & 82.8 & 80.8 & 84.4 & 92.2 & 78.2 & 77.4 & 79.6 & 87.3 \\
\hline Only with someone else & 14.7 & 15.5 & 13.8 & 11.1 & 13.3 & 14.5 & 12.4 & 7.6 & 15.3 & 15.8 & 14.6 & 12.7 \\
\hline Not allowed & 5.7 & 6.3 & 4.9 & 0.0 & 3.8 & 4.8 & 3.2 & 0.2 & 6.4 & 6.8 & 5.8 & 0.0 \\
\hline \multicolumn{13}{|c|}{$\begin{array}{l}\text { Visit friend/relative within village/ } \\
\text { neighbourhood }\end{array}$} \\
\hline Alone & 71.6 & 68.5 & 75.7 & 80.3 & 74.0 & 69.9 & 77.2 & 82.9 & 70.6 & 68.0 & 74.8 & 79.2 \\
\hline Only with someone else & 26.2 & 29.0 & 22.5 & 17.9 & 23.9 & 28.0 & 20.7 & 16.8 & 27.1 & 29.3 & 23.6 & 18.3 \\
\hline Not allowed & 2.2 & 2.5 & 1.7 & 1.9 & 2.1 & 2.1 & 2.1 & 0.3 & 2.3 & 2.7 & 1.6 & 2.5 \\
\hline \multicolumn{13}{|c|}{$\begin{array}{l}\text { Attend programme within village/ } \\
\text { neighbourhood }\end{array}$} \\
\hline Alone & 9.3 & 9.8 & 8.7 & 60.9 & 7.7 & 7.2 & 8.0 & 64.2 & 10.0 & 10.6 & 9.0 & 59.5 \\
\hline Only with someone else & 76.1 & 75.8 & 76.4 & 33.4 & 78.5 & 80.2 & 77.1 & 31.1 & 75.1 & 74.6 & 75.9 & 34.3 \\
\hline Not allowed & 14.6 & 14.3 & 15.0 & 5.7 & 13.8 & 12.6 & 14.9 & 4.8 & 14.9 & 14.8 & 15.1 & 6.2 \\
\hline \multicolumn{13}{|c|}{$\begin{array}{l}\text { Visit friend/relative outside village/ } \\
\text { neighbourhood }\end{array}$} \\
\hline Alone & 21.9 & 22.4 & 21.4 & 71.4 & 20.3 & 20.2 & 20.5 & 71.4 & 22.6 & 23.0 & 22.0 & 71.3 \\
\hline Only with someone else & 72.3 & 72.1 & 72.6 & 27.9 & 73.9 & 74.7 & 73.3 & 28.3 & 71.6 & 71.3 & 72.1 & 27.7 \\
\hline Not allowed & 5.8 & 5.6 & 6.0 & 0.8 & 5.8 & 5.1 & 6.3 & 0.3 & 5.7 & 5.7 & 5.8 & 1.0 \\
\hline \multicolumn{13}{|c|}{$\begin{array}{l}\text { Visit nearby village/neighbourhood for } \\
\text { entertainment }\end{array}$} \\
\hline Alone & 3.3 & 3.3 & 3.1 & 55.3 & 3.3 & 3.0 & 3.5 & 60.1 & 3.2 & 3.4 & 2.9 & 53.2 \\
\hline Only with someone else & 84.9 & 85.0 & 84.5 & 43.1 & 89.1 & 90.3 & 88.2 & 39.0 & 83.1 & 83.5 & 82.4 & 45.0 \\
\hline Not allowed & 11.9 & 11.6 & 12.3 & 1.6 & 7.5 & 6.7 & 8.3 & 1.0 & 13.7 & 13.1 & 14.7 & 1.9 \\
\hline \multicolumn{13}{|c|}{$\begin{array}{l}\text { Attend programme outside village/ } \\
\text { neighbourhood }\end{array}$} \\
\hline Alone & 3.7 & 4.1 & 3.3 & 54.6 & 3.0 & 2.9 & 3.1 & 57.0 & 4.1 & 4.5 & 3.4 & 53.7 \\
\hline Only with someone else & 69.7 & 70.0 & 69.1 & 39.1 & 71.5 & 73.2 & 70.1 & 37.3 & 68.9 & 69.1 & 68.6 & 39.8 \\
\hline Not allowed & 26.6 & 25.8 & 27.6 & 6.3 & 25.5 & 24.0 & 26.8 & 5.7 & 27.0 & 26.4 & 28.1 & 6.5 \\
\hline \multicolumn{13}{|l|}{ Visit health facility } \\
\hline $\begin{array}{l}\text { Alone } \\
\text { Only with someone else }\end{array}$ & $\begin{array}{l}10.4 \\
89.1\end{array}$ & $\begin{array}{l}12.8 \\
86.9\end{array}$ & $\begin{array}{r}7.3 \\
92.1\end{array}$ & $\begin{array}{l}42.4 \\
57.5\end{array}$ & $\begin{array}{l}10.0 \\
89.5\end{array}$ & $\begin{array}{l}12.6 \\
87.0\end{array}$ & $\begin{array}{r}8.0 \\
91.5\end{array}$ & $\begin{array}{l}44.4 \\
55.5\end{array}$ & $\begin{array}{l}10.6 \\
89.0\end{array}$ & $\begin{array}{l}12.9 \\
86.9\end{array}$ & $\begin{array}{r}6.9 \\
92.4\end{array}$ & $\begin{array}{l}41.5 \\
58.4\end{array}$ \\
\hline Not allowed & 0.4 & 0.3 & 0.6 & 0.1 & 0.4 & 0.4 & 0.4 & 0.2 & 0.4 & 0.3 & 0.6 & 0.1 \\
\hline Number of respondents & 4,848 & 2,330 & 2,518 & 2,077 & 2,126 & 1,037 & 1,089 & 1,132 & 2,722 & 1,293 & 1,429 & 945 \\
\hline
\end{tabular}

Note: All Ns are unweighted. Column totals may not equal 100\% due to missing cases or "don't know" responses. Questions regarding freedom of movement were not asked of married men, as their mobility is generally unrestricted. 
Figure 7.2: Percentage of youth allowed to visit selected places within and outside the village/neighbourhood unescorted, Andhra Pradesh, 2007-08

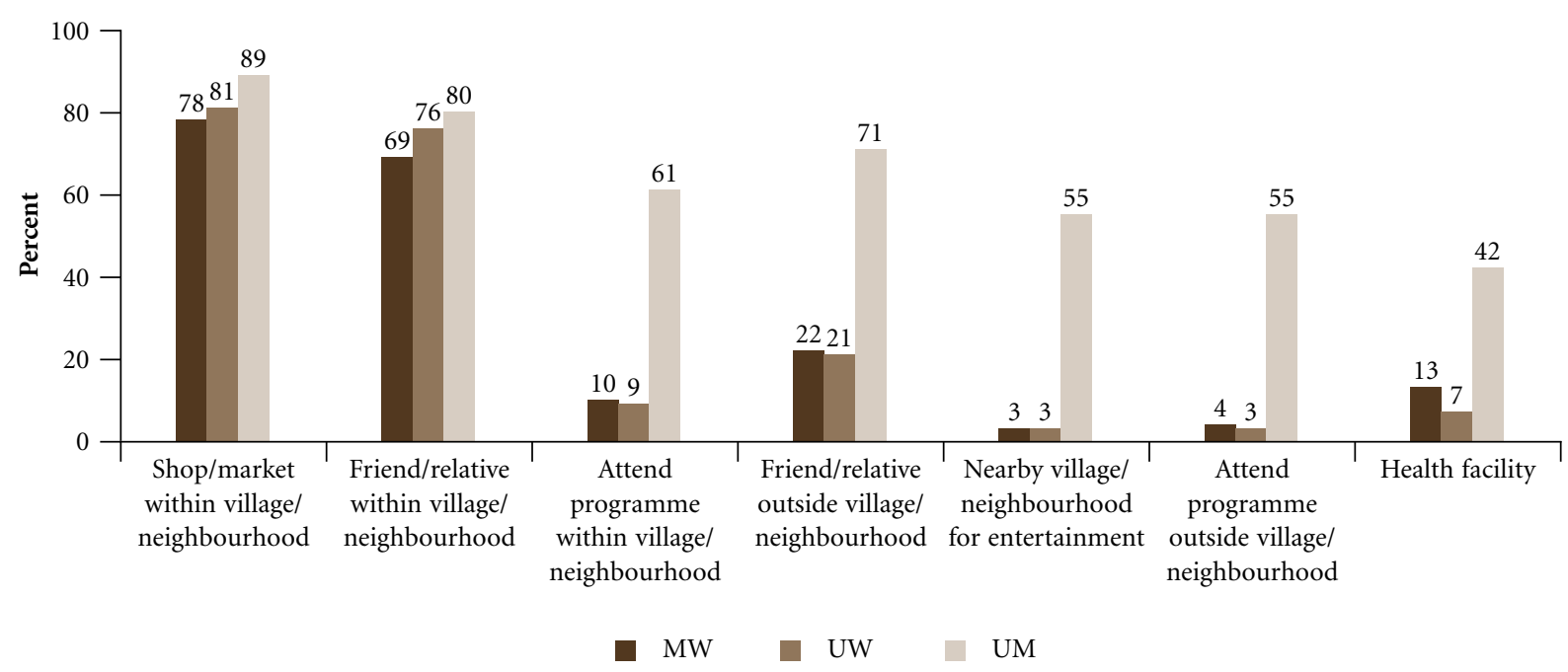

Note: Questions regarding freedom of movement were not asked of married men, as their mobility is generally unrestricted.

With regard to freedom to visit a health facility unescorted, findings, presented in Table 7.3, reveal that just one-tenth of young women, compared with $42 \%$ of unmarried young men, reported that they could do so.

Among young women, differences by marital status were by and large mild. Differences by rural-urban residence were, likewise, muted among young women as well as unmarried young men.

Summary measures have been created from the range of questions relating to freedom to visit places unescorted within and outside the village or neighbourhood; namely, the percentages who were free to visit at least one place within the village or neighbourhood; at least one place outside the village or neighbourhood; and a health facility. Table 7.4 presents percentages of youth reporting each of these summary measures of freedom of movement by selected socio-economic and demographic characteristics.

As shown in Table 7.4, 93\% and 76\% of unmarried young men had the freedom to visit unescorted at least one place within and outside the village or neighbourhood, respectively, and $42 \%$, to visit a health facility. In comparison, only $84 \%$ and $23 \%$ of young women reported freedom to visit unescorted at least one place within and outside the village or neighbourhood, respectively, and $10 \%$ to visit a health facility. As observed earlier, differences by marital status among young women, and differences by rural-urban residence among both young women and unmarried young men, were muted.

Findings reveal that among young women and unmarried young men, socio-demographic differentials were, by and large, narrow in the case of mobility within the village. The only notable difference observed was that Muslims were less likely than those belonging to Hindu and other religions to report freedom to visit at least one location within the village or neighbourhood.

Freedom to visit places outside the village or neighbourhood, was, in contrast, associated with several more background characteristics. For example, this freedom increased with age and was greater among working youth than those who were not working. In addition, differences by religion suggest that Muslim youth were less likely than others to report mobility outside the village or neighbourhood. Caste-wise differences were not evident among 
Table 7.4: Freedom of movement by selected background characteristics

Percentage of youth who could visit various places unescorted by selected background characteristics, according to residence, Andhra Pradesh, 2007-08

\begin{tabular}{|c|c|c|c|c|c|c|c|c|c|c|c|c|}
\hline \multirow[t]{2}{*}{$\begin{array}{l}\text { Background } \\
\text { characteristics (\%) }\end{array}$} & $\begin{array}{c}\mathrm{W} \\
15-24 \\
\end{array}$ & $\begin{array}{c}\text { MW } \\
15-24 \\
\end{array}$ & $\begin{array}{c}\text { UW } \\
15-24 \\
\end{array}$ & $\begin{array}{c}\text { UM } \\
15-24 \\
\end{array}$ & $\begin{array}{c}W \\
15-24 \\
\end{array}$ & $\begin{array}{c}\text { MW } \\
15-24 \\
\end{array}$ & $\begin{array}{c}\text { UW } \\
15-24 \\
\end{array}$ & $\begin{array}{c}\text { UM } \\
15-24 \\
\end{array}$ & $\begin{array}{c}\text { W } \\
15-24 \\
\end{array}$ & $\begin{array}{c}\text { MW } \\
15-24 \\
\end{array}$ & $\begin{array}{c}\text { UW } \\
15-24 \\
\end{array}$ & $\begin{array}{c}\text { UM } \\
15-24 \\
\end{array}$ \\
\hline & \multicolumn{4}{|c|}{ Within village/neighbourhood } & \multicolumn{4}{|c|}{ Outside village/neighbourhood } & \multicolumn{4}{|c|}{ Health facility } \\
\hline \multicolumn{13}{|c|}{ Combined } \\
\hline \multicolumn{13}{|l|}{ Age (years) } \\
\hline $15-19$ & 82.8 & 78.1 & 85.1 & 91.8 & 18.0 & 15.7 & 19.2 & 73.0 & 7.2 & 9.8 & 5.9 & 35.3 \\
\hline $20-24$ & 85.0 & 84.0 & 88.7 & 94.1 & 28.6 & 27.1 & 35.4 & 80.6 & 13.8 & 14.1 & 12.4 & 52.0 \\
\hline \multicolumn{13}{|l|}{ Religion } \\
\hline Hindu & 84.9 & 83.1 & 87.3 & 93.6 & 23.7 & 23.8 & 23.6 & 77.4 & 10.6 & 12.8 & 7.6 & 42.0 \\
\hline Muslim & 69.9 & 66.2 & 73.6 & 86.2 & 18.1 & 19.6 & 16.8 & 68.3 & 11.4 & 15.0 & 7.6 & 41.3 \\
\hline Other ${ }^{1}$ & 84.6 & 85.2 & 84.1 & 93.1 & 22.8 & 25.2 & 19.4 & 73.3 & 8.4 & 11.4 & 4.0 & 52.5 \\
\hline \multicolumn{13}{|l|}{ Caste } \\
\hline SC & 82.9 & 81.4 & 84.7 & 94.7 & 21.8 & 23.2 & 20.5 & 77.4 & 9.4 & 11.8 & 6.3 & 43.4 \\
\hline ST & 88.7 & 87.3 & 91.7 & 97.1 & 19.5 & 21.8 & 14.2 & 85.7 & 8.9 & 10.3 & 5.8 & 48.6 \\
\hline $\mathrm{OBC}$ & 85.1 & 82.9 & 88.1 & 92.0 & 23.8 & 24.2 & 23.2 & 77.5 & 10.8 & 13.5 & 6.8 & 42.8 \\
\hline General $^{2}$ & 80.8 & 79.4 & 82.1 & 93.3 & 24.3 & 23.5 & 25.3 & 72.8 & 11.2 & 12.8 & 9.2 & 40.7 \\
\hline \multicolumn{13}{|c|}{ Educational level (years) } \\
\hline None $^{3}$ & 83.6 & 83.4 & 85.2 & 92.5 & 22.4 & 24.1 & 12.7 & 86.0 & 15.3 & 16.9 & 6.1 & 47.7 \\
\hline $1-7$ & 83.7 & 83.5 & 84.1 & 93.3 & 22.1 & 23.7 & 17.9 & 73.3 & 8.6 & 9.5 & 6.3 & 43.8 \\
\hline $8-11$ & 82.6 & 78.9 & 85.0 & 92.5 & 20.9 & 22.8 & 19.8 & 74.4 & 8.3 & 12.7 & 5.3 & 37.8 \\
\hline 12 and above & 87.5 & 82.6 & 89.5 & 92.9 & 32.5 & 24.2 & 36.0 & 79.8 & 12.5 & 11.8 & 12.7 & 49.3 \\
\hline \multicolumn{13}{|c|}{ Worked in last 12 months } \\
\hline Yes & 85.1 & 84.2 & 86.6 & 94.1 & 25.5 & 26.4 & 24.1 & 79.9 & 12.7 & 15.3 & 8.6 & 47.9 \\
\hline No & 82.6 & 80.2 & 85.4 & 90.3 & 21.2 & 20.8 & 21.7 & 69.2 & 8.4 & 10.2 & 6.3 & 31.5 \\
\hline \multicolumn{13}{|l|}{ Wealth quintile } \\
\hline First & 82.3 & 82.4 & 82.0 & 93.7 & 21.8 & 22.7 & 20.4 & 78.6 & 11.9 & 14.4 & 7.0 & 50.2 \\
\hline Second & 86.0 & 86.2 & 85.6 & 92.6 & 25.0 & 28.0 & 19.9 & 74.9 & 12.2 & 16.4 & 5.2 & 42.2 \\
\hline Third & 84.3 & 82.5 & 86.7 & 91.0 & 21.4 & 21.9 & 20.7 & 69.9 & 9.4 & 10.9 & 7.0 & 35.9 \\
\hline Fourth & 83.5 & 81.6 & 85.5 & 93.1 & 22.5 & 23.4 & 21.6 & 77.9 & 8.3 & 9.7 & 6.7 & 44.8 \\
\hline Fifth & 82.7 & 76.0 & 87.6 & 93.7 & 25.5 & 21.4 & 28.6 & 79.8 & 10.8 & 12.6 & 9.6 & 42.4 \\
\hline Total & 83.8 & 82.2 & 85.9 & 92.8 & 23.2 & 23.6 & 22.8 & 76.3 & 10.4 & 12.8 & 7.3 & 42.4 \\
\hline \multicolumn{13}{|c|}{ Urban } \\
\hline \multicolumn{13}{|l|}{ Age (years) } \\
\hline $15-19$ & 84.8 & 78.0 & 86.6 & 94.4 & 17.4 & 18.6 & 17.0 & 72.6 & 7.3 & 11.1 & 6.3 & 33.6 \\
\hline $20-24$ & 86.6 & 85.0 & 89.7 & 94.5 & 25.6 & 21.9 & 33.0 & 82.9 & 12.5 & 13.0 & 11.7 & 55.5 \\
\hline \multicolumn{13}{|l|}{ Religion } \\
\hline Hindu & 88.0 & 85.6 & 90.1 & 95.3 & 23.5 & 22.5 & 24.2 & 79.0 & 9.5 & 11.5 & 7.8 & 45.6 \\
\hline Muslim & 72.1 & 70.9 & 73.1 & 92.2 & 15.1 & 16.4 & 13.4 & 75.6 & 14.0 & 21.8 & 8.4 & 40.0 \\
\hline Other $^{1}$ & 82.6 & 77.8 & 86.8 & 90.3 & 14.2 & 13.3 & 15.8 & 63.3 & 9.2 & 11.1 & 9.2 & 38.7 \\
\hline \multicolumn{13}{|l|}{ Caste } \\
\hline SC & 82.9 & 78.3 & 86.5 & 95.1 & 15.0 & 13.3 & 16.5 & 75.5 & 8.7 & 10.8 & 6.5 & 44.1 \\
\hline ST & (93.5) & $(88.2)$ & * & (100.0) & (29.0) & $(25.0)$ & * & $(90.0)$ & $(10.0)$ & $(17.6)$ & * & $(45.0)$ \\
\hline $\mathrm{OBC}$ & 89.7 & 87.7 & 91.3 & 95.0 & 23.0 & 23.1 & 23.0 & 78.9 & 9.0 & 10.4 & 7.4 & 47.7 \\
\hline General $^{2}$ & 81.4 & 78.1 & 83.3 & 93.2 & 24.0 & 24.2 & 24.1 & 76.1 & 12.9 & 18.0 & 10.0 & 40.3 \\
\hline \multicolumn{13}{|c|}{ Educational level (years) } \\
\hline None $^{3}$ & 80.6 & 81.3 & $(76.3)$ & (90.5) & 15.3 & 16.7 & (7.9) & $(76.2)$ & 13.3 & 14.4 & (7.9) & $(42.9)$ \\
\hline $1-7$ & 85.7 & 85.6 & 85.7 & 96.2 & 19.4 & 18.8 & 21.0 & 77.4 & 9.3 & 11.2 & 5.9 & 48.1 \\
\hline $8-11$ & 84.7 & 81.8 & 86.6 & 94.7 & 18.1 & 22.9 & 15.1 & 75.1 & 7.7 & 12.5 & 4.7 & 39.0 \\
\hline 12 and above & 89.1 & 84.3 & 90.5 & 93.7 & 30.8 & 26.8 & 32.3 & 81.2 & 12.9 & 14.5 & 12.4 & 49.8 \\
\hline \multicolumn{13}{|c|}{ Worked in last 12 months } \\
\hline Yes & 88.4 & 87.6 & 89.3 & 95.8 & 28.7 & 28.9 & 28.3 & 83.3 & 15.9 & 17.4 & 15.0 & 52.8 \\
\hline No & 84.8 & 82.4 & 87.0 & 92.9 & 19.4 & 18.8 & 20.0 & 69.6 & 8.1 & 11.2 & 5.6 & 32.0 \\
\hline
\end{tabular}

Cont'd on next page... 


\begin{tabular}{|c|c|c|c|c|c|c|c|c|c|c|c|c|}
\hline \multirow[t]{2}{*}{$\begin{array}{l}\text { Background } \\
\text { characteristics (\%) }\end{array}$} & $\begin{array}{c}W \\
15-24\end{array}$ & $\begin{array}{c}\text { MW } \\
15-24\end{array}$ & $\begin{array}{l}\text { UW } \\
15-24\end{array}$ & $\begin{array}{c}\text { UM } \\
15-24 \\
\end{array}$ & $\begin{array}{c}W \\
15-24\end{array}$ & $\begin{array}{c}\text { MW } \\
15-24\end{array}$ & $\begin{array}{c}\text { UW } \\
15-24\end{array}$ & $\begin{array}{c}\text { UM } \\
15-24\end{array}$ & $\begin{array}{c}\mathrm{W} \\
15-24 \\
\end{array}$ & $\begin{array}{c}\text { MW } \\
15-24 \\
\end{array}$ & $\begin{array}{c}\text { UW } \\
15-24\end{array}$ & $\begin{array}{c}\text { UM } \\
15-24 \\
\end{array}$ \\
\hline & \multicolumn{4}{|c|}{ Within village/neighbourhood } & \multicolumn{4}{|c|}{ Outside village/neighbourhood } & \multicolumn{4}{|c|}{ Health facility } \\
\hline \multicolumn{13}{|c|}{ Urban } \\
\hline \multicolumn{13}{|l|}{ Wealth quintile } \\
\hline First & 78.6 & 80.9 & 72.9 & $(95.5)$ & 18.4 & 21.3 & 14.9 & $(81.8)$ & 10.1 & 8.5 & 10.6 & $(61.9)$ \\
\hline Second & 85.4 & 87.3 & 82.3 & 91.3 & 20.2 & 19.4 & 21.3 & 72.3 & 11.5 & 16.1 & 3.3 & 38.3 \\
\hline Third & 88.4 & 87.9 & 89.1 & 91.7 & 17.4 & 18.2 & 16.7 & 75.0 & 11.1 & 14.1 & 7.6 & 45.2 \\
\hline Fourth & 85.2 & 82.1 & 87.8 & 95.2 & 21.5 & 23.4 & 19.8 & 78.8 & 9.9 & 13.7 & 6.5 & 46.0 \\
\hline Fifth & 86.5 & 80.7 & 89.3 & 95.5 & 24.4 & 20.8 & 26.0 & 78.2 & 9.5 & 10.0 & 9.3 & 42.8 \\
\hline Total & 85.7 & 83.4 & 87.6 & 94.6 & 21.7 & 21.1 & 22.1 & 77.7 & 10.0 & 12.6 & 8.0 & 44.4 \\
\hline \multicolumn{13}{|c|}{ Rural } \\
\hline Age (years) & & & & & & & & & & & & \\
\hline $15-19$ & 82.0 & 78.1 & 84.5 & 90.8 & 18.3 & 15.3 & 20.2 & 73.2 & 7.2 & 9.4 & 5.7 & 35.8 \\
\hline $20-24$ & 84.2 & 83.7 & 87.5 & 93.8 & 30.0 & 28.8 & 38.1 & 79.4 & 14.4 & 14.5 & 13.2 & 50.1 \\
\hline \multicolumn{13}{|l|}{ Religion } \\
\hline Hindu & 83.7 & 82.4 & 85.8 & 93.0 & 23.8 & 24.1 & 23.3 & 76.8 & 11.0 & 13.1 & 7.5 & 40.5 \\
\hline Muslim & 67.3 & 63.4 & 74.4 & 81.9 & 21.1 & 20.7 & 21.8 & 63.0 & 8.8 & 10.9 & 6.4 & 41.7 \\
\hline Other ${ }^{1}$ & 85.5 & 86.7 & 82.4 & $(93.0)$ & 26.1 & 28.7 & 21.6 & $(77.5)$ & 7.8 & 11.4 & 0.8 & $(57.7)$ \\
\hline \multicolumn{13}{|l|}{ Caste } \\
\hline SC & 82.9 & 82.2 & 83.7 & 94.6 & 24.8 & 26.2 & 22.6 & 78.0 & 9.7 & 12.1 & 6.0 & 43.3 \\
\hline ST & 88.2 & 87.2 & 90.8 & 96.5 & 18.3 & 20.9 & 11.9 & 84.7 & 8.8 & 10.1 & 6.4 & 49.4 \\
\hline OBC & 83.4 & 81.6 & 86.5 & 90.5 & 24.1 & 24.5 & 23.4 & 76.9 & 11.5 & 14.4 & 6.5 & 40.5 \\
\hline General $^{2}$ & 80.4 & 79.8 & 81.1 & 93.3 & 24.6 & 23.2 & 26.6 & 70.9 & 10.1 & 11.0 & 8.5 & 40.9 \\
\hline \multicolumn{13}{|c|}{ Educational level(years) } \\
\hline None $^{3}$ & 84.1 & 83.7 & 87.4 & 92.9 & 23.6 & 25.1 & 13.3 & 88.4 & 15.6 & 17.2 & 5.6 & 48.2 \\
\hline $1-7$ & 83.2 & 83.0 & 83.7 & 92.3 & 22.9 & 24.9 & 16.9 & 71.9 & 8.4 & 9.2 & 6.4 & 42.5 \\
\hline $8-11$ & 81.5 & 77.5 & 84.3 & 91.7 & 22.3 & 22.7 & 22.0 & 74.2 & 8.6 & 12.8 & 5.6 & 37.4 \\
\hline 12 and above & 85.4 & 79.7 & 88.0 & 92.3 & 34.9 & 21.5 & 41.0 & 78.6 & 11.9 & 8.9 & 13.1 & 48.9 \\
\hline \multicolumn{13}{|c|}{ Worked in last 12 months } \\
\hline Yes & 84.6 & 83.8 & 85.9 & 93.4 & 24.9 & 26.1 & 23.0 & 78.6 & 12.1 & 15.1 & 6.9 & 46.1 \\
\hline No & 81.0 & 79.1 & 83.9 & 89.0 & 22.5 & 22.0 & 23.2 & 69.0 & 8.6 & 9.7 & 6.8 & 31.2 \\
\hline \multicolumn{13}{|l|}{ Wealth quintile } \\
\hline First & 82.8 & 82.6 & 83.6 & 93.5 & 22.3 & 22.8 & 21.4 & 78.3 & 12.2 & 15.1 & 6.0 & 48.6 \\
\hline Second & 86.1 & 86.1 & 86.2 & 92.8 & 25.7 & 29.1 & 19.6 & 75.4 & 12.4 & 16.3 & 5.3 & 42.6 \\
\hline Third & 83.2 & 81.4 & 86.2 & 90.8 & 22.5 & 22.7 & 21.9 & 68.4 & 9.0 & 10.2 & 6.9 & 33.6 \\
\hline Fourth & 82.5 & 81.4 & 83.8 & 92.2 & 23.1 & 23.2 & 22.9 & 77.3 & 7.3 & 7.5 & 6.8 & 44.0 \\
\hline Fifth & 77.8 & 72.4 & 84.5 & 91.4 & 26.9 & 21.9 & 33.3 & 82.3 & 12.7 & 14.6 & 10.5 & 42.1 \\
\hline Total & 83.0 & 81.9 & 85.0 & 92.0 & 23.9 & 24.4 & 23.1 & 75.6 & 10.6 & 12.9 & 6.9 & 41.5 \\
\hline
\end{tabular}

Note: ( ) Based on 25-49 unweighted cases. ${ }^{*}$ Percentage not shown, based on fewer than 25 unweighted cases. Questions regarding freedom of movement were not asked of married men, as their mobility is generally unrestricted. OBC: Other backward caste. SC: Scheduled caste. ST: Scheduled tribe. ${ }^{1}$ Includes Christian, Buddhist, Neo-Buddhist, Sikh, Jain, Jewish, Parsi/Zoroastrian and no specified religion. ${ }^{2}$ Includes all those not belonging to SC, ST or OBC. ${ }^{3}$ Includes non-literate and literate with no formal schooling.

young women but young men belonging to scheduled tribes were more likely than others to report freedom to visit at least one location outside the village or neighbourhood unescorted. Associations were less consistent in terms of such background characteristics as education and household economic status, however, among young women, those with 12 or more years of education were markedly more likely than other young women to report freedom to visit at least one location outside the village or neighbourhood. The patterns remained by and large similar in both urban and rural areas.

Freedom to visit a health facility was also associated with age and work status for both young men and women. Associations were by and large less consistent with regard to all other background characteristics, although among unmarried young men, Hindus and Muslims were less likely than those belonging to other religions to report freedom to visit a health centre unescorted and those belonging to scheduled tribes were more likely than others to report so. 
For the most part, patterns did not differ between married and unmarried young women with regard to most background characteristics. One notable exception was the consistent positive association between education and freedom to visit places outside the village unescorted among unmarried young women. Patterns of socio-demographic differentials were, by and large, similar among rural and urban respondents, as seen in Table 7.4.

\subsection{Access to money}

In order to understand access to financial resources among youth, information was obtained on whether they had any savings, whether they owned an account in a bank or a post office and if so, whether they operated the account themselves. Results are presented in Table 7.5.

\section{Table 7.5: Access to money}

Percentage of youth who reported having any savings, owning an account in a bank or post office and operating the account themselves, according to residence, Andhra Pradesh, 2007-08

\begin{tabular}{|c|c|c|c|c|c|c|}
\hline Savings indicators (\%) & $\begin{array}{c}M \\
15-24\end{array}$ & $\begin{array}{c}\mathrm{W} \\
15-24\end{array}$ & $\begin{array}{c}\text { MM } \\
15-29\end{array}$ & $\begin{array}{c}\text { MW } \\
15-24\end{array}$ & $\begin{array}{c}\text { UM } \\
15-24\end{array}$ & $\begin{array}{c}\text { UW } \\
15-24\end{array}$ \\
\hline \multicolumn{7}{|c|}{ Combined } \\
\hline Has savings of any amount & 24.2 & 25.2 & 37.2 & 24.0 & 22.7 & 26.6 \\
\hline Ownership of a bank/post office account: & & & & & & \\
\hline In own (respondent's) name & 17.8 & 12.8 & 22.4 & 9.3 & 18.7 & 17.4 \\
\hline Jointly with someone else & 0.7 & 4.3 & 0.4 & 6.5 & 0.7 & 1.5 \\
\hline No account & 81.5 & 82.9 & 77.2 & 84.2 & 80.6 & 81.2 \\
\hline Number of respondents & 2,479 & 4,848 & 1,405 & 2,330 & 2,077 & 2,518 \\
\hline Operates bank/post office account themselves & 93.3 & 60.8 & 98.1 & 49.6 & 92.8 & 72.8 \\
\hline Number with an account & 511 & 852 & 370 & 362 & 444 & 490 \\
\hline \multicolumn{7}{|c|}{ Urban } \\
\hline Has savings of any amount & 28.8 & 29.9 & 45.1 & 25.1 & 27.3 & 33.8 \\
\hline Ownership of a bank/post office account: & & & & & & \\
\hline In own (respondent's) name & 24.7 & 17.2 & 33.9 & 10.3 & 25.2 & 22.8 \\
\hline Jointly with someone else & 0.7 & 2.9 & 0.3 & 5.3 & 0.8 & 1.0 \\
\hline No account & 74.6 & 80.1 & 65.8 & 84.8 & 74.2 & 76.3 \\
\hline Number of respondents & 1,289 & 2,126 & 648 & 1,037 & 1,132 & 1,089 \\
\hline Operates bank/post office account themselves & 95.0 & 74.0 & 99.0 & 63.0 & 94.5 & 79.9 \\
\hline Number with an account & 323 & 418 & 222 & 158 & 288 & 260 \\
\hline \multicolumn{7}{|c|}{ Rural } \\
\hline Has savings of any amount & 22.5 & 23.3 & 34.9 & 23.7 & 20.6 & 22.6 \\
\hline Ownership of a bank/post office account: & & & & & & \\
\hline In own (respondent's) name & 15.1 & 11.0 & 19.2 & 9.0 & 15.9 & 14.4 \\
\hline Jointly with someone else & 0.7 & 4.9 & 0.5 & 6.9 & 0.6 & 1.7 \\
\hline No account & 84.2 & 84.0 & 80.3 & 84.1 & 83.4 & 83.9 \\
\hline Number of respondents & 1,190 & 2,722 & 757 & 1,293 & 945 & 1,429 \\
\hline Operates bank/post office account themselves & 92.3 & 53.9 & 97.2 & 46.0 & 91.7 & 67.1 \\
\hline Number with an account & 188 & 434 & 148 & 204 & 156 & 230 \\
\hline
\end{tabular}

Note: All Ns are unweighted. Column totals may not equal 100\% due to missing cases or "don't know" responses. 
Findings suggest that young men were as likely as young women to have reported some savings (24-25\%). While similar proportions of young women and unmarried young men reported having savings (23-27\%), considerably more married young men reported so (37\%). Moreover, while marital status differences were muted for the overall and rural samples of young women, unmarried young women in urban areas were more likely than the married to report savings (34\% versus 25\%). Differences by rural-urban residence indicate that somewhat more urban youth reported some savings than did rural youth (29-30\% versus $23 \%)$.

Fewer youth reported owning a bank/post office account (17-19\%). Gender differences were narrow for the overall population; however, differences were apparent among the married among whom men were somewhat more likely than women to own an account. Differences by marital status were muted for the overall and rural samples, but notable for the urban sample. In urban areas, while married young men were somewhat more likely than their unmarried counterparts to own an account (34\% versus $26 \%$ ), a reverse pattern was evident among young women ( $16 \%$ and $24 \%$ of married and unmarried young women, respectively). Rural-urban differences were apparent among young men, with urban young men considerably more likely than rural young men to report owning a bank account ( $25 \%$ versus $16 \%)$; but difference were negligible among young women (20\% versus $16 \%)$.

With regard to operation of the account, gender differences were stark. While $93 \%$ of young men who owned an account operated it themselves, only $61 \%$ of young women who owned an account did so. Marital status differences were modest among young men; however among young women, the unmarried were more likely than the married to operate their account on their own (73\% versus 50\%). Likewise, rural-urban differences were negligible among young men, but wide among young women, among whom those in urban areas were more likely than their rural counterparts to operate their account on their own $(74 \%$ versus $54 \%)$.

\subsection{Gender role attitudes}

In order to understand gender role attitudes, youth were asked seven questions reflecting attitudes, including the relative importance attached to educating boys versus girls, the role of the husband as the main decision-maker with regard to spending money, girls' participation in decisions about their own marriages, a woman's need to take permission from her husband for any activity, the comparative performance of girls versus boys in studies, gender roles in domestic work and whether girls who dress provocatively deserve to be teased. Findings, presented in Table 7.6, suggest a mixed scenario.

Questions that were most likely to elicit egalitarian attitudes from both young men and women included whether educating boys is more important than educating girls, whether the husband should be the main decision-maker with regard to spending money, whether girls should be allowed to decide about their own marriage and whether girls who dress provocatively deserve to be teased; $64-70 \%$ of young men and $66-84 \%$ of young women expressed egalitarian views on these matters. In addition, almost three-fifths of young men and women (56-58\%) expressed egalitarian views about whether girls are usually as good as boys in studies. Questions that were least likely to elicit egalitarian responses from youth included whether women should obtain their husbands' permission for most things ( $44 \%$ of young men and $31 \%$ of young women disagreed with the statement) and whether boys should do as much domestic work as girls (17\% of young men and $30 \%$ of young women agreed with the statement).

Young men were more likely than young women to report unequal gender role attitudes in relation to four out of the seven statements and less likely to do so in relation to just one statement. For example, even though large proportions of youth believed that educating boys is no more important than educating girls, more young men than women (31\% versus $16 \%)$ expressed the traditional attitude that educating boys is more important than educating girls. Nonetheless, roughly similar percentages of young men and women agreed that the husband alone should not be the main decision-maker with regard to spending money $(70 \%$ and $66 \%$, respectively) and that girls are usually as good as boys in studies ( $56 \%$ and $58 \%$, respectively). Finally, considerably more young men than women agreed on one statement - that a woman need not obtain her husband's permission for most things (44\% versus $31 \%$ ). Patterns remained, by and large, similar in both urban and rural settings. 
Table 7.6: Gender role attitudes

Percent distribution of youth by attitudes towards gender roles, according to residence, Andhra Pradesh, 2007-08

\begin{tabular}{|c|c|c|c|c|c|c|}
\hline Gender role attitudes (\%) & $\begin{array}{c}\mathrm{M} \\
15-24\end{array}$ & $\begin{array}{c}\text { W } \\
15-24\end{array}$ & $\begin{array}{c}\text { MM } \\
15-29\end{array}$ & $\begin{array}{c}\text { MW } \\
15-24\end{array}$ & $\begin{array}{c}\text { UM } \\
15-24\end{array}$ & $\begin{array}{l}\text { UW } \\
15-24\end{array}$ \\
\hline \multicolumn{7}{|c|}{ Combined } \\
\hline \multicolumn{7}{|c|}{$\begin{array}{l}\text { Educating boys is more important than } \\
\text { educating girls }\end{array}$} \\
\hline Yes & 30.9 & 15.8 & 35.1 & 18.9 & 29.3 & 11.9 \\
\hline No & 67.5 & 83.6 & 62.6 & 80.4 & 69.1 & 87.6 \\
\hline \multicolumn{7}{|c|}{$\begin{array}{l}\text { Husband alone/mainly should decide about } \\
\text { spending money }\end{array}$} \\
\hline Yes & 29.4 & 33.5 & 34.0 & 40.9 & 28.6 & 24.0 \\
\hline No & 69.6 & 66.2 & 65.9 & 59.0 & 70.2 & 75.4 \\
\hline \multicolumn{7}{|c|}{$\begin{array}{l}\text { Girls should be allowed to decide about their } \\
\text { own marriage }\end{array}$} \\
\hline Yes & 63.6 & 77.8 & 60.8 & 76.1 & 64.6 & 80.0 \\
\hline No & 33.6 & 21.6 & 37.0 & 23.3 & 32.5 & 19.5 \\
\hline \multicolumn{7}{|c|}{$\begin{array}{l}\text { A woman should obtain her husband's permission } \\
\text { for most things }\end{array}$} \\
\hline Yes & 53.8 & 68.9 & 56.6 & 77.3 & 52.8 & 58.2 \\
\hline No & 44.3 & 30.8 & 42.8 & 22.6 & 45.0 & 41.3 \\
\hline \multicolumn{7}{|c|}{ Girls are usually as good as boys in studies } \\
\hline Yes & 56.1 & 58.4 & 61.4 & 54.0 & 54.7 & 64.1 \\
\hline No & 41.1 & 38.0 & 33.9 & 40.9 & 42.7 & 34.1 \\
\hline \multicolumn{7}{|c|}{ Boys should do as much domestic work as girls } \\
\hline Yes & 17.0 & 30.4 & 16.7 & 24.8 & 16.6 & 37.5 \\
\hline No & 82.7 & 69.1 & 82.8 & 74.6 & 83.1 & 62.2 \\
\hline \multicolumn{7}{|c|}{ Girls who dress provocatively deserve to be teased } \\
\hline Yes & 30.3 & 20.2 & 25.5 & 22.0 & 31.1 & 17.8 \\
\hline No & 69.1 & 78.9 & 72.6 & 76.8 & 68.7 & 81.7 \\
\hline Number of respondents & 2,479 & 4,848 & 1,405 & 2,330 & 2,077 & 2,518 \\
\hline \multicolumn{7}{|c|}{ Urban } \\
\hline \multicolumn{7}{|c|}{$\begin{array}{l}\text { Educating boys is more important than } \\
\text { educating girls }\end{array}$} \\
\hline Yes & 24.1 & 9.8 & 28.8 & 12.4 & 23.5 & 7.7 \\
\hline No & 73.7 & 90.0 & 69.3 & 87.2 & 74.5 & 92.1 \\
\hline \multicolumn{7}{|c|}{$\begin{array}{l}\text { Husband alone/mainly should decide about } \\
\text { spending money }\end{array}$} \\
\hline Yes & 25.6 & 21.6 & 31.7 & 26.7 & 25.2 & 17.4 \\
\hline No & 73.1 & 77.9 & 68.0 & 73.1 & 73.4 & 82.0 \\
\hline \multicolumn{7}{|c|}{$\begin{array}{l}\text { Girls should be allowed to decide about their } \\
\text { own marriage }\end{array}$} \\
\hline Yes & 68.5 & 83.3 & 66.3 & 81.9 & 68.5 & 84.4 \\
\hline No & 28.8 & 16.3 & 31.0 & 17.5 & 28.5 & 15.4 \\
\hline \multicolumn{7}{|c|}{$\begin{array}{l}\text { A woman should obtain her husband's permission } \\
\text { for most things }\end{array}$} \\
\hline Yes & 47.5 & 59.4 & 52.0 & 72.2 & 46.2 & 49.0 \\
\hline No & 50.8 & 40.2 & 47.7 & 27.4 & 51.9 & 50.5 \\
\hline \multicolumn{7}{|c|}{ Girls are usually as good as boys in studies } \\
\hline Yes & 54.3 & 62.6 & 56.0 & 57.7 & 53.0 & 66.6 \\
\hline No & 43.3 & 34.3 & 39.1 & 37.5 & 44.8 & 31.7 \\
\hline
\end{tabular}




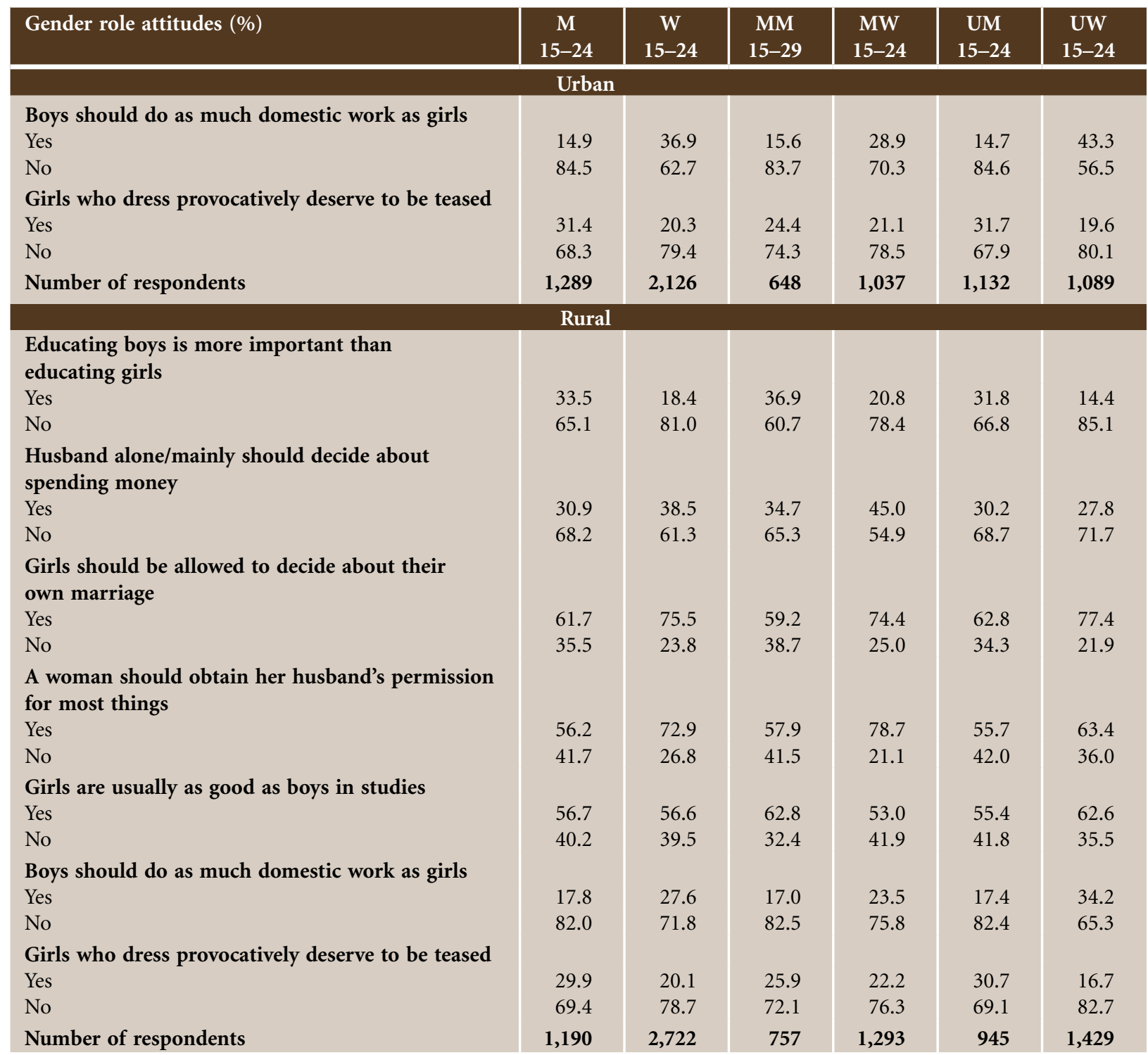

Note: All Ns are unweighted. Column totals may not equal 100\% due to missing cases, “don't know" or "unsure" responses.

Differences by marital status were modest among young men. Among young women, however, the unmarried expressed more egalitarian attitudes than the married with regard to all seven statements (see also Figure 7.3). Differences by rural-urban residence indicate that urban youth were more likely than their rural counterparts to express gender egalitarian attitudes in relation to most topics. These differences were more pronounced among young women than men. For example among young women, larger proportions of those in urban areas than in rural areas expressed egalitarian attitudes on all topics except on whether girls who dress provocatively deserve to be teased about which an equal percentage disagreed with the statement (79\%). Among young men, somewhat larger proportions of those in urban areas than in rural areas expressed egalitarian attitudes on four out of the seven topics and roughly similar percentages of urban and rural young men expressed egalitarian attitudes on the remaining three topics. 
Figure 7.3: Percentage of youth who expressed egalitarian gender role attitudes on selected issues, Andhra Pradesh, 2007-08

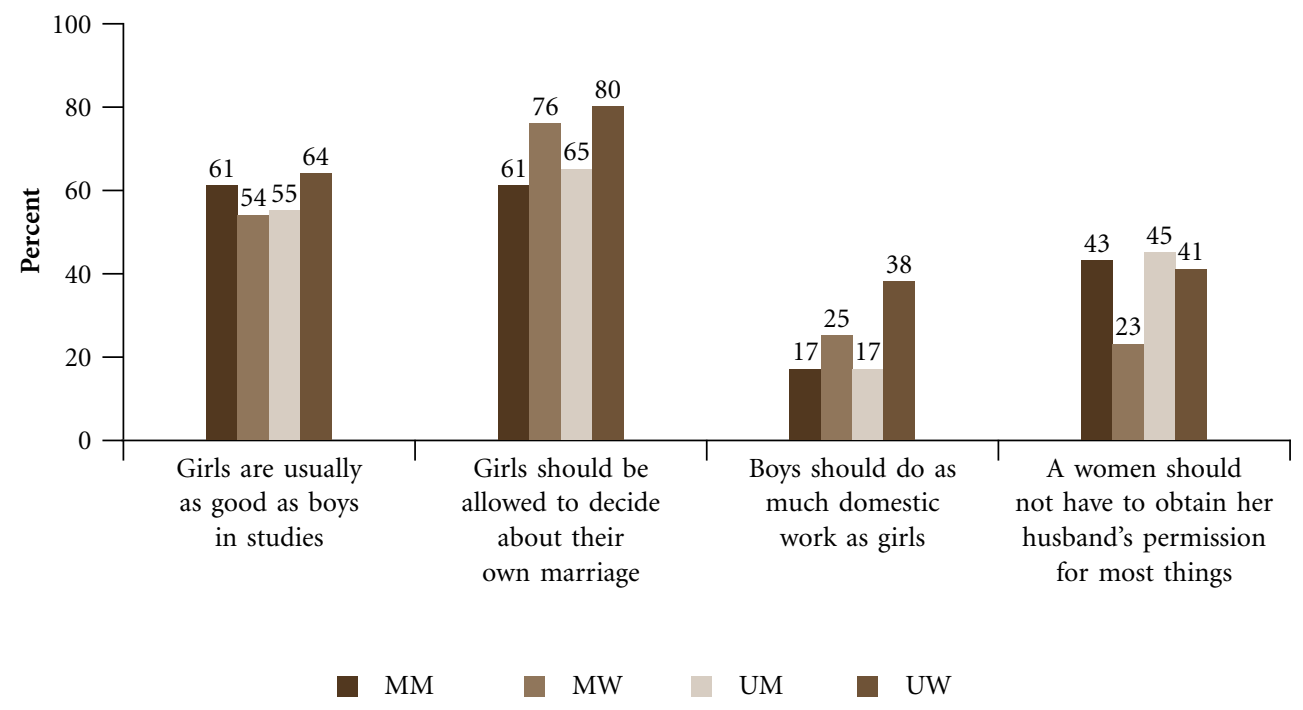

\subsection{Attitudes towards wife beating}

Youth were asked a number of questions to gauge the extent to which beating one's wife was perceived to be an acceptable behaviour. Young people were asked whether they agreed that wife beating was a way of expressing love, and whether wife beating was justified in four situations, including refusal to have sex with the husband. Findings are presented in Table 7.7. Although large proportions of youth (73\% of young men and $84 \%$ of young women) disagreed that wife beating was a sign of love, it is notable that $21 \%$ of young men and $13 \%$ of young women did conform to this view. Differences by marital status and rural-urban residence were mild.

Findings show, moreover, that the majority of youth justified wife beating. Gender differences were stark, with considerably larger percentages of young women $(88 \%)$ than young men $(63 \%)$ justifying wife beating in at least one of the four situations about which information was sought.

Differences by marital status were modest; rural-urban differences, however, suggest that more rural than urban youth justified wife beating (66\% versus 55\% among young men and $90 \%$ versus $81 \%$ among young women).

Of the four situations posed, young people, irrespective of sex, marital status and rural-urban residence, were most likely to perceive that wife beating was justified if the husband suspected that the wife had been unfaithful ( $50 \%$ of young men and $77 \%$ of young women). Young men were least likely to justify wife beating if a woman refused to have sexual relations with her husband and if a woman went out without telling her husband (25\%). Young women were least likely to justify wife beating if a woman refused to have sexual relations with her husband $(30 \%)$. Patterns remained the same among married and unmarried, and rural and urban youth. However, a larger percentage of married than unmarried young women and a larger proportion of rural than urban youth agreed that wife beating was justified in each of the four situations posed. 
Table 7.7: Attitudes towards wife beating

Percent distribution of youth by attitudes towards wife beating in selected situations, according to residence, Andhra Pradesh, 2007-08

\begin{tabular}{|c|c|c|c|c|c|c|}
\hline Attitudes towards wife beating (\%) & $\begin{array}{c}M \\
15-24\end{array}$ & $\begin{array}{c}W \\
15-24\end{array}$ & $\begin{array}{c}\text { MM } \\
15-29\end{array}$ & $\begin{array}{c}\text { MW } \\
15-24\end{array}$ & $\begin{array}{l}\text { UM } \\
15-24\end{array}$ & $\begin{array}{l}\text { UW } \\
15-24\end{array}$ \\
\hline \multicolumn{7}{|c|}{ Combined } \\
\hline Beating wife means husband loves her & & & & & & \\
\hline Agree & 21.0 & 13.2 & 24.7 & 13.7 & 20.4 & 12.5 \\
\hline Disagree & 73.3 & 84.1 & 71.7 & 84.4 & 73.2 & 83.7 \\
\hline Don't know/can't say & 5.4 & 2.7 & 2.8 & 1.8 & 6.2 & 3.8 \\
\hline \multicolumn{7}{|l|}{ Beating wife is justified if: } \\
\hline \multicolumn{7}{|l|}{ Husband suspects wife has been unfaithful } \\
\hline Yes & 50.3 & 77.3 & 51.2 & 80.8 & 49.2 & 73.0 \\
\hline No & 47.9 & 22.3 & 48.3 & 19.1 & 48.6 & 26.3 \\
\hline Don't know/can't say & 1.7 & 0.4 & 0.6 & 0.1 & 2.1 & 0.7 \\
\hline \multicolumn{7}{|l|}{ Wife goes out without telling husband } \\
\hline Yes & 25.4 & 41.9 & 24.2 & 46.3 & 25.5 & 36.4 \\
\hline No & 73.4 & 57.4 & 75.4 & 53.0 & 73.1 & 62.7 \\
\hline Don't know/can't say & 1.2 & 0.8 & 0.4 & 0.6 & 1.4 & 1.0 \\
\hline \multicolumn{7}{|l|}{ Wife disagrees with husband's opinion } \\
\hline Yes & 38.5 & 46.8 & 40.4 & 50.9 & 37.1 & 41.9 \\
\hline No & 59.4 & 52.3 & 58.9 & 48.4 & 60.5 & 56.9 \\
\hline Don't know/can't say & 2.1 & 0.9 & 0.8 & 0.7 & 2.4 & 1.2 \\
\hline \multicolumn{7}{|l|}{ Wife refuses to have sexual relations with husband } \\
\hline Yes & 24.9 & 30.3 & 26.7 & 35.0 & 24.3 & 24.3 \\
\hline No & 70.0 & 65.7 & 71.9 & 64.1 & 69.8 & 67.4 \\
\hline Don't know/can't say & 5.2 & 4.1 & 1.4 & 0.9 & 5.9 & 8.3 \\
\hline $\begin{array}{l}\text { Believed that wife beating is justified in at least one of } \\
\text { the above situations }\end{array}$ & 63.3 & 87.5 & 65.4 & 89.9 & 61.5 & 84.7 \\
\hline Number of respondents & 2,479 & 4,848 & 1,405 & 2,330 & 2,077 & 2,518 \\
\hline \multicolumn{7}{|c|}{ Urban } \\
\hline $\begin{array}{l}\text { Beating wife means husband loves her } \\
\text { Agree }\end{array}$ & 18.6 & 13.8 & 18.9 & 17.1 & 17.8 & 11.1 \\
\hline Disagree & 74.6 & 83.5 & 77.9 & 80.8 & 74.8 & 85.7 \\
\hline Don't know/can't say & 6.5 & 2.6 & 2.9 & 1.9 & 7.3 & 3.2 \\
\hline \multicolumn{7}{|l|}{$\begin{array}{l}\text { Beating wife is justified if: } \\
\text { Husband suspects wife has been unfaithful }\end{array}$} \\
\hline Yes & 42.7 & 71.3 & 43.0 & 74.5 & 41.5 & 68.8 \\
\hline No & 54.3 & 28.3 & 56.7 & 25.5 & 55.0 & 30.7 \\
\hline Don't know/can't say & 3.0 & 0.3 & 0.3 & 0.0 & 3.5 & 0.6 \\
\hline \multicolumn{7}{|l|}{ Wife goes out without telling husband } \\
\hline Yes & 20.3 & 29.9 & 18.6 & 33.2 & 19.8 & 27.2 \\
\hline No & 77.9 & 69.5 & 80.8 & 66.6 & 78.0 & 71.9 \\
\hline Don't know/can't say & 1.8 & 0.6 & 0.7 & 0.2 & 2.2 & 0.9 \\
\hline \multicolumn{7}{|l|}{ Wife disagrees with husband's opinion } \\
\hline Yes & 33.3 & 35.8 & 31.4 & 40.9 & 32.7 & 31.6 \\
\hline No & 64.3 & 63.4 & 68.0 & 58.4 & 64.6 & 67.5 \\
\hline Don't know/can't say & 2.4 & 0.8 & 0.7 & 0.8 & 2.7 & 0.9 \\
\hline \multicolumn{7}{|l|}{ Wife refuses to have sexual relations with husband } \\
\hline Yes & 22.8 & 22.2 & 19.3 & 27.4 & 22.7 & 18.1 \\
\hline No & 72.4 & 73.1 & 79.4 & 71.8 & 72.2 & 74.1 \\
\hline Don't know/can't say & 4.8 & 4.7 & 1.3 & 0.8 & 5.1 & 7.8 \\
\hline $\begin{array}{l}\text { Believed that wife beating is justified in at least one of } \\
\text { the above situations }\end{array}$ & 55.2 & 81.2 & 54.2 & 84.6 & 54.6 & 78.5 \\
\hline Number of respondents & 1,289 & 2,126 & 648 & 1,037 & 1,132 & 1,089 \\
\hline
\end{tabular}


Table 7.7: (Cont'd)

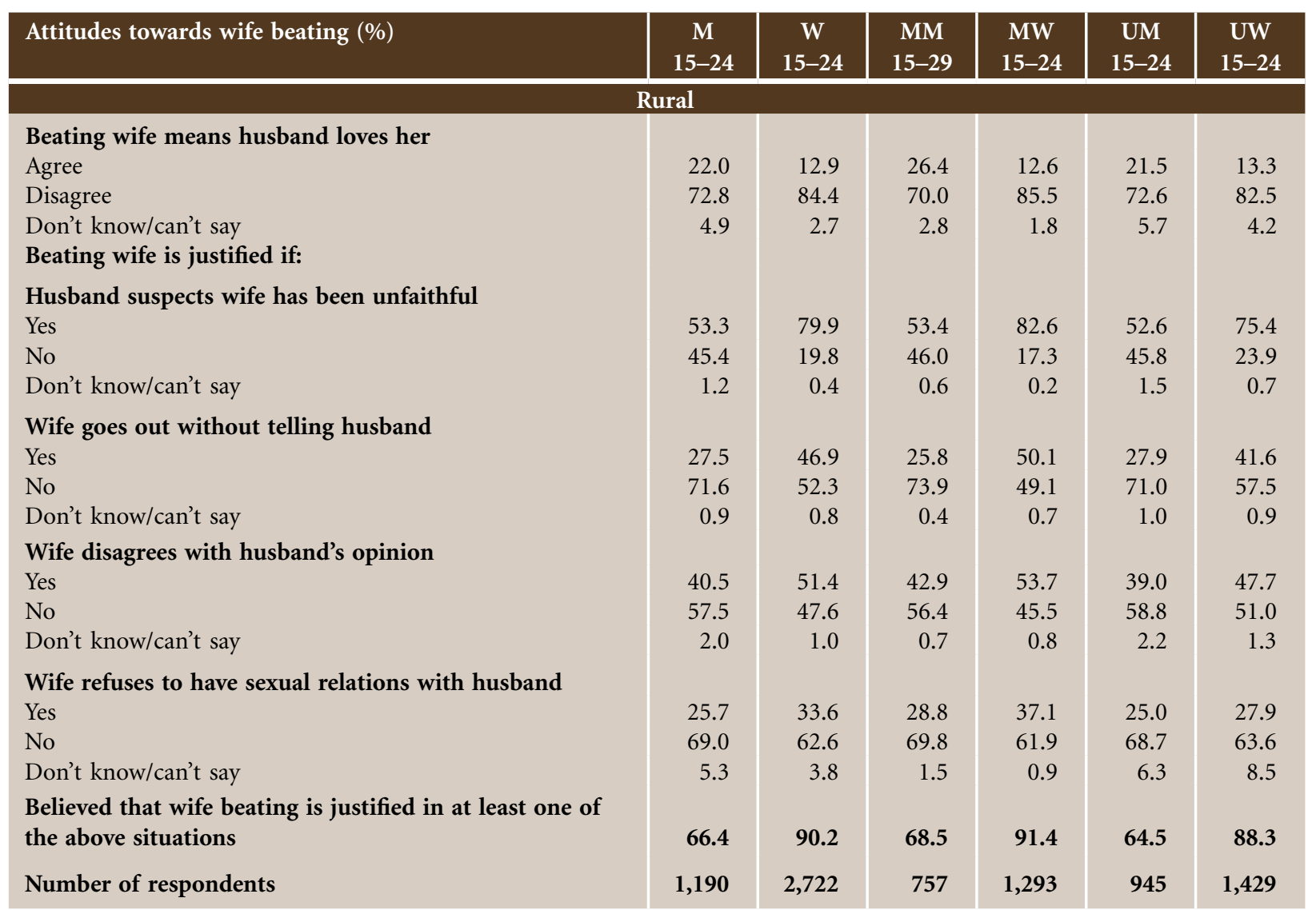

Note: All Ns are unweighted. Column totals may not equal 100\% due to missing cases.

Figure 7.4: Percentage of youth who believed wife beating is justified in selected situations, Andhra Pradesh, 2007-08

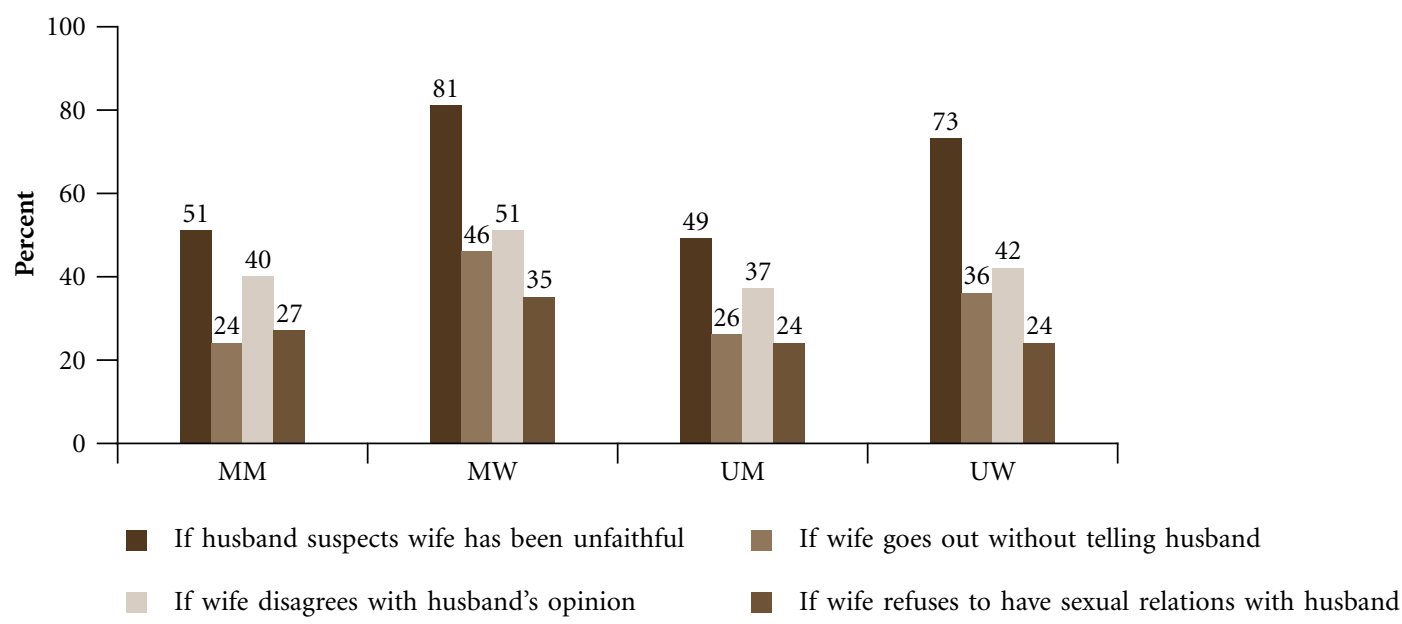




\subsection{Summary}

Findings highlight young women's limited agency. For example, just one in four young women reported independent decision-making on all three issues explored in the survey, namely, choice of friends, spending one's own money and purchase of clothes for oneself. Likewise, freedom of movement even within the village or neighbourhood was not universal among young women; just four in five young women had the freedom to visit even locations within their own village or neighbourhood unescorted. Many fewer, just one in four young women reported freedom to visit at least one place outside their village or neighbourhood unescorted, and one in 10 could visit a health facility unescorted. Access to and control over financial resources tended to be limited among young women; just one in four reported some savings and one in six owned a bank or post office savings account. Of those who owned an account, just three in five young women operated it themselves.

Within the sub-group of young women, findings indicate that the married were more disadvantaged, in some respects, than the unmarried. For example, compared to the unmarried, married young women were less likely to exercise control over financial resources, and at the same time, more likely to hold unequal gender role attitudes and to justify wife-beating. They were, however, about as likely as the unmarried to report independent decision-making and freedom of movement.

Also notable from the findings is the striking gender divide in most dimensions of young people's agency explored in the survey. Young women were far more disadvantaged than young men. For example, even the least educated young men were more likely than the most educated women to report independent decision-making on all three issues explored in the survey. Although young women were as likely as young men to have some money saved ( $25 \%$ and $24 \%$, respectively) and own a bank or post office savings account (17\% and $19 \%$, respectively), they were less likely than young men to operate these accounts themselves (61\% and $93 \%$, respectively, of those who had an account).

While young men were not as disadvantaged as young women, findings indicate that many young men were also not able to exercise agency in their everyday lives. For example, only 55\% of young men reported independent decision-making on all three issues explored in the survey. Similarly, young men's freedom to visit selected localities was far from universal; for example, just over half of unmarried young men $(55 \%)$ were allowed to visit a place of entertainment or to attend a programme conducted outside their village or neighbourhood unescorted, and just two-fifths were allowed to visit a health facility unescorted.

Relatively large proportions of youth espoused egalitarian gender role attitudes on such issues as the relative importance attached to educating boys versus girls, the role of the husband as the main decision-maker with regard to spending money, girls' participation in decisions about their own marriages and so on. Even so, it is notable that young men were generally more likely than young women to report unequal gender role attitudes. A somewhat different picture emerged, however, with regard to attitudes to wife-beating. Findings highlight widespread acceptance of violence within marriage among youth, and in this case, young women were more likely than young men to so perceive; $88 \%$ of young women compared to $63 \%$ of young men justified wife beating in at least one situation explored in the survey. 


\section{Awareness of sexual and} reproductive health matters

A considerable body of research, including the NFHS (IIPS and Macro International, 2007a), has highlighted relatively low levels of awareness regarding selected sexual and reproductive health issues in both the general and youth populations. The Youth Study sought to explore awareness of a wide range of issues relating to sex, pregnancy, contraception and STIs, including HIV/AIDS, as well as knowledge of laws governing age at marriage and abortion. Where possible, further questions were posed to assess the extent of in-depth awareness of these matters. Along with the results of these items, this chapter presents findings on communication about and sources of information for sexual and reproductive health matters, as well as youth perceptions and experiences of family life or sex education.

\subsection{Awareness of sex and pregnancy, contraception, STIs and HIV}

In this section, we present evidence of the extent to which young people were aware of or held misconceptions about various issues related to sex and pregnancy, contraception, STIs and HIV.

\subsubsection{Sex and pregnancy}

In order to assess young people's knowledge about sex and pregnancy, the Youth Study asked youth whether they agreed or disagreed with four statements: (a) a woman can get pregnant after kissing or hugging; (b) a woman is most likely to get pregnant if she has sex half-way between her periods; (c) a woman has to bleed at first intercourse; and (d) a woman can get pregnant at first sex. Given the prevalence of sex-selective abortions in the country (Bhat and Zavier, 2007; Dagar, 2007), we also asked whether youth were aware of any tests that could determine the sex of the foetus.

Findings, presented in Table 8.1, clearly suggest that awareness of sex- and pregnancy-related matters among youth was limited. The one exception was knowledge that women cannot become pregnant after kissing or hugging; $98 \%$ of young men and $96 \%$ of young women were aware of this and no differences in awareness were observed by marital status or rural-urban residence.

Awareness of other matters was reported by far smaller proportions of youth. Over two-fifths of young men and women were aware that women are most likely to become pregnant if they engage in sexual relations mid-cycle ( $45 \%$ and $41 \%$, respectively). Awareness that a woman can get pregnant at first sex was correctly reported by just $42 \%$ of young men and $62 \%$ of young women. Finally, awareness that a woman does not have to bleed at first intercourse was reported by even fewer (33\% of young men and $22 \%$ of young women).

Marital status differences were evident, with the married consistently more likely to report awareness than the unmarried. For example, more married than unmarried youth (61\% versus $41 \%$ among young men and $54 \%$ versus $24 \%$ among young women) were aware that women are most likely to become pregnant if they engage in sexual relations mid-cycle (see Figure 8.1). Likewise, more married than unmarried youth ( $49 \%$ versus $41 \%$ among young men and $70 \%$ versus $51 \%$ among young women) reported correctly that a woman can become pregnant at first sex, and that a woman does not have to bleed at first intercourse (46\% versus $31 \%$ among young men and $32 \%$ versus $10 \%$ among young women). 
Table 8.1: Awareness of sex- and pregnancy-related matters

Percent distribution of youth by awareness of sex- and pregnancy-related matters, according to residence, Andhra Pradesh, 2007-08

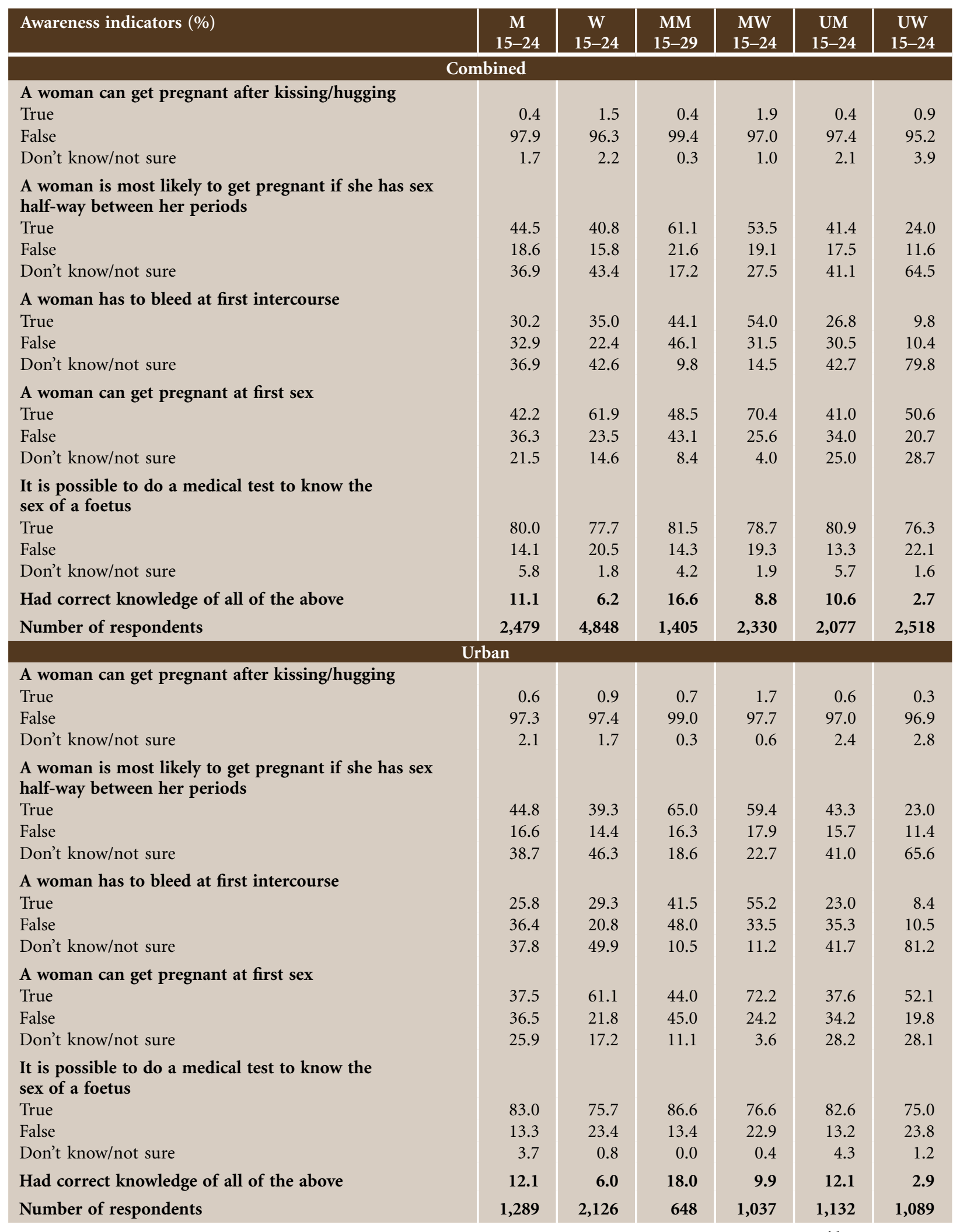

Cont'd on next page... 
Table 8.1: (Cont'd)

\begin{tabular}{|c|c|c|c|c|c|c|}
\hline Awareness indicators (\%) & $\underset{15-24}{M}$ & $\begin{array}{c}\text { W } \\
15-24\end{array}$ & $\begin{array}{c}\text { MM } \\
15-29\end{array}$ & $\begin{array}{c}\text { MW } \\
15-24\end{array}$ & $\begin{array}{c}\text { UM } \\
15-24\end{array}$ & $\begin{array}{c}\text { UW } \\
15-24\end{array}$ \\
\hline \multicolumn{7}{|c|}{ Rural } \\
\hline $\begin{array}{l}\text { A woman can get pregnant after kissing/ht } \\
\text { True }\end{array}$ & 0.2 & 1.7 & 0.3 & 2.0 & 0.3 & 1.2 \\
\hline False & 98.2 & 95.8 & 99.5 & 96.8 & 97.7 & 94.2 \\
\hline Don't know/not sure & 1.6 & 2.5 & 0.3 & 1.2 & 2.0 & 4.6 \\
\hline \multicolumn{7}{|c|}{$\begin{array}{l}\text { A woman is most likely to get pregnant if she has sex } \\
\text { half-way between her periods }\end{array}$} \\
\hline True & 44.4 & 41.4 & 60.0 & 51.7 & 40.6 & 24.5 \\
\hline False & 19.4 & 16.4 & 23.2 & 19.4 & 18.3 & 11.6 \\
\hline Don't know/not sure & 36.2 & 42.1 & 16.8 & 28.9 & 41.1 & 63.9 \\
\hline \multicolumn{7}{|l|}{ A woman has to bleed at first intercourse } \\
\hline True & 31.9 & 37.4 & 44.9 & 53.7 & 28.5 & 10.7 \\
\hline False & 31.6 & 23.1 & 45.5 & 30.9 & 28.4 & 10.3 \\
\hline Don't know/not sure & 36.5 & 39.5 & 9.6 & 15.4 & 43.1 & 79.0 \\
\hline \multicolumn{7}{|l|}{ A woman can get pregnant at first sex } \\
\hline True & 44.0 & 62.2 & 49.8 & 69.9 & 42.5 & 49.8 \\
\hline False & 36.2 & 24.2 & 42.6 & 26.0 & 34.0 & 21.1 \\
\hline Don't know/not sure & 19.8 & 13.6 & 7.6 & 4.1 & 23.6 & 29.1 \\
\hline \multicolumn{7}{|l|}{$\begin{array}{l}\text { It is possible to do a medical test to know the } \\
\text { sex of a foetus }\end{array}$} \\
\hline True & 78.8 & 78.5 & 80.0 & 79.4 & 80.1 & 77.0 \\
\hline False & 14.4 & 19.3 & 14.6 & 18.2 & 13.5 & 21.1 \\
\hline Don't know/not sure & 6.7 & 2.2 & 5.4 & 2.4 & 6.3 & 1.9 \\
\hline Had correct knowledge of all of the above & 10.8 & 6.3 & 16.2 & 8.5 & 10.0 & 2.7 \\
\hline Number of respondents & 1,190 & 2,722 & 757 & 1,293 & 945 & 1,429 \\
\hline
\end{tabular}

Note: All Ns are unweighted. Column totals may not equal $100 \%$ due to missing cases.

Figure 8.1: Percentage of youth reporting awareness of selected sex- and pregnancy-related matters, according to residence, Andhra Pradesh, 2007-08

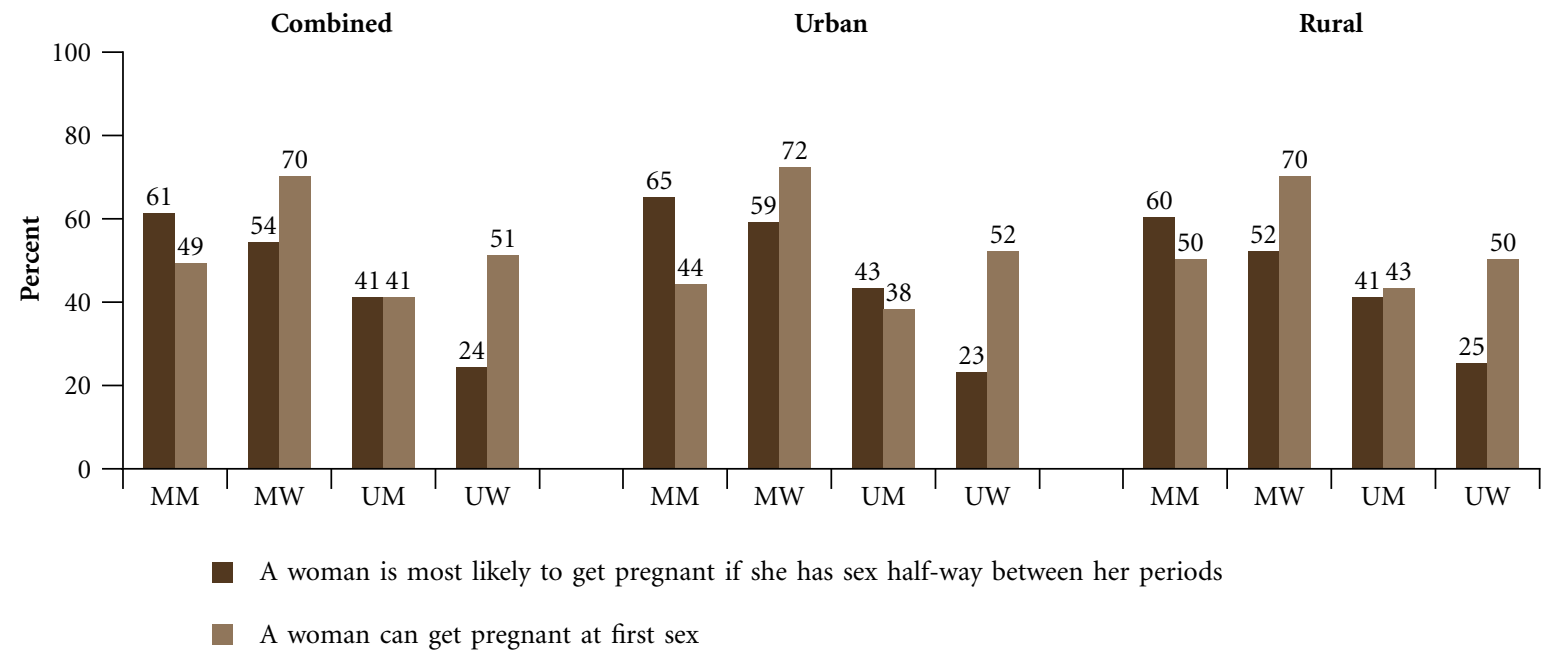

Differences by rural-urban residence were negligible on most issues among both young men and women. Indeed, the widest difference, observed among young men, suggests that those in rural areas were somewhat more likely than their urban counterparts to report correctly that a woman can become pregnant at first sex (44\% versus 38\%). 
Almost four-fifths (78-80\%) of youth were aware of the availability of tests to determine the sex of the foetus. Differences by sex, marital status and rural-urban residence were narrow.

In order to examine overall knowledge regarding sex and pregnancy, a summary measure was computed that assessed the percentage of youth who were aware of all five matters, and is presented in Table 8.1. Findings show that just $11 \%$ of young men and $6 \%$ of young women had correct knowledge of all five matters. Differences by marital status were noted; more married than unmarried youth had correct knowledge of all five issues ( $17 \%$ versus $11 \%$ among young men and $9 \%$ versus 3\% among young women). Differences by rural-urban residence were muted.

\subsubsection{Socio-demographic differentials in awareness of sex- and pregnancy-related matters}

Differentials in awareness, measured with respect to the percentage aware of all five issues relating to sex and pregnancy discussed above, are presented in Table 8.2. Levels of awareness did not differ much by background characteristics, which is not surprising given the low level of awareness observed for the overall population. However, some notable differences were apparent, particularly among young men. For example among young men, larger proportions of Hindu and Muslim men than those belonging to other religions reported awareness of all five matters, irrespective of marital status (12\% versus 3\%). Findings also show that awareness of all matters increased from 5\% among uneducated young men to $15 \%$ among those with 12 or more years of education; the differences were wider among the married than the unmarried. Although awareness of all five matters did not increase steadily with household economic status, larger proportions of those belonging to the wealthiest quintile than others reported awareness of all five matters (17\% versus $7-13 \%)$. Differentials with regard to other socio-demographic characteristics were narrow and less consistent. Among young women, too, differentials were typically narrow and consistent, except that among married young women, those belonging to scheduled tribe were less likely than others to be aware of all five matters (2\% versus $8-11 \%)$. Patterns remained more or less similar in both rural and urban settings.

\subsubsection{Awareness of contraceptive methods}

The Youth Study explored young people's awareness of contraceptive methods in several ways. First, youth were asked to list all contraceptive methods about which they had heard; second, interviewers gave respondents a brief description of a variety of non-terminal contraceptive methods not mentioned spontaneously and inquired whether the respondent had heard of each; and third, further questioning probed for specific knowledge regarding the use of oral pills, emergency contraception, condoms, the intra-uterine device (IUD) and withdrawal. Table 8.3 presents percentages of youth reporting awareness—spontaneously or on prompting — of condoms, oral contraceptives, emergency contraception, the IUD and withdrawal; and those spontaneously reporting awareness of such methods as sterilisation, implants, vaginal methods, injectables and herbal and other traditional methods. Also presented are percentages of respondents reporting correct specific knowledge of the five methods indicated above.

The vast majority of youth reported awareness (spontaneous or prompted) of at least one method of contraception and a similar range of youth were aware of at least one modern contraceptive method (97-98\%). Differences in awareness of any method, specifically, any modern method, by sex, marital status and rural-urban residence of the respondent were muted. The most widely known spacing methods were condoms (reported by $94 \%$ and $69 \%$ of young men and women, respectively) and oral contraceptives (reported by 63-65\%). Fewer youth reported awareness of emergency contraception ( $28 \%$ of young men and 18\% of young women), the IUD (5\% of young men and $22 \%$ of young women) or implants or injectables (3\%). Terminal method awareness was not probed, even so, female sterilisation was spontaneously reported by $79 \%$ of young men and $93 \%$ of young women. Fewer young people, however, spontaneously reported awareness of male sterilisation- $53 \%$ of young men and $44 \%$ of young women.

Compared to awareness of modern methods, awareness of traditional methods was reported by far fewer youth, with somewhat more young men than women reporting awareness (17\% versus $10 \%)$. The most widely known traditional method was withdrawal, reported by $16 \%$ of young men and $7 \%$ of young women. Married youth were more likely than the unmarried to report awareness of at least one traditional method ( $32 \%$ versus $14 \%$ among young men and $14 \%$ versus $5 \%$ among young women). Rural-urban differences were generally narrow, except that 
Table 8.2: Awareness of sex- and pregnancy-related matters by selected background characteristics

Percentage of youth who had correct knowledge of all five sex- and pregnancy-related matters by selected background characteristics, according to residence, Andhra Pradesh, 2007-08

\begin{tabular}{|c|c|c|c|c|c|c|}
\hline Background characteristics (\%) & $\begin{array}{c}\mathrm{M} \\
15-24\end{array}$ & $\begin{array}{c}\text { W } \\
15-24\end{array}$ & $\begin{array}{c}\text { MM } \\
15-29\end{array}$ & $\begin{array}{c}\text { MW } \\
15-24\end{array}$ & $\begin{array}{c}\text { UM } \\
15-24\end{array}$ & $\begin{array}{c}\text { UW } \\
15-24\end{array}$ \\
\hline \multicolumn{7}{|c|}{ Combined } \\
\hline \multicolumn{7}{|l|}{ Age (years) } \\
\hline $15-19$ & 9.6 & 3.9 & * & 6.6 & 9.7 & 2.5 \\
\hline $20-24$ & 12.5 & 8.6 & 13.4 & 9.8 & 12.0 & 3.6 \\
\hline $25-29$ & NA & NA & 18.1 & NA & NA & NA \\
\hline \multicolumn{7}{|l|}{ Religion } \\
\hline Hindu & 11.5 & 6.0 & 16.9 & 8.5 & 11.1 & 2.7 \\
\hline Muslim & 12.1 & 5.0 & 22.1 & 7.5 & 11.0 & 2.5 \\
\hline Other $^{1}$ & 3.0 & 8.9 & 5.7 & 12.3 & 3.0 & 4.0 \\
\hline \multicolumn{7}{|l|}{ Caste } \\
\hline SC & 12.1 & 6.8 & 14.9 & 11.0 & 11.1 & 1.6 \\
\hline ST & 7.7 & 2.4 & 12.3 & 1.8 & 10.5 & 3.3 \\
\hline $\mathrm{OBC}$ & 10.9 & 6.0 & 17.1 & 8.4 & 10.2 & 2.5 \\
\hline General $^{2}$ & 11.7 & 6.9 & 19.2 & 9.9 & 11.2 & 4.0 \\
\hline \multicolumn{7}{|l|}{ Educational level (years) } \\
\hline None $^{3}$ & 4.6 & 7.0 & 8.8 & 8.1 & 7.5 & 0.6 \\
\hline $1-7$ & 9.7 & 6.1 & 14.4 & 8.1 & 9.0 & 0.9 \\
\hline $8-11$ & 11.2 & 5.7 & 19.4 & 9.5 & 10.1 & 3.1 \\
\hline 12 and above & 14.8 & 6.4 & 26.7 & 12.4 & 13.5 & 4.0 \\
\hline \multicolumn{7}{|l|}{ Worked in last 12 months } \\
\hline Yes & 11.2 & 5.6 & 16.5 & 8.2 & 10.5 & 1.4 \\
\hline No & 11.1 & 6.8 & * & 9.4 & 11.0 & 3.8 \\
\hline \multicolumn{7}{|l|}{ Wealth quintile } \\
\hline First & 7.8 & 4.5 & 6.3 & 6.4 & 8.2 & 0.9 \\
\hline Second & 7.1 & 7.2 & 11.4 & 10.3 & 6.1 & 1.8 \\
\hline Third & 12.5 & 6.5 & 23.0 & 8.2 & 10.7 & 3.9 \\
\hline Fourth & 9.5 & 5.7 & 15.0 & 8.5 & 9.3 & 2.5 \\
\hline Fifth & 16.5 & 6.7 & 24.1 & 10.9 & 16.5 & 3.4 \\
\hline Total & 11.1 & 6.2 & 16.6 & 8.8 & 10.6 & 2.7 \\
\hline \multicolumn{7}{|c|}{ Urban } \\
\hline \multicolumn{7}{|l|}{ Age (years) } \\
\hline $15-19$ & 9.6 & 3.8 & * & 8.5 & 9.7 & 2.6 \\
\hline $20-24$ & 14.1 & 8.0 & 12.2 & 10.3 & 14.6 & 3.4 \\
\hline $25-29$ & NA & NA & 19.9 & NA & NA & NA \\
\hline \multicolumn{7}{|l|}{ Religion } \\
\hline Hindu & 12.6 & 5.7 & 18.7 & 9.6 & 12.7 & 2.4 \\
\hline Muslim & 10.1 & 5.2 & 8.6 & 9.1 & 11.1 & 1.7 \\
\hline Other ${ }^{1}$ & 8.6 & 10.0 & $(28.6)$ & 11.4 & 6.5 & 9.2 \\
\hline \multicolumn{7}{|l|}{ Caste } \\
\hline SC & 16.4 & 6.2 & 18.0 & 10.0 & 16.7 & 3.5 \\
\hline ST & $(8.7)$ & (6.7) & $(14.3)$ & (5.9) & $(10.0)$ & * \\
\hline $\mathrm{OBC}$ & 12.5 & 5.4 & 19.4 & 9.3 & 11.9 & 1.6 \\
\hline General $^{2}$ & 9.9 & 6.5 & 14.9 & 11.7 & 10.2 & 3.5 \\
\hline
\end{tabular}


Table 8.2: (Cont'd)

\begin{tabular}{|c|c|c|c|c|c|c|}
\hline Background characteristics (\%) & $\begin{array}{c}\mathrm{M} \\
15-24\end{array}$ & $\begin{array}{c}\text { W } \\
15-24\end{array}$ & $\begin{array}{c}\text { MM } \\
15-29\end{array}$ & $\begin{array}{c}\text { MW } \\
15-24\end{array}$ & $\begin{array}{c}\text { UM } \\
15-24\end{array}$ & $\begin{array}{c}\text { UW } \\
\text { 15-24 }\end{array}$ \\
\hline \multicolumn{7}{|c|}{ Urban } \\
\hline \multicolumn{7}{|l|}{ Educational level (years) } \\
\hline None $^{3}$ & 8.8 & 7.6 & 14.3 & 10.0 & $(9.5)$ & $(0.0)$ \\
\hline $1-7$ & 12.4 & 5.3 & 17.6 & 8.1 & 13.3 & 0.0 \\
\hline $8-11$ & 11.1 & 6.0 & 15.9 & 9.9 & 10.6 & 3.2 \\
\hline 12 and above & 13.7 & 6.0 & 24.2 & 13.3 & 13.9 & 3.7 \\
\hline \multicolumn{7}{|l|}{ Worked in last 12 months } \\
\hline Yes & 13.1 & 4.0 & 17.7 & 8.3 & 13.3 & 0.9 \\
\hline No & 10.5 & 6.6 & * & 10.4 & 10.3 & 3.6 \\
\hline \multicolumn{7}{|l|}{ Wealth quintile } \\
\hline First & 0.0 & 4.1 & $(5.9)$ & 6.4 & $(0.0)$ & 0.0 \\
\hline Second & 10.2 & 7.0 & 18.8 & 11.3 & 8.7 & 1.6 \\
\hline Third & 10.8 & 7.6 & 20.8 & 12.1 & 7.2 & 2.5 \\
\hline Fourth & 9.4 & 5.3 & 13.3 & 8.4 & 10.1 & 2.3 \\
\hline Fifth & 16.1 & 6.0 & 22.9 & 10.7 & 15.9 & 3.8 \\
\hline Total & 12.1 & 6.0 & 18.0 & 9.9 & 12.1 & 2.9 \\
\hline \multicolumn{7}{|c|}{ Rural } \\
\hline \multicolumn{7}{|l|}{ Age (years) } \\
\hline $15-19$ & 9.7 & 4.0 & * & 6.4 & 9.7 & 2.4 \\
\hline $20-24$ & 11.8 & 8.8 & 13.7 & 9.5 & 10.6 & 3.9 \\
\hline $25-29$ & NA & NA & 17.5 & NA & NA & NA \\
\hline \multicolumn{7}{|l|}{ Religion } \\
\hline Hindu & 11.1 & 6.1 & 16.4 & 8.2 & 10.4 & 2.8 \\
\hline Muslim & 12.9 & 4.7 & $(29.0)$ & 6.5 & 10.2 & 2.6 \\
\hline Other $^{1}$ & 1.0 & 8.4 & 1.4 & 12.7 & (1.4) & 0.8 \\
\hline \multicolumn{7}{|l|}{ Caste } \\
\hline SC & 11.2 & 7.0 & 14.0 & 11.3 & 9.7 & 0.5 \\
\hline ST & 7.5 & 1.9 & 12.0 & 2.0 & 10.5 & 1.8 \\
\hline $\mathrm{OBC}$ & 10.2 & 6.3 & 16.4 & 8.2 & 9.3 & 3.0 \\
\hline General $^{2}$ & 12.9 & 7.2 & 21.1 & 9.2 & 11.8 & 4.2 \\
\hline \multicolumn{7}{|l|}{ Educational level (years) } \\
\hline None $^{3}$ & 3.7 & 6.9 & 8.2 & 7.8 & 7.1 & 0.7 \\
\hline $1-7$ & 8.8 & 6.4 & 13.7 & 8.1 & 7.4 & 1.2 \\
\hline $8-11$ & 11.2 & 5.6 & 20.5 & 9.4 & 10.0 & 3.0 \\
\hline 12 and above & 15.5 & 6.8 & 28.1 & 11.5 & 13.1 & 4.4 \\
\hline \multicolumn{7}{|l|}{ Worked in last 12 months } \\
\hline Yes & 10.6 & 5.9 & 16.1 & 8.2 & 9.5 & 1.6 \\
\hline No & 11.4 & 6.9 & * & 8.8 & 11.2 & 4.0 \\
\hline \multicolumn{7}{|l|}{ Wealth quintile } \\
\hline First & 8.6 & 4.7 & 6.3 & 6.5 & 9.2 & 1.1 \\
\hline Second & 6.7 & 7.3 & 10.9 & 10.3 & 5.4 & 1.9 \\
\hline Third & 13.0 & 6.3 & 23.3 & 7.5 & 11.5 & 4.1 \\
\hline Fourth & 9.5 & 6.0 & 15.7 & 8.5 & 8.9 & 2.6 \\
\hline Fifth & 17.4 & 7.5 & 25.0 & 10.9 & 17.2 & 3.2 \\
\hline Total & 10.8 & 6.3 & 16.2 & 8.5 & 10.0 & 2.7 \\
\hline
\end{tabular}

Note: ( ) Based on 25-49 unweighted cases. *Percentage not shown, based on fewer than 25 unweighted cases. NA: Not applicable. OBC: Other backward caste. SC: Scheduled caste. ST: Scheduled tribe. ${ }^{1}$ Includes Christian, Buddhist, Neo-Buddhist, Sikh, Jain, Jewish, Parsi/Zoroastrian and no specified religion. ${ }^{2}$ Includes all those not belonging to SC, ST or OBC. ${ }^{3}$ Includes non-literate and literate with no formal schooling. 
married young women in urban areas were somewhat more likely than their rural counterparts to report awareness of traditional methods (19\% versus $12 \%)$.

Findings also show significant gender differences in terms of awareness of individual contraceptive methods; larger percentages of young men than women were aware of most methods (emergency contraceptive pills, condoms, male sterilisation and withdrawal). Young women, in contrast, were more likely than young men to be aware of IUDs and female sterilisation and about as likely to be aware of oral pills and implants or injectables. Differences by marital status were narrow among young men, except that more married than unmarried young men reported awareness of female sterilisation, male sterilisation and withdrawal. Among young women, the differences were more pronounced. More married than unmarried young women reported awareness of oral pills, IUDs, male sterilisation and withdrawal and conversely, more unmarried than married young women reported awareness of condoms. Rural-urban differences were modest among young men, except that those in urban areas were more likely than their rural counterparts to report awareness of emergency contraceptive pills and conversely less likely to report awareness of female sterilisation. The differences were pronounced among young women among whom those in urban areas were more likely than their rural counterparts to be aware of most methods.

In order to assess the extent to which youth had correct specific knowledge of contraceptive methods, and had not just heard of various methods, the Youth Study inquired whether youth were aware of the frequency with which oral contraceptives must be consumed (daily or weekly); the number of sex acts for which one condom could be used (one); the number of hours following sex that emergency contraceptive pills could be consumed (72 hours); where the IUD is placed (uterus); and when a man practising withdrawal should pull out of a woman (prior to ejaculation). Panel B of Table 8.3 presents percentages of youth reporting correct specific knowledge of these five methods.

Correct specific knowledge of at least one of these five methods was reported by four-fifths of young men and about one-third of young women. While differences by marital status and rural-urban differences were muted among young men, married and urban young women were more likely than their corresponding counterparts to report correct specific knowledge of at least one method (34\% and $25 \%$ of married and unmarried young women, respectively; $37 \%$ and $27 \%$ of urban and rural young women, respectively).

Differentials in correct specific knowledge of contraceptive methods by sex, marital status and rural-urban residence are evident from findings presented in Table 8.3. In general, young men were more likely than young women to report correct specific knowledge of male-oriented methods such as condoms (80\% versus $21 \%$ ) and withdrawal (15\% versus $4 \%)$. While somewhat more young women than men reported correct specific knowledge of the IUD (11\% versus $2 \%)$, roughly similar percentages of young women and men reported correct specific knowledge of oral contraceptives (15-17\%) and emergency contraceptive pills (7-9\%). Differences by marital status were mild, except that married young men were more likely than their unmarried counterparts to report specific knowledge of withdrawal (30\% versus 12\%) and married young women were more likely than the unmarried to report specific knowledge of oral contraceptives (18\% versus 10\%) and the IUD (13\% versus 8\%) (see also Figure 8.2). Differences by marital status among young women were particularly evident in urban areas where somewhat larger proportions of married young women, reported correct specific knowledge of condoms and withdrawal, compared to their unmarried counterparts. Likewise, rural-urban differences were mild, except that urban youth were somewhat more likely than their rural counterparts to report correct specific knowledge of emergency contraceptive pills and urban young women, in addition, were more likely to report correct specific knowledge of condoms.

\subsubsection{Condom-related perceptions}

Among youth who reported awareness of condoms, the Youth Study probed perceptions regarding three specific aspects of this method, namely, whether condoms are a suitable method for preventing pregnancy, whether condoms can slip off a man and disappear inside a woman's body and whether condoms reduce sexual pleasure. Findings, presented in Table 8.4, show that nine in ten young men (91\%) and three in four young women (78\%) agreed that condoms are a suitable method for preventing pregnancy. However, just $42 \%$ of young men and $13 \%$ of young 
Table 8.3: Awareness of contraceptive methods

Percentage of youth who reported awareness and correct specific knowledge of various contraceptive methods, according to residence, Andhra Pradesh, 2007-08

\begin{tabular}{|c|c|c|c|c|c|c|}
\hline Awareness indicators (\%) & $\begin{array}{c}\mathrm{M} \\
15-24\end{array}$ & $\begin{array}{c}\text { W } \\
15-24\end{array}$ & $\begin{array}{c}\text { MM } \\
15-29\end{array}$ & $\begin{array}{c}\text { MW } \\
15-24\end{array}$ & $\begin{array}{c}\text { UM } \\
15-24\end{array}$ & $\begin{array}{c}\text { UW } \\
15-24\end{array}$ \\
\hline \multicolumn{7}{|c|}{ A. Awareness } \\
\hline \multicolumn{7}{|c|}{ Combined } \\
\hline Any method & 96.9 & 97.6 & 98.2 & 98.0 & 96.7 & 97.1 \\
\hline Any modern method & 96.9 & 97.5 & 98.2 & 97.9 & 96.7 & 97.1 \\
\hline Oral pills & 65.2 & 62.6 & 65.8 & 65.6 & 66.0 & 58.5 \\
\hline Emergency contraceptive pills & 28.3 & 18.4 & 26.8 & 17.0 & 28.9 & 19.8 \\
\hline Condom & 93.5 & 68.8 & 92.5 & 63.3 & 94.0 & 75.8 \\
\hline IUD & 4.5 & 22.4 & 6.6 & 27.2 & 4.4 & 15.8 \\
\hline Female sterilisation & 79.2 & 93.2 & 86.8 & 94.2 & 77.5 & 91.9 \\
\hline Male sterilisation & 53.3 & 44.0 & 59.6 & 46.9 & 52.2 & 39.9 \\
\hline Implant/vaginal methods/injectables & 2.9 & 2.8 & 3.7 & 2.8 & 2.7 & 2.7 \\
\hline Any traditional method & 16.5 & 9.8 & 32.2 & 13.6 & 14.3 & 4.6 \\
\hline Withdrawal & 15.7 & 6.7 & 31.4 & 10.3 & 13.5 & 1.9 \\
\hline Safe period & 1.0 & 3.1 & 1.3 & 3.3 & 1.1 & 2.7 \\
\hline Traditional/herbal methods & 0.4 & 0.6 & 0.6 & 0.7 & 0.5 & 0.5 \\
\hline Number of respondents & 2,479 & 4,848 & 1,405 & 2,330 & 2,077 & 2,518 \\
\hline \multicolumn{7}{|c|}{ Urban } \\
\hline Any method & 96.9 & 98.9 & 98.7 & 99.2 & 96.7 & 98.7 \\
\hline Any modern method & 96.9 & 98.7 & 98.7 & 98.9 & 96.7 & 98.7 \\
\hline Oral pills & 67.9 & 66.9 & 73.5 & 72.4 & 68.1 & 62.4 \\
\hline Emergency contraceptive pills & 34.6 & 26.4 & 37.1 & 26.5 & 35.7 & 26.5 \\
\hline Condom & 95.8 & 79.1 & 96.7 & 75.0 & 95.9 & 82.4 \\
\hline IUD & 6.4 & 25.7 & 9.8 & 35.7 & 7.0 & 17.5 \\
\hline Female sterilisation & 73.9 & 94.1 & 84.0 & 95.0 & 72.6 & 93.4 \\
\hline Male sterilisation & 54.2 & 48.9 & 61.2 & 54.8 & 52.9 & 44.2 \\
\hline Implant/vaginal methods/injectables & 4.3 & 2.6 & 6.2 & 2.3 & 3.8 & 3.0 \\
\hline Any traditional method & 16.9 & 11.8 & 34.2 & 18.5 & 15.1 & 6.3 \\
\hline Withdrawal & 16.7 & 7.3 & 32.7 & 13.4 & 14.9 & 2.3 \\
\hline Safe period & 1.0 & 4.6 & 2.9 & 5.7 & 1.1 & 3.7 \\
\hline Traditional/herbal methods & 0.3 & 1.0 & 0.3 & 1.3 & 0.3 & 0.8 \\
\hline Number of respondents & 1,289 & 2,126 & 648 & 1,037 & 1,132 & 1,089 \\
\hline \multicolumn{7}{|c|}{ Rural } \\
\hline Any method & 96.9 & 97.0 & 98.0 & 97.6 & 96.8 & 96.1 \\
\hline Any modern method & 96.9 & 97.0 & 98.0 & 97.6 & 96.8 & 96.1 \\
\hline Oral pills & 64.1 & 60.9 & 63.7 & 63.7 & 65.1 & 56.3 \\
\hline Emergency contraceptive pills & 25.8 & 15.0 & 23.8 & 14.3 & 26.0 & 16.0 \\
\hline Condom & 92.6 & 64.5 & 91.3 & 59.8 & 93.2 & 72.1 \\
\hline IUD & 3.7 & 21.0 & 5.7 & 24.8 & 3.3 & 14.9 \\
\hline Female sterilisation & 81.3 & 92.8 & 87.5 & 93.9 & 79.6 & 91.0 \\
\hline Male sterilisation & 52.9 & 41.9 & 59.1 & 44.6 & 51.9 & 37.4 \\
\hline Implant/vaginal methods/injectables & 2.4 & 2.8 & 3.1 & 2.9 & 2.2 & 2.6 \\
\hline Any traditional method & 16.3 & 8.9 & 31.7 & 12.2 & 14.0 & 3.6 \\
\hline Withdrawal & 15.3 & 6.5 & 31.0 & 9.5 & 12.9 & 1.6 \\
\hline Safe period & 1.1 & 2.4 & 0.8 & 2.7 & 1.0 & 2.1 \\
\hline Traditional/herbal methods & 0.5 & 0.4 & 0.7 & 0.6 & 0.7 & 0.3 \\
\hline Number of respondents & 1,190 & 2,722 & 757 & 1,293 & 945 & 1,429 \\
\hline
\end{tabular}


Table 8.3: (Cont'd)

\begin{tabular}{|c|c|c|c|c|c|c|}
\hline Awareness indicators (\%) & $\begin{array}{c}\text { M } \\
15-24\end{array}$ & $\begin{array}{c}\text { W } \\
15-24\end{array}$ & $\begin{array}{c}\text { MM } \\
15-29\end{array}$ & $\begin{array}{c}\text { MW } \\
15-24\end{array}$ & $\begin{array}{c}\text { UM } \\
15-24\end{array}$ & $\begin{array}{c}\text { UW } \\
\text { 15-24 }\end{array}$ \\
\hline \multicolumn{7}{|c|}{ B. Correct specific knowledge ${ }^{1}$} \\
\hline \multicolumn{7}{|c|}{ Combined } \\
\hline Any method & 81.3 & 30.2 & 84.3 & 33.6 & 81.0 & 25.3 \\
\hline At least one modern method & 80.8 & 29.4 & 82.6 & 32.4 & 80.6 & 25.2 \\
\hline Oral pills & 16.8 & 14.8 & 18.6 & 18.2 & 17.0 & 10.2 \\
\hline Emergency contraceptive pills & 8.9 & 7.0 & 7.3 & 6.5 & 9.7 & 7.5 \\
\hline Condoms & 80.3 & 20.5 & 82.0 & 21.1 & 80.2 & 19.5 \\
\hline IUD & 2.2 & 10.9 & 3.9 & 13.4 & 2.2 & 7.5 \\
\hline $\begin{array}{l}\text { Any traditional method } \\
\text { Withdrawal }\end{array}$ & 14.7 & 3.5 & 29.5 & 5.8 & 12.4 & 0.6 \\
\hline Number of respondents & 2,479 & 4,848 & 1,405 & 2,330 & 2,077 & 2,518 \\
\hline \multicolumn{7}{|c|}{ Urban } \\
\hline Any method & 81.7 & 37.1 & 89.5 & 44.4 & 81.3 & 31.2 \\
\hline At least one modern method & 81.2 & 36.0 & 87.9 & 42.1 & 81.0 & 31.1 \\
\hline Oral pills & 19.4 & 16.5 & 24.1 & 22.9 & 19.7 & 11.4 \\
\hline Emergency contraceptive pills & 12.9 & 10.8 & 12.4 & 9.5 & 13.6 & 11.8 \\
\hline Condoms & 80.7 & 25.9 & 87.9 & 29.5 & 80.5 & 23.1 \\
\hline IUD & 3.4 & 12.4 & 5.6 & 16.4 & 4.0 & 9.1 \\
\hline Any traditional method & & & & & & \\
\hline Withdrawal & 15.3 & 4.3 & 31.6 & 8.8 & 13.3 & 0.8 \\
\hline Number of respondents & 1,289 & 2,126 & 648 & 1,037 & 1,132 & 1,089 \\
\hline \multicolumn{7}{|c|}{ Rural } \\
\hline Any method & 81.2 & 27.3 & 83.0 & 30.6 & 80.8 & 21.9 \\
\hline At least one modern method & 80.7 & 26.6 & 81.1 & 29.5 & 80.5 & 21.9 \\
\hline Oral pills & 15.7 & 14.1 & 17.0 & 16.9 & 15.8 & 9.4 \\
\hline Emergency contraceptive pills & 7.4 & 5.4 & 5.8 & 5.5 & 8.0 & 5.0 \\
\hline Condoms & 80.0 & 18.2 & 80.3 & 18.7 & 80.0 & 17.5 \\
\hline IUD & 1.7 & 10.3 & 3.5 & 12.5 & 1.5 & 6.7 \\
\hline \multicolumn{7}{|l|}{ Any traditional method } \\
\hline Withdrawal & 14.4 & 3.2 & 28.9 & 4.9 & 12.0 & 0.5 \\
\hline Number of respondents & 1,190 & 2,722 & 757 & 1,293 & 945 & 1,429 \\
\hline
\end{tabular}

Note: All Ns are unweighted. ${ }^{1}$ Correct specific knowledge was assessed for oral pills, emergency contraceptive pills, condoms, IUD and withdrawal. The following questions were asked (correct answers in brackets)_Oral pills: How often should a woman take pills? [Daily/Weekly]; Emergency contraceptive pills: How soon after sexual intercourse should these pills be taken? [72 hours]; Condoms: For how many acts of sexual intercourse can one condom be used? [One]; IUD: Where is the IUD placed? [Uterus]; Withdrawal: When should a man pull out of a woman during sexual intercourse? [Prior to ejaculation].

women were aware that condoms cannot disappear inside a woman's body and just $23 \%$ and $11 \%$, respectively, felt that condoms do not reduce sexual pleasure. Notably, $40-50 \%$ of young men and $77-80 \%$ of young women reported that they were unsure about these two aspects.

Marital status differences suggest that the married were better informed about condoms than were the unmarried. For example, $52 \%$ of married young men compared to $40 \%$ of unmarried young men were aware that condoms cannot disappear inside a woman's body; the corresponding percentages among young women were $17 \%$ and $9 \%$. Likewise, $30 \%$ of married young men compared to $21 \%$ of their unmarried counterparts, and $14 \%$ of married young women compared to $8 \%$ of unmarried young women believed that condoms do not reduce sexual pleasure. Rural-urban differences were negligible, except that somewhat more urban than rural young women agreed that condoms are a suitable method for preventing pregnancy ( $82 \%$ versus $76 \%)$. 
Figure 8.2: Percentage of youth who reported correct specific knowledge of oral pills and condoms, according to residence, Andhra Pradesh, 2007-08

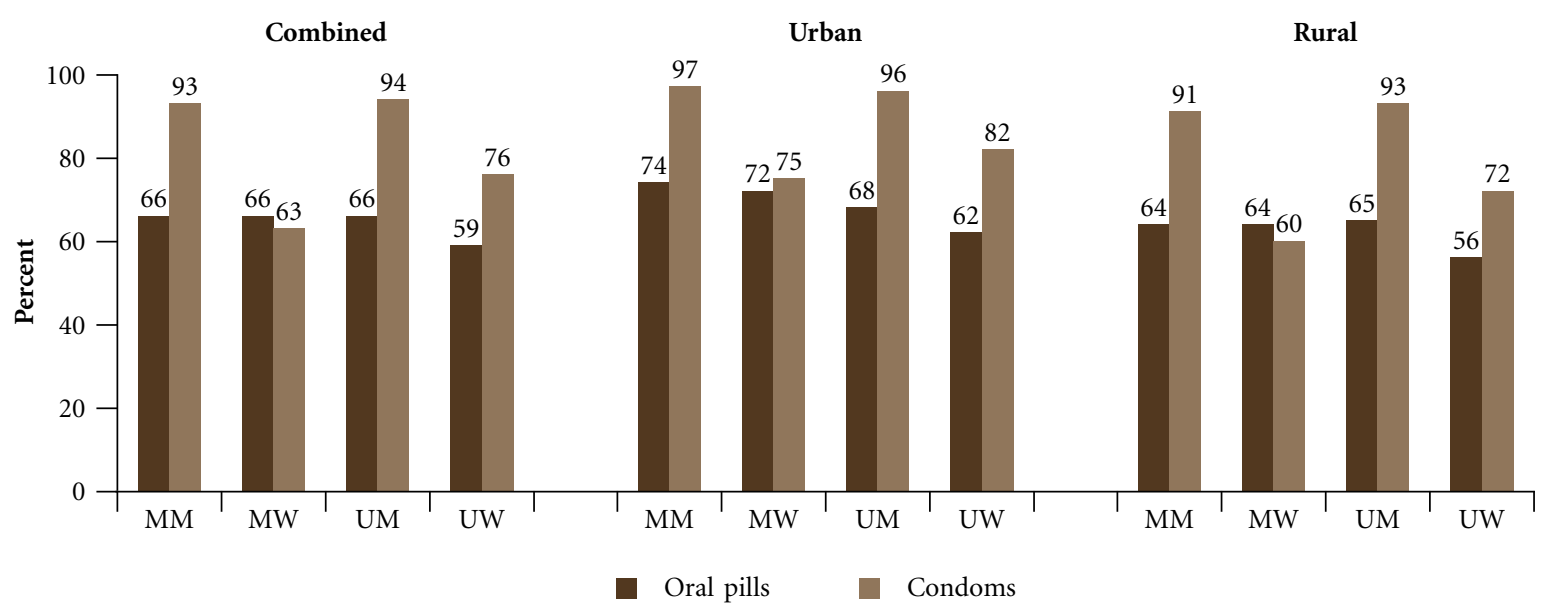

\subsubsection{Awareness of contraception prior to marriage}

Married youth were specifically asked whether they had been aware of contraception or had known where to obtain contraceptives prior to their marriage. Findings, presented in Table 8.5, suggest that among those who were aware of at least one method of contraception at the time of the interview, pre-marital awareness of any method was far from universal and young women were particularly poorly informed. For example, three-fifths of young men $(60 \%)$ compared to only one-fifth of young women (22\%) had been aware of a contraceptive method before marriage. Rural-urban differences were evident: more urban than rural youth had been aware of a contraceptive method before marriage (68\% and $58 \%$ of urban and rural young men, respectively; and $28 \%$ and $20 \%$ of urban and rural young women, respectively were aware of a contraceptive method prior to marriage). Somewhat fewer married youth knew, before marriage, about where to obtain contraceptives- $58 \%$ of young men and $17 \%$ of young women. Rural-urban differentials, noted above, persisted.

\subsubsection{Awareness of medical abortion}

Given that medical abortion, that is, the mifepristone-misoprostol regimen, has been approved for use in India since 2002, youth were asked if they were aware of "any pills" that a woman could take to terminate a pregnancy. As evident from Figure 8.3, over two-fifths of youth (42-44\%) reported that they were aware of such a method (since we did not probe further, some of these positive responses may not have specifically referred to the mifepristone-misoprostol combination, but rather to the variety of herbal and ayurvedic medications and other home remedies available). About $30 \%$ of young men and $41 \%$ of young women reported that they were unsure whether such a means of inducing abortion existed. Somewhat more married than unmarried youth were aware of medical abortion (46-47\% versus $40-42 \%$ ). Rural-urban differences were negligible; however, married young men and women in urban areas were somewhat more likely than their rural counterparts to report awareness (52-53\% compared to $45 \%)$.

\subsubsection{Awareness of sexually transmitted infections (STIs) and HIV/AIDS}

The Youth Study inquired whether youth had ever heard of infections that were transmitted through sexual contact. Findings, presented in Table 8.6, suggest that awareness of STIs other than HIV/AIDS was extremely limited. Indeed, just $30 \%$ of young men and $16 \%$ of young women reported awareness of STIs. Differences by marital status and rural-urban residence were muted, except that somewhat more urban than rural young men reported awareness of STIs (34\% versus 28\%). Rural-urban differences were particularly pronounced among married young men ( $41 \%$ versus $32 \%$ among married young men, compared to $34 \%$ versus $29 \%$ among unmarried young men). 
Table 8.4: Perceptions of selected issues related to condom use

Percent distribution of youth by their perceptions of condom use, according to residence, Andhra Pradesh, 2007-08

\begin{tabular}{|c|c|c|c|c|c|c|}
\hline Perceptions (\%) & $\begin{array}{c}M \\
15-24\end{array}$ & $\begin{array}{c}\text { W } \\
15-24\end{array}$ & $\begin{array}{c}\text { MM } \\
15-29\end{array}$ & $\begin{array}{l}\text { MW } \\
15-24\end{array}$ & $\begin{array}{c}\text { UM } \\
15-24\end{array}$ & $\begin{array}{l}\text { UW } \\
15-24\end{array}$ \\
\hline \multicolumn{7}{|c|}{ Combined } \\
\hline $\begin{array}{l}\text { Condoms are a suitable met } \\
\text { preventing pregnancy }\end{array}$ & & & & & & \\
\hline Agree & 91.0 & 77.8 & 92.5 & 79.1 & 90.5 & 76.0 \\
\hline Disagree & 2.6 & 4.0 & 4.0 & 3.3 & 2.5 & 4.8 \\
\hline Don't know/can't say & 6.4 & 18.2 & 3.2 & 17.5 & 7.0 & 19.1 \\
\hline \multicolumn{7}{|c|}{$\begin{array}{l}\text { Condoms can slip off a man and disappear } \\
\text { inside a woman's body }\end{array}$} \\
\hline Agree & 7.7 & 6.4 & 9.8 & 9.0 & 6.7 & 3.4 \\
\hline Disagree & 42.2 & 13.2 & 51.8 & 16.8 & 40.0 & 9.2 \\
\hline Don't know/can't say & 49.9 & 80.4 & 38.2 & 74.1 & 53.2 & 87.4 \\
\hline \multicolumn{7}{|c|}{ Condoms reduce sexual pleasure } \\
\hline Agree & 37.3 & 11.6 & 47.8 & 15.8 & 35.6 & 7.0 \\
\hline Disagree & 22.9 & 11.3 & 29.7 & 14.4 & 21.1 & 7.8 \\
\hline Don’t know/can't say & 39.6 & 77.1 & 22.2 & 69.8 & 43.3 & 85.1 \\
\hline Number aware of condoms & 2,339 & 3,492 & 1,319 & 1,558 & 1,967 & 1,934 \\
\hline \multicolumn{7}{|c|}{ Urban } \\
\hline \multicolumn{7}{|c|}{$\begin{array}{l}\text { Condoms are a suitable method for } \\
\text { preventing pregnancy }\end{array}$} \\
\hline Agree & 91.3 & 82.2 & 95.6 & 84.3 & 91.2 & 80.3 \\
\hline Disagree & 2.7 & 3.6 & 1.7 & 3.3 & 2.6 & 4.0 \\
\hline Don’t know/can't say & 6.1 & 14.1 & 2.4 & 12.1 & 6.1 & 15.6 \\
\hline \multicolumn{7}{|c|}{$\begin{array}{l}\text { Condoms can slip off a man and disappear } \\
\text { inside a woman's body }\end{array}$} \\
\hline Agree & 6.8 & 5.5 & 8.1 & 8.6 & 6.1 & 3.2 \\
\hline Disagree & 42.8 & 12.2 & 58.3 & 17.2 & 40.5 & 8.3 \\
\hline Don’t know/can't say & 50.2 & 82.3 & 33.6 & 73.9 & 53.2 & 88.4 \\
\hline \multicolumn{7}{|c|}{ Condoms reduce sexual pleasure } \\
\hline Agree & 38.3 & 9.6 & 52.0 & 14.7 & 36.7 & 5.9 \\
\hline Disagree & 22.0 & 11.4 & 30.1 & 17.0 & 21.0 & 7.2 \\
\hline Don’t know/can't say & 39.5 & 78.9 & 17.9 & 68.0 & 42.1 & 86.8 \\
\hline Number aware of condoms & 1,234 & 1,677 & 625 & 778 & 1,085 & 899 \\
\hline \multicolumn{7}{|c|}{ Rural } \\
\hline \multicolumn{7}{|c|}{$\begin{array}{l}\text { Condoms are a suitable method for } \\
\text { preventing pregnancy }\end{array}$} \\
\hline Agree & 90.9 & 75.5 & 91.5 & 77.1 & 90.3 & 73.3 \\
\hline Disagree & 2.6 & 4.2 & 4.8 & 3.4 & 2.3 & 5.3 \\
\hline Don't know/can't say & 6.5 & 20.3 & 3.4 & 19.4 & 7.4 & 21.4 \\
\hline \multicolumn{7}{|c|}{$\begin{array}{l}\text { Condoms can slip off a man and disappear } \\
\text { inside a woman's body }\end{array}$} \\
\hline Agree & 8.0 & 6.8 & 10.4 & 9.3 & 7.0 & 3.5 \\
\hline Disagree & 42.0 & 13.7 & 49.8 & 16.7 & 39.8 & 9.7 \\
\hline Don't know/can't say & 49.8 & 79.5 & 39.5 & 74.1 & 53.2 & 86.6 \\
\hline \multicolumn{7}{|c|}{ Condoms reduce sexual pleasure } \\
\hline Agree & 36.9 & 12.6 & 46.6 & 16.2 & 35.1 & 7.8 \\
\hline Disagree & 23.2 & 11.2 & 29.6 & 13.4 & 21.1 & 8.2 \\
\hline Don't know/can't say & 39.7 & 76.1 & 23.5 & 70.4 & 43.8 & 84.0 \\
\hline Number aware of condoms & 1,105 & 1,815 & 694 & 780 & 882 & 1,035 \\
\hline
\end{tabular}

Note: All Ns are unweighted. Column totals may not equal 100\% due to missing cases. 
Table 8.5: Awareness of contraception prior to marriage

Percentage of married youth aware of any contraceptive method prior to marriage and percentage aware of a source of contraceptive supplies at that time, according to residence, Andhra Pradesh, 2007-08

\begin{tabular}{|c|c|c|c|c|c|c|}
\hline \multirow[t]{2}{*}{ Knowledge (\%) } & $\begin{array}{c}\mathrm{MM} \\
15-29\end{array}$ & $\begin{array}{c}\text { MW } \\
15-24\end{array}$ & $\begin{array}{c}\text { MM } \\
15-29\end{array}$ & $\begin{array}{c}\text { MW } \\
15-24\end{array}$ & $\begin{array}{c}\text { MM } \\
15-29\end{array}$ & $\begin{array}{c}\text { MW } \\
15-24\end{array}$ \\
\hline & \multicolumn{2}{|c|}{ Combined } & \multicolumn{2}{|c|}{ Urban } & \multicolumn{2}{|c|}{ Rural } \\
\hline $\begin{array}{l}\text { Aware of any contraceptive method } \\
\text { before marriage }\end{array}$ & 60.2 & 21.8 & 68.2 & 28.0 & 57.9 & 20.0 \\
\hline Aware of a contraceptive source before marriage & 58.3 & 17.3 & 66.7 & 21.7 & 55.9 & 15.9 \\
\hline $\begin{array}{l}\text { Number currently aware of at least one contraceptive } \\
\text { method }\end{array}$ & 1,382 & 2,292 & 640 & 1,029 & 742 & 1,263 \\
\hline
\end{tabular}

Note: All Ns are unweighted.

Figure 8.3: Percent distribution of youth by awareness of medical abortion, according to residence, Andhra Pradesh, 2007-08

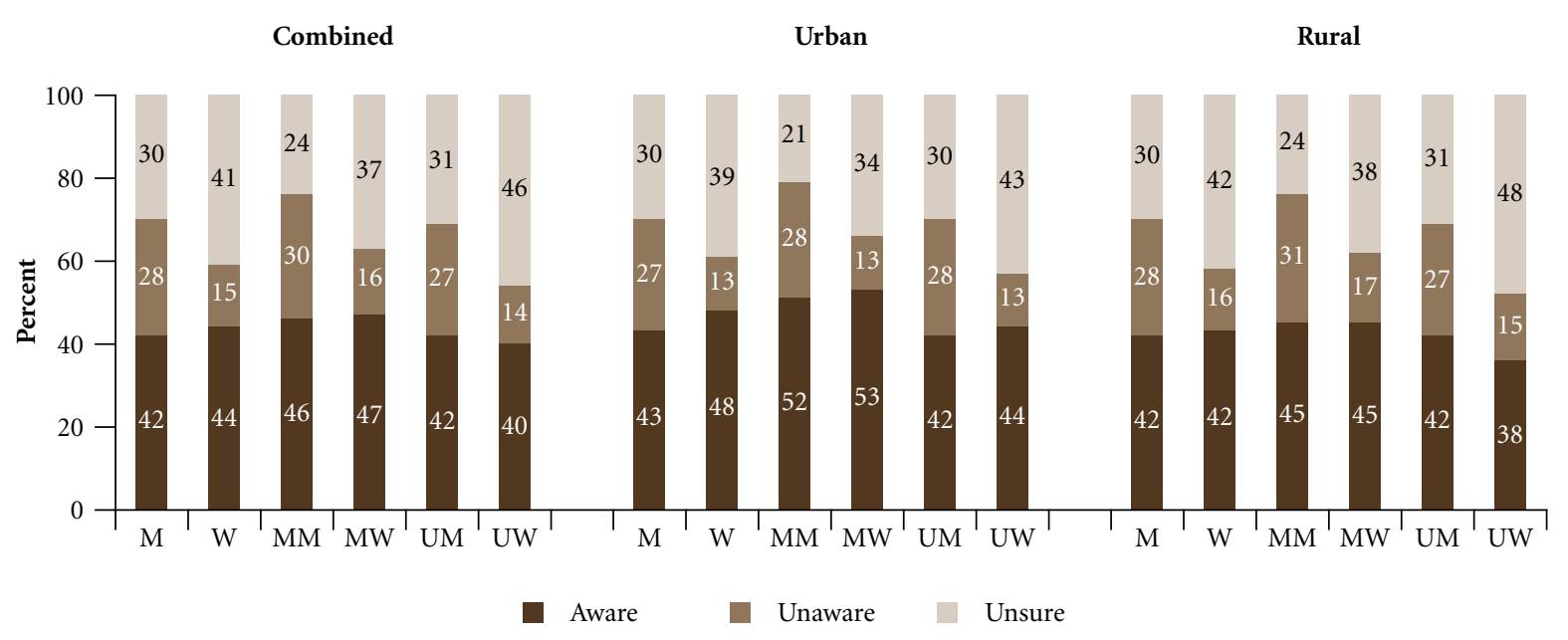

Note: Percentages may not equal 100.0 because of rounding.

Among those who were aware of STIs other than HIV, awareness of at least one symptom of infection was widespread: $94 \%$ of young men and $80 \%$ of young women could identify at least one such symptom. Differences by marital status and rural-urban residence were muted, except that more rural than urban married young men reported awareness of at least one symptom of infection ( $98 \%$ versus $89 \%)$.

Questions exploring young people's awareness of HIV/AIDS were adapted from those used in the NFHS (IIPS and Macro International, 2007b). Findings, presented in Table 8.6, show that awareness of HIV/AIDS was nearly universal; $95-96 \%$ of youth had heard of HIV/AIDS. Differences by marital status and rural-urban residence were negligible.

Notwithstanding these high overall levels of awareness of HIV, among those who reported awareness of HIV/AIDS, knowledge of specific aspects of the disease was not complete, particularly among young women. For example, $94 \%$ of young men and $83 \%$ of young women who had heard of HIV/AIDS were aware that one can reduce the chances of getting HIV by being faithful to a single partner. Awareness that one can reduce the chances of contracting HIV by using a condom every time one has sex was reported by $88 \%$ of young men and $57 \%$ of young 
Table 8.6: Awareness of STIs and HIV/AIDS

Percent distribution of youth who had heard of and had specific knowledge about STIs and HIV/AIDS, according to residence, Andhra Pradesh, 2007-08

\begin{tabular}{|c|c|c|c|c|c|c|c|c|c|}
\hline \multirow[t]{2}{*}{ Awareness among young men (\%) } & $\begin{array}{c}\text { M } \\
15-24\end{array}$ & $\begin{array}{c}\text { MM } \\
15-29\end{array}$ & $\begin{array}{c}\text { UM } \\
15-24\end{array}$ & $\begin{array}{c}\text { M } \\
15-24\end{array}$ & $\begin{array}{c}\text { MM } \\
15-29\end{array}$ & $\begin{array}{l}\text { UM } \\
15-24\end{array}$ & $\begin{array}{c}\mathrm{M} \\
15-24\end{array}$ & $\begin{array}{c}\text { MM } \\
15-29\end{array}$ & $\begin{array}{c}\text { UM } \\
15-24 \\
\end{array}$ \\
\hline & \multicolumn{3}{|c|}{ Combined } & \multicolumn{3}{|c|}{ Urban } & \multicolumn{3}{|c|}{ Rural } \\
\hline Heard about STIs ${ }^{1}$ & 29.6 & 34.4 & 30.2 & 33.8 & 41.2 & 33.6 & 27.9 & 32.4 & 28.7 \\
\hline Number of respondents & 2,479 & 1,405 & 2,077 & 1,289 & 648 & 1,132 & 1,190 & 757 & 945 \\
\hline Could identify at least one symptom of STIs & 93.8 & 95.2 & 93.1 & 90.8 & 88.9 & 91.5 & 95.0 & 97.8 & 94.0 \\
\hline Number who had heard about STIs & 766 & 511 & 651 & 432 & 267 & 378 & 334 & 244 & 273 \\
\hline Heard about HIV/AIDS & 96.4 & 94.8 & 96.8 & 97.6 & 96.4 & 97.9 & 96.0 & 94.4 & 96.3 \\
\hline Number of respondents & 2,479 & 1,405 & 2,077 & 1,289 & 648 & 1,132 & 1,190 & 757 & 945 \\
\hline \multicolumn{10}{|l|}{$\begin{array}{l}\text { Of respondents who had heard about } \\
\text { HIV/AIDS, those reporting that: }\end{array}$} \\
\hline $\begin{array}{l}\text { One can reduce one's chances of getting HIV } \\
\text { by having a single sexual partner }\end{array}$ & 93.5 & 93.2 & 93.0 & 92.7 & 93.9 & 92.6 & 93.9 & 93.0 & 93.3 \\
\hline $\begin{array}{l}\text { One can reduce one's chances of getting HIV } \\
\text { by consistent use of condoms }\end{array}$ & 87.7 & 85.5 & 88.8 & 87.8 & 89.8 & 88.2 & 87.7 & 84.2 & 88.9 \\
\hline One cannot get HIV through mosquito bites & 81.3 & 76.3 & 83.6 & 82.7 & 79.7 & 84.0 & 80.8 & 75.4 & 83.3 \\
\hline $\begin{array}{l}\text { One cannot get HIV by sharing food with an } \\
\text { HIV-positive person }\end{array}$ & 92.7 & 90.6 & 93.8 & 94.9 & 92.9 & 95.5 & 91.7 & 90.0 & 93.0 \\
\hline $\begin{array}{l}\text { One cannot get HIV by hugging an } \\
\text { HIV-positive person }\end{array}$ & 93.3 & 90.5 & 94.3 & 95.5 & 92.9 & 95.8 & 92.5 & 89.8 & 93.6 \\
\hline $\begin{array}{l}\text { One cannot tell if a person is HIV-positive by } \\
\text { just looking at him/her }\end{array}$ & 85.8 & 84.1 & 86.8 & 88.1 & 86.8 & 88.5 & 84.8 & 83.5 & 86.0 \\
\hline Number who had heard about HIV/AIDS & 2,403 & 1,340 & 2,020 & 1,259 & 623 & 1,110 & 1,144 & 717 & 910 \\
\hline \multirow[t]{2}{*}{ Awareness among young women (\%) } & $\begin{array}{c}W \\
15-24 \\
\end{array}$ & $\begin{array}{c}\text { MW } \\
15-24 \\
\end{array}$ & $\begin{array}{c}\text { UW } \\
15-24 \\
\end{array}$ & $\begin{array}{c}W \\
15-24 \\
\end{array}$ & $\begin{array}{c}\text { MW } \\
15-24 \\
\end{array}$ & $\begin{array}{c}\text { UW } \\
15-24 \\
\end{array}$ & $\begin{array}{c}W \\
15-24 \\
\end{array}$ & $\begin{array}{c}\text { MW } \\
15-24 \\
\end{array}$ & $\begin{array}{c}\text { UW } \\
15-24\end{array}$ \\
\hline & \multicolumn{3}{|c|}{ Combined } & \multicolumn{3}{|c|}{ Urban } & \multicolumn{3}{|c|}{ Rural } \\
\hline Heard about STIs ${ }^{1}$ & 15.6 & 15.8 & 15.3 & 16.6 & 18.1 & 15.4 & 15.2 & 15.1 & 15.3 \\
\hline Number of respondents & 4,848 & 2,330 & 2,518 & 2,126 & 1,037 & 1,089 & 2,722 & 1,293 & 1,429 \\
\hline Could identify at least one symptom of STIs & 80.4 & 80.9 & 80.1 & 78.9 & 77.7 & 80.0 & 81.3 & 82.0 & 80.1 \\
\hline Number who had heard about STIs & 768 & 381 & 387 & 353 & 185 & 168 & 415 & 196 & 219 \\
\hline Heard about HIV/AIDS & 94.6 & 93.0 & 96.5 & 97.9 & 97.9 & 97.9 & 93.1 & 91.6 & 95.7 \\
\hline Number of respondents & 4,848 & 2,330 & 2,518 & 2,126 & 1,037 & 1,089 & 2,722 & 1,293 & 1,429 \\
\hline \multicolumn{10}{|l|}{$\begin{array}{l}\text { Of respondents who had heard about } \\
\text { HIV/AIDS, those reporting that: }\end{array}$} \\
\hline $\begin{array}{l}\text { One can reduce one's chances of getting HIV } \\
\text { by having single sexual partner }\end{array}$ & 82.5 & 81.9 & 83.2 & 85.5 & 84.8 & 86.0 & 81.1 & 80.9 & 81.5 \\
\hline $\begin{array}{l}\text { One can reduce one's chances of getting HIV } \\
\text { by consistent use of condoms }\end{array}$ & 56.6 & 52.8 & 61.1 & 65.9 & 63.5 & 67.9 & 52.5 & 49.5 & 57.2 \\
\hline One cannot get HIV through mosquito bites & 67.5 & 62.4 & 73.9 & 75.6 & 71.2 & 79.1 & 64.0 & 59.6 & 70.8 \\
\hline $\begin{array}{l}\text { One cannot get HIV by sharing food with an } \\
\text { HIV-positive person }\end{array}$ & 83.1 & 79.0 & 88.1 & 89.7 & 85.0 & 93.4 & 80.2 & 77.1 & 85.1 \\
\hline $\begin{array}{l}\text { One cannot get HIV by hugging an } \\
\text { HIV-positive person }\end{array}$ & 80.0 & 75.3 & 85.7 & 86.8 & 83.1 & 89.8 & 76.9 & 72.8 & 83.3 \\
\hline $\begin{array}{l}\text { One cannot tell if a person is HIV-positive by } \\
\text { just looking at him/her }\end{array}$ & 88.8 & 86.7 & 91.2 & 92.6 & 91.6 & 93.5 & 87.1 & 85.2 & 89.9 \\
\hline Number who had heard about HIV/AIDS & 4,639 & 2,203 & 2,436 & 2,082 & 1,015 & 1,067 & 2,557 & 1,188 & 1,369 \\
\hline
\end{tabular}

Note: All Ns are unweighted. ${ }^{1}$ Other than HIV. 
women. Differences by marital status were narrow, except that somewhat more unmarried than married young women reported awareness of consistent condom use as a way of reducing HIV transmission (61\% versus 53\%). Rural-urban differences were muted among young men, but notable among young women among whom larger proportions of those in urban than rural areas reported awareness of consistent condom use as a way of reducing HIV transmission (66\% versus 53\%).

Findings show, moreover, that rejection of common misconceptions about modes of transmission was far from universal, and that in several instances, more young men than young women rejected these misconceptions. For example, among those aware of HIV/AIDS, $86-93 \%$ of young men and $80-89 \%$ of young women were correctly aware that one cannot get HIV by sharing food with or hugging an infected person and that one cannot tell if a person is HIV-positive by just looking at him/her. As many as $81 \%$ of young men compared to $68 \%$ of young women believed, moreover, that one cannot acquire HIV through mosquito bites. Misconceptions about modes of transmission were somewhat more likely to be rejected by unmarried than married youth, particularly among young women. For example, $84 \%$ of unmarried young men compared to $76 \%$ of married young men were aware that one cannot acquire HIV through mosquito bites; the corresponding percentages among young women were $74 \%$ and $62 \%$, respectively. Rural-urban differences suggest that more urban than rural youth, particularly young women, rejected these misconceptions.

\subsubsection{Comprehensive awareness of HIV/AIDS}

We measure comprehensive awareness of HIV/AIDS on the basis of information obtained regarding respondents' knowledge of HIV prevention and transmission. Comprehensive awareness is defined as knowledge of two ways of preventing HIV (specifically, condom use and single partner relations), rejection of common misconceptions about HIV transmission (namely, that HIV can be transmitted through mosquito bites, sharing food or hugging) and awareness that one cannot tell by looking at a person whether he or she has HIV. Findings are presented in Table 8.7 and suggest limited comprehensive awareness of HIV/AIDS, particularly among young women. For example, young men were almost twice as likely as young women to report comprehensive awareness (62\% versus $33 \%)$. More unmarried than married youth reported comprehensive awareness; for example, $64 \%$ of unmarried young men compared to $55 \%$ married young men, and $41 \%$ of unmarried young women compared to $27 \%$ married young women, respectively, reported so. Likewise, urban youth, particularly young women, were more likely to report comprehensive awareness than rural youth (66\% versus $60 \%$ among young men and $46 \%$ versus $28 \%$ among young women).

Differences in comprehensive awareness of HIV/AIDS by age were narrow for the overall sample; however, when the married and unmarried were analysed separately, a positive association was observed, particularly among both the married and the unmarried in urban areas and among unmarried young women, irrespective of rural-urban residence. Differences by religion suggest that Hindu and Muslim young men were more likely than those belonging to other religions to report comprehensive awareness of HIV/AIDS; however among young women, the association was less consistent. Caste wise differences indicate that comprehensive awareness of HIV/AIDS was most limited among youth belonging to scheduled tribes and most widespread among those belonging to general castes.

Comprehensive awareness of HIV/AIDS was greater among better educated and economically better off youth than others in all groups of youth. For example, as shown in Figure 8.4a, 33\% of married young men with no formal education displayed comprehensive HIV/AIDS awareness compared to $87 \%$ of those with 12 or more years of education; comprehensive HIV/AIDS awareness was reported likewise by just 9\% of married young women with no education compared to $63 \%$ of those with 12 or more years of education. Likewise, Figure $8.4 \mathrm{~b}$ shows that comprehensive awareness increased from $54 \%$ among unmarried young men in the poorest (first) wealth quintile to $74 \%$ among those in the wealthiest (fifth) quintile, and from $22 \%$ to $55 \%$, respectively, among unmarried young women.

Differentials with regard to religion were mild among young women; however, more Muslim and Hindu young men reported comprehensive awareness of HIV/AIDS than did those from other religions (62\% and 53\%, respectively). Caste-wise differences indicate that youth from scheduled tribes were considerably less likely to report comprehensive awareness than were those from other castes (50\% and 61-65\% respectively, among young men; $22 \%$ and $31-42 \%$, 
Table 8.7: Comprehensive knowledge of HIV/AIDS by selected background characteristics

Percentage of youth who had comprehensive knowledge of HIV/AIDS by selected background characteristics, according to residence, Andhra Pradesh, 2007-08

\begin{tabular}{|c|c|c|c|c|c|c|}
\hline Background characteristics (\%) & $\begin{array}{c}\text { M } \\
15-24\end{array}$ & $\begin{array}{c}\text { W } \\
15-24\end{array}$ & $\begin{array}{c}\text { MM } \\
15-29\end{array}$ & $\begin{array}{c}\text { MW } \\
15-24\end{array}$ & $\begin{array}{c}\text { UM } \\
15-24\end{array}$ & $\begin{array}{c}\text { UW } \\
15-24\end{array}$ \\
\hline \multicolumn{7}{|c|}{ Combined } \\
\hline $\begin{array}{l}\text { Age (years) } \\
15-19 \\
20-24 \\
25-29\end{array}$ & $\begin{array}{r}62.6 \\
61.1 \\
\text { NA }\end{array}$ & $\begin{array}{r}34.1 \\
31.9 \\
\text { NA }\end{array}$ & $\begin{array}{r}* \\
49.6 \\
57.2\end{array}$ & $\begin{array}{r}25.0 \\
27.3 \\
\text { NA }\end{array}$ & $\begin{array}{r}62.4 \\
67.1 \\
\text { NA }\end{array}$ & $\begin{array}{r}38.6 \\
49.8 \\
\text { NA }\end{array}$ \\
\hline $\begin{array}{l}\text { Religion } \\
\text { Hindu } \\
\text { Muslim } \\
\text { Other }^{1}\end{array}$ & $\begin{array}{l}62.3 \\
61.8 \\
53.4\end{array}$ & $\begin{array}{l}33.6 \\
31.3 \\
29.2\end{array}$ & $\begin{array}{l}55.2 \\
66.3 \\
41.4\end{array}$ & $\begin{array}{l}27.1 \\
25.2 \\
22.4\end{array}$ & $\begin{array}{l}65.0 \\
62.7 \\
57.4\end{array}$ & $\begin{array}{l}41.6 \\
37.1 \\
39.3\end{array}$ \\
\hline $\begin{array}{l}\text { Caste } \\
\text { SC } \\
\text { ST } \\
\text { OBC } \\
\text { General }^{2}\end{array}$ & $\begin{array}{l}62.6 \\
49.7 \\
61.0 \\
65.2\end{array}$ & $\begin{array}{l}30.9 \\
21.6 \\
31.1 \\
42.0\end{array}$ & $\begin{array}{l}51.6 \\
39.5 \\
56.2 \\
63.3\end{array}$ & $\begin{array}{l}25.1 \\
15.8 \\
25.5 \\
34.6\end{array}$ & $\begin{array}{l}66.5 \\
50.9 \\
63.9 \\
65.7\end{array}$ & $\begin{array}{l}37.9 \\
33.3 \\
38.8 \\
49.0\end{array}$ \\
\hline $\begin{array}{l}\text { Educational level (years) } \\
\text { None }^{3} \\
1-7 \\
8-11 \\
12 \text { and above }\end{array}$ & $\begin{array}{l}30.8 \\
44.5 \\
66.3 \\
80.7\end{array}$ & $\begin{array}{r}8.0 \\
20.8 \\
44.7 \\
63.8\end{array}$ & $\begin{array}{l}32.8 \\
43.3 \\
68.2 \\
87.1\end{array}$ & $\begin{array}{r}8.8 \\
23.6 \\
42.7 \\
62.7\end{array}$ & $\begin{array}{l}29.9 \\
47.9 \\
65.6 \\
80.9\end{array}$ & $\begin{array}{r}3.3 \\
13.4 \\
45.8 \\
63.9\end{array}$ \\
\hline $\begin{array}{l}\text { Worked in last } 12 \text { months } \\
\text { Yes } \\
\text { No }\end{array}$ & $\begin{array}{l}56.8 \\
75.2\end{array}$ & $\begin{array}{l}24.1 \\
41.0\end{array}$ & $\begin{array}{r}55.0 \\
*\end{array}$ & $\begin{array}{l}19.5 \\
33.9\end{array}$ & $\begin{array}{l}59.0 \\
75.3\end{array}$ & $\begin{array}{l}31.1 \\
49.0\end{array}$ \\
\hline $\begin{array}{l}\text { Wealth quintile } \\
\text { First } \\
\text { Second } \\
\text { Third } \\
\text { Fourth } \\
\text { Fifth }\end{array}$ & $\begin{array}{l}51.1 \\
56.9 \\
58.1 \\
64.2 \\
71.9\end{array}$ & $\begin{array}{l}16.6 \\
24.9 \\
31.3 \\
39.9 \\
49.3\end{array}$ & $\begin{array}{l}45.1 \\
39.6 \\
54.4 \\
62.2 \\
72.3\end{array}$ & $\begin{array}{l}13.8 \\
22.5 \\
25.9 \\
33.3 \\
41.3\end{array}$ & $\begin{array}{l}53.9 \\
62.1 \\
60.6 \\
64.5 \\
73.5\end{array}$ & $\begin{array}{l}22.0 \\
29.0 \\
39.7 \\
46.9 \\
54.5\end{array}$ \\
\hline Total & 61.8 & 33.0 & 55.2 & 26.6 & 64.4 & 41.1 \\
\hline \multicolumn{7}{|c|}{ Urban } \\
\hline $\begin{array}{l}\text { Age (years) } \\
15-19 \\
20-24 \\
25-29\end{array}$ & $\begin{array}{r}63.5 \\
68.0 \\
\text { NA }\end{array}$ & $\begin{array}{r}43.7 \\
48.2 \\
\text { NA }\end{array}$ & $\begin{array}{r}* \\
54.1 \\
65.9\end{array}$ & $\begin{array}{r}28.0 \\
44.3 \\
\text { NA }\end{array}$ & $\begin{array}{r}63.6 \\
72.3 \\
\text { NA }\end{array}$ & $\begin{array}{r}48.1 \\
55.7 \\
\text { NA }\end{array}$ \\
\hline $\begin{array}{l}\text { Religion } \\
\text { Hindu } \\
\text { Muslim } \\
\text { Other }^{1}\end{array}$ & $\begin{array}{l}66.8 \\
63.6 \\
61.8\end{array}$ & $\begin{array}{l}47.7 \\
40.7 \\
37.5\end{array}$ & $\begin{array}{r}63.4 \\
62.9 \\
(57.1)\end{array}$ & $\begin{array}{l}42.3 \\
34.5 \\
31.8\end{array}$ & $\begin{array}{l}68.8 \\
64.4 \\
63.3\end{array}$ & $\begin{array}{l}52.4 \\
44.5 \\
42.1\end{array}$ \\
\hline $\begin{array}{l}\text { Caste } \\
\text { SC } \\
\text { ST } \\
\text { OBC } \\
\text { General }^{2}\end{array}$ & $\begin{array}{r}69.6 \\
(41.7) \\
64.5 \\
69.8\end{array}$ & $\begin{array}{r}38.3 \\
(32.3) \\
45.2 \\
53.9\end{array}$ & $\begin{array}{r}62.0 \\
(28.6) \\
63.2 \\
69.0\end{array}$ & $\begin{array}{r}33.3 \\
(29.4) \\
40.0 \\
49.6\end{array}$ & $\begin{array}{r}69.6 \\
(45.0) \\
66.9 \\
70.7\end{array}$ & $\begin{array}{r}42.5 \\
* \\
50.3 \\
56.3\end{array}$ \\
\hline $\begin{array}{l}\text { Educational level (years) } \\
\text { None }^{3} \\
1-7 \\
8-11 \\
12 \text { and above }\end{array}$ & $\begin{array}{l}38.2 \\
50.7 \\
67.1 \\
78.1\end{array}$ & $\begin{array}{l}12.5 \\
24.1 \\
50.7 \\
67.7\end{array}$ & $\begin{array}{l}38.1 \\
48.9 \\
66.4 \\
92.5\end{array}$ & $\begin{array}{l}14.4 \\
28.1 \\
51.6 \\
67.5\end{array}$ & $\begin{array}{r}(42.9) \\
51.9 \\
67.6 \\
78.0\end{array}$ & $\begin{array}{r}(5.3) \\
16.8 \\
50.0 \\
67.7\end{array}$ \\
\hline
\end{tabular}

Cont'd on next page... 
Table 8.7: (Cont'd)

\begin{tabular}{|c|c|c|c|c|c|c|}
\hline Background characteristics (\%) & $\begin{array}{c}\mathrm{M} \\
15-24\end{array}$ & $\begin{array}{c}\text { W } \\
15-24\end{array}$ & $\begin{array}{c}\mathrm{MM} \\
15-29\end{array}$ & $\begin{array}{c}\text { MW } \\
15-24\end{array}$ & $\begin{array}{c}\text { UM } \\
15-24\end{array}$ & $\begin{array}{c}\text { UW } \\
15-24\end{array}$ \\
\hline \multicolumn{7}{|c|}{ Urban } \\
\hline \multicolumn{7}{|l|}{ Worked in last 12 months } \\
\hline Yes & 61.4 & 37.8 & 63.0 & 33.9 & 63.4 & 40.8 \\
\hline No & 74.5 & 48.8 & * & 42.7 & 74.7 & 53.8 \\
\hline \multicolumn{7}{|l|}{ Wealth quintile } \\
\hline First & 53.6 & 20.4 & $(41.2)$ & 19.1 & $(54.5)$ & 23.4 \\
\hline Second & 50.0 & 27.9 & 43.8 & 29.0 & 53.2 & 26.2 \\
\hline Third & 64.7 & 37.3 & 53.2 & 37.4 & 69.0 & 37.0 \\
\hline Fourth & 64.6 & 45.0 & 65.7 & 41.1 & 65.1 & 48.9 \\
\hline Fifth & 71.8 & 59.4 & 74.3 & 54.0 & 72.8 & 62.1 \\
\hline Total & 66.1 & 46.1 & 63.1 & 40.6 & 67.8 & 50.5 \\
\hline \multicolumn{7}{|c|}{ Rural } \\
\hline \multicolumn{7}{|l|}{ Age (years) } \\
\hline $15-19$ & 62.2 & 30.4 & * & 24.4 & 62.0 & 34.3 \\
\hline $20-24$ & 58.1 & 24.4 & 48.7 & 21.6 & 64.3 & 43.2 \\
\hline $25-29$ & NA & NA & 54.6 & NA & NA & NA \\
\hline \multicolumn{7}{|l|}{ Religion } \\
\hline Hindu & 60.7 & 28.1 & 53.0 & 23.0 & 63.5 & 36.1 \\
\hline Muslim & 60.5 & 22.1 & $(68.1)$ & 19.6 & 61.9 & 25.6 \\
\hline Other $^{1}$ & 50.5 & 25.7 & 38.4 & 19.9 & $(54.3)$ & 37.3 \\
\hline \multicolumn{7}{|l|}{ Caste } \\
\hline SC & 60.8 & 27.6 & 49.7 & 22.4 & 65.4 & 35.4 \\
\hline ST & 51.1 & 20.3 & 41.6 & 14.2 & 52.3 & 33.0 \\
\hline OBC & 59.6 & 25.7 & 54.0 & 21.4 & 62.5 & 33.2 \\
\hline General $^{2}$ & 62.6 & 34.7 & 61.0 & 29.4 & 63.0 & 42.4 \\
\hline \multicolumn{7}{|l|}{ Educational level (years) } \\
\hline None $^{3}$ & 29.2 & 7.3 & 31.9 & 8.0 & 27.1 & 2.1 \\
\hline $1-7$ & 42.5 & 19.8 & 42.1 & 22.5 & 46.3 & 12.2 \\
\hline $8-11$ & 66.0 & 41.7 & 68.8 & 38.5 & 64.9 & 43.9 \\
\hline 12 and above & 82.5 & 58.5 & 84.4 & 58.2 & 82.7 & 59.0 \\
\hline \multicolumn{7}{|l|}{ Worked in last 12 months } \\
\hline Yes & 55.2 & 21.7 & 52.8 & 17.8 & 57.5 & 28.5 \\
\hline No & 75.4 & 35.3 & * & 29.2 & 75.6 & 44.4 \\
\hline \multicolumn{7}{|l|}{ Wealth quintile } \\
\hline First & 50.8 & 16.0 & 45.6 & 13.2 & 53.8 & 21.7 \\
\hline Second & 57.9 & 24.5 & 39.1 & 21.6 & 63.4 & 29.4 \\
\hline Third & 56.6 & 29.8 & 54.4 & 23.3 & 58.6 & 40.6 \\
\hline Fourth & 63.9 & 36.3 & 60.8 & 29.1 & 64.2 & 45.6 \\
\hline Fifth & 72.1 & 35.4 & 70.6 & 31.8 & 74.2 & 40.2 \\
\hline Total & 60.1 & 27.5 & 53.0 & 22.5 & 62.9 & 35.7 \\
\hline
\end{tabular}

Note: ( ) Based on 25-49 unweighted cases. ${ }^{*}$ Percentage not shown, based on fewer than 25 unweighted cases. Comprehensive knowledge of HIV/AIDS includes: (1) identifying two major ways of preventing HIV (using condoms and limiting sex to one partner); (2) rejecting three common misconceptions about HIV transmission (that HIV can be transmitted through mosquito bites, sharing food with a person who has HIV and hugging someone who has HIV); and (3) knowing that a healthy looking person can be HIV-positive. NA: Not applicable. OBC: Other backward caste. SC: Scheduled caste. ST: Scheduled tribe. ${ }^{1}$ Includes Christian, Buddhist, Neo-Buddhist, Sikh, Jain, Jewish, Parsi/Zoroastrian and no specified religion. ${ }^{2}$ Includes all those not belonging to SC, ST or OBC. ${ }^{3}$ Includes non-literate and literate with no formal schooling. 
Figure 8.4a: Comprehensive knowledge of HIV/AIDS by educational level, Andhra Pradesh, 2007-08

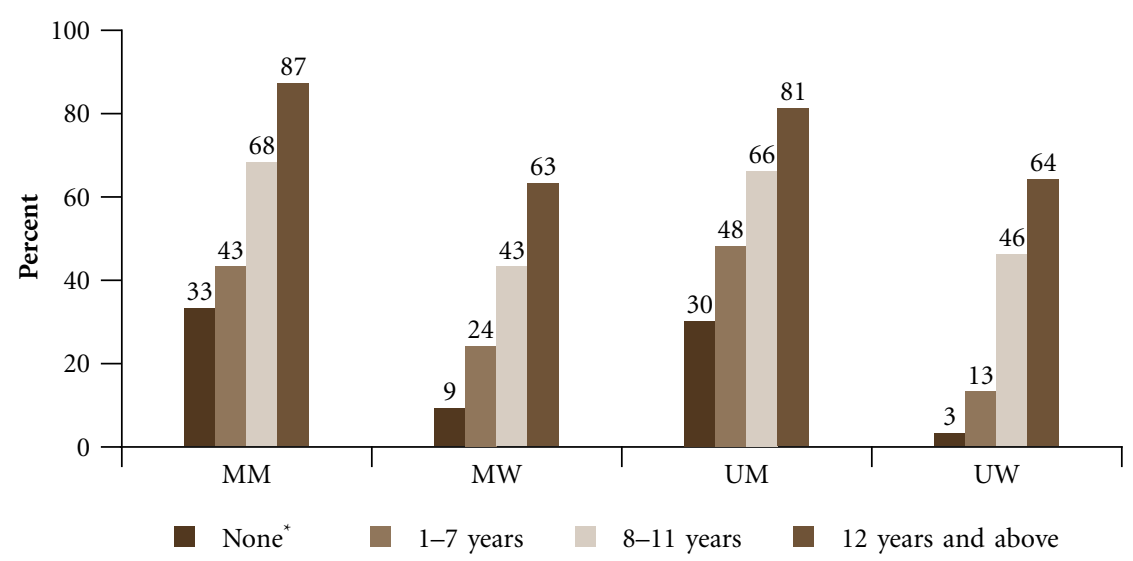

Note: ${ }^{*}$ Includes non-literate and literate with no formal schooling.

Figure 8.4b: Comprehensive knowledge of HIV/AIDS by wealth quintile, Andhra Pradesh, 2007-08

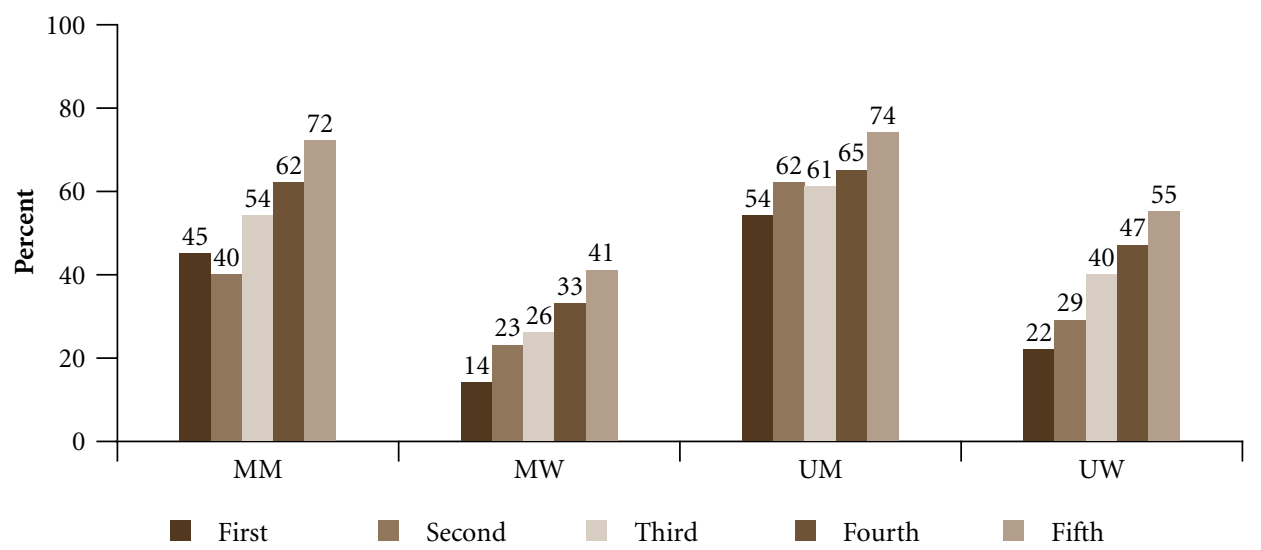

respectively, among young women). Differences by current economic activity indicate that those not engaged in any economic activity were better informed about HIV/AIDS than those who were engaged in work $(75 \%$ and $57 \%$, respectively, among young men, and $41 \%$ and $24 \%$, respectively, among young women), perhaps because many of those who were not working were in school or college and therefore more likely to be exposed to HIV-related information. Socio-demographic differences among married and unmarried youth, and rural and urban youth, more or less mirrored the pattern observed for the combined sample.

A comparison of awareness of HIV and other STIs, presented in Figure 8.5, shows that awareness of STIs other than HIV among youth was far more limited than awareness of HIV/AIDS and even comprehensive awareness of HIV/AIDS.

\subsection{Knowledge of legal issues related to marriage and abortion}

Lack of awareness of such issues as the legal minimum age at marriage and the fact that abortion services are legally available may pose barriers to health promoting behaviours. In this section, we present young people's awareness about the law on each of these issues. 
Figure 8.5: Percentage of youth by awareness of HIV/AIDS, comprehensive knowledge about HIV/AIDS and awareness of STIs, Andhra Pradesh, 2007-08

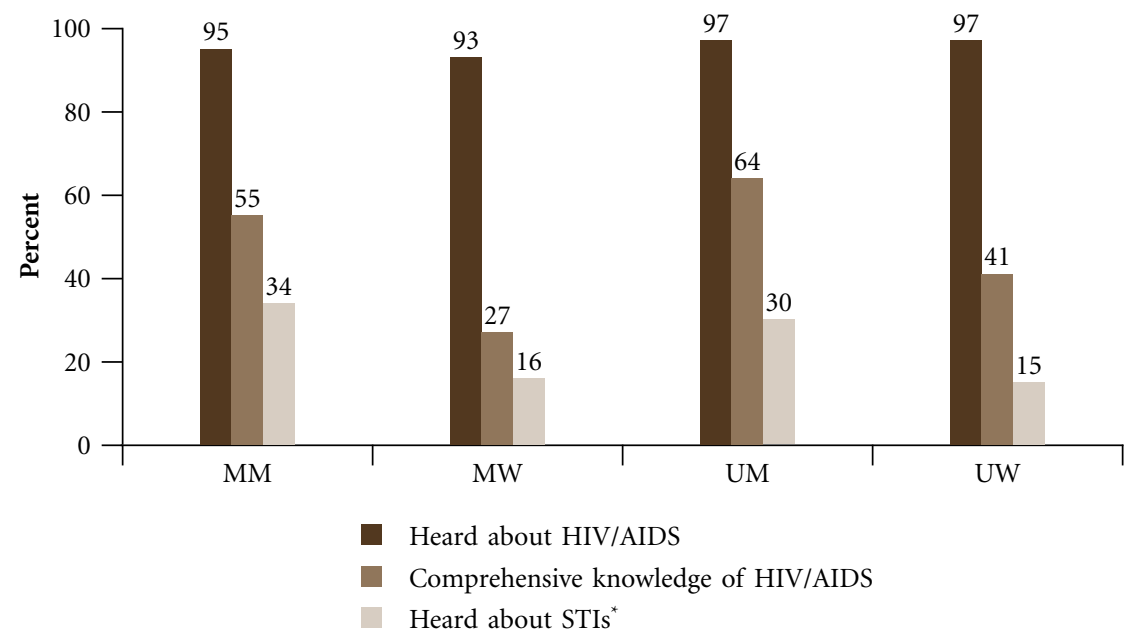

Note: ${ }^{*}$ Other than HIV.

\subsubsection{Knowledge of the legal minimum age at marriage}

The Youth Study collected information on whether youth were aware of the existence of laws relating to the legal minimum age at marriage for males and females in India and probed specific knowledge of these laws. Findings are presented in Table 8.8 and suggest that the majority of youth were aware of the existence of laws governing the minimum age at marriage for both males and females. Gender differences were, however, apparent; young men were more likely than young women to be aware of such laws, with $96 \%$ of young men compared to $88-89 \%$ of young women reporting that there is a legal age at marriage for males and females. Differences by marital status were negligible among young men; in contrast, married young women were somewhat less likely than unmarried young women to be aware of such laws (84-85\% versus $93-94 \%)$. These differences were more pronounced in rural than urban areas. Likewise, rural-urban differences were muted among young men but notable among young women among whom more urban than rural women were aware of such laws (94\% versus $86-87 \%$ ).

The correct legal minimum age at marriage for females and especially males was however, far less likely to be known. Moreover, young women were less likely than young men to be aware of the correct legal minimum age at marriage. For example, $62 \%$ of young men compared to $51 \%$ of young women correctly reported that 18 years is the legal minimum age at marriage for females; in contrast, only $38 \%$ of young men and $31 \%$ of young women correctly reported that 21 is the legal minimum age at marriage for males. Differences by marital status were mild among both young men and women. Rural-urban differences were, likewise, negligible with respect to awareness of legal minimum age at marriage for females; however, somewhat more urban than rural youth reported awareness of the correct legal minimum age at marriage for males (43\% versus $36 \%$ among young men and $36 \%$ versus $28 \%$ among young women).

\subsubsection{Awareness of the conditions under which abortion is legal}

The Youth Study posed a number of questions to gauge youth awareness of conditions under which abortion is legal, for example, if the woman is married, if the woman is unmarried, if the pregnancy exceeds 20 weeks and if the foetus is female but the couple wants a son. Findings are presented in Table 8.9.

Of the four conditions probed, the largest percentages of youth-87-92\% of young men and women-were aware that sex-selective abortion is illegal, presumably the result of widespread information campaigns against sex-selective abortion. A second issue about which large percentages of youth (74-79\%) were aware was that it is illegal to terminate a pregnancy that has gone beyond 20 weeks. Fewer youth, just $43 \%$ of young men and $71 \%$ of young women, were aware that an unmarried woman is legally entitled to undergo an abortion. Even fewer youth were 
Table 8.8: Knowledge of the legal minimum age at marriage

Percentage of youth who had correct knowledge of the legal minimum age at marriage in India, according to residence, Andhra Pradesh, 2007-08

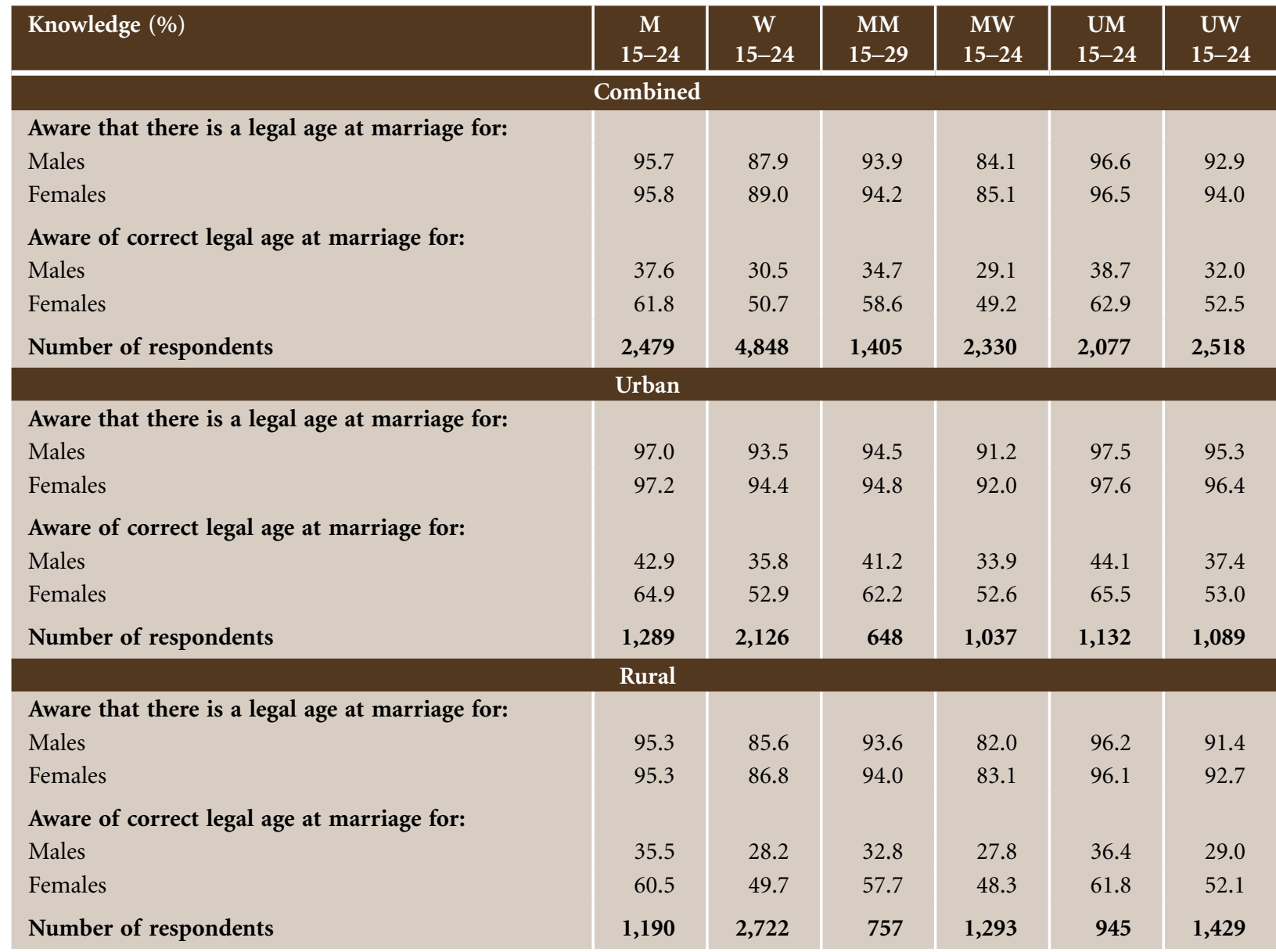

Note: All Ns are unweighted.

aware that a married woman is legally entitled to undergo an abortion (16-20\%). Gender differences were narrow, except that young women were more likely than young men to report that an unmarried woman is legally entitled to undergo abortion (71\% versus $43 \%)$. Differences by marital status and rural-urban residence were modest; however, some exceptions were notable. Among married young men, those in urban areas were somewhat less likely than their rural counterparts to be aware of unmarried women's legal entitlement to abortion (40\% versus $47 \%$ ). Among married young men and women, those in urban areas were more likely than their rural counterparts to be aware that abortion of the foetus after 20 weeks gestation are not legally permitted ( $83 \%$ versus $74 \%$ among young men; $85 \%$ versus $79 \%$ among young women).

As is evident from Table 8.9 , just $4 \%$ of young men and $8 \%$ of young women could correctly report the legality of all four conditions probed. Differences were mild by marital status and rural-urban residence.

\subsection{Sources of information on sex and reproduction}

The Youth Study questionnaire asked respondents about their sources of information on sexual matters and contraception. For the married, questions about sources of information on sexual matters referred to the situation prior to marriage; in contrast, questions relating to sources of information about contraception referred to the current situation, that is, around the time of the interview. 


\subsubsection{Sources of information on sexual matters}

Findings, presented in Table 8.10, suggest that young women had few sources of information on sex and reproduction. Indeed, half of young women reported that they had never received any information on sexual matters (prior to marriage among the married). While young men were far more likely to have received information, one-tenth reported that they had never received information on sex or reproduction (prior to marriage among the married). The married were more likely than the unmarried to report that they had never received any information on sexual matters (15\% versus 9\% among young men, and 63\% versus 36\% among young women). While rural-urban differences were muted among young men, somewhat larger percentages of rural than young urban women reported that they had never received any information on sexual matters (53\% and $47 \%$, respectively).

Table 8.9: Awareness of the conditions under which abortion is legal

Percentage of youth by knowledge of the conditions under which abortion is legal, according to residence, Andhra Pradesh, 2007-08

\begin{tabular}{|c|c|c|c|c|c|c|}
\hline Knowledge (\%) & $\begin{array}{c}M \\
15-24\end{array}$ & $\begin{array}{c}\text { W } \\
15-24\end{array}$ & $\begin{array}{c}\text { MM } \\
15-29\end{array}$ & $\begin{array}{c}\text { MW } \\
15-24\end{array}$ & $\begin{array}{c}\mathrm{UM} \\
15-24\end{array}$ & $\begin{array}{c}\text { UW } \\
15-24\end{array}$ \\
\hline \multicolumn{7}{|c|}{ Combined } \\
\hline $\begin{array}{l}\text { Agree that it is legal for a married woman to } \\
\text { terminate a pregnancy }\end{array}$ & 20.0 & 15.7 & 19.8 & 15.9 & 19.7 & 15.4 \\
\hline $\begin{array}{l}\text { Agree that it is legal for an unmarried woman to } \\
\text { terminate a pregnancy }\end{array}$ & 42.6 & 70.8 & 45.4 & 72.2 & 41.4 & 69.1 \\
\hline $\begin{array}{l}\text { Agree that it is illegal to undergo abortion after } \\
20 \text { weeks of gestation }\end{array}$ & 74.3 & 78.7 & 75.8 & 80.1 & 73.5 & 76.8 \\
\hline $\begin{array}{l}\text { Disagree that it is legal to abort a pregnancy if the } \\
\text { foetus is female but the couple wants a son }\end{array}$ & 87.0 & 92.3 & 89.3 & 92.2 & 86.6 & 92.3 \\
\hline Had correct knowledge of all of the above & 4.0 & 7.6 & 3.3 & 7.6 & 3.9 & 7.5 \\
\hline Number of respondents & 2,479 & 4,848 & 1,405 & 2,330 & 2,077 & 2,518 \\
\hline \multicolumn{7}{|c|}{ Urban } \\
\hline $\begin{array}{l}\text { Agree that it is legal for a married woman to } \\
\text { terminate a pregnancy }\end{array}$ & 19.5 & 16.1 & 19.9 & 17.0 & 19.7 & 15.5 \\
\hline $\begin{array}{l}\text { Agree that it is legal for an unmarried woman to } \\
\text { terminate a pregnancy }\end{array}$ & 40.3 & 69.7 & 39.5 & 71.2 & 39.3 & 68.3 \\
\hline $\begin{array}{l}\text { Agree that it is illegal to undergo abortion after } \\
20 \text { weeks of gestation }\end{array}$ & 76.3 & 82.0 & 83.3 & 84.8 & 75.3 & 79.8 \\
\hline $\begin{array}{l}\text { Disagree that it is legal to abort a pregnancy if the } \\
\text { foetus is female but the couple wants a son }\end{array}$ & 88.2 & 92.2 & 92.2 & 91.2 & 87.8 & 93.0 \\
\hline Had correct knowledge of all of the above & 4.7 & 9.1 & 4.9 & 9.0 & 4.9 & 9.2 \\
\hline Number of respondents & 1,289 & 2,126 & 648 & 1,037 & 1,132 & 1,089 \\
\hline \multicolumn{7}{|c|}{ Rural } \\
\hline $\begin{array}{l}\text { Agree that it is legal for a married woman to } \\
\text { terminate a pregnancy }\end{array}$ & 20.2 & 15.5 & 19.7 & 15.6 & 19.7 & 15.4 \\
\hline $\begin{array}{l}\text { Agree that it is legal for an unmarried woman to } \\
\text { terminate a pregnancy }\end{array}$ & 43.5 & 71.4 & 47.1 & 72.5 & 42.3 & 69.5 \\
\hline $\begin{array}{l}\text { Agree that it is illegal to undergo abortion after } \\
20 \text { weeks of gestation }\end{array}$ & 73.5 & 77.3 & 73.9 & 78.7 & 72.6 & 75.1 \\
\hline $\begin{array}{l}\text { Disagree that it is legal to abort a pregnancy if the } \\
\text { foetus is female but the couple wants a son }\end{array}$ & 86.4 & 92.4 & 88.5 & 92.5 & 86.1 & 91.9 \\
\hline Had correct knowledge of all of the above & 3.7 & 7.0 & 2.9 & 7.3 & 3.4 & 6.6 \\
\hline Number of respondents & 1,190 & 2,722 & 757 & 1,293 & 945 & 1,429 \\
\hline
\end{tabular}

Note: All Ns are unweighted. 
Figure 8.6: Percentage of youth who were aware of selected conditions under which abortion is legal, Andhra Pradesh, 2007-08

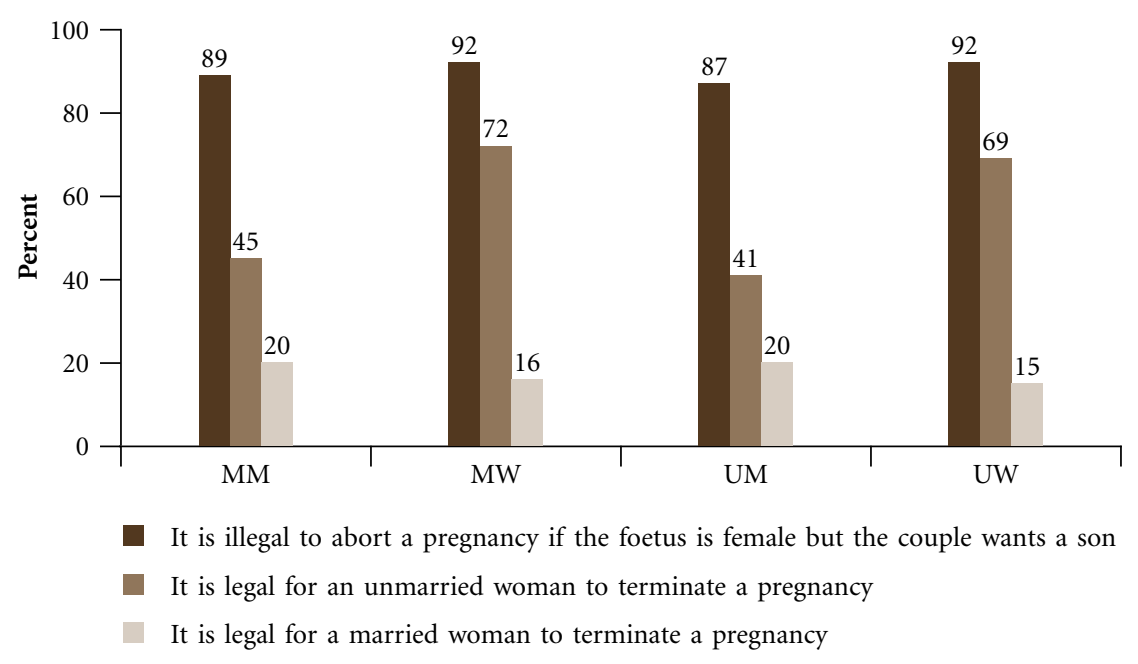

Leading sources of information on sex and reproduction varied considerably between young men and women. Among young men, the leading sources of information reported were friends and neighbours $(68 \%)$, followed by the media (47\%). Additionally, $14-18 \%$ each of young men cited teachers and schools, health care providers and posters/billboards as a source of information. Among young women, leading sources of information were the media $(21 \%)$, teachers and schools $(18 \%)$ and friends and neighbours (16\%). Notably, 5\% or less of youth cited family members and $6 \%$ cited a health care provider as a source of information on sex and reproduction.

Differences by marital status indicate that more unmarried than married youth cited the mass media (50\% versus $37 \%$ among young men, and $29 \%$ versus $15 \%$ among young women) and teachers and school (21\% versus $7 \%$ among young men and 33\% versus 7\% among young women) as a source of information. Among young women, in addition, the unmarried were more likely than the married to mention friends and neighbours as a source of information (20\% versus $13 \%)$.

Patterns were, by and large, similar in urban and rural areas, yet some differences were notable among young women. For example, urban young women were more likely than rural young women to cite teachers and schools ( $25 \%$ versus $15 \%)$ and the media (26\% versus $19 \%)$ as the main source of information.

\subsubsection{Current sources of information on contraception}

Table 8.11 describes current sources of information on contraception as reported by youth who were aware of at least one contraceptive method. Findings reiterate, as above, that friends and the media played an important role in conveying contraception-related information to young people.

Sources of information on contraception did, however, vary by sex of the respondent. Among young men, for example, key sources of information were male friends (76\%) and the media (53\%). In addition, 16-19\% each reported posters or billboards, health care providers and teachers and schools as current sources of information on contraception. Young women, in contrast, had a greater variety of sources of information on contraceptives than men. While friends and neighbours and the media continued to be leading sources of information on contraception among young women as well ( $48 \%$ and $39 \%$, respectively), sizeable proportions also cited such sources as family members other than spouse (29\%), health care providers (18\%) and teachers and schools (17\%). Of interest also is the finding that among married young women, a leading source of information was their husband (33\%); married young men, in contrast, rarely reported their wife as a source of information on contraception (1\%). 
Table 8.10: Sources of information on sexual matters before marriage

Percentage of youth by sources of information on sexual matters before marriage, according to residence, Andhra Pradesh, 2007-08

\begin{tabular}{|c|c|c|c|c|c|c|}
\hline Sources of information (\%) & $\begin{array}{c}\mathrm{M} \\
15-24\end{array}$ & $\begin{array}{c}\text { W } \\
15-24\end{array}$ & $\begin{array}{c}\text { MM } \\
15-29\end{array}$ & $\begin{array}{c}\text { MW } \\
15-24\end{array}$ & $\begin{array}{c}\text { UM } \\
15-24\end{array}$ & $\begin{array}{c}\text { UW } \\
15-24\end{array}$ \\
\hline \multicolumn{7}{|c|}{ Combined } \\
\hline Never received information & 9.9 & 50.9 & 14.7 & 62.7 & 8.9 & 35.5 \\
\hline A family member other than spouse & 2.6 & 5.1 & 5.1 & 6.2 & 1.5 & 3.8 \\
\hline Spouse/partner & 0.1 & 1.3 & 0.2 & 2.2 & 0.0 & 0.0 \\
\hline Friend/neighbour & 67.6 & 16.2 & 70.5 & 13.3 & 68.1 & 20.2 \\
\hline Teacher/school & 18.1 & 18.2 & 6.5 & 7.3 & 20.6 & 32.5 \\
\hline Health care provider & 13.9 & 6.0 & 12.5 & 6.0 & 13.4 & 5.9 \\
\hline Mass media ${ }^{1}$ & 47.2 & 21.1 & 37.4 & 15.4 & 49.6 & 28.6 \\
\hline Youth/mahila mandal/NGO worker & 0.9 & 0.5 & 1.2 & 0.6 & 1.0 & 0.4 \\
\hline Poster/billboard & 16.2 & 2.1 & 14.7 & 1.5 & 16.7 & 2.9 \\
\hline Don't remember & 1.6 & 4.2 & 1.0 & 4.6 & 1.6 & 3.7 \\
\hline Number of respondents & 2,479 & 4,848 & 1,405 & 2,330 & 2,077 & 2,518 \\
\hline \multicolumn{7}{|c|}{ Urban } \\
\hline Never received information & 10.9 & 46.9 & 13.1 & 61.0 & 10.3 & 35.5 \\
\hline A family member other than spouse & 3.1 & 4.4 & 5.5 & 5.7 & 2.2 & 3.3 \\
\hline Spouse/partner & 0.0 & 0.7 & 0.7 & 1.5 & 0.0 & 0.0 \\
\hline Friend/neighbour & 70.4 & 14.6 & 73.2 & 12.8 & 70.8 & 16.0 \\
\hline Teacher/school & 19.8 & 25.1 & 10.8 & 11.6 & 21.2 & 35.9 \\
\hline Health care provider & 10.6 & 5.1 & 15.7 & 5.3 & 9.5 & 4.8 \\
\hline Mass media ${ }^{1}$ & 50.4 & 26.4 & 47.4 & 19.7 & 51.7 & 31.8 \\
\hline Youth/mahila mandal/NGO worker & 1.1 & 0.2 & 2.3 & 0.4 & 1.0 & 0.1 \\
\hline Poster/billboard & 18.3 & 2.0 & 17.3 & 1.7 & 18.3 & 2.4 \\
\hline Don't remember & 1.1 & 3.4 & 0.7 & 3.6 & 1.3 & 3.3 \\
\hline Number of respondents & 1,289 & 2,126 & 648 & 1,037 & 1,132 & 1,089 \\
\hline \multicolumn{7}{|c|}{ Rural } \\
\hline Never received information & 9.6 & 52.6 & 15.1 & 63.2 & 8.3 & 35.4 \\
\hline A family member other than spouse & 2.4 & 5.4 & 4.9 & 6.3 & 1.1 & 4.0 \\
\hline Spouse/partner & 0.1 & 1.5 & 0.1 & 2.4 & 0.0 & 0.0 \\
\hline Friend/neighbour & 66.5 & 16.9 & 69.8 & 13.4 & 66.9 & 22.6 \\
\hline Teacher/school & 17.5 & 15.3 & 5.3 & 6.0 & 20.3 & 30.6 \\
\hline Health care provider & 15.2 & 6.3 & 11.6 & 6.2 & 15.1 & 6.5 \\
\hline Mass media ${ }^{1}$ & 46.0 & 18.9 & 34.6 & 14.1 & 48.6 & 26.7 \\
\hline Youth/mahila mandal/NGO worker & 0.8 & 0.6 & 0.9 & 0.6 & 1.0 & 0.6 \\
\hline Poster/billboard & 15.4 & 2.1 & 14.0 & 1.4 & 16.0 & 3.1 \\
\hline Don't remember & 1.7 & 4.5 & 1.1 & 4.9 & 1.9 & 4.0 \\
\hline Number of respondents & 1,190 & 2,722 & 757 & 1,293 & 945 & 1,429 \\
\hline
\end{tabular}

Note: All Ns are unweighted. Column totals may exceed 100\% due to multiple responses. For married respondents, questions referred to the period prior to marriage. ${ }^{1}$ Includes newspapers, books/magazines, radio/television and the internet. 
Differences by marital status suggest that unmarried youth were more likely than the married to cite the media (56\% versus $43 \%$ among young men and 50\% versus $32 \%$ among young women) and teachers and schools ( $19 \%$ versus $5 \%$ among young men and $33 \%$ versus $5 \%$ among young women), and less likely to cite health care providers (16\% versus $23 \%$ among young men and $9 \%$ versus $25 \%$ among young women) as a source of information on contraception. In addition, married young men were more likely than the unmarried to mention friends and neighbours ( $82 \%$ versus $75 \%$ ), and married young women were more likely than the unmarried to report family members (34\% versus $23 \%$ ) and friends and neighbours (51\% versus $45 \%$ ), as a source of information on contraception.

Rural-urban differences were mild among young men but wide among young women. Among young men, those in urban areas were more likely than their rural counterparts to cite the media as a source of information on contraception (62\% versus 50\%). Among young women, those in urban areas were more likely than their rural counterparts to obtain information from teachers and schools (24\% versus $15 \%$ ) and the media (54\% versus $33 \%$ ), and conversely, less likely to rely on family members other than spouse (24\% versus $31 \%$ ), friends and neighbours (41\% versus $51 \%$ ) and health care providers (13\% versus $21 \%)$.

It is evident that current leading sources of information on contraception among young people who were aware of at least one contraceptive method were largely similar to the sources of information on sexual matters reported by all youth (prior to marriage for the married) reported in the previous section. Among the leading sources of information on both contraception and sexual matters were peers and the media, although among young women, teachers and schools ranked second as a leading source of information on sexual matters. Of note are findings that health care providers reached just $6-14 \%$ of youth on sexual matters and $17-18 \%$ on contraception. Indeed, no more than a quarter of married youth and no more than one-sixth of unmarried youth obtained information on sexual matters or contraception from health care providers, possibly a consequence of the lack of attention that the $\mathrm{RCH}$ Programme has paid, thus far, to young people. Although teachers, charged with providing family life education to youth, did indeed reach considerable proportions of unmarried youth and were the leading source of information on sexual matters among unmarried young women, their reach was far from universal. Teachers were cited as a source of information on sexual matters or contraception by only between one-fifth and one-third of unmarried youth and by just $5-7 \%$ of married youth. So too, family members, often considered a credible source of information, were almost never a source of information about sex or contraception to young men, and rarely a source of information on sexual matters to young women. In short, health care providers, teachers and family members-often assumed to be more reliable sources of information than peers and the media-were relatively infrequently cited as sources of information on these sensitive topics by young people.

\subsection{Perceptions and experience of family life or sex education}

In the Youth Study, we asked respondents about their views on the importance of imparting family life or sex education to youth, the ideal age at which youth should receive information about sexual matters and the best person to provide that information. We also asked youth whether they had received formal family life or sex education and if so, the source of this education and their opinion about its quality.

Table 8.12 presents findings on young people's perceptions of family life or sex education. The majority of youth felt that it is important to impart family life or sex education to youth. Young men were more likely than young women to report so $(88 \%$ compared to $79 \%)$. Differences by marital status were modest, although the unmarried were somewhat more likely than the married to report so. Urban youth, irrespective of sex, were somewhat more likely than their rural counterparts to report this perception (92\% versus $86 \%$ among young men and $83 \%$ versus $78 \%$ among young women).

Of those who perceived family life or sex education to be important, half or more youth reported that such education should be provided to young people at ages between 15 and 17 years (56\% and 50\% among young men and women, respectively). Young women were more likely than young men to believe that information on sexual matters should be provided from an earlier age: $19 \%$ of young women compared to $9 \%$ of young men believed that this information 
Table 8.11: Current sources of information on contraception

Percentage of youth reporting awareness of contraceptives by current sources of information, according to residence, Andhra Pradesh, 2007-08

\begin{tabular}{|c|c|c|c|c|c|c|}
\hline Current sources of information (\%) & $\begin{array}{c}\mathrm{M} \\
15-24\end{array}$ & $\begin{array}{c}\text { W } \\
15-24\end{array}$ & $\begin{array}{c}\text { MM } \\
15-29\end{array}$ & $\begin{array}{c}\text { MW } \\
15-24\end{array}$ & $\begin{array}{c}\text { UM } \\
15-24\end{array}$ & $\begin{array}{c}\text { UW } \\
15-24\end{array}$ \\
\hline \multicolumn{7}{|c|}{ Combined } \\
\hline Family member other than spouse & 3.5 & 28.9 & 5.4 & 33.7 & 2.7 & 22.6 \\
\hline Spouse/partner & 0.2 & 18.9 & 1.2 & 32.9 & 0.0 & 0.2 \\
\hline Female friend/neighbour & 4.9 & 48.0 & 0.7 & 50.9 & 5.9 & 44.5 \\
\hline Male friend/neighbour & 76.4 & 1.2 & 81.7 & 1.1 & 75.2 & 1.6 \\
\hline Teacher/school/college & 16.2 & 17.2 & 4.6 & 5.1 & 19.0 & 33.3 \\
\hline Health care provider & 17.1 & 18.4 & 22.7 & 25.4 & 15.7 & 9.3 \\
\hline Mass media ${ }^{1}$ & 53.0 & 39.4 & 43.2 & 31.5 & 56.2 & 49.5 \\
\hline Poster/billboard & 19.4 & 3.5 & 15.8 & 2.4 & 20.9 & 5.0 \\
\hline Youth/mahila mandal/NGO worker & 0.9 & 0.4 & 1.1 & 0.4 & 0.9 & 0.3 \\
\hline Number aware of contraceptives & 2,402 & 4,745 & 1,382 & 2,292 & 2,009 & 2,453 \\
\hline \multicolumn{7}{|c|}{ Urban } \\
\hline Family member other than spouse & 3.4 & 24.0 & 6.6 & 30.7 & 2.6 & 18.6 \\
\hline Spouse/partner & 0.4 & 16.8 & 3.6 & 37.3 & 0.0 & 0.1 \\
\hline Female friend/neighbour & 4.7 & 40.8 & 0.7 & 48.0 & 5.4 & 34.9 \\
\hline Male friend/neighbour & 77.0 & 1.2 & 83.1 & 1.3 & 75.9 & 1.1 \\
\hline Teacher/school/college & 17.3 & 23.5 & 6.6 & 7.9 & 19.3 & 36.1 \\
\hline Health care provider & 14.5 & 13.4 & 22.1 & 20.7 & 13.0 & 7.4 \\
\hline Mass media ${ }^{1}$ & 61.6 & 54.0 & 52.8 & 45.1 & 64.3 & 61.3 \\
\hline Poster/billboard & 23.3 & 5.1 & 20.1 & 4.2 & 24.1 & 5.9 \\
\hline Youth/mahila mandal/NGO worker & 0.9 & 0.2 & 1.0 & 0.4 & 1.0 & 0.1 \\
\hline Number aware of contraceptives & 1,248 & 2,104 & 640 & 1,029 & 1,094 & 1,075 \\
\hline \multicolumn{7}{|c|}{ Rural } \\
\hline Family member other than spouse & 3.6 & 31.0 & 5.1 & 34.6 & 2.9 & 25.0 \\
\hline Spouse/partner & 0.1 & 19.8 & 0.6 & 31.6 & 0.1 & 0.3 \\
\hline Female friend/neighbour & 4.9 & 51.1 & 0.7 & 51.7 & 6.0 & 50.0 \\
\hline Male friend/neighbour & 76.1 & 1.3 & 81.2 & 1.0 & 74.9 & 1.7 \\
\hline Teacher/school/college & 15.7 & 14.6 & 4.0 & 4.3 & 18.9 & 31.7 \\
\hline Health care provider & 18.2 & 20.6 & 22.8 & 26.8 & 16.9 & 10.4 \\
\hline Mass media ${ }^{1}$ & 49.7 & 33.2 & 40.6 & 27.5 & 52.6 & 42.7 \\
\hline Poster/billboard & 17.8 & 2.9 & 14.6 & 1.9 & 19.5 & 4.5 \\
\hline Youth/mahila mandal/NGO worker & 0.9 & 0.5 & 1.1 & 0.5 & 0.9 & 0.5 \\
\hline Number aware of contraceptives & 1,154 & 2,641 & 742 & 1,263 & 915 & 1,378 \\
\hline
\end{tabular}

Note: All Ns are unweighted. Column totals may not equal 100\% due to multiple responses. ${ }^{1}$ Includes newspapers, books/magazines, radio/television and the internet.

should be provided to youth before they reached age 15. By and large, differences by marital status and rural-urban residence were narrow, although married young men were more likely than unmarried young men to believe that information on sexual matters should be provided at ages 18 or above ( $43 \%$ versus $32 \%)$.

In terms of youth perceptions about the best person to impart education on sex or family life matters, young men and women revealed fairly different preferences. Findings suggest that while young women would like to obtain information on sex or family life matters from their parents, this was not the case for young men, who would prefer 
Table 8.12: Perceptions about family life or sex education

Percentage of youth by perceptions about family life or sex education, according to residence, Andhra Pradesh, 2007-08

\begin{tabular}{|c|c|c|c|c|c|c|}
\hline Perceptions (\%) & $\begin{array}{c}M \\
15-24\end{array}$ & $\begin{array}{c}\text { W } \\
15-24\end{array}$ & $\begin{array}{c}\text { MM } \\
15-29\end{array}$ & $\begin{array}{c}\text { MW } \\
15-24\end{array}$ & $\begin{array}{c}\text { UM } \\
15-24\end{array}$ & $\begin{array}{c}\text { UW } \\
\text { 15-24 }\end{array}$ \\
\hline \multicolumn{7}{|c|}{ Combined } \\
\hline Perceived family life/sex education to be important & 87.9 & 79.2 & 85.1 & 76.5 & 89.0 & 82.6 \\
\hline Number of respondents & 2,479 & 4,848 & 1,405 & 2,330 & 2,077 & 2,518 \\
\hline \multicolumn{7}{|l|}{$\begin{array}{l}\text { Perceived that family life/sex education } \\
\text { should be provided at age (years): }\end{array}$} \\
\hline Below 12 & 0.5 & 0.9 & 1.0 & 0.9 & 0.3 & 0.8 \\
\hline $12-14$ & 8.4 & 17.6 & 5.7 & 16.1 & 9.1 & 19.6 \\
\hline $15-17$ & 55.6 & 49.8 & 47.1 & 49.2 & 57.5 & 50.6 \\
\hline 18 or above & 34.2 & 31.1 & 43.1 & 33.1 & 32.2 & 28.5 \\
\hline \multicolumn{7}{|l|}{$\begin{array}{l}\text { Perceived that the best person to provide family } \\
\text { life/sex education was: }\end{array}$} \\
\hline Parent & 2.9 & 38.8 & 4.8 & 43.6 & 2.4 & 32.9 \\
\hline Sibling/sister-in-law & 0.3 & 1.4 & 0.7 & 1.1 & 0.3 & 1.7 \\
\hline Spouse/partner & 0.0 & 3.9 & 0.0 & 6.6 & 0.1 & 0.7 \\
\hline Teacher & 44.8 & 25.3 & 33.6 & 16.1 & 48.4 & 36.5 \\
\hline Friend & 29.2 & 17.2 & 35.2 & 16.4 & 28.4 & 18.1 \\
\hline Health care provider/expert & 21.6 & 12.2 & 24.8 & 15.0 & 19.1 & 8.9 \\
\hline Youth club/mandal/NGO worker & 0.5 & 0.3 & 0.5 & 0.3 & 0.5 & 0.2 \\
\hline $\begin{array}{l}\text { Number who perceived family life/sex } \\
\text { education to be important }\end{array}$ & 2,215 & 3,917 & 1,218 & 1,827 & 1,872 & 2,090 \\
\hline \multicolumn{7}{|c|}{ Urban } \\
\hline Perceived family life/sex education to be important & 91.8 & 83.1 & 90.2 & 82.3 & 91.9 & 83.7 \\
\hline Number of respondents & 1,289 & 2,126 & 648 & 1,037 & 1,132 & 1,089 \\
\hline \multicolumn{7}{|l|}{$\begin{array}{l}\text { Perceived that family life/sex education } \\
\text { should be provided at age (years): }\end{array}$} \\
\hline Below 12 & 0.3 & 0.8 & 0.7 & 1.2 & 0.2 & 0.4 \\
\hline $12-14$ & 9.1 & 16.8 & 6.9 & 13.4 & 9.3 & 19.5 \\
\hline $15-17$ & 56.0 & 49.8 & 49.6 & 50.2 & 57.2 & 49.5 \\
\hline 18 or above & 34.0 & 32.3 & 42.0 & 34.7 & 32.6 & 30.4 \\
\hline \multicolumn{7}{|l|}{$\begin{array}{l}\text { Perceived that the best person to provide family } \\
\text { life/sex education was: }\end{array}$} \\
\hline Parent & 4.0 & 39.0 & 5.5 & 43.3 & 3.6 & 35.6 \\
\hline Sibling/sister-in-law & 0.2 & 1.7 & 0.4 & 0.9 & 0.2 & 2.2 \\
\hline Spouse/partner & 0.2 & 3.1 & 0.0 & 6.0 & 0.2 & 0.8 \\
\hline Teacher & 47.5 & 29.2 & 36.4 & 20.1 & 49.9 & 36.3 \\
\hline Friend & 31.0 & 17.9 & 34.5 & 19.0 & 30.6 & 17.0 \\
\hline Health care provider/expert & 16.3 & 8.6 & 22.9 & 10.0 & 14.5 & 7.5 \\
\hline Youth club/mandal/NGO worker & 0.3 & 0.1 & 0.4 & 0.0 & 0.3 & 0.1 \\
\hline $\begin{array}{l}\text { Number who perceived family life/sex } \\
\text { education to be important }\end{array}$ & 1,183 & 1,770 & 582 & 854 & 1,041 & 916 \\
\hline
\end{tabular}


Table 8.12: (Cont'd)

\begin{tabular}{|c|c|c|c|c|c|c|}
\hline Perceptions (\%) & $\begin{array}{c}\mathrm{M} \\
15-24\end{array}$ & $\begin{array}{c}\text { W } \\
15-24\end{array}$ & $\begin{array}{c}\text { MM } \\
15-29\end{array}$ & $\begin{array}{c}\text { MW } \\
15-24\end{array}$ & $\begin{array}{c}\text { UM } \\
15-24\end{array}$ & $\begin{array}{c}\text { UW } \\
15-24\end{array}$ \\
\hline \multicolumn{7}{|c|}{ Rural } \\
\hline Perceived family life/sex education to be important & 86.4 & 77.5 & 83.8 & 74.8 & 87.8 & 81.9 \\
\hline Number of respondents & 1,190 & 2,722 & 757 & 1,293 & 945 & 1,429 \\
\hline \multicolumn{7}{|l|}{$\begin{array}{l}\text { Perceived that family life/sex education } \\
\text { should be provided at age (years): }\end{array}$} \\
\hline Below 12 & 0.5 & 0.9 & 1.1 & 0.9 & 0.4 & 1.0 \\
\hline $12-14$ & 8.1 & 18.0 & 5.3 & 16.9 & 9.0 & 19.6 \\
\hline $15-17$ & 55.5 & 49.8 & 46.4 & 48.8 & 57.6 & 51.3 \\
\hline 18 or above & 34.2 & 30.5 & 43.3 & 32.6 & 32.0 & 27.4 \\
\hline \multicolumn{7}{|l|}{$\begin{array}{l}\text { Perceived that the best person to provide family } \\
\text { life/sex education was: }\end{array}$} \\
\hline Parent & 2.4 & 38.7 & 4.7 & 43.7 & 1.9 & 31.3 \\
\hline Sibling/sister-in-law & 0.4 & 1.2 & 0.8 & 1.1 & 0.4 & 1.4 \\
\hline Spouse/partner & 0.0 & 4.3 & 0.0 & 6.7 & 0.0 & 0.6 \\
\hline Teacher & 43.7 & 23.6 & 32.9 & 14.8 & 47.8 & 36.7 \\
\hline Friend & 28.5 & 16.9 & 35.5 & 15.5 & 27.4 & 18.8 \\
\hline Health care provider/expert & 23.8 & 13.9 & 25.4 & 16.6 & 21.2 & 9.8 \\
\hline Youth club/mandal/NGO worker & 0.5 & 0.4 & 0.4 & 0.4 & 0.6 & 0.3 \\
\hline $\begin{array}{l}\text { Number who perceived family life/sex } \\
\text { education to be important }\end{array}$ & 1,032 & 2,147 & 636 & 973 & 831 & 1,174 \\
\hline
\end{tabular}

Note: All Ns are unweighted. Column totals may not equal 100\% due to missing cases and "don't know" responses.

Figure 8.7: Percentage of youth who received family life or sex education, according to residence, Andhra Pradesh, 2007-08

Combined

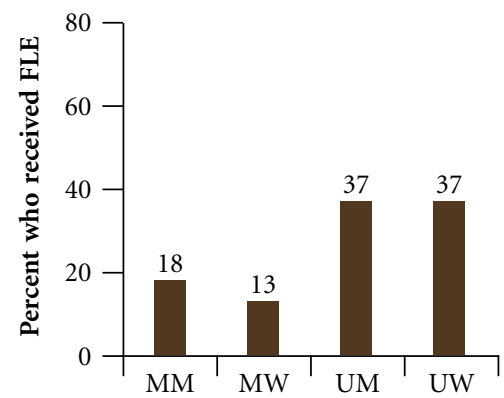

Urban

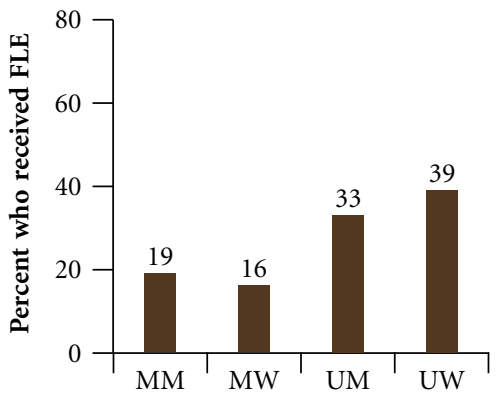

Rural

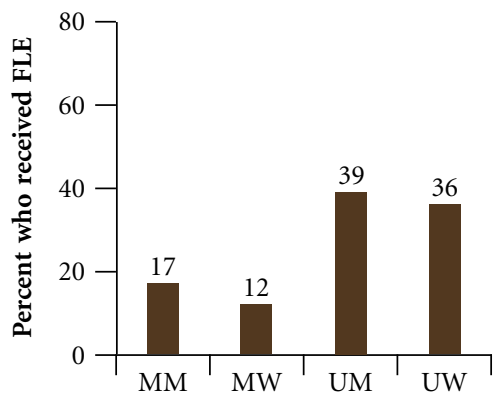

Note: FLE: Family life or sex education.

to consult teachers, health care providers or other knowledgeable persons, on one hand, and peers, on the other. As shown in Table 8.12, among young men who perceived family life or sex education to be important, leading preferred sources of such education were teachers (45\%), friends (29\%) and health care providers and other experts (22\%). Although substantial proportions of young women who perceived family life or sex education to be important also cited teachers $(25 \%)$, friends $(17 \%)$ and health care providers and other experts $(12 \%)$ as preferred sources, the most commonly cited preferred source was parents, reported by $39 \%$ of young women.

Differences by marital status suggest that larger proportions of unmarried than married youth considered teachers best equipped to provide family life or sex education (48\% versus 34\% among young men and $37 \%$ versus $16 \%$ among young women); conversely, somewhat more married than unmarried youth considered health care providers 
best equipped to provide family life or sex education (25\% versus $19 \%$ among young men and $15 \%$ versus $9 \%$ among young women). Among young men, in addition, somewhat more married than the unmarried cited peers as ideal for this role (35\% versus 28\%) and among young women, larger proportions of the married than the unmarried cited parents as ideal for this role (44\% versus 33\%). Rural-urban differences were negligible, although somewhat more rural than urban youth reported that health care providers and other experts were best equipped to provide family life or sex education (24\% versus $16 \%$ among young men and $14 \%$ versus $9 \%$ among young women) and somewhat fewer young women in rural than urban areas reported that teachers were best equipped to do so $(24 \%$ versus $29 \%)$.

Few youth reported that they had received family life or sex education in school or through special programmes sponsored by the government or NGOs. As seen in Table 8.13, just one-third of young men and one-quarter of young women had received any formal family life or sex education. Differences by marital status were wide (see also Figure 8.7). The unmarried were more likely than the married to have received such education (37\% versus $13-18 \%$ ). While roughly similar proportions of urban and rural young men had ever received family life or sex education (31\% and $34 \%$, respectively), young women in urban settings were somewhat more likely than their rural counterparts to have received this education ( $29 \%$ and $21 \%$, respectively).

The large majority of youth who had received family life or sex education had done so in school or college ( $78 \%$ of young men and $85 \%$ of young women). Married youth and rural youth were less likely than the unmarried

Table 8.13: Experiences of family life or sex education Percentage of youth by experiences of family life or sex education, according to residence,
Andhra Pradesh, 2007-08

\begin{tabular}{|c|c|c|c|c|c|c|}
\hline Experiences (\%) & $\begin{array}{c}\text { M } \\
15-24\end{array}$ & $\begin{array}{c}\text { W } \\
15-24\end{array}$ & $\begin{array}{c}\text { MM } \\
15-29\end{array}$ & $\begin{array}{c}\text { MW } \\
15-24\end{array}$ & $\begin{array}{c}\text { UM } \\
15-24\end{array}$ & $\begin{array}{c}\text { UW } \\
\text { 15-24 }\end{array}$ \\
\hline \multicolumn{7}{|c|}{ Combined } \\
\hline Received formal family life/sex education & 33.4 & 23.5 & 17.5 & 13.0 & 36.9 & 37.4 \\
\hline Number of respondents & 2,479 & 4,848 & 1,405 & 2,330 & 2,077 & 2,518 \\
\hline \multicolumn{7}{|l|}{ Source of family life/sex education } \\
\hline NGO programme/camp & 4.2 & 6.0 & 10.2 & 13.6 & 3.9 & 2.4 \\
\hline Government programme/camp & 21.7 & 16.5 & 29.4 & 33.0 & 21.3 & 9.0 \\
\hline School/college & 78.1 & 85.3 & 64.5 & 67.2 & 78.9 & 93.4 \\
\hline \multicolumn{7}{|l|}{ Opinion about family life/sex education received } \\
\hline It answered many queries & 95.1 & 92.6 & 95.9 & 94.4 & 94.7 & 91.6 \\
\hline Teacher/trainer explained well & 95.0 & 92.9 & 89.8 & 92.4 & 94.8 & 93.0 \\
\hline Respondent felt embarrassed & 13.5 & 32.0 & 8.2 & 30.0 & 13.8 & 33.1 \\
\hline Number who received family life/sex education & 814 & 1,280 & 253 & 326 & 744 & 954 \\
\hline \multicolumn{7}{|c|}{ Urban } \\
\hline Received formal family life/sex education & 30.9 & 28.8 & 18.6 & 16.2 & 33.3 & 39.1 \\
\hline Number of respondents & 1,289 & 2,126 & 648 & 1,037 & 1,132 & 1,089 \\
\hline \multicolumn{7}{|l|}{ Source of family life/sex education } \\
\hline NGO programme/camp & 5.0 & 3.4 & 19.3 & 8.2 & 4.3 & 1.7 \\
\hline Government programme/camp & 18.0 & 8.5 & 26.3 & 16.5 & 18.1 & 5.6 \\
\hline School/college & 81.6 & 94.4 & 58.6 & 88.1 & 82.4 & 96.6 \\
\hline \multicolumn{7}{|l|}{ Opinion about family life/sex education received } \\
\hline It answered many queries & 92.2 & 92.3 & 93.0 & 94.0 & 91.9 & 91.8 \\
\hline Teacher/trainer explained well & 93.1 & 94.4 & 92.9 & 94.1 & 92.9 & 94.7 \\
\hline Respondent felt embarrassed & 12.8 & 30.9 & 7.0 & 30.6 & 13.3 & 31.2 \\
\hline Number who received family life/sex education & 400 & 596 & 121 & 167 & 378 & 429 \\
\hline
\end{tabular}


Table 8.13: (Cont'd)

\begin{tabular}{|c|c|c|c|c|c|c|}
\hline Experiences (\%) & $\begin{array}{c}M \\
15-24\end{array}$ & $\begin{array}{c}\text { W } \\
15-24\end{array}$ & $\begin{array}{c}\text { MM } \\
15-29\end{array}$ & $\begin{array}{c}\text { MW } \\
15-24\end{array}$ & $\begin{array}{c}\text { UM } \\
15-24\end{array}$ & $\begin{array}{c}\text { UW } \\
\text { 15-24 }\end{array}$ \\
\hline \multicolumn{7}{|c|}{ Rural } \\
\hline Received formal family life/sex education & 34.4 & 21.3 & 17.2 & 12.1 & 38.6 & 36.4 \\
\hline Number of respondents & 1,190 & 2,722 & 757 & 1,293 & 945 & 1,429 \\
\hline \multicolumn{7}{|l|}{ Source of family life/sex education } \\
\hline NGO programme/camp & 3.9 & 7.4 & 7.4 & 16.1 & 3.6 & 2.9 \\
\hline Government programme/camp & 22.9 & 21.0 & 30.3 & 39.4 & 22.4 & 11.1 \\
\hline School/college & 76.9 & 80.1 & 66.1 & 59.2 & 77.6 & 91.5 \\
\hline \multicolumn{7}{|l|}{ Opinion about family life/sex education received } \\
\hline It answered many queries & 96.0 & 92.7 & 96.8 & 94.5 & 95.5 & 91.7 \\
\hline Teacher/trainer explained well & 95.6 & 91.9 & 88.9 & 91.7 & 95.3 & 92.0 \\
\hline Respondent felt embarrassed & 13.7 & 32.6 & 8.5 & 29.8 & 13.8 & 34.2 \\
\hline Number who received family life/sex education & 414 & 684 & 132 & 159 & 366 & 525 \\
\hline
\end{tabular}

Note: All Ns are unweighted. Column totals may not equal 100\% due to missing cases or "don't know" responses.

Figure 8.8: Percentage of youth reporting knowledge of selected sexual and reproductive health matters according to whether they had or had not received family life or sex education, Andhra Pradesh, 2007-08

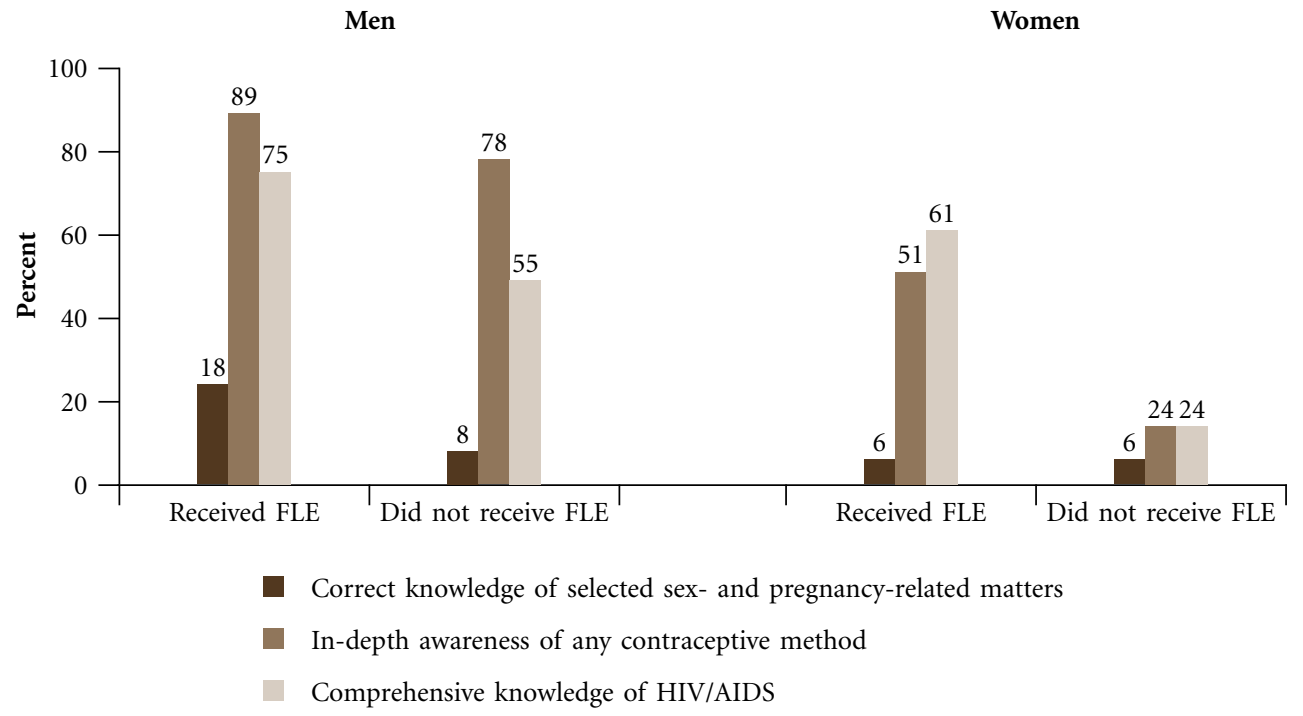

Note: FLE: Family life or sex education.

and the urban, respectively, to have obtained such education in school/college, and conversely, more likely to have received this education through special programmes sponsored by the government or NGOs.

Of those who reported receiving formal family life or sex education, almost all felt that it had answered many of their questions (93-95\%) and that teachers or trainers had explained matters well (93-95\%). Differences by marital status and rural-urban residence of respondents were negligible. Despite the fact that youth gave a generally positive assessment of the education they had received, $14 \%$ young men and $32 \%$ young women reported feeling uncomfortable or embarrassed in the course of family life or sex education, raising questions about the extent to which they were indeed able to participate freely and clarify doubts. Difference by marital status and rural-urban 
residence were generally negligible except that unmarried men were somewhat more likely than married men to report feeling uncomfortable or embarrassed ( $14 \%$ versus $8 \%)$.

Figure 8.8 compares the extent to which those who had received family life or sex education differed in terms of correct knowledge of selected sexual and reproductive health matters with those who had not (see Sections 8.1.1, 8.1.3 and 8.1.8 for details of items considered in each summary measure). Findings suggest that youth who had received family life or sex education were more likely than those who had not to report in-depth awareness of contraception and comprehensive knowledge of HIV/AIDS. Young men who received such education, in addition, were more likely to report correct knowledge of selected sex and pregnancy matters. This pattern was, by and large, evident among both rural and urban respondents (not shown in figure).

\subsection{Summary}

Findings presented in this chapter underscore young people's limited awareness of most sexual and reproductive matters, ranging from how pregnancy occurs to contraception, HIV and safe sex practices. For example, just over two-fifths of young men and over three-fifths of young women were aware that a woman can get pregnant at first sex, and just $30 \%$ of young men and $16 \%$ of young women reported awareness of STIs other than HIV.

Even on topics about which young people were generally aware, findings show that in-depth understanding was limited. For example, while $97-98 \%$ of youth reported awareness of at least one modern contraceptive method, in-depth awareness of condoms and oral contraceptives, the methods most familiar to youth, was reported by just $80 \%$ and $17 \%$ of young men and $21 \%$ and $15 \%$ of young women, respectively. Likewise, although $94-95 \%$ of youth had heard of HIV/AIDS, just $62 \%$ of young men and $33 \%$ of young women had comprehensive knowledge of HIV/AIDS.

Youth, particularly young women had few sources of information on sex and reproduction. Indeed, half of young women and one-tenth of young men reported that they had never received any information on sexual matters (prior to marriage among the married).

Leading sources of information on sex and reproduction varied considerably between young men and women. Among young men, the leading sources of information reported were friends and neighbours (68\%), followed by the media (47\%). Among young women, leading sources of information were the media (21\%), teachers and schools (18\%) and friends and neighbours (16\%). Notably, just 3-5\% cited a family member. Among the leading current sources of information on contraception among young people who were aware of at least one method were similarly, peers and the media. Teachers and health care providers were relatively infrequently reported as such. Indeed, health care providers were cited as an important source of information on contraception by only a quarter of married youth; they were far less likely to have provided information to the unmarried (9-16\%). Likewise, teachers were cited by just $16-17 \%$ of youth. However considerable proportions of young women (29\%) but hardly any young men $(4 \%)$ cited family members as a leading source of information about contraception. In short, health care providers, teachers and family members-often assumed to be more reliable sources of information than peers or the media-were infrequently and inconsistently cited as sources of information on sensitive topics such as sexual matters and contraception by young people.

Few youth had attended family life or sex education programmes either in or outside the school setting-just one-third of young men and almost one-quarter of young women. Despite this, youth were overwhelmingly in favour of the provision of family life or sex education to young people; while young men preferred to receive this education from a professional (health care provider or teacher) and peers, young women preferred parents and teachers. Findings suggest, moreover, that youth who had undergone family life or sex education were indeed more knowledgeable about sexual and reproductive matters than those not exposed to this education. 


\section{Pre-marital romantic and} sexual relationships

While evidence is sparse, several studies have noted that despite socio-cultural taboos, youth in India do find opportunities to mix and form romantic relationships, and to engage in pre-marital sex with a range of partners and in a variety of situations (Abraham, 2001; 2002; Abraham and Kumar, 1999; Alexander et al., 2006a; 2006b; Awasthi, Nichter and Pande, 2000). This chapter begins by describing the development of questions intended to capture these youth relationships. The chapter then explores young people's attitudes towards pre-marital physical intimacy and sex, and the extent and nature of their pre-marital romantic experiences, followed by a description of their pre-marital sexual experiences, including those within romantic partnerships and other situations. Finally, the chapter compares reports of pre-marital romantic and sexual experiences derived using three different methodological approaches, that is, face-to-face interviews, anonymous reporting of respondents' own experiences using a sealed envelope and anonymous third-party reporting of the experiences of respondents' friends.

\subsection{Development of the questionnaire module on pre-marital romantic and sexual relationships}

In view of the fact that social norms prohibiting pre-marital opposite-sex mixing may result in serious under-reporting of romantic and sexual relationships by youth, the Youth Study initiated the development of this module with a series of focus group discussions among married and unmarried young men and women. In the course of these focus group discussions, youth confirmed that romantic relationships were indeed formed, and mapped a range of places in which youth met their romantic partners secretly. They also listed the vocabulary used by youth to describe their romantic relationships, including the commonly used term "to give a proposal" to describe the act of conveying romantic intentions to opposite-sex individuals.

Building on these insights, a romantic relationship was defined as one comprising a boyfriend-girlfriend relationship (worded culturally appropriately) in which an emotional, physical or sexual relationship was experienced; one in which a "proposal" was accepted or one in which the couple spent time together alone and secretly. Correspondingly, all respondents were asked questions on whether or not they had ever had a boy- or girl-friend; whether they had ever "proposed" to anyone of the opposite sex or whether someone of the opposite sex had ever "proposed" to them and whether the "proposal" was accepted; and whether they had spent time alone and secretly with an opposite-sex person. Youth who reported any of the above experiences were considered to have experienced a romantic relationship. We note that our definition of romantic relationships precluded the possibility of reporting same-sex romantic relationships.

All respondents who had reported a romantic partner were then probed regarding the nature of the relationship and the extent of physical contact experienced in the relationship. Questions probing respondents' experience with physical intimacy were posed on a continuum, starting with hand-holding and extending to sexual relations. Thus, the instrument sought to ask potentially sensitive or embarrassing questions in a gradual way, thereby also enabling the interviewer to build rapport with the respondent. Detailed questions concerning the nature of the relationship were asked with reference to the first romantic partner as well as the most recent, if more than one was reported.

Pre-survey focus group discussions also probed the nature of situations in which sex was experienced. Participants discussed an array of partners with whom youth engaged in sexual relations, including romantic and casual, 
heterosexual and homosexual, sex workers and older married women. Situations of forced and exchange sex were also discussed. Our survey, correspondingly, inquired about each of these different types of relationships after we had obtained detailed information on the nature of relationships with romantic partners.

Additionally, recognising the reluctance of youth to disclose sexual experience in a survey situation, at the conclusion of the interview, all respondents were asked a single question ["Have you ever had sex with anyone?" (for the unmarried)/"Did you ever have sex with anyone before marriage?" (for the married)] and asked to mark a blank card with a " $\checkmark$ " or a "X," place the card in an envelope, seal it and return it to the interviewer. Respondents were informed that the envelope would not be opened in the field, and that only the principal investigators would be able to link the information provided in the envelope with what was provided in the main body of the questionnaire.

We also recognised that despite significant rapport building and a well-developed sequence of questions eliciting sexual behaviours, young people may not have wished to disclose sexual activity in either of the above formats. Other researchers have observed that respondents may be more forthcoming about reporting sensitive behaviours among their peer networks than about themselves and that responses relating to the peer network correspond closely to their own experiences (Rossier, 2003). Hence the Youth Study incorporated anonymous third-party reporting questions, in which respondents reported the romantic and sexual experiences of up to five same-sex peers.

In addition, efforts were made to ensure that youth were comfortable revealing sensitive behaviours. Interviewers were young and trained to build rapport, discuss sensitive experiences in empathetic and matter-of-fact ways and generally make respondents feel comfortable about the topics to be discussed during the interview. As far as possible, interviews were held at times and places that assured the respondent maximum confidentiality. In cases in which family members attempted to participate in or overhear the interview, another interviewer was called upon to conduct an informal discussion or interview with other family members so as to ensure privacy for the interview. Nevertheless, we acknowledge that ensuring privacy may have been a problem, especially in low-income urban settings characterised by cramped housing conditions or that some youth may not have felt entirely at ease despite the extensive efforts made to ensure confidentiality. While findings are indeed in line with those observed in other small-scale and less representative studies (see Jejeebhoy and Sebastian, 2004 for a review), we acknowledge that romantic and sexual experiences may have been under-reported in the survey, notably by young women, and suggest that percentages presented here may be interpreted as conservative estimates.

\subsection{Attitudes toward pre-marital physical intimacy and sexual relations}

The Youth Study included a number of questions to assess young people's attitudes regarding the acceptability of pre-marital physical intimacy and sexual activity. Findings, presented in Table 9.1, suggest that young people's attitudes towards pre-marital physical intimacy and sex were generally negative; that is, most disapproved of kissing a partner before marriage, and agreed that a young person's future would be ruined if he or she had sex before marriage. Even so, notable proportions of young men and women considered pre-marital kissing and sexual activity acceptable. Young men were more likely than young women to condone such behaviours. For example, $40 \%$ of young men compared to just $13 \%$ of young women felt that it is all right for a boy and girl to kiss each other before marriage. Findings also indicate gendered attitudes towards pre-marital physical intimacy and sex. While about one-quarter of young men and one in ten young women condoned pre-marital sexual activity among young men, far fewer- $11 \%$ of young men and just $3 \%$ of young women-considered such behaviour acceptable among young women.

Differences by marital status were mild, although in urban areas, somewhat more married than unmarried young men considered it unacceptable for a boy and girl to kiss each other before marriage (60\% versus 53\%) and in rural areas, somewhat more married than unmarried young men condoned pre-marital sexual activity among young men (27\% compared to $21 \%)$. Rural-urban differences were, likewise, negligible; however, somewhat more urban than rural young men expressed the view that it is all right for a boy and girl to kiss each other before marriage ( $44 \%$ versus $38 \%)$ and considered pre-marital sexual activity acceptable among young men (28\% versus $23 \%)$. 
Table 9.1: Attitudes toward pre-marital physical intimacy and sexual relations

Percent distribution of youth by attitudes towards pre-marital physical intimacy and sexual relations, according to residence, Andhra Pradesh, 2007-08

\begin{tabular}{|c|c|c|c|c|c|c|}
\hline Attitudes (\%) & $\begin{array}{c}\mathrm{M} \\
15-24\end{array}$ & $\begin{array}{c}\text { W } \\
15-24\end{array}$ & $\begin{array}{c}\text { MM } \\
15-29\end{array}$ & $\begin{array}{c}\text { MW } \\
15-24\end{array}$ & $\begin{array}{c}\text { UM } \\
15-24\end{array}$ & $\begin{array}{c}\text { UW } \\
15-24\end{array}$ \\
\hline \multicolumn{7}{|c|}{ Combined } \\
\hline \multicolumn{7}{|c|}{ Kissing before marriage is all right } \\
\hline Agree & 39.8 & 13.0 & 37.2 & 11.4 & 40.4 & 15.0 \\
\hline Disagree & 57.6 & 86.5 & 61.4 & 88.1 & 56.8 & 84.5 \\
\hline \multicolumn{7}{|c|}{$\begin{array}{l}\text { A boy's future would be ruined if he has sex } \\
\text { before marriage }\end{array}$} \\
\hline Agree & 71.6 & 89.2 & 69.5 & 89.4 & 72.7 & 89.0 \\
\hline Disagree & 24.1 & 10.2 & 27.6 & 10.1 & 23.0 & 10.2 \\
\hline \multicolumn{7}{|c|}{$\begin{array}{l}\text { A girl's future would be ruined if she has sex } \\
\text { before marriage }\end{array}$} \\
\hline Agree & 84.4 & 96.1 & 87.1 & 96.4 & 84.0 & 95.8 \\
\hline Disagree & 10.7 & 3.3 & 9.6 & 3.2 & 10.9 & 3.4 \\
\hline Number of respondents & 2,479 & 4,848 & 1,405 & 2,330 & 2,077 & 2,518 \\
\hline \multicolumn{7}{|c|}{ Urban } \\
\hline \multicolumn{7}{|c|}{ Kissing before marriage is all right } \\
\hline Agree & 43.5 & 13.7 & 38.9 & 11.6 & 44.8 & 15.4 \\
\hline Disagree & 54.2 & 86.0 & 59.5 & 88.2 & 52.9 & 84.3 \\
\hline \multicolumn{7}{|c|}{$\begin{array}{l}\text { A boy's future would be ruined if he has sex } \\
\text { before marriage }\end{array}$} \\
\hline Agree & 69.3 & 88.2 & 69.0 & 88.6 & 69.5 & 87.9 \\
\hline Disagree & 27.5 & 11.4 & 29.4 & 10.9 & 27.0 & 11.8 \\
\hline \multicolumn{7}{|c|}{$\begin{array}{l}\text { A girl's future would be ruined if she has sex } \\
\text { before marriage }\end{array}$} \\
\hline Agree & 82.7 & 96.7 & 86.3 & 96.9 & 81.8 & 96.5 \\
\hline Disagree & 12.8 & 3.1 & 11.1 & 2.9 & 13.1 & 3.2 \\
\hline Number of respondents & 1,289 & 2,126 & 648 & 1,037 & 1,132 & 1,089 \\
\hline \multicolumn{7}{|c|}{ Rural } \\
\hline \multicolumn{7}{|c|}{ Kissing before marriage is all right } \\
\hline Agree & 38.3 & 12.6 & 36.7 & 11.2 & 38.5 & 14.8 \\
\hline Disagree & 59.0 & 86.7 & 62.0 & 88.1 & 58.6 & 84.5 \\
\hline \multicolumn{7}{|c|}{$\begin{array}{l}\text { A boy's future would be ruined if he has sex } \\
\text { before marriage }\end{array}$} \\
\hline Agree & 72.6 & 89.6 & 69.7 & 89.6 & 74.0 & 89.6 \\
\hline Disagree & 22.8 & 9.7 & 27.1 & 9.9 & 21.2 & 9.3 \\
\hline \multicolumn{7}{|c|}{$\begin{array}{l}\text { A girl's future would be ruined if she has sex } \\
\text { before marriage }\end{array}$} \\
\hline Agree & 85.0 & 95.9 & 87.3 & 96.2 & 84.9 & 95.3 \\
\hline Disagree & 9.9 & 3.4 & 9.1 & 3.3 & 9.9 & 3.6 \\
\hline Number of respondents & 1,190 & 2,722 & 757 & 1,293 & 945 & 1,429 \\
\hline
\end{tabular}

Note: All Ns are unweighted. Column totals may not equal 100\% due to missing cases or "can't say" responses. 


\subsection{Pre-marital romantic relationships}

In this section we present findings on the prevalence of pre-marital opposite-sex romantic relationships among youth and a profile of those who engaged in such relationships. The section also describes parent and peer awareness of pre-marital romantic relationships, youth intentions regarding marriage with their romantic partners and the extent of physical contact experienced in these relationships.

\subsubsection{Prevalence of pre-marital romantic relationships}

Despite the fact that youth tended to report relatively traditional attitudes, opportunities to form romantic relationships did exist for them, irrespective of sex or rural-urban residence. As shown in Table 9.2, about a quarter of youth $(23 \%)$ had either made a romantic "proposal" to an opposite-sex individual or had received such a "proposal". Very few young women reported "proposing" to a man (2\%); among young men, however, almost as many reported making a "proposal" as receiving one (13\% and 15\%, respectively, had made and received a proposal) (not shown in tabular form), suggesting the possibility that young men may have exaggerated the extent of their interaction with women, or young women may have concealed behaviour that may be considered socially unacceptable.

Patterns of experience in initiating pre-marital romantic relationships by marital status indicate that while differences were narrow among young men, fewer married than unmarried young women reported making or receiving a "proposal" (19\% versus 27\%) (see also Figure 9.1), a difference attributable perhaps to the limited number of years married young women had spent as an unmarried adolescent. Rural-urban differences suggest that larger percentages of urban youth compared to their rural counterparts had made or received a "proposal" (27\% versus $22 \%$ among young men and $29 \%$ versus $20 \%$ among young women).

"Proposals" were often conveyed through an intermediary-a friend, relative or sibling. Indeed, 6-7\% of all youth reported that they had made or received a "proposal" conveyed through a mediator. This corresponds to one-fourth of young men and one-third of women who had ever made or received a "proposal" (not shown in tabular form). Differences by marital status and rural-urban residence were negligible.

Compared to those who had made or received "proposals," fewer youth, particularly young women, reported the acceptance of such a "proposal". One-sixth of young men (16\%) and one-tenth of young women (10\%) reported that they had accepted a "proposal" or that their own "proposal" had been accepted. Similar percentages reported that they had met an opposite-sex individual secretly. In total, in response to the direct or indirect questions, $17 \%$ of young men and $11 \%$ of young women acknowledged the experience of a romantic partnership. Few young men $(3 \%)$ and hardly any young women $(0.1 \%)$ reported more than one romantic partner.

Figure 9.1: Percentage of youth who had made or received a "proposal" for romantic partnership formation and percentage who had an opposite-sex romantic partner, according to residence, Andhra Pradesh, 2007-08

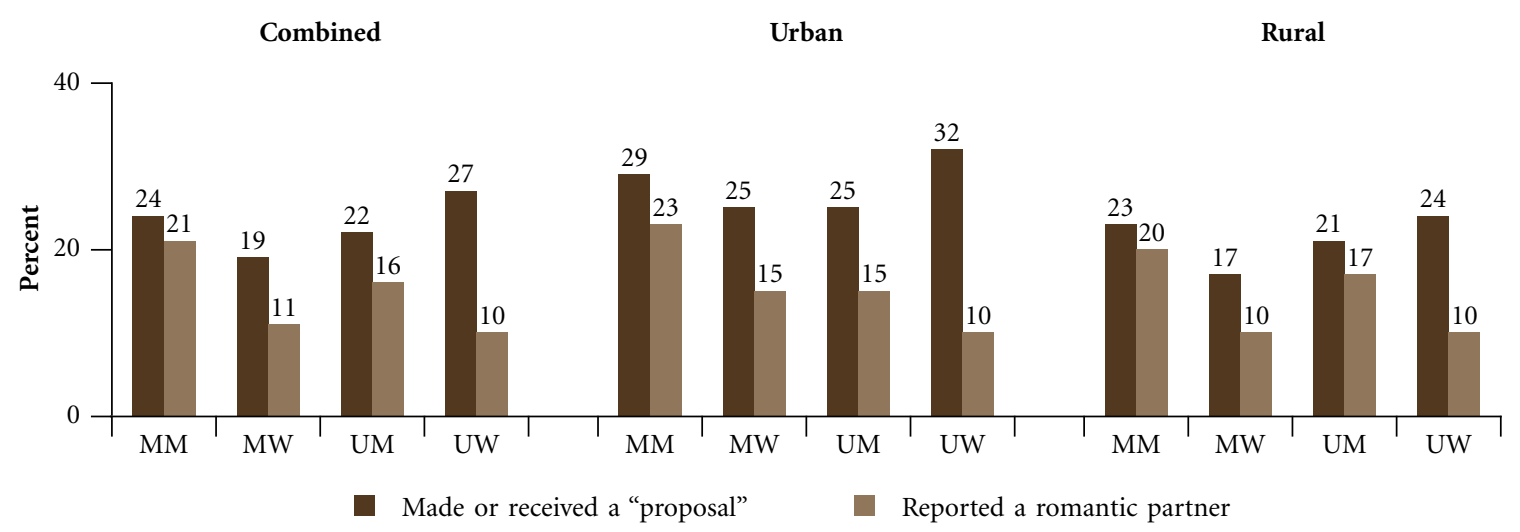


Table 9.2: Pre-marital romantic relationships

Percentage of youth reporting a pre-marital romantic relationship by relationship characteristics, according to residence, Andhra Pradesh, 2007-08

\begin{tabular}{|c|c|c|c|c|c|c|}
\hline Characteristics (\%) & $\begin{array}{c}M \\
15-24\end{array}$ & $\begin{array}{c}W \\
15-24\end{array}$ & $\begin{array}{c}\text { MM } \\
15-29\end{array}$ & $\begin{array}{c}\text { MW } \\
15-24\end{array}$ & $\begin{array}{c}\text { UM } \\
15-24\end{array}$ & $\begin{array}{c}\text { UW } \\
15-24\end{array}$ \\
\hline \multicolumn{7}{|c|}{ Combined } \\
\hline \multicolumn{7}{|l|}{ "Proposals" made/received and accepted } \\
\hline Made or received a "proposal" & 23.0 & 22.7 & 24.3 & 19.2 & 22.1 & 27.0 \\
\hline Made or received a "proposal" through a mediator & 6.3 & 7.2 & 6.1 & 5.8 & 6.0 & 8.9 \\
\hline Accepted a "proposal”/“proposal" was accepted & 16.2 & 10.3 & 19.1 & 10.6 & 15.1 & 9.8 \\
\hline \multicolumn{7}{|l|}{ Secret meetings with an opposite-sex individual } \\
\hline \multicolumn{7}{|l|}{$\begin{array}{l}\text { Reported romantic relationships in one of the above } \\
\text { or in direct question }{ }^{2}\end{array}$} \\
\hline Reported a romantic partner & 17.4 & 10.5 & 20.5 & 10.7 & 16.3 & 10.1 \\
\hline Reported more than one romantic partner & 2.8 & 0.1 & 3.0 & 0.1 & 2.4 & 0.2 \\
\hline Number of respondents & 2,479 & 4,848 & 1,405 & 2,330 & 2,077 & 2,518 \\
\hline \multicolumn{7}{|c|}{ Urban } \\
\hline \multicolumn{7}{|l|}{ “Proposals" made/received and accepted } \\
\hline Made or received a "proposal" & 26.9 & 28.8 & 29.4 & 25.3 & 25.1 & 31.6 \\
\hline Made or received a "proposal" through a mediator & 7.0 & 9.6 & 8.2 & 8.2 & 6.5 & 10.7 \\
\hline Accepted a "proposal"/“proposal" was accepted & 16.3 & 12.1 & 22.1 & 14.7 & 14.3 & 10.0 \\
\hline Secret meetings with an opposite-sex individual & & & & & & \\
\hline Met secretly in any of five selected places ${ }^{1}$ & 15.6 & 11.1 & 20.5 & 13.5 & 13.9 & 9.0 \\
\hline \multicolumn{7}{|l|}{$\begin{array}{l}\text { Reported romantic relationships in one of the above } \\
\text { or in direct question }{ }^{2}\end{array}$} \\
\hline Reported a romantic partner & 17.0 & 12.5 & 23.1 & 15.0 & 15.1 & 10.3 \\
\hline Reported more than one romantic partner & 1.6 & 0.1 & 2.6 & 0.2 & 1.3 & 0.2 \\
\hline Number of respondents & 1,289 & 2,126 & 648 & 1,037 & 1,132 & 1,089 \\
\hline \multicolumn{7}{|c|}{ Rural } \\
\hline \multicolumn{7}{|l|}{ "Proposals" made/received and accepted } \\
\hline Made or received a "proposal" & 21.5 & 20.1 & 22.9 & 17.4 & 20.7 & 24.4 \\
\hline Made or received a "proposal" through a mediator & 6.0 & 6.2 & 5.6 & 5.1 & 5.7 & 8.0 \\
\hline Accepted a "proposal"/“proposal” was accepted & 16.1 & 9.5 & 18.3 & 9.4 & 15.4 & 9.7 \\
\hline Secret meetings with an opposite-sex individual & & & & & & \\
\hline Met secretly in any of five selected places ${ }^{1}$ & 16.2 & 8.5 & 19.4 & 8.5 & 15.1 & 8.3 \\
\hline \multicolumn{7}{|l|}{$\begin{array}{l}\text { Reported romantic relationships in one of the above } \\
\text { or in direct question }{ }^{2}\end{array}$} \\
\hline Reported a romantic partner & 17.5 & 9.6 & 19.8 & 9.5 & 16.8 & 9.9 \\
\hline Reported more than one romantic partner & 3.2 & 0.1 & 3.1 & 0.1 & 2.8 & 0.1 \\
\hline Number of respondents & 1,190 & 2,722 & 757 & 1,293 & 945 & 1,429 \\
\hline
\end{tabular}

Note: All Ns are unweighted. ${ }^{1}$ Behind or around a temple/mosque/church; around a school/college; at own or someone else's home in the absence of parents; in fields/grazing areas (rural) and restaurants (urban); or in a garden/park/maidan/market or haat. ${ }^{2}$ Respondents were asked a direct question on whether or not they had ever had a boyfriend/girlfriend. 
Differentials by marital status in reporting a romantic relationship for the combined sample were negligible among young women but evident among young men, with the married somewhat more likely than the unmarried to report a romantic relationship (21\% versus $16 \%$ ). Differences by marital status were also notable for the urban sample. In urban areas, the married were more likely than their unmarried counterparts to report a romantic relationship ( $23 \%$ versus $15 \%$ among young men and $15 \%$ versus $10 \%$ among young women). Rural-urban differences were negligible, although married young women in urban areas were somewhat more likely than their rural counterparts ( $15 \%$ versus $10 \%)$, to acknowledge the experience of a romantic partnership.

Table 9.3 presents the percentage of youth reporting pre-marital romantic relationships by background characteristics. We note that such characteristics as work status and household economic status reflect the situation of youth at the time of interview, and not necessarily at the time when romantic relationships were formed. Age profiles indicate a positive association between age and the formation of romantic relationships among young men; this association was evident among the unmarried but not among the married. Among young women, differences by age were negligible.

Differentials by religion suggest that Hindu and Muslim young men were considerably less likely than those belonging to the other religions to report a pre-marital romantic relationship, irrespective of marital status (17\% versus $25 \%)$. Although a similar pattern was evident among young women, the differences were much narrow (8-10\% versus 14\%). Caste-wise differences indicate that young men belonging to scheduled tribes, general castes or other backward castes were less likely than those belonging to scheduled castes to report a pre-marital romantic relationship. Among young women, those belonging to both scheduled castes and tribes were more likely than those belonging to general castes or other backward castes to report a romantic relationship. This association was consistently observed among both the married and the unmarried.

Findings also show a largely positive association between schooling and the formation of romantic relationship, perhaps a result of greater opportunities for mobility and social mixing offered by schooling. For example, the percentage of young men who reported a romantic partner increased from $12 \%$ among those without formal schooling to $21 \%$ among those who had completed 12 or more years of schooling. The corresponding percentages among young women were $7 \%$ and $14 \%$, respectively. While a positive association between work participation and the formation of romantic relationship was evident among young men (20\% of those who worked in the 12 months preceding the survey versus $12 \%$ of those who did not), no such differences were evident among young women. The association between household economic status and the formation of romantic partnership was inconsistent among both young men and women, although among unmarried young women, those belonging to the poorest quintile were more likely than others to report a romantic partner (16\% versus $8-10 \%)$.

Similar patterns of socio-demographic differentials were observed, by and large, among rural and urban respondents. Some exceptions were, however, notable. For example, the association between schooling and the formation of romantic relationship was found to be inverse in urban areas, but positive in rural areas, as observed for the overall samples. Similarly, an inverse association between household economic status and the formation of romantic relationship was evident in urban areas, in contrast to the inconsistent pattern observed for the overall and rural samples.

\subsubsection{Characteristics of pre-marital romantic relationships}

Selected characteristics of reported pre-marital romantic relationships are presented in Table 9.4; in cases in which more than one romantic partner was reported, only information relating to the respondent's first romantic relationship was included. Age at initiation of pre-marital romantic relationships was measured by the age at which youth first spent time alone with their partner.

Findings indicate that relationships were initiated at a young age for substantial proportions of youth who had experienced pre-marital romantic relationships. One-fifth of young men and two-fifths of young women reported that they had spent time alone with their first romantic partner at age 15 or below. Among young men, the 
Table 9.3: Prevalence of pre-marital romantic relationships by selected background characteristics

Percentage of youth reporting a pre-marital romantic relationship by selected background characteristics, according to residence, Andhra Pradesh, 2007-08

\begin{tabular}{|c|c|c|c|c|c|c|}
\hline Background characteristics (\%) & $\begin{array}{c}\mathrm{M} \\
15-24\end{array}$ & $\begin{array}{c}\text { W } \\
15-24\end{array}$ & $\begin{array}{c}\text { MM } \\
15-29\end{array}$ & $\begin{array}{c}\text { MW } \\
15-24\end{array}$ & $\begin{array}{c}\text { UM } \\
15-24\end{array}$ & $\begin{array}{c}\text { UW } \\
15-24\end{array}$ \\
\hline \multicolumn{7}{|c|}{ Combined } \\
\hline \multicolumn{7}{|l|}{ Age (years) } \\
\hline $15-19$ & 12.4 & 10.1 & * & 11.4 & 11.8 & 9.4 \\
\hline $20-24$ & 21.8 & 10.8 & 20.7 & 10.5 & 22.2 & 12.4 \\
\hline $25-29$ & NA & NA & 19.9 & NA & NA & NA \\
\hline \multicolumn{7}{|l|}{ Religion } \\
\hline Hindu & 17.0 & 10.3 & 20.3 & 10.6 & 15.9 & 10.0 \\
\hline Muslim & 16.6 & 7.6 & 13.5 & 7.4 & 16.1 & 8.1 \\
\hline Other $^{1}$ & 24.8 & 13.9 & 31.0 & 14.3 & 24.5 & 13.4 \\
\hline \multicolumn{7}{|l|}{ Caste } \\
\hline SC & 26.2 & 16.0 & 29.7 & 17.8 & 24.1 & 13.8 \\
\hline ST & 17.8 & 19.5 & 26.3 & 18.9 & 14.3 & 20.8 \\
\hline OBC & 14.3 & 7.8 & 17.2 & 7.8 & 13.8 & 7.8 \\
\hline General $^{2}$ & 14.7 & 8.4 & 14.7 & 7.8 & 14.6 & 8.9 \\
\hline \multicolumn{7}{|l|}{ Educational level (years) } \\
\hline None $^{3}$ & 11.8 & 7.0 & 16.9 & 6.7 & 11.2 & 8.3 \\
\hline $1-7$ & 18.0 & 9.5 & 21.1 & 9.2 & 15.3 & 10.1 \\
\hline $8-11$ & 16.3 & 12.0 & 20.3 & 15.9 & 15.2 & 9.3 \\
\hline 12 or more & 20.9 & 13.5 & 24.8 & 17.4 & 20.2 & 12.0 \\
\hline \multicolumn{7}{|l|}{ Worked in last 12 months } \\
\hline Yes & 19.6 & 10.0 & 20.3 & 9.6 & 19.0 & 10.6 \\
\hline No & 11.5 & 10.9 & * & 11.9 & 11.0 & 9.7 \\
\hline \multicolumn{7}{|l|}{ Wealth quintile } \\
\hline First & 19.8 & 13.0 & 22.3 & 11.8 & 16.0 & 15.5 \\
\hline Second & 14.3 & 9.8 & 16.1 & 9.9 & 14.5 & 9.6 \\
\hline Third & 19.2 & 9.5 & 22.7 & 10.2 & 18.3 & 8.4 \\
\hline Fourth & 18.4 & 10.2 & 20.6 & 11.2 & 17.1 & 9.1 \\
\hline Fifth & 15.6 & 10.3 & 21.6 & 10.8 & 14.9 & 9.9 \\
\hline Total & 17.4 & 10.5 & 20.5 & 10.7 & 16.3 & 10.1 \\
\hline \multicolumn{7}{|c|}{ Urban } \\
\hline \multicolumn{7}{|l|}{ Age (years) } \\
\hline $15-19$ & 8.6 & 11.1 & * & 16.1 & 8.4 & 9.7 \\
\hline $20-24$ & 23.8 & 13.7 & 30.1 & 14.7 & 21.9 & 11.7 \\
\hline $25-29$ & NA & NA & 20.7 & NA & NA & NA \\
\hline \multicolumn{7}{|l|}{ Religion } \\
\hline Hindu & 15.9 & 12.4 & 22.9 & 14.1 & 13.9 & 10.9 \\
\hline Muslim & 20.2 & 8.1 & 20.0 & 10.7 & 17.8 & 6.7 \\
\hline Other $^{1}$ & 26.5 & 19.2 & $(38.5)$ & 29.5 & 23.3 & 10.5 \\
\hline \multicolumn{7}{|l|}{ Caste } \\
\hline SC & 24.3 & 19.3 & 34.0 & 28.3 & 21.6 & 12.0 \\
\hline ST & (13.0) & (13.3) & $(28.6)$ & $(17.6)$ & $(10.0)$ & * \\
\hline $\mathrm{OBC}$ & 16.6 & 10.8 & 18.7 & 11.2 & 14.9 & 10.5 \\
\hline General $^{2}$ & 14.0 & 9.7 & 24.4 & 10.2 & 12.2 & 9.4 \\
\hline
\end{tabular}


Table 9.3: (Cont'd)

\begin{tabular}{|c|c|c|c|c|c|c|}
\hline Background characteristics (\%) & $\begin{array}{c}\text { M } \\
15-24\end{array}$ & $\begin{array}{c}\text { W } \\
15-24\end{array}$ & $\begin{array}{c}\text { MM } \\
15-29\end{array}$ & $\begin{array}{c}\text { MW } \\
15-24\end{array}$ & $\begin{array}{c}\text { UM } \\
15-24\end{array}$ & $\begin{array}{c}\text { UW } \\
15-24\end{array}$ \\
\hline \multicolumn{7}{|c|}{ Urban } \\
\hline \multicolumn{7}{|l|}{ Educational level (years) } \\
\hline None $^{3}$ & 25.7 & 14.0 & 23.8 & 15.4 & $(14.3)$ & $(10.5)$ \\
\hline $1-7$ & 19.7 & 14.0 & 24.2 & 14.4 & 17.0 & 13.4 \\
\hline $8-11$ & 15.0 & 11.4 & 19.6 & 15.6 & 13.8 & 8.7 \\
\hline 12 or more & 16.7 & 12.2 & 26.9 & 15.7 & 15.8 & 11.0 \\
\hline \multicolumn{7}{|l|}{ Worked in last 12 months } \\
\hline Yes & 20.7 & 14.0 & 23.0 & 16.4 & 18.3 & 12.4 \\
\hline No & 10.5 & 11.9 & * & 14.6 & 10.3 & 9.6 \\
\hline \multicolumn{7}{|l|}{ Wealth quintile } \\
\hline First & 24.1 & 21.4 & $(17.6)$ & 25.5 & $(22.7)$ & 17.0 \\
\hline Second & 23.7 & 14.0 & 25.0 & 14.5 & 21.3 & 12.9 \\
\hline Third & 21.6 & 13.3 & 31.3 & 17.3 & 16.7 & 8.4 \\
\hline Fourth & 16.9 & 11.5 & 23.8 & 13.7 & 14.8 & 9.6 \\
\hline Fifth & 13.8 & 10.9 & 18.1 & 12.0 & 13.1 & 10.3 \\
\hline Total & 17.0 & 12.5 & 23.1 & 15.0 & 15.1 & 10.3 \\
\hline \multicolumn{7}{|c|}{ Rural } \\
\hline \multicolumn{7}{|l|}{ Age (years) } \\
\hline $15-19$ & 13.8 & 9.7 & * & 10.4 & 13.1 & 9.3 \\
\hline $20-24$ & 20.9 & 9.6 & 18.5 & 9.0 & 22.5 & 13.2 \\
\hline $25-29$ & NA & NA & 19.7 & NA & NA & NA \\
\hline \multicolumn{7}{|l|}{ Religion } \\
\hline Hindu & 17.4 & 9.6 & 19.7 & 9.6 & 16.7 & 9.5 \\
\hline Muslim & 14.2 & 7.0 & $(10.1)$ & 5.4 & 14.2 & 10.3 \\
\hline Other $^{1}$ & 24.5 & 11.8 & 30.1 & 10.2 & $(23.9)$ & 15.1 \\
\hline \multicolumn{7}{|l|}{ Caste } \\
\hline SC & 26.7 & 14.6 & 29.1 & 14.5 & 24.9 & 14.7 \\
\hline ST & 18.8 & 20.3 & 26.0 & 18.9 & 16.3 & 22.9 \\
\hline $\mathrm{OBC}$ & 13.4 & 6.7 & 16.8 & 6.8 & 13.2 & 6.5 \\
\hline General $^{2}$ & 15.2 & 7.7 & 10.6 & 7.0 & 15.9 & 8.5 \\
\hline \multicolumn{7}{|l|}{ Educational level (years) } \\
\hline None $^{3}$ & 8.7 & 5.8 & 15.4 & 5.5 & 9.4 & 7.7 \\
\hline $1-7$ & 17.5 & 8.2 & 20.4 & 8.1 & 14.7 & 8.7 \\
\hline $8-11$ & 16.7 & 12.2 & 20.5 & 16.0 & 15.7 & 9.6 \\
\hline 12 or more & 23.7 & 15.6 & 23.7 & 20.3 & 23.3 & 13.5 \\
\hline \multicolumn{7}{|l|}{ Worked in last 12 months } \\
\hline Yes & 19.3 & 9.3 & 19.5 & 8.8 & 19.3 & 10.1 \\
\hline No & 12.1 & 10.1 & * & 10.4 & 11.4 & 9.7 \\
\hline \multicolumn{7}{|l|}{ Wealth quintile } \\
\hline First & 19.5 & 11.9 & 22.8 & 10.2 & 15.7 & 15.3 \\
\hline Second & 12.9 & 9.1 & 15.0 & 9.0 & 13.5 & 9.3 \\
\hline Third & 18.6 & 8.5 & 21.5 & 8.6 & 18.7 & 8.4 \\
\hline Fourth & 19.3 & 9.3 & 19.2 & 10.1 & 18.3 & 8.5 \\
\hline Fifth & 17.8 & 9.5 & 24.1 & 9.9 & 17.6 & 9.1 \\
\hline Total & 17.5 & 9.6 & 19.8 & 9.5 & 16.8 & 9.9 \\
\hline
\end{tabular}

Note: ( ) Based on 25-49 unweighted cases. ${ }^{*}$ Percentage not shown, based on fewer than 25 unweighted cases. NA: Not applicable. OBC: Other backward caste. SC: Scheduled caste. ST: Scheduled tribe. ${ }^{1}$ Includes Christian, Buddhist, Neo-Buddhist, Sikh, Jain, Jewish, Parsi/Zoroastrian and no specified religion. ${ }^{2}$ Includes all those not belonging to SC, ST or OBC. ${ }^{3}$ Includes non-literate and literate with no formal schooling. 
unmarried were more likely than the married to have initiated their romantic relationship at age 15 or below (21\% versus $10 \%)$; a reverse pattern was, however, evident among young women (48\% and $32 \%$ of married and unmarried young women, respectively). Youth in rural areas were more likely than those in urban areas to have initiated a pre-marital romantic relationship at age 15 or below $(21 \%$ compared to $13 \%$ among young men and $47 \%$ compared to $31 \%$ among young women). Median ages of respondents when they first spent time alone with their pre-marital romantic partner were two years older among young men than among young women (18 years and 16 years, respectively), one year older among unmarried young women compared to married young women (16 and 15, respectively) and identical among married and unmarried young men (18 years). Irrespective of marital status, youth in urban areas initiated their relationships one year later than did their rural counterparts (18 versus 17 years among young men, and 16 versus15 years among young women). Information on the relative ages of reported partners suggests that male partners were, for the most part, older than female partners. For example, $79 \%$ of young men reported a female partner who was younger than they were, while $89 \%$ of young women reported a male partner who was older than they were. While differences by marital status and rural-urban residence were negligible among young women, unmarried young men and those in rural areas were considerably more likely than their married and urban counterparts, respectively to report a pre-marital romantic relationship with a young woman of their own age (21\% versus 9\% among the unmarried and married; $20 \%$ and $13 \%$ among rural and urban young men, respectively). Overwhelmingly, the partner was unmarried, reported by $97-98 \%$ of youth.

The first reported romantic partner was typically a neighbour or friend (40\%), or a fellow student or colleague $(27 \%)$ among young men, and a relative $(38 \%)$ or a neighbour or friend (31\%) among young women. Findings, moreover, suggest that young men were more likely than young women to cite a fellow student or colleague (27\% versus $15 \%$ ), a neighbour or friend (40\% versus $31 \%$ ) and an acquaintance from outside the village/urban community (20 versus $16 \%$ ), and conversely less likely to report a relative (13\% versus $38 \%$ ). This gender difference may be attributed to young women's relatively limited mobility and fewer opportunities for social mixing as compared to young men, described in Chapter 7.

This pattern was fairly consistent among both married and unmarried youth. Nonetheless, married youth were less likely than the unmarried to report a fellow student or colleague (14\% versus $32 \%$ among young men and $11 \%$ versus $20 \%$ among young women) and conversely, somewhat more likely to report an acquaintance from outside the village/urban community (24\% versus $18 \%$ among young men and $19 \%$ versus $11 \%$ among young women) as the first romantic partner. Married young men, in addition, were more likely than their unmarried counterparts to report a neighbour or friend from the village or urban community (47\% versus $37 \%$ ). Marital status differences were more pronounced in urban than rural areas. Rural-urban differences were mild, although urban young men were somewhat more likely than their rural counterparts to report a fellow student or colleague (31\% versus $25 \%$ ) and considerably less likely to report an acquaintance from outside the village/urban community ( $9 \%$ versus $24 \%$ ), and urban young women were somewhat less likely than their rural counterparts to report a relative (33\% versus $40 \%$ ) as the first romantic partner.

Respondents had typically been acquainted with their first romantic partner for one year or more before becoming romantically linked; this was consistently observed in all groups, irrespective of sex, marital status and rural-urban residence. Many young men and women (29-31\%) reported that they had been acquainted with their partner since childhood, a finding not surprising given that a sizeable proportion of partners were either from the same neighbourhood or, among young women, relatives. Differences by marital status and rural-urban residence suggest that married and rural youth were somewhat more likely than unmarried and urban youth to report that they had been acquainted with their first romantic partner since childhood.

The majority of youth reported that their partners came from religious and caste backgrounds similar to their own. Nevertheless, it is notable that considerable proportions of young people did engage in a romantic relationship with someone of a different religion and caste. For example, $18 \%$ of young men and $24 \%$ of young women reported that their first romantic partner was someone from a different religion and over two-fifths of young men (44\%) and one-third of young women (34\%) reported that their first romantic partner was someone from a different caste. 
Table 9.4: Characteristics of pre-marital romantic relationships and partners

Percentage of youth reporting a pre-marital romantic relationship by age at initiation of relationship, partner's socio-economic and demographic characteristics, and nature and duration of prior acquaintance, according to residence, Andhra Pradesh, 2007-08

\begin{tabular}{|c|c|c|c|c|c|c|}
\hline Characteristics $(\%)^{1}$ & $\begin{array}{c}M \\
15-24\end{array}$ & $\begin{array}{c}\text { W } \\
15-24\end{array}$ & $\begin{array}{c}\text { MM } \\
15-29\end{array}$ & $\begin{array}{c}\text { MW } \\
15-24\end{array}$ & $\begin{array}{c}\text { UM } \\
15-24\end{array}$ & $\begin{array}{c}\text { UW } \\
15-24\end{array}$ \\
\hline \multicolumn{7}{|c|}{ Combined } \\
\hline \multicolumn{7}{|c|}{$\begin{array}{l}\text { Age when respondent first spent time alone with } \\
\text { partner (years) }\end{array}$} \\
\hline 15 or below & 19.1 & 41.3 & 10.1 & 48.2 & 21.0 & 32.0 \\
\hline $\begin{array}{l}\text { Median age when respondent first spent tim } \\
\text { with partner }\end{array}$ & 18.0 & 16.0 & 18.0 & 15.0 & 18.0 & 16.0 \\
\hline \multicolumn{7}{|l|}{ Age of partner } \\
\hline Younger than respondent & 78.9 & 0.0 & 88.2 & 0.0 & 75.7 & 0.0 \\
\hline Same age as respondent & 18.1 & 3.0 & 8.7 & 2.4 & 21.3 & 4.0 \\
\hline Older than respondent & 1.6 & 89.2 & 2.4 & 87.6 & 1.8 & 91.3 \\
\hline Don't remember & 1.4 & 7.9 & 0.7 & 10.0 & 1.2 & 4.7 \\
\hline \multicolumn{7}{|l|}{ Partner's marital status } \\
\hline Unmarried & 96.6 & 98.0 & 94.1 & 98.4 & 97.3 & 97.6 \\
\hline Married & 3.4 & 2.0 & 5.6 & 1.6 & 2.7 & 2.4 \\
\hline \multicolumn{7}{|l|}{ Nature of prior acquaintance with first partner } \\
\hline Relative & 13.3 & 37.5 & 14.6 & 39.8 & 12.1 & 34.6 \\
\hline Fellow student/colleague & 26.8 & 14.6 & 13.9 & 10.8 & 32.2 & 19.7 \\
\hline Neighbour/friend & 39.7 & 31.2 & 47.4 & 30.1 & 37.2 & 32.7 \\
\hline Family friend & 0.2 & 0.2 & 0.0 & 0.0 & 0.3 & 0.4 \\
\hline Person from outside village/neighbourhood & 19.7 & 15.6 & 24.0 & 18.9 & 18.0 & 10.6 \\
\hline Other $^{2}$ & 0.2 & 1.0 & 0.0 & 0.4 & 0.3 & 2.0 \\
\hline \multicolumn{7}{|l|}{ Duration of acquaintance } \\
\hline Less than 1 month & 3.2 & 2.6 & 4.5 & 3.2 & 2.4 & 1.6 \\
\hline $1-11$ months & 8.0 & 9.6 & 11.5 & 7.6 & 7.4 & 13.0 \\
\hline 12 months or more & 59.3 & 57.3 & 50.0 & 54.4 & 62.7 & 61.0 \\
\hline Since childhood & 29.4 & 30.5 & 34.0 & 34.8 & 27.5 & 24.4 \\
\hline \multicolumn{7}{|l|}{ Partner's religion } \\
\hline Same as respondent & 82.3 & 75.4 & 81.2 & 83.2 & 81.7 & 64.4 \\
\hline Different from respondent & 17.5 & 24.2 & 18.8 & 16.8 & 18.0 & 34.8 \\
\hline \multicolumn{7}{|l|}{ Partner's caste } \\
\hline Same as respondent & 54.8 & 65.2 & 59.0 & 73.2 & 52.2 & 54.2 \\
\hline Different from respondent & 44.0 & 34.3 & 41.0 & 26.8 & 46.3 & 44.7 \\
\hline \multicolumn{7}{|l|}{ Partner's socio-economic status } \\
\hline Same as respondent & 44.4 & 32.7 & 51.7 & 34.8 & 42.6 & 29.6 \\
\hline Better than respondent & 35.2 & 48.0 & 27.1 & 44.8 & 36.7 & 53.0 \\
\hline Worse than respondent & 19.3 & 17.3 & 20.5 & 19.6 & 19.8 & 13.8 \\
\hline Number reporting a romantic relationship & 426 & 535 & 297 & 281 & 329 & 254 \\
\hline
\end{tabular}




\begin{tabular}{|c|c|c|c|c|c|c|}
\hline Characteristics $(\%)^{1}$ & $\underset{15-24}{M}$ & $\underset{15-24}{W}$ & $\begin{array}{c}\text { MM } \\
15-29\end{array}$ & $\begin{array}{c}\text { MW } \\
15-24\end{array}$ & $\begin{array}{c}\text { UM } \\
15-24\end{array}$ & $\begin{array}{l}\text { UW } \\
15-24\end{array}$ \\
\hline \multicolumn{7}{|c|}{ Urban } \\
\hline \multicolumn{7}{|c|}{$\begin{array}{l}\text { Age when respondent first spent time alone with } \\
\text { partner (years) }\end{array}$} \\
\hline 15 or below & 13.3 & 30.7 & 7.0 & 38.5 & 14.7 & 21.3 \\
\hline $\begin{array}{l}\text { Median age when respondent first spent tim } \\
\text { with partner }\end{array}$ & 18.0 & 16.0 & 19.0 & 16.0 & 18.0 & 17.0 \\
\hline \multicolumn{7}{|l|}{ Age of partner } \\
\hline Younger than respondent & 80.8 & 0.0 & 88.7 & 0.0 & 76.8 & 0.0 \\
\hline Same age as respondent & 13.3 & 3.9 & 5.6 & 1.3 & 16.8 & 6.5 \\
\hline Older than respondent & 4.2 & 88.8 & 4.2 & 89.9 & 5.3 & 89.2 \\
\hline Don't remember & 1.7 & 7.3 & 1.4 & 8.9 & 1.1 & 4.3 \\
\hline \multicolumn{7}{|l|}{ Partner's marital status } \\
\hline Unmarried & 95.8 & 98.3 & 93.0 & 97.5 & 96.8 & 97.9 \\
\hline Married & 4.2 & 1.7 & 5.6 & 2.5 & 3.2 & 2.1 \\
\hline \multicolumn{7}{|l|}{ Nature of prior acquaintance with first partner } \\
\hline Relative & 15.7 & 33.1 & 20.0 & 39.2 & 12.5 & 25.3 \\
\hline Fellow student/colleague & 30.6 & 17.4 & 17.1 & 8.9 & 37.5 & 28.4 \\
\hline Neighbour/friend & 43.0 & 32.6 & 48.6 & 29.1 & 39.6 & 36.8 \\
\hline Family friend & 0.8 & 0.6 & 0.0 & 0.0 & 1.0 & 0.0 \\
\hline Person from outside village/neighbourhood & 9.1 & 15.2 & 14.3 & 21.5 & 8.3 & 7.4 \\
\hline Other $^{2}$ & 0.8 & 1.1 & 0.0 & 1.3 & 1.0 & 2.1 \\
\hline \multicolumn{7}{|l|}{ Duration of acquaintance } \\
\hline Less than 1 month & 1.7 & 2.2 & 4.2 & 1.3 & 1.1 & 3.2 \\
\hline $1-11$ months & 10.0 & 10.6 & 14.1 & 10.0 & 7.4 & 11.7 \\
\hline 12 months or more & 65.8 & 60.9 & 54.9 & 55.0 & 73.6 & 68.1 \\
\hline Since childhood & 22.5 & 26.3 & 26.8 & 33.8 & 18.9 & 17.0 \\
\hline \multicolumn{7}{|l|}{ Partner's religion } \\
\hline Same as respondent & 77.5 & 71.9 & 81.4 & 77.2 & 73.7 & 64.9 \\
\hline Different from respondent & 21.7 & 27.0 & 18.6 & 22.8 & 25.3 & 33.0 \\
\hline \multicolumn{7}{|l|}{ Partner's caste } \\
\hline Same as respondent & 45.5 & 56.2 & 50.0 & 67.1 & 39.6 & 43.6 \\
\hline Different from respondent & 51.2 & 42.7 & 50.0 & 32.9 & 56.3 & 54.3 \\
\hline \multicolumn{7}{|l|}{ Partner's socio-economic status } \\
\hline Same as respondent & 50.8 & 36.9 & 48.6 & 43.0 & 50.5 & 29.8 \\
\hline Better than respondent & 30.8 & 45.3 & 27.1 & 38.0 & 32.6 & 54.3 \\
\hline Worse than respondent & 16.7 & 16.2 & 24.3 & 19.0 & 14.7 & 12.8 \\
\hline Number reporting a romantic relationship & 218 & 273 & 147 & 159 & 170 & 114 \\
\hline
\end{tabular}


Table 9.4: (Cont'd)

\begin{tabular}{|c|c|c|c|c|c|c|}
\hline Characteristics $(\%)^{1}$ & $\underset{15-24}{M}$ & $\begin{array}{c}\text { W } \\
15-24\end{array}$ & $\begin{array}{c}\text { MM } \\
15-29\end{array}$ & $\begin{array}{c}\text { MW } \\
15-24\end{array}$ & $\begin{array}{c}\text { UM } \\
15-24\end{array}$ & $\begin{array}{c}\text { UW } \\
15-24\end{array}$ \\
\hline \multicolumn{7}{|c|}{ Rural } \\
\hline \multicolumn{7}{|c|}{$\begin{array}{l}\text { Age when respondent first spent time alone with } \\
\text { partner (years) }\end{array}$} \\
\hline 15 or below & 21.3 & 47.1 & 11.1 & 52.9 & 23.5 & 38.4 \\
\hline $\begin{array}{l}\text { Median age when respondent first spent tim } \\
\text { with partner }\end{array}$ & 17.0 & 15.0 & 18.0 & 15.0 & 17.0 & 16.0 \\
\hline \multicolumn{7}{|l|}{ Age of partner } \\
\hline Younger than respondent & 78.1 & 0.0 & 88.0 & 0.0 & 75.0 & 0.0 \\
\hline Same age as respondent & 20.0 & 2.4 & 9.7 & 2.9 & 23.4 & 1.9 \\
\hline Older than respondent & 0.3 & 89.4 & 1.9 & 86.6 & 0.4 & 93.1 \\
\hline Don't remember & 1.6 & 8.2 & 0.5 & 10.5 & 1.2 & 5.0 \\
\hline \multicolumn{7}{|l|}{ Partner's marital status } \\
\hline Unmarried & 97.1 & 97.9 & 94.5 & 98.2 & 97.5 & 96.9 \\
\hline Married & 2.9 & 2.1 & 5.5 & 1.8 & 2.5 & 3.1 \\
\hline \multicolumn{7}{|l|}{ Nature of prior acquaintance with first partner } \\
\hline Relative & 12.4 & 39.8 & 13.3 & 39.8 & 12.3 & 40.0 \\
\hline Fellow student/colleague & 25.2 & 13.1 & 12.4 & 11.7 & 29.9 & 14.4 \\
\hline Neighbour/friend & 38.5 & 30.4 & 47.2 & 31.0 & 36.1 & 30.0 \\
\hline Family friend & 0.0 & 0.3 & 0.0 & 0.0 & 0.0 & 0.6 \\
\hline Person from outside village/neighbourhood & 23.9 & 15.8 & 27.1 & 17.5 & 21.7 & 13.1 \\
\hline Other $^{2}$ & 0.0 & 0.6 & 0.0 & 0.0 & 0.0 & 1.9 \\
\hline \multicolumn{7}{|l|}{ Duration of acquaintance } \\
\hline Less than 1 month & 3.5 & 2.7 & 4.1 & 4.1 & 3.3 & 0.6 \\
\hline $1-11$ months & 7.6 & 9.1 & 10.6 & 6.5 & 7.4 & 13.2 \\
\hline 12 months or more & 56.8 & 55.3 & 48.8 & 54.1 & 58.4 & 57.2 \\
\hline Since childhood & 32.1 & 32.8 & 36.4 & 35.3 & 30.9 & 28.9 \\
\hline \multicolumn{7}{|l|}{ Partner's religion } \\
\hline Same as respondent & 84.1 & 77.3 & 81.1 & 86.0 & 84.8 & 64.2 \\
\hline Different from respondent & 15.9 & 22.7 & 18.9 & 14.0 & 15.2 & 35.8 \\
\hline \multicolumn{7}{|l|}{ Partner's caste } \\
\hline Same as respondent & 58.6 & 69.9 & 61.8 & 76.0 & 56.8 & 60.4 \\
\hline Different from respondent & 41.1 & 29.8 & 38.2 & 24.0 & 42.8 & 39.0 \\
\hline \multicolumn{7}{|l|}{ Partner's socio-economic status } \\
\hline Same as respondent & 41.9 & 30.1 & 53.0 & 30.6 & 39.5 & 29.4 \\
\hline Better than respondent & 36.8 & 49.8 & 27.2 & 48.2 & 38.3 & 51.9 \\
\hline Worse than respondent & 20.3 & 17.9 & 19.4 & 20.0 & 21.8 & 15.0 \\
\hline Number reporting a romantic relationship & 208 & 262 & 150 & 122 & 159 & 140 \\
\hline
\end{tabular}

Note: All Ns are unweighted. Column totals may not equal 100\% due to missing cases or "don't know" responses. ${ }^{1}$ First romantic partner, if more than one romantic partner reported. ${ }^{2}$ Includes employee, employer, teacher, other acquaintance and stranger.

In contrast, the majority of youth reported partners from a different socio-economic background. Over half of young men $(55 \%)$ and two-thirds of young women $(65 \%)$ reported that the partner was from a family that was either socio-economically better or worse off than their own.

Differentials by marital status indicate that unmarried young women were considerably more likely than the married to report a partner from a different religion (35\% versus $17 \%)$; no such differences were evident among young men. 
Findings also show that the unmarried were more likely than the married to report a partner from a different caste ( $46 \%$ versus $41 \%$ among young men and $45 \%$ versus $27 \%$ among young women). Unmarried young men were, likewise, more likely than their married counterparts to report a partner from a different socio-economic background (57\% versus $48 \%$ ); differences were narrower among young women. Rural-urban differences were mild with regard to partner's religious background, although somewhat more urban than rural young men reported a partner from a different religion (22\% versus 16\%). Rural-urban differences in the partner's caste and socio-economic background were wide. More urban than rural youth reported a partner from a caste other than their own (51\% versus $41 \%$ among young men and $43 \%$ versus $30 \%$ among young women). In contrast, more rural than urban youth reported a partner from a different socio-economic background (57\% versus $48 \%$ among young men and $68 \%$ versus $62 \%$ among young women).

Table 9.5 presents youth responses to questions regarding places in which youth met their first romantic partner secretly, without adults present. The majority of youth who reported romantic relationships met their first romantic partner in each other's homes when other family members were absent ( $44 \%$ of young men and $51 \%$ of young women). At the same time, sizeable proportions of youth met secretly in places that offered them privacy or anonymity, including parks and gardens (39\% of young men and $28 \%$ of young women), movie theatres (35\% of young men and $26 \%$ of young women), places of worship (28\% of young men and $39 \%$ of young women) and fields or grazing areas (22-24\%). About $8-12 \%$ of youth reported not meeting their romantic partner in secret, without adults present, with unmarried youth somewhat more likely than married youth to report so ( $9 \%$ versus $4 \%$ among young men and $15 \%$ versus $10 \%$ among young women).

\subsubsection{Parental and peer awareness of romantic partnerships}

Table 9.6 reports findings on peer and parental awareness of young people's romantic partnerships. Youth overwhelmingly ( $94 \%$ of young men and $82 \%$ of young women) reported that their peers were aware of their romantic relationships. A larger percentage of unmarried than married young men reported peer awareness of romantic relationships ( $95 \%$ versus $86 \%$ ); among young women, however, somewhat more married than the unmarried so reported (84\% versus $79 \%)$. Rural-urban differences were negligible, although somewhat more married young men and unmarried young women in urban than rural areas reported peer awareness of their romantic relationships, and conversely, more married young women in rural than urban areas so reported.

Relatively few youth reported that their parents were aware of these partnerships. Young women were more likely than young men to report that parents were aware of their relationship (59\% and $40 \%$, respectively), and the married were considerably more likely to report parental awareness than the unmarried ( $49 \%$ and $35 \%$ of young men and $74 \%$ and $38 \%$ of young women, respectively). Rural-urban differences were mild, although somewhat more urban than rural married young men reported parental awareness of their relationship (59\% versus $46 \%$ ). Gender differences may be attributed to the likelihood that young women, who tend to be more strictly supervised, have fewer opportunities to hide a relationship from their parents than young men. Differences by marital status may be attributed to the likelihood that revelation of the relationship could itself have triggered marriage, either to the same person or someone else, as seen in the panel on parental reactions.

The reported reactions of those parents who became aware of their children's pre-marital romantic relationships are presented in Table 9.6. Given the small numbers, we provide findings for the rural and urban populations together. Parents' reported reactions varied between young men and young women. Large proportions of young men and women reported negative reactions. Half of young men and over one-third of young women (36\%) reported that their parents had shouted at them and one-fifth (18\% and $21 \%$, respectively) reported that their parents had beaten them. Some $7-12 \%$ of youth were not permitted to go out and $12-15 \%$ were forbidden from meeting their partner. Among young women, $2 \%$ were withdrawn from school as a consequence.

For considerable percentages of young women, parents reacted by arranging their marriages, more often to the romantic partner $(42 \%)$ than to someone else $(12 \%)$, perhaps in order to protect the family's reputation. In contrast, few young men reported that their parents reacted in this way $(2-6 \%)$. 
Table 9.5: Meeting places with pre-marital romantic partners

Percentage of youth reporting a pre-marital romantic relationship by places where they met their partner secretly, according to residence, Andhra Pradesh, 2007-08

\begin{tabular}{|c|c|c|c|c|c|c|}
\hline Meeting places $(\%)^{1}$ & $\begin{array}{c}\mathrm{M} \\
15-24\end{array}$ & $\begin{array}{c}\text { W } \\
15-24\end{array}$ & $\begin{array}{c}\text { MM } \\
15-29\end{array}$ & $\begin{array}{c}\text { MW } \\
15-24\end{array}$ & $\begin{array}{c}\text { UM } \\
15-24\end{array}$ & $\begin{array}{c}\text { UW } \\
15-24\end{array}$ \\
\hline \multicolumn{7}{|c|}{ Combined } \\
\hline Each other's home & 43.7 & 50.8 & 51.4 & 55.6 & 39.1 & 44.3 \\
\hline Temple/mosque/church & 28.0 & 38.7 & 25.6 & 39.6 & 26.9 & 37.4 \\
\hline Cinema/theatre & 34.5 & 25.6 & 33.7 & 26.8 & 34.6 & 23.6 \\
\hline Park/garden & 38.6 & 28.3 & 36.8 & 29.6 & 38.9 & 26.0 \\
\hline Restaurant/eating place & 11.5 & 11.2 & 9.7 & 8.4 & 13.3 & 15.0 \\
\hline Jungle/riverside & 7.4 & 2.4 & 7.3 & 2.4 & 6.2 & 2.0 \\
\hline Field/grazing area & 24.1 & 22.0 & 36.5 & 26.8 & 22.4 & 15.8 \\
\hline Never met unaccompanied & 7.6 & 12.0 & 3.5 & 10.0 & 8.6 & 15.0 \\
\hline Number reporting a romantic relationship & 426 & 535 & 297 & 281 & 329 & 254 \\
\hline \multicolumn{7}{|c|}{ Urban } \\
\hline Each other's home & 45.0 & 46.9 & 43.7 & 54.4 & 42.1 & 37.2 \\
\hline Temple/mosque/church & 31.7 & 44.9 & 32.4 & 46.8 & 31.6 & 42.6 \\
\hline Cinema/theatre & 32.2 & 30.3 & 44.3 & 36.7 & 30.5 & 23.4 \\
\hline Park/garden & 47.5 & 36.3 & 42.3 & 36.7 & 51.6 & 36.2 \\
\hline Restaurant/eating place & 17.5 & 20.2 & 16.9 & 19.0 & 19.1 & 22.3 \\
\hline Jungle/riverside & 4.1 & 3.4 & 5.6 & 3.8 & 2.1 & 3.2 \\
\hline Field/grazing area & 8.3 & 7.8 & 11.4 & 11.4 & 6.3 & 3.2 \\
\hline Never met unaccompanied & 6.7 & 12.9 & 5.6 & 10.1 & 6.4 & 17.0 \\
\hline Number reporting a romantic relationship & 218 & 273 & 147 & 159 & 170 & 114 \\
\hline \multicolumn{7}{|c|}{ Rural } \\
\hline Each other's home & 42.9 & 52.9 & 53.9 & 56.1 & 37.9 & 48.1 \\
\hline Temple/mosque/church & 26.7 & 35.3 & 23.0 & 35.7 & 25.1 & 34.4 \\
\hline Cinema/theatre & 35.2 & 23.1 & 30.0 & 22.8 & 36.2 & 23.8 \\
\hline Park/garden & 35.2 & 23.9 & 34.9 & 26.3 & 33.7 & 20.0 \\
\hline Restaurant/eating place & 9.2 & 6.4 & 7.4 & 3.5 & 11.1 & 10.7 \\
\hline Jungle/riverside & 8.6 & 1.5 & 7.8 & 1.8 & 7.8 & 1.3 \\
\hline Field/grazing area & 30.2 & 29.8 & 44.5 & 33.9 & 28.7 & 23.1 \\
\hline Never met unaccompanied & 7.9 & 11.6 & 2.8 & 9.9 & 9.4 & 14.4 \\
\hline Number reporting a romantic relationship & 208 & 262 & 150 & 122 & 159 & 140 \\
\hline
\end{tabular}

Note: All Ns are unweighted. Column totals may exceed $100 \%$ due to multiple responses. ${ }^{1}$ First romantic partner, if more than one romantic partner reported.

Notably, many parents of young men either accepted the situation or did not react at all to knowledge of their child's romantic relationship (37\%), or reacted by advising their son, for example, not to let school performance suffer as a result of the relationship (21\%). In contrast, relatively fewer young women reported these parental reactions ( $28 \%$ and $12 \%$, respectively).

\subsubsection{Marriage intentions and duration of pre-marital romantic relationships}

The questionnaire probed all respondents who reported a relationship about their intentions to marry their romantic partner. Findings are reported in Table 9.7 and suggest that the majority of youth did intend to marry either 
Table 9.6: Peer and parental awareness of first pre-marital romantic relationship

Percentage of youth reporting a pre-marital romantic relationship by peer and parental awareness of the first romantic relationship and parents' reaction, according to residence, Andhra Pradesh, 2007-08

\begin{tabular}{|c|c|c|c|c|c|c|}
\hline Awareness and reactions $(\%)^{1}$ & $\begin{array}{c}M \\
15-24\end{array}$ & $\begin{array}{c}\text { W } \\
15-24\end{array}$ & $\begin{array}{c}\text { MM } \\
15-29\end{array}$ & $\begin{array}{c}\text { MW } \\
15-24\end{array}$ & $\begin{array}{c}\text { UM } \\
15-24\end{array}$ & $\begin{array}{c}\text { UW } \\
15-24\end{array}$ \\
\hline \multicolumn{7}{|c|}{ Combined } \\
\hline Friends aware of relationship & 94.3 & 82.1 & 85.5 & 84.0 & 94.7 & 79.4 \\
\hline Parents aware of relationship & 40.2 & 59.4 & 48.8 & 74.4 & 34.9 & 37.9 \\
\hline Number reporting a romantic relationship & 426 & 535 & 297 & 281 & 329 & 254 \\
\hline \multicolumn{7}{|c|}{ Urban } \\
\hline Friends aware of relationship & 94.2 & 80.4 & 91.4 & 79.5 & 94.7 & 83.0 \\
\hline Parents aware of relationship & 43.3 & 57.9 & 59.2 & 73.8 & 35.8 & 39.4 \\
\hline Number reporting a romantic relationship & 218 & 273 & 147 & 159 & 170 & 114 \\
\hline \multicolumn{7}{|c|}{ Rural } \\
\hline Friends aware of relationship & 94.3 & 82.9 & 83.9 & 87.1 & 94.7 & 76.9 \\
\hline Parents aware of relationship & 38.9 & 60.2 & 45.6 & 74.9 & 34.8 & 37.5 \\
\hline Number reporting a romantic relationship & 208 & 262 & 150 & 122 & 159 & 140 \\
\hline \multicolumn{7}{|c|}{ Combined } \\
\hline Parents' reaction & & & & & & \\
\hline Shouted at respondent & 49.7 & 35.9 & 56.0 & 35.3 & 52.5 & 37.1 \\
\hline Beat respondent & 17.7 & 20.6 & 12.0 & 19.4 & 24.6 & 24.0 \\
\hline Did not allow respondent to go out & 6.9 & 11.6 & 5.7 & 11.8 & 5.9 & 11.3 \\
\hline Stopped respondent from meeting partner & 14.9 & 11.9 & 15.5 & 10.2 & 12.7 & 16.5 \\
\hline Forced respondent to discontinue education & 0.0 & 2.0 & 0.0 & 1.1 & 0.0 & 4.2 \\
\hline Reported to/shouted at partner's family & 2.9 & 4.7 & 5.7 & 3.8 & 3.4 & 8.2 \\
\hline Arranged marriage with partner & 5.7 & 42.2 & 8.5 & 49.5 & 4.2 & 22.7 \\
\hline Arranged marriage with someone else & 2.3 & 12.0 & 6.4 & 15.1 & 0.0 & 3.1 \\
\hline No reaction/accepted the situation & 36.6 & 28.2 & 32.6 & 23.7 & 34.7 & 40.6 \\
\hline $\begin{array}{l}\text { Advised respondent, including not to let school/colleg } \\
\text { performance suffer }\end{array}$ & 20.6 & 12.0 & 12.8 & 11.3 & 21.2 & 14.4 \\
\hline Other $^{2}$ & 0.0 & 0.3 & 0.0 & 0.5 & 0.0 & 0.0 \\
\hline Number whose parents were aware of relationship & 173 & 305 & 155 & 209 & 114 & 96 \\
\hline
\end{tabular}

Note: All Ns are unweighted. Columns totals may exceed 100\% due to multiple responses. Reporting of parents' reactions is presented for rural and urban combined due to small numbers. ${ }^{1}$ First romantic partner, if more than one romantic partner reported. ${ }^{2}$ Includes, for example, taking the matter to the panchayat or the police.

their first or most recent partner. Far larger percentages of young women than young men reported an intention to marry their partner (92\% versus $72 \%$ ), a finding observed in other studies as well (Alexander et al., 2006a; 2006b). Differences by marital status were mild, although somewhat more married than unmarried young women reported this intention (95\% versus 88\%). Findings suggest, moreover, that the pattern differed among young men, when analysed separately for urban and rural samples; the married were more likely than the unmarried to report marriage intentions in urban areas $(82 \%$ versus $75 \%)$ and less likely to so report in rural areas $(63 \%$ versus $71 \%)$. While urban young men were more likely than rural young men to report marriage intentions $(78 \%$ versus $70 \%$ among young men), the differences were muted among young women.

Reality, in terms of outcomes of romantic relationships, was different from intention. For example, while 95\% of married young women had intended to marry their pre-marital partner, just $70 \%$ reported having done so; $68 \%$ of married young men reported an intention to marry their pre-marital partner, yet only $23 \%$ reported doing so. 
Rural-urban differences were apparent; more urban than rural youth had married their pre-marital romantic partner ( $42 \%$ versus $17 \%$ among young men and $80 \%$ versus $66 \%$ among young women).

The majority of unmarried youth were still in a relationship at the time of the interview (57\% of young men and $79 \%$ of young women); unmarried young women (but not young men) in urban areas were slightly more likely than those in rural areas to be in a relationship. Some $7-8 \%$ of married young men and women reported continuing a relationship with their pre-marital partner even after marriage; more rural than urban married youth reported so (9-10\% versus $3 \%)$.

Among those who had discontinued their romantic relationship (the longest one, if more than one romantic partner was reported), relationships extended over fairly long time periods (more than one year for $48-50 \%$ of young men and women). Differences by marital status suggest that married young women (but not young men) were more likely than their unmarried counterparts to report that relationships extended over a year (52\% versus $42 \%)$. Rural-urban differences varied between young men and young women; while somewhat more urban than rural young men reported that relationships extended over a year (55\% versus $48 \%$ ), a reverse pattern was evident among young women (36\% versus $51 \%$ ).

\subsubsection{Pre-marital physical intimacy and sex with a romantic partner}

Respondents who reported a pre-marital romantic relationship were asked whether they had engaged in a number of intimate behaviours with their romantic partner. These ranged from behaviours reflecting minimal physical intimacy (hand-holding, hugging) to those reflecting increased physical intimacy (kissing on the lips) and finally, engaging in sexual relations. Findings, presented in Table 9.8, refer to youth experiences of physical intimacy with their first and/or most recent romantic partner, if more than one.

While the large majority of youth had held hands with a romantic partner, consistently fewer reported progressively more intimate behaviours. Wide gender differences were apparent in reporting of such experiences as hand-holding, hugging and kissing on the lips. For example, $95 \%$ of young men compared to $82 \%$ of young women reported holding hands with a romantic partner and $77 \%$ and $63 \%$, respectively, reported kissing their romantic partner on the lips. No such gender differences were, however, evident in the case of sexual experiences with a romantic partner, an equal percentage of young men and women reported such an experience (46\%). Gender differences were however apparent when the married and unmarried, and the rural and urban were analysed separately. Among the married, for example, young men were consistently more likely than young women to report each of these forms of behaviour. Among urban youth, too, young men were more likely than young women to have held hands, hugged and kissed their romantic partner, they were somewhat less likely than young women to have engaged in sex (33\% versus $38 \%)$.

Differences by marital status suggest that more married than unmarried youth reported each of these intimate behaviours (see also Figure 9.2). Differences by rural-urban residence differed somewhat between young men and women. Among young men, the differences were by and large mild, although rural young men were more likely than their urban counterparts to have engaged in sex with a romantic partner. Among both young men and women, larger proportions of those in rural than urban areas reported engaging in sex with a romantic partner (51\% versus 33\% among young men; $50 \%$ versus $38 \%$ among young women); young women in rural areas were also more likely than their urban counterparts to have hugged and kissed their romantic partners. Rural-urban differences, especially in the case of engaging in sex, may be attributed to the greater opportunities for privacy in rural than urban areas.

In short, findings confirm that pre-marital romantic relationships among youth almost always included some form of physical intimacy. Indeed, nearly half $(46 \%)$ of youth who reported a pre-marital romantic relationship had experienced sex with a romantic partner.

\subsubsection{Characteristics of sexual experiences within pre-marital romantic relationships}

The Youth Study asked all respondents reporting pre-marital sex with a romantic partner about fears of pregnancy or infection at the time of first sex, condom and contraceptive decision-making and use at first and subsequent 
Table 9.7: Marriage intentions and duration of pre-marital romantic relationships

Percentage of youth reporting a pre-marital romantic relationship by intention to marry partner, current relationship status and duration of relationship, according to residence, Andhra Pradesh, 2007-08

\begin{tabular}{|c|c|c|c|c|c|c|}
\hline $\begin{array}{l}\text { Marriage intentions, relationship status and duration } \\
\text { of relationship }{ }^{1}(\%)\end{array}$ & $\underset{15-24}{M}$ & $\begin{array}{c}W \\
15-24\end{array}$ & $\begin{array}{c}\text { MM } \\
15-29\end{array}$ & $\begin{array}{c}\text { MW } \\
15-24\end{array}$ & $\begin{array}{c}\text { UM } \\
15-24\end{array}$ & $\begin{array}{c}\text { UW } \\
15-24\end{array}$ \\
\hline \multicolumn{7}{|c|}{ Combined } \\
\hline Marriage intentions and outcomes & & & & & & \\
\hline Intended to marry pre-marital partner & 72.0 & 92.3 & 67.8 & 95.2 & 71.9 & 87.8 \\
\hline Married pre-marital partner & NA & NA & 22.9 & 70.4 & NA & NA \\
\hline \multicolumn{7}{|l|}{ Relationship status } \\
\hline Currently continuing relationship & 46.0 & 37.1 & 8.3 & 7.2 & 57.4 & 79.4 \\
\hline Number reporting a romantic relationship & 426 & 535 & 297 & 281 & 329 & 254 \\
\hline \multicolumn{7}{|l|}{ Duration of longest reported relationship (months) } \\
\hline Less than 1 & 1.5 & 0.9 & 2.0 & 1.8 & 0.7 & $(0.0)$ \\
\hline $1-6$ & 13.6 & 16.4 & 14.5 & 14.3 & 13.3 & $(18.9)$ \\
\hline $7-12$ & 35.0 & 34.5 & 30.5 & 32.1 & 32.2 & $(39.6)$ \\
\hline $13-24$ & 31.6 & 32.7 & 27.0 & 35.7 & 34.3 & $(26.4)$ \\
\hline 25 or more & 18.4 & 15.5 & 26.0 & 16.1 & 19.6 & $(15.1)$ \\
\hline Number who discontinued relationship & 195 & 107 & 192 & 58 & 139 & 49 \\
\hline \multicolumn{7}{|c|}{ Urban } \\
\hline Marriage intentions and outcomes & & & & & & \\
\hline Intended to marry pre-marital partner & 78.3 & 93.9 & 81.7 & 98.7 & 74.5 & 88.3 \\
\hline Married pre-marital partner & NA & NA & 42.3 & 79.7 & NA & $\mathrm{NA}$ \\
\hline \multicolumn{7}{|l|}{ Relationship status } \\
\hline Currently continuing relationship & 45.8 & 40.8 & 2.8 & 2.5 & 58.9 & 86.2 \\
\hline Number reporting a romantic relationship & 218 & 273 & 147 & 159 & 170 & 114 \\
\hline \multicolumn{7}{|l|}{ Duration of longest reported relationship (months) } \\
\hline Less than 1 & 0.0 & $(0.0)$ & 2.6 & $(0.0)$ & 0.0 & * \\
\hline $1-6$ & 11.8 & $(28.6)$ & 10.3 & $(28.6)$ & 10.0 & * \\
\hline $7-12$ & 33.3 & $(35.7)$ & 30.8 & $(35.7)$ & 32.5 & * \\
\hline $13-24$ & 35.3 & $(17.9)$ & 33.3 & $(21.4)$ & 37.5 & * \\
\hline 25 or more & 19.6 & $(17.9)$ & 23.1 & $(14.3)$ & 20.0 & * \\
\hline Number who discontinued relationship & 93 & 43 & 81 & 27 & 71 & 16 \\
\hline \multicolumn{7}{|c|}{ Rural } \\
\hline Marriage intentions and outcomes & & & & & & \\
\hline Intended to marry pre-marital partner & 69.5 & 91.5 & 63.3 & 93.6 & 70.9 & 87.5 \\
\hline Married pre-marital partner & NA & NA & 16.6 & 65.5 & NA & NA \\
\hline \multicolumn{7}{|l|}{ Relationship status } \\
\hline Currently continuing relationship & 46.0 & 35.2 & 10.1 & 9.4 & 57.0 & 75.6 \\
\hline Number reporting a romantic relationship & 208 & 262 & 150 & 122 & 159 & 140 \\
\hline \multicolumn{7}{|l|}{ Duration of longest reported relationship (months) } \\
\hline Less than 1 & 1.9 & 1.2 & 1.9 & $(2.3)$ & 1.0 & $(0.0)$ \\
\hline $1-6$ & 14.2 & 12.3 & 15.6 & $(9.3)$ & 15.2 & $(15.0)$ \\
\hline $7-12$ & 35.5 & 35.8 & 30.0 & $(30.2)$ & 32.4 & $(42.5)$ \\
\hline $13-24$ & 31.0 & 37.0 & 25.6 & $(41.9)$ & 32.4 & $(30.0)$ \\
\hline 25 or more & 17.4 & 13.6 & 26.9 & $(16.3)$ & 19.0 & (12.5) \\
\hline Number who discontinued relationship & 102 & 64 & 111 & 31 & 68 & 33 \\
\hline
\end{tabular}

Note: All Ns are unweighted. Column totals may not equal 100\% due to missing cases or "don't know" responses. ( ) Based on 25-49 unweighted cases. ${ }^{*}$ Percentage not shown, based on fewer than 25 unweighted cases. NA: Not applicable. ${ }^{1}$ Data on marriage intentions, relationship status and duration of relationship were collected only with regard to the first and/or most recent partner. These data were not available for 20 young men and 2 young women who reported more than two romantic partners. 
Table 9.8: Physical intimacy and sexual experiences in pre-marital romantic relationships

Percentage of youth reporting a pre-marital romantic relationship by experiences of physical intimacy and sex with their partner, according to residence, Andhra Pradesh, 2007-08

\begin{tabular}{|c|c|c|c|c|c|c|}
\hline Physical intimacy $(\%)^{1}$ & $\begin{array}{c}\mathrm{M} \\
15-24\end{array}$ & $\begin{array}{c}\mathrm{W} \\
15-24\end{array}$ & $\begin{array}{c}\text { MM } \\
15-29\end{array}$ & $\begin{array}{c}\text { MW } \\
15-24\end{array}$ & $\begin{array}{c}\text { UM } \\
15-24\end{array}$ & $\begin{array}{c}\text { UW } \\
15-24\end{array}$ \\
\hline \multicolumn{7}{|c|}{ Combined } \\
\hline Ever held hands & 94.5 & 81.7 & 96.5 & 85.6 & 93.8 & 76.0 \\
\hline Ever hugged & 81.6 & 63.0 & 86.8 & 68.8 & 80.2 & 55.1 \\
\hline Ever kissed & 76.8 & 62.6 & 83.3 & 69.2 & 74.9 & 53.9 \\
\hline Ever had sexual relations & 45.9 & 45.9 & 63.7 & 50.0 & 41.3 & 40.6 \\
\hline Number reporting a romantic relationship & 426 & 535 & 297 & 281 & 329 & 254 \\
\hline \multicolumn{7}{|c|}{ Urban } \\
\hline Ever held hands & 93.3 & 80.4 & 94.4 & 84.8 & 93.7 & 75.5 \\
\hline Ever hugged & 79.3 & 54.5 & 78.9 & 59.5 & 81.1 & 48.9 \\
\hline Ever kissed & 77.5 & 53.4 & 77.5 & 59.5 & 77.9 & 45.7 \\
\hline Ever had sexual relations & 33.3 & 38.0 & 43.7 & 41.8 & 31.6 & 33.0 \\
\hline Number reporting a romantic relationship & 218 & 273 & 147 & 159 & 170 & 114 \\
\hline \multicolumn{7}{|c|}{ Rural } \\
\hline Ever held hands & 94.9 & 82.4 & 97.2 & 86.5 & 93.8 & 76.1 \\
\hline Ever hugged & 82.2 & 67.8 & 89.0 & 73.1 & 79.8 & 59.1 \\
\hline Ever kissed & 76.5 & 67.8 & 85.3 & 73.7 & 73.7 & 58.8 \\
\hline Ever had sexual relations & 50.5 & 50.2 & 70.2 & 53.2 & 45.3 & 45.0 \\
\hline Number reporting a romantic relationship & 208 & 262 & 150 & 122 & 159 & 140 \\
\hline
\end{tabular}

Note: All Ns are unweighted. ${ }^{1}$ Data on ever held hands, ever hugged and ever kissed pertain to the first or most recent partner, if more than one partner was reported. Data on pre-marital sexual relations pertain not only to the first or most recent partner, but also to other romantic partners, if more than two romantic partners were reported.

Figure 9.2: Percentage of youth reporting experiences of physical intimacy and sex with a pre-marital romantic partner, Andhra Pradesh, 2007-08

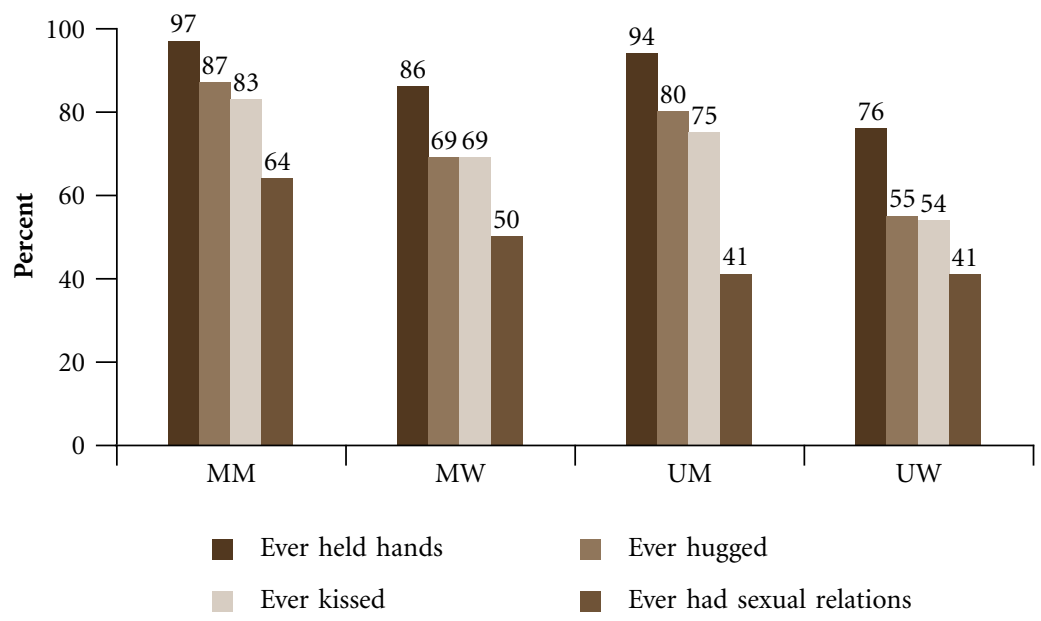

sexual encounters with a romantic sexual partner, and the consensual nature of first sex. Findings are presented in Table 9.9. Given the small numbers of respondents reporting pre-marital sexual experience, we provide combined rural-urban findings for married and unmarried young men and women. 
Among those who reported sexual experience within pre-marital romantic relationships, many more young women than men reported fear of pregnancy or infection at the time of first sex. For example, fear of pregnancy was reported by $28 \%$ of young men and $49 \%$ of young women; far fewer young men and women reported fear of infection, $6 \%$ and $14 \%$, respectively. Unmarried young men were somewhat more likely than married young men to report fear of pregnancy (32\% versus $26 \%$ ), but were as likely to report fear of infection (4-5\%). Among young women, the unmarried were more likely than the married to report fear of both pregnancy ( $54 \%$ and $46 \%$, respectively) and infection (18\% and $11 \%$, respectively).

Reported contraceptive use at first pre-marital sex with a romantic partner and consistent contraceptive use in subsequent sexual encounters were limited. In total, just $24 \%$ of young men and $9 \%$ of young women reported using contraception at first sex, and $19 \%$ and 3\%, respectively, reported that contraception was consistently practised in all sexual encounters with their romantic opposite-sex partner(s). More unmarried than married youth reported using contraception at first sex (26\% compared to $20 \%$ among young men and $15 \%$ compared to $6 \%$ among young women) and consistent contraceptive use (20\% versus $10 \%$ among young men and $7 \%$ versus $1 \%$ among young women).

Condom use was limited. Just $20 \%$ of young men and $6 \%$ of young women had used a condom during their first sexual encounter with a romantic partner. Even so, it is clear that the majority of those who practised contraception at first sex used a condom ( $85 \%$ of young men and $70 \%$ of young women). Of note, however, is that fewer married than unmarried youth who practised contraception at first sex used a condom (57-69\% compared to $80-85 \%$ ). Somewhat fewer- $16 \%$ of young men and $3 \%$ of young women-reported that they had used condoms in all sexual encounters with their romantic partner(s). Virtually all young men and women who used a condom at first sex, irrespective of marital status, reported doing so to prevent pregnancy, many fewer reported doing so to prevent infection as well.

Youth reports of decision-making regarding contraceptive use at first pre-marital sex with an opposite-sex romantic partner reveal that, in many cases, the female partner was involved in the decision to practise contraception at first sex. While $24 \%$ of young men and $9 \%$ of young women had practised contraception at first sex with an opposite-sex romantic partner, as many as $16 \%$ and $6 \%$, respectively, reported that the female partner had participated in the decision.

While the majority of young men and women reported that their first experience of pre-marital sex with an opposite-sex romantic partner was consensual, several youth reported that it occurred without consent. Gender differences were modest. For example, $81 \%$ of young men and $76 \%$ of young women reported that sex was consensual. A small minority of young women (6\%) reported that their opposite-sex romantic partner had forced them to engage in sex the first time and 3\% of young men acknowledged that they had forced their partner to do so. Somewhat larger percentages of young men (10\%) and women (14\%) reported that the male partner had persuaded the female partner to engage in sexual relations. Very few youth reported that the female partner had persuaded $(3-4 \%)$ or forced $(1-2 \%)$ the male partner to engage in sex.

Differences by marital status were mild, although more married than unmarried young men reported that their first experience of pre-marital sex with an opposite-sex romantic partner was consensual (88\% versus $77 \%$ ) and more unmarried than married young women reported that male partner had forced the female partner to engage in sexual relations $(12 \%$ versus $3 \%)$.

\subsection{Pre-marital sexual experiences within romantic and other relationships}

Aside from the heterosexual romantic partnerships discussed in previous sections, the Youth Study also probed youth experiences of pre-marital sex with other partners, including casual partners and spouse before marriage, and in situations characterised by force and exchange of gifts or favours. In addition, in the case of male respondents, questions were asked about pre-marital sex with same-sex partners, sex workers and married women. In this and subsequent sections of this chapter, we present findings on the prevalence of pre-marital sexual experiences (irrespective of whether such experiences took place within romantic or other partnerships) among all youth in the sample. 
Table 9.9: Characteristics of sexual experiences within pre-marital romantic relationships

Percentage of youth reporting pre-marital sexual experiences with an opposite-sex romantic partner by selected characteristics of their first and subsequent sexual encounters with the partner, Andhra Pradesh, 2007-08

\begin{tabular}{|c|c|c|c|c|c|c|}
\hline Characteristics $(\%)^{1}$ & $\underset{15-24}{M}$ & $\begin{array}{c}\text { W } \\
15-24\end{array}$ & $\begin{array}{c}\text { MM } \\
15-29\end{array}$ & $\begin{array}{c}\text { MW } \\
15-24\end{array}$ & $\begin{array}{c}\text { UM } \\
15-24\end{array}$ & $\begin{array}{l}\text { UW } \\
15-24\end{array}$ \\
\hline Anxiety associated with first sex & & & & & & \\
\hline Afraid of getting pregnant at first sex & 28.3 & 49.4 & 25.8 & 46.4 & 31.7 & 54.4 \\
\hline Afraid of getting infection at first sex & 5.6 & 13.7 & 5.0 & 11.2 & 4.3 & 18.4 \\
\hline Contraceptive use & & & & & & \\
\hline Practised contraception at first sex & 23.7 & 8.6 & 19.9 & 5.6 & 25.9 & 14.6 \\
\hline $\begin{array}{l}\text { Practised contraception in all sexual } \\
\text { encounters }{ }^{2}\end{array}$ & 18.7 & 3.4 & 10.4 & 0.8 & 20.0 & 6.9 \\
\hline Condom use & & & & & & \\
\hline Used a condom at first sex to: & 20.2 & 6.0 & 13.7 & 3.2 & 22.1 & 11.7 \\
\hline Avoid pregnancy & 19.7 & 6.0 & 12.6 & 3.2 & 20.9 & 11.7 \\
\hline Avoid infection & 15.7 & 3.9 & 11.5 & 3.2 & 17.1 & 5.8 \\
\hline Used condoms in all sexual encounters ${ }^{2}$ & 15.7 & 3.4 & 8.7 & 0.8 & 16.4 & 6.8 \\
\hline $\begin{array}{l}\text { Decision to use contraception at first sex } \\
\text { taken by: }\end{array}$ & & & & & & \\
\hline Respondent & 7.6 & 0.4 & 5.6 & 0.0 & 8.6 & 0.0 \\
\hline Partner & 2.0 & 2.6 & 0.6 & 1.6 & 2.9 & 4.9 \\
\hline Jointly & 14.1 & 6.0 & 13.3 & 4.0 & 14.4 & 9.7 \\
\hline Consensuality of first sex & & & & & & \\
\hline Mutual consent & 80.9 & 75.5 & 88.4 & 74.4 & 76.6 & 76.7 \\
\hline Male partner forced & 3.0 & 6.4 & 2.2 & 3.2 & 3.5 & 11.7 \\
\hline Female partner forced & 2.0 & 1.3 & 0.6 & 2.4 & 2.8 & 0.0 \\
\hline Male partner persuaded & 10.1 & 14.2 & 7.7 & 16.0 & 11.3 & 11.7 \\
\hline Female partner persuaded & 4.0 & 2.6 & 1.1 & 4.0 & 5.7 & 0.0 \\
\hline $\begin{array}{l}\text { Number reporting pre-marital sex with an } \\
\text { opposite-sex romantic partner }\end{array}$ & 177 & 234 & 168 & 132 & 125 & 102 \\
\hline
\end{tabular}

Note: All Ns are unweighted. Column totals may not equal 100\% due to missing cases or "don't know" responses. ${ }^{1}$ In-depth probing of sexual experiences was restricted to respondents' first or most recent romantic partner. Therefore, if a respondent reported his/her first sexual experience as occurring with a romantic partner other than the first or the most recent, then age, consensuality and other characteristics at first sex were unknown. Information was not available in 1 such case. ${ }^{2}$ Data were missing for 8 young men and 1 young woman who reported sexual experiences with a romantic partner other than the first or most recent partner.

\subsubsection{Extent of pre-marital sexual experiences}

Table 9.10 reports percentages of respondents reporting pre-marital sex in any of the situations described above. For $8 \%$ of young men and $5 \%$ of young women, pre-marital sex occurred in a romantic relationship with a person of the opposite sex. In addition, $0.4 \%$ of young men and women reported that they were forced to engage in sex, and $0.5 \%$ of young men reported that they had forced sex on a female partner. Sex in exchange for money or favours was reported by $0.1 \%$ of youth. Casual sex was reported by $2.4 \%$ of young men and $0.1 \%$ of young women.

Young men were asked, in addition, about same-sex relations, relations with sex workers and married women (excluding their own wife, if married). Just $0.2 \%$ of young men reported having experienced same-sex relations. Relations with sex workers and married women were reported by $3 \%$ of young men. Some $3-4 \%$ of married young men and women reported sex with their spouse before marriage (some of these included those who had sex with a romantic partner whom they later married). In this way, a total of $13 \%$ of young men and $5 \%$ of young women reported pre-marital sexual relations in the course of face-to-face interviews. 
Several youth, particularly young men who had not admitted sexual experience in the face-to-face interview did so in the anonymous sealed envelope. Including these, in total, $16 \%$ of young men and $6 \%$ of young women had reported any pre-marital sexual experience. Although the Youth Study findings fall within ranges observed in a variety of small case studies (15-30\% for males and fewer than 10\% for females; Jejeebhoy and Sebastian, 2004), the possibility that youth opted not to disclose sexual experience in various situations cannot be discounted, particularly in the case of reporting by young women, and in the reporting of forced, same-sex or sex worker relations.

Married young men were twice as likely as the unmarried to report any pre-marital sexual experience ( $27 \%$ versus $14 \%$ ), however, the differences were relatively narrow among young women ( $6 \%$ and $5 \%$, respectively) (see also Figure 9.3). Rural-urban differences were muted among both young men and women; however, rural young men were somewhat more likely than their urban counterparts to report sex with a romantic opposite sex partner and this was particularly evident among the married (14\% versus $10 \%)$.

Table 9.11 presents percentages reporting pre-marital sexual experience by selected socio-demographic characteristics of youth. As indicated earlier, such background characteristics as work status and household economic status reflect the situation of youth at the time of interview, and not necessarily at the time when pre-marital sex was experienced. In view of the small numbers of respondents reporting such experiences, findings are presented for all married and unmarried young men and women; findings for rural and urban respondents are not separately provided.

Age profiles confirm the positive association between age and pre-marital sexual experience among young men, with those aged $20-24$ being twice as likely as younger respondents to report sexual experience (22\% versus $10 \%$ ). Differences were, however, muted among young women. Differentials by religion and caste were pronounced among both young men and women. Findings consistently show that Hindu and Muslim young men and women were less likely than those belonging to the other religions to report pre-marital sex, although the differences were narrow among young women. Among young men, for example, 15-16\% of Hindus and Muslims reported pre-marital sex compared with $26 \%$ of those belonging to the other religions. Caste-wise differences suggest that young men from scheduled castes were more likely than others to report pre-marital sexual experience (24\% versus $12-17 \%)$. Among young women, those from scheduled tribes were more likely than others to report pre-marital sex (13\% versus 4-9\%).

Associations between reported pre-marital sexual experience and both educational attainment and wealth quintile were inconsistent among both young men and women. However, among unmarried young women, those belonging to the poorest quintile were more likely than others to report pre-marital sexual experience (10\% versus $4-6 \%$ ). A strong positive relationship was observed with economic activity status among young men, a finding that may be attributed to the greater mobility and relative freedom from parental supervision experienced by working young men as compared to non-working young men. Young men who had worked in the last year were considerably more likely than non-working young men to have experienced pre-marital sex (20\% versus $7 \%)$. No such differences were, however, apparent among young women.

Socio-demographic differentials described above were by and large observed among both married and unmarried youth.

\subsubsection{Age at initiation of pre-marital sex}

Table 9.12 presents cumulative percentages of youth who experienced first pre-marital sex at selected ages (among all youth in the sample) calculated using life table techniques, with censoring taking place at the time of marriage for married youth and at the time of the interview for unmarried youth. For youth who reported pre-marital sex only through the anonymous sealed envelope method, age at first premarital sex was imputed conservatively, using age at marriage (for the married) and current age (for the unmarried) as age at initiation of pre-marital sex.

Several findings are notable. First, roughly similar proportions of young men and women-3\% and 5\%, respectively - had initiated first sex before age 18 . Second, youth in rural areas were considerably more likely to initiate pre-marital sexual relations in adolescence than their urban counterparts; for example, $10-12 \%$ of rural youth compared to $6-7 \%$ of urban youth had their sexual debut in adolescence, that is, before they were aged 20 . Third, findings indicate notable increases in the initiation of pre-marital sexual activity as young people transitioned 
Table 9.10: Overall pre-marital sexual experiences

Percentage of youth reporting pre-marital sexual experiences with any partner and via different reporting methods, according to residence, Andhra Pradesh, 2007-08

\begin{tabular}{|c|c|c|c|c|c|c|}
\hline $\begin{array}{l}\text { Pre-marital sexual experiences and } \\
\text { reporting methods }(\%)\end{array}$ & $\begin{array}{c}M \\
15-24\end{array}$ & $\begin{array}{c}\text { W } \\
15-24\end{array}$ & $\begin{array}{c}\text { MM } \\
15-29\end{array}$ & $\begin{array}{l}\text { MW } \\
15-24\end{array}$ & $\begin{array}{c}\text { UM } \\
15-24\end{array}$ & $\begin{array}{c}\text { UW } \\
15-24\end{array}$ \\
\hline \multicolumn{7}{|c|}{ Combined } \\
\hline $\begin{array}{l}\text { Reported pre-marital sex with: } \\
\text { Opposite-sex romantic partner } \\
\text { Same-sex partner } \\
\text { Someone who forced respondent to have sex } \\
\text { Girl whom respondent forced } \\
\text { Someone in exchange for money/favour } \\
\text { Sex worker } \\
\text { Married woman } \\
\text { Casual partner } \\
\text { Spouse before marriage }\end{array}$ & $\begin{array}{r}8.0 \\
0.2 \\
0.4 \\
0.5 \\
0.1 \\
2.6 \\
2.8 \\
2.4 \\
\text { NA }\end{array}$ & $\begin{array}{r}4.7 \\
\text { NA } \\
0.4 \\
\text { NA } \\
0.1 \\
\text { NA } \\
\text { NA } \\
0.1 \\
\text { NA }\end{array}$ & $\begin{array}{r}13.0 \\
0.1 \\
0.1 \\
0.0 \\
0.2 \\
5.2 \\
4.6 \\
2.1 \\
2.8\end{array}$ & $\begin{array}{r}5.2 \\
\text { NA } \\
0.3 \\
\text { NA } \\
0.0 \\
\text { NA } \\
\text { NA } \\
0.0 \\
3.9\end{array}$ & $\begin{array}{r}6.7 \\
0.3 \\
0.4 \\
0.6 \\
0.1 \\
2.4 \\
2.6 \\
2.2 \\
\text { NA }\end{array}$ & $\begin{array}{r}4.1 \\
\text { NA } \\
0.6 \\
\text { NA } \\
0.1 \\
\text { NA } \\
\text { NA } \\
0.3 \\
\text { NA }\end{array}$ \\
\hline $\begin{array}{l}\text { Reported any pre-marital sex via: } \\
\text { Face-to-face interview } \\
\text { Anonymous format (sealed envelope) } \\
\text { Face-to-face interview or anonymous format } \\
\quad \text { (sealed envelope) } \\
\text { Number of respondents }\end{array}$ & $\begin{array}{r}16.4 \\
2,479\end{array}$ & $\begin{array}{r}5.8 \\
\mathbf{4 , 8 4 8}\end{array}$ & $\begin{array}{r}26.6 \\
1,405\end{array}$ & $\begin{array}{r}6.3 \\
2,330\end{array}$ & $\begin{array}{r}14.4 \\
2,077\end{array}$ & $\begin{array}{r}5.2 \\
2,518\end{array}$ \\
\hline \multicolumn{7}{|c|}{ Urban } \\
\hline $\begin{array}{l}\text { Reported pre-marital sex with: } \\
\text { Opposite-sex romantic partner } \\
\text { Same-sex partner } \\
\text { Someone who forced respondent to have sex } \\
\text { Girl whom respondent forced } \\
\text { Someone in exchange for money/favour } \\
\text { Sex worker } \\
\text { Married woman }{ }^{1} \\
\text { Casual partner } \\
\text { Spouse before marriage }\end{array}$ & $\begin{array}{r}5.7 \\
0.1 \\
0.1 \\
0.1 \\
0.0 \\
3.3 \\
2.1 \\
2.3 \\
\text { NA }\end{array}$ & $\begin{array}{r}4.7 \\
\text { NA } \\
0.6 \\
\text { NA } \\
0.1 \\
\text { NA } \\
\text { NA } \\
0.1 \\
\text { NA }\end{array}$ & $\begin{array}{l}9.8 \\
0.0 \\
0.3 \\
0.0 \\
0.0 \\
5.9 \\
4.9 \\
2.3 \\
3.6\end{array}$ & $\begin{array}{r}6.3 \\
\text { NA } \\
0.6 \\
\text { NA } \\
0.0 \\
\text { NA } \\
\text { NA } \\
0.0 \\
5.5\end{array}$ & $\begin{array}{l}4.8 \\
0.2 \\
0.2 \\
0.2 \\
0.0 \\
3.5 \\
1.7 \\
2.2 \\
\text { NA }\end{array}$ & $\begin{array}{r}3.4 \\
\text { NA } \\
0.7 \\
\text { NA } \\
0.2 \\
\text { NA } \\
\text { NA } \\
0.2 \\
\text { NA }\end{array}$ \\
\hline $\begin{array}{l}\text { Reported any pre-marital sex via: } \\
\text { Face-to-face interview } \\
\text { Anonymous format (sealed envelope) } \\
\text { Face-to-face interview or anonymous format } \\
\quad \text { (sealed envelope) } \\
\text { Number of respondents }\end{array}$ & $\begin{array}{r}11.5 \\
12.5 \\
14.4 \\
\mathbf{1 . 2 8 9}\end{array}$ & $\begin{array}{r}6.1 \\
2126\end{array}$ & $\begin{array}{r}19.9 \\
24.8 \\
26.7 \\
648\end{array}$ & $\begin{array}{r}8.0 \\
1.037\end{array}$ & $\begin{array}{r}10.5 \\
11.0 \\
12.9 \\
1.32\end{array}$ & $\begin{array}{r}3.9 \\
4.0 \\
4.6 \\
\\
1089\end{array}$ \\
\hline \multicolumn{7}{|c|}{ Rural } \\
\hline $\begin{array}{l}\text { Reported pre-marital sex with: } \\
\text { Opposite-sex romantic partner } \\
\text { Same-sex partner } \\
\text { Someone who forced respondent to have sex } \\
\text { Girl whom respondent forced } \\
\text { Someone in exchange for money/favour } \\
\text { Sex worker } \\
\text { Married woman }{ }^{1} \\
\text { Casual partner } \\
\text { Spouse before marriage }\end{array}$ & $\begin{array}{r}8.8 \\
0.3 \\
0.4 \\
0.6 \\
0.2 \\
2.3 \\
3.1 \\
2.5 \\
\text { NA }\end{array}$ & $\begin{array}{r}4.7 \\
\text { NA } \\
0.3 \\
\text { NA } \\
0.1 \\
\text { NA } \\
\text { NA } \\
0.1 \\
\text { NA }\end{array}$ & $\begin{array}{r}13.8 \\
0.1 \\
0.1 \\
0.0 \\
0.3 \\
5.1 \\
4.5 \\
2.0 \\
2.5\end{array}$ & $\begin{array}{c}4.8 \\
\text { NA } \\
0.2 \\
\text { NA } \\
0.1 \\
\text { NA } \\
\text { NA } \\
0.0 \\
3.5\end{array}$ & $\begin{array}{l}7.6 \\
0.3 \\
0.6 \\
0.8 \\
0.2 \\
1.9 \\
2.8 \\
2.1 \\
\text { NA }\end{array}$ & $\begin{array}{r}4.5 \\
\text { NA } \\
0.5 \\
\text { NA } \\
0.1 \\
\text { NA } \\
\text { NA } \\
0.4 \\
\text { NA }\end{array}$ \\
\hline $\begin{array}{l}\text { Reported any pre-marital sex via: } \\
\text { Face-to-face interview } \\
\text { Anonymous format (sealed envelope) } \\
\text { Face-to-face interview or anonymous format } \\
\quad \text { (sealed envelope) }\end{array}$ & $\begin{array}{l}14.1 \\
15.3\end{array}$ & $\begin{array}{l}5.1 \\
5.0\end{array}$ & $\begin{array}{l}21.7 \\
23.5\end{array}$ & $\begin{array}{l}5.3 \\
5.3\end{array}$ & $\begin{array}{l}12.4 \\
13.7\end{array}$ & $\begin{array}{l}4.7 \\
4.7\end{array}$ \\
\hline Number of respondents & 1,190 & 2,722 & 757 & 1,293 & 945 & 1,429 \\
\hline
\end{tabular}

Note: All Ns are unweighted. NA: Not applicable. ${ }^{1}$ Sex with a married woman excludes sex with wife before marriage. 
Figure 9.3: Percentage of youth reporting any pre-marital sexual experiences (in face-to-face interview or sealed envelope), according to residence, Andhra Pradesh, 2007-08
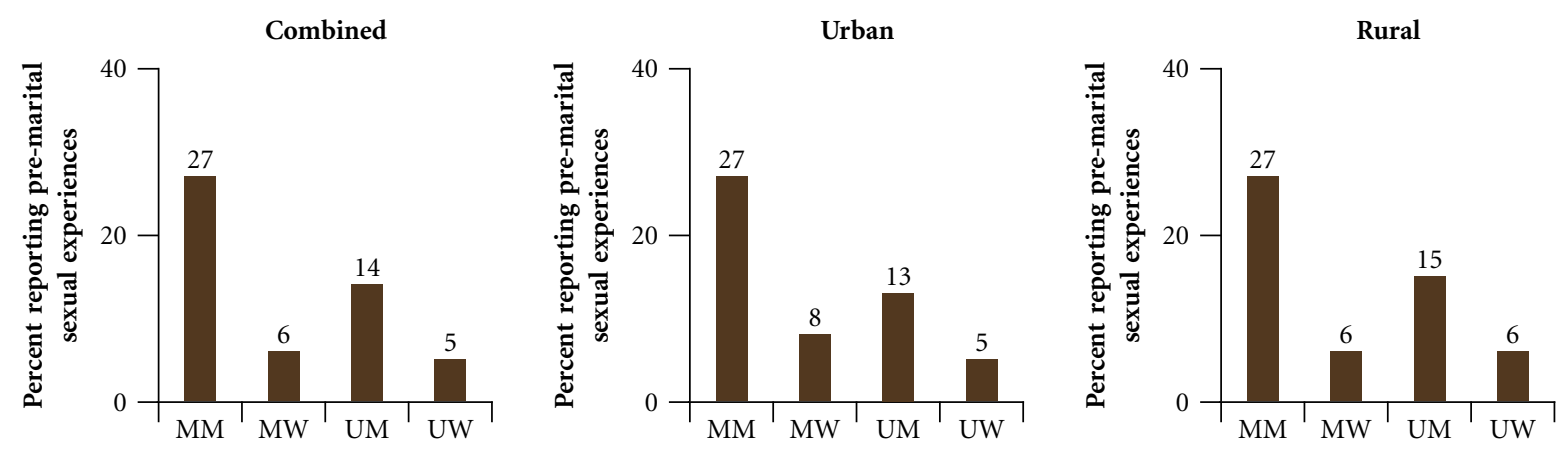

Table 9.11: Overall pre-marital sexual experiences by selected background characteristics

Percentage of youth reporting any pre-marital sexual experiences by selected background characteristics, Andhra Pradesh, 2007-08

\begin{tabular}{|c|c|c|c|c|c|c|}
\hline Background characteristics (\%) & $\begin{array}{c}\mathrm{M} \\
15-24\end{array}$ & $\begin{array}{c}\text { W } \\
15-24\end{array}$ & $\begin{array}{c}\mathrm{MM} \\
15-29\end{array}$ & $\begin{array}{c}\text { MW } \\
15-24\end{array}$ & $\begin{array}{c}\text { UM } \\
15-24\end{array}$ & $\begin{array}{c}\text { UW } \\
\text { 15-24 }\end{array}$ \\
\hline \multicolumn{7}{|l|}{ Age (years) } \\
\hline $15-19$ & 10.1 & 5.6 & * & 7.3 & 9.7 & 4.7 \\
\hline $20-24$ & 22.1 & 6.0 & 24.6 & 5.9 & 20.7 & 6.6 \\
\hline $25-29$ & NA & NA & 27.3 & NA & NA & NA \\
\hline \multicolumn{7}{|l|}{ Religion } \\
\hline Hindu & 16.0 & 5.8 & 26.6 & 6.4 & 14.0 & 5.0 \\
\hline Muslim & 14.6 & 3.5 & 22.1 & 3.4 & 12.9 & 4.0 \\
\hline Other $^{1}$ & 25.6 & 7.7 & 32.2 & 7.6 & 23.8 & 8.0 \\
\hline \multicolumn{7}{|l|}{ Caste } \\
\hline SC & 23.7 & 8.7 & 34.7 & 10.4 & 21.5 & 6.4 \\
\hline ST & 17.2 & 13.4 & 29.8 & 12.7 & 13.3 & 15.0 \\
\hline $\mathrm{OBC}$ & 14.8 & 4.4 & 25.4 & 4.6 & 12.7 & 4.1 \\
\hline General $^{2}$ & 12.3 & 4.1 & 18.5 & 4.1 & 11.6 & 4.2 \\
\hline \multicolumn{7}{|l|}{ Educational level (years) } \\
\hline None $^{3}$ & 16.4 & 5.1 & 24.3 & 5.2 & 14.0 & 3.9 \\
\hline $1-7$ & 19.9 & 6.7 & 28.0 & 6.9 & 17.4 & 6.5 \\
\hline $8-11$ & 15.8 & 5.9 & 26.4 & 7.0 & 14.0 & 5.1 \\
\hline 12 and above & 14.0 & 4.9 & 27.4 & 5.6 & 13.1 & 4.7 \\
\hline \multicolumn{7}{|l|}{ Worked in last 12 months } \\
\hline Yes & 20.0 & 6.2 & 26.6 & 6.3 & 18.2 & 6.1 \\
\hline No & 6.9 & 5.5 & * & 6.4 & 6.8 & 4.4 \\
\hline \multicolumn{7}{|l|}{ Wealth quintile } \\
\hline First & 20.8 & 7.0 & 26.9 & 5.8 & 16.4 & 9.5 \\
\hline Second & 12.1 & 6.0 & 22.8 & 7.0 & 10.6 & 4.3 \\
\hline Third & 17.9 & 5.1 & 30.2 & 6.0 & 15.0 & 3.7 \\
\hline Fourth & 18.4 & 6.3 & 26.4 & 6.8 & 16.6 & 5.8 \\
\hline Fifth & 13.9 & 4.8 & 27.0 & 6.1 & 13.7 & 4.1 \\
\hline Total & 16.4 & 5.8 & 26.6 & 6.3 & 14.4 & 5.2 \\
\hline
\end{tabular}

Note: ${ }^{*}$ Percentage not shown, based on fewer than 25 unweighted cases. NA: Not applicable. OBC: Other backward caste. SC: Scheduled caste. ST: Scheduled tribe. ${ }^{1}$ Includes Christian, Buddhist, Neo-Buddhist, Sikh, Jain, Jewish, Parsi/Zoroastrian and no specified religion. ${ }^{2}$ Includes all those not belonging to SC, ST or OBC. ${ }^{3}$ Includes non-literate and literate with no formal schooling. 
from early adolescence (before age 15) into late adolescence (before age 20) into young adulthood (before age 25). For example, while just $0.2 \%$ of young men and $0.5 \%$ of young women initiated pre-marital sexual relations before age $15,9-10 \%$ of young men and women experienced first pre-marital sex before age 20 , and $35 \%$ of young men and $20 \%$ of young women initiated pre-marital sexual relations before age 25 .

The age-specific increase in cumulative percentages of those who had initiated pre-marital sexual relations was steeper among rural than among urban youth. Among rural young men, for example, while just $0.3 \%$ had experienced first sex before age 15, as many as $12 \%$ had experienced pre-marital sex before age 20 and $37 \%$ before age 25 . The corresponding percentages among urban young men were $0.1 \%, 6 \%$ and $32 \%$, respectively. Although levels of pre-marital sex were lower among young women, the same pattern held true. Among rural young women, $0.6 \%$ had initiated sex before age 15 and this percentage increased to $10 \%$ and further to $25 \%$ before ages 20 and 25 , respectively. Increases among young women in urban areas, in contrast, were relatively mild (from $0.3 \%$ before age 15 to $7 \%$ before age 20 and $16 \%$ before age 25 ).

\subsubsection{Pre-marital sexual risk behaviours}

Table 9.13 presents findings relating to sexual risk behaviours of those reporting pre-marital sexual experience, including multiple partner relations and inconsistent condom use. Findings confirm that where youth engaged in pre-marital sex, it was generally under unsafe conditions.

A larger percentage of sexually experienced young men than women had engaged in sex with multiple partners before marriage (22\% versus $7 \%$ ). Among young men, the unmarried were almost as likely as the married to report multiple partner relationships ( $22 \%$ and 19\%, respectively); among young women, in contrast, the unmarried were more likely than the married to report multiple partner relationships (13\% versus $5 \%$ ). Unmarried youth were further probed about the number of partners with whom they had engaged in sex over the 12 months preceding the interview; $11 \%$ and $2 \%$ of sexually experienced unmarried young men and women, respectively, reported multiple sexual partners in the last year.

The Youth Study questionnaire probed consistent condom use only with regard to sex with the first and/or most recent romantic partner, in exchange sex encounters, with sex workers and with married women. Information on condom use was not obtained for pre-marital sexual experiences with romantic partners other than the first or most recent, same-sex romantic partners, casual sex partners, spouse before marriage or among those who reported the experience of forced sex. Although few youth reported these latter relationships, we acknowledge that our consistent condom use indicator may not be comprehensive.

Table 9.12: Age at initiation of pre-marital sex

Cumulative percentage of youth by age at first pre-marital sexual experience, according to residence, Andhra Pradesh, 2007-08

\begin{tabular}{|c|c|c|c|c|c|c|}
\hline \multirow[t]{2}{*}{ Age at first pre-marital sex $(\%)^{1}$} & $\begin{array}{c}M \\
15-24\end{array}$ & $\begin{array}{c}\text { W } \\
15-24\end{array}$ & $\begin{array}{c}M \\
15-24\end{array}$ & $\begin{array}{c}\text { W } \\
15-24\end{array}$ & $\begin{array}{c}M \\
15-24\end{array}$ & $\begin{array}{c}W \\
15-24\end{array}$ \\
\hline & \multicolumn{2}{|c|}{ Combined } & \multicolumn{2}{|c|}{ Urban } & \multicolumn{2}{|c|}{ Rural } \\
\hline $\begin{array}{l}\text { First pre-marital sex occurred before age (years): } \\
15\end{array}$ & 0.2 & 0.5 & 0.1 & 0.3 & 0.3 & 0.6 \\
\hline 18 & 2.7 & 4.6 & 1.0 & 3.1 & 3.3 & 5.4 \\
\hline 20 & 10.1 & 8.6 & 5.8 & 6.9 & 11.9 & 9.5 \\
\hline 21 & 14.9 & 9.7 & 10.0 & 8.2 & 16.8 & 10.5 \\
\hline 25 & 35.4 & 20.3 & 31.7 & 16.2 & 37.0 & 25.3 \\
\hline Number of respondents & 2,479 & 4,848 & 1,289 & 2,126 & 1,190 & 2,722 \\
\hline
\end{tabular}

Note: All Ns are unweighted. ${ }^{1}$ Calculated using life table techniques. Age at first pre-marital sex among those who reported pre-marital sex only through the anonymous sealed envelope method was imputed conservatively, using age at marriage (for the married) and current age (for the unmarried). 
Findings suggest that among youth who reported pre-marital sex in the face-to-face interview, consistent condom use was extremely limited; only $17 \%$ of young men and $2 \%$ of young women reported that they had always used a condom. While differences by marital status were muted among young men, among young women, the unmarried were more likely than the married to report consistent condom use (5\% versus $0 \%)$. Condom use during the last pre-marital sexual encounter, assessed for unmarried respondents, suggests that one-third (33\%) of unmarried young men and $11 \%$ of unmarried young women reported condom use at last sex.

\subsubsection{Non-consensual sexual experiences}

The Youth Study questionnaire also probed the extent to which young people had experienced such non-consensual sexual experiences as verbal harassment of a sexual nature, non-consensual sexual touch or forced sex. In addition, young men were asked whether they had ever verbally harassed a girl or perpetrated non-consensual sexual touch or forced sex. Findings on non-consensual sexual experiences are presented in Table 9.14. For the married, these refer to the period before marriage. We acknowledge that forced sex is an extremely sensitive issue and hence, very likely to have been under-reported.

Verbal harassment was experienced by small percentages of youth ( $7 \%$ of young men and $2 \%$ of young women). Marital status differences and rural-urban differences were mild.

Non-consensual sexual touch was measured by questions that probed whether the respondent had ever been a victim of unwanted hugging or kissing in a sexual way, whether someone had touched their private parts without consent or had forced them to touch the perpetrator's private parts, and finally, whether someone had attempted to have sex with the respondent against her/his will using physical force or threats. As shown in Table 9.14, few respondents- $5 \%$ of young men and 3\% of young women—admitted the experience of unwanted touch measured in these ways. Differences by marital status and rural-urban residence were negligible.

Questions on forced sex were posed in two ways: in relation to first sex with a romantic opposite- or same-sex partner, on the one hand, and with any non-romantic partner, on the other. Even measured in this way, forced sex was rarely reported, that is, by $0.4 \%$ of young men and women.

Table 9.13: Pre-marital sexual risk behaviours

\section{Percentage of sexually experienced youth who had pre-marital sex by number of partners and condom} use, Andhra Pradesh, 2007-08

\begin{tabular}{|c|c|c|c|c|c|c|}
\hline Sexual behaviours (\%) & $\begin{array}{c}\text { M } \\
15-24\end{array}$ & $\begin{array}{c}\text { W } \\
15-24\end{array}$ & $\begin{array}{c}\text { MM } \\
15-29\end{array}$ & $\begin{array}{c}\text { MW } \\
15-24\end{array}$ & $\begin{array}{l}\text { UM } \\
15-24\end{array}$ & $\begin{array}{l}\text { UW } \\
15-24\end{array}$ \\
\hline $\begin{array}{l}\text { Total number of pre-marital sexual partners } \\
1 \\
2 \text { or more }\end{array}$ & $\begin{array}{l}78.1 \\
21.9\end{array}$ & $\begin{array}{r}92.6 \\
7.4\end{array}$ & $\begin{array}{l}80.7 \\
19.3\end{array}$ & $\begin{array}{r}95.3 \\
4.7\end{array}$ & $\begin{array}{l}78.0 \\
22.0\end{array}$ & $\begin{array}{l}87.4 \\
12.6\end{array}$ \\
\hline Consistent condom use with pre-marital sexual partners ${ }^{1}$ & 17.0 & 1.6 & 18.7 & 0.0 & 17.1 & 4.5 \\
\hline $\begin{array}{l}\text { Number reporting pre-marital sex in face-to-face interview } \\
\text { Number of sexual partners in last } 12 \text { months }\end{array}$ & 313 & 248 & 294 & 137 & 234 & 111 \\
\hline None & NA & NA & NA & NA & 40.2 & 17.1 \\
\hline 1 & NA & NA & NA & NA & 48.8 & 81.1 \\
\hline $\begin{array}{l}2 \text { or more } \\
\text { Condom used at last pre-marital sex }\end{array}$ & NA & NA & NA & NA & 11.0 & 1.8 \\
\hline $\begin{array}{l}\text { Number of unmarried respondents reporting pre-marital } \\
\text { sex in face-to-face interview }\end{array}$ & NA & NA & NA & NA & 234 & 111 \\
\hline
\end{tabular}

Note: All Ns are unweighted. NA: Not applicable. ${ }^{1}$ Questions on consistent condom use were asked only with regard to sexual relationships with first and/or most recent romantic partner, exchange sex partner, sex worker or married woman and excluded experiences with romantic partners other than first or most recent romantic partner, same-sex romantic partner, casual partner, spouse before marriage and experiences of forced sex. 
Young men's reports of perpetration of these acts, presented in Table 9.14, suggest, however, that non-consensual sexual experiences may well have been under-reported by young women. Indeed, as many as $43 \%$ of young men admitted that they had ever verbally harassed a girl. Moreover, 31\% of young men admitted touching or brushing past a girl without her consent. Unmarried young men were more likely than married young men to report perpetration of verbal harassment (44\% versus 37\%), but as likely to report perpetration of unwanted touch (30-31\%). Rural-urban differences were negligible.

\subsection{Triangulation of data on pre-marital sexual experiences among young people}

Acknowledging that young people may have been reluctant to disclose behaviours perceived as socially unacceptable such as pre-marital sex, the Youth Study included three approaches to elicit data on sexual behaviours. These were face-to-face interviews, anonymous reporting of respondents' own experiences via the sealed envelope and anonymous third-party reporting of peer experiences. Anonymous third-party reporting of peer experiences is a useful method by which to assess sensitive behaviours that individuals may be reluctant to disclose about themselves; findings are intended to shed light on the behaviours of the peer network and not necessarily on those of the individual himself or herself (Rossier, 2003).

We note that in anonymous third-party reporting, respondents may have reported as peers individuals whose ages fell outside our sample ages (15-24 and, in the case of married males, 15-29); therefore, in estimating pre-marital romantic and sexual experiences of young people using this reporting method, these individuals were excluded. In addition, we recognise that in anonymous third-party reporting, friends reported by one respondent may also be reported by others. In estimating pre-marital romantic and sexual experiences of young people using this reporting method, our analysis sought to minimise the chances that the experience of an individual belonging to more than one peer network would be included multiple times. Specifically, we inversely weighted the total sample of friends by the number of friends reported by each respondent. As a result, each respondent's network was given equal weight irrespective of its size.

Findings, presented in Table 9.15, compare the levels of pre-marital romantic and sexual experiences obtained through these different approaches. Specifically, three indicators are presented: (a) percent reporting a pre-marital romantic relationship, (b) percent reporting the experience of pre-marital sex with a romantic opposite-sex partner, and (c) percent reporting any pre-marital sexual experience. For indicators $a-b$, we compare two sets of estimates derived from the face-to-face interview: respondents' reports of their own experiences as well as third-party reporting of the experiences of their peers. For indicator $c$, we compare three sets of estimates: any pre-marital sex as reported in the face-to-face format; any pre-marital sex among peers as assessed through anonymous third-party reporting; and any pre-marital sex as reported in the face-to-face interview supplemented by reports of pre-marital sexual experience recorded in the anonymous format, using the sealed envelope.

Comparisons indicate differences in reporting level by sex of the respondent and type of behaviour under consideration. In terms of pre-marital romantic relationships, anonymous third-party reporting yielded higher rates than did face-to-face reporting for both young men and women. Differences were more apparent among young women than men (18\% and 11\%, respectively, among young women, and $20 \%$ and $17 \%$, respectively, among young men), and similar patterns were observed among both married and unmarried young men and women. Differences in reporting were similar among young women in urban and rural areas, but wider among young men in urban than rural areas.

As far as reporting of experience of pre-marital sex with a romantic partner was concerned, differences were narrow. Young men and women were about as likely to report sexual relations with a romantic partner in the face-to-face interview as in the anonymous third-party reporting method. Among married young men in urban areas, however, the anonymous third-party reporting method provided mildly higher estimates of sexual experiences with a romantic partner than did the face-to-face interview. 
Table 9.14: Pre-marital non-consensual sexual experiences

Percentage of youth reporting various pre-marital non-consensual sexual experiences, according to residence, Andhra Pradesh, 2007-08

\begin{tabular}{|c|c|c|c|c|c|c|}
\hline Non-consensual sexual experiences (\%) & $\begin{array}{c}\text { M } \\
15-24\end{array}$ & $\begin{array}{c}\text { W } \\
15-24\end{array}$ & $\begin{array}{c}\text { MM } \\
15-29\end{array}$ & $\begin{array}{c}\text { MW } \\
15-24\end{array}$ & $\begin{array}{c}\text { UM } \\
15-24\end{array}$ & $\begin{array}{c}\text { UW } \\
15-24\end{array}$ \\
\hline \multicolumn{7}{|c|}{ Combined } \\
\hline \multicolumn{7}{|l|}{ Ever experienced } \\
\hline Verbal harassment & 6.8 & 2.0 & 4.6 & 1.1 & 7.3 & 3.2 \\
\hline Any non-consensual sexual touch ${ }^{1}$ & 5.0 & 2.7 & 3.7 & 1.9 & 5.2 & 3.7 \\
\hline Any forced sex & 0.4 & 0.4 & 0.1 & 0.3 & 0.4 & 0.6 \\
\hline \multicolumn{7}{|l|}{ Ever perpetrated the following: } \\
\hline Verbally harassed anyone ${ }^{2}$ & 43.4 & NA & 37.2 & NA & 43.6 & NA \\
\hline Touched or brushed past a girl ${ }^{2}$ & 30.6 & NA & 30.5 & NA & 30.2 & NA \\
\hline Forced sex on a girl & 0.5 & NA & 0.0 & NA & 0.6 & NA \\
\hline Number of respondents & 2,479 & 4,848 & 1,405 & 2,330 & 2,077 & 2,518 \\
\hline \multicolumn{7}{|c|}{ Urban } \\
\hline \multicolumn{7}{|l|}{ Ever experienced } \\
\hline Verbal harassment & 6.4 & 2.3 & 5.6 & 1.3 & 6.3 & 3.1 \\
\hline Any non-consensual sexual touch ${ }^{1}$ & 5.5 & 2.7 & 4.9 & 2.1 & 5.2 & 3.3 \\
\hline Any forced sex & 0.1 & 0.6 & 0.3 & 0.6 & 0.2 & 0.7 \\
\hline \multicolumn{7}{|l|}{ Ever perpetrated the following: } \\
\hline Verbally harassed anyone ${ }^{2}$ & 45.8 & NA & 38.9 & NA & 46.1 & NA \\
\hline Touched or brushed past a girl ${ }^{2}$ & 31.6 & NA & 28.1 & NA & 31.9 & NA \\
\hline Forced sex on a girl & 0.1 & $\mathrm{NA}$ & 0.0 & NA & 0.2 & NA \\
\hline Number of respondents & 1,289 & 2,126 & 648 & 1,037 & 1,132 & 1,089 \\
\hline \multicolumn{7}{|c|}{ Rural } \\
\hline \multicolumn{7}{|l|}{ Ever experienced } \\
\hline Verbal harassment & 7.0 & 1.9 & 4.3 & 1.1 & 7.7 & 3.3 \\
\hline Any non-consensual sexual touch ${ }^{1}$ & 4.8 & 2.7 & 3.4 & 1.9 & 5.3 & 4.0 \\
\hline Any forced sex & 0.4 & 0.3 & 0.1 & 0.2 & 0.6 & 0.5 \\
\hline \multicolumn{7}{|l|}{ Ever perpetrated the following: } \\
\hline Verbally harassed anyone ${ }^{2}$ & 42.4 & NA & 36.7 & NA & 42.4 & $\mathrm{NA}$ \\
\hline Touched or brushed past a girl ${ }^{2}$ & 30.1 & NA & 31.1 & NA & 29.5 & NA \\
\hline Forced sex on a girl & 0.6 & NA & 0.0 & NA & 0.8 & NA \\
\hline Number of respondents & 1,190 & 2,722 & 757 & 1,293 & 945 & 1,429 \\
\hline
\end{tabular}

Note: All Ns are unweighted. NA: Not applicable. ${ }^{1}$ Includes hugging in a sexual way, kissing in a sexual way, touching of private parts and attempted forced sex. ${ }^{2}$ It is possible that married young men may have reported the occurrence of these events post-marriage since age at occurrence was not probed.

With regard to reporting of any pre-marital sexual experience, anonymous third-party reporting of peer behaviours yielded, among both young men and young women, rates that were more or less similar to those self-reported in response to questions posed face-to-face. While differences were mild, it is notable that pre-marital sexual experience was almost always more likely to be reported in self-reports than in anonymous third-party reporting (with the exception of young men in urban areas).

At the same time, a comparison of any pre-marital sexual experience reported in face-to-face interviews and via the anonymous sealed envelope format suggests that several youth, particularly young men who had not admitted sexual 
Table 9.15: Levels of pre-marital romantic and sexual experiences by different reporting methods

Percentage of youth reporting pre-marital romantic relationships and percentage reporting sexual experiences within pre-marital romantic and other relationships by reporting method, according to residence, Andhra Pradesh, 2007-08

\begin{tabular}{|c|c|c|c|c|c|c|}
\hline Indicators (\%) & $\begin{array}{c}\text { M } \\
15-24\end{array}$ & $\begin{array}{c}\text { W } \\
15-24\end{array}$ & $\begin{array}{c}\text { MM } \\
15-29\end{array}$ & $\begin{array}{c}\text { MW } \\
15-24\end{array}$ & $\begin{array}{c}\text { UM } \\
15-24\end{array}$ & $\begin{array}{c}\text { UW } \\
15-24\end{array}$ \\
\hline \multicolumn{7}{|c|}{ Combined } \\
\hline \multicolumn{7}{|l|}{ Reported a pre-marital opposite-sex romantic partner via: } \\
\hline Face-to-face interview & 17.4 & 10.5 & 20.5 & 10.7 & 16.3 & 10.1 \\
\hline Anonymous third-party reporting & 20.2 & 18.0 & 23.6 & 17.2 & 19.6 & 19.0 \\
\hline \multicolumn{7}{|l|}{ Reported pre-marital sex with a romantic opposite-sex partner via: } \\
\hline Face-to-face interview & 8.0 & 4.7 & 13.0 & 5.2 & 6.7 & 4.1 \\
\hline Anonymous third-party reporting & 7.9 & 4.1 & 12.6 & 4.5 & 6.9 & 3.5 \\
\hline \multicolumn{7}{|l|}{ Reported any pre-marital sexual experience via: } \\
\hline Face-to-face interview & 13.4 & 5.1 & 21.3 & 5.5 & 11.8 & 4.4 \\
\hline Anonymous third-party reporting & 11.6 & 4.4 & 19.7 & 5.0 & 10.2 & 3.8 \\
\hline $\begin{array}{l}\text { Face-to-face interview or anonymous reporting through } \\
\text { sealed envelope }\end{array}$ & 16.4 & 5.8 & 26.6 & 6.3 & 14.4 & 5.2 \\
\hline Number of respondents & 2,479 & 4,848 & 1,405 & 2,330 & 2,077 & 2,518 \\
\hline \multicolumn{7}{|c|}{ Urban } \\
\hline \multicolumn{7}{|l|}{ Reported a pre-marital opposite-sex romantic partner via: } \\
\hline Face-to-face interview & 17.0 & 12.5 & 23.1 & 15.0 & 15.1 & 10.3 \\
\hline Anonymous third-party reporting & 23.8 & 19.6 & 27.1 & 19.6 & 22.9 & 19.7 \\
\hline \multicolumn{7}{|l|}{ Reported pre-marital sex with a romantic opposite-sex partner via: } \\
\hline Face-to-face interview & 5.7 & 4.7 & 9.8 & 6.3 & 4.8 & 3.4 \\
\hline Anonymous third-party reporting & 7.9 & 3.4 & 13.2 & 4.0 & 6.7 & 2.8 \\
\hline \multicolumn{7}{|l|}{ Reported any pre-marital sexual experience via: } \\
\hline Face-to-face interview & 11.5 & 5.1 & 19.9 & 6.5 & 10.5 & 3.9 \\
\hline Anonymous third-party reporting & 12.1 & 3.5 & 22.1 & 4.3 & 10.7 & 2.9 \\
\hline $\begin{array}{l}\text { Face-to-face interview or anonymous reporting through } \\
\text { sealed envelope }\end{array}$ & 14.4 & 6.1 & 26.7 & 8.0 & 12.9 & 4.6 \\
\hline Number of respondents & 1,289 & 2,126 & 648 & 1,037 & 1,132 & 1,089 \\
\hline \multicolumn{7}{|c|}{ Rural } \\
\hline \multicolumn{7}{|l|}{ Reported a pre-marital opposite-sex romantic partner via: } \\
\hline Face-to-face interview & 17.5 & 9.6 & 19.8 & 9.5 & 16.8 & 9.9 \\
\hline Anonymous third-party reporting & 18.8 & 17.4 & 22.5 & 16.6 & 18.1 & 18.6 \\
\hline \multicolumn{7}{|l|}{ Reported pre-marital sex with a romantic opposite-sex partner via: } \\
\hline Face-to-face interview & 8.8 & 4.7 & 13.8 & 4.8 & 7.6 & 4.5 \\
\hline Anonymous third-party reporting & 7.9 & 4.3 & 12.3 & 4.7 & 6.9 & 3.8 \\
\hline \multicolumn{7}{|l|}{ Reported any pre-marital sexual experience via: } \\
\hline Face-to-face interview & 14.1 & 5.1 & 21.7 & 5.3 & 12.4 & 4.7 \\
\hline Anonymous third-party reporting & 11.4 & 4.8 & 19.1 & 5.2 & 9.9 & 4.2 \\
\hline $\begin{array}{l}\text { Face-to-face interview or anonymous reporting through } \\
\text { sealed envelope }\end{array}$ & 17.2 & 5.7 & 26.6 & 5.9 & 15.1 & 5.5 \\
\hline Number of respondents & 1,190 & 2,722 & 757 & 1,293 & 945 & 1,429 \\
\hline
\end{tabular}

Note: All Ns are unweighted. Detailed information on friends' romantic and sexual experiences was collected for up to five of the respondent's closest same-sex friends. 
experience in the face-to-face interview did so in the anonymous format: $3 \%$ of young men and less than $1 \%$ of young women. Indeed, $18 \%$ of young men and $14 \%$ of young women who reported sexual experience did so only in this more anonymous format (not shown in tabular form). It would appear, therefore, that self-reports of sexual experience, supplemented by self-reported experience in an anonymous format, provide consistently higher estimates of pre-marital sex among youth than does anonymous third-party reporting of pre-marital sexual experience among peers: for example, while $12 \%$ of young men reported pre-marital sex through anonymous third-party reporting, $16 \%$ acknowledged the experience through direct reporting. Among young women too, direct reporting yielded somewhat higher estimates than anonymous third-party reporting (6\% versus $4 \%)$.

\subsection{Summary}

Findings confirm that despite strict norms prohibiting pre-marital opposite-sex mixing, opportunities do exist for the formation of pre-marital romantic relationships. Indeed, significant minorities of young men and women have made or received a "proposal" for a romantic relationship (23\%), and noteworthy, if smaller, percentages reported that they had been involved in a romantic partnership (17\% and $11 \%$ of young men and women, respectively). Patterns of pre-marital romantic partnerships suggest that where partnerships occurred, they were initiated at an early age by many, were hidden from parents by many, but were rarely hidden from peers. Notable disparities in expectations of a longer-term commitment emerged that show that young women were more likely than young men to have expected a romantic relationship to lead to marriage. The experiences of the married suggest, moreover, a disconnect between intentions and reality: although almost all (95\%) young women and two-thirds (68\%) of young men reported the intention to marry their pre-marital partner, fewer- $70 \%$ of young women and $23 \%$ of young men-had done so.

While the majority- $95 \%$ of young men and $82 \%$ of young women — had held hands with a romantic partner, fewer- $46 \%$ - had engaged in sexual relations with their partner. It is notable that unlike the findings of many previous studies, no gender differences in reports of pre-marital sex with a romantic partner were apparent. Findings suggest, moreover, that the vast majority had engaged in unprotected sex. However, many young men and women who reported contraception at first sex reported that the female partner was involved in the decision. Very few young women who had engaged in sexual relations with a romantic partner reported that their opposite-sex romantic partner had forced them to engage in sex the first time.

In total, $16 \%$ of young men and $6 \%$ of young women reported the experience of pre-marital sex within romantic and/or other partnerships. Roughly similar proportions of young men and women-3\% and 5\%, respectively-had initiated first sex before age 18; however, larger proportions of youth in rural than urban areas had initiated pre-marital sexual relations in adolescence. Moreover, initiation into pre-marital sexual activity increased as young people transitioned from early into late adolescence and further as they transitioned into young adulthood.

While sex with a romantic partner characterised pre-marital sexual experiences for many of the sexually experienced, findings suggest that young men, but not young women, also engaged in sex in other contexts; other partners reported by young men included, mainly, sex workers, married women and casual partners. Many of the pre-marital sexual experiences reported by youth were risky, for example, one-fifth of young men and $7 \%$ of young women reporting pre-marital sex had engaged in sex with more than one partner. Moreover, consistent condom use was limited—only $17 \%$ of young men and $2 \%$ of young women reported condom use in all the pre-marital encounters reported.

We acknowledge that youth, especially young women, may not report sexual experience in a survey situation. Hence, the Youth Study supplemented a series of direct questions with an opportunity to report sexual experience in an anonymous format. Surprisingly, findings suggest that a larger percentage of sexually active young men than women reported pre-marital sex only in the anonymous format. In total, direct reporting yielded somewhat higher estimates than anonymous third-party reporting, although the differences were narrow among young women. However, direct questioning supplemented by self-reporting in an anonymous format provided higher estimates of sexual experience than did face-to-face questioning alone or anonymous third-party reporting of peer behaviours. 


\section{Transition to marriage and early} married life

As is well known, the transition to marriage occurs early in India, both for young men and young women. The recent NFHS (IIPS and Macro International, 2007a) shows, for example, that $47 \%$ of young women aged 20-24 had married before the age of 18, the minimum legal age at marriage for females; $32 \%$ of young men aged 25-29 had, likewise, married before they reached the age of 21, the legal minimum age at marriage for males. While marriage occurs early, marriage-related planning occurs even earlier, often as soon as a girl reaches menarche and, in many cases, even before she does so and without her participation. Moreover, while the consummation of marriage generally occurs following menarche, early married life tends to be isolating and frightening for many adolescent girls and young women. This chapter captures some of these experiences, including young people's preferences regarding the timing and type of marriage, marriage preparation and planning, and young people's participation in these processes, as well as their experiences in early married life and the fertility and contraceptive behaviours of young couples in the state of Andhra Pradesh.

\subsection{Young people's preferences regarding timing and type of marriage}

The Youth Study sought to assess young people's preferences about the age at which to marry and, among the unmarried, their preferences for love or arranged marriages. While it is possible that youth who were married in adolescence might have reported the age at which they married as the preferred age, findings, presented in Table 10.1 , indicate that most youth preferred to marry after age 18. This preference was indicated by all youth, irrespective of sex, marital status or rural-urban residence. Even so, it is notable that substantial proportions of young women (26\%) and just $1 \%$ of young men preferred to marry before age 20 , that is, while still adolescent. The majority of young men $(66 \%)$ preferred to marry at age 25 or later, a preference articulated by $10 \%$ of young women as well. Notably, $9 \%$ of young women and $12 \%$ of married young women expressed a preference not to marry at all; just $3 \%$ of young men expressed this view.

Differentials by marital status and rural-urban residence of respondents were notable. Married young women were more likely than the unmarried to report that they preferred to marry before age 20 (35\% versus 15\%). Conversely, more unmarried than married youth preferred to marry at age 25 or later $(73 \%$ versus $51 \%$ among young men and $18 \%$ versus $4 \%$ among young women). A larger proportion of rural than urban young women expressed a preference to marry before age 20 (31\% versus $14 \%)$. Conversely, a smaller proportion of rural than urban youth expressed a preference to marry late; for example, $63 \%$ of rural young men compared to $74 \%$ of urban young men preferred to marry at age 25 or later, as did $7 \%$ and $19 \%$, respectively, of young women.

Findings also show that the vast majority of unmarried youth preferred to have an arranged rather than love marriage. For example, just $11 \%$ of young men and $8 \%$ of young women reported that they would prefer a love marriage. Rural-urban differences were muted.

\subsection{Marriage planning and extent of youth involvement}

Several questions were put to both married and unmarried youth to understand the process of marriage planning as well as their involvement in it. While questions on marriage planning were similar for the married and the unmarried, some questions were unique to one or the other group. For example, the Youth Study asked unmarried 
Table 10.1: Preferences regarding timing and type of marriage

Percent distribution of youth reporting preferences regarding timing of marriage and percentage preferring a love marriage, according to residence, Andhra Pradesh, 2007-08

\begin{tabular}{|c|c|c|c|c|c|c|}
\hline Indicators (\%) & $\begin{array}{c}\mathrm{M} \\
15-24\end{array}$ & $\begin{array}{c}\text { W } \\
15-24\end{array}$ & $\begin{array}{c}\text { MM } \\
15-29\end{array}$ & $\begin{array}{c}\text { MW } \\
15-24\end{array}$ & $\begin{array}{c}\text { UM } \\
15-24\end{array}$ & $\begin{array}{c}\text { UW } \\
\text { 15-24 }\end{array}$ \\
\hline \multicolumn{7}{|c|}{ Combined } \\
\hline \multicolumn{7}{|l|}{ Preferred to marry at age: } \\
\hline 17 or below & 0.2 & 4.7 & 0.6 & 7.4 & 0.1 & 1.3 \\
\hline 18 & 0.4 & 16.4 & 1.3 & 22.1 & 0.0 & 9.2 \\
\hline 19 & 0.3 & 4.6 & 0.9 & 5.0 & 0.0 & 4.0 \\
\hline 20 & 4.4 & 28.6 & 9.5 & 32.0 & 2.2 & 24.4 \\
\hline 21 & 3.5 & 12.0 & 6.3 & 10.1 & 2.7 & 14.5 \\
\hline 22 & 6.9 & 6.7 & 10.6 & 4.5 & 5.7 & 9.5 \\
\hline 23 & 6.3 & 4.2 & 6.7 & 1.8 & 5.3 & 7.4 \\
\hline 24 & 8.9 & 3.6 & 9.7 & 1.2 & 8.4 & 6.5 \\
\hline 25 or above & 66.3 & 10.2 & 51.4 & 3.7 & 73.0 & 18.4 \\
\hline Preferred not to marry & 2.9 & 9.0 & 3.0 & 12.2 & 2.5 & 4.8 \\
\hline Preferred a love marriage ${ }^{1}$ & NA & NA & NA & NA & 10.8 & 7.8 \\
\hline Number of respondents & 2,479 & 4,848 & 1,405 & 2,330 & 2,077 & 2,518 \\
\hline \multicolumn{7}{|c|}{ Urban } \\
\hline \multicolumn{7}{|l|}{ Preferred to marry at age: } \\
\hline 17 or below & 0.1 & 1.8 & 0.0 & 3.2 & 0.0 & 0.6 \\
\hline 18 & 0.0 & 9.3 & 0.7 & 16.6 & 0.0 & 3.5 \\
\hline 19 & 0.3 & 3.3 & 0.7 & 5.3 & 0.2 & 1.8 \\
\hline 20 & 2.8 & 22.3 & 7.2 & 30.5 & 1.1 & 15.7 \\
\hline 21 & 2.3 & 13.2 & 4.6 & 13.3 & 1.3 & 13.1 \\
\hline 22 & 4.2 & 9.4 & 8.5 & 8.2 & 3.5 & 10.5 \\
\hline 23 & 4.5 & 7.6 & 6.2 & 3.4 & 4.0 & 11.0 \\
\hline 24 & 7.6 & 8.2 & 9.8 & 3.8 & 7.4 & 11.8 \\
\hline 25 or above & 74.4 & 18.8 & 59.2 & 6.5 & 79.1 & 28.6 \\
\hline Preferred not to marry & 3.7 & 6.1 & 3.3 & 9.1 & 3.5 & 3.5 \\
\hline Preferred a love marriage ${ }^{1}$ & NA & NA & NA & NA & 12.0 & 8.1 \\
\hline Number of respondents & 1,289 & 2,126 & 648 & 1,037 & 1,132 & 1,089 \\
\hline \multicolumn{7}{|c|}{ Rural } \\
\hline \multicolumn{7}{|l|}{ Preferred to marry at age: } \\
\hline 17 or below & 0.3 & 6.0 & 0.7 & 8.6 & 0.1 & 1.7 \\
\hline 18 & 0.5 & 19.4 & 1.5 & 23.7 & 0.1 & 12.4 \\
\hline 19 & 0.3 & 5.1 & 1.0 & 4.9 & 0.0 & 5.3 \\
\hline 20 & 4.9 & 31.2 & 10.3 & 32.5 & 2.8 & 29.2 \\
\hline 21 & 4.0 & 11.5 & 6.8 & 9.1 & 3.4 & 15.4 \\
\hline 22 & 7.9 & 5.5 & 11.2 & 3.4 & 6.6 & 9.0 \\
\hline 23 & 6.9 & 2.8 & 6.8 & 1.3 & 5.9 & 5.3 \\
\hline 24 & 9.4 & 1.7 & 9.7 & 0.6 & 8.8 & 3.5 \\
\hline 25 or above & 63.1 & 6.6 & 49.1 & 2.9 & 70.2 & 12.6 \\
\hline Preferred not to marry & 2.7 & 10.2 & 2.9 & 13.0 & 2.1 & 5.6 \\
\hline Preferred a love marriage ${ }^{1}$ & NA & NA & NA & NA & 10.2 & 7.6 \\
\hline Number of respondents & 1,190 & 2,722 & 757 & 1,293 & 945 & 1,429 \\
\hline
\end{tabular}

Note: All Ns are unweighted. NA: Not applicable. ${ }^{1}$ Excludes those who reported a preference not to marry. 
respondents whether their parents or family members had begun discussing plans for their marriage; and asked all married youth, and those unmarried youth for whom discussion had been initiated, about their age at that time and whether their parents had sought their opinion about the age at which they wished to marry.

Findings presented in Table 10.2 indicate vast gender differences in the age of young people when discussions were initiated regarding the planning of their marriage. Among those whose parents had ever initiated marriage-related discussions (almost all married youth and 9\% and 39\% of unmarried young men and women, respectively), findings suggest that marriage-related discussions were initiated at young ages for considerable proportions of young women. For example, three-fourths of all young women (73\%) compared to one-tenth of young men (11\%) reported that marriage-related discussions were initiated before age 18 . Indeed, for $43 \%$ of young women (compared to $2 \%$ of young men), such discussions were initiated as early as 15 years or below. Marital status differences were insignificant for young men, but discussions were initiated earlier for currently married young women compared to the unmarried. For example, for half of married young women, compared to one-fifth of the unmarried, discussions were initiated at age 15 or earlier. Likewise, discussions on marriage were initiated earlier for rural than urban young women; here too, differences were negligible among young men.

Among those whose parents had initiated marriage-related discussions, the majority of young men reported that they were consulted on the timing of marriage (70\%), but fewer young women so reported (26\%). Disparities by marital status were observed, particularly among young women (see Figure 10.1). For example, a much smaller proportion of married than unmarried young women were consulted about when they wished to marry (21\% versus $40 \%)$, a finding that may be attributed to the more traditional family background of young women who were married early than those who were unmarried. Rural-urban differences were evident among young women, and among unmarried youth. A larger proportion of young women in urban areas compared to rural areas reported that their opinions were sought (30\% versus 24\%); differences were wider among unmarried young men and women (80\% versus $69 \%$ among young men and $47 \%$ versus $37 \%$ among young women).

The Youth Study also sought to assess the extent to which youth (other than married young men) perceived they could express or, among the married, could have expressed to their parents their preference not to marry the prospective spouse selected for them. Findings suggest that the majority of youth perceived that it would not be difficult to oppose their parents if they did not approve of the match chosen for them; even so, $25 \%$ of young women and $13 \%$ of unmarried young men perceived difficulty in opposing their parents. Marital status differences suggest that unmarried young women were somewhat less likely than the married to perceive difficulty in opposing their parents $(23 \%$ and $28 \%$, respectively). Finally, and perhaps for reasons pertaining to the traditional nature of family life in rural areas, rural young women were more likely than their urban counterparts to report difficulty

Figure 10.1: Percentage of youth reporting that their parents had ever sought their opinion on timing of marriage, according to residence, Andhra Pradesh, 2007-08
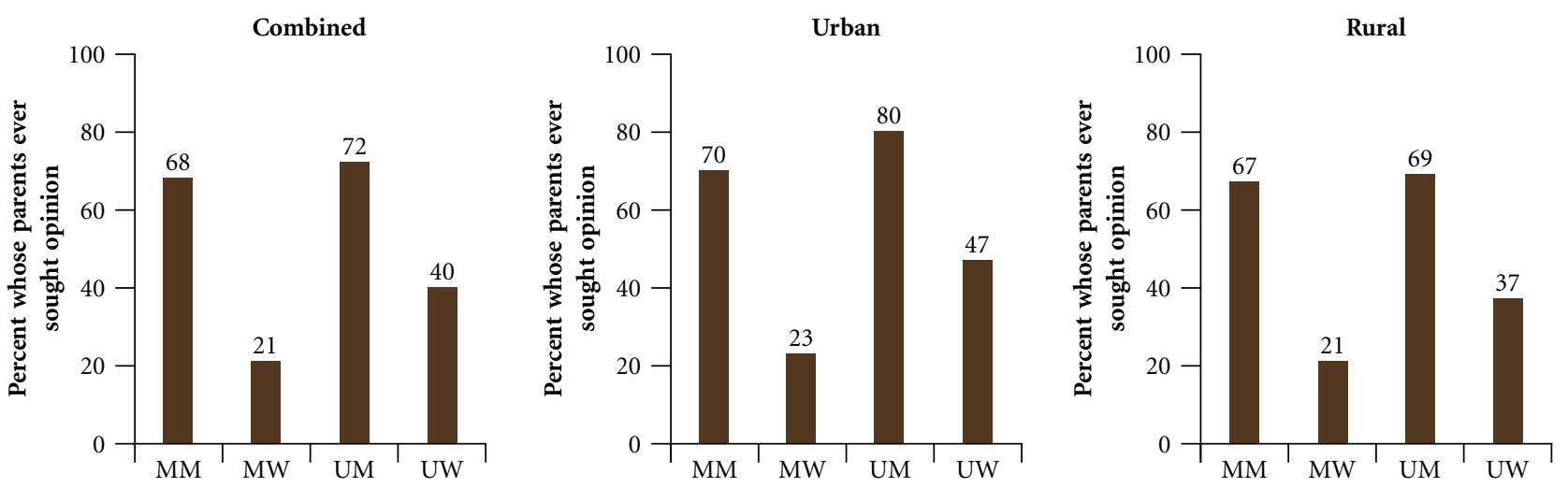

Note: Includes respondents whose parents had initiated marriage-related discussion. 
Table 10.2: Initiation of discussion on marriage and extent of youth involvement

Percent distribution of youth by age at initiation of marriage-related discussion, percentage whose opinion had been sought on timing of marriage and percentage who would find it difficult to tell parents if they did not like the match chosen, according to residence, Andhra Pradesh, 2007-08

\begin{tabular}{|c|c|c|c|c|c|c|}
\hline Marriage discussion (\%) & $\begin{array}{c}\mathrm{M} \\
15-24\end{array}$ & $\begin{array}{c}W \\
15-24\end{array}$ & $\begin{array}{c}\text { MM } \\
15-29\end{array}$ & $\begin{array}{c}\text { MW } \\
15-24\end{array}$ & $\begin{array}{c}\text { UM } \\
15-24\end{array}$ & $\begin{array}{l}\text { UW } \\
15-24\end{array}$ \\
\hline \multicolumn{7}{|c|}{ Combined } \\
\hline Parents ever initiated discussion on marriage & 25.9 & 72.5 & 97.5 & 97.8 & 9.4 & 39.2 \\
\hline Number of respondents & 2,479 & 4,848 & 1,405 & 2,330 & 2,077 & 2,518 \\
\hline \multicolumn{7}{|l|}{ Discussion on marriage initiated at age (years) } \\
\hline 13 or below & 0.0 & 13.4 & 0.1 & 17.1 & 0.0 & 1.3 \\
\hline $14-15$ & 2.0 & 29.2 & 1.5 & 32.6 & 2.1 & 18.2 \\
\hline $16-17$ & 9.1 & 30.4 & 6.9 & 29.8 & 7.7 & 32.5 \\
\hline 18 or above & 88.3 & 25.7 & 91.3 & 18.9 & 88.2 & 47.6 \\
\hline $\begin{array}{l}\text { Parents ever sought respondent's opinion about when } \\
\text { to get married }\end{array}$ & 69.9 & 25.6 & 67.7 & 21.2 & 72.4 & 40.1 \\
\hline Number whose parents had initiated discussion on marriage & 581 & 3,244 & 1,365 & 2,271 & 196 & 973 \\
\hline $\begin{array}{l}\text { Would find/have found it difficult to tell parents if } \\
\text { respondent did not like the match chosen }\end{array}$ & NA & 25.4 & NA & 27.8 & 13.2 & 22.5 \\
\hline Number of respondents & 2,479 & 4,848 & 1,405 & 2,330 & 2,077 & 2,518 \\
\hline \multicolumn{7}{|c|}{ Urban } \\
\hline Parents ever initiated discussion on marriage & 20.4 & 62.3 & 96.4 & 97.1 & 9.5 & 34.2 \\
\hline Number of respondents & 1,289 & 2,126 & 648 & 1,037 & 1,132 & 1,089 \\
\hline \multicolumn{7}{|l|}{ Discussion on marriage initiated at age (years) } \\
\hline 13 or below & 0.0 & 6.9 & 0.0 & 9.6 & 0.0 & 1.0 \\
\hline $14-15$ & 1.4 & 21.5 & 0.7 & 26.4 & 1.7 & 10.3 \\
\hline $16-17$ & 5.6 & 30.3 & 3.1 & 32.3 & 6.7 & 25.7 \\
\hline 18 or above & 91.0 & 40.6 & 96.3 & 30.9 & 86.7 & 62.7 \\
\hline $\begin{array}{l}\text { Parents ever sought respondent's opinion about when to } \\
\text { get married }\end{array}$ & 73.1 & 30.3 & 70.4 & 23.3 & 80.3 & 46.6 \\
\hline Number whose parents had initiated discussion on marriage & 256 & 1,380 & 624 & 1,005 & 108 & 375 \\
\hline $\begin{array}{l}\text { Would find/have found it difficult to tell parents if } \\
\text { respondent did not like the match chosen }\end{array}$ & NA & 18.7 & NA & 21.1 & 11.3 & 16.7 \\
\hline Number of respondents & 1,289 & 2,126 & 648 & 1,037 & 1,132 & 1,089 \\
\hline \multicolumn{7}{|c|}{ Rural } \\
\hline Parents ever initiated discussion on marriage & 28.0 & 76.8 & 97.8 & 98.0 & 9.4 & 42.1 \\
\hline Number of respondents & 1,190 & 2,722 & 757 & 1,293 & 945 & 1,429 \\
\hline \multicolumn{7}{|l|}{ Discussion on marriage initiated at age (years) } \\
\hline 13 or below & 0.0 & 15.6 & 0.1 & 19.3 & 0.0 & 1.3 \\
\hline $14-15$ & 2.2 & 31.8 & 1.7 & 34.4 & 2.2 & 22.0 \\
\hline $16-17$ & 10.1 & 30.4 & 7.9 & 29.1 & 8.1 & 35.5 \\
\hline 18 or above & 87.3 & 20.6 & 90.0 & 15.3 & 88.2 & 40.6 \\
\hline $\begin{array}{l}\text { Parents ever sought respondent's opinion about when to } \\
\text { get married }\end{array}$ & 68.8 & 24.0 & 67.1 & 20.6 & 68.6 & 37.1 \\
\hline Number whose parents had initiated discussion on marriage & 325 & 1,864 & 741 & 1,266 & 88 & 598 \\
\hline $\begin{array}{l}\text { Would find/have found it difficult to tell parents if } \\
\text { respondent did not like the match chosen }\end{array}$ & NA & 28.2 & NA & 29.7 & 14.0 & 25.8 \\
\hline Number of respondents & 1,190 & 2,722 & 757 & 1,293 & 945 & 1,429 \\
\hline
\end{tabular}

Note: All Ns are unweighted. Column totals may not equal 100\% due to missing cases or "don't know"/“don't remember" responses. NA: Not applicable. 
in confronting their parents on marriage-related issues (28\% compared to 19\%); differences were negligible among unmarried young men ( $14 \%$ and $11 \%$, respectively).

\subsection{Age at marriage and cohabitation}

Youth Study findings underscore the early age at marriage among young women in Andhra Pradesh (Table 10.3). Of those aged 20-24, almost one-fifth (18\%) of young women were married before age 15, over half (54\%) before age 18 and almost three-quarters $(72 \%)$ before age 20 . In rural areas, as many as $63 \%$ and $81 \%$ of women aged 20-24 years were married before age 18 and 20, respectively; the corresponding percentages in urban areas were $34 \%$ and $51 \%$. Findings from the NFHS-3 also indicate that as many as 55\% of $20-24$ year-old women in Andhra Pradesh were married before age 18 (IIPS and Macro International, 2008). Youth Study findings, moreover, indicate that even though there were indications of a decline in very early marriage (before age 15) among young women, significant minorities continued to marry before age 15: $18 \%$ of those aged 20-24, compared to $9 \%$ of those aged 15-19, were married before age 15.

Early marriage was less prevalent among young men, only $2 \%$ young men aged $20-24$ years were married before age 18. Even so, one in ten young men (11\%) was married in adolescence. In rural areas, $3 \%$ and $13 \%$ of men aged 20-24 were married before age 18 and 20 , respectively.

Almost all married youth (99.5\%) had been married just once (not shown in tabular form). The mean age at marriage among those who were married was 21.5 years among young men and 16.2 years among young women. As expected, rural youth married earlier than urban youth; the mean age at marriage among rural youth was one year earlier than that of urban youth (21.3 years versus 22.4 years for young men and 15.9 years versus 17.2 years for young women). The mean age at cohabitation was almost the same as the mean age at marriage.

\subsection{Marriage preparedness}

Several questions were put to both married and unmarried youth who were engaged to be married to understand their preparedness for marriage. Questions ranged from whether the proposed spouse was chosen by the young person or by his/her parents; whether the young person's approval of the prospective spouse, if chosen by the parents, was sought; and how much contact the young person and the prospective spouse had prior to marriage. As fewer than $1 \%$ and $4 \%$ of unmarried young men and women, respectively, reported that they were engaged to be married (not shown in tabular form), we restrict our discussion to the currently married.

Table 10.4 describes marriage-related preparedness among the married. An overwhelming proportion (93-95\%) of respondents, whether male or female, had married a partner chosen by their parents. While the young person's approval of the prospective spouse had been sought in the majority of cases (86-89\%), 6-7\% of young men and women reported that their parents had not sought their approval. Five percent of young men and $7 \%$ of young women reported having chosen their marriage partner on their own. While gender differences were negligible, urban youth were somewhat more likely than their rural counterparts to report so (9-11\% versus $4-6 \%)$. Also indicative of the lack of preparedness was the short gap between the point at which marriage arrangements were fixed and the date of the actual marriage: on average one month; over three in four young men and four in five young women reporting the occurrence of their marriage within three months of the completion of marriage negotiations (not shown in tabular form).

Reported pre-marital acquaintance was limited, and highlights that even those who reported that they had approved their parents' choice of spouse had rarely had an opportunity to meet their prospective spouse prior to marriage. Indeed, a little over one-quarter of young men (29\%) and one-fifth of young women (19\%) reported that they had ever had a chance to meet and interact with their spouse-to-be alone prior to marriage. Rural-urban differences suggest that more urban than rural youth had met or talked with their fiancé/fiancée alone before marriage (39\% versus $26 \%$ among young men and $27 \%$ versus $16 \%$ among young women). Over two in five married youth (43-44\%) reported that they had met their spouse for the first time on the wedding day. About one-third of youth 
Table 10.3: Age at marriage and cohabitation

Percentage of youth aged 15-24 who were married before selected ages, percentage never married and mean age at marriage and cohabitation among those married, according to current age and residence, Andhra Pradesh, 2007-08

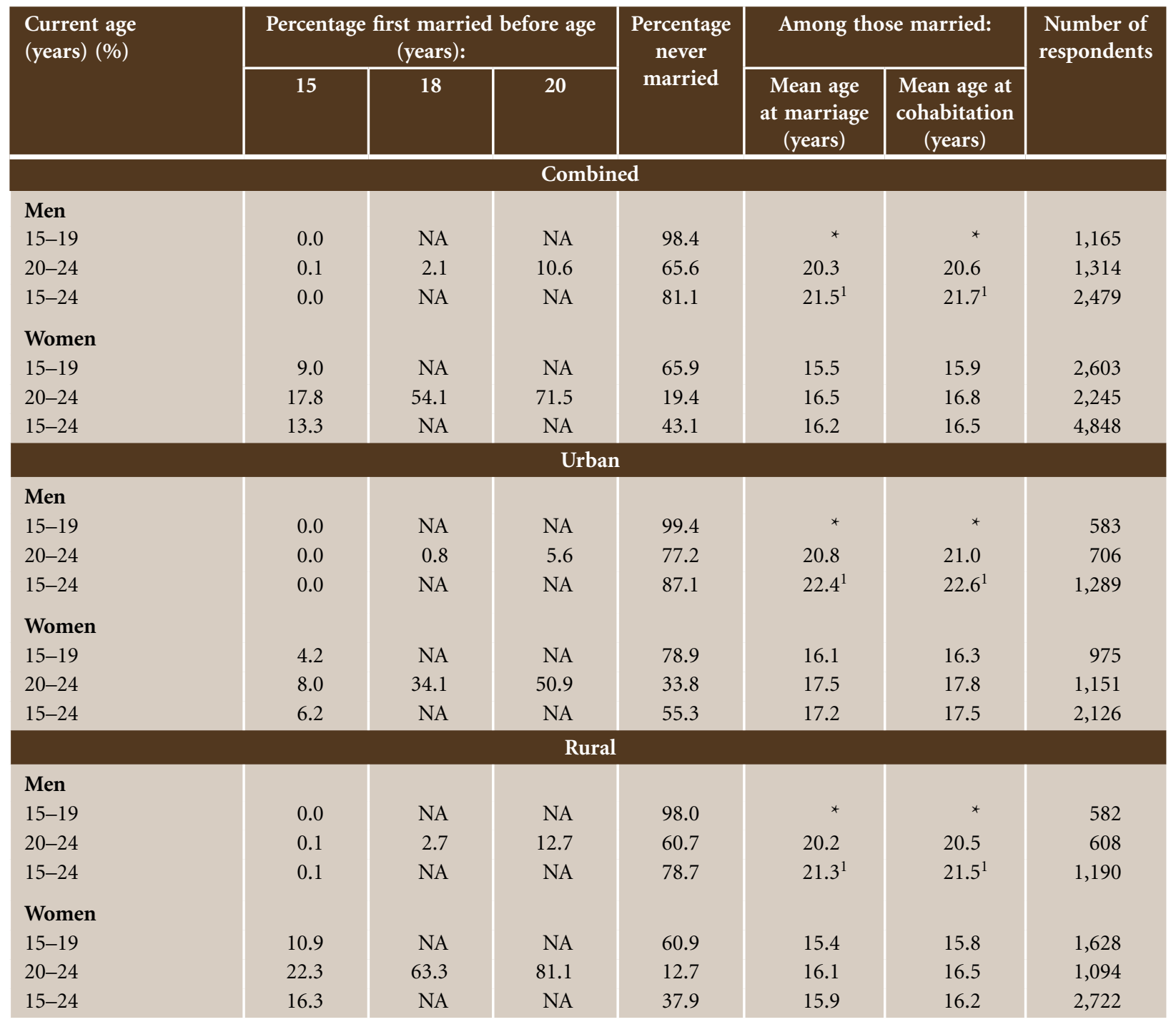

Note: All Ns are unweighted. ${ }^{*}$ Mean not shown, based on fewer than 25 unweighted cases. NA: Not applicable due to censoring. ${ }^{1}$ Includes married men aged 25-29 years.

(29-33\%) reported that they knew their spouse only a little before marriage and a quarter (24-26\%) reported that they knew their spouse well prior to marriage (see Figure 10.2). Rural-urban differences were narrow. In short, findings underscore that considerable proportions of youth, and especially young women, were excluded from marriage-related decision-making and were married to relative strangers.

Compounding this lack of pre-marital acquaintance, large proportions of youth $(71 \%$ of young men and $85 \%$ of young women) who had begun cohabiting with their spouse reported they were unaware at the time of their marriage of what to expect of married life. Rural-urban differences suggest that rural youth were more likely than urban youth to so report ( $74 \%$ versus $61 \%$ among young men and $87 \%$ versus $80 \%$ among young women). Two-thirds $(67 \%)$ of young men and over four-fifths $(83 \%)$ of young women reported that young people in general were poorly informed about married life prior to marriage, highlighting the need for family life or sex education and pre-marital counselling for young people. Rural-urban differences were mild. 
Table 10.4: Marriage preparedness

Percent distribution of married youth by type of marriage and selected indicators of their preparedness for marriage, according to residence, Andhra Pradesh, 2007-08

\begin{tabular}{|c|c|c|c|c|c|c|}
\hline \multirow[t]{2}{*}{ Marriage indicators (\%) } & $\begin{array}{c}\mathrm{MM} \\
15-29\end{array}$ & $\begin{array}{c}\text { MW } \\
15-24\end{array}$ & $\begin{array}{c}\text { MM } \\
15-29\end{array}$ & $\begin{array}{c}\text { MW } \\
15-24\end{array}$ & $\begin{array}{c}\text { MM } \\
15-29\end{array}$ & $\begin{array}{c}\text { MW } \\
15-24\end{array}$ \\
\hline & \multicolumn{2}{|c|}{ Combined } & \multicolumn{2}{|c|}{ Urban } & \multicolumn{2}{|c|}{ Rural } \\
\hline Type of marriage & & & & & & \\
\hline $\begin{array}{l}\text { Marriage fixed by respondent himself/herself } \\
\text { (love marriage) }\end{array}$ & 4.8 & 7.0 & 9.1 & 11.4 & 3.5 & 5.8 \\
\hline $\begin{array}{l}\text { Marriage arranged by parents, with respondent's } \\
\text { approval of choice of spouse }\end{array}$ & 89.3 & 85.7 & 85.7 & 83.6 & 90.4 & 86.3 \\
\hline Marriage fixed by parents without respondent's approval & 5.9 & 7.3 & 5.2 & 5.0 & 6.1 & 7.9 \\
\hline $\begin{array}{l}\text { Ever had a chance to meet/talk with fiancé/fiancée alone } \\
\text { Acquaintance with spouse before marrige }\end{array}$ & 29.0 & 18.6 & 38.8 & 26.9 & 26.2 & 16.2 \\
\hline Met on wedding day & 43.3 & 44.4 & 45.9 & 43.6 & 42.5 & 44.6 \\
\hline Knew somewhat before wedding day & 32.7 & 29.4 & 29.6 & 29.0 & 33.5 & 29.6 \\
\hline Knew well before wedding day & 23.5 & 26.1 & 23.8 & 27.2 & 23.4 & 25.8 \\
\hline Feelings about getting married & & & & & & \\
\hline Excited/looked forward to it & 51.7 & 17.3 & 56.2 & 23.2 & 50.5 & 15.5 \\
\hline Nothing special & 39.7 & 24.7 & 37.3 & 20.8 & 40.4 & 25.9 \\
\hline Very scared & 6.5 & 53.1 & 4.6 & 51.2 & 7.0 & 53.7 \\
\hline Anxious & 1.0 & 3.6 & 1.0 & 4.0 & 1.0 & 3.5 \\
\hline Unhappy & 0.9 & 1.1 & 0.7 & 0.4 & 0.9 & 1.3 \\
\hline Number of respondents & 1,405 & 2,330 & 648 & 1,037 & 757 & 1,293 \\
\hline Did not know what to expect of married life & 70.9 & 85.1 & 61.2 & 80.0 & 73.6 & 86.6 \\
\hline $\begin{array}{l}\text { Agree that youth do not get accurate information } \\
\text { about married life before marriage }\end{array}$ & 67.4 & 82.7 & 64.2 & 80.7 & 68.3 & 83.3 \\
\hline Number who had begun cohabiting & 1,404 & 2,325 & 648 & 1,036 & 756 & 1,289 \\
\hline
\end{tabular}

Note: All Ns are unweighted. Column totals may not equal 100\% due to missing cases or "don't know" responses.

Figure 10.2: Percent distribution of married youth by degree of acquaintance with future spouse before marriage, according to residence, Andhra Pradesh, 2007-08

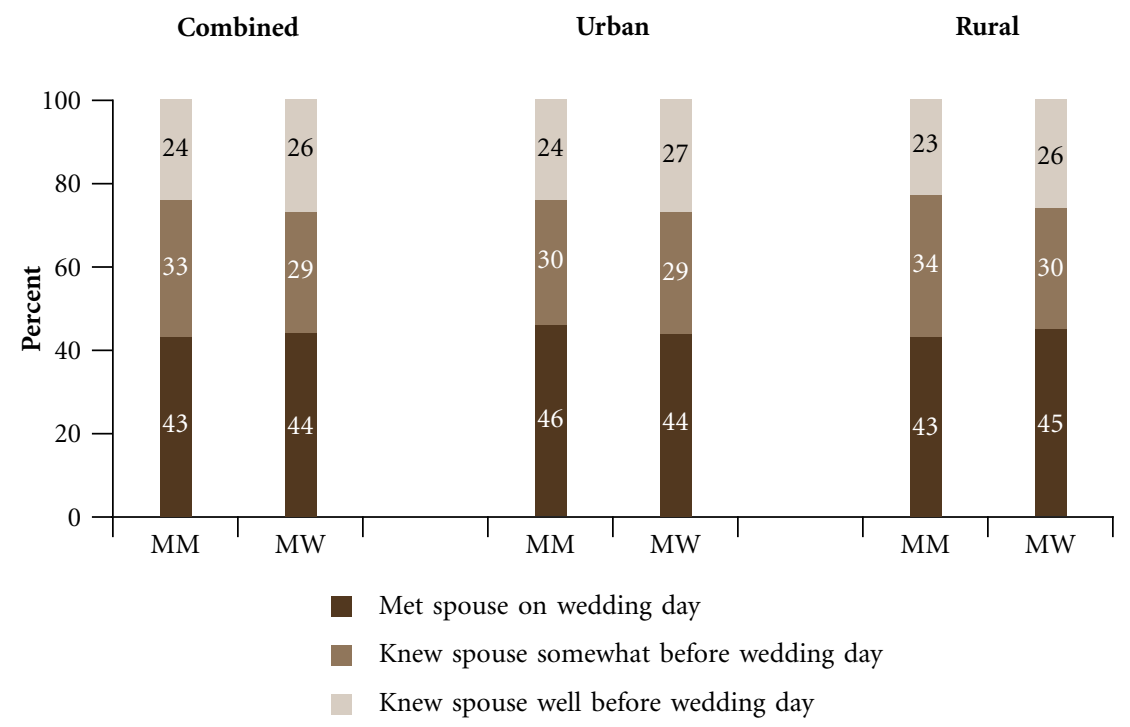

Note: Percentages may not equal 100.0 because of rounding. 
Commensurate with low levels of marriage preparedness, not all youth reported that they had looked forward to or had been excited about their marriage. Gender differences were pronounced: while 52\% of young men reported that they had been excited about their marriage, only 17\% of young women so reported. Rural-urban differences were mild, although urban youth were somewhat more likely than rural youth to report so. Large proportions of young women reported that they had been very scared about getting married, reported by 53\% of young women compared to just $7 \%$ of young men, levels that were observed in both rural and urban areas.

\subsection{Payment of dowry}

Despite the existence of laws against dowry, Figure 10.3 shows that $81 \%$ and $86 \%$ of young married men and women reported taking and giving dowry, respectively. Rural-urban differences in dowry reporting were not evident, highlighting that the practice of dowry remained just as strong among families of urban youth as among their rural counterparts.

Figure 10.3: Percentage of married youth who reported receiving or giving dowry, according to residence, Andhra Pradesh, 2007-08

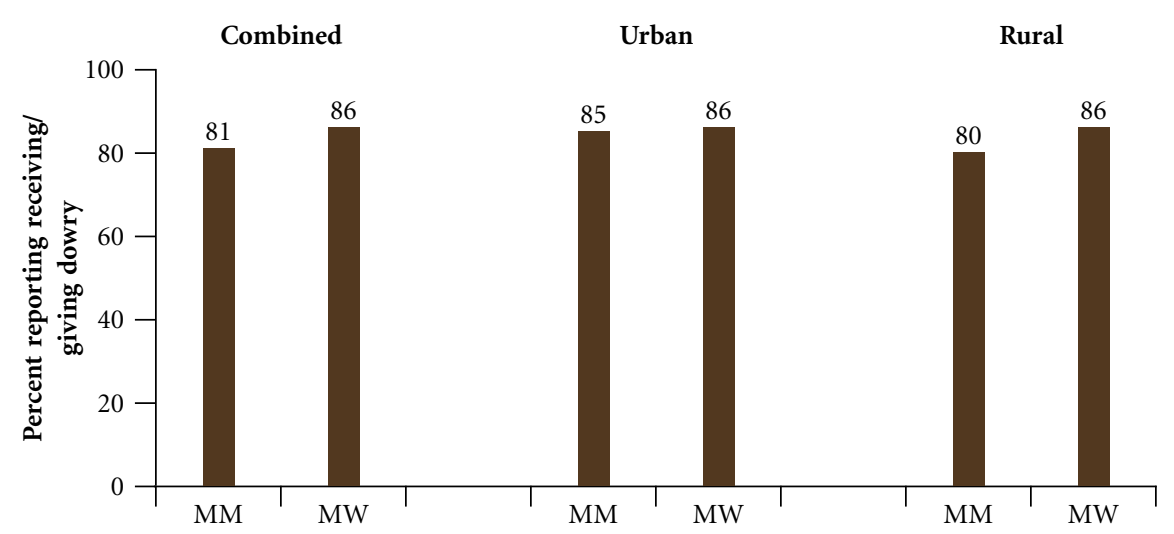

\subsection{Early marital experiences: Spousal communication and interaction}

Table 10.5 describes the extent of communication and interaction among young couples and suggests that spousal communication was common. The extent of communication, however, varied considerably by topic and sex of the respondent (see also Figure 10.4). For example, $81 \%$ of young men compared to $90 \%$ of young women reported regularly discussing how to spend money; in contrast, $49 \%$ of young men compared to $72 \%$ of young women reported regularly discussing in-law issues with their spouse. Rural-urban differences suggest that urban youth were by and large more likely than rural youth to report spousal communication, although among young men, an equal percentage of those in urban and rural areas reported regularly discussing in-law issues with their wife.

Likewise, $80-90 \%$ of youth reported that they had communicated with their spouse on when and/or whether to have children and how many children to have. Somewhat more young women than men (88-90\% versus $80-84 \%)$, and urban than rural youth (84-95\% versus 79-89\%), reported having discussed these topics with their spouse. In contrast, discussion was more limited on the topic of contraception and notably, far fewer young men (12\%) than women (34\%) reported that they had ever discussed contraception with their spouse. Here again, rural-urban differences were wide. While $20 \%$ of urban young men compared to $10 \%$ of rural young men reported such communication, corresponding percentages among young women were $42 \%$ and $32 \%$, respectively.

Spousal interaction was measured by questions regarding whether, in the six months preceding the interview, respondents had gone with their spouse to a movie, been on an outing or gone to their own (for young women) or wife's (for young men) natal home. These types of interaction were commonly reported, but varied by type of 
Table 10.5: Early marital experiences

Percentage of married youth by selected characteristics of the marital relationship, according to residence, Andhra Pradesh, 2007-08

\begin{tabular}{|c|c|c|c|c|c|c|}
\hline \multirow[t]{2}{*}{ Characteristics (\%) } & $\begin{array}{c}\text { MM } \\
15-29\end{array}$ & $\begin{array}{c}\text { MW } \\
15-24\end{array}$ & $\begin{array}{c}\mathrm{MM} \\
15-29\end{array}$ & $\begin{array}{c}\text { MW } \\
15-24\end{array}$ & $\begin{array}{c}\text { MM } \\
15-29\end{array}$ & $\begin{array}{c}\text { MW } \\
15-24\end{array}$ \\
\hline & \multicolumn{2}{|c|}{ Combined } & \multicolumn{2}{|c|}{ Urban } & \multicolumn{2}{|c|}{ Rural } \\
\hline \multicolumn{7}{|l|}{ Usually communicates with spouse on: } \\
\hline How to spend money & 81.2 & 89.5 & 88.9 & 93.1 & 79.0 & 88.4 \\
\hline In-law issues & 49.2 & 71.7 & 49.2 & 77.1 & 49.2 & 70.2 \\
\hline \multicolumn{7}{|l|}{ Ever communicated with spouse on: } \\
\hline When/whether to have a baby & 79.8 & 87.5 & 83.7 & 90.8 & 78.8 & 86.5 \\
\hline Number of children to have & 84.3 & 90.4 & 90.5 & 94.8 & 82.5 & 89.2 \\
\hline Contraceptive use & 12.2 & 34.0 & 19.9 & 41.8 & 10.0 & 31.7 \\
\hline \multicolumn{7}{|l|}{ Went with spouse to the following in last 6 months: } \\
\hline Theatre/video parlour & 68.0 & 52.7 & 81.0 & 70.9 & 64.4 & 47.3 \\
\hline Festival/yatra/tamasha/play/tour/picnic/restaurant & 69.0 & 52.3 & 73.9 & 64.2 & 67.6 & 48.8 \\
\hline Woman's/wife's natal home & 89.5 & 93.2 & 88.2 & 92.4 & 89.8 & 93.5 \\
\hline \multicolumn{7}{|l|}{ Assessment of married life } \\
\hline Very happy & 58.4 & 47.7 & 63.7 & 60.1 & 56.9 & 44.1 \\
\hline Reasonably happy & 39.7 & 48.2 & 35.6 & 37.6 & 40.8 & 51.3 \\
\hline Unhappy & 1.7 & 3.7 & 0.7 & 1.7 & 2.0 & 4.3 \\
\hline Very unhappy & 0.2 & 0.3 & 0.0 & 0.6 & 0.3 & 0.3 \\
\hline Number who had begun cohabiting & 1,404 & 2,325 & 648 & 1,036 & 756 & 1,289 \\
\hline
\end{tabular}

Note: All Ns are unweighted. Column totals may not equal 100\% due to missing cases or "don't know" responses.

Figure 10.4: Percentage of married youth who reported spousal communication on selected topics, according to residence, Andhra Pradesh, 2007-08
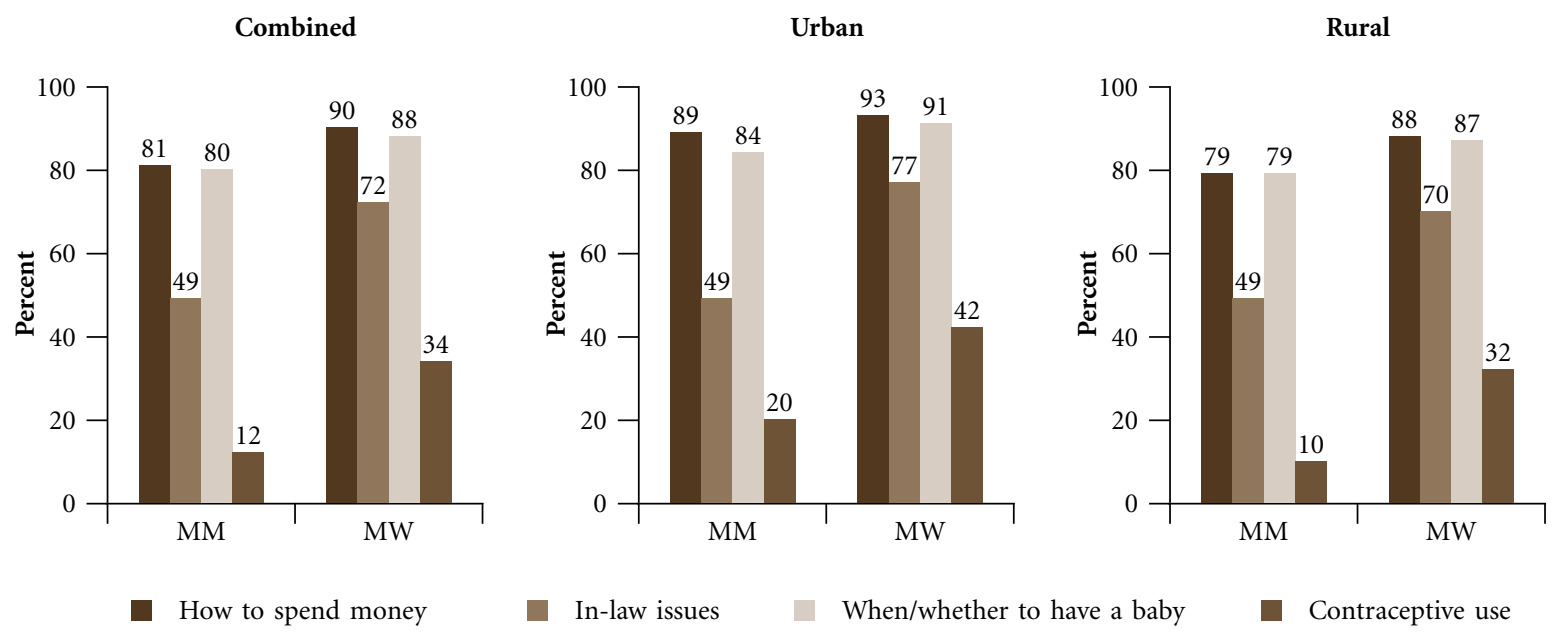

interaction. For example, $90 \%$ or more of youth had visited their own/ wife's natal home together with the spouse. Somewhat fewer youth-68-69\% of young men and $52-53 \%$ of young women-had been together with their spouse on an outing (festival, picnic, etc) and to a movie or other places of entertainment. Gender and rural-urban differences were apparent, with young women and rural youth less likely than their respective counterparts to report 
going on an outing or to places of entertainment. For example, while $69 \%$ of young men reported that they had been together with their wife on an outing, only $52 \%$ of young women reported that they had done so with their husband; while $74 \%$ of young men in urban areas reported that they had been on an outing with their wife, only $68 \%$ of their rural counterparts had done so; among young women, $64 \%$ and $49 \%$, correspondingly, so reported.

Youth were also asked to assess their relative contentment with married life. Almost all youth ( $98 \%$ of young men and $96 \%$ of young women) reported that they were very happy or reasonably happy.

\subsection{Nature of marital sexual experiences}

In several previous studies, significant minorities of young women reported the experience of forced sex within marriage, including at initiation (see for example, Santhya and Jejeebhoy, 2006; Santhya et al., 2007). The Youth Study explored the extent to which early marital sexual experiences were enjoyable or forced. Findings, presented in Table 10.6, suggest that while virtually all young men (98\%) reported enjoying their first marital sexual experience, fewer young women so reported $(76 \%)$, with little rural-urban variation. A comparison of responses to questions regarding whether the spouse had enjoyed the first sexual experience suggests a disconnect: $92 \%$ of young men perceived that their wife had enjoyed the first sexual experience (compared to $76 \%$ of young women who reported that they had enjoyed the first sexual experience), and $89 \%$ young women perceived that their husband had enjoyed the experience (less than that reported by young men themselves-98\%).

For many young women, the first marital sexual experience was painful (43\%); many fewer young men (28\%), however, perceived that the first experience was painful for their wife. Forced sex at initiation was reported by $6 \%$ of young women, with little rural-urban variation (3-6\%). A similar percentage of young men (8\%) reported that they had forced their wife to engage in sex the first time, with little rural-urban variation $(5-8 \%)$.

Over the course of their marital lives, somewhat more young people acknowledged the experience (young women) or perpetration (young men) of forced sex within marriage: $10 \%$ of young women reported that they had ever experienced forced sex within marriage and $15 \%$ of young men reported that they had ever perpetrated it. Rural young women were somewhat more likely than urban young women to report having experienced forced sex within marriage (12\% versus $6 \%)$, but young men in rural areas were as likely as those in urban areas to report having perpetrated it (14-15\%). Recent experience/perpetration of such an incident, that is, in the 12 months preceding the interview, was reported by $5 \%$ of young men and women who had cohabited for at least one year.

Table 10.6: Sexual experiences within marriage

Percentage of married youth by nature of first and lifetime sexual experiences with spouse, according to residence, Andhra Pradesh, 2007-08

\begin{tabular}{|c|c|c|c|c|c|c|}
\hline \multirow[t]{2}{*}{ Experiences (\%) } & $\begin{array}{c}\text { MM } \\
15-29\end{array}$ & $\begin{array}{c}\text { MW } \\
15-24\end{array}$ & $\begin{array}{c}\mathrm{MM} \\
15-29\end{array}$ & $\begin{array}{c}\text { MW } \\
15-24\end{array}$ & $\begin{array}{c}\text { MM } \\
15-29\end{array}$ & $\begin{array}{c}\text { MW } \\
15-24\end{array}$ \\
\hline & \multicolumn{2}{|c|}{ Combined } & \multicolumn{2}{|c|}{ Urban } & \multicolumn{2}{|c|}{ Rural } \\
\hline Nature of first sexual experience with spouse & & & & & & \\
\hline Respondent enjoyed it & 97.6 & 75.9 & 99.7 & 78.6 & 97.1 & 75.2 \\
\hline Spouse enjoyed it & 91.6 & 88.6 & 92.5 & 90.1 & 91.3 & 88.1 \\
\hline Wife cried & 12.8 & 26.5 & 13.1 & 20.4 & 12.8 & 28.2 \\
\hline Painful for wife & 28.3 & 43.3 & 32.0 & 38.0 & 27.2 & 44.9 \\
\hline Wife unwilling and husband forced her & 7.6 & 5.6 & 4.6 & 3.1 & 8.4 & 6.4 \\
\hline Husband ever forced wife to have sex & 14.7 & 10.2 & 13.7 & 5.9 & 14.9 & 11.5 \\
\hline Number who had begun cohabiting & 1,404 & 2,325 & 648 & 1,036 & 756 & 1,289 \\
\hline Husband forced wife to have sex in last 12 months & 4.8 & 5.3 & 4.5 & 2.8 & 4.9 & 6.1 \\
\hline Number who had cohabited for at least 12 months & 1,247 & 2,089 & 568 & 918 & 679 & 1,171 \\
\hline
\end{tabular}

Note: All Ns are unweighted. 


\subsection{Experience of domestic violence within marriage}

The Youth Study explored the extent of domestic violence or verbal abuse perpetrated by young men on their wife and in less detail, by women on their husband. Table 10.7 shows that $1 \%$ or fewer young women had either verbally humiliated their husband in the presence of others or perpetrated violence on him in any way. No differences were observed by respondents' sex or place of residence.

In contrast, somewhat larger proportions of young men were reported to have verbally humiliated their wife in the presence of others or perpetrated some form of physical violence on their wife. Verbal humiliation was reported by $9 \%$ young men and women. One-fifth of young men $(20 \%)$ and $23 \%$ of young women reported the experience (women) or perpetration (men) of some form of physical violence. Rural-urban differences were narrow among young men, but among young women, somewhat more from rural than urban areas reported the experience of physical violence (24\% versus $19 \%$ ) (see also Figure 10.5$)$.

Figure 10.5: Percentage of married young women reporting experience of physical violence perpetrated by their husband and percentage of married young men reporting perpetration of physical violence against their wife, according to residence, Andhra Pradesh, 2007-08

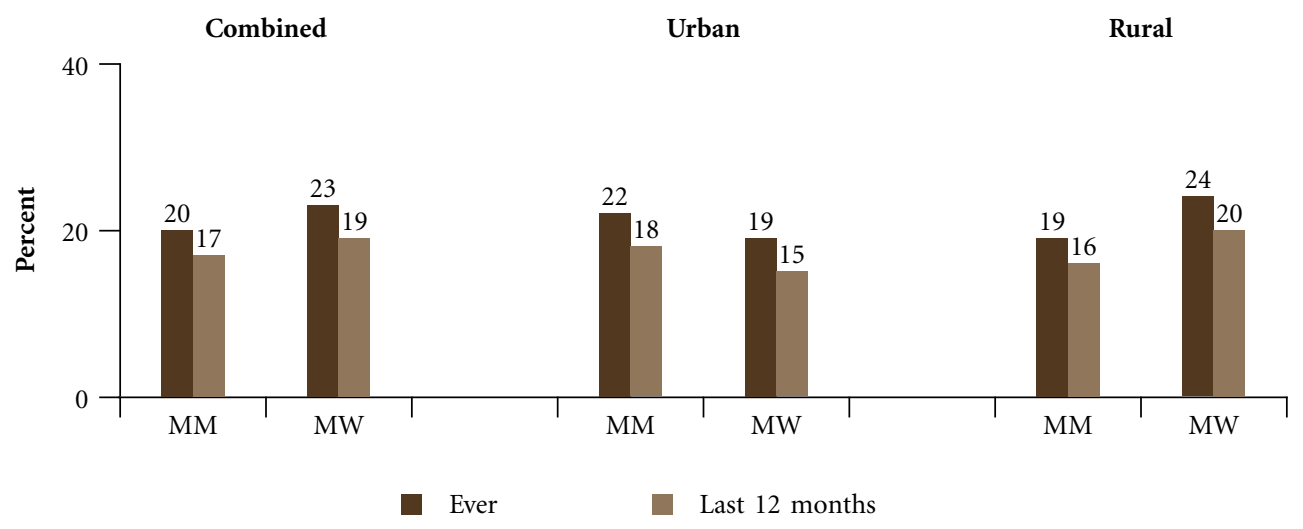

Of all forms of physical violence, slapping was most commonly reported (19-22\%), followed by twisting of the wife's arm or pulling her hair $(6-10 \%)$. Between $3 \%$ and $6 \%$ of young women and men also reported the experience/perpetration of being pushed, shaken or having something thrown at them, punched, kicked, dragged or beaten. One percent of young women reported that they had been burnt or choked by their husband. It is notable that $9 \%$ of young men reported perpetrating more than one form of violence on their wife and $13 \%$ of young women reported experiencing more than one form of violence perpetrated by their husband.

Perpetration or experience of any form of physical violence within marriage in the 12 months preceding the interview was reported by $17 \%$ of young men and $19 \%$ young women. As earlier, slapping was most commonly reported; $15 \%$ of young men reported slapping their wife in the last year and $17 \%$ of young women reported being slapped by their husband in the 12 months preceding the interview.

Findings on the occurrence of physical violence within the first year of marriage (among those who had cohabited for at least one year) indicate that a small minority of youth (7-10\%) reported perpetration or experience of physical violence within a year of marriage. Rural-urban differences were muted.

\subsection{Extent and nature of extra-marital sexual relations}

The Youth Study did not probe as extensively about extra-marital sexual experiences as it did about pre-marital sex, discussed in Chapter 9. A single direct question was asked to all married youth about whether they had experienced sexual relations with someone other than their spouse following marriage. In addition, youth reporting same-sex, 
Table 10.7: Domestic violence within marriage

Percentage of married youth reporting experience of verbal abuse or physical violence within marriage by type of violence, according to residence, Andhra Pradesh, 2007-08

\begin{tabular}{|c|c|c|c|c|c|c|}
\hline \multirow[t]{2}{*}{ Types of violence (\%) } & $\begin{array}{c}\text { MM } \\
15-29\end{array}$ & $\begin{array}{c}\text { MW } \\
15-24\end{array}$ & $\begin{array}{c}\text { MM } \\
15-29\end{array}$ & $\begin{array}{c}\text { MW } \\
15-24\end{array}$ & $\begin{array}{c}\text { MM } \\
15-29\end{array}$ & $\begin{array}{c}\text { MW } \\
15-24\end{array}$ \\
\hline & \multicolumn{2}{|c|}{ Combined } & \multicolumn{2}{|c|}{ Urban } & \multicolumn{2}{|c|}{ Rural } \\
\hline \multicolumn{7}{|c|}{ A. Verbal abuse or physical violence perpetrated by wife } \\
\hline Wife verbally abused husband in the presence of others & 1.2 & 0.9 & 1.6 & 0.8 & 1.1 & 0.9 \\
\hline Wife ever perpetrated any physical violence on husband & 0.4 & 0.6 & 0.7 & 0.4 & 0.4 & 0.6 \\
\hline $\begin{array}{l}\text { Wife perpetrated any physical violence on husband in } \\
\text { last } 12 \text { months }\end{array}$ & 0.2 & 0.2 & 0.0 & 0.0 & 0.3 & 0.2 \\
\hline Number who had begun cohabiting & 1,404 & 2,325 & 648 & 1,036 & 756 & 1,289 \\
\hline \multicolumn{7}{|c|}{ B. Verbal abuse or physical violence perpetrated by husband } \\
\hline Husband verbally abused wife in the presence of others & 8.6 & 8.9 & 9.1 & 6.7 & 8.5 & 9.6 \\
\hline Physical violence ever perpetrated by husband & & & & & & \\
\hline Slapped wife & 18.6 & 21.5 & 19.9 & 18.5 & 18.1 & 22.3 \\
\hline Twisted wife's arm or pulled her hair & 5.8 & 10.4 & 6.2 & 7.3 & 5.7 & 11.3 \\
\hline Pushed/shook or threw something at wife & 4.0 & 6.4 & 4.6 & 4.4 & 3.8 & 7.1 \\
\hline Punched wife & 5.5 & 5.4 & 5.9 & 2.9 & 5.4 & 6.1 \\
\hline Kicked, dragged or beat wife & 3.3 & 5.7 & 3.6 & 4.2 & 3.2 & 6.1 \\
\hline Choked or burnt wife on purpose & 0.1 & 1.1 & 0.0 & 0.6 & 0.1 & 1.2 \\
\hline Threatened or attacked wife with knife/gun & 0.0 & 0.3 & 0.0 & 0.2 & 0.0 & 0.4 \\
\hline $\begin{array}{l}\text { Perpetrated/experienced at least one of the above } \\
\text { forms of violence }\end{array}$ & 19.7 & 22.9 & 21.5 & 19.1 & 19.2 & 24.1 \\
\hline $\begin{array}{l}\text { Perpetrated/experienced more than one of the above } \\
\text { forms of violence }\end{array}$ & 8.5 & 13.1 & 8.5 & 9.3 & 8.6 & 14.2 \\
\hline \multirow{2}{*}{\multicolumn{7}{|c|}{$\begin{array}{l}\text { Experience of violence perpetrated by husband in } \\
\text { last } 12 \text { months } \\
\text { Slapped wife }\end{array}$}} \\
\hline & & & & & & \\
\hline Never & 84.4 & 82.7 & 82.7 & 85.7 & 84.9 & 81.8 \\
\hline Sometimes & 15.3 & 16.1 & 17.3 & 13.4 & 14.8 & 16.9 \\
\hline Often & 0.1 & 1.1 & 0.0 & 0.8 & 0.2 & 1.2 \\
\hline \multicolumn{7}{|l|}{ Twisted wife's arm or pulled her hair } \\
\hline Never & 95.3 & 91.5 & 94.8 & 94.1 & 95.5 & 90.7 \\
\hline Sometimes & 4.7 & 7.6 & 5.2 & 5.5 & 4.5 & 8.2 \\
\hline Often & 0.0 & 0.9 & 0.0 & 0.4 & 0.0 & 1.1 \\
\hline \multicolumn{7}{|l|}{ Pushed/shook or threw something at wife } \\
\hline Never & 96.3 & 94.7 & 95.8 & 96.6 & 96.4 & 94.2 \\
\hline Sometimes & 3.7 & 4.7 & 4.2 & 3.2 & 3.6 & 5.1 \\
\hline Often & 0.0 & 0.5 & 0.0 & 0.2 & 0.0 & 0.6 \\
\hline \multicolumn{7}{|l|}{ Punched wife } \\
\hline Never & 95.1 & 95.4 & 94.8 & 97.5 & 95.1 & 94.7 \\
\hline Sometimes & 4.9 & 4.1 & 5.2 & 2.1 & 4.9 & 4.8 \\
\hline Often & 0.0 & 0.5 & 0.0 & 0.4 & 0.0 & 0.5 \\
\hline
\end{tabular}


Table 10.7: (Cont'd)

\begin{tabular}{|c|c|c|c|c|c|c|}
\hline \multirow[t]{2}{*}{ Types of violence $(\%)$} & $\begin{array}{c}\mathrm{MM} \\
15-29\end{array}$ & $\begin{array}{c}\text { MW } \\
15-24\end{array}$ & $\begin{array}{c}\mathrm{MM} \\
15-29\end{array}$ & $\begin{array}{c}\text { MW } \\
15-24\end{array}$ & $\begin{array}{c}\mathrm{MM} \\
15-29\end{array}$ & $\begin{array}{c}\text { MW } \\
15-24\end{array}$ \\
\hline & \multicolumn{2}{|c|}{ Combined } & \multicolumn{2}{|c|}{ Urban } & \multicolumn{2}{|c|}{ Rural } \\
\hline \multicolumn{7}{|c|}{ B. Verbal abuse or physical violence perpetrated by husband } \\
\hline \multicolumn{7}{|l|}{ Kicked, dragged or beat wife } \\
\hline Never & 96.9 & 95.1 & 96.7 & 96.8 & 97.0 & 94.7 \\
\hline Sometimes & 3.1 & 4.3 & 3.3 & 2.9 & 3.0 & 4.8 \\
\hline Often & 0.0 & 0.5 & 0.0 & 0.4 & 0.0 & 0.4 \\
\hline \multicolumn{7}{|l|}{ Choked or burnt wife on purpose } \\
\hline Never & 99.9 & 99.0 & 100.0 & 99.4 & 99.9 & 98.9 \\
\hline Sometimes & 0.1 & 0.8 & 0.0 & 0.6 & 0.1 & 0.9 \\
\hline Often & 0.0 & 0.2 & 0.0 & 0.0 & 0.0 & 0.2 \\
\hline \multicolumn{7}{|l|}{ Threatened or attacked wife with knife/gun } \\
\hline Never & 100.0 & 99.7 & 100.0 & 99.8 & 100.0 & 99.7 \\
\hline Sometimes & 0.0 & 0.1 & 0.0 & 0.2 & 0.0 & 0.1 \\
\hline Often & 0.0 & 0.1 & 0.0 & 0.0 & 0.0 & 0.1 \\
\hline $\begin{array}{l}\text { Perpetrated/experienced at least one of the above for } \\
\text { of violence in last } 12 \text { months }\end{array}$ & 16.5 & 18.6 & 18.3 & 14.7 & 16.0 & 19.8 \\
\hline Number who had begun cohabiting & 1,404 & 2,325 & 648 & 1,036 & 756 & 1,289 \\
\hline Experienced violence in first 12 months of marriage & 9.5 & 7.0 & 11.2 & 8.2 & 9.0 & 6.7 \\
\hline Number who had cohabited for at least 12 months & 1,247 & 2,089 & 568 & 918 & 679 & 1,171 \\
\hline
\end{tabular}

Note: All Ns are unweighted. Column totals may not equal 100\% due to missing cases or "don't know" responses.

exchange, forced or sex worker sex were probed about the timing of the first such encounter; for very few, it occurred following marriage. Given the lack of extensive probing, we caution readers that percentages of youth reporting extra-marital sexual experience, indicated in Table 10.8 , may be particularly under-reported.

Hardly any young women $(0.8 \%)$ reported an extra-marital sexual partner. In contrast, $6 \%$ of young men, irrespective of rural-urban residence, reported an extra-marital sexual partner. Among young men, $2 \%$ reported extra-marital sex in the one year (or months since marriage for those married for less than one year) preceding the interview.

\section{Table 10.8: Extent of extra-marital sexual experiences}

Percentage of married youth by extent of extra-marital sexual experiences, according to residence, Andhra Pradesh, 2007-08

\begin{tabular}{|c|c|c|c|c|c|c|}
\hline \multirow[t]{2}{*}{ Experiences (\%) } & $\begin{array}{c}\text { MM } \\
15-29\end{array}$ & $\begin{array}{l}\text { MW } \\
15-24\end{array}$ & $\begin{array}{c}\text { MM } \\
15-29\end{array}$ & $\begin{array}{c}\text { MW } \\
15-24\end{array}$ & $\begin{array}{c}\mathrm{MM} \\
15-29\end{array}$ & $\begin{array}{c}\text { MW } \\
15-24\end{array}$ \\
\hline & \multicolumn{2}{|c|}{ Combined } & \multicolumn{2}{|c|}{ Urban } & \multicolumn{2}{|c|}{ Rural } \\
\hline Had sex with someone other than spouse after marriage & 6.2 & 0.8 & 5.9 & 0.2 & 6.3 & 0.9 \\
\hline $\begin{array}{l}\text { Reported at least one extra-marital sexual partner in last } \\
12 \text { months }\end{array}$ & 2.3 & 0.6 & 1.6 & 0.2 & 2.4 & 0.7 \\
\hline Number who had begun cohabiting & 1,404 & 2,325 & 648 & 1,036 & 756 & 1,289 \\
\hline
\end{tabular}

Note: All Ns are unweighted. 


\subsection{Contraceptive practice within marriage: Lifetime, current and prior to first pregnancy}

The practise of contraception at any time during marital life was limited, reported by $10 \%$ of young men and $23 \%$ of young women (see Table 10.9 and Figure 10.6) Urban youth were somewhat more likely than rural youth to report use of contraceptives (13\% versus $9 \%$ among young men; $27 \%$ versus $22 \%$ among young women). A similar proportion of youth reported use of modern contraceptive methods; the use of traditional contraceptive methods was reported by less than $1 \%$ of youth. It is notable that despite the young age of our sample, the method most likely to be used was female sterilization, reported by $6 \%$ of young men and $18 \%$ of young women, a finding that may be attributed to the skewed focus of the family welfare programme in the state on terminal methods. Among non-terminal modern methods, condoms were most likely to have been used by both men and women (3\%), followed by oral pills $(2 \%)$.

Relatively few youth reported practising contraception at the time of the interview: $8 \%$ of young men and $21 \%$ of young women. Rural-urban differences were muted. Reporting of methods currently used mirrored the pattern described above with regard to ever use of contraception.

The duration between marriage and first use of contraception was also explored. Findings suggest that the use of contraception within the first year of marriage was almost non-existent, reported by just 2-3\% of young men and women. About 1-3\% of youth reported initiation of contraceptive use between 1 and 3 years following marriage, and $5 \%$ of young men and $15 \%$ of young women, three or more years following marriage. Indeed, of those who reported ever having practised contraception, half of young men and about two-thirds of young women had initiated contraceptive use three or more years following marriage. Differences by sex and place of residence of the respondent were negligible. Consistent with this profile, just 2-3\% of youth reported the practise of contraception to delay the first pregnancy.

\subsection{Reproductive history}

This section addresses young people's reproductive history, namely, the first pregnancy and its outcome, children ever born and surviving, experience of pregnancy loss and the wantedness of recent pregnancies. As reported in Table $10.10,80-81 \%$ of young women and men reported that they or their wife had experienced at least one pregnancy. Rural-urban differences were not observed among young women, but somewhat more rural than urban young men reported that their wife had ever been pregnant ( $81 \%$ versus $75 \%)$.

Figure 10.6: Percentage of married youth reporting lifetime and current use of contraceptive methods within marriage, Andhra Pradesh, 2007-08

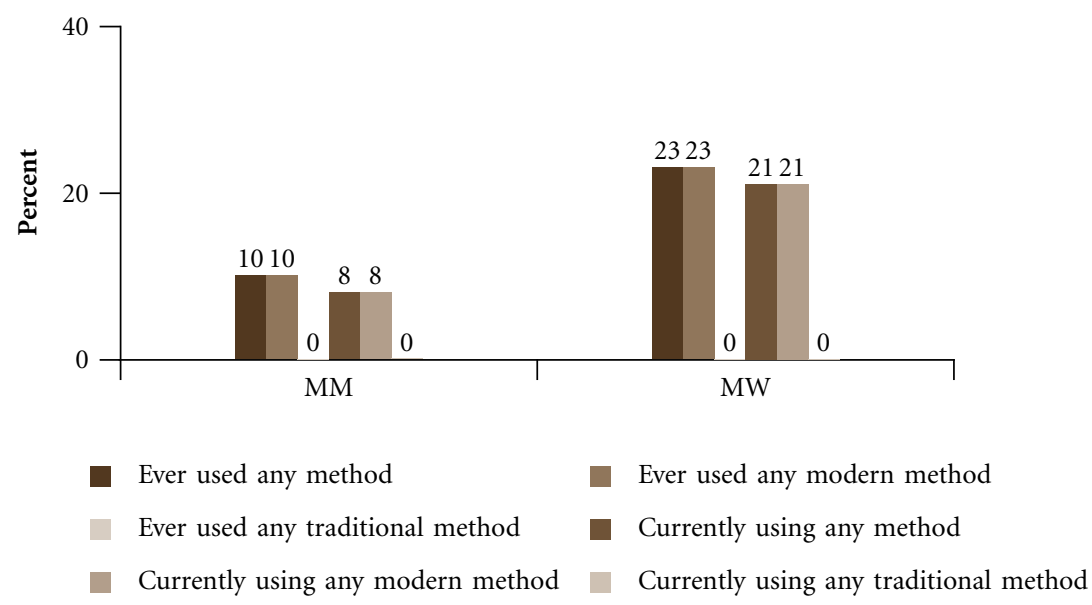


Table 10.9: Contraceptive use within marriage

Percentage of married youth by ever and current contraceptive use, percent distribution by duration between marriage and initiation of contraceptive use and percentage who used different contraceptive methods to delay first pregnancy, according to residence, Andhra Pradesh, 2007-08

\begin{tabular}{|c|c|c|c|c|c|c|}
\hline \multirow[t]{2}{*}{ Contraceptive use (\%) } & $\begin{array}{c}\text { MM } \\
15-29\end{array}$ & $\begin{array}{c}\text { MW } \\
15-24\end{array}$ & $\begin{array}{c}\text { MM } \\
15-29\end{array}$ & $\begin{array}{c}\text { MW } \\
15-24\end{array}$ & $\begin{array}{c}\text { MM } \\
15-29\end{array}$ & $\begin{array}{c}\text { MW } \\
15-24\end{array}$ \\
\hline & \multicolumn{2}{|c|}{ Combined } & \multicolumn{2}{|c|}{ Urban } & \multicolumn{2}{|c|}{ Rural } \\
\hline \multicolumn{7}{|c|}{ Ever use of contraception } \\
\hline Any method & 10.0 & 23.1 & 12.7 & 26.5 & 9.3 & 22.1 \\
\hline Any modern method & 9.9 & 22.9 & 12.4 & 26.3 & 9.3 & 21.9 \\
\hline Female sterilisation & 6.0 & 18.0 & 5.9 & 17.6 & 6.0 & 18.2 \\
\hline Male sterilisation & 0.2 & 0.8 & 0.0 & 0.6 & 0.3 & 0.9 \\
\hline Oral pills & 1.5 & 2.0 & 2.0 & 3.4 & 1.4 & 1.6 \\
\hline IUD & 0.3 & 0.8 & 0.3 & 1.3 & 0.3 & 0.6 \\
\hline Condom & 2.8 & 2.6 & 4.9 & 5.5 & 2.1 & 1.8 \\
\hline Other $^{1}$ & 0.0 & 0.2 & 0.0 & 0.2 & 0.0 & 0.2 \\
\hline Any traditional method ${ }^{2}$ & 0.3 & 0.4 & 0.3 & 1.0 & 0.3 & 0.2 \\
\hline Number who had begun cohabiting & 1,404 & 2,325 & 648 & 1,036 & 756 & 1,289 \\
\hline \multicolumn{7}{|c|}{ Current use of contraception } \\
\hline Any method & 7.8 & 21.4 & 8.8 & 23.3 & 7.6 & 20.9 \\
\hline Any modern method & 7.8 & 21.3 & 8.8 & 23.1 & 7.6 & 20.7 \\
\hline Female sterilisation & 6.0 & 18.0 & 5.9 & 17.6 & 6.0 & 18.2 \\
\hline Male sterilisation & 0.2 & 0.8 & 0.0 & 0.6 & 0.3 & 0.9 \\
\hline Oral pills & 0.7 & 0.7 & 1.0 & 1.5 & 0.7 & 0.6 \\
\hline IUD & 0.2 & 0.6 & 0.0 & 1.3 & 0.3 & 0.4 \\
\hline Condom & 1.0 & 1.3 & 2.0 & 2.7 & 0.7 & 0.9 \\
\hline Other $^{1}$ & 0.0 & 0.0 & 0.0 & 0.0 & 0.0 & 0.0 \\
\hline Any traditional method ${ }^{2}$ & 0.2 & 0.3 & 0.0 & 0.6 & 0.3 & 0.2 \\
\hline Number who had begun cohabiting & 1,404 & 2,325 & 648 & 1,036 & 756 & 1,289 \\
\hline \multicolumn{7}{|c|}{ Duration between marriage and first use of contraception } \\
\hline \multicolumn{7}{|l|}{ Duration } \\
\hline Less than 6 months & 2.4 & 1.8 & 3.9 & 4.0 & 2.0 & 1.1 \\
\hline 6-11 months & 0.2 & 0.4 & 0.0 & 0.4 & 0.3 & 0.4 \\
\hline 12 months to 3 years & 0.9 & 2.7 & 2.6 & 5.2 & 0.4 & 2.1 \\
\hline More than 3 years & 5.1 & 14.9 & 5.2 & 13.5 & 5.2 & 15.4 \\
\hline Don't know/don't remember & 1.3 & 3.2 & 1.0 & 3.4 & 1.4 & 3.1 \\
\hline Never used contraception & 90.1 & 76.9 & 87.3 & 73.5 & 90.8 & 77.9 \\
\hline Number who had begun cohabiting & 1,404 & 2,325 & 648 & 1,036 & 756 & 1,289 \\
\hline \multicolumn{7}{|c|}{ Ever use of contraception to delay first pregnancy } \\
\hline Any method & 2.7 & 2.1 & 4.6 & 4.2 & 2.2 & 1.6 \\
\hline Any modern method & 2.6 & 2.0 & 4.2 & 4.0 & 2.2 & 1.4 \\
\hline Oral pills & 1.3 & 0.9 & 1.3 & 1.7 & 1.3 & 0.8 \\
\hline IUD & 0.1 & 0.1 & 0.0 & 0.4 & 0.1 & 0.1 \\
\hline Condom & 1.8 & 1.2 & 3.3 & 2.5 & 1.4 & 0.8 \\
\hline Other $^{1}$ & 0.0 & 0.0 & 0.0 & 0.0 & 0.0 & 0.0 \\
\hline Any traditional method ${ }^{2}$ & 0.2 & 0.3 & 0.0 & 0.4 & 0.3 & 0.2 \\
\hline Number who had begun cohabiting & 1,404 & 2,325 & 648 & 1,036 & 756 & 1,289 \\
\hline
\end{tabular}

Note: All Ns are unweighted. Column totals may not equal 100\% due to missing cases. ${ }^{1}$ Includes female condoms, injectables, implants, diaphragm and foam/jelly. ${ }^{2}$ Includes periodic abstinence/rhythm and withdrawal. 
Table 10.10: First pregnancy experience

Percent distribution of married youth by duration from cohabitation to first pregnancy, outcome of first pregnancy, place of first delivery and type of attendance at first delivery, according to residence, Andhra Pradesh, 2007-08

\begin{tabular}{|c|c|c|c|c|c|c|}
\hline \multirow[t]{2}{*}{ First pregnancy experience (\%) } & $\begin{array}{c}\text { MM } \\
15-29\end{array}$ & $\begin{array}{c}\text { MW } \\
15-24\end{array}$ & $\begin{array}{c}\text { MM } \\
15-29\end{array}$ & $\begin{array}{c}\text { MW } \\
15-24\end{array}$ & $\begin{array}{c}\text { MM } \\
15-29\end{array}$ & $\begin{array}{c}\text { MW } \\
15-24\end{array}$ \\
\hline & \multicolumn{2}{|c|}{ Combined } & \multicolumn{2}{|c|}{ Urban } & \multicolumn{2}{|c|}{ Rural } \\
\hline Ever been pregnant & 79.6 & 81.2 & 75.2 & 80.4 & 80.9 & 81.5 \\
\hline Number who had begun cohabiting & 1,404 & 2,325 & 648 & 1,036 & 756 & 1,289 \\
\hline Currently pregnant for the first time & 8.2 & 6.4 & 9.5 & 7.8 & 7.9 & 6.0 \\
\hline \multicolumn{7}{|l|}{ Duration from cohabitation to first pregnancy (months) } \\
\hline Up to 3 & 6.4 & 11.5 & 6.9 & 15.8 & 6.2 & 10.2 \\
\hline $3-6$ & 30.5 & 33.8 & 35.5 & 36.4 & 29.2 & 33.2 \\
\hline $7-12$ & 17.7 & 16.4 & 16.0 & 16.3 & 18.2 & 16.4 \\
\hline $13-24$ & 24.5 & 17.5 & 23.8 & 14.7 & 24.7 & 18.3 \\
\hline More than 24 & 15.2 & 13.8 & 11.3 & 9.5 & 16.3 & 15.0 \\
\hline Do not know/can't remember & 5.6 & 7.0 & 6.5 & 7.3 & 5.4 & 6.9 \\
\hline Median duration & 12.0 & 7.0 & 8.0 & 6.0 & 12.0 & 8.0 \\
\hline Number who had ever been pregnant & 1,101 & 1,885 & 487 & 836 & 614 & 1,049 \\
\hline \multicolumn{7}{|l|}{ Outcome of first pregnancy } \\
\hline Live birth & 89.1 & 87.3 & 89.5 & 87.7 & 89.0 & 87.2 \\
\hline Still birth & 2.2 & 3.2 & 1.0 & 2.6 & 2.6 & 3.3 \\
\hline Induced abortion & 0.3 & 0.5 & 1.0 & 0.5 & 0.1 & 0.5 \\
\hline Miscarriage & 8.4 & 9.0 & 8.6 & 9.2 & 8.3 & 9.0 \\
\hline Number who completed first pregnancy & 1,009 & 1,756 & 444 & 771 & 565 & 985 \\
\hline \multicolumn{7}{|l|}{ Place of first delivery } \\
\hline Respondent's parental home & 6.9 & 18.1 & 5.3 & 11.9 & 7.4 & 19.8 \\
\hline Spouse's parental home & 21.6 & 5.9 & 10.6 & 2.6 & 24.3 & 6.8 \\
\hline Health institution & 71.5 & 76.0 & 84.1 & 85.5 & 68.3 & 73.3 \\
\hline In transit & 0.0 & 0.1 & 0.0 & 0.0 & 0.0 & 0.1 \\
\hline \multicolumn{7}{|l|}{ Type of attendance at first delivery ${ }^{1}$} \\
\hline Doctor/ANM/Nurse/LHV & 78.9 & 81.5 & 90.0 & 89.7 & 76.2 & 79.2 \\
\hline Midwife (trained) & 8.1 & 6.8 & 3.2 & 4.6 & 9.4 & 7.4 \\
\hline Other health personnel & 2.9 & 0.6 & 1.6 & 0.6 & 3.1 & 0.6 \\
\hline Dai/traditional birth attendant & 5.9 & 5.4 & 3.2 & 3.1 & 6.6 & 6.1 \\
\hline Friend/relative & 4.1 & 5.4 & 2.1 & 2.0 & 4.6 & 6.4 \\
\hline Other person ${ }^{2}$ & 0.1 & 0.1 & 0.0 & 0.0 & 0.1 & 0.1 \\
\hline None & 0.1 & 0.1 & 0.0 & 0.0 & 0.1 & 0.2 \\
\hline $\begin{array}{l}\text { Number whose first pregnancy outcome was a live or } \\
\text { still birth }\end{array}$ & 919 & 1,587 & 402 & 696 & 517 & 891 \\
\hline
\end{tabular}

Note: All Ns are unweighted. Column totals may not equal 100\% due to missing cases or "don't know" responses. ANM: Auxiliary nurse midwife; LHV: Lady health visitor. ${ }^{1}$ If the respondent reported that the delivery had occurred in a health institution, then it was assumed that a Doctor/ANM/Nurse/LHV had attended the birth. ${ }^{2}$ If the delivery was reported in transit, attendance at delivery was categorised as "other person". 


\subsubsection{First pregnancy experiences}

Of those who reported that they or their wife had ever been pregnant, significant minorities reported a current first pregnancy ( $6 \%$ of young women and $8 \%$ of young men, respectively). Among those who had experienced at least one pregnancy, the first pregnancy had occurred within a year of marriage for over half of young men (55\%) and three-fifths of young women (62\%). Rural-urban differences suggest that the first pregnancy had occurred within a year of marriage for somewhat larger proportions of urban than rural youth (58-69\% versus 54-60\%). The median duration between marriage and first pregnancy was 12 months among young men and 7 months among young women, with rural youth reporting a somewhat longer median duration than their urban counterparts (8-12 months reported by rural youth compared to 6-8 months reported by urban youth).

Pregnancy outcomes were reported by all respondents who had completed their first pregnancy. The vast majority had experienced a live birth in every group, irrespective of sex or rural-urban residence. Among other outcomes, less than 1\% had aborted their first pregnancy; for 2-3\% the pregnancy had ended in a stillbirth and for $8-9 \%$ in a miscarriage. Differences by sex and rural-urban residence were negligible.

The large majority reported institutional delivery; even so, 24-29\% reported that the first delivery had taken place at home. Rural-urban differences were pronounced: $84-86 \%$ of urban youth, compared to $68-73 \%$ of rural youth, reported an institutional delivery.

The vast majority of youth (89-90\%) reported skilled attendance at first delivery. Rural-urban differences were evident; $95 \%$ of urban youth reported skilled attendance at delivery, compared to $87-89 \%$ of rural youth.

\subsubsection{Children ever born and surviving}

Findings, revealed in Table 10.11, show that youth had experienced an average of about 1.4-1.5 pregnancies and just over one live birth. Gender and rural-urban differences were negligible, although somewhat more urban than rural young men reported no live births (34\% versus $29 \%$ ).

Few youth reported an infant or child death. The distribution of respondents by number of surviving children resembles that of children ever born, reported above. Youth typically reported about as many daughters as sons.

Somewhat more youth reported the experience of pregnancy loss. For example, stillbirths were reported by $2-3 \%$ of youth, and disparities by respondents' sex and residence were negligible. The situation was different in the case of miscarriage; $7 \%$ of young men and $11 \%$ of young women reported at least one miscarriage; the slight gender differences observed reflect perhaps the relatively limited communication between spouses on reproductive health matters. Finally, induced abortion was reported by less than $1 \%$ of young men and women, with little rural-urban variation.

Table 10.12 reports mean numbers of children ever born and surviving by respondents' socio-demographic characteristics. As expected, age was positively associated with both fertility indicators. Religion- and caste-specific differences were narrow. The number of years of schooling completed was inversely associated with both fertility indicators, but was more consistently so among young women than young men, and the inverse association was observed, irrespective of rural-urban residence. Differentials in fertility indicators by work status and household economic status were narrow, although among young men in urban areas, an inverse association between household economic status and both fertility indicators was apparent. Patterns remained roughly similar in both urban and rural settings.

\subsubsection{Wantedness of recent pregnancies}

All youth who reported one or more pregnancies were asked about the wantedness of their last pregnancy or current pregnancy (for those currently pregnant). Findings, presented in Table 10.13, suggest substantial levels of unplanned pregnancy especially reported by young women. For example, among young men whose wife was not pregnant and young women who were not pregnant at the time of the interview, $15 \%$ of young men and $23 \%$ of young women 
Table 10.11: Reproductive history

Mean number of pregnancies experienced, percent distribution by children ever born and children surviving, and mean number of child deaths, stillbirths, miscarriages and abortions among married youth, according to residence, Andhra Pradesh, 2007-08

\begin{tabular}{|c|c|c|c|c|c|c|}
\hline \multirow[t]{2}{*}{ Pregnancy outcomes (\%) } & $\begin{array}{c}\text { MM } \\
15-29\end{array}$ & $\begin{array}{c}\text { MW } \\
\text { 15-24 }\end{array}$ & $\begin{array}{c}\text { MM } \\
15-29\end{array}$ & $\begin{array}{c}\text { MW } \\
15-24\end{array}$ & $\begin{array}{c}\text { MM } \\
15-29\end{array}$ & $\begin{array}{c}\text { MW } \\
\text { 15-24 }\end{array}$ \\
\hline & \multicolumn{2}{|c|}{ Combined } & \multicolumn{2}{|c|}{ Urban } & \multicolumn{2}{|c|}{ Rural } \\
\hline Mean number of lifetime pregnancies & 1.4 & 1.5 & 1.3 & 1.4 & 1.4 & 1.6 \\
\hline \multicolumn{7}{|l|}{ Number of children ever born } \\
\hline 0 & 30.3 & 28.2 & 34.3 & 30.3 & 29.2 & 27.6 \\
\hline 1 & 31.3 & 28.8 & 32.0 & 31.9 & 31.2 & 28.0 \\
\hline 2 & 31.4 & 35.3 & 29.1 & 32.4 & 32.0 & 36.1 \\
\hline 3 & 5.8 & 6.5 & 3.6 & 4.6 & 6.5 & 7.1 \\
\hline 4 or more & 1.1 & 1.1 & 1.0 & 0.8 & 1.2 & 1.2 \\
\hline Mean number of children ever born & 1.2 & 1.2 & 1.1 & 1.1 & 1.2 & 1.3 \\
\hline \multicolumn{7}{|l|}{ Number of children surviving } \\
\hline 0 & 30.7 & 29.4 & 35.0 & 31.1 & 29.5 & 28.9 \\
\hline 1 & 31.9 & 30.0 & 31.7 & 31.7 & 32.0 & 29.6 \\
\hline 2 & 32.3 & 34.8 & 30.1 & 32.6 & 32.9 & 35.4 \\
\hline 3 & 4.6 & 5.1 & 2.9 & 4.0 & 5.1 & 5.4 \\
\hline 4 or more & 0.5 & 0.6 & 0.3 & 0.6 & 0.5 & 0.7 \\
\hline Mean number of children surviving & 1.1 & 1.2 & 1.0 & 1.1 & 1.2 & 1.2 \\
\hline Mean number of sons surviving & 0.6 & 0.6 & 0.5 & 0.6 & 0.6 & 0.6 \\
\hline Mean number of daughters surviving & 0.6 & 0.6 & 0.5 & 0.5 & 0.6 & 0.6 \\
\hline Mean number of children dead & 0.0 & 0.1 & 0.0 & 0.0 & 0.0 & 0.1 \\
\hline Reported one or more still births & 2.1 & 3.2 & 1.3 & 2.9 & 2.3 & 3.4 \\
\hline Reported one or more miscarriages & 7.3 & 10.7 & 7.5 & 9.9 & 7.2 & 10.9 \\
\hline Reported one or more induced abortions & 0.6 & 0.9 & 1.0 & 1.5 & 0.4 & 0.7 \\
\hline Number who had begun cohabiting & 1,404 & 2,325 & 648 & 1,036 & 756 & 1,289 \\
\hline
\end{tabular}

Note: All Ns are unweighted. Column totals may not equal 100\% due to missing cases or "don't know" responses.

Table 10.12: Children ever born and surviving by selected background characteristics

Mean number of children ever born and children surviving among married youth by selected background characteristics, according to residence, Andhra Pradesh, 2007-08

\begin{tabular}{|c|c|c|c|c|c|c|c|c|c|c|c|c|}
\hline \multirow{3}{*}{$\begin{array}{l}\text { Background } \\
\text { characteristics } \\
\text { (mean number) }\end{array}$} & \multicolumn{2}{|c|}{$\begin{array}{c}\text { MM } \\
15-29\end{array}$} & \multicolumn{2}{|c|}{$\begin{array}{c}\text { MW } \\
15-24\end{array}$} & \multicolumn{2}{|c|}{$\begin{array}{c}\text { MM } \\
15-29\end{array}$} & \multicolumn{2}{|c|}{$\begin{array}{c}\text { MW } \\
\text { 15-24 }\end{array}$} & \multicolumn{2}{|c|}{$\begin{array}{c}\text { MM } \\
15-29\end{array}$} & \multicolumn{2}{|c|}{$\begin{array}{c}\text { MW } \\
15-24\end{array}$} \\
\hline & \multicolumn{4}{|c|}{ Combined } & \multicolumn{4}{|c|}{ Urban } & \multicolumn{4}{|c|}{ Rural } \\
\hline & CEB & CS & CEB & CS & CEB & CS & CEB & CS & CEB & CS & CEB & CS \\
\hline Age (years) & & & & & & & & & & & & \\
\hline $15-19$ & * & * & 0.6 & 0.6 & * & * & 0.6 & 0.6 & * & * & 0.6 & 0.6 \\
\hline $20-24$ & 0.7 & 0.7 & 1.5 & 1.4 & 0.6 & 0.6 & 1.3 & 1.3 & 0.7 & 0.7 & 1.6 & 1.5 \\
\hline $25-29$ & 1.4 & 1.3 & NA & NA & 1.2 & 1.2 & NA & NA & 1.4 & 1.4 & NA & NA \\
\hline Religion & & & & & & & & & & & & \\
\hline Hindu & 1.2 & 1.1 & 1.2 & 1.2 & 1.1 & 1.0 & 1.1 & 1.1 & 1.2 & 1.1 & 1.3 & 1.2 \\
\hline Muslim & 1.1 & 1.1 & 1.4 & 1.4 & 1.0 & 0.9 & 1.3 & 1.3 & $(1.2)$ & $(1.2)$ & 1.5 & 1.5 \\
\hline Other $^{1}$ & 1.4 & 1.3 & 1.3 & 1.2 & $(1.2)$ & (1.2) & 1.2 & 1.2 & 1.4 & 1.3 & 1.3 & 1.2 \\
\hline
\end{tabular}


Table 10.12: (Cont'd)

\begin{tabular}{|c|c|c|c|c|c|c|c|c|c|c|c|c|}
\hline \multirow{3}{*}{$\begin{array}{l}\text { Background } \\
\text { characteristics } \\
\text { (mean number) }\end{array}$} & \multicolumn{2}{|c|}{$\begin{array}{c}\text { MM } \\
15-29\end{array}$} & \multicolumn{2}{|c|}{$\begin{array}{c}\text { MW } \\
15-24\end{array}$} & \multicolumn{2}{|c|}{$\begin{array}{c}\mathrm{MM} \\
15-29\end{array}$} & \multicolumn{2}{|c|}{$\begin{array}{c}\text { MW } \\
15-24\end{array}$} & \multicolumn{2}{|c|}{$\begin{array}{c}\mathrm{MM} \\
15-29\end{array}$} & \multicolumn{2}{|c|}{$\begin{array}{c}\text { MW } \\
15-24\end{array}$} \\
\hline & \multicolumn{4}{|c|}{ Combined } & \multicolumn{4}{|c|}{ Urban } & \multicolumn{4}{|c|}{ Rural } \\
\hline & CEB & CS & CEB & CS & CEB & CS & CEB & CS & CEB & CS & CEB & CS \\
\hline Caste & & & & & & & & & & & & \\
\hline SC & 1.2 & 1.1 & 1.3 & 1.2 & 1.2 & 1.1 & 1.2 & 1.2 & 1.2 & 1.1 & 1.3 & 1.2 \\
\hline ST & 1.4 & 1.3 & 1.4 & 1.3 & $(1.4)$ & $(1.3)$ & $(1.1)$ & (1.1) & 1.4 & 1.3 & 1.4 & 1.3 \\
\hline $\mathrm{OBC}$ & 1.2 & 1.1 & 1.2 & 1.2 & 1.0 & 1.0 & 1.2 & 1.2 & 1.2 & 1.2 & 1.2 & 1.2 \\
\hline General $^{2}$ & 1.1 & 1.1 & 1.2 & 1.2 & 1.0 & 0.9 & 1.0 & 1.0 & 1.2 & 1.2 & 1.3 & 1.2 \\
\hline $\begin{array}{l}\text { Educational } \\
\text { level (years) }\end{array}$ & & & & & & & & & & & & \\
\hline None $^{3}$ & 1.3 & 1.3 & 1.5 & 1.4 & 1.5 & 1.5 & 1.4 & 1.4 & 1.3 & 1.2 & 1.5 & 1.4 \\
\hline $1-7$ & 1.3 & 1.2 & 1.3 & 1.2 & 1.1 & 1.1 & 1.3 & 1.2 & 1.3 & 1.2 & 1.3 & 1.2 \\
\hline $8-11$ & 1.0 & 1.0 & 1.0 & 1.0 & 1.0 & 1.0 & 1.1 & 1.1 & 1.1 & 1.0 & 1.0 & 0.9 \\
\hline 12 and above & 1.0 & 1.0 & 0.8 & 0.7 & 0.8 & 0.8 & 0.7 & 0.7 & 1.1 & 1.1 & 0.8 & 0.8 \\
\hline $\begin{array}{l}\text { Worked in last } \\
12 \text { months }\end{array}$ & & & & & & & & & & & & \\
\hline Yes & 1.2 & 1.1 & 1.3 & 1.2 & 1.1 & 1.0 & 1.1 & 1.0 & 1.2 & 1.2 & 1.3 & 1.2 \\
\hline No & * & * & 1.2 & 1.2 & * & * & 1.2 & 1.1 & * & * & 1.3 & 1.2 \\
\hline Wealth quintile & & & & & & & & & & & & \\
\hline First & 1.3 & 1.2 & 1.4 & 1.3 & $(1.5)$ & $(1.3)$ & 1.2 & 1.2 & 1.2 & 1.2 & 1.4 & 1.3 \\
\hline Second & 1.3 & 1.2 & 1.3 & 1.2 & 1.2 & 1.1 & 1.1 & 1.1 & 1.3 & 1.2 & 1.3 & 1.3 \\
\hline Third & 1.2 & 1.1 & 1.2 & 1.2 & 1.2 & 1.2 & 1.3 & 1.2 & 1.2 & 1.1 & 1.2 & 1.2 \\
\hline Fourth & 1.2 & 1.1 & 1.1 & 1.1 & 1.0 & 1.0 & 1.2 & 1.1 & 1.2 & 1.2 & 1.1 & 1.0 \\
\hline Fifth & 1.0 & 1.0 & 1.1 & 1.1 & 0.9 & 0.9 & 1.0 & 1.0 & 1.1 & 1.0 & 1.2 & 1.2 \\
\hline Total & 1.2 & 1.1 & 1.2 & 1.2 & 1.1 & 1.0 & 1.1 & 1.1 & 1.2 & 1.2 & 1.3 & 1.2 \\
\hline
\end{tabular}

Note: ( ) Based on 25-49 unweighted cases. ${ }^{*}$ Mean not shown, based on fewer than 25 unweighted cases. CEB: Children ever born. CS: Children surviving. NA: Not applicable. OBC: Other backward caste. SC: Scheduled caste. ST: Scheduled tribe. ${ }^{1}$ Includes Christian, Buddhist, Neo-Buddhist, Sikh, Jain, Jewish, Parsi/Zoroastrian and no specified religion. ${ }^{2}$ Includes all those not belonging to SC, ST or OBC. ${ }^{3}$ Includes non-literate and literate with no formal schooling.

Table 10.13: Wantedness of most recent pregnancy

Percent distribution of married youth by wantedness of most recent pregnancy in the three years preceding the interview, according to residence, Andhra Pradesh, 2007-08

\begin{tabular}{|c|c|c|c|c|c|c|}
\hline \multirow[t]{2}{*}{ Status (\%) } & $\begin{array}{c}\text { MM } \\
15-29\end{array}$ & $\begin{array}{c}\text { MW } \\
\text { 15-24 }\end{array}$ & $\begin{array}{c}\text { MM } \\
15-29\end{array}$ & $\begin{array}{c}\text { MW } \\
15-24\end{array}$ & $\begin{array}{c}\text { MM } \\
15-29\end{array}$ & $\begin{array}{c}\text { MW } \\
15-24\end{array}$ \\
\hline & \multicolumn{2}{|c|}{ Combined } & \multicolumn{2}{|c|}{ Urban } & \multicolumn{2}{|c|}{ Rural } \\
\hline \multicolumn{7}{|l|}{ Wantedness status of last pregnancy ${ }^{1}$} \\
\hline Planned & 84.8 & 77.4 & 90.9 & 77.7 & 83.1 & 77.3 \\
\hline Mistimed & 10.1 & 20.1 & 5.3 & 20.0 & 11.4 & 20.1 \\
\hline Unwanted & 5.1 & 2.4 & 3.8 & 2.1 & 5.5 & 2.5 \\
\hline Number who had experienced at least one pregnancy & 1,008 & 1,756 & 443 & 771 & 565 & 985 \\
\hline \multicolumn{7}{|l|}{ Wantedness status of current pregnancy } \\
\hline Planned & 84.3 & 78.7 & 89.7 & 78.1 & 82.8 & 78.5 \\
\hline Mistimed & 12.8 & 18.0 & 10.3 & 20.3 & 13.4 & 17.7 \\
\hline Unwanted & 2.9 & 2.9 & 0.0 & 1.6 & 3.7 & 3.3 \\
\hline Number currently pregnant & 172 & 277 & 78 & 126 & 94 & 151 \\
\hline
\end{tabular}

Note: All Ns are unweighted. Column totals may not equal 100\% due to missing cases or “don't know" responses. ${ }^{1}$ Excludes respondents/respondents' wives currently pregnant for the first time or never been pregnant. 
Table 10.14: Ideal family size

Percent distribution of married youth by their reported ideal number of children and mean ideal number of children, according to residence, Andhra Pradesh, 2007-08

\begin{tabular}{|c|c|c|c|c|c|c|}
\hline \multirow[t]{2}{*}{ Ideal family size (\%) } & $\begin{array}{c}\text { MM } \\
15-29\end{array}$ & $\begin{array}{c}\text { MW } \\
15-24\end{array}$ & $\begin{array}{c}\text { MM } \\
15-29\end{array}$ & $\begin{array}{c}\text { MW } \\
15-24\end{array}$ & $\begin{array}{c}\text { MM } \\
15-29\end{array}$ & $\begin{array}{c}\text { MW } \\
15-24\end{array}$ \\
\hline & \multicolumn{2}{|c|}{ Combined } & \multicolumn{2}{|c|}{ Urban } & \multicolumn{2}{|c|}{ Rural } \\
\hline Ideal number of children: & & & & & & \\
\hline 1 & 2.1 & 3.2 & 3.3 & 6.3 & 1.8 & 2.3 \\
\hline 2 & 78.8 & 84.4 & 81.8 & 83.2 & 77.9 & 84.7 \\
\hline 3 or more & 11.3 & 8.9 & 10.4 & 8.4 & 11.5 & 9.1 \\
\hline Other $^{1}$ & 7.9 & 3.4 & 4.6 & 2.1 & 8.8 & 3.8 \\
\hline Mean ideal number of children ${ }^{2}$ & 2.1 & 2.1 & 2.1 & 2.0 & 2.1 & 2.1 \\
\hline Number who had begun cohabiting & 1,404 & 2,325 & 648 & 1,036 & 756 & 1,289 \\
\hline
\end{tabular}

Note: All Ns are unweighted. Column totals may not equal 100\% due to missing cases or "don't know" responses. ${ }^{1}$ Includes "it's up to God," "difficult to say," etc. ${ }^{2}$ Includes only respondents who gave numeric responses.

reported that the last pregnancy was mistimed or unwanted. Rural-urban differences were muted among young women, but more rural than urban young men reported that the last pregnancy was unplanned (17\% versus $9 \%$ ). A similar pattern emerged with regard to the wantedness of current pregnancy among those pregnant at the time of the interview or whose wife was pregnant at the time of the interview: of those young men who reported that their wife was pregnant at the time of the interview, $16 \%$ reported that the pregnancy was either unwanted or wanted at a later time, and $21 \%$ of young women pregnant at the time of the interview reported that the pregnancy was unwanted or mistimed. Rural-urban differences were, again, muted among young women, but notable among young men among whom those in rural areas were more likely than the urban to report that the current pregnancy was mistimed or unwanted (17\% versus $10 \%)$.

\subsection{Ideal family size}

All respondents were asked to report the number of children they considered ideal, and among these the number of sons and daughters considered ideal. As several respondents reported that they were unconcerned about the sex of children, a third response "children of either sex" was also recorded. As seen in Table 10.14, young men and young women typically considered 2.1 children ideal, irrespective of rural-urban residence.

Tables $10.15 \mathrm{a}$ and $10.15 \mathrm{~b}$ present the ideal number of sons and daughters reported by young men and women by selected socio-demographic characteristics. The majority of youth- $84 \%$ of young men and $90 \%$ of young women-reported a preference for an equal number of sons and daughters, with an average of slightly less than one of each. There was, however, a slight indication of son preference, for example, $11 \%$ of young men and $8 \%$ of young women reported a preference for more sons than daughters; in contrast, fewer youth reported a preference for more daughters than sons (3-5\%). Rural-urban differences were negligible. This pattern persisted, irrespective of socio-demographic characteristics under consideration. Even so, son preference was somewhat less common among those belonging to general castes than others and among the better educated than the less educated. Among young men, in addition, son preference was less common among those belonging to the wealthiest quintile than among others. 
Table 10.15a: Married young men's preferences for sons and daughters by selected background characteristics

Mean ideal number of sons, daughters and children of either sex and some indicators of sex preference by selected background characteristics of married young men, according to residence, Andhra Pradesh, 2007-08

\begin{tabular}{|c|c|c|c|c|c|c|c|}
\hline \multirow[t]{3}{*}{ Background characteristics } & \multirow{2}{*}{\multicolumn{3}{|c|}{ Mean ideal number of: }} & \multirow{2}{*}{\multicolumn{4}{|c|}{$\begin{array}{c}\text { Indicators of sex preference } \\
\text { Percent who wanted: }\end{array}$}} \\
\hline & & & & & & & \\
\hline & Sons & Daughters & $\begin{array}{l}\text { Children } \\
\text { of either } \\
\text { sex }\end{array}$ & $\begin{array}{c}\text { More } \\
\text { sons than } \\
\text { daughters }\end{array}$ & $\begin{array}{c}\text { More } \\
\text { daughters } \\
\text { than sons }\end{array}$ & $\begin{array}{c}\text { At least } \\
\text { one } \\
\text { son }\end{array}$ & $\begin{array}{c}\text { At least } \\
\text { one } \\
\text { daughter }\end{array}$ \\
\hline \multicolumn{8}{|l|}{ Residence } \\
\hline Urban & 0.7 & 0.7 & 0.7 & 8.9 & 3.4 & 67.2 & 65.2 \\
\hline Rural & 0.8 & 0.8 & 0.5 & 11.2 & 5.6 & 74.2 & 72.1 \\
\hline \multicolumn{8}{|l|}{ Age (years) } \\
\hline $15-19$ & * & * & * & * & * & * & * \\
\hline $20-24$ & 0.8 & 0.7 & 0.6 & 11.5 & 2.9 & 74.0 & 69.4 \\
\hline $25-29$ & 0.8 & 0.8 & 0.5 & 10.4 & 6.1 & 72.1 & 70.9 \\
\hline \multicolumn{8}{|l|}{ Religion } \\
\hline Hindu & 0.8 & 0.8 & 0.5 & 10.9 & 5.1 & 73.5 & 71.3 \\
\hline Muslim & 0.7 & 0.7 & 0.8 & 7.3 & 7.2 & 61.9 & 59.8 \\
\hline Other $^{1}$ & 0.9 & 0.8 & 0.6 & 11.8 & 2.4 & 74.1 & 71.4 \\
\hline \multicolumn{8}{|l|}{ Caste } \\
\hline SC & 0.9 & 0.8 & 0.5 & 12.7 & 5.1 & 74.1 & 71.2 \\
\hline ST & 1.0 & 0.9 & 0.4 & 14.9 & 5.3 & 79.8 & 78.7 \\
\hline OBC & 0.8 & 0.8 & 0.5 & 11.0 & 4.3 & 73.9 & 71.0 \\
\hline General $^{2}$ & 0.7 & 0.7 & 0.6 & 6.3 & 7.0 & 65.8 & 65.8 \\
\hline \multicolumn{8}{|l|}{ Educational level (years) } \\
\hline None $^{3}$ & 0.9 & 0.8 & 0.5 & 15.6 & 5.3 & 75.8 & 73.3 \\
\hline $1-7$ & 0.9 & 0.8 & 0.5 & 12.4 & 5.4 & 75.7 & 72.1 \\
\hline $8-11$ & 0.8 & 0.7 & 0.6 & 8.0 & 3.8 & 69.6 & 67.4 \\
\hline 12 and above & 0.7 & 0.8 & 0.6 & 6.6 & 7.1 & 68.4 & 70.4 \\
\hline \multicolumn{8}{|l|}{ Worked in last 12 months } \\
\hline Yes & 0.8 & 0.8 & 0.6 & 10.5 & 5.0 & 72.2 & 70.2 \\
\hline No & * & * & * & * & * & * & * \\
\hline \multicolumn{8}{|l|}{ Wealth quintile } \\
\hline First & 1.0 & 0.8 & 0.4 & 15.3 & 4.3 & 80.5 & 78.7 \\
\hline Second & 0.8 & 0.7 & 0.6 & 12.0 & 5.4 & 72.1 & 66.7 \\
\hline Third & 0.8 & 0.8 & 0.5 & 10.5 & 4.7 & 74.2 & 73.2 \\
\hline Fourth & 0.8 & 0.7 & 0.6 & 10.2 & 4.4 & 69.9 & 67.0 \\
\hline Fifth & 0.8 & 0.8 & 0.6 & 6.0 & 6.9 & 69.7 & 70.9 \\
\hline Total & 0.8 & 0.8 & 0.5 & 10.7 & 5.1 & 72.6 & 70.5 \\
\hline
\end{tabular}

Note: ${ }^{*}$ Percentage not shown, based on fewer than 25 unweighted cases. OBC: Other backward caste. SC: Scheduled caste. ST: Scheduled tribe. ${ }^{1}$ Includes Christian, Buddhist, Neo-Buddhist, Sikh, Jain, Jewish, Parsi/Zoroastrian and no specified religion.

${ }^{2}$ Includes all those not belonging to SC, ST or OBC. ${ }^{3}$ Includes non-literate and literate with no formal schooling. 
Table 10.15b: Married young women's preferences for sons and daughters by selected background characteristics

Mean ideal number of sons, daughters and children of either sex and some indicators of sex preference by selected background characteristics of married young women, according to residence, Andhra Pradesh, 2007-08

\begin{tabular}{|c|c|c|c|c|c|c|c|}
\hline \multirow[t]{3}{*}{ Background characteristics } & \multirow{2}{*}{\multicolumn{3}{|c|}{ Mean ideal number of: }} & \multirow{2}{*}{\multicolumn{4}{|c|}{$\begin{array}{c}\text { Indicators of sex preference } \\
\text { Percent who wanted: }\end{array}$}} \\
\hline & & & & & & & \\
\hline & Sons & Daughters & $\begin{array}{l}\text { Children } \\
\text { of either } \\
\text { sex }\end{array}$ & $\begin{array}{c}\text { More } \\
\text { sons than } \\
\text { daughters }\end{array}$ & $\begin{array}{c}\text { More } \\
\text { daughters } \\
\text { than sons }\end{array}$ & $\begin{array}{c}\text { At least } \\
\text { one } \\
\text { son }\end{array}$ & $\begin{array}{c}\text { At least } \\
\text { one } \\
\text { daughter }\end{array}$ \\
\hline \multicolumn{8}{|l|}{ Residence } \\
\hline Urban & 0.7 & 0.6 & 0.7 & 6.8 & 3.7 & 62.4 & 61.3 \\
\hline Rural & 0.8 & 0.7 & 0.6 & 7.7 & 2.4 & 69.4 & 67.5 \\
\hline \multicolumn{8}{|l|}{ Age (years) } \\
\hline $15-19$ & 0.7 & 0.7 & 0.7 & 6.8 & 2.1 & 65.7 & 64.4 \\
\hline $20-24$ & 0.8 & 0.7 & 0.6 & 7.8 & 2.9 & 68.7 & 66.9 \\
\hline \multicolumn{8}{|l|}{ Religion } \\
\hline Hindu & 0.7 & 0.7 & 0.6 & 7.4 & 2.5 & 67.1 & 65.3 \\
\hline Muslim & 0.8 & 0.7 & 0.7 & 7.2 & 2.2 & 66.7 & 66.2 \\
\hline Other $^{1}$ & 0.8 & 0.8 & 0.5 & 9.4 & 5.0 & 74.8 & 73.8 \\
\hline \multicolumn{8}{|l|}{ Caste } \\
\hline SC & 0.8 & 0.7 & 0.5 & 8.0 & 2.7 & 72.8 & 71.1 \\
\hline ST & 0.9 & 0.8 & 0.5 & 10.4 & 3.1 & 74.8 & 73.8 \\
\hline OBC & 0.7 & 0.7 & 0.7 & 8.1 & 2.3 & 66.8 & 64.8 \\
\hline General $^{2}$ & 0.7 & 0.6 & 0.7 & 4.7 & 3.4 & 62.4 & 61.5 \\
\hline \multicolumn{8}{|l|}{ Educational level (years) } \\
\hline None $^{3}$ & 0.8 & 0.7 & 0.7 & 11.5 & 2.2 & 69.4 & 67.1 \\
\hline $1-7$ & 0.7 & 0.7 & 0.6 & 6.7 & 1.8 & 68.4 & 66.1 \\
\hline $8-11$ & 0.7 & 0.7 & 0.6 & 4.1 & 3.9 & 67.8 & 68.2 \\
\hline 12 and above & 0.6 & 0.6 & 0.8 & 6.8 & 4.3 & 57.8 & 54.0 \\
\hline \multicolumn{8}{|l|}{ Worked in last 12 months } \\
\hline Yes & 0.8 & 0.7 & 0.6 & 8.1 & 2.7 & 69.7 & 67.9 \\
\hline No & 0.7 & 0.7 & 0.7 & 7.0 & 2.7 & 65.8 & 64.3 \\
\hline \multicolumn{8}{|l|}{ Wealth quintile } \\
\hline First & 0.9 & 0.8 & 0.5 & 8.8 & 4.2 & 77.3 & 76.5 \\
\hline Second & 0.7 & 0.7 & 0.7 & 8.4 & 1.4 & 66.1 & 64.1 \\
\hline Third & 0.7 & 0.7 & 0.6 & 7.1 & 1.9 & 67.6 & 65.6 \\
\hline Fourth & 0.7 & 0.6 & 0.7 & 6.9 & 2.2 & 64.7 & 62.5 \\
\hline Fifth & 0.7 & 0.6 & 0.7 & 6.1 & 4.5 & 62.1 & 61.5 \\
\hline Total & 0.7 & 0.7 & 0.6 & 7.5 & 2.7 & 67.8 & 66.1 \\
\hline
\end{tabular}

Note: OBC: Other backward caste. SC: Scheduled caste. ST: Scheduled tribe. ${ }^{1}$ Includes Christian, Buddhist, Neo-Buddhist, Sikh, Jain, Jewish, Parsi/Zoroastrian and no specified religion. ${ }^{2}$ Includes all those not belonging to SC, ST or OBC. ${ }^{3}$ Includes non-literate and literate with no formal schooling. 


\subsection{Summary}

Findings indicate that although most youth preferred to marry after age 18, early marriage continues to characterise the lives of many young women, and, to a certain extent, the lives of young men as well. Among young women aged 20-24 years, almost one in five young women (18\%) was married before age 15, 54\% before age 18 and almost three in four $(72 \%)$ before age 20 . Even though early marriage was less prevalent among young men, one in ten young men aged 20-24 years was married before age 20.

Almost all youth reported arranged marriages; it is notable, however, that $86-89 \%$ of youth were given an opportunity to participate in the decision on the choice of their spouse. At the same time, reported pre-marital acquaintance was limited. Just a little over one-quarter of young men and one-fifth of young women reported that they had ever had a chance to meet and interact alone with their spouse-to-be prior to marriage. Over two in five married youth reported that they had met their spouse for the first time on the wedding day. Compounding the lack of pre-marital acquaintance was the lack of awareness of what to expect of married life, reported by over two-thirds of young men and over four-fifths of young women.

Despite the existence of laws against the payment of dowry, this practice characterised the marriages of $81 \%$ of young men and $86 \%$ of young women. Findings also show that families of urban youth appeared as likely as their rural counterparts to conform to traditional practices, such as payment of dowry.

Reports of marital life suggest that married life was characterised by considerable inter-spousal communication on most topics, yet, just one in eight young men and one in three young women had ever discussed matters relating to contraception with their spouse, clearly undermining married young people's ability to adopt protective actions.

Physical violence and forced sex within marriage were reported by sizeable proportions of youth. For example, a little less than one-quarter of young women reported that they had ever faced physical violence perpetrated by their husband and one-fifth of young men reported perpetrating violence on their wife. Recent violence was likewise reported by about one-sixth of young men and about one-fifth of young women. Sexual violence was also reported by significant minorities of youth. One in ten young women reported ever being forced to engage in sex by their husband; one in seven young men reported forcing their wife to engage in sex. Fewer youth (5\%) reported recent sexual violence.

While the Youth Study did not explore extra-marital sexual experiences in detail, the available data indicate that $6 \%$ of young men reported an extra-marital sexual encounter. In contrast, less than 1\% of young women reported an extra-marital sexual encounter.

Contraceptive use at any time within marriage was relatively limited, reported by $10 \%$ of young men and $23 \%$ of young women. Moreover, just $8 \%$ of young men and $21 \%$ of young women reported current use of contraception. Among contraceptive methods typically used, female sterilisation was mostly likely to be reported; indeed, over three-quarters of young men and over four-fifths of young women who were practising contraception at the time of the interview reported that their wife or they had been sterilised. Just 2-3\% of young people practised contraception to delay the first birth. Not surprisingly, pregnancy typically occurred within a year of marriage for over three-fifths of young women and over half of young men who reported that they or their wife had been pregnant at least once. Moreover, substantial proportions of youth reported experiencing unintended pregnancy. For example, among young women who were not pregnant at the time of the interview and among young men whose wife was not pregnant at the time of the interview, $23 \%$ and $15 \%$, respectively, reported that the last pregnancy was mistimed or unwanted.

Circumstances of the first birth suggest that the overwhelming majority of first births were delivered in a health care facility (72-76\%) and $89-90 \%$ of first births were delivered by a skilled attendant.

Findings also show that most respondents wanted one child of each sex and just a small minority of young men and women expressed a preference for more sons than daughters. 


\section{Health and health seeking} behaviour

This chapter focuses on young people's patterns of substance use, health status and treatment-seeking for health problems experienced. The Youth Study probed alcohol, drug and tobacco use as well as, among those who reported substance use, consumption characteristics, including recent use and extent of use. The Study also included questions relating to the experience of problems in the areas of general, sexual and reproductive health and mental health. It also explored young people's care seeking practices for general and sexual and reproductive health problems as well as their attitudes towards pre-marital HIV testing for boys and girls and the extent to which youth had undergone HIV testing. Where numbers are small, we present combined findings for rural and urban respondents.

\subsection{Substance use}

Research has shown that substance use can directly compromise young people's health. For example, evidence suggests that the use of alcohol and drugs among youth is associated with physical fights, risky sexual activity, depression and suicide as well as irregular school or work attendance and other negative outcomes (DiClemente, 1992; Ellickson, Saner and McGuigan, 1997; Gruber et al., 1996; Lowry et al., 1994; Mohan, Sankara Sarma and Thankappan, 2005; Singh and Saini, 2007).

Youth Study findings on the extent of substance use among young people themselves suggest that hardly any young men and not a single young woman reported drug use (including, for example, ganja, charas, brown sugar, cocaine and bhang). While hardly any young women reported consumption of tobacco or alcohol, a substantial proportion of young men reported doing so (Table 11.1). For example, 20\% of young men and less than $1 \%$ of young women had ever consumed tobacco products. Most of those young men who had ever consumed tobacco products reported that they had done so once a week or more frequently in the month prior to the interview. Married young men were far more likely than the unmarried to report having ever used tobacco products ( $42 \%$ and $15 \%$, respectively) and recent tobacco use (37\% and $13 \%$, respectively). Rural-urban differences were negligible.

As far as alcohol consumption is concerned, just two percent of young women, including $1 \%$ of urban young women and $2 \%$ of rural young women, reported alcohol consumption. In contrast to young women, larger percentages of young men reported that they had ever consumed (27\%) or had recently consumed (9\%) alcohol. As in the case of tobacco use, married young men were far more likely to have ever consumed alcohol than the unmarried (49\% and 23\%, respectively). Recent alcohol use-once a week or more frequently in the month prior to the interview-was reported by many fewer: $22 \%$ and $6 \%$ of married and unmarried young men, respectively. Young men in urban settings-both the married and the unmarried-were more likely than their rural counterparts to report having ever consumed alcohol (61\% and $28 \%$ among married and unmarried young men in urban settings, respectively, and $46 \%$ and $21 \%$ of married and unmarried young men in rural settings). The large majority of young men who had ever consumed alcohol reported that they usually consumed alcohol with peers $(91 \%$ and $93 \%$ of the married and unmarried, respectively) and over two-fifths ( $42 \%$ and $44 \%$ of the married and unmarried, respectively) reported that they sometimes or often became drunk (not shown in tabular form). 
Table 11.1: Substance use

Percentage of youth reporting lifetime and recent substance use, according to residence, Andhra Pradesh, 2007-08

\begin{tabular}{|c|c|c|c|c|c|c|}
\hline Substance use (\%) & $\begin{array}{c}\mathrm{M} \\
15-24\end{array}$ & $\begin{array}{c}\text { W } \\
15-24\end{array}$ & $\begin{array}{c}\text { MM } \\
15-29\end{array}$ & $\begin{array}{c}\text { MW } \\
\text { 15-24 }\end{array}$ & $\begin{array}{c}\text { UM } \\
15-24\end{array}$ & $\begin{array}{c}\text { UW } \\
\text { 15-24 }\end{array}$ \\
\hline \multicolumn{7}{|c|}{ Combined } \\
\hline \multicolumn{7}{|l|}{ Ever consumed } \\
\hline Tobacco and its products & 19.5 & 0.3 & 41.5 & 0.4 & 15.4 & 0.2 \\
\hline Alcohol & 27.3 & 1.5 & 48.9 & 2.3 & 23.3 & 0.6 \\
\hline Drugs $^{1}$ & 0.0 & 0.0 & 0.1 & 0.0 & 0.0 & 0.0 \\
\hline \multicolumn{7}{|c|}{ Consumed once a week or more frequently in last month } \\
\hline Tobacco and its products & 16.7 & 0.2 & 37.1 & 0.3 & 12.8 & 0.0 \\
\hline Alcohol & 9.0 & 0.4 & 22.1 & 0.6 & 6.4 & 0.1 \\
\hline Drugs $^{1}$ & 0.0 & 0.0 & 0.0 & 0.0 & 0.0 & 0.0 \\
\hline Number of respondents & 2,479 & 4,848 & 1,405 & 2,330 & 2,077 & 2,518 \\
\hline \multicolumn{7}{|c|}{ Urban } \\
\hline \multicolumn{7}{|l|}{ Ever consumed } \\
\hline Tobacco and its products & 20.5 & 0.2 & 45.0 & 0.4 & 17.6 & 0.1 \\
\hline Alcohol & 31.9 & 0.6 & 60.8 & 1.0 & 28.2 & 0.3 \\
\hline Drugs $^{1}$ & 0.0 & 0.0 & 0.0 & 0.0 & 0.0 & 0.0 \\
\hline \multicolumn{7}{|c|}{ Consumed once a week or more frequently in last month } \\
\hline Tobacco and its products & 16.9 & 0.1 & 37.5 & 0.0 & 14.3 & 0.0 \\
\hline Alcohol & 11.0 & 0.1 & 25.5 & 0.4 & 8.6 & 0.0 \\
\hline Drugs $^{1}$ & 0.0 & 0.0 & 0.0 & 0.0 & 0.0 & 0.0 \\
\hline Number of respondents & 1,289 & 2,126 & 648 & 1,037 & 1,132 & 1,089 \\
\hline \multicolumn{7}{|c|}{ Rural } \\
\hline \multicolumn{7}{|l|}{ Ever consumed } \\
\hline Tobacco and its products & 19.1 & 0.3 & 40.5 & 0.4 & 14.5 & 0.2 \\
\hline Alcohol & 25.5 & 1.9 & 45.6 & 2.6 & 21.2 & 0.7 \\
\hline Drugs $^{1}$ & 0.0 & 0.0 & 0.1 & 0.0 & 0.0 & 0.0 \\
\hline \multicolumn{7}{|c|}{ Consumed once a week or more frequently in last month } \\
\hline Tobacco and its products & 16.7 & 0.2 & 37.0 & 0.3 & 12.2 & 0.1 \\
\hline Alcohol & 8.2 & 0.5 & 21.1 & 0.6 & 5.4 & 0.2 \\
\hline Drugs $^{1}$ & 0.0 & 0.0 & 0.0 & 0.0 & 0.0 & 0.0 \\
\hline Number of respondents & 1,190 & 2,722 & 757 & 1,293 & 945 & 1,429 \\
\hline
\end{tabular}

Note: All Ns are unweighted. ${ }^{1}$ Includes ganja, charas, brown sugar, cocaine, bhang, etc.

\subsection{General and sexual and reproductive health problems}

General health problems about which youth were questioned included high fever and injury. Sexual and reproductive health problems included symptoms of genital infection (burning during urination, genital ulcers, genital itching, and swelling in the groin, and genital discharge, for example), anxiety about nocturnal emission or swapnadosh (for young men) and menstrual problems (for young women). Findings related to recent experiences of various general health problems, and sexual and reproductive health problems are presented in Table 11.2.

\subsubsection{General health problems}

Findings show that $22 \%$ of young men and $33 \%$ of young women had experienced high fever in the three months preceding the survey. Differences by marital status were negligible among both young men and women. Rural-urban differences were also negligible among young women; however, somewhat more young men in rural than urban areas reported experiencing high fever. 
Injuries were experienced by a minority of respondents in the three months preceding the survey, specifically, $7 \%$ of young men and $2 \%$ of young women. Differences by marital status and rural-urban residences were narrow.

\subsubsection{Sexual and reproductive health problems}

Table 11.2 presents young people's reported experiences of symptoms of genital infection in the three months preceding the survey. We note that these findings are based on self-reports and not on clinical examination or laboratory testing and therefore must be interpreted with caution. Young women were more likely than young men to report symptoms of genital infection (11\% versus $1 \%)$. Differences by marital status and rural-urban residence were negligible.

Previous research has documented the extent to which semen loss is associated with anxiety regarding masculine weakness and ill-health in South Asian cultures (Bhatia and Choudhary, 1998; Bhatia and Malik, 1991; Bhende, 1995; Collumbien et al., 2004; Khan et al., 2006; Pelto, 1999; Verma et al., 2003). Youth Study findings

Table 11.2: Self-reported health problems

Percentage of youth reporting recent experiences of selected general and sexual and reproductive health problems, according to residence, Andhra Pradesh, 2007-08

\begin{tabular}{|c|c|c|c|c|c|c|}
\hline $\begin{array}{l}\text { General/sexual and reproductive health } \\
\text { problems experienced }(\%)\end{array}$ & $\begin{array}{c}M \\
15-24\end{array}$ & $\begin{array}{c}\text { W } \\
15-24\end{array}$ & $\begin{array}{c}\text { MM } \\
15-29\end{array}$ & $\begin{array}{l}\text { MW } \\
15-24\end{array}$ & $\begin{array}{c}\text { UM } \\
15-24\end{array}$ & $\begin{array}{l}\text { UW } \\
15-24\end{array}$ \\
\hline \multicolumn{7}{|c|}{ Combined } \\
\hline High fever in last 3 months & 22.2 & 33.1 & 21.4 & 33.8 & 21.5 & 32.2 \\
\hline Injury in last 3 months & 7.3 & 2.0 & 6.3 & 1.4 & 7.0 & 2.7 \\
\hline Symptoms of genital infection in last 3 months $^{1}$ & 1.1 & 10.9 & 0.9 & 11.5 & 1.1 & 10.2 \\
\hline $\begin{array}{l}\text { Anxiety about swapnadosh/nocturnal emission in last } \\
12 \text { months }\end{array}$ & 18.0 & NA & 4.3 & NA & 21.2 & NA \\
\hline Menstrual problems in last 3 months & NA & 11.7 & NA & 10.0 & NA & 13.9 \\
\hline Number of respondents & 2,479 & 4,848 & 1,405 & 2,330 & 2,077 & 2,518 \\
\hline \multicolumn{7}{|c|}{ Urban } \\
\hline High fever in last 3 months & 17.6 & 31.9 & 16.3 & 33.7 & 17.1 & 30.4 \\
\hline Injury in last 3 months & 5.5 & 1.9 & 3.6 & 1.0 & 5.9 & 2.6 \\
\hline Symptoms of genital infection in last 3 months $^{1}$ & 0.9 & 9.3 & 0.7 & 10.1 & 0.8 & 8.8 \\
\hline $\begin{array}{l}\text { Anxiety about swapnadosh/nocturnal emission in last } \\
12 \text { months }\end{array}$ & 20.3 & NA & 6.2 & NA & 21.9 & NA \\
\hline Menstrual problems in last 3 months & NA & 11.9 & NA & 9.5 & NA & 13.9 \\
\hline Number of respondents & 1,289 & 2,126 & 648 & 1,037 & 1,132 & 1,089 \\
\hline \multicolumn{7}{|c|}{ Rural } \\
\hline High fever in last 3 months & 24.1 & 33.6 & 22.8 & 33.8 & 23.4 & 33.2 \\
\hline Injury in last 3 months & 7.9 & 2.0 & 7.0 & 1.6 & 7.5 & 2.7 \\
\hline Symptoms of genital infection in last 3 months $^{1}$ & 1.2 & 11.6 & 0.9 & 12.0 & 1.2 & 11.0 \\
\hline $\begin{array}{l}\text { Anxiety about swapnadosh/nocturnal emission in last } \\
12 \text { months }\end{array}$ & 17.1 & NA & 3.7 & NA & 20.9 & NA \\
\hline Menstrual problems in last 3 months & NA & 11.6 & NA & 10.1 & NA & 13.9 \\
\hline Number of respondents & 1,190 & 2,722 & 757 & 1,293 & 945 & 1,429 \\
\hline
\end{tabular}

Note: All Ns are unweighted. NA: Not applicable. ${ }^{1}$ Includes genital ulcers, genital itching, swelling in the groin, discharge, burning during urination, etc. 
suggest that $18 \%$ of young men had indeed experienced anxiety about swapnadosh or nocturnal emission in the 12 months preceding the interview. Vast differences were, however, observed by marital status: $4 \%$ of married men compared to $21 \%$ of unmarried young men reported anxiety about nocturnal emission. Differences by rural-urban residence were muted.

With regard to young women's experience of other reproductive health problems in the three months preceding the interview, findings suggest that $12 \%$ of young women experienced menstrual problems. A somewhat larger percentage of unmarried than married young women experienced menstrual problem (14\% versus 10\%); rural-urban differences were, however, not evident.

\subsection{Mental health disorders}

The mental health status of young people was assessed based on their responses to the General Health Questionnaire (GHQ-12) (Goldberg, 1992; Patel and Andrew, 2001). This questionnaire, designed to identify the presence of possible mental health disorders, is based on 12 questions that assess the extent to which a respondent experienced, for example, happiness, depression, anxiety and sleep disturbance in the one month preceding the interview. Threshold scores of 2, 3, 4 or more have been variously used to identify the possible presence of common mental health disorders (Bashir et al., 1996; Donath, 2001; Jacob, Bhugra and Mann, 1997). Table 11.3 presents responses on each item of the GHQ-12, and a summary measure indicating the percentage who gave three or more responses suggestive of mental health disorders.

Relatively small proportions of young men and women reported responses suggestive of mental disorders on any of the 12 items and gender disparities in response patterns were narrow. Indeed, for the most part, fewer than $10 \%$ of young men and women reported responses likely to suggest mental health disorders. On just one item did more than $10 \%$ of young men and women report so: $12 \%$ of young men reported feeling that they were constantly under strain and $12 \%$ of young women reported feeling that they were incapable of making decisions. Differences by marital status and rural-urban residence were narrow although more married young men in rural than urban areas reported losing sleep over worry $(12 \%$ versus $5 \%)$ and not feeling reasonably happy, all things considered (11\% versus $5 \%)$.

Overall, $11 \%$ of young men and $10 \%$ young women reported three or more of the 12 symptoms/behaviours probed in the GHQ-12, indicative of mental disorders. Marital status differences and rural-urban differences were mild (see Figure 11.1). Even so, somewhat more married young men in rural than urban areas displayed scores indicative of mental health disorders (14\% versus $7 \%)$.

\subsection{Care and advice seeking}

Young people who reported physical or sexual and reproductive health problems were probed about whether they had sought care or advice for the problem and the source of this care or advice. Findings are presented in Table 11.4 and suggest that care and advice seeking differed by the kind of problem experienced as well as, in several instances, sex and marital status of the respondent.

\subsubsection{General health problems}

According to findings presented in Table 11.4, almost all (93-96\%) of young people experiencing high fever had sought treatment. Differences by marital status were negligible. Treatment was sought from a government health care facility or provider by a relatively small percentage of those who sought care: $24 \%$ of young men and $21 \%$ of young women. The majority of respondents reportedly sought care from private sector providers (60\% of young men and $64 \%$ of young women), reflecting the pattern in health care seeking behaviour in India more generally. We note, however, that respondents may not always have been able to discern whether the private sector provider 
Table 11.3: Reported symptoms or behaviours suggestive of mental health disorders

Percentage of youth reporting symptoms or behaviours suggestive of mental health disorders experienced in the month preceding the interview, according to residence, Andhra Pradesh, 2007-08

\begin{tabular}{|c|c|c|c|c|c|c|}
\hline Symptoms/behaviours (\%) & $\begin{array}{c}\mathrm{M} \\
15-24\end{array}$ & $\begin{array}{c}\text { W } \\
15-24\end{array}$ & $\begin{array}{c}\text { MM } \\
15-29\end{array}$ & $\begin{array}{c}\text { MW } \\
15-24\end{array}$ & $\begin{array}{c}\text { UM } \\
15-24\end{array}$ & $\begin{array}{c}\text { UW } \\
15-24\end{array}$ \\
\hline \multicolumn{7}{|c|}{ Combined } \\
\hline Unable to concentrate on whatever he/she was doing & 2.7 & 3.5 & 2.4 & 4.1 & 2.8 & 2.8 \\
\hline Lost much sleep over worry & 9.2 & 6.9 & 10.5 & 7.8 & 8.5 & 5.7 \\
\hline Felt that he/she was not playing a useful role & 3.1 & 3.6 & 2.8 & 4.1 & 3.2 & 2.9 \\
\hline Felt incapable of making decisions & 6.5 & 11.6 & 6.5 & 12.7 & 5.8 & 10.2 \\
\hline Felt constantly under strain & 11.6 & 7.1 & 12.4 & 8.2 & 11.3 & 5.7 \\
\hline Felt that he/she could not overcome his/her difficulties & 4.7 & 7.8 & 4.5 & 8.6 & 4.7 & 6.6 \\
\hline Unable to enjoy normal day-to-day activities & 6.2 & 5.0 & 6.7 & 5.9 & 5.6 & 3.9 \\
\hline Unable to face up to his/her problems & 7.2 & 7.8 & 5.6 & 8.4 & 7.5 & 6.9 \\
\hline Been feeling unhappy and depressed & 7.8 & 7.6 & 7.4 & 8.5 & 7.6 & 6.4 \\
\hline Been losing confidence in himself/herself & 6.0 & 4.4 & 5.8 & 5.1 & 5.8 & 3.5 \\
\hline Been thinking of himself/herself as a worthless person & 5.0 & 3.6 & 4.2 & 4.2 & 4.9 & 2.8 \\
\hline Not feeling reasonably happy, all things considered & 7.6 & 7.5 & 9.6 & 8.2 & 7.0 & 6.7 \\
\hline Three or more symptoms/behaviours & 10.6 & 10.1 & 12.5 & 11.1 & 9.7 & 8.9 \\
\hline Number of respondents & 2,479 & 4,848 & 1,405 & 2,330 & 2,077 & 2,518 \\
\hline \multicolumn{7}{|c|}{ Urban } \\
\hline Unable to concentrate on whatever he/she was doing & 2.7 & 2.3 & 1.3 & 2.1 & 2.9 & 2.5 \\
\hline Lost much sleep over worry & 7.1 & 5.6 & 5.2 & 5.3 & 7.4 & 5.8 \\
\hline Felt that he/she was not playing a useful role & 2.8 & 2.1 & 2.3 & 2.3 & 2.9 & 2.0 \\
\hline Felt incapable of making decisions & 5.2 & 9.8 & 3.6 & 10.3 & 4.9 & 9.4 \\
\hline Felt constantly under strain & 11.5 & 5.8 & 9.2 & 6.5 & 11.6 & 5.2 \\
\hline Felt that he/she could not overcome his/her difficulties & 3.7 & 6.5 & 1.6 & 7.4 & 4.1 & 5.7 \\
\hline Unable to enjoy normal day-to-day activities & 5.8 & 2.8 & 5.2 & 2.5 & 5.1 & 3.2 \\
\hline Unable to face up to his/her problems & 5.8 & 7.3 & 3.9 & 8.0 & 6.0 & 6.6 \\
\hline Been feeling unhappy and depressed & 5.4 & 5.8 & 4.6 & 5.3 & 5.2 & 6.2 \\
\hline Been losing confidence in himself/herself & 3.5 & 3.0 & 2.6 & 3.4 & 3.2 & 2.8 \\
\hline Been thinking of himself/herself as a worthless person & 3.0 & 2.9 & 1.3 & 3.0 & 3.2 & 2.9 \\
\hline Not feeling reasonably happy, all things considered & 6.4 & 5.8 & 5.2 & 5.3 & 6.3 & 6.2 \\
\hline Three or more symptoms/behaviours & 8.1 & 8.3 & 7.2 & 8.4 & 7.6 & 8.3 \\
\hline Number of respondents & 1,289 & 2,126 & 648 & 1,037 & 1,132 & 1,089 \\
\hline \multicolumn{7}{|c|}{ Rural } \\
\hline Unable to concentrate on whatever he/she was doing & 2.7 & 4.0 & 2.8 & 4.7 & 2.8 & 3.0 \\
\hline Lost much sleep over worry & 10.1 & 7.4 & 12.1 & 8.5 & 8.9 & 5.6 \\
\hline Felt that he/she was not playing a useful role & 3.2 & 4.2 & 2.8 & 4.7 & 3.4 & 3.5 \\
\hline Felt incapable of making decisions & 7.0 & 12.4 & 7.5 & 13.4 & 6.2 & 10.8 \\
\hline Felt constantly under strain & 11.7 & 7.6 & 13.3 & 8.6 & 11.1 & 5.9 \\
\hline Felt that he/she could not overcome his/her difficulties & 5.1 & 8.3 & 5.2 & 9.0 & 5.0 & 7.1 \\
\hline Unable to enjoy normal day-to-day activities & 6.3 & 5.9 & 7.1 & 6.9 & 5.8 & 4.2 \\
\hline Unable to face up to his/her problems & 7.8 & 8.0 & 6.1 & 8.5 & 8.2 & 7.1 \\
\hline Been feeling unhappy and depressed & 8.8 & 8.3 & 8.2 & 9.4 & 8.6 & 6.5 \\
\hline Been losing confidence in himself/herself & 7.0 & 5.0 & 6.6 & 5.7 & 6.9 & 3.9 \\
\hline Been thinking of himself/herself as a worthless person & 5.7 & 3.9 & 5.0 & 4.7 & 5.7 & 2.8 \\
\hline Not feeling reasonably happy, all things considered & 8.0 & 8.2 & 10.8 & 9.0 & 7.3 & 7.0 \\
\hline Three or more symptoms/behaviours & 11.6 & 10.9 & 13.9 & 11.9 & 10.6 & 9.3 \\
\hline Number of respondents & 1,190 & 2,722 & 757 & 1,293 & 945 & 1,429 \\
\hline
\end{tabular}

Note: All Ns are unweighted. 
Figure 11.1: Percentage of youth reporting symptoms/behaviours suggestive of mental health disorders in the month preceding the interview, according to residence, Andhra Pradesh, 2007-08
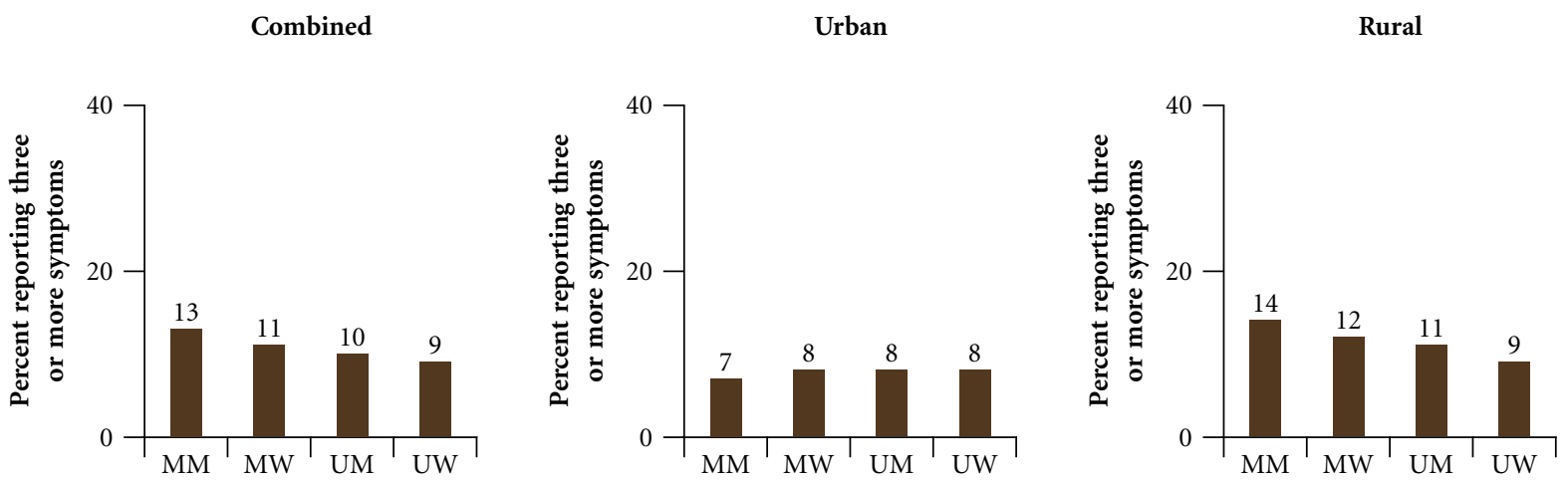

from whom they had sought care had been trained and was licensed to provide such care. Some $15 \%$ had sought treatment from traditional health care providers or relied on home remedies. For the most part, both married and unmarried youth sought treatment from similar sources; the only exception was that more unmarried than married young men had relied on traditional health care providers or home remedies (16\% versus $11 \%)$.

Fewer youth had sought care for their reported injuries (86\% of young men and $70 \%$ of young women). Marital status differences were negligible. Among youth who had sought treatment, gender differences were evident with regard to place of treatment, nevertheless, private sector providers were most often sought by both young men and young women. Young men were, however, more likely than young women to have opted for treatment in the private sector $(56 \%$ versus $42 \%)$ and less likely to have done so in a government facility ( $26 \%$ versus $38 \%$ ). The sources from which young people sought care differed somewhat between the married and the unmarried. Among young men, more unmarried than married young men had sought care from traditional health care providers or relied on home remedies (23\% versus $13 \%$ ). Among young women, the married were more likely than the unmarried to rely on government health care facility or provider ( $42 \%$ versus $34 \%$ ), and conversely, less likely to rely on private sector providers $(31 \%$ versus $50 \%)$.

\subsubsection{Sexual and reproductive health problems}

Responses regarding treatment seeking for sexual and reproductive health problems depict a somewhat different picture than that for general health ailments. In general, fewer young people had sought care for these problems than for general health problems.

Indeed, just $30 \%$ of young men who experienced symptoms of genital infection had sought treatment for their symptom. Young men who experienced anxiety about swapnadosh or nocturnal emission were asked, moreover, if they had sought advice for this anxiety. Three-fifths of all young men had done so. The most common source was friends, from whom $94 \%$ of young men reported seeking advice. In contrast, just $2 \%$ sought advice from a medical professional and hardly any reported that they had sought advice from a traditional health care provider generally known to "treat" such symptoms $(1 \%)$. While more unmarried than married young men had sought advice for their anxiety, friends were the leading source of advice for both (94-97\%).

Seeking treatment for sexual and reproductive health problems was also limited among young women. For example, $57 \%$ of women experiencing menstrual problems had sought care for this problem as did half of those experiencing symptoms of genital infection. The married were considerably more likely than the unmarried to have sought treatment for menstrual problems (65\% versus $48 \%$ ) and symptoms of genital infection (61\% versus $34 \%$ ) a finding that can be attributed to the likelihood that sexual and reproductive conditions evoke greater embarrassment among the unmarried and their families than their married counterparts. 
Table 11.4: Care and advice seeking for reported health problems

Percentage of youth who experienced selected health problems by reported care and advice seeking and place of treatment, Andhra Pradesh, 2007-08

\begin{tabular}{|c|c|c|c|c|c|c|}
\hline Care and advice seeking (\%) & $\begin{array}{c}\mathrm{M} \\
15-24\end{array}$ & $\begin{array}{c}\text { W } \\
15-24\end{array}$ & $\begin{array}{c}\text { MM } \\
15-29\end{array}$ & $\begin{array}{c}\text { MW } \\
15-24\end{array}$ & $\begin{array}{c}\text { UM } \\
15-24\end{array}$ & $\begin{array}{c}\text { UW } \\
\text { 15-24 }\end{array}$ \\
\hline Sought treatment for high fever & 96.4 & 93.3 & 96.3 & 93.0 & 96.2 & 93.7 \\
\hline Number reporting high fever & 511 & 1,593 & 275 & 790 & 416 & 803 \\
\hline $\begin{array}{l}\text { Place treatment sought for high fever } \\
\text { Government facility/doctor } \\
\text { Private facility/doctor } \\
\text { Other }^{2}\end{array}$ & $\begin{array}{l}24.1 \\
59.7 \\
15.4\end{array}$ & $\begin{array}{l}20.7 \\
64.4 \\
14.6\end{array}$ & $\begin{array}{l}26.9 \\
61.7 \\
11.4\end{array}$ & $\begin{array}{l}21.2 \\
63.9 \\
14.3\end{array}$ & $\begin{array}{l}23.5 \\
59.0 \\
16.4\end{array}$ & $\begin{array}{l}20.3 \\
64.6 \\
15.1\end{array}$ \\
\hline Number who sought treatment for high fever & 492 & 1,485 & 266 & 735 & 400 & 750 \\
\hline Sought treatment for injury & 86.3 & 70.4 & 86.8 & $(68.6)$ & 84.8 & 71.0 \\
\hline Number reporting injury & 165 & 102 & 79 & 33 & 136 & 69 \\
\hline $\begin{array}{l}\text { Place treatment sought for injury } \\
\text { Government facility/doctor } \\
\text { Private facility/doctor } \\
\text { Other }^{2}\end{array}$ & $\begin{array}{l}25.5 \\
56.1 \\
18.5\end{array}$ & $\begin{array}{l}37.5 \\
41.7 \\
16.7\end{array}$ & $\begin{array}{l}27.5 \\
57.5 \\
12.5\end{array}$ & $\begin{array}{l}(42.3) \\
(30.8) \\
(19.2)\end{array}$ & $\begin{array}{l}25.2 \\
52.0 \\
22.8\end{array}$ & $\begin{array}{l}34.0 \\
50.0 \\
14.0\end{array}$ \\
\hline Number who sought treatment for injury & 143 & 75 & 71 & 25 & 116 & 50 \\
\hline Sought treatment for symptoms of genital infection ${ }^{3}$ & $(29.6)$ & 50.1 & * & 61.0 & * & 33.6 \\
\hline Number reporting symptoms of genital infection & 26 & 510 & 12 & 259 & 20 & 251 \\
\hline $\begin{array}{l}\text { Place treatment sought for symptoms of genital infection }{ }^{1,3,4} \\
\text { Government facility/doctor } \\
\text { Private facility/doctor } \\
\text { Other }{ }^{2}\end{array}$ & $\begin{array}{l}* \\
* \\
*\end{array}$ & $\begin{array}{r}17.0 \\
78.6 \\
5.3\end{array}$ & $\begin{array}{l}* \\
* \\
*\end{array}$ & $\begin{array}{r}15.9 \\
82.3 \\
3.0\end{array}$ & $\begin{array}{l}* \\
* \\
*\end{array}$ & $\begin{array}{l}19.8 \\
68.6 \\
11.6\end{array}$ \\
\hline $\begin{array}{l}\text { Number who sought treatment for symptoms of } \\
\text { genital infection }\end{array}$ & 9 & 243 & 5 & 158 & 7 & 85 \\
\hline Sought advice on swapnadosh/nocturnal emission & 59.7 & NA & 50.8 & NA & 59.4 & NA \\
\hline $\begin{array}{l}\text { Number reporting anxiety over swapnadosh/nocturnal emission } \\
\text { Person from whom advice was sought on swapnadosh/ } \\
\text { nocturnal emission }\end{array}$ & 472 & NA & 68 & NA & 449 & NA \\
\hline $\begin{array}{l}\text { Friend } \\
\text { Parent } \\
\text { Relative } \\
\text { Traditional healer } \\
\text { Medical professional }\end{array}$ & $\begin{array}{r}93.7 \\
1.1 \\
2.2 \\
1.1 \\
1.5\end{array}$ & $\begin{array}{l}\text { NA } \\
\text { NA } \\
\text { NA } \\
\text { NA } \\
\text { NA }\end{array}$ & $\begin{array}{r}(96.7) \\
(0.0) \\
(0.0) \\
(3.3) \\
(0.0)\end{array}$ & $\begin{array}{l}\text { NA } \\
\text { NA } \\
\text { NA } \\
\text { NA } \\
\text { NA }\end{array}$ & $\begin{array}{r}94.3 \\
1.1 \\
2.3 \\
0.8 \\
1.5\end{array}$ & $\begin{array}{l}\text { NA } \\
\text { NA } \\
\text { NA } \\
\text { NA } \\
\text { NA }\end{array}$ \\
\hline Number who sought advice for swapnadosh/nocturnal emission & 281 & NA & 36 & NA & 267 & NA \\
\hline Sought treatment for menstrual problems & NA & 56.8 & NA & 65.4 & NA & 48.4 \\
\hline Number reporting menstrual problems & NA & 585 & NA & 231 & NA & 354 \\
\hline $\begin{array}{l}\text { Place treatment sought for menstrual problems }{ }^{1} \\
\text { Government facility/doctor } \\
\text { Private facility/doctor } \\
\text { Other }{ }^{2}\end{array}$ & $\begin{array}{l}\text { NA } \\
\text { NA } \\
\text { NA }\end{array}$ & $\begin{array}{r}21.5 \\
71.4 \\
6.5\end{array}$ & $\begin{array}{l}\text { NA } \\
\text { NA } \\
\text { NA }\end{array}$ & $\begin{array}{r}18.7 \\
71.6 \\
8.4\end{array}$ & $\begin{array}{l}\text { NA } \\
\text { NA } \\
\text { NA }\end{array}$ & $\begin{array}{r}25.3 \\
70.6 \\
4.1\end{array}$ \\
\hline Number who sought treatment for menstrual problems & NA & 321 & NA & 145 & NA & 176 \\
\hline
\end{tabular}

Note: All Ns are unweighted. Column totals may not equal 100\% due to missing cases or "don't know" responses. ( ) Based on 25-49 unweighted cases. ${ }^{*}$ Percentage not shown, based on fewer than 25 unweighted cases. NA: Not applicable. ${ }^{1}$ Refers to the last time the respondent sought treatment. ${ }^{2}$ Includes registered medical practitioner, unregistered medical practitioner, vaid/traditional healer and home remedies. ${ }^{3}$ Includes genital ulcers, genital itching, swelling in the groin, genital discharge, burning during urination, etc. ${ }^{4}$ Multiple responses were given. 
Among young women, as in the case of general health problems, care was most likely to be sought from a private sector provider: $79 \%$ of young women who had sought care for symptoms of genital infection and $71 \%$ of young women who had sought care for menstrual problems had approached a private sector provider. Just 5-7\% had relied on traditional health care providers or home remedies for these problems. Marital status differences were evident. More married than unmarried young women had sought care from the private sector for symptoms of genital infection (82\% versus 69\%) and fewer married than unmarried young women had sought care from a traditional health care provider (3\% versus $12 \%$ ). In the case of treatment seeking for menstrual problems, fewer married than unmarried young women had sought treatment from a government facility (19\% versus $25 \%$ ).

\subsection{Hesitation to access contraceptive supplies}

In order to capture the extent to which young people perceived that they could approach health care professionals for sexual and reproductive health services, the Youth Study posed two questions relating to accessing contraceptives, namely, whether the respondent would feel shy to approach a health care provider and a pharmacist, respectively, for contraceptives. Findings are presented in Table 11.5 and confirm that large proportions of young people would indeed feel shy to approach a health care provider or pharmacy/medical shop for contraceptive supplies. Young women were somewhat more likely than young men to report discomfort in approaching a health care provider (44\% versus 38\%). With regard to approaching a pharmacy/medical shop for contraceptive supplies, similarly, more young women than men reported discomfort (58\% versus $35 \%$ ) and this pattern was evident among both the married and the unmarried.

Marital status differences suggest that married youth were less likely than the unmarried to report discomfort; for example, among young men, $26 \%$ of the married compared to $40 \%$ of the unmarried reported feeling shy to approach a health care provider for contraceptive supplies and $23 \%$ and $36 \%$, respectively, reported discomfort in

\section{Table 11.5: Hesitation to access contraceptive supplies}

\section{Percentage of youth reporting hesitation to access contraceptive supplies from a health care provider or medical shop, according to residence, Andhra Pradesh, 2007-08}

\begin{tabular}{|c|c|c|c|c|c|c|}
\hline Indicators (\%) & $\begin{array}{c}\text { M } \\
15-24\end{array}$ & $\begin{array}{c}\text { W } \\
15-24\end{array}$ & $\begin{array}{c}\text { MM } \\
15-29\end{array}$ & $\begin{array}{c}\text { MW } \\
15-24\end{array}$ & $\begin{array}{c}\text { UM } \\
15-24\end{array}$ & $\begin{array}{c}\text { UW } \\
15-24\end{array}$ \\
\hline \multicolumn{7}{|c|}{ Combined } \\
\hline $\begin{array}{l}\text { Would feel shy to approach an HCP for contraceptives } \\
\text { Would feel shy to approach a pharmacy/medical shop for } \\
\text { contraceptives }\end{array}$ & 38.4 & 44.2 & 25.8 & 40.3 & 39.5 & $\begin{array}{l}49.5 \\
59.3\end{array}$ \\
\hline Number of respondents & 2,479 & 4,848 & 1,405 & 2,330 & 2,077 & 2,518 \\
\hline \multicolumn{7}{|c|}{ Urban } \\
\hline $\begin{array}{l}\text { Would feel shy to approach an HCP for contraceptives } \\
\text { Would feel shy to approach a pharmacy/medical shop for } \\
\text { contraceptives }\end{array}$ & 38.1 & 57.2 & 18.3 & 55.0 & 31.7 & 55.7 \\
\hline Number of respondents & 1,289 & 2,126 & 648 & 1,037 & 1,132 & 1,089 \\
\hline \multicolumn{7}{|c|}{ Rural } \\
\hline $\begin{array}{l}\text { Would feel shy to approach an HCP for contraceptives } \\
\text { Would feel shy to approach a pharmacy/medical shop for } \\
\text { contraceptives }\end{array}$ & 33.2 & 45.1 & 26.7 & 41.1 & 38.5 & $\begin{array}{l}51.6 \\
59.4\end{array}$ \\
\hline Number of respondents & 1,190 & 2,722 & 757 & 1,293 & 945 & 1,429 \\
\hline
\end{tabular}

Note: All Ns are unweighted. HCP: Health care provider. 
approaching a pharmacy/medical shop for contraceptive supplies. Among young women, differences were evident with regard to approaching a health care provider (40\% of the married compared with $50 \%$ of the unmarried, reported discomfort), but marital status differences were not evident with regard to reports of discomfort in approaching a pharmacy/medical shop for contraceptive supplies (reported by $57-59 \%$ of married and unmarried young women). Rural-urban differences were mild. Findings confirm that many youth would indeed find it difficult to seek appropriate services for sexual and reproductive matters.

\subsection{Attitudes towards pre-marital HIV testing and extent of HIV testing}

Youth who were aware of HIV/AIDS were asked whether they approved of pre-marital HIV testing for boys and girls, and whether they had ever undergone an HIV test. Findings, presented in Table 11.6, suggest that 78-84\% of youth agreed that boys and girls should be tested for HIV before marriage. While differences were narrow, larger percentages of unmarried than married youth expressed favourable attitudes towards HIV testing. While rural-urban differences were not observed among young men, young women in urban areas were somewhat more likely than their rural counterparts to favour pre-marital HIV testing. Despite positive attitudes towards HIV testing, just $5 \%$ of young men and $24 \%$ of young women had undergone HIV testing. Married youth were more likely than unmarried youth to report having undergone an HIV test; indeed, of all the categories of youth, it was married young women who were most likely to have an HIV test (42\%), most likely as part of antenatal check-ups. Rural-urban differences were mild.

Table 11.6: Attitudes towards pre-marital HIV testing and extent of HIV testing

Percent distribution of youth aware of HIV/AIDS who believe that boys/girls should be tested for HIV before marriage and percentage who have ever had an HIV test, according to residence, Andhra Pradesh, 2007-08

\begin{tabular}{|c|c|c|c|c|c|c|}
\hline Attitudes/experiences (\%) & $\underset{15-24}{\mathrm{M}}$ & $\begin{array}{c}\text { W } \\
15-24\end{array}$ & $\begin{array}{c}\text { MM } \\
15-29\end{array}$ & $\begin{array}{c}\text { MW } \\
15-24\end{array}$ & $\begin{array}{c}\text { UM } \\
15-24\end{array}$ & $\begin{array}{c}\text { UW } \\
15-24\end{array}$ \\
\hline \multicolumn{7}{|c|}{ Combined } \\
\hline \multicolumn{7}{|c|}{ Boys should be tested for HIV before marriage } \\
\hline Yes & 84.3 & 81.4 & 79.8 & 77.7 & 86.0 & 86.1 \\
\hline No & 14.2 & 15.4 & 18.0 & 18.5 & 12.6 & 11.5 \\
\hline \multicolumn{7}{|c|}{ Girls should be tested for HIV before marriage } \\
\hline Yes & 82.8 & 77.6 & 77.9 & 73.6 & 84.6 & 82.7 \\
\hline No & 15.7 & 19.2 & 19.6 & 22.6 & 13.9 & 15.0 \\
\hline Youth who underwent an HIV test & 5.2 & 24.1 & 10.4 & 41.6 & 4.9 & 1.8 \\
\hline Number aware of HIV/AIDS & 2,403 & 4,639 & 1,340 & 2,203 & 2,020 & 2,436 \\
\hline \multicolumn{7}{|c|}{ Urban } \\
\hline \multicolumn{7}{|c|}{ Boys should be tested for HIV before marriage } \\
\hline Yes & 84.6 & 85.6 & 83.1 & 83.8 & 86.2 & 87.1 \\
\hline No & 14.2 & 13.1 & 15.9 & 14.8 & 12.6 & 11.8 \\
\hline \multicolumn{7}{|c|}{ Girls should be tested for HIV before marriage } \\
\hline Yes & 83.2 & 81.7 & 80.4 & 79.7 & 85.1 & 83.4 \\
\hline No & 15.7 & 17.0 & 18.6 & 18.7 & 13.8 & 15.5 \\
\hline Youth who underwent an HIV test & 6.8 & 21.0 & 12.8 & 44.4 & 6.9 & 2.0 \\
\hline Number aware of HIV/AIDS & 1,259 & 2,082 & 623 & 1,015 & 1,110 & 1,067 \\
\hline
\end{tabular}


Table 11.6: $\left(\right.$ Cont'd $\left.^{\mathrm{t}}\right)$

\begin{tabular}{|c|c|c|c|c|c|c|}
\hline Attitudes/experiences (\%) & $\begin{array}{c}\mathrm{M} \\
15-24\end{array}$ & $\begin{array}{c}\text { W } \\
15-24\end{array}$ & $\begin{array}{c}\text { MM } \\
15-29\end{array}$ & $\begin{array}{c}\text { MW } \\
15-24\end{array}$ & $\begin{array}{c}\text { UM } \\
15-24\end{array}$ & $\begin{array}{c}\text { UW } \\
15-24\end{array}$ \\
\hline \multicolumn{7}{|c|}{ Rural } \\
\hline \multicolumn{7}{|c|}{ Boys should be tested for HIV before marriage } \\
\hline Yes & 84.2 & 79.6 & 78.9 & 75.8 & 85.9 & 85.6 \\
\hline No & 14.1 & 16.4 & 18.6 & 19.6 & 12.6 & 11.3 \\
\hline \multicolumn{7}{|c|}{ Girls should be tested for HIV before marriage } \\
\hline Yes & 82.6 & 75.9 & 77.1 & 71.7 & 84.5 & 82.3 \\
\hline No & 15.6 & 20.2 & 19.9 & 23.8 & 14.0 & 14.7 \\
\hline Youth who underwent an HIV test & 4.6 & 25.5 & 9.7 & 40.7 & 4.0 & 1.6 \\
\hline Number aware of HIV/AIDS & 1,144 & 2,557 & 717 & 1,188 & 910 & 1,369 \\
\hline
\end{tabular}

Note: All Ns are unweighted. Column totals may not equal 100\% due to missing cases or "don't know" responses.

\subsection{Summary}

Findings show that substantial proportions of young men reported the consumption of tobacco and alcohol; one-fifth of young men reported tobacco consumption and over one-fourth reported alcohol consumption. In contrast, consumption of tobacco products and alcohol was reported by very few young women (less than 2\%). Drug use was reported by very few young men and not a single young woman.

Although youth is a generally healthy period of life, significant minorities reported experiencing general, mental, and sexual and reproductive health problems in the period preceding the interview. For example, $22 \%$ of young men and $33 \%$ of young women had experienced high fever, $7 \%$ and $2 \%$, respectively, had experienced an injury, and $1 \%$ and $11 \%$, respectively, reported experience of symptoms of genital infection. Moreover, $12 \%$ of young women reported menstrual problems; at the same time, $18 \%$ of young men reported anxiety about nocturnal emission. Finally, responses indicative of mental health disorders were reported by $10-11 \%$ of young men and women.

With regard to care seeking for general and sexual and reproductive health problems, patterns varied by type of problem experienced. While the large majority of those who had experienced high fever, for example, had sought care, many fewer had sought care for sexual and reproductive health problems. Of those who had sought treatment, the majority had sought advice or treatment from a private facility or provider, irrespective of the type of problem experienced. It is notable, however, that in the case of anxiety about nocturnal emission, young men rarely sought advice from a health care provider, preferring to do so from peers.

Findings suggest that youth were shy about seeking sexual and reproductive health services. For example, many youth, including the married, reported that they would indeed hesitate to approach a health care provider or a pharmacy/medical shop for contraceptive supplies.

Finally, although youth were overwhelmingly in favour of pre-marital HIV testing, relatively few had undergone HIV testing. Just $5 \%$ of young men and almost one-quarter of young women had undergone HIV testing. Married young women were far more likely than any other group to have done so; over two-fifths of married young women had undergone HIV testing, most likely as part of antenatal check-ups. 


\section{Participation in civil society} and political life

The National Youth Policy 2003 has underscored the role of India's youth in political decision-making and argued for greater representation of youth in appropriate bodies and more extensive youth participation in the design and implementation of programmes (Ministry of Youth Affairs and Sports, 2003). Indeed, there is a recognition that today's youth, who have better access to skills and information than those of earlier generations, can play an important role in influencing political processes and the socio-economic development of the country.

This chapter presents a profile of youth involvement in government- and NGO-sponsored programmes, community activities and political processes. It also explores young people's behaviours and attitudes towards individuals of different religions and caste groups, violence within their community and their own participation in such violence, and their perceptions about the most important problem facing youth in India.

\subsection{Awareness of and participation in government- and NGO-sponsored programmes}

Youth were asked whether they were aware of programmes in which youth could participate that had taken place in their village or urban neighbourhood in the three years preceding the interview. They were also asked whether they had participated in these programmes and whether these programmes had been organised by government agencies or NGOs. Findings are presented in Table 12.1.

Programme awareness was considerably more likely to be reported by young women than men: two-fifths of young men and two-thirds of young women reported awareness of one or more programmes that addressed youth needs organised in the three years prior to the interview (see also Figure 12.1). Differences by marital status were negligible, except that among young men, the unmarried were somewhat more likely than the married to be aware of one or more programmes ( $40 \%$ versus $35 \%$ ). However, rural youth were considerably more likely than urban youth to be aware of one or more programmes ( $45 \%$ versus $25 \%$ of young men; $71 \%$ versus $48 \%$ of young women). It is evident, therefore, that community-level programmes were more likely to be directed toward young women than young men and more likely to be conducted in rural than urban settings.

Findings suggest a gender divide also in the kinds of programmes about which youth were aware. The leading programmes about which young men were aware related to health promotion (19\%), leadership and life skills $(14 \%)$ and employment $(10 \%)$. In contrast, the largest proportions of young women were aware of self-help groups (43\%), health promotion (19\%) and leadership and life skills (14\%) programmes. Marital status differences were negligible among young men; however, among young women, the unmarried were more likely than the married to be aware of leadership and life skills programmes (17\% versus 12\%), and less likely to be aware of self-help groups ( $38 \%$ versus $48 \%$ ); unmarried young women in rural areas were also more likely than their married counterparts to report awareness of health promotion and literacy programmes (23\% versus $18 \%$ and $16 \%$ versus $9 \%$, respectively). Rural-urban differences were also apparent, with rural youth more aware of almost every type of programme than urban youth and these differences were more pronounced among young men than young women. 
Table 12.1: Awareness of and participation in government-and NGO-sponsored programmes

Percentage of youth reporting awareness of and participation in government- and NGO-sponsored programmes conducted in the village/neighbourhood in the three years preceding the interview, according to residence, Andhra Pradesh, 2007-08

\begin{tabular}{|c|c|c|c|c|c|c|}
\hline $\begin{array}{l}\text { Awareness of and participation in } \\
\text { programmes (\%) }\end{array}$ & $\begin{array}{c}M \\
15-24\end{array}$ & $\begin{array}{c}\text { W } \\
15-24\end{array}$ & $\begin{array}{c}\text { MM } \\
15-29\end{array}$ & $\begin{array}{l}\text { MW } \\
15-24\end{array}$ & $\begin{array}{l}\text { UM } \\
15-24\end{array}$ & $\begin{array}{l}\text { UW } \\
15-24\end{array}$ \\
\hline \multicolumn{7}{|c|}{ Combined } \\
\hline Aware of programme(s) held & 39.2 & 64.5 & 35.3 & 65.6 & 39.5 & 63.7 \\
\hline \multicolumn{7}{|l|}{ Focus of programmes held } \\
\hline Health promotion & 19.1 & 19.2 & 18.1 & 17.9 & 19.4 & 21.0 \\
\hline Awareness/leadership/vocational/life skills & 14.4 & 14.2 & 13.4 & 12.3 & 14.9 & 16.8 \\
\hline Employment $^{1}$ & 9.9 & 7.6 & 9.5 & 7.4 & 10.0 & 7.9 \\
\hline Self-help group & 3.0 & 43.2 & 3.7 & 47.9 & 2.9 & 37.5 \\
\hline Literacy & 8.0 & 9.9 & 7.1 & 8.3 & 8.5 & 12.3 \\
\hline Sports and recreation & 3.2 & 0.2 & 1.9 & 0.0 & 3.5 & 0.4 \\
\hline Number of respondents & 2,479 & 4,848 & 1,405 & 2,330 & 2,077 & 2,518 \\
\hline \multicolumn{7}{|l|}{ Organising agency } \\
\hline Government & 85.2 & 94.7 & 88.9 & 95.7 & 85.5 & 93.4 \\
\hline NGO & 15.3 & 7.2 & 11.1 & 6.0 & 16.0 & 8.7 \\
\hline Don’t know & 1.0 & 2.4 & 1.0 & 2.1 & 0.6 & 2.7 \\
\hline Number aware of any programme(s) & 852 & 2,990 & 432 & 1,424 & 715 & 1,566 \\
\hline Participated in programme(s) held & 19.4 & 21.8 & 18.3 & 30.7 & 19.7 & 10.2 \\
\hline Number of respondents & 2,479 & 4,848 & 1,405 & 2,330 & 2,077 & 2,518 \\
\hline \multicolumn{7}{|l|}{ Participation in specific programmes } \\
\hline Health promotion & 37.7 & 16.7 & 40.9 & 15.3 & 38.3 & 21.8 \\
\hline Awareness/leadership/vocational/life skills & 34.6 & 9.4 & 31.9 & 5.6 & 35.2 & 24.5 \\
\hline Employment $^{1}$ & 16.7 & 6.3 & 24.9 & 5.2 & 15.6 & 11.3 \\
\hline Self-help group & 1.2 & 69.4 & 3.9 & 79.3 & 1.2 & 30.0 \\
\hline Literacy & 14.0 & 6.3 & 15.6 & 5.3 & 13.9 & 10.5 \\
\hline Sports and recreation & 12.6 & 0.3 & 7.4 & 0.1 & 13.4 & 1.2 \\
\hline Number who participated in any programme(s) & 424 & 903 & 221 & 653 & 358 & 250 \\
\hline \multicolumn{7}{|c|}{ Urban } \\
\hline Aware of programme(s) held & 25.0 & 48.0 & 20.3 & 50.1 & 25.4 & 46.4 \\
\hline \multicolumn{7}{|l|}{ Focus of programmes held } \\
\hline Health promotion & 14.6 & 17.6 & 12.4 & 17.1 & 14.9 & 17.9 \\
\hline Awareness/leadership/vocational/life skills & 9.6 & 11.7 & 7.2 & 11.0 & 10.1 & 12.2 \\
\hline Employment $^{1}$ & 2.6 & 5.0 & 2.9 & 4.2 & 2.7 & 5.6 \\
\hline Self-help group & 2.0 & 28.1 & 1.6 & 32.8 & 2.1 & 24.2 \\
\hline Literacy & 3.3 & 5.9 & 2.3 & 5.0 & 3.6 & 6.6 \\
\hline Sports and recreation & 3.8 & 0.3 & 2.0 & 0.2 & 4.0 & 0.3 \\
\hline Number of respondents & 1,289 & 2,126 & 648 & 1,037 & 1,132 & 1,089 \\
\hline \multicolumn{7}{|l|}{ Organising agency } \\
\hline Government & 76.1 & 93.0 & 75.8 & 94.3 & 77.4 & 91.9 \\
\hline NGO & 26.7 & 6.8 & 27.0 & 5.3 & 25.8 & 8.1 \\
\hline Don't know & 1.1 & 2.8 & 0.0 & 2.3 & 1.3 & 3.3 \\
\hline
\end{tabular}


Table 12.1: (Cont'd)

\begin{tabular}{|c|c|c|c|c|c|c|}
\hline $\begin{array}{l}\text { Awareness of and participation in } \\
\text { programmes }(\%)\end{array}$ & $\begin{array}{c}\text { M } \\
15-24\end{array}$ & $\begin{array}{c}\text { W } \\
15-24\end{array}$ & $\begin{array}{c}\text { MM } \\
15-29\end{array}$ & $\begin{array}{c}\text { MW } \\
15-24\end{array}$ & $\begin{array}{c}\text { UM } \\
15-24\end{array}$ & $\begin{array}{c}\text { UW } \\
15-24\end{array}$ \\
\hline \multicolumn{7}{|c|}{ Urban } \\
\hline Number aware of any programme(s) & 320 & 1,026 & 131 & 514 & 285 & 512 \\
\hline Participated in programme(s) held & 12.6 & 13.4 & 9.8 & 22.1 & 12.7 & 6.5 \\
\hline Number of respondents & 1,289 & 2,126 & 648 & 1,037 & 1,132 & 1,089 \\
\hline \multicolumn{7}{|l|}{ Participation in specific programmes } \\
\hline Health promotion & 47.7 & 29.2 & 53.3 & 29.6 & 48.1 & 27.6 \\
\hline Awareness/leadership/vocational/life skills & 36.4 & 10.9 & 30.0 & 6.0 & 36.3 & 24.1 \\
\hline Employment ${ }^{1}$ & 4.5 & 1.6 & 10.0 & 0.9 & 5.0 & 3.4 \\
\hline Self-help group & 2.2 & 58.0 & 6.7 & 69.0 & 1.2 & 28.8 \\
\hline Literacy & 7.9 & 5.7 & 6.7 & 2.6 & 7.5 & 15.3 \\
\hline Sports and recreation & 22.5 & 0.5 & 10.0 & 0.9 & 24.7 & 1.7 \\
\hline Number who participated in any programme(s) & 160 & 299 & 63 & 227 & 142 & 72 \\
\hline \multicolumn{7}{|c|}{ Rural } \\
\hline Aware of programme(s) held & 44.7 & 71.4 & 39.5 & 70.1 & 45.7 & 73.5 \\
\hline \multicolumn{7}{|l|}{ Focus of programmes held } \\
\hline Health promotion & 20.8 & 19.9 & 19.7 & 18.2 & 21.4 & 22.7 \\
\hline Awareness/leadership/vocational/life skills & 16.2 & 15.2 & 15.0 & 12.6 & 16.9 & 19.3 \\
\hline Employment $^{1}$ & 12.7 & 8.7 & 11.4 & 8.3 & 13.2 & 9.3 \\
\hline Self-help group & 3.4 & 49.5 & 4.3 & 52.2 & 3.3 & 45.0 \\
\hline Literacy & 9.8 & 11.6 & 8.5 & 9.3 & 10.6 & 15.5 \\
\hline Sports and recreation & 2.9 & 0.2 & 1.9 & 0.0 & 3.3 & 0.5 \\
\hline Number of respondents & 1,190 & 2,722 & 757 & 1,293 & 945 & 1,429 \\
\hline \multicolumn{7}{|l|}{ Organising agency } \\
\hline Government & 87.2 & 95.2 & 90.8 & 96.0 & 87.4 & 93.9 \\
\hline NGO & 12.8 & 7.3 & 9.0 & 6.2 & 13.6 & 9.1 \\
\hline Don't know & 1.0 & 2.3 & 1.2 & 2.1 & 0.5 & 2.5 \\
\hline Number aware of any programme(s) & 532 & 1,964 & 301 & 910 & 430 & 1,054 \\
\hline Participated in programme(s) held & 22.1 & 25.3 & 20.7 & 33.2 & 22.8 & 12.3 \\
\hline Number of respondents & 1,190 & 2,722 & 757 & 1,293 & 945 & 1,429 \\
\hline \multicolumn{7}{|l|}{ Participation in specific programmes } \\
\hline Health promotion & 35.5 & 13.9 & 39.2 & 12.5 & 35.9 & 20.2 \\
\hline Awareness/leadership/vocational/life skills & 34.3 & 9.2 & 32.2 & 5.7 & 34.8 & 24.2 \\
\hline Employment $^{1}$ & 19.4 & 7.4 & 27.0 & 6.0 & 18.2 & 13.6 \\
\hline Self-help group & 1.0 & 72.0 & 3.1 & 81.3 & 1.2 & 30.3 \\
\hline Literacy & 15.4 & 6.5 & 16.3 & 5.8 & 15.5 & 9.1 \\
\hline Sports and recreation & 10.3 & 0.2 & 6.6 & 0.0 & 10.9 & 1.0 \\
\hline Number who participated in any programme(s) & 264 & 604 & 158 & 426 & 216 & 178 \\
\hline
\end{tabular}

Note: All Ns are unweighted. Column total may exceed 100\% due to multiple responses. ${ }^{1}$ Includes Employment Guarantee Scheme (EGS), Jawahar Rozgar Yojana (JRY), National Rural Employment Programme (NREP), Pradhan Mantri Rozgar Yojana (PMRY), Training for Rural Youth for Self Employment (TRYSEM), etc. 
Figure 12.1: Percentage of youth reporting awareness of and participation in government- and NGO-sponsored programmes in the three years preceding the interview, according to residence, Andhra Pradesh, 2007-08

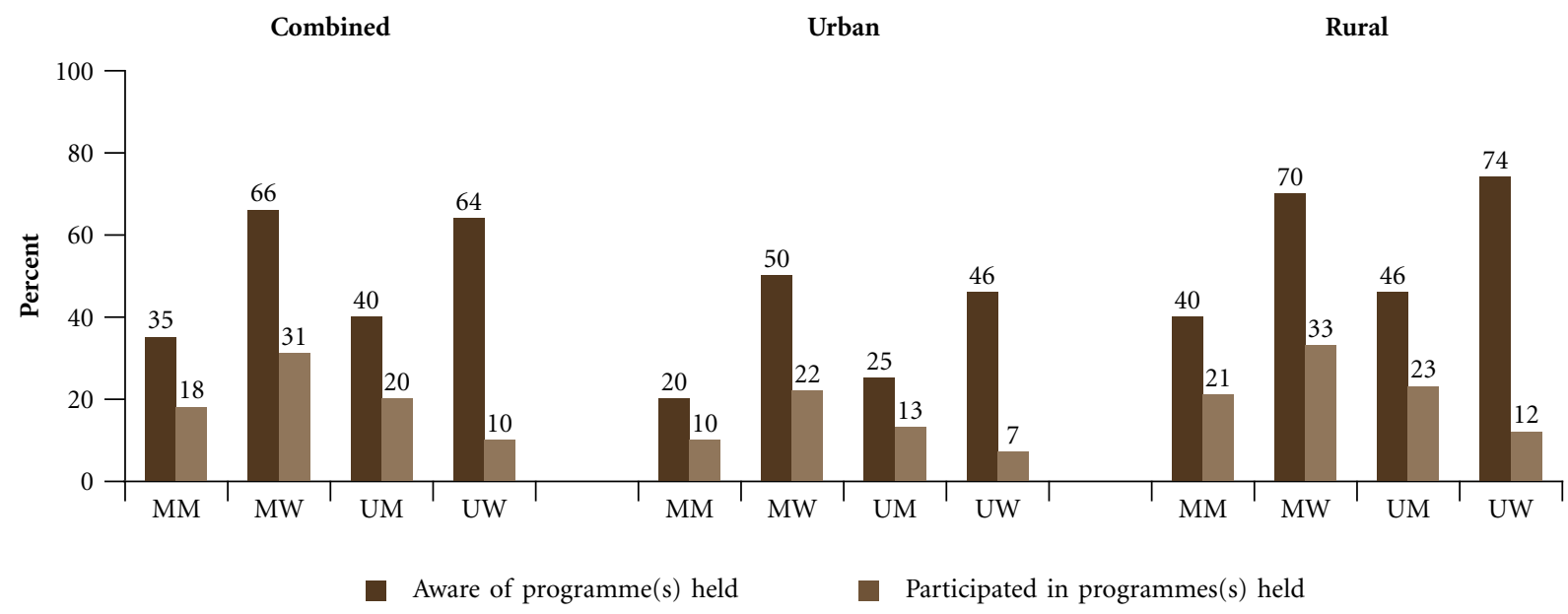

As many as $85 \%$ of young men and $95 \%$ of young women who were familiar with such programmes reported that these were organized by government agencies. Far fewer youth $(15 \%$ of young men and $7 \%$ of young women) reported awareness of programmes organised by the NGO sector. Marital status differences were generally mild; however, somewhat more unmarried than married young men reported awareness of NGO programmes ( $16 \%$ versus $11 \%$ ), a difference that was also observed among young men in rural areas. Rural-urban differences were also generally mild; however, young men in urban areas were more likely than those in rural areas to report awareness of NGO-sponsored programmes (27\% versus $13 \%)$.

Although young women were far more likely than young men to be aware of programmes, they were no more likely than young men to have participated in any programmes in the preceding three years. Indeed, just one-fifth of young men and women reported participation in a programme in the preceding three years (19\% and $22 \%$, respectively). Differences by marital status were negligible for young men, but considerably more married than unmarried young women reported participation in a programme (31\% versus $10 \%)$. Rural-urban differences were evident for both young men and women: far more rural than urban youth reported participation in a programme (22\% versus $13 \%$ among young men; $25 \%$ versus $13 \%$ among young women).

The kinds of programmes in which young men and women participated differed. Among young men, 35-38\% had participated in health promotion and leadership and life skills programmes, and $13-17 \%$ in employment, literacy and sports and recreation programmes. Among young women in contrast, the large majority (69\%) had participated in self-help groups, with somewhat fewer reporting participation in health promotion and leadership and life skills programmes (17\% and $9 \%$, respectively). Differences by marital status were evident among both young men and women. Among young men, the married were more likely than the unmarried to have participated in employment related programmes $(25 \%$ versus $16 \%)$ and less likely than them to have participated in sports and recreation programmes (7\% versus 13\%). Among young women, the married were far more likely than the unmarried to have participated in self-help group programmes (79\% versus 30\%), and much less likely to have participated in most others, notably, for example, awareness/leadership/vocational/life skills programmes (6\% versus $25 \%$ ), health promotion programmes (15\% versus $22 \%$ ), employment related programmes (5\% versus $11 \%$ ) and literacy programmes (5\% versus $11 \%$ ). Similar differences by marital status were by and large observed among youth in rural and urban areas as well. 
Rural-urban differences were also evident among both young men and women. For example, more urban than rural young men reported participation in health promotion programmes and sports and recreation programmes ( $48 \%$ versus $36 \%$ and $23 \%$ versus $10 \%$, respectively). Conversely, fewer urban than rural young men reported participation in employment and literacy programmes (5\% versus $19 \%$ and $8 \%$ versus $15 \%$, respectively). Among young women, more of those in urban than rural settings reported participation in health promotion programmes (29\% versus $14 \%)$ and fewer urban than rural young women reported participation in self-help groups (58\% versus $72 \%)$.

\subsection{Participation in community- or panchayat-sponsored programmes}

In many villages and urban neighbourhoods, community-led activities include, for example, cleanliness drives, health promotion activities, and the celebration of festivals and national days. As part of the Youth Study, youth were asked whether they had participated in any community-led activities organised by the panchayat/community leaders in the 12 months prior to the interview. Findings, reported in Table 12.2, suggest that almost three-fifths of

Table 12.2: Participation in community-led programmes

Percentage of youth who had attended community-led programmes in the village/urban neighbourhood and types of programmes attended in the 12 months preceding the interview, according to residence, Andhra Pradesh, 2007-08

\begin{tabular}{|c|c|c|c|c|c|c|}
\hline Participation in community-led programmes (\%) & $\begin{array}{c}M \\
15-24\end{array}$ & $\begin{array}{c}\text { W } \\
15-24\end{array}$ & $\begin{array}{c}\text { MM } \\
15-29\end{array}$ & $\begin{array}{c}\text { MW } \\
15-24\end{array}$ & $\begin{array}{c}\text { UM } \\
15-24\end{array}$ & $\begin{array}{c}\text { UW } \\
15-24\end{array}$ \\
\hline \multicolumn{7}{|c|}{ Combined } \\
\hline Attended any programme(s) organised & 58.9 & 17.4 & 51.2 & 11.6 & 60.7 & 25.2 \\
\hline Number of respondents & 2,479 & 4,848 & 1,405 & 2,330 & 2,077 & 2,518 \\
\hline \multicolumn{7}{|l|}{ Specific programmes attended } \\
\hline Cleanliness/sanitation & 21.9 & 7.0 & 21.8 & 4.8 & 22.0 & 8.3 \\
\hline Health promotion & 7.2 & 4.5 & 9.2 & 6.3 & 7.8 & 3.6 \\
\hline Festival celebration & 55.4 & 55.2 & 58.0 & 80.0 & 54.4 & 40.5 \\
\hline National day celebration & 70.5 & 49.9 & 65.8 & 24.4 & 71.6 & 65.4 \\
\hline Number who attended above programmes & 1,421 & 877 & 687 & 258 & 1,215 & 619 \\
\hline \multicolumn{7}{|c|}{ Urban } \\
\hline Attended any programme $(s)$ organised & 53.8 & 14.7 & 42.8 & 9.9 & 54.4 & 18.6 \\
\hline Number of respondents & 1,289 & 2,126 & 648 & 1,037 & 1,132 & 1,089 \\
\hline \multicolumn{7}{|l|}{ Specific programmes attended } \\
\hline Cleanliness/sanitation & 17.9 & 7.6 & 16.8 & 3.8 & 17.8 & 9.4 \\
\hline Health promotion & 8.4 & 0.9 & 10.7 & 1.9 & 8.7 & 0.6 \\
\hline Festival celebration & 63.4 & 42.9 & 69.7 & 73.1 & 62.7 & 30.2 \\
\hline National day celebration & 66.6 & 59.0 & 62.9 & 30.8 & 67.3 & 71.0 \\
\hline Number who attended above programmes & 691 & 309 & 278 & 103 & 613 & 206 \\
\hline \multicolumn{7}{|c|}{ Rural } \\
\hline Attended any programme(s) organised & 60.9 & 18.5 & 53.5 & 12.1 & 63.5 & 28.9 \\
\hline Number of respondents & 1,190 & 2,722 & 757 & 1,293 & 945 & 1,429 \\
\hline \multicolumn{7}{|l|}{ Specific programmes attended } \\
\hline Cleanliness/sanitation & 23.3 & 6.8 & 23.0 & 5.1 & 23.6 & 8.0 \\
\hline Health promotion & 6.8 & 5.7 & 8.7 & 7.3 & 7.5 & 4.7 \\
\hline Festival celebration & 52.6 & 59.4 & 55.4 & 81.7 & 51.4 & 44.1 \\
\hline National day celebration & 71.9 & 46.8 & 66.5 & 22.9 & 73.2 & 63.3 \\
\hline Number who attended above programmes & 730 & 568 & 409 & 155 & 602 & 413 \\
\hline
\end{tabular}

Note: All Ns are unweighted. Column totals may exceed 100\% due to multiple responses. 
young men $(59 \%)$ compared to just one-sixth of young women (17\%) reported having participated in a communityled programme in the last year. Participation was more likely to be reported by unmarried than married youth ( $61 \%$ versus $51 \%$ among young men, and $25 \%$ versus $12 \%$ among young women) and more likely to be reported by rural than urban residents (61\% versus $54 \%$ of young men and $19 \%$ versus $15 \%$ of young women).

Findings reveal that among those who participated in community-led programmes, the activities in which the largest percentage of youth participated were the celebration of national days $(71 \%$ and $50 \%$ of young men and women, respectively) and festivals (55\% of both young men and women). In addition, $22 \%$ of young men (and just $7 \%$ of young women) participated in cleanliness drives. More married than unmarried youth, particularly among young women, participated in the celebration of festivals (58\% versus $54 \%$ among young men, and $80 \%$ versus $41 \%$ among young women). In contrast, more unmarried than married youth participated in the celebration of national days ( $72 \%$ versus $66 \%$ among young men, and $65 \%$ versus $24 \%$ among young women).

\subsection{Membership in organised groups}

Youth were asked whether they belonged to any organised group, ranging from self-help groups to youth groups to sports and social clubs. Findings, reported in Table 12.3, suggest that young women were more likely than young men to report membership in any group (26\% compared to $10 \%)$. Marital status and rural-urban differences were negligible among young men but wide among young women. Indeed, group membership was reported by $40 \%$ of married young women, compared to just $7 \%$ of unmarried young women, and by $30 \%$ of rural young women, compared to $14 \%$ of their urban counterparts.

The types of groups in which youth reported membership varied widely by sex of the respondent. Young men were most likely to report membership in youth groups or yuva/tarun/kishor/kishori mandals (7\%), with little variation by marital status or rural-urban residence. Young women, in contrast, were most likely to report membership in self-help groups $(20 \%)$, followed by membership in mahila mandals (5\%). Married young women were far more likely than their unmarried counterparts to report membership in self-help groups and mahila mandals $(33 \%$ versus $4 \%$ and $8 \%$ versus $1 \%$, respectively). Likewise, young women in rural areas were more likely than their urban counterparts to report membership in self-help groups (24\% versus $10 \%)$. Fewer than $1 \%$ of young men reported membership in self-help groups, and similarly, fewer than $1 \%$ of young women reported membership in youth groups.

Among married young men who reported group membership, almost three in five had become members prior to marriage (58\%) - about three in five of those in rural areas (57\%) and two-thirds of those in urban areas (67\%). The opposite was true among married young women, of whom almost all (97\%), irrespective of rural-urban residence, had joined an organised group after marriage, confirming the limited duration of exposure prior to marriage of young women to organised groups.

\subsection{Perceptions about action taken by panchayats in addressing defiance of social norms}

In the course of the pre-survey qualitative investigations, researchers noted that in several rural areas, village panchayats took action in various situations in which youth did not adhere to social norms. Hence, youth in rural areas were asked whether they believed that the panchayat in their village had ever taken action if someone was reported to have teased a girl or woman, if parents refused to permit their sons or daughters to marry someone of their choice, if youth were found to have engaged in pre- or extra-marital sex or if an unmarried girl became pregnant. Responses are reported in Table 12.4.

Considerable proportions of youth perceived that their village panchayats would punish those accused of teasing a girl or woman $(63 \%$ and $61 \%$ of young men and women, respectively) and would fine youth who had engaged in pre- or extra-marital sex $(51 \%$ and $42 \%$ of young men and women, respectively). They were less likely to report that the local panchayat would arrange the marriage of youth whose parents had refused to permit them to marry 
Table 12.3: Membership in organised groups

Percentage of youth reporting membership in organised groups, according to residence, Andhra Pradesh, 2007-08

\begin{tabular}{|c|c|c|c|c|c|c|}
\hline Membership in organised groups (\%) & $\begin{array}{c}M \\
15-24\end{array}$ & $\begin{array}{c}\text { W } \\
15-24\end{array}$ & $\begin{array}{c}\text { MM } \\
15-29\end{array}$ & $\begin{array}{c}\text { MW } \\
15-24\end{array}$ & $\begin{array}{c}\text { UM } \\
15-24\end{array}$ & $\begin{array}{c}\text { UW } \\
15-24\end{array}$ \\
\hline \multicolumn{7}{|c|}{ Combined } \\
\hline Member of an organised group & 9.6 & 25.5 & 10.2 & 40.0 & 10.3 & 6.5 \\
\hline Self-help group & 0.3 & 20.2 & 1.3 & 32.6 & 0.3 & 4.0 \\
\hline Mahila mandal & NA & 4.7 & NA & 7.6 & NA & 1.0 \\
\hline Social or sports club & 2.6 & 0.5 & 1.4 & 0.1 & 2.9 & 1.0 \\
\hline Youth group/yuva/tarun/kishor/kishori mandal & 6.7 & 0.4 & 7.6 & 0.2 & 7.0 & 0.6 \\
\hline Number of respondents & 2,479 & 4,848 & 1,405 & 2,330 & 2,077 & 2,518 \\
\hline \multicolumn{7}{|l|}{ Became member of an organised group ${ }^{1}$} \\
\hline Before marriage & NA & NA & 58.3 & 2.7 & NA & NA \\
\hline After marriage & NA & NA & 41.7 & 97.2 & NA & NA \\
\hline Number reporting membership in an organised group & NA & NA & 137 & 840 & NA & NA \\
\hline \multicolumn{7}{|c|}{ Urban } \\
\hline Member of an organised group & 8.6 & 13.9 & 7.2 & 26.7 & 9.5 & 3.7 \\
\hline Self-help group & 0.0 & 10.3 & 0.7 & 21.0 & 0.0 & 1.7 \\
\hline Mahila mandal & NA & 3.1 & NA & 6.1 & NA & 0.6 \\
\hline Social or sports club & 2.3 & 0.6 & 2.0 & 0.0 & 2.5 & 1.0 \\
\hline Youth group/yuva/tarun/kishor/kishori mandal & 6.4 & 0.3 & 4.9 & 0.0 & 7.0 & 0.6 \\
\hline Number of respondents & 1,289 & 2,126 & 648 & 1,037 & 1,132 & 1,089 \\
\hline \multicolumn{7}{|l|}{ Became member of an organised group ${ }^{1}$} \\
\hline Before marriage & NA & NA & $(66.7)$ & 5.8 & NA & NA \\
\hline After marriage & NA & NA & $(33.3)$ & 94.2 & NA & NA \\
\hline Number reporting membership in an organised group & NA & NA & 45 & 272 & NA & NA \\
\hline \multicolumn{7}{|c|}{ Rural } \\
\hline Member of an organised group & 10.0 & 30.3 & 11.2 & 44.0 & 10.6 & 8.0 \\
\hline Self-help group & 0.4 & 24.3 & 1.5 & 36.0 & 0.4 & 5.3 \\
\hline Mahila mandal & NA & 5.4 & NA & 8.0 & NA & 1.2 \\
\hline Social or sports club & 2.7 & 0.5 & 1.3 & 0.1 & 3.1 & 1.1 \\
\hline Youth group/yuva/tarun/kishor/kishori mandal & 6.9 & 0.4 & 8.4 & 0.3 & 7.1 & 0.6 \\
\hline Number of respondents & 1,190 & 2,722 & 757 & 1,293 & 945 & 1,429 \\
\hline \multicolumn{7}{|l|}{ Became member of an organised group ${ }^{1}$} \\
\hline Before marriage & NA & NA & 57.0 & 2.1 & NA & NA \\
\hline After marriage & NA & NA & 43.0 & 97.7 & NA & NA \\
\hline Number reporting membership in an organised group & NA & NA & 92 & 568 & NA & NA \\
\hline
\end{tabular}

Note: All Ns are unweighted. ( ) Based on 25-49 unweighted cases. NA: Not applicable. ${ }^{1}$ Column total may not equal 100\% due to missing cases.

someone of their choice (39\% and $34 \%$, respectively) or that the panchayat would force a boy to marry a girl whom he had made pregnant (35\% and $30 \%$ of young men and women, respectively). In most cases, young men were more likely than young women to perceive panchayat involvement. Differences by marital status were negligible, except that more married than unmarried young men perceived that the panchayat would force a boy to marry a girl whom he had made pregnant (39\% versus $32 \%)$. 
Table 12.4: Perceptions about actions taken by the panchayat in case of defiance of social norms

Percent distribution of youth by their perceptions about actions taken by the panchayat in case of defiance of social norms in selected situations, Andhra Pradesh (rural), 2007-08

\begin{tabular}{|c|c|c|c|c|c|c|}
\hline Perceptions (\%) & $\begin{array}{c}\mathrm{M} \\
15-24\end{array}$ & $\begin{array}{c}\text { W } \\
15-24\end{array}$ & $\begin{array}{c}\text { MM } \\
15-29\end{array}$ & $\begin{array}{c}\text { MW } \\
15-24\end{array}$ & $\begin{array}{c}\text { UM } \\
15-24\end{array}$ & $\begin{array}{c}\text { UW } \\
\text { 15-24 }\end{array}$ \\
\hline \multicolumn{7}{|c|}{$\begin{array}{l}\text { Panchayat would punish anyone who teases a } \\
\text { girl/woman }\end{array}$} \\
\hline Yes & 62.8 & 60.5 & 63.1 & 59.1 & 62.9 & 62.8 \\
\hline No & 32.3 & 35.3 & 32.9 & 36.3 & 32.0 & 33.6 \\
\hline Can't say & 4.9 & 4.2 & 4.0 & 4.5 & 5.1 & 3.6 \\
\hline \multicolumn{7}{|c|}{$\begin{array}{l}\text { Panchayat would fine a boy/girl who had } \\
\text { engaged in pre-/extra-marital sexual relations }\end{array}$} \\
\hline Yes & 50.8 & 42.2 & 51.6 & 40.9 & 50.1 & 44.4 \\
\hline No & 38.9 & 41.5 & 41.8 & 41.8 & 39.2 & 41.0 \\
\hline Can't say & 10.3 & 16.3 & 6.6 & 17.3 & 10.7 & 14.6 \\
\hline \multicolumn{7}{|c|}{$\begin{array}{l}\text { Panchayat would arrange the marriages of youth } \\
\text { if parents refused to let them marry }\end{array}$} \\
\hline Yes & 38.6 & 34.1 & 40.9 & 32.8 & 37.8 & 36.2 \\
\hline No & 52.1 & 54.9 & 53.3 & 55.6 & 52.0 & 53.8 \\
\hline Can't say & 9.3 & 11.0 & 5.8 & 11.6 & 10.2 & 10.0 \\
\hline \multicolumn{7}{|c|}{$\begin{array}{l}\text { Panchayat had ever forced a boy to marry a } \\
\text { girl who he had made pregnant }\end{array}$} \\
\hline Yes & 34.5 & 30.1 & 39.2 & 30.2 & 32.1 & 29.8 \\
\hline No & 53.9 & 58.2 & 52.6 & 57.0 & 55.4 & 60.3 \\
\hline Can't say & 11.6 & 11.7 & 8.3 & 12.8 & 12.5 & 9.9 \\
\hline Number of respondents & 1,190 & 2,722 & 757 & 1,293 & 945 & 1,429 \\
\hline
\end{tabular}

Note: All Ns are unweighted. Column totals may not equal 100\% due to missing cases. Questions were asked only of respondents in rural areas.

\subsection{Voting behaviour and perceptions of political matters}

Table 12.5 presents the percentage of eligible youth — that is, those at least 20 years of age at the time of the interview who would have been eligible to vote prior to the interview-who had voted in the last election. Findings suggest that voting behaviour was far from universal. About three-in five youth had voted and gender differences were negligible (62-63\%). However, voting behaviour varied considerably by marital status and rural-urban residence (see also Figure 12.2). Larger proportions of married than unmarried youth reported that they had voted in the last election. Specifically, $88 \%$ of eligible married young men compared to $56 \%$ of unmarried young men reported that they had voted in the last election. The corresponding percentages among young women were $68 \%$ and $38 \%$, respectively. Rural-urban differences suggest, moreover, that considerably more rural than urban youth had voted in the last election: $69 \%$ versus $49 \%$ among young men, and $71 \%$ versus $42 \%$ among young women.

Table 12.5 also reports youth perceptions about political processes, notably the extent of disillusionment with the ability of any political party to achieve change at the community level and the extent to which respondents believed that people could vote freely and without fear, pressure or influence.

Considerable proportions of youth reported disillusionment with the political process. Gender differences were, however, apparent. Larger proportions of young men than women $(60 \%$ and $53 \%$, respectively) agreed that 
there would be no improvement in their village/neighbourhood irrespective of the political party governing the state. Differences by marital status and rural-urban residence were negligible, except that more rural than urban young women reported disillusionment with political parties' commitment to improving local conditions (55\% versus $48 \%)$. At the same time, most young people- $89-90 \%$ of young men and women-felt that one could vote freely and without fear or pressure. Indeed, just $8-9 \%$ of youth felt that one could not vote freely. Differences by marital status and rural-urban residence were negligible.

Figure 12.2: Percentage of youth aged 20 or above who had voted in the last election, according to residence, Andhra Pradesh, 2007-08
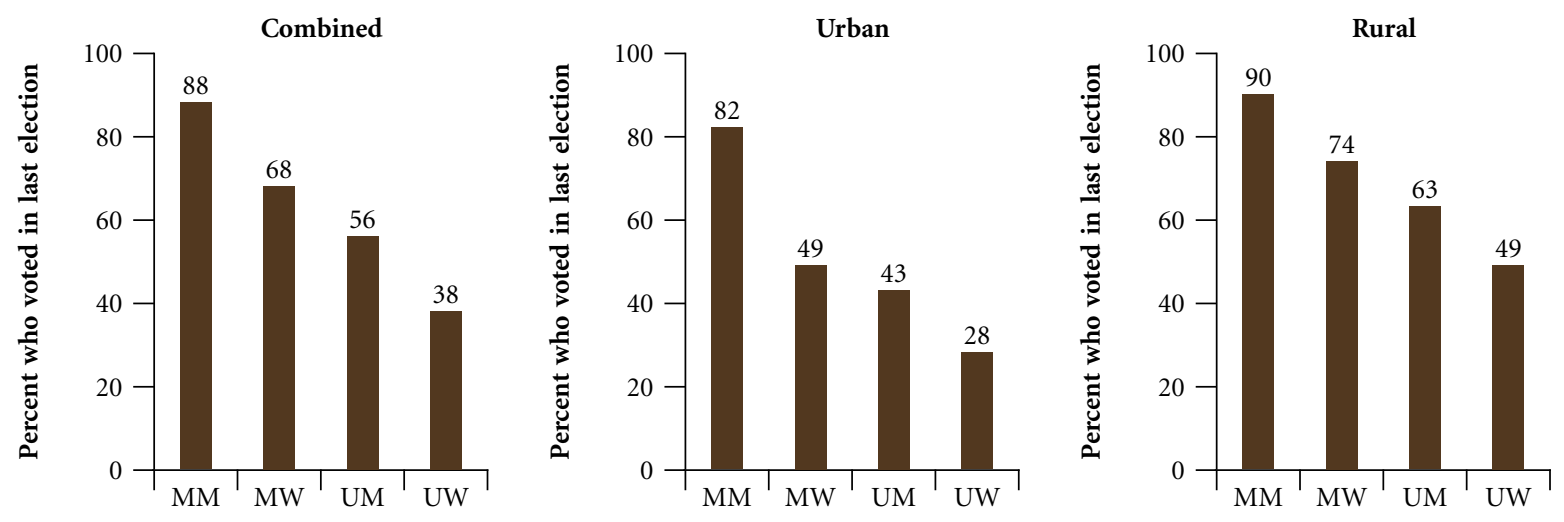

\subsection{Expression of secular attitudes}

In order to gauge attitudes regarding social interaction with individuals of different castes and religions, the Youth Study inquired whether youth mixed freely with those of other castes and religions, whether they would eat together with a person from a different caste or religion, whether they would talk to someone who had an inter-caste marriage and whether they considered it acceptable to punish someone who showed disrespect for their religion. Findings, presented in Table 12.6, suggest that youth expressed secular attitudes on most topics, with little variation, for the most part, by sex of the respondent, marital status or rural-urban residence.

In response to specific issues, both young men and young women were most likely to report that they mixed freely with individuals of different castes (97-98\%) and religions (96-97\%), that they would talk to a person who had an inter-caste marriage (93-95\%) and that they would eat together with a person from a different caste or religion ( $93 \%$ of young men and $88 \%$ of young women). Considerable percentages of youth also felt that it was not acceptable to punish someone who had shown disrespect to their religion and young women were more likely than young men to report so (34\% of young men and $57 \%$ of young women).

Differences in the nature of behaviours and attitudes towards individuals of different castes and religions were negligible by marital status, except that more unmarried than married young women felt that it was not acceptable to punish someone who had shown disrespect to their religion (60\% versus 55\%). Differences by rural-urban residence were also largely negligible, except that young women in urban areas were more likely than their rural counterparts to express secular attitudes on such issues as eating with those of other castes and religions ( $93 \%$ versus $86 \%$ ), and tolerating without punishment someone who had shown disrespect to their religion (61\% versus 56\%). 
Table 12.5: Voting behaviour of eligible youth and perceptions about political matters

Percentage of youth aged 20 or above who had voted in the last election and percent distribution of all youth by their perceptions about political matters, according to residence, Andhra Pradesh, 2007-08

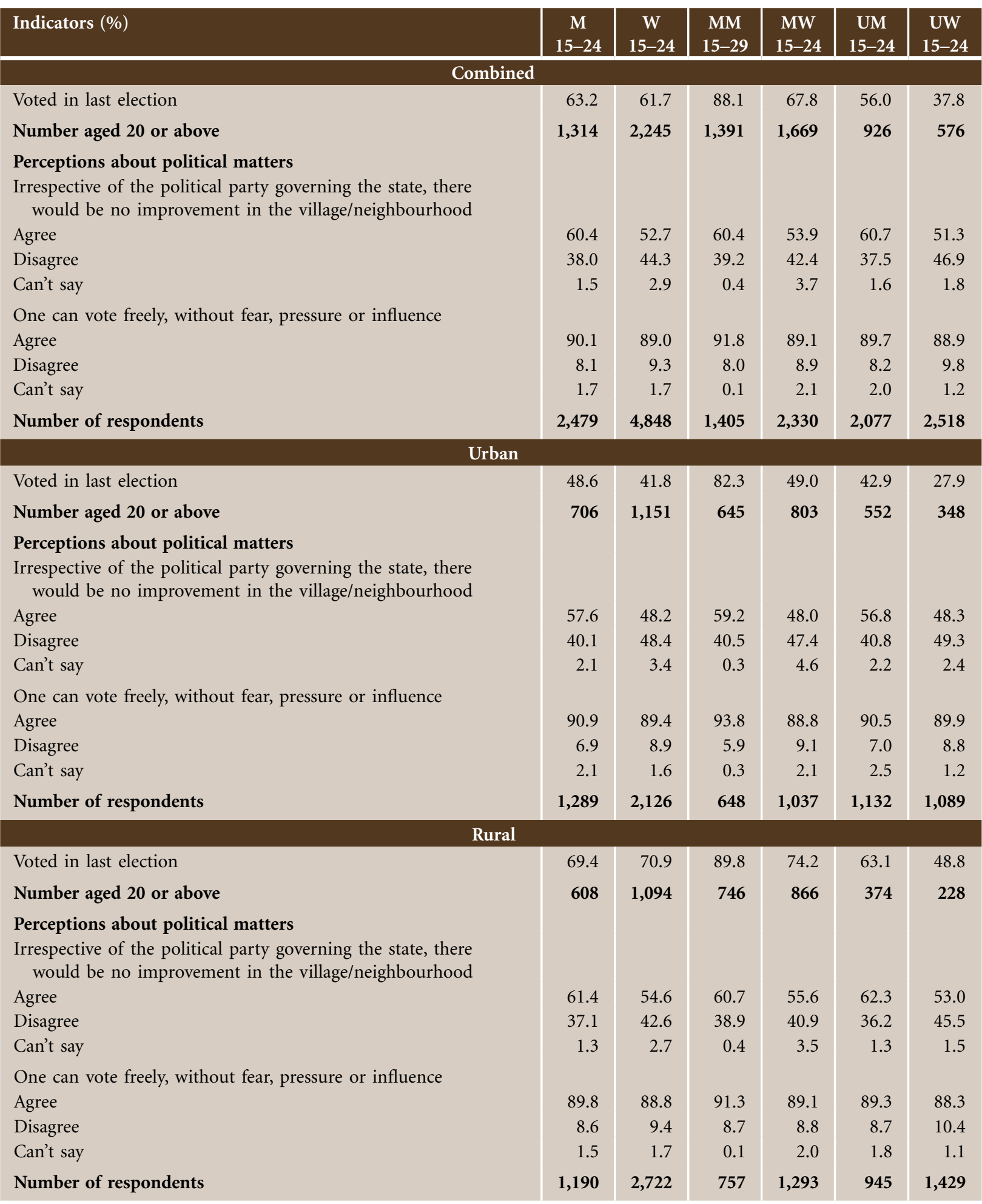

Note: All Ns are unweighted. Column totals may not equal 100\% due to missing cases. 
Table 12.6: Expression of secular attitudes

Percent distribution of youth by reported behaviours and attitudes towards interaction with people of different castes and religions, according to residence, Andhra Pradesh, 2007-08

\begin{tabular}{|c|c|c|c|c|c|c|}
\hline Behaviours/attitudes (\%) & $\underset{15-24}{M}$ & $\begin{array}{c}\text { W } \\
15-24\end{array}$ & $\begin{array}{c}\mathrm{MM} \\
15-29\end{array}$ & $\begin{array}{c}\text { MW } \\
15-24\end{array}$ & $\begin{array}{c}\text { UM } \\
15-24\end{array}$ & $\begin{array}{l}\text { UW } \\
15-24\end{array}$ \\
\hline \multicolumn{7}{|c|}{ Combined } \\
\hline \multicolumn{7}{|c|}{ Mixes freely with people of other castes } \\
\hline Yes & 97.0 & 97.6 & 97.4 & 97.4 & 96.9 & 97.8 \\
\hline No & 2.8 & 2.4 & 2.5 & 2.6 & 2.9 & 2.2 \\
\hline \multicolumn{7}{|c|}{ Mixes freely with people of other religions } \\
\hline Yes & 96.8 & 95.6 & 96.9 & 95.1 & 96.6 & 96.3 \\
\hline No & 3.1 & 4.4 & 3.1 & 4.9 & 3.2 & 3.7 \\
\hline \multicolumn{7}{|c|}{ Would eat together with a person of another caste/religion } \\
\hline Yes & 93.4 & 88.3 & 93.3 & 87.0 & 93.9 & 90.0 \\
\hline No & 6.3 & 11.5 & 6.5 & 12.8 & 5.8 & 10.0 \\
\hline \multicolumn{7}{|c|}{ Would talk to a person who has had an inter-caste marriage } \\
\hline Yes & 95.3 & 93.4 & 95.9 & 93.5 & 95.4 & 93.2 \\
\hline No & 4.1 & 6.3 & 4.0 & 6.2 & 3.9 & 6.6 \\
\hline \multicolumn{7}{|c|}{$\begin{array}{l}\text { Believes it is acceptable to punish someone who shows } \\
\text { disrespect to respondent's religion }\end{array}$} \\
\hline Yes & 65.5 & 42.3 & 64.6 & 44.9 & 66.0 & 39.1 \\
\hline No & 33.5 & 57.1 & 34.8 & 54.5 & 32.9 & 60.4 \\
\hline Number of respondents & 2,479 & 4,848 & 1,405 & 2,330 & 2,077 & 2,518 \\
\hline \multicolumn{7}{|c|}{ Urban } \\
\hline \multicolumn{7}{|c|}{ Mixes freely with people of other castes } \\
\hline Yes & 99.0 & 98.0 & 99.0 & 97.5 & 99.0 & 98.3 \\
\hline No & 0.9 & 2.0 & 1.0 & 2.5 & 0.8 & 1.7 \\
\hline \multicolumn{7}{|c|}{ Mixes freely with people of other religions } \\
\hline Yes & 98.6 & 96.9 & 97.7 & 96.2 & 98.6 & 97.5 \\
\hline No & 1.3 & 3.1 & 2.3 & 3.8 & 1.3 & 2.5 \\
\hline \multicolumn{7}{|c|}{ Would eat together with a person of another caste/religion } \\
\hline Yes & 96.2 & 93.0 & 95.8 & 92.4 & 96.5 & 93.5 \\
\hline No & 3.5 & 7.0 & 4.2 & 7.6 & 3.2 & 6.5 \\
\hline \multicolumn{7}{|c|}{ Would talk to a person who had an inter-caste marriage } \\
\hline Yes & 96.2 & 94.4 & 96.1 & 95.2 & 96.0 & 93.6 \\
\hline No & 3.1 & 5.5 & 3.6 & 4.8 & 3.2 & 6.2 \\
\hline \multicolumn{7}{|c|}{$\begin{array}{l}\text { Believes it is acceptable to punish someone who shows } \\
\text { disrespect to respondent's religion }\end{array}$} \\
\hline Yes & 62.7 & 38.8 & 61.1 & 44.4 & 63.9 & 34.4 \\
\hline No & 36.0 & 60.6 & 38.9 & 55.2 & 34.7 & 65.0 \\
\hline Number of respondents & 1,289 & 2,126 & 648 & 1,037 & 1,132 & 1,089 \\
\hline
\end{tabular}


Table 12.6: (Cont'd)

\begin{tabular}{|c|c|c|c|c|c|c|}
\hline Behaviours/attitudes (\%) & $\begin{array}{c}\mathrm{M} \\
15-24\end{array}$ & $\begin{array}{c}\text { W } \\
15-24\end{array}$ & $\begin{array}{c}\mathrm{MM} \\
15-29\end{array}$ & $\begin{array}{c}\text { MW } \\
15-24\end{array}$ & $\begin{array}{c}\text { UM } \\
15-24\end{array}$ & $\begin{array}{c}\text { UW } \\
15-24\end{array}$ \\
\hline \multicolumn{7}{|c|}{ Rural } \\
\hline \multicolumn{7}{|c|}{ Mixes freely with people of other castes } \\
\hline Yes & 96.2 & 97.4 & 97.0 & 97.3 & 96.0 & 97.5 \\
\hline No & 3.6 & 2.6 & 2.9 & 2.7 & 3.9 & 2.4 \\
\hline \multicolumn{7}{|c|}{ Mixes freely with people of other religions } \\
\hline Yes & 96.2 & 95.1 & 96.7 & 94.7 & 95.8 & 95.6 \\
\hline No & 3.7 & 4.9 & 3.3 & 5.3 & 4.1 & 4.4 \\
\hline \multicolumn{7}{|c|}{ Would eat together with a person of another caste/religion } \\
\hline Yes & 92.3 & 86.4 & 92.7 & 85.4 & 92.7 & 87.9 \\
\hline No & 7.3 & 13.4 & 7.2 & 14.3 & 6.9 & 12.0 \\
\hline \multicolumn{7}{|c|}{ Would talk to a person who had an inter-caste marriage } \\
\hline Yes & 94.9 & 92.9 & 95.7 & 93.0 & 95.2 & 93.0 \\
\hline No & 4.4 & 6.7 & 4.2 & 6.6 & 4.1 & 6.8 \\
\hline \multicolumn{7}{|c|}{$\begin{array}{l}\text { Believes it is acceptable to punish someone who shows } \\
\text { disrespect to respondent's religion }\end{array}$} \\
\hline Yes & 66.5 & 43.8 & 65.6 & 45.0 & 66.9 & 41.8 \\
\hline No & 32.5 & 55.6 & 33.7 & 54.2 & 32.2 & 57.9 \\
\hline Number of respondents & 1,190 & 2,722 & 757 & 1,293 & 945 & 1,429 \\
\hline
\end{tabular}

Note: All Ns are unweighted. Column totals may not equal 100\% due to missing cases or "don't know" responses.

\subsection{Physical fights in the village or urban neighbourhood}

All respondents were asked whether physical fights—-more specifically, youth beating, slapping or pulling the hair of others-were common among young men and women, respectively, in their village or neighbourhood. Findings, presented in Table 12.7, suggest that physical fights were reported to be more common among young men than young women, and that young women were more likely than young men to report that physical fights took place sometimes or often among both men and women. For example, $64 \%$ of young men and $70 \%$ of young women reported that young men engaged in physical fights sometimes or often, and $32 \%$ of young men and $48 \%$ of young women reported the same for young women. Differences by marital status were negligible in reports of physical fighting among both young men and women. In contrast, rural-urban differences were evident, with rural youth consistently more likely than their urban counterparts to report occasional or frequent physical fighting among both young men and women (66\% versus $60 \%$ among young men, and $75 \%$ versus $60 \%$ among young women with regard to physical fights among young men; $35 \%$ versus $25 \%$ and $51 \%$ versus $41 \%$, respectively, among young women).

Youth were also asked a direct question about their own involvement in physical fights with anyone within the village or urban neighbourhood in the 12 months preceding the interview. The question did not probe further and hence we acknowledge that responses may include fights among family members and others. Few youth $-8 \%$ of young men and $3 \%$ of young women reported that they had been involved in physical fights. Marital status differences and rural-urban differences were negligible. 
Table 12.7: Physical fights in village/neighbourhood

Percentage of youth reporting perceptions of youth involvement in physical fights in their village/neighbourhood and percentage of youth themselves involved in physical fights in the last 12 months, according to residence, Andhra Pradesh, 2007-08

\begin{tabular}{|c|c|c|c|c|c|c|}
\hline Perceptions/experiences of physical fights (\%) & $\begin{array}{c}\text { M } \\
15-24\end{array}$ & $\begin{array}{c}\mathrm{W} \\
15-24\end{array}$ & $\begin{array}{c}\text { MM } \\
15-29\end{array}$ & $\begin{array}{c}\text { MW } \\
15-24\end{array}$ & $\begin{array}{c}\text { UM } \\
15-24\end{array}$ & $\begin{array}{c}\text { UW } \\
15-24\end{array}$ \\
\hline \multicolumn{7}{|c|}{ Combined } \\
\hline \multicolumn{7}{|l|}{ Respondents' perceptions of the extent to which: } \\
\hline \multicolumn{7}{|l|}{ Young men in the area engaged in physical fights } \\
\hline Never & 35.6 & 29.8 & 38.3 & 28.3 & 35.2 & 31.3 \\
\hline Sometimes & 63.1 & 63.9 & 60.0 & 65.0 & 63.5 & 62.9 \\
\hline Often & 1.3 & 6.3 & 1.6 & 6.7 & 1.3 & 5.8 \\
\hline \multicolumn{7}{|c|}{ Young women in the area engaged in physical fights } \\
\hline Never & 67.9 & 51.7 & 67.2 & 50.1 & 69.4 & 53.6 \\
\hline Sometimes & 31.7 & 42.5 & 32.5 & 43.4 & 30.2 & 41.5 \\
\hline Often & 0.4 & 5.8 & 0.3 & 6.5 & 0.3 & 4.8 \\
\hline $\begin{array}{l}\text { Respondents themselves involved in physical fights in } \\
\text { last } 12 \text { months }\end{array}$ & 8.0 & 3.1 & 5.2 & 3.7 & 8.2 & 2.3 \\
\hline Number of respondents & 2,479 & 4,848 & 1,405 & 2,330 & 2,077 & 2,518 \\
\hline \multicolumn{7}{|c|}{ Urban } \\
\hline \multicolumn{7}{|l|}{ Respondents' perception of the extent to which: } \\
\hline \multicolumn{7}{|l|}{ Young men in the area engaged in physical fights } \\
\hline Never & 39.7 & 40.3 & 42.3 & 36.6 & 39.8 & 43.3 \\
\hline Sometimes & 57.8 & 54.1 & 54.7 & 57.3 & 57.9 & 51.5 \\
\hline Often & 2.5 & 5.5 & 2.9 & 6.1 & 2.2 & 5.1 \\
\hline \multicolumn{7}{|l|}{ Young women in the area engaged in physical fights } \\
\hline Never & 74.8 & 59.3 & 75.9 & 56.2 & 75.3 & 61.7 \\
\hline Sometimes & 24.7 & 36.3 & 23.1 & 39.2 & 24.2 & 34.0 \\
\hline Often & 0.6 & 4.3 & 1.0 & 4.6 & 0.5 & 4.2 \\
\hline \multicolumn{7}{|c|}{ Respondents themselves involved in physical fights in } \\
\hline Number of respondents & 1,289 & 2,126 & 648 & 1,037 & 1,132 & 1,089 \\
\hline \multicolumn{7}{|c|}{ Rural } \\
\hline \multicolumn{7}{|l|}{ Respondents' perception of the extent to which: } \\
\hline \multicolumn{7}{|l|}{ Young men in the area engaged in physical fights } \\
\hline Sometimes & $\begin{array}{l}34.0 \\
65.1\end{array}$ & $\begin{array}{l}25.4 \\
68.0\end{array}$ & $\begin{array}{l}3 / .2 \\
61.5\end{array}$ & $\begin{array}{l}25.9 \\
67.3\end{array}$ & $\begin{array}{l}33.2 \\
65.9\end{array}$ & $\begin{array}{l}24.5 \\
69.3\end{array}$ \\
\hline Often & 0.8 & 6.6 & 1.3 & 6.9 & 1.0 & 6.2 \\
\hline \multicolumn{7}{|c|}{ Young women in the area engaged in physical fights } \\
\hline Never & 65.3 & 48.6 & 64.8 & 48.4 & 66.8 & 48.9 \\
\hline Sometimes & 34.4 & 45.0 & 35.2 & 44.6 & 32.8 & 45.8 \\
\hline Often & 0.3 & 6.4 & 0.1 & 7.0 & 0.2 & 5.3 \\
\hline \multicolumn{6}{|c|}{ Respondents themselves involved in physical fights in } & 2.6 \\
\hline Number of respondents & 1,190 & 2,722 & 757 & 1,293 & 945 & 1,429 \\
\hline
\end{tabular}

Note: All Ns are unweighted. Column totals may not equal 100\% due to missing cases or "don't know" responses. 


\subsection{Perceptions of the leading problems facing youth}

Finally, youth were asked to give their opinion on the most important problem facing youth in their village or urban neighbourhood. Findings presented in Table 12.8 clearly suggest that young men and women had different perceptions. For example, young men described unemployment and poverty more generally as leading problems (reported by 36-38\%), followed by lack of educational opportunities (8\%). Together, these three issues were expressed by $82 \%$ of young men. Poverty was also described by young women as a leading problem facing youth (30\%); however, young women also described a range of other problems, including concerns about lack of amenities or infrastructure, i.e., water and sanitation, roads and electricity (19\%), lack of educational opportunities (15\%) and unemployment $(13 \%)$. Together, these four issues were expressed by $77 \%$ of young women.

Differences by marital status were evident. Compared to unmarried youth, the married were more likely to report poverty ( $42 \%$ versus $35 \%$ among young men; $33 \%$ versus $27 \%$ among young women) and less likely to report concerns about unemployment (33\% versus $40 \%$ among young men; $11 \%$ versus $16 \%$ among young women) as leading problems facing youth. Married young women, in addition, were more likely than unmarried young women to report concerns about lack of amenities or infrastructure (23\% versus $14 \%)$ and less likely to report concerns about lack of educational opportunities (13\% versus $18 \%)$.

Urban youth were more likely than rural youth to feel that difficulty in finding employment was a leading problem facing youth ( $45 \%$ versus $35 \%$ among young men, and $25 \%$ versus $9 \%$ among young women), and less likely to feel that poverty was a leading problem ( $27 \%$ versus $40 \%$ among young men; $25 \%$ versus $32 \%$ among young women). Among young women, moreover, more of those from rural than urban areas reported lack of amenities/infrastructure $(23 \%$ versus $8 \%)$ as a leading problem facing youth. Young women in urban areas were more likely than those in rural areas to cite lack of safety for girls as a leading problem (7\% versus $2 \%)$.

Table 12.8: Perceptions about the leading problem facing youth

Percent distribution of youth by their perceptions of the leading problem facing youth, according to residence, Andhra Pradesh, 2007-08

\begin{tabular}{|c|c|c|c|c|c|c|}
\hline Leading problem (\%) & $\begin{array}{c}\mathrm{M} \\
15-24 \\
\end{array}$ & $\begin{array}{c}W \\
15-24\end{array}$ & $\begin{array}{c}\text { MM } \\
15-29\end{array}$ & $\begin{array}{c}\text { MW } \\
15-24\end{array}$ & $\begin{array}{c}\text { UM } \\
15-24\end{array}$ & $\begin{array}{c}\text { UW } \\
15-24\end{array}$ \\
\hline \multicolumn{7}{|c|}{ Combined } \\
\hline Finding a job/unemployment & 37.8 & 13.4 & 33.3 & 11.0 & 39.7 & 16.3 \\
\hline Poverty & 36.4 & 30.2 & 41.5 & 33.0 & 34.7 & 26.6 \\
\hline $\begin{array}{l}\text { Lack of amenities/infrastructure (water/toilets/ } \\
\text { roads/electricity) }\end{array}$ & 3.8 & 18.8 & 4.3 & 22.5 & 3.7 & 14.2 \\
\hline Health-/health service-related concerns & 0.5 & 1.0 & 0.1 & 1.1 & 0.6 & 0.9 \\
\hline Security of girls/law and order & 0.2 & 3.5 & 0.4 & 1.7 & 0.2 & 5.8 \\
\hline Finding a good spouse/dowry & 0.1 & 5.1 & 0.2 & 4.5 & 0.1 & 5.8 \\
\hline Lack of educational opportunities & 7.5 & 14.9 & 5.8 & 12.5 & 7.7 & 18.3 \\
\hline Lack of career counselling/vocational training & 5.3 & 6.7 & 7.0 & 6.5 & 5.1 & 6.9 \\
\hline Alcohol/drug abuse & 2.4 & 1.5 & 2.9 & 1.8 & 2.1 & 1.2 \\
\hline Lack of sex education & 0.3 & 0.4 & 0.0 & 0.4 & 0.4 & 0.4 \\
\hline Other ${ }^{1}$ & 2.0 & 0.7 & 1.3 & 0.6 & 2.3 & 0.8 \\
\hline Don't know/can't say & 3.7 & 3.9 & 3.2 & 4.6 & 3.4 & 2.9 \\
\hline Number of respondents & 2,479 & 4,848 & 1,405 & 2,330 & 2,077 & 2,518 \\
\hline
\end{tabular}




\begin{tabular}{|c|c|c|c|c|c|c|}
\hline Leading problem (\%) & $\begin{array}{c}\mathrm{M} \\
15-24\end{array}$ & $\begin{array}{c}W \\
15-24\end{array}$ & $\begin{array}{c}\text { MM } \\
15-29\end{array}$ & $\begin{array}{c}\text { MW } \\
15-24\end{array}$ & $\begin{array}{c}\text { UM } \\
15-24\end{array}$ & $\begin{array}{c}\text { UW } \\
15-24\end{array}$ \\
\hline \multicolumn{7}{|c|}{ Urban } \\
\hline Finding a job/unemployment & 44.8 & 24.5 & 40.8 & 22.9 & 45.6 & 25.9 \\
\hline Poverty & 26.7 & 25.4 & 35.0 & 31.3 & 26.3 & 20.7 \\
\hline $\begin{array}{l}\text { Lack of amenities/infrastructure (water/toilets/ } \\
\text { roads/electricity) }\end{array}$ & 2.4 & 8.0 & 2.6 & 10.5 & 2.7 & 6.1 \\
\hline Health-/health service-related concerns & 0.4 & 1.1 & 0.3 & 0.6 & 0.5 & 1.5 \\
\hline Security of girls/law and order & 0.4 & 6.8 & 1.0 & 3.6 & 0.3 & 9.4 \\
\hline Finding a good spouse/dowry & 0.1 & 7.1 & 0.0 & 6.3 & 0.2 & 7.8 \\
\hline Lack of educational opportunities & 8.5 & 12.6 & 4.9 & 9.5 & 8.4 & 15.0 \\
\hline Lack of career counselling/vocational training & 6.1 & 7.5 & 7.2 & 7.8 & 5.9 & 7.4 \\
\hline Alcohol/drug abuse & 3.1 & 1.1 & 2.9 & 1.3 & 2.7 & 0.9 \\
\hline Lack of sex education & 0.3 & 0.3 & 0.0 & 0.6 & 0.5 & 0.2 \\
\hline Other $^{1}$ & 2.8 & 1.3 & 2.0 & 0.8 & 2.9 & 1.5 \\
\hline Don't know/can't say & 4.2 & 4.2 & 3.3 & 4.8 & 4.1 & 3.7 \\
\hline Number of respondents & 1,289 & 2,126 & 648 & 1,037 & 1,132 & 1,089 \\
\hline \multicolumn{7}{|c|}{ Rural } \\
\hline Finding a job/unemployment & 35.0 & 8.8 & 31.2 & 7.5 & 37.0 & 10.9 \\
\hline Poverty & 40.2 & 32.2 & 43.4 & 33.5 & 38.4 & 30.0 \\
\hline $\begin{array}{l}\text { Lack of amenities/infrastructure (water/toilets/ } \\
\text { roads/electricity) }\end{array}$ & 4.3 & 23.3 & 4.7 & 26.0 & 4.1 & 18.8 \\
\hline Health-/health service-related concerns & 0.6 & 0.9 & 0.0 & 1.2 & 0.7 & 0.5 \\
\hline Security of girls/law and order & 0.1 & 2.2 & 0.3 & 1.2 & 0.1 & 3.8 \\
\hline Finding a good spouse/dowry & 0.1 & 4.2 & 0.3 & 4.0 & 0.1 & 4.6 \\
\hline Lack of educational opportunities & 7.1 & 15.9 & 6.0 & 13.3 & 7.3 & 20.1 \\
\hline Lack of career counselling/vocational training & 5.0 & 6.3 & 6.9 & 6.1 & 4.7 & 6.7 \\
\hline Alcohol/drug abuse & 2.2 & 1.7 & 2.9 & 1.9 & 1.9 & 1.4 \\
\hline Lack of sex education & 0.3 & 0.4 & 0.0 & 0.3 & 0.4 & 0.6 \\
\hline Other $^{1}$ & 1.7 & 0.5 & 1.0 & 0.5 & 2.1 & 0.4 \\
\hline Don't know/can't say & 3.4 & 3.7 & 3.2 & 4.6 & 3.2 & 2.4 \\
\hline Number of respondents & 1,190 & 2,722 & 757 & 1,293 & 945 & 1,429 \\
\hline
\end{tabular}

Note: All Ns are unweighted. Column totals may not equal 100\% due to missing cases. ${ }^{1}$ Includes lack of recreational/sports facilities, lack of political participation, gambling, corruption, child marriage, lack of loan services, limited freedom for girls, social conflicts, generation gap, parents not allowing love marriage, caste differences, etc.

\subsection{Summary}

Findings highlight somewhat limited participation of youth in civil society. Although a number of programmes are organised by the government or NGOs at the community level in which youth can participate, familiarity with these programmes was far from universal. Just two-fifths of young men and two-thirds of young women reported familiarity with these programmes and even fewer youth-19\% of young men $22 \%$ of young women-reported participating in such programmes. Considerably more young men (59\%), but fewer young women (17\%) reported that they had participated in community-led activities such as cleanliness drives, and the celebration of festivals and national days. Finally, just $10 \%$ of young men compared to $26 \%$ of young women reported membership in organised groups. 
Findings suggest that voting behaviour was far from universal. Among those eligible to vote, 62-63\% of young men and women had cast their vote in the most recent election. Also of note is that while most youth (89-90\%) perceived that one could vote freely and without fear and pressure, as many as $60 \%$ of young men and 53\% of young women reported disillusionment with the commitment of political parties to work for change at the community level.

By and large, youth reported secular attitudes; $88-98 \%$ reported that they mixed freely and would eat together with individuals of different religions and castes, and that they would talk to a person who has had an inter-caste marriage. However, only one-third of young men and three-fifths of young women agreed that it was best to tolerate rather than punish someone who had shown disrespect to their religion.

Considerable proportions of young men and women acknowledged that physical fights among young men and also among young women did occur in their villages or urban neighbourhoods. However, few youth-8\% of young men and 3\% of young women-reported that they had been involved in a physical fight in the year preceding the interview.

Youth reported a number of leading problems facing youth and gender differences were evident. Among young men, the majority reported difficulty in finding employment and concerns about poverty more generally as leading problems. In contrast, leading problems expressed by young women were poverty and to a lesser extent, lack of amenities and infrastructure, lack of opportunities for education and difficulty in finding employment. 


\section{Chapter 13}

\section{Looking forward}

Findings of the Youth Study presented in earlier chapters highlight the situation of young men and women in Andhra Pradesh. They underscore the fact that youth are a heterogeneous group with correspondingly diverse needs, and identify numerous challenges youth face in making the transition to adulthood. Findings suggest several priority programmatic areas for action as well as several themes requiring research attention, which are highlighted in this chapter.

\subsection{Recommendations for programmes}

Findings suggest a number of key programme areas for intervention at the youth, family and service delivery levels.

\section{Address obstacles to universal school enrolment and secondary school completion}

Youth Study findings highlight that school enrolment was far from universal among young people in the state: indeed, one in twelve young men and one in five young women had never been enrolled in school. School completion rates were also relatively low, particularly among young women; just $52 \%$ of young men and $36 \%$ of young women had completed high school. The state government has articulated its commitment to improving schooling situation in the state, and what is required now, particularly if the state is to achieve the Millennium Development Goal of ensuring universal primary school completion, are actions to implement these commitments. While the achievement of universal primary school completion is a key goal, the importance of high school education in enabling youth to make a successful transition to adulthood underscores the need, at the same time, for efforts to overcome barriers to high school completion. The gender divide and rural-urban divide observed in school enrolment and completion call, moreover, for efforts that target female children and children in rural areas.

A number of factors have been identified in the Youth Study that inhibit school completion; leading among these were economic reasons; attitudes and perceptions; and, among young women, school-related reasons. Multiple activities are needed to address these barriers. Efforts must be made, for example, to address the economic pressures that dissuade parents from enrolling their children in school and from keeping them in school once enrolled. While a number of state government programmes are ongoing that aim to reduce the cost of education, additional inputs by way of conditional grants that encourage school completion among disadvantaged groups also need to be considered. Moreover, there is a need to ensure that ongoing government programmes do indeed reach the most disadvantaged groups. At the same time, activities are needed that raise awareness among parents about the importance of a secondary school education in expanding their children's livelihood opportunities.

Activities must also address school-level barriers, notably, poor infrastructure, quality of education and academic failure, particularly among young women. There is a need to incorporate livelihoods skills building models within the school setting that will not only raise young people's aspirations regarding their education and careers but also provide them opportunities to gain market-driven job skills. There is also a need to focus on providing better training and ensuring accountability for teachers-investments that are likely to improve the quality of the schooling experience for youth. 
Findings suggesting that married young men and women remain considerably disadvantaged call for interventions that give the married a second chance to continue their education. Likewise, evidence that rural youth, particularly young women, were more disadvantaged with regard to educational opportunities than their urban counterparts calls for efforts to provide those out of school an opportunity to complete their schooling.

\section{Strengthen efforts to prevent child labour}

Findings of the Youth Study that almost two in five young men and women had initiated work in childhood or in early adolescence (before age 15) reiterate the recommendation highlighted above regarding the need to provide conditional grants and targeted subsidies to disadvantaged groups, which would encourage parents to opt for schooling over work for their children. At the same time, it is important to vigorously enforce existing laws that prohibit child labour.

\section{Enable opportunities for employment}

Although unemployment rates were relatively low (7-10\%) among youth, findings that unemployment rates were particularly high among the educated suggest a possible disconnect between youth skills and market needs. Indeed, few youth were aware of employment generation programmes and even fewer had availed of these or vocational skills training. It is notable that while considerable proportions of urban youth reported exposure to computer skills, English language skills and so on, rural youth tended to opt for relatively traditional vocational skills and may not have had the opportunity to learn about market needs or develop appropriate skills for which a demand exists. Formal mechanisms need to be developed that enable youth-and particularly rural youth-to acquire skills for which there is an established market demand, and that link eligible youth to market opportunities.

\section{Build upon youth's growing access to the internet}

Findings suggesting that one quarter of young men and one in ten young women with five or more years of education - and considerably more in urban areas—-had accessed the internet, highlight the role that this medium can play in building youth awareness of the world around them and the opportunities available to them. The development of youth-friendly web-sites in Telugu may be a useful way to convey such information to youth.

Promote agency and gender equitable norms among youth

Findings highlight the limited agency of young women and the persistence of gender double standards among youth. Young women were particularly disadvantaged in terms of school completion, mobility, opportunities to build peer networks, decision-making authority in matters relating to their own lives and control over resources. And although young women were more likely to express equitable gender role attitudes than young men, they were more likely than young men to express traditional attitudes concerning wife beating. These findings call for attention to promote life skills education programmes for young women, both unmarried and married, that will not only raise their awareness of new ideas and the world around them but also enable them to put information into practice, encourage them to question gender stereotypes, develop self-esteem and strengthen their skills in problem-solving, decision-making, communication and inter-personal relations and negotiation. Safe spaces should be identified in which young women can build social networks and find social support among peers.

Interventions intended to build life skills must also be inclusive of young men. Indeed, findings that inegalitarian gender role attitudes were expressed by many young men, on the one hand, and that considerable numbers of young men were not able to exercise agency in their everyday lives, on the other, call for programmes that build their life skills, promote new concepts of masculinity and femininity and at the same time, promote messages that build egalitarian relations between women and men. 
Promoting gender equitable norms and practices requires active engagement with the community. It is essential that programmes for youth work with key community members, such as parents and political and religious leaders in the community, to critically examine prevailing gender norms and forces that perpetuate such norms.

An increasing number of intervention models to build agency and promote egalitarian gender role attitudes among young people have been tested in India. These models could be reviewed and replicated or scaled up as appropriate.

\section{Provide opportunities for formal saving, especially for young women}

Findings suggest that just one-quarter of young men and women had some savings and few youth owned a bank or post office account (just 19\% of young men and $17 \%$ of young women). Despite the fact that young women were as likely as young men to report savings and to own a savings account, they were considerably less likely to operate the account independently. Programmes are needed that inculcate a savings orientation among young people, that offer savings products that are attractive and appropriate to the small and erratic savings patterns of young people and that enable young women in particular to overcome obstacles related to owning and controlling savings products.

\section{Promote youth participation in civil society and political processes and reinforce secular attitudes}

Findings have noted that large proportions of youth have exercised their right to vote, that the majority hold secular attitudes with regard to mixing with a person from another caste and religion, and few engage in community-level violence. Nevertheless, not all youth expressed secular attitudes-two thirds of young men and about two-fifths of young women reported that they would endorse violence against someone who showed disrespect to their religion. Moreover, relatively few had taken part in civil society, that is, government- or NGO-sponsored programmes or community-led activities. Programmes are needed—at the school, college and community levels, through national service programmes, sports and other non-formal mechanisms - that encourage civic participation, incorporate value building components and reinforce secular attitudes and values that espouse responsible citizenship.

Strengthen family life or sex education for those in school and out of school

Youth Study findings provide considerable evidence suggesting that family life or sex education is urgently needed among youth, both for those in school and those who have discontinued their education. Findings demonstrate a limited understanding of sexual and reproductive matters among young people, including the married. Misconceptions abound on most topics: sex and pregnancy, contraceptive methods including condoms, STIs and HIV/AIDS and the conditions under which abortion is legally available or restricted. Where awareness exists, it is superficial in many cases.

Youth themselves have called for family life or sex education. Findings highlight that large proportions of youth recognised the need for information and education on these issues, and indicated a preference for receiving this education from teachers, health care providers or other experts; many youth also indicated a preference for receiving this education from friends, and large proportions of young women, from parents. However, no more than one in three youth had been exposed to family life or sex education, notwithstanding the School AIDS Education Programme, the Red Ribbon Clubs and special programmes for out-of-school youth. Indeed, substantial proportions of married young women (and some young men) reported entering marriage completely unaware of what marriage entailed. At the same time, several young people had engaged in sexual risk taking.

As mentioned above, a number of state government programmes are ongoing that aim to impart sexual and reproductive health information to young people. What is needed is a strong commitment to ensuring that these programmes do indeed reach young people, both those in school and out-of-school, married and unmarried, and rural and urban. Moreover, there is a need to expand the contents of existing awareness raising programmes to include not just HIV-related information but broader sexual and reproductive topics. These programmes should 
be designed not only to raise awareness among youth but also to enable young people to correctly understand and assess the risks they face and to adopt appropriate protective actions.

In addition, special attention needs to be paid to the training of trainers. It is important that teachers, health care providers and other experts undergo training that enables them to overcome their reluctance to communicate with youth on sensitive sexual and reproductive matters, dispels their misconceptions on these matters, and enhances their technical knowledge of these issues. Peers and, in the case of young women, parents have also been identified as acceptable sources of information and efforts must therefore be made that identify youth leaders and build networks of peer educators. Also needed are efforts to engage parents-providing them with accurate information and working with them to overcome inhibitions about imparting this information to their children, particularly their daughters.

\section{Ensure that the transition to sexual life is safe and wanted}

While for the vast majority of young women sexual activity is initiated within the context of marriage, findings show that small proportions of young men and women had engaged in sex before marriage. As documented in this report, many youth had initiated sexual activities uninformed, reiterating the need for providing family life or sex education to young people. Moreover, the finding that for many youth, pre-marital sexual experiences were unsafe, and for some unwanted, calls for programmes that focus on building sexual and reproductive health awareness among young people, and developing their skills in negotiating safe sex and communicating with their partners on sexual and reproductive health matters. At the same time, programmes must make available appropriate family planning and infection prevention services for both married and unmarried young men and women in a manner acceptable to them.

\section{Intensify efforts to eliminate the practice of early marriage}

Findings indicate that although most youth prefer to marry after age 18, the practice of early marriage is widespread among young women. These findings call for measures that go beyond information campaigns to address the underlying factors—social norms and economic constraints—driving early marriage in the state.

There is a need for a multi-pronged approach to eliminate the practice of early marriage. Strategies are needed that mobilise communities to help parents resist pressures that foster the practice of early marriage; that establish new norms and practices; as well as that initiate campaigns highlighting the adverse consequences of early marriage and how it is a violation of the rights of the child. Community mobilisation efforts must involve youth themselves, their families, as well as influential persons in the community, including religious and political leaders.

Equally important is to ensure greater commitment on the part of law enforcement agencies to enforce existing laws on the minimum age at marriage and the registration of marriages, and to levy penalties on violators. Allowing anonymous reporting, making law enforcement agencies and others aware that the practice of early marriage is not a minor violation, and making the guidelines for penalties clear to enforcement agencies and the wider community are possible steps in this direction.

Efforts to delay marriage also require providing girls with viable alternatives to marriage in the form of accessible and quality schooling and opportunities to build and use livelihood skills. Working with the education sector to make schooling for girls more accessible, and to make classrooms gender-sensitive and responsive to the needs of girls and the concerns of their parents is important. At the same time, it is necessary to provide livelihoods training within and outside the educational system.

Findings that most marriages were arranged with the participation of young people themselves are encouraging. However, the finding that few young people had an opportunity to meet their spouse-to-be prior to the wedding day call for actions to sensitise parents of the need to enable their children to interact with their prospective spouse 
prior to the wedding day. Parents must also be made aware of the physical and mental health consequences of early marriage and the adverse experiences of many young women (and some young men) who were married early or who were unprepared for marriage.

\section{Support newly-weds to postpone the first pregnancy}

Findings show that the social pressure to bear children as soon as possible following marriage persists. Contraceptives were rarely used to postpone the first pregnancy and many young women experienced their first pregnancy soon after marriage. It would appear that numerous forces work against delaying the first pregnancy—young people's lack of awareness of appropriate methods of contraception and access to supplies, their limited skills in countering social expectations and negotiating pregnancy postponement, overwhelming pressure from the family and the community to bear children as soon as possible after marriage, and the lack of attention from health care providers.

Programmes are needed that inform youth about their pregnancy postponement options and enable them to access appropriate contraception. At the same time, providers must be trained and charged with the responsibility of reaching married young women and men-including those who have not yet experienced pregnancy-with information regarding contraception and other reproductive health matters as well as contraceptive supplies. Many married young women lack the freedom of movement to seek health care, underscoring the need for health workers to reach these women — particularly those newly married and first time pregnant—in their homes.

\section{Address power imbalances within marriage}

Findings confirm that within marriage, large proportions reported communication and interaction with their spouse; nevertheless communication on sensitive matters such as contraception was limited for many. In addition, married life was marked by violence for many; for example, notable proportions of young women had suffered physical and sexual violence perpetrated by their husband.

Efforts are needed to encourage couple communication on sensitive issues (contraception, for example), negotiation and conflict management skills early in marriage. Efforts are also needed to inform married young women of their rights so that they have the opportunity to exercise control over their own lives; at the same time, efforts must be made to promote new concepts of masculinity and femininity and egalitarian couple relations among young men and women. Intervention models exist in India that have attempted to address these needs; these should be reviewed and up-scaled as appropriate.

\section{Create a supportive family environment}

Findings highlight the limited interaction and social distance between parents and young people while growing up and the gendered nature of perceptions regarding parental controls on youth behaviours. Efforts must be made to create a supportive environment for young people. While evidence on models that are effective in bridging the distance between parents and children or that enable parents to adopt more gender-egalitarian socialisation practices is not currently available, findings presented in this report call for programmes that address parental inhibitions about discussing sexual matters with their children, encourage greater openness and interaction between parents and children, and enable the adoption of gender-egalitarian child-rearing practices. Programmes that aim to encourage universal education or eliminate child marriage must, likewise, address parental concerns about the potential consequences of keeping daughters in school or delaying their marriage.

\section{Reorient service provision to address the unique needs of unmarried and married young women and men}

Although the RCH Programme has advocated special services for youth, including the unmarried, these services had not reached the youth in our survey. For example, relatively small percentages of young people have ever practised contraception and the method most likely to be adopted was sterilisation. Few had sought care for symptoms of 
STI or gynaecological problems, and most youth who had sought care for the latter preferred private to public sector facilities. Lack of care seeking and the disconnect between the public health sector and youth underscores the need to sensitise health care providers about the special needs, heterogeneity and vulnerability of unmarried and married young women and men, and to orient them to the need for developing appropriate strategies to reach these diverse groups, including young newlyweds. It raises, at the same time, the need to explore the feasibility of implementing various financing strategies, for example, health insurance, competitive voucher schemes and community financing schemes, which will allow youth to have a wider choice of providers and enhance the possibility of obtaining quality care.

Programmes must be inclusive of unmarried as well as married young people and recognise their need and right to sexual and reproductive health and related information and services. Counselling and contraceptive services must be made available to all young people, including the unmarried, in a non-threatening, non-judgmental and confidential environment. Indeed, these findings call for the implementation of strategies outlined under the National Rural Health Mission's RCH Programme.

Findings that one in ten young men and women reported symptoms suggestive of mental health disorders suggest that there is a need to establish systems that address young people's mental health. Efforts are needed to screen young people_-particularly the married among whom symptoms were more likely to be reported-for mental health disorders when they avail of other primary health services, including, for example, sexual and reproductive health services, and to refer youth with such symptoms to appropriate health facilities and providers.

\subsection{Directions for future research}

Findings presented in this report provide a broad picture of youth in Andhra Pradesh. At the same time, however, they have raised a number of issues that require further investigation, particularly with regard to the determinants and consequences of youth behaviours and practices during their transition to adulthood. While the Youth Study is indeed a rich source of data that will enable investigators to fill many of the information gaps identified, there are several gaps in knowledge that will require additional research efforts.

A general research recommendation is the urgent need for prospective or panel study designs that follow a cohort of adolescents at regular intervals up to age 24. Thus far, research has relied on cross-sectional data. While these data are valuable in describing the levels and trends in key markers of transitions to adulthood, they rarely capture the ways in which the situation and experiences of youth influence their life courses at later ages. Moreover, drawing causal inferences from cross-sectional surveys has several limitations.

\section{Barriers to school completion}

While evidence presented in this report sheds light on the reasons for school discontinuation, further research is needed that profiles youth at risk of discontinuation, that defines underlying obstacles to school continuation and that identifies strategies to mitigate these barriers. Moreover, even though sizeable proportions of young women reported early transition to marriage as a significant factor for school discontinuation, research is needed that explores whether it is early marriage that curtails schooling for young women or whether it is such reasons as poverty and school-related factors, including access and quality, that lead to school discontinuation and thereby perpetuate early marriage. In addition, operations research is required that evaluates the reach, effectiveness and feasibility of existing programmes, including those implemented by the Andhra Pradesh government, intended to address barriers to school continuation. Moreover, as suggested in the section on recommendations for programmes, a variety of interventions need to be implemented that address school quality issues, enhance parental involvement in children's education, provide the out-of-school, and especially married young women, a second chance to continue their education, provide conditional grants to disadvantaged groups and impart livelihoods skills to young people in school; operations research is required to be in-built in these programmes to assess their effectiveness and reach. 


\section{Transition to work}

Several questions related to transition to work remain unanswered. For example, the finding that unemployment rates were particularly high among the educated calls for further research to better understand the difficulties experienced by educated youth in finding suitable employment opportunities. Further research is needed that explores the links between education attained or vocational training acquired and work patterns. With regard to vocational skills building, research is needed that explores the factors underlying the findings that few young people received vocational skills training even though large proportions were in favour of receiving such training, and that despite the availability of a range of vocational skills training opportunities, many young women continued to opt for training in traditional vocational skills. Equally important is the need for operations research that will test models intended to enable youth to acquire skills for which there is an established market demand and link eligible youth to market opportunities.

\section{Socialisation experiences, interaction with parents}

Youth Study findings are mixed with respect to socialisation experiences and interaction with parents. For example, considerable proportions of young people reported that their parents did not discriminate between sons and daughters in terms of freedom of movement and expectations regarding housework. At the same time, findings suggest that parents were far more controlling of their daughter's behaviours than those of their sons and that far more young women than young men were not allowed to visit locations within or outside the village or neighbourhood unescorted. Qualitative studies would help shed light on these socialisation experiences, and this observed disconnect.

Findings also suggest that parent-child interaction was limited on sensitive sexual and reproductive matters. Little is known, however, about the ways in which limited interaction may influence young people's lives, for example, their sexual behaviours or their ability to exercise informed choices in their lives. Similarly, there is a dearth of research on parents' perspectives on the socialisation of sons and daughters, the ways in which parents communicate sensitive matters to their adolescent children, and the factors inhibiting parents from adopting gender-egalitarian socialisation practices and communicating with their adolescent children on sexual and reproductive matters. As mentioned earlier, there is a need to design and test interventions intended to involve parents more meaningfully in young people's transitions to adulthood in terms of educational attainment, work, marriage and entry into sexual relations.

Findings also suggested that large proportions of youth had witnessed or observed family violence. Research is needed that assesses whether and how these experiences of violence within the family while growing up affects young people's transitions to adulthood.

\section{Sexual risk behaviours}

Research is needed that explores the correlates of behaviours that undermine healthy development among young people, for example, sexual risk behaviours and substance use, and the linkages between them. At the same time, it would be useful to identify the characteristics of youth who make the transition in a safe and healthy way, for example, practise consistent condom use and seek appropriate care.

The Youth Study has raised serious methodological concerns that need to be addressed. For example, despite the fact that the Youth Study did employ such methods as gradual sequencing of questions to include progressively more sensitive topics (with regard to romantic and sexual relationships), anonymous third-party reporting and anonymous sealed envelope reporting; as in many studies pre-marital sexual experience was far less likely to be reported by young women than young men. Moreover, sex worker, exchange, forced and same-sex relationships were rarely reported. Such findings emphasise the need to continue the search for appropriate methodologies to measure sensitive behaviours among youth; computer-assisted survey interviews are one such option. Indeed, methodological studies that compare estimates derived using different approaches could provide an insight into efforts to refine measures of reporting of sensitive behaviours among youth. 


\section{Early marriage and childbearing}

Research is needed that explores the extent to which early marriage compromises young people's lives. For example, does early marriage impede young women's ability to exercise agency in the marital home? How prepared for marriage are those who marry early and how does preparedness or lack thereof influence married life?

Many youth reported that they had not used a contraceptive to delay the first pregnancy and consequently they or their wife had experienced pregnancy soon after marriage. Further research is needed that sheds light on the factors that undermine young people's ability to delay the first pregnancy.

Several promising interventions have been implemented that are intended to address the social isolation experienced by married young women and/or their reproductive health needs, including delaying pregnancy or making pregnancy safe. Few of these interventions have been rigorously evaluated and there is a need for research that assesses the feasibility and possibility of scaling up such interventions.

\section{Partner violence}

Youth Study findings have documented physical and sexual violence perpetrated by young men on their wives, as well as forced sex experienced in romantic and non-romantic situations by a small number of young women prior to marriage. Findings call for research that explores the factors underlying these experiences of violence, documents their health and social consequences for young women and men and their children, and tests interventions that enable youth to prevent such violence, on the one hand, and to overcome obstacles to seeking prompt and appropriate care, on the other.

\section{Family life or sex education}

Findings suggest that in-depth awareness about sexual and reproductive matters was limited, posing an obstacle to the ability of youth to make informed choices. Research is needed that explores the extent to which young people's awareness of sexual and reproductive matters and of sexual and reproductive rights varies according to the sources from which they derive their knowledge. Equally important are studies that examine the sexual and reproductive knowledge and technical competence of those from whom information is sought-including, for example, teachers, health care providers and parents— to communicate sensitive sexual matters to young people.

There has been reticence in several states of the country to impart school-based family life or sex education to youth on the assumption-disproved in some settings - that such education will encourage youth to engage in risky sexual behaviours. Research is needed that explores the extent to which exposure to school- or community-based family life or sex education does indeed enable youth to make informed decisions and adopt safe behaviours in the area of sexual and reproductive health. Research is also needed that explores whether the transition into married life is safer and healthier among those-particularly young women-who were exposed to such education. In addition, operations research is required that evaluates the reach, quality and effectiveness of existing programmes intended to impart sexual and reproductive health information to young people.

\section{Agency and gender role attitudes}

While findings confirm young women's limited agency and gender inegalitarian attitudes held by youth, particularly young men but also young women, several gaps remain in our understanding of the ways in which these affect young people's transitions to adulthood. Further research is needed, for example, that identifies the factors underlying the expression of unequal and equal gender role attitudes by young men and women, particularly the role of parents, teachers, peers and media and that explores the ways in which inegalitarian gender role attitudes and limited agency compromise youth development, including in the area of sexual and reproductive health. 
Methodological issues also arise. There is a need to refine measures of agency as applicable to young men and women. The Youth Study has obtained data on multiple dimensions of agency among young men and women, the married and the unmarried and those from rural and urban areas. These data lend themselves to methodological exercises that measure agency among youth, that assess the extent to which key components of agency may differ across different categories of youth and that explore whether a single summary measure of youth agency can be developed.

\section{Access to and control over resources}

Findings that few youth, irrespective of sex, owned a savings account, and among those who did own an account, young women were far less likely than young men to operate the account independently, call for efforts to map savings and credit options available to youth. At the same time, research is needed that assesses the financial literacy skills of youth, their savings and spending patterns, barriers faced in accessing existing financial products, and ways in which these barriers can be overcome.

\section{Mental health disorders}

Findings suggest that one in 10 youth experienced symptoms suggestive of mental health disorders. Research is needed that explores young people's mental health profile in greater depth and that assesses the linkages between sexual and reproductive health, on the one hand, and mental health, on the other.

\section{Health seeking for sexual and reproductive health symptoms}

Findings suggesting that health care seeking, particularly for sexual and reproductive matters, was limited, highlight the need for research that explores the factors inhibiting youth from seeking care. Youth Study data will enable, as a start, exploration of the factors distinguishing those who sought care from those who did not, in terms of both socio-economic factors as well as parental and peer interaction levels and youth inhibitions about seeking services relating to sexual matters. At the same time, research is needed that assesses the perspectives of providers with regard to the barriers they face in providing services to youth and ways to overcome these barriers.

In brief, the Youth Study has documented, for the first time, the multi-faceted situation of youth in Andhra Pradesh. The study highlights several positive aspects of young people's lives but also alerts us to the many challenges confronting youth and their ability to make a successful transition to adulthood. It emphasises the heterogeneity of youth, not only in terms of their situation but also with regard to their stated needs and preferred mechanisms to address these needs. Programmes must recognise the heterogeneity of young people, and interventions and delivery mechanisms should be appropriately tailored to meet their special needs. Evidence presented here provides not only a blue-print for the programming needs of youth in Andhra Pradesh but also a base-line by which to measure the impact of programmes intended to address youth needs. 


\section{References}

Abraham, L. 2001. "Redrawing the lakshman rekha: Gender differences and cultural constructions in youth sexuality in urban India," South Asia 24:133-56.

Abraham, L. 2002. "Bhai-behen, true love, time pass: Friendships and sexual partnerships among youth in an Indian metropolis," Culture, Health, and Sexuality 4(3):337-53.

Abraham, L. and K.A. Kumar. 1999. "Sexual experiences and their correlates among college students in Mumbai city, India," International Family Planning Perspectives 25(3):139-46.

Alexander, M., L. Garda, S. Kanade et al. 2003. Formation of partnerships among young women and men in Pune district, Maharashtra; Survey Questionnaire, unpublished. New Delhi: Population Council.

Alexander, M., L. Garda, S. Kanade et al. 2006a. Formation of Partnerships among Young Women and Men in Pune District, Maharashtra. New Delhi: Population Council.

Alexander, M., L. Garda, S. Kanade et al. 2006b. "Romance and sex: Pre-marital partnership formation among young women and men, Pune district, India," Reproductive Health Matters 14(28):144-55.

Andhra Pradesh State AIDS Control Society (APSACS). 2005. HIV/AIDS in Andhra Pradesh Situation and Response: A Report. Hyderabad: Government of Andhra Pradesh.

Andrew, G., V. Patel and J. Ramakrishna. 2003. "Sex, studies or strife? What to integrate in adolescent health services," Reproductive Health Matters 11(21):120-29.

Awasthi, S., M. Nichter and V.K. Pande. 2000. "Developing an interactive STD prevention programme for youth: Lessons from a north Indian slum,” Studies in Family Planning 31(2):138-50.

Bashir, K., R. Blizard, R. Jenkins et al. 1996. "Validation of the 12-item General Health Questionnaire in British general practice," Primary Care Psychiatry 2:4-7.

Bhat, P.N.M. and A.J.F. Zavier. 2007. "Factors influencing the use of prenatal diagnostic techniques and sex ratio at birth in India," in Watering the Neighbour's Garden: The Growing Demographic Female Deficit in Asia, eds. I. Attane and C.Z. Guilmoto. Paris: Committee for International Cooperation in National Research in Demography, pp. 131-60.

Bhatia, M.S. and S. Choudhary. 1998. "Dhat symptoms: A culture-bound sex neurosis," Indian Journal of Medical Science 52:30-35.

Bhatia, M.S. and S.C. Malik. 1991. "Dhat syndrome: A useful diagnostic entity in Indian culture," British Journal of Psychiatry 159:691-95.

Bhende, A.A. 1995. "Evolving a model for AIDS prevention education among underprivileged adolescent girls in urban India," Women and AIDS Research Program Report, Series No. 5. Washington, DC: International Centre for Research on Women. 
Bhuiya, I., U. Rob, M.E. Khan et al. 2003. "Reproductive health services for adolescents: Recent experiences from a pilot project in Bangladesh," in Towards Adulthood: Exploring the Sexual and Reproductive Health of Adolescents in South Asia, eds. S. Bott, S.J. Jejeebhoy, I. Shah et al. Geneva: World Health Organisation, pp. 203-6.

Centre for Good Governance. 2007. Strategy for Girl Child Education for Andhra Pradesh. Accessed on 15 October, 2009 at <http://unpan1.un.org/intradoc/groups/public/documents/CGG/UNPAN027150.pdf>.

Chandrasekhar, C.P., J. Ghosh and A. Roychowdhury. 2006. “The 'demographic dividend' and young India's economic future," Economic and Political Weekly 41(49): 5055-63.

Cleland, J. 2001. "Illustrative questionnaire for interview-surveys with young people," in Asking Young People about Sexual and Reproductive Behaviours: Illustrative Core Instruments, eds. J. Cleland, R. Ingham and N. Stone. Geneva: World Health Organisation, pp. 5-16.

Cochran, W.G. 1977. Sampling Techniques. New York: John Wiley \& Sons.

Collumbien, M., N. Bohidar, R. Das et al. 2004. "Etic and emic categories in male sexual health: A case study from Orissa," in Categories and Contexts: Anthropological and Historical Studies in Critical Demography, eds. S. Szreter, H. Sholkamy and A. Dharmalingam. New York: Oxford University Press, pp. 199-221.

Dagar, R. 2007. "Rethinking female foeticide: Perspective and issues," in Sex-selective Abortion in India: Gender,Society and New Reproductive Technologies, ed. T. Patel. New Delhi: Sage Publications, pp. 91-134.

Demographic Research and Development Foundation, Inc. (DRDF) and University of the Philippines Population Institute (UPPI). 2002. Young Adult Fertility and Sexuality Study [Married Female] Questionnaire. Quezon City, Philippines: DRDF and UPPI.

Department of Women Development and Child Welfare, Government of Andhra Pradesh n.d. Adolescent Girls Scheme- Balika Mandals. Accessed on 15 October, 2009 at <http://anganwadi.ap.nic.in/icds_programs.html>.

DiClemente, R.J. 1992. "Psychosocial determinants of condom use among adolescents," in Adolescents and AIDS: A Generation in Jeopardy, ed. R.J. DiClemente. Newbury Park, CA: Sage, pp. 34-51.

Donath, S. 2001. "The validity of the 12-item General Health Questionnaire in Australia: A comparison between three scoring methods," Australian and New Zealand Journal of Psychiatry 35(2):231-35.

Ellickson, P., H. Saner and K.A. McGuigan. 1997. "Profiles of violent youth: Substance use and other concurrent problems," American Journal of Public Health 87:985-91.

Goldberg, D. 1992. General Health Questionnaire (GHQ-12). Windsor: NFER-Nelson.

Government of Andhra Pradesh. 1997. Andhra Pradesh State Population Policy: A Statement and a Strategy. Hyderabad: Department of Health, Medical and Family Welfare, Government of Andhra Pradesh.

Government of Andhra Pradesh. 1999. Andhra Pradesh Vision 2020. Accessed on 18 February, 2007 at $<$ www.aponline.gov.in/vision2020.html $>$.

Government of Andhra Pradesh. 2004. Guidelines and Prevention Education Programme (APEP) 2004-05. Hyderabad: Department of School Education, Government of Andhra Pradesh.

Government of Andhra Pradesh. 2006. State Programme Implementation Plan: Reproductive and Child Health, RCH II. Hyderabad: Department of Health, Medical and Family Welfare, Government of Andhra Pradesh. 
Guttmacher Institute. 2004a. 2004 Ghana National Survey of Adolescents: Survey Questionnaire, unpublished. New York: Guttmacher Institute.

Guttmacher Institute. 2004b. 2004 Malawi National Survey of Adolescents: Survey Questionnaire, unpublished. New York: Guttmacher Institute.

Guttmacher Institute. 2004c. 2004 Uganda National Survey of Adolescents: Survey Questionnaire, unpublished. New York: Guttmacher Institute.

International Institute for Population Sciences (IIPS) and Macro International. 2007a. National Family Health Survey (NFHS-3), 2005-06: India, Volume 1. Mumbai: IIPS.

International Institute for Population Sciences (IIPS) and Macro International. 2007b. National Family Health Survey (NFHS-3), 2005-06: India, Volume II. Mumbai: IIPS.

International Institute for Population Sciences (IIPS) and Macro International. 2008. National Family Health Survey (NFHS-3), India: 2005-06: Andhra Pradesh. Mumbai: IIPS.

International Institute for Population Sciences (IIPS) and Population Council. 2002. First-time Parents project: Baseline Survey Questionnaire, unpublished. Mumbai: IIPS.

International Labour Organisation (ILO). 2006. Global Employment Trends for Youth. Geneva: ILO.

Jacob, K.S., D. Bhugra and A.H. Mann. 1997. "The validation of the 12-item General Health Questionnaire among ethnic Indian women living in the United Kingdom," Psychological Medicine 27(5):1215-17.

Jejeebhoy, S.J. and M.P. Sebastian. 2003. "Actions that protect: Promoting sexual and reproductive health and choice among young people in India," Regional Working Papers, No. 18. New Delhi: Population Council.

Jejeebhoy, S.J. and M.P. Sebastian. 2004. "Young people's sexual and reproductive health," in Looking Back, Looking Forward: A Profile of Sexual and Reproductive Health in India, ed. S.J. Jejeebhoy. New Delhi: Population Council, pp. 138-68.

Khan, S.I., N. Hudson-Rodd, S. Saggers et al. 2006. "Semen contains vitality and heredity, not germs': Seminal discourse in the AIDS era," Journal of Health, Population and Nutrition 24(4):426-37.

Kish, L. 1995. “Methods for design effects,” Journal of Official Statistics 11:55-77.

Laird, R.D., G.S. Pettit, K.A. Dodge et al. 2003. "Change in parents' monitoring knowledge: Links with parenting, relationship quality, adolescent beliefs, and antisocial behaviour," Social Development 12(3):401-19.

Lambert, H. and K. Wood. 2005. "A comparative analysis of communication about sex, health and sexual health in India and South Africa: Implications for HIV prevention,” Culture, Health and Sexuality 7(6):527-41.

Lowry, R., D. Holtzman, B.I. Truman et al. 1994. "Substance use and HIV-related sexual behaviours among US high school students: Are they related?” American Journal of Public Health 84:1116-20.

Marta, E. 1997. "Parent-adolescent interactions and psychosocial risk in adolescents: An analysis of communication, support and gender," Journal of Adolescence 20(5):473-87.

Mehra, S., R. Savithri and L. Coutinho. 2002. "Sexual behaviour among unmarried adolescents in Delhi, India: Opportunities despite parental controls," Paper presented at the IUSSP Regional Population Conference, Bangkok, June. 
Mensch, B.S., D.N. Anh and W.H. Clark. 2000. Adolescents and Social Change in Vietnam: Preliminary Report. Hanoi: Population Council and Institute of Sociology.

Ministry of Finance. 2008. Economic Survey of India 2007-2008. New Delhi: Ministry of Finance, Government of India.

Ministry of Health and Family Welfare (MOHFW). 2000. National Population Policy 2000. New Delhi: Department of Family Welfare, MOHFW, Government of India.

Ministry of Health and Family Welfare (MOHFW). 2005. National Rural Health Mission: Meeting People's Health Needs, Framework for Implementation 2005-2012. New Delhi: MOHFW, Government of India. Accessed on 1 January, 2008 at <www.mohfw.nic.in/NRHM/Documents/NRHM-Framework for Implementation.pdf $>$.

Ministry of Health and Family Welfare (MOHFW). 2006. RCH Phase II: National Programme Implementation Plan. New Delhi: MOHFW, Government of India.

Ministry of Human Resource Development (MHRD), National AIDS Control Organisation (NACO) and United Nations Children's Fund (UNICEF). 2005. Adolescence Education: National Framework and State Action Plan (2005-06). New Delhi: MOHRD, NACO and UNICEF.

Ministry of Human Resource Development (MHRD). 2007. Selected Educational Statistics 2004-2005. New Delhi: Ministry of Human Resource Development, Government of India.

Ministry of Statistics and Programme Implementation. 2008. Statement: Gross State Domestic Product at Current Prices as on 28 February. Accessed 31 March 2009 at <http://mospi.nic.in/6_gsdp_cur_9394 ser.htm>.

Ministry of Youth Affairs and Sports. 2003. National Youth Policy 2003. New Delhi: Government of India.

Mohan, S., P. Sankara Sarma and K.R. Thankappan. 2005. "Access to pocket money and low educational performance predict tobacco use among adolescent boys in Kerala, India," Preventive Medicine 41(2):685-92.

National Institute of Medical Statistics and National AIDS Control Organisation (NACO). 2008. National Behavioural Surveillance Survey (NBSS), 2006; Youth (15-24 Years). New Delhi: NACO, Ministry of Health and Family Welfare, Government of India. Accessed on 5 January, 2009 at <http://www.nacoonline.org/Quick_Links/Publication/ME_ and_Research_Surveillance/Reports_and_Surveys/National_BSS_20062/>.

National Research Council and Institute of Medicine. 2005. Growing Up Global: The Changing Transition to Adulthood in Developing Countries. Washington, DC: National Academies Press.

National Sample Survey Organisation (NSSO). 2006. Employment and Unemployment Situation in India 2004-05, Part 1, NSS 61st Round. New Delhi: NSSO, Government of India.

Office of the Registrar General, India. 2003. Sample Registration System, Statistical Report, 2003. New Delhi: Office of the Registrar General, India.

Office of the Registrar General, India (RGI). 2008a. SRS Based Abridged Life Tables 2002-06. New Delhi: RGI.

Office of the Registrar General, India (RGI). 2008b. Sample Registration System, Statistical Report, 2007, Report No. 2 of 2008. New Delhi: RGI.

Office of the Registrar General and Census Commissioner. 2001a. Age Groups. Accessed on 27 November, 2008 at $<$ http://www.censusindia.gov.in/Census_Data_2001/Census_Data_Online/Social_and_cultural/Age_Groups.aspx $>$. 
Office of the Registrar General and Census Commissioner. 2001b. Primary Census Abstract, Total Population: Table $A-5$, Series 1. New Delhi: Office of the Registrar General and Census Commissioner.

Office of the Registrar General and Census Commissioner. 2001c. Religious Compositions. Accessed on 27 November, 2008 at <http://www.censusindia.gov.in/Census_Data_2001/Census_Data_Online/Social_and_cultural/Religion. $\operatorname{aspx}>$.

Office of the Registrar General and Census Commissioner, 2001d. Duration of Residence for Migrants by Last Residence. Accessed on 27 November, 2008 at <http://www.censusindia.gov.in/Census_Data_2001/Census_Data_Online/ Online_Migration/Duration_of_Residence_By_LastResidence.aspx>.

Office of the Registrar General and Census Commissioner. 2001e. Educational Level by Age and Sex for Population Aged 7 and Above, Table C-8. New Delhi: Office of the Registrar General and Census Commissioner.

Office of the Registrar General and Census Commissioner. 2006. Population Projections for India and States 2001-2026 (Revised December 2006). New Delhi: Office of the Registrar General and Census Commissioner.

Patel, V. and G. Andrew. 2001. "Gender, sexual abuse and risk behaviours: A survey of school-based adolescents in Goa," National Medical Journal of India 14:263-67.

Pelto, P.J. 1999. "Sexuality and sexual behaviour: The current discourse," in Implementing a Reproductive Health Agenda in India: The Beginning, ed. S. Pachauri. New Delhi: Population Council, pp. 539-86.

Planning Commission. 2002. Tenth Five Year Plan (2002-2007), Volume II, Sectoral Policies and Programmes. New Delhi: Government of India.

Planning Commission. 2006. Towards Faster and More Inclusive Growth: An Approach to the 11th Five Year Plan (2007-2012). New Delhi: Government of India.

Planning Commission. 2007. Poverty Estimates for 2004-05, Press release, March. New Delhi: Government of India.

Planning Department, Government of Andhra Pradesh. 2007. Socio Economic Survey 2006-07. Accessed on 27 November, 2008 at <http://www.aponline.gov.in/Apportal/SocioSurvery0607/economic_Survey.html>.

Ram, F., R.K. Sinha, S.K. Mohanty et al. 2006. Marriage and Motherhood: An Exploratory Study of the Social and Reproductive Health Status of Married Young Women in Gujarat and West Bengal, India. New Delhi: Population Council.

Rossier, C. 2003. “Estimating induced abortion rates: A review”, Studies in Family Planning 34(2):87-102.

Sachdev, P. 1998. "Sex on campus: A preliminary study of knowledge, attitudes and behaviour of university students in Delhi, India," Journal of Biosocial Science 30:95-105.

Santhya, K.G. and S.J. Jejeebhoy. 2006. "Young women's experience of forced sex within marriage: Evidence from India," in Sex without Consent: Young People in Developing Countries, eds. S.J. Jejeebhoy, I. Shah and S. Thapa. London: Zed Books, pp. 59-73.

Santhya, K.G., N. Haberland, F. Ram et al. 2007. "Consent and coercion: Examining unwanted sex among married young women in India," International Family Planning Perspectives 33(3):124-32.

Santhya, K.G. and S.J. Jejeebhoy. 2007. Young people's sexual and reproductive health I India: Policies, programmes and realities. Regional Working Paper, No. 19, New Delhi, Population Council. 
Santhya, K.G., S.J. Jejeebhoy and S. Ghosh. 2008. Early Marriage and Sexual and Reproductive Health Risks: Experiences of Young Women and Men in Andhra Pradesh and Madhya Pradesh, India. New Delhi: Population Council.

Sathar, Z.A., M. Haque, A. Faizunnissa et al. 2003. Adolescents and Youth in Pakistan, 2001-02. Islamabad: Population Council.

Sebastian, M.P., M. Grant and B. Mensch. 2005. Integrating Adolescent Livelihood Activities Within a Reproductive Health Programme for Urban Slum Dwellers in India. New Delhi: Population Council.

Sebastian, M.P., M. Grant, B. Mensch et al. 2003. Integrating adolescent livelihood activities within a reproductive health programme for urban slum dwellers in India, Survey Questionnaire, unpublished. New Delhi: Population Council.

Singh, S. and S. Saini. 2007. "HIV risk perception in relation to peer pressure and drug abuse behavior among adolescents," Indian Journal of Sexually Transmitted Diseases 28:53-54.

Sroufe, J.W. 1991. "Assessment of parent-adolescent relationships: Implications for adolescent development," Journal of Family Psychology 5(1):21-45.

Ul Haque, M. and A. Faizunnisa. 2003. "Access to reproductive health information in Punjab and Sindh, Pakistan: The perspectives of adolescents and parents," in Towards Adulthood: Exploring the Sexual and Reproductive Health of Adolescents in South Asia, eds. S. Bott, S.J. Jejeebhoy, I. Shah et al. Geneva: World Health Organisation, pp. 153-55.

United Nations Development Programme (UNDP). 2000. Millennium Development Goals. United Nations website. Accessed on 8 January, 2008 at $<$ http://www.un.org/millenniumgoals/goals.html $>$.

Verma, R.K., S. Sharma, R. Singh et al. 2003. "Beliefs concerning sexual health problems and treatment seeking among men in an Indian slum community," Culture, Health and Sexuality 5(3):265-76.

Wolter, K.M. 1985. Introduction to Variance Estimation. New York: Springer. 


\section{Appendix A \\ Principal investigators and authors}

Usha Ram, Associate Professor, Department of Public Health and Mortality Studies, International Institute for Population Sciences, Mumbai

S.K. Mohanty, Reader, Department of Fertility Studies, International Institute for Population Sciences, Mumbai

Abhishek Singh, Assistant Professor, Department of Public Health and Mortality Studies, International Institute for Population Sciences, Mumbai

F. Ram, Director and Senior Professor, International Institute for Population Sciences, Mumbai

K.G. Santhya, Associate, Population Council, New Delhi

Shireen J. Jejeebhoy, Senior Associate, Population Council, New Delhi

Rajib Acharya, Senior Programme Officer, Population Council, New Delhi 


\section{Appendix A \\ Andhra Pradesh study staff}

Senior Research Officers

Shreeparna Ghosh

Research Officers

P. Ganesh

K.S. Bharat Kumar

S. Jareena

C. Latha

Himanshu Katyan

Santosh Kumar Singh

Abhishek Kumar

Prashant Kumar Singh

Rajesh Kumar

Assistant Research Officers

B. Sd. Imran

E. Suneetha

T. Pradeep Kumar

Finance Staff

Jeba Kumar

Anil Paul

Administrative Staff

Nazrul Haque Ansari

Avadhesh Prajapati

Pranita Dalvi

Namrata Ghadge

M.A. Jose

Shyam V. Rathod

Komal Saxena

Usha Sonavane

\section{Editorial Staff}

Shanti Menon

Data Entry Operators

Sumita D. Choudhary

Aparna R Narvekar

Sarika D. Dethe

Manisha D. Esave

Sayali P. Shivalkar

Sarita S. Mohane

Prasad Surve 
Field Supervisor
S. K. Ameenasaheb
B. Ravindranath
S. K. Bhasha
G. Ashok Reddy
K. Narendra Kumar
M. Srinivasulu Reddy
S. Narayana Murthy
T. Suresh
C. Bala Ram
P. Yadaiah

S. Venkata Rao

\section{Field Editor}

P. Suresh Babu

B. Bharthi

K. Chakrapani

M. Dhanlakshmi

M. Gattaiah

N. Aruna Jyothi

Office Editors

Rakesh M. Pawar

Household Listers and Mappers

P. Baskar

R. Obularao Bukka

R. Chandrappa

Raju Doddi

Hemraj Daima

M. Dalaiah

Dongari Govandhan

Chavali Hemachandar

G. Hema Santosh Kumar

A. Dilip Kumar

Field Investigators

P. Satish Babu

Shiv Chandra

B. Laxmi Devi

G. Narsim Das

Chandrashekhar Goud

T. Grace

M. Gurudevi

K.Hemalatha

B. Hemavathi

R. Haritha

T. Jayamani

P. Geetha Kumari

V. Aravind Kumar

A. Lakshmi
B. Aruna Kumari

M. Ratna Mala

N. Govinda Reddy

M. Satish Reddy

G. Sridevi

Shantanu G. Sawant

Avvaru Pundari Kaksha Kundavaram Obaiah

Hari Prasad

Chelli Raju

S. Ramanjaneyulu Challa Ramana

A. Nagesgwar Rao Kiran Kumar Soma

A. Venu

K. Roja

V. Rajitha

P. Rajesh

G. Solomon Raju

N. Chandrashekhara Rao

T. Eswara Rao

T. Rama Rao

P. Rama Rao

V. Sanyasi Rao

T. Govinda Rao

R. Ramesh

P. Ramesh

G. Rukmini

K. Sailaja 


\author{
S. Malathi Latha \\ V. Madhavi \\ B. Uma Maheshwari \\ A. G. Manorama \\ Irshad Mohammad \\ M. Nagaraju \\ Ashok Nagilla \\ A. Surya Narayana \\ M. Venkat Narayana \\ D. Sanyasi Naidu \\ P. Nirmala \\ E. Paleena \\ G. Padma \\ V. Surya Prakash \\ G. Kumar Raja \\ P. Rajeswari \\ Ch. Upendra Rao
}

\author{
P. Raja Shekhar \\ P. Simhachalam \\ V. Srinivas \\ K. Sumanlatha \\ T. Sushma \\ Y. Sujatha \\ S. Kumara Swamy \\ Nirmala Tandi \\ P. Trimurthulu \\ Preeti Devi Verma \\ V. Vani \\ S. Venu \\ M. Yadiah \\ N. Yesuprema
}




\section{Advisory committees}

\section{Project Advisory Committee}

Additional Secretary

Ministry of Health and Family Welfare

Government of India

\section{Chief Director}

Ministry of Health and Family Welfare

Government of India

Deputy Secretary

Information, Education, Communication

Ministry of Health and Family Welfare

Government of India

\section{Director}

Directorate of Health Services

Government of Andhra Pradesh

\section{Director}

Directorate of Health Services

Government of Bihar

\section{Director}

Directorate of Health Services

Government of Jharkhand

Director

Directorate of Health Services

Government of Maharashtra

Director

Directorate of Health Services

Government of Rajasthan

\section{Director}

Directorate of Health Services

Government of Tamil Nadu

\section{Director}

Nehru Yuvak Kendra Sangatan

Ministry of Sports and Youth Affairs

Government of India

\section{Director}

National Institute of Health and Family Welfare,

New Delhi

Country Director (India)

John D. and Catherine T. Mac Arthur Foundation, New Delhi

\section{Country Programme Advisor (India)}

David and Lucile Packard Foundation,

New Delhi

\section{Director}

International Institute for Population Sciences, Mumbai

\section{Regional Director}

Population Council, New Delhi

\section{NFHS Coordinator}

International Institute for Population Sciences, Mumbai

Shalini Bharat

Professor and Dean

School of Health Systems Studies

Tata Institute of Social Sciences, Mumbai

P.M. Kulkarni

Professor

Centre for the Study of Regional Development Jawaharlal Nehru University, New Delhi 
Arvind Pandey

Director

Institute for Research in Medical Statistics, New Delhi

G. Rama Rao

Former Officiating Director

International Institute for Population Sciences, Mumbai

\section{Technical Advisory Committee}

Shalini Bharat

Professor and Dean

School of Health Systems Studies

Tata Institute of Social Sciences, Mumbai

Director

International Institute for Population Sciences, Mumbai

P.M. Kulkarni

Professor

Centre for the Study of Regional Development Jawaharlal Nehru University, New Delhi

Arvind Pandey

Director

Institute for Research in Medical Statistics, New Delhi
T.K. Roy

Former Director

International Institute for Population Sciences, Mumbai

Leela Visaria

National Professor

Gujarat Institute of Development Research, Ahmedabad

Pertti J. Pelto

Professor Emeritus

Medical Anthropology

University of Connecticut, USA

T.K. Roy

Former Director

International Institute for Population Sciences, Mumbai

Leela Visaria

National Professor

Gujarat Institute of Development Research, Ahmedabad 


\section{Estimates of sampling errors}

As in the case of any sample survey, estimates from the Youth Study in Andhra Pradesh, as presented in Chapters 1-12, are affected by two major sources of errors: non-sampling and sampling errors. Non-sampling errors are generally the result of procedural mistakes made during data collection and data processing, such as, the inability to locate and interview the correct household or individual, failure to conform to standard survey procedures laid out by the central office, misunderstanding of questions on the part of either the interviewer or the respondent, and data entry errors. At the same time, because of the inclusion of numerous sensitive issues, the Youth Study faced the risk of other non-sampling errors as well, such as, the deliberate skipping of sensitive questions by the interviewer or refusal to answer sensitive questions by the respondent. In order to minimise non-sampling errors, a number of precautions were taken during the implementation of the study, which are described in detail in Chapter 1 . However, we acknowledge that despite these efforts, non-sampling errors are impossible to avoid; they are, moreover, extremely difficult to evaluate statistically.

Sampling errors, on the other hand, can be evaluated statistically. These errors, as the name suggests, result from the choice of the particular sample selected. The sample of respondents selected in the Youth Study is only one of many possible samples that could have been selected from the population of Andhra Pradesh, using the same design and expected sample size. Each of these samples would have yielded results that differed somewhat from the results of the sample selected. The sampling error is a measure of variability among all possible samples. Although the degree of variability may not be known exactly, it can be estimated from the survey results using standard statistical procedures.

A sampling error, usually measured in terms of the standard error for a particular statistic (mean, percentage, ratio, etc.), is the square root of the variance of that statistic. The standard error can be used to calculate confidence intervals within which the true value for the population can reasonably be assumed to fall. For example, for any given statistic calculated from the survey, the value of that statistic will fall within a range of plus or minus two times the standard error of that statistic in $95 \%$ of all possible samples of identical size and design.

If the sample of respondents had been selected as a simple random sample, it would have been possible to use straightforward formulae for calculating the variance of the statistic and consequently, sampling errors. However, the Youth Study sample is the result of a multi-stage stratified design, and consequently, it was necessary to use more complex formulae. The variance estimators that were used can be found in Cochran (1977) and Wolter (1985). The computer software used to calculate sampling errors for the Youth Study was programmed in STATA SE 8.2. This procedure uses the Taylor linearisation method for variance estimation for survey estimates that are means, proportions or ratios.

The Taylor linearisation method treats any percentage or average as a ratio estimate. Let $r=y / x$ be our sample estimate of the population ratio (mean or percentage) denoted by $\mathrm{R}=\mathrm{Y} / \mathrm{X}$, where $\mathrm{y}$ represents the total sample value for variable $\mathrm{Y}$, and $\mathrm{x}$ represents the total number of sample cases in the group or sub-group under consideration. 
Using first order Taylor expansion, it can be shown that the approximate variance of distribution of $r$ (square root of which is the standard error) is as below:

$$
\operatorname{Var}(r)=\frac{1-f}{x^{2}} \sum_{h=1}^{L}\left[\frac{n_{h}}{n_{h}-1}\left(\sum_{i=1}^{n_{h}} z_{h i}^{2}-\frac{z_{h}^{2}}{n_{h}}\right)\right]
$$

in which $\mathrm{z}_{h i}=\mathrm{y}_{h i}-\mathrm{rx}_{h i}$ and $\mathrm{z}_{h}=\mathrm{y}_{h}-\mathrm{rx}_{h}$

where $h$ represents the sampling stratum which varies from 1 to $\mathrm{L}$,

$n_{h} \quad$ is the number of PSUs selected in the $\mathrm{h}^{\text {th }}$ stratum,

$y_{h i} \quad$ is the sum of the weighted values of variable $\mathrm{Y}$ in the $\mathrm{i}^{\text {th }} \mathrm{PSU}$ in the $\mathrm{h}^{\text {th }}$ stratum,

$x_{h i} \quad$ is the sum of the weighted number of cases in the $\mathrm{i}^{\text {th }}$ PSU in the $\mathrm{h}^{\text {th }}$ stratum,

$f \quad$ is the overall sampling fraction, which is so small that it is ignored.

In addition to the standard error, the design effect (DEFT) for each estimate was also computed, which is defined as the ratio between the standard error using the given sample design and the standard error that would result if a simple random sample had been used (Kish, 1995) represented by the following simple formula:

$$
D E F T=\sqrt{\frac{\operatorname{Var}(r)}{\operatorname{Var}_{\text {srswr }}\left(r_{\text {srs }}\right)}}
$$

where $\operatorname{Var}(r)$ is a design-based estimate of variance for the parameter $r$, $\operatorname{Var}_{\text {srswr }}\left(r_{\text {srs }}\right)$ is an estimate of the variance for an estimator $r_{\text {srs }}$ that would be obtained from a similar hypothetical survey conducted using simple random sampling (srs) with replacement (wr).

A DEFT value of 1.0 indicates that the sample design is as efficient as a simple random sample, while a value greater than 1.0 indicates the increase in the sampling error due to the use of a more complex and less statistically efficient design. Relative standard error (SE/R) and 95\% confidence limits for each estimate were also computed.

Sampling errors for the Youth Study were calculated for selected variables and results are presented in this appendix for each sex and marital status sub-group of respondents for the state as a whole, and for those in urban and rural areas, respectively. For each variable, the type of statistic (mean, proportion or ratio) and the base population are given in Table B.1. Table B.2 presents the value of the statistic (R), its standard error (SE), the number of unweighted $(\mathrm{N})$ and weighted $(\mathrm{WN})$ cases, the design effect (DEFT), the relative standard error (SE/R) and the 95\% confidence limits, for each variable. 
Table B.1: List of selected variables for sampling errors, Andhra Pradesh, 2007-08

\begin{tabular}{|c|c|c|}
\hline Variables & Estimates & Base Population \\
\hline Sex ratio (females per 1,000 males) & Ratio & De jure household population, all ages \\
\hline Sex ratio (females per 1,000 males) & Ratio & De jure household population, aged 0-6 \\
\hline $\begin{array}{l}\text { Currently married, including married but not yet } \\
\text { cohabiting }\end{array}$ & Proportion & De jure household population, aged $20-24$ \\
\hline No education & Proportion & De jure household population, aged 6 or above \\
\hline No education & Proportion & Young men and women \\
\hline Completed 12 or more years of education & Proportion & Young men and women \\
\hline Ever worked in last 12 months & Proportion & Young men and women \\
\hline Unemployed & Proportion & Young men and women in labour force \\
\hline Discussed friendships with father & Proportion & $\begin{array}{l}\text { Young men and women whose father was alive } \\
\text { at the time of interview }\end{array}$ \\
\hline Discussed friendships with mother & Proportion & $\begin{array}{l}\text { Young men and women whose mother was alive } \\
\text { at the time of interview }\end{array}$ \\
\hline $\begin{array}{l}\text { Independently makes decisions on choice of friends, } \\
\text { spending money and buying clothes for oneself }\end{array}$ & Proportion & Young men and women \\
\hline $\begin{array}{l}\text { Can visit places outside village or neighbourhood } \\
\text { unescorted }\end{array}$ & Proportion & Young men and women \\
\hline Has savings of any amount & Proportion & Young men and women \\
\hline Justified wife beating in at least one situation & Proportion & Young men and women \\
\hline Awareness of sex- and pregnancy-related matters & Proportion & Young men and women \\
\hline $\begin{array}{l}\text { Correct specific knowledge of at least one } \\
\text { contraceptive method }\end{array}$ & Proportion & Young men and women \\
\hline Correct specific knowledge of condoms & Proportion & Young men and women \\
\hline Ever heard of HIV/AIDS & Proportion & Young men and women \\
\hline Comprehensive knowledge of HIV/AIDS & Proportion & Young men and women \\
\hline Ever heard of STIs other than HIV & Proportion & Young men and women \\
\hline $\begin{array}{l}\text { Correct knowledge of the conditions under which } \\
\text { abortion is legal }\end{array}$ & Proportion & Young men and women \\
\hline Ever received family life or sex education & Proportion & Young men and women \\
\hline Ever had an opposite-sex romantic partner & Proportion & Young men and women \\
\hline Ever had sex with an opposite-sex romantic partner & Proportion & Young men and women \\
\hline Ever had pre-marital sex & Proportion & Young men and women \\
\hline Used condom consistently in pre-marital relations & Proportion & $\begin{array}{l}\text { Young men and women who reported } \\
\text { pre-marital sex in face-to-face interview }\end{array}$ \\
\hline Ever communicated with spouse on contraception & Proportion & $\begin{array}{l}\text { Married young men and women who had } \\
\text { begun cohabiting }\end{array}$ \\
\hline Husband ever forced wife to have sex & Proportion & $\begin{array}{l}\text { Married young men and women who had } \\
\text { begun cohabiting }\end{array}$ \\
\hline Husband ever perpetrated physical violence on wife & Proportion & $\begin{array}{l}\text { Married young men and women who had } \\
\text { begun cohabiting }\end{array}$ \\
\hline $\begin{array}{l}\text { Husband ever perpetrated physical violence on wife in } \\
\text { last } 12 \text { months }\end{array}$ & Proportion & $\begin{array}{l}\text { Married young men and women who had } \\
\text { begun cohabiting }\end{array}$ \\
\hline Currently using any modern contraceptive method & Proportion & $\begin{array}{l}\text { Married young men and women who had } \\
\text { begun cohabiting }\end{array}$ \\
\hline
\end{tabular}


Table B.1: (Cont'd)

\section{Variables}

First delivery in a health institution

Mean number of children ever born

Mean number of children surviving

Mean ideal number of children

Experienced 3 or more symptoms or behaviours suggestive of mental health disorders in the month preceding the interview

Ever consumed alcohol

Participated in a government- /NGO-sponsored programme in the 3 years preceding the interview Voted in last election

\section{\begin{tabular}{l|l} 
Estimates & Base Population
\end{tabular}}

Proportion Married young men and women whose first pregnancy outcome was a live or still birth

Mean $\quad$ Married young men and women who had begun cohabiting

Mean $\quad$ Married young men and women who had begun cohabiting

Mean

Proportion Young men and women

Married young men and women who had begun cohabiting and gave a numeric response

Proportion

Young men and women

Proportion

Young men and women

Proportion Young men and women, aged 20 or above 
Table B.2: Sampling errors, Andhra Pradesh, 2007-08

\begin{tabular}{|c|c|c|c|c|c|c|c|c|}
\hline \multirow{2}{*}{$\begin{array}{l}\text { Variable/ } \\
\text { respondent } \\
\text { category }\end{array}$} & \multirow{2}{*}{$\begin{array}{l}\text { Value } \\
(\mathbf{R})\end{array}$} & \multirow{2}{*}{$\begin{array}{c}\text { Standard } \\
\text { error } \\
\text { (SE) }\end{array}$} & \multicolumn{2}{|c|}{ Number of cases } & \multirow{2}{*}{$\begin{array}{c}\text { Design } \\
\text { effect } \\
\text { (DEFT) }\end{array}$} & \multirow{2}{*}{$\begin{array}{l}\text { Relative } \\
\text { standard } \\
\text { error } \\
(\mathrm{SE} / \mathrm{R})\end{array}$} & \multicolumn{2}{|c|}{ 95\% Confidence limits } \\
\hline & & & $\begin{array}{l}\text { Unweighted } \\
(\mathbf{N})\end{array}$ & $\begin{array}{l}\text { Weighted } \\
(\mathbf{W N})\end{array}$ & & & Lower & Upper \\
\hline \multicolumn{9}{|c|}{ Sex ratio } \\
\hline Combined & 1.0183 & 0.0061 & 63,011 & 62,621 & 1.5147 & 0.0060 & 1.0063 & 1.0303 \\
\hline Urban & 0.9888 & 0.0095 & 25,743 & 16,821 & 1.4835 & 0.0096 & 0.9702 & 1.0074 \\
\hline Rural & 1.0291 & 0.0076 & 37,268 & 45,798 & 1.4662 & 0.0074 & 1.0142 & 1.0441 \\
\hline \multicolumn{9}{|c|}{$\begin{array}{l}\text { Sex ratio } \\
\text { de jure household population, aged } 0-6 \text { ) }\end{array}$} \\
\hline Combined & 0.9696 & 0.0177 & 7,378 & 7,473 & 1.1010 & 0.0183 & 0.9347 & 1.0045 \\
\hline Urban & 0.9646 & 0.0260 & 2,940 & 1,920 & 1.0194 & 0.0270 & 0.9134 & 1.0158 \\
\hline Rural & 0.9713 & 0.0221 & 4,438 & 5,553 & 1.0648 & 0.0228 & 0.9278 & 1.0148 \\
\hline \multicolumn{9}{|c|}{$\begin{array}{l}\text { Currently married, including married but not yet cohabiting } \\
\text { (de jure household population, aged } 20-24 \text { ) }\end{array}$} \\
\hline \multicolumn{9}{|c|}{ Combined } \\
\hline Male & 0.2846 & 0.0104 & 5,827 & 5,568 & 1.7546 & 0.0364 & 0.2641 & 0.3050 \\
\hline Female & 0.7575 & 0.0080 & 5,826 & 5,824 & 1.4333 & 0.0106 & 0.7416 & 0.7733 \\
\hline \multicolumn{9}{|c|}{ Urban } \\
\hline Male & 0.1602 & 0.0118 & 2,560 & 1,659 & 1.6325 & 0.0739 & 0.1369 & 0.1835 \\
\hline Female & 0.6329 & 0.0139 & 2,534 & 1,672 & 1.4555 & 0.0220 & 0.6054 & 0.6603 \\
\hline \multicolumn{9}{|c|}{ Rural } \\
\hline Male & 0.3374 & 0.0140 & 3,267 & 3,908 & 1.6921 & 0.0415 & 0.3098 & 0.3649 \\
\hline Female & 0.8077 & 0.0099 & 3,292 & 4,153 & 1.4446 & 0.0123 & 0.7881 & 0.8272 \\
\hline
\end{tabular}

\begin{tabular}{|c|c|c|c|c|c|c|c|c|}
\hline & & (de jure & $\begin{array}{r}\text { No } \\
\text { sehold }\end{array}$ & $\begin{array}{l}\text { cation } \\
\text { lation, a }\end{array}$ & 6 or abo & & & \\
\hline & & & & ined & & & & \\
\hline Male & 0.2919 & 0.0062 & 56,812 & 56,337 & 3.2297 & 0.0211 & 0.2798 & 0.3041 \\
\hline Female & 0.4735 & 0.0069 & 57,049 & 57,776 & 3.2942 & 0.0145 & 0.4600 & 0.4871 \\
\hline & & & & & & & & \\
\hline Male & 0.1516 & 0.0081 & 23,292 & 15,218 & 3.4544 & 0.0535 & 0.1356 & 0.1676 \\
\hline Female & 0.3010 & 0.0105 & 22,972 & 15,087 & 3.4785 & 0.0350 & 0.2803 & 0.3217 \\
\hline & & & & & & & & \\
\hline Male & 0.3439 & 0.0080 & 33,520 & 41,120 & 3.0888 & 0.0233 & 0.3281 & 0.3596 \\
\hline Female & 0.5345 & 0.0089 & 34,077 & 42,689 & 3.2797 & 0.0166 & 0.5170 & 0.5519 \\
\hline
\end{tabular}


Table B.2: (Cont'd)

\begin{tabular}{|c|c|c|c|c|c|c|c|c|}
\hline \multirow{2}{*}{$\begin{array}{l}\text { Variable/ } \\
\text { respondent } \\
\text { category }\end{array}$} & \multirow{2}{*}{$\begin{array}{l}\text { Value } \\
(\mathbf{R})\end{array}$} & \multirow{2}{*}{$\begin{array}{l}\text { Standard } \\
\text { error } \\
\text { (SE) }\end{array}$} & \multicolumn{2}{|c|}{ Number of cases } & \multirow{2}{*}{$\begin{array}{l}\text { Design } \\
\text { effect } \\
\text { (DEFT) }\end{array}$} & \multirow{2}{*}{$\begin{array}{c}\text { Relative } \\
\text { standard } \\
\text { error } \\
(\mathrm{SE} / \mathrm{R})\end{array}$} & \multicolumn{2}{|c|}{ 95\% Confidence limits } \\
\hline & & & $\begin{array}{c}\text { Unweighted } \\
(\mathbf{N})\end{array}$ & $\begin{array}{l}\text { Weighted } \\
\text { (WN) }\end{array}$ & & & Lower & Upper \\
\hline \multicolumn{9}{|c|}{ No education (young men and women) } \\
\hline \multicolumn{9}{|c|}{ Combined } \\
\hline M (15-24) & 0.0779 & 0.0083 & 2,479 & 2,504 & 1.5438 & 0.1067 & 0.0615 & 0.0942 \\
\hline W (15-24) & 0.2079 & 0.0141 & 4,848 & 4,848 & 2.4146 & 0.0677 & 0.1802 & 0.2356 \\
\hline MM (15-29) & 0.2107 & 0.0189 & 1,405 & 1,405 & 1.7391 & 0.0898 & 0.1735 & 0.2480 \\
\hline MW (15-24) & 0.3127 & 0.0199 & 2,330 & 2,330 & 2.0760 & 0.0638 & 0.2734 & 0.3520 \\
\hline UM (15-24) & 0.0514 & 0.0068 & 2,077 & 2,077 & 1.3978 & 0.1318 & 0.0381 & 0.0647 \\
\hline UW (15-24) & 0.0719 & 0.0075 & 2,518 & 2,518 & 1.4618 & 0.1047 & 0.0571 & 0.0868 \\
\hline \multicolumn{9}{|c|}{ Urban } \\
\hline M (15-24) & 0.0482 & 0.0081 & 1,289 & 706 & 1.3484 & 0.1669 & 0.0324 & 0.0641 \\
\hline W (15-24) & 0.1002 & 0.0137 & 2,126 & 1,434 & 2.1033 & 0.1368 & 0.0732 & 0.1271 \\
\hline MM (15-29) & 0.1371 & 0.0163 & 648 & 306 & 1.2056 & 0.1189 & 0.1050 & 0.1692 \\
\hline MW (15-24) & 0.1721 & 0.0214 & 1,037 & 525 & 1.8271 & 0.1245 & 0.1299 & 0.2143 \\
\hline UM (15-24) & 0.0335 & 0.0067 & 1,132 & 631 & 1.2607 & 0.2013 & 0.0202 & 0.0468 \\
\hline UW (15-24) & 0.0419 & 0.0086 & 1,089 & 909 & 1.4096 & 0.2044 & 0.0250 & 0.0587 \\
\hline \multicolumn{9}{|c|}{ Rural } \\
\hline$M(15-24)$ & 0.0895 & 0.0110 & 1,190 & 1,799 & 1.3299 & 0.1230 & 0.0678 & 0.1111 \\
\hline $\mathrm{W}(15-24)$ & 0.2532 & 0.0186 & 2,722 & 3,414 & 2.2332 & 0.0735 & 0.2165 & 0.2898 \\
\hline MM (15-29) & 0.2313 & 0.0236 & 757 & 1,099 & 1.5359 & 0.1018 & 0.1849 & 0.2777 \\
\hline MW (15-24) & 0.3536 & 0.0245 & 1,293 & 1,805 & 1.8447 & 0.0694 & 0.3053 & 0.4019 \\
\hline UM (15-24) & 0.0592 & 0.0093 & 945 & 1,446 & 1.2048 & 0.1563 & 0.0410 & 0.0774 \\
\hline UW (15-24) & 0.0889 & 0.0106 & 1,429 & 1,609 & 1.4109 & 0.1195 & 0.0680 & 0.1098 \\
\hline \multicolumn{9}{|c|}{ Completed 12 or more years of education (young men and women) } \\
\hline \multicolumn{9}{|c|}{ Combined } \\
\hline$M(15-24)$ & 0.2274 & 0.0126 & 2,479 & 2,504 & 1.5008 & 0.0556 & 0.2025 & 0.2523 \\
\hline W (15-24) & 0.1439 & 0.0087 & 4,848 & 4,848 & 1.7333 & 0.0607 & 0.1267 & 0.1611 \\
\hline MM (15-29) & 0.1436 & 0.0119 & 1,405 & 1,405 & 1.2698 & 0.0828 & 0.1202 & 0.1670 \\
\hline MW (15-24) & 0.0691 & 0.0065 & 2,330 & 2,330 & 1.2277 & 0.0934 & 0.0564 & 0.0818 \\
\hline UM (15-24) & 0.2579 & 0.0137 & 2,077 & 2,077 & 1.4260 & 0.0531 & 0.2309 & 0.2848 \\
\hline UW (15-24) & 0.2376 & 0.0131 & 2,518 & 2,518 & 1.5383 & 0.0549 & 0.2119 & 0.2633 \\
\hline \multicolumn{9}{|c|}{ Urban } \\
\hline M (15-24) & 0.3225 & 0.0202 & 1,289 & 706 & 1.5543 & 0.0628 & 0.2826 & 0.3623 \\
\hline $\mathrm{W}(15-24)$ & 0.2811 & 0.0200 & 2,126 & 1,434 & 2.0461 & 0.0710 & 0.2419 & 0.3204 \\
\hline MM (15-29) & 0.2169 & 0.0219 & 648 & 306 & 1.3495 & 0.1008 & 0.1739 & 0.2600 \\
\hline MW (15-24) & 0.1568 & 0.0188 & 1,037 & 525 & 1.6683 & 0.1202 & 0.1197 & 0.1939 \\
\hline UM (15-24) & 0.3531 & 0.0215 & 1,132 & 631 & 1.5160 & 0.0610 & 0.3107 & 0.3955 \\
\hline UW (15-24) & 0.3819 & 0.0220 & 1,089 & 909 & 1.4924 & 0.0576 & 0.3386 & 0.4251 \\
\hline \multicolumn{9}{|c|}{ Rural } \\
\hline$M(15-24)$ & 0.1901 & 0.0155 & 1,190 & 1,799 & 1.3602 & 0.0814 & 0.1596 & 0.2206 \\
\hline W (15-24) & 0.0863 & 0.0068 & 2,722 & 3,414 & 1.2689 & 0.0792 & 0.0728 & 0.0997 \\
\hline MM (15-29) & 0.1231 & 0.0137 & 757 & 1,099 & 1.1448 & 0.1111 & 0.0962 & 0.1501 \\
\hline MW (15-24) & 0.0436 & 0.0059 & 1,293 & 1,805 & 1.0434 & 0.1359 & 0.0319 & 0.0553 \\
\hline UM (15-24) & 0.2163 & 0.0173 & 945 & 1,446 & 1.2878 & 0.0798 & 0.1824 & 0.2503 \\
\hline UW (15-24) & 0.1561 & 0.0124 & 1,429 & 1,609 & 1.2918 & 0.0795 & 0.1317 & 0.1806 \\
\hline
\end{tabular}


Table B.2: (Cont'd)

\begin{tabular}{|c|c|c|c|c|c|c|c|c|}
\hline \multirow{2}{*}{$\begin{array}{l}\text { Variable/ } \\
\text { respondent } \\
\text { category }\end{array}$} & \multirow{2}{*}{$\begin{array}{l}\text { Value } \\
\text { (R) }\end{array}$} & \multirow{2}{*}{$\begin{array}{l}\text { Standard } \\
\text { error } \\
\text { (SE) }\end{array}$} & \multicolumn{2}{|c|}{ Number of cases } & \multirow{2}{*}{$\begin{array}{l}\text { Design } \\
\text { effect } \\
\text { (DEFT) }\end{array}$} & \multirow{2}{*}{$\begin{array}{l}\text { Relative } \\
\text { standard } \\
\text { error } \\
(\mathrm{SE} / \mathrm{R})\end{array}$} & \multicolumn{2}{|c|}{ 95\% Confidence limits } \\
\hline & & & $\begin{array}{c}\text { Unweighted } \\
\text { (N) }\end{array}$ & $\begin{array}{l}\text { Weighted } \\
\text { (WN) }\end{array}$ & & & Lower & Upper \\
\hline \multicolumn{9}{|c|}{ Ever worked in last 12 months (young men and women) } \\
\hline \multicolumn{9}{|c|}{ Combined } \\
\hline$M(15-24)$ & 0.7257 & 0.0155 & 2,479 & 2,504 & 1.7239 & 0.0213 & 0.6953 & 0.7562 \\
\hline $\mathrm{W}(15-24)$ & 0.4737 & 0.0178 & 4,848 & 4,848 & 2.4843 & 0.0376 & 0.4386 & 0.5088 \\
\hline MM (15-29) & 0.9843 & 0.0046 & 1,405 & 1,405 & 1.3800 & 0.0047 & 0.9753 & 0.9933 \\
\hline MW (15-24) & 0.5050 & 0.0221 & 2,330 & 2,330 & 2.1344 & 0.0438 & 0.4614 & 0.5485 \\
\hline UM (15-24) & 0.6663 & 0.0160 & 2,077 & 2,077 & 1.5505 & 0.0241 & 0.6347 & 0.6979 \\
\hline UW (15-24) & 0.4410 & 0.0184 & 2,518 & 2,518 & 1.8567 & 0.0417 & 0.4048 & 0.4772 \\
\hline \multicolumn{9}{|c|}{ Urban } \\
\hline M (15-24) & 0.6503 & 0.0202 & 1,289 & 706 & 1.5196 & 0.0310 & 0.6106 & 0.6901 \\
\hline W (15-24) & 0.2451 & 0.0150 & 2,126 & 1,434 & 1.6123 & 0.0614 & 0.2155 & 0.2747 \\
\hline MM (15-29) & 0.9954 & 0.0026 & 648 & 306 & 0.9854 & 0.0026 & 0.9902 & 1.0005 \\
\hline MW (15-24) & 0.2313 & 0.0199 & 1,037 & 525 & 1.5180 & 0.0860 & 0.1921 & 0.2704 \\
\hline UM (15-24) & 0.5988 & 0.0210 & 1,132 & 631 & 1.4381 & 0.0350 & 0.5576 & 0.6401 \\
\hline UW (15-24) & 0.2563 & 0.0176 & 1,089 & 909 & 1.3266 & 0.0685 & 0.2218 & 0.2909 \\
\hline \multicolumn{9}{|c|}{ Rural } \\
\hline$M(15-24)$ & 0.7553 & 0.0200 & 1,190 & 1,799 & 1.6032 & 0.0265 & 0.7160 & 0.7947 \\
\hline W (15-24) & 0.5697 & 0.0218 & 2,722 & 3,414 & 2.3020 & 0.0384 & 0.5266 & 0.6127 \\
\hline MM (15-29) & 0.9812 & 0.0058 & 757 & 1,099 & 1.1772 & 0.0059 & 0.9698 & 0.9927 \\
\hline MW (15-24) & 0.5845 & 0.0258 & 1,293 & 1,805 & 1.8831 & 0.0442 & 0.5337 & 0.6353 \\
\hline UM (15-24) & 0.6957 & 0.0214 & 945 & 1,446 & 1.4275 & 0.0307 & 0.6536 & 0.7378 \\
\hline UW (15-24) & 0.5453 & 0.0240 & 1,429 & 1,609 & 1.8183 & 0.0439 & 0.4982 & 0.5925 \\
\hline \multicolumn{9}{|c|}{ Unemployed (young men and women in labour force) } \\
\hline \multicolumn{9}{|c|}{ Combined } \\
\hline$M(15-24)$ & 0.0675 & 0.0081 & 1,551 & 1,625 & 1.2728 & 0.1201 & 0.0516 & 0.0835 \\
\hline $\mathrm{W}(15-24)$ & 0.1009 & 0.0102 & 1,553 & 1,716 & 1.3301 & 0.1008 & 0.0809 & 0.1209 \\
\hline MM (15-29) & 0.0108 & 0.0036 & 1,372 & 1,362 & 1.2857 & 0.3318 & 0.0038 & 0.0179 \\
\hline MW (15-24) & 0.0771 & 0.0118 & 758 & 881 & 1.2127 & 0.1525 & 0.0540 & 0.1002 \\
\hline UM (15-24) & 0.0912 & 0.0108 & 1,162 & 1,199 & 1.2813 & 0.1187 & 0.0698 & 0.1125 \\
\hline UW (15-24) & 0.1346 & 0.0144 & 795 & 823 & 1.1922 & 0.1073 & 0.1062 & 0.1631 \\
\hline \multicolumn{9}{|c|}{ Urban } \\
\hline M (15-24) & 0.0619 & 0.0096 & 752 & 415 & 1.0924 & 0.1552 & 0.0430 & 0.0808 \\
\hline W (15-24) & 0.2322 & 0.0278 & 451 & 305 & 1.3954 & 0.1196 & 0.1776 & 0.2869 \\
\hline MM (15-29) & 0.0063 & 0.0030 & 642 & 304 & 0.9710 & 0.4810 & 0.0003 & 0.0123 \\
\hline MW (15-24) & 0.2693 & 0.0421 & 209 & 107 & 1.3678 & 0.1562 & 0.1865 & 0.3521 \\
\hline UM (15-24) & 0.0791 & 0.0125 & 595 & 333 & 1.1248 & 0.1574 & 0.0546 & 0.1037 \\
\hline UW (15-24) & 0.2046 & 0.0316 & 242 & 200 & 1.2141 & 0.1542 & 0.1425 & 0.2667 \\
\hline \multicolumn{9}{|c|}{ Rural } \\
\hline M (15-24) & 0.0695 & 0.0104 & 799 & 1,210 & 1.1544 & 0.1496 & 0.0490 & 0.0899 \\
\hline W (15-24) & 0.0725 & 0.0102 & 1,102 & 1,411 & 1.3016 & 0.1403 & 0.0525 & 0.0925 \\
\hline MM (15-29) & 0.0121 & 0.0046 & 730 & 1,058 & 1.1231 & 0.3754 & 0.0032 & 0.0211 \\
\hline MW (15-24) & 0.0507 & 0.0115 & 549 & 775 & 1.2259 & 0.2266 & 0.0281 & 0.0733 \\
\hline UM (15-24) & 0.0958 & 0.0142 & 567 & 866 & 1.1467 & 0.1481 & 0.0678 & 0.1237 \\
\hline UW (15-24) & 0.1121 & 0.0157 & 553 & 622 & 1.1682 & 0.1399 & 0.0812 & 0.1430 \\
\hline
\end{tabular}


Table B.2: (Cont'd)

\begin{tabular}{|c|c|c|c|c|c|c|c|c|}
\hline \multirow{2}{*}{$\begin{array}{l}\text { Variable/ } \\
\text { respondent } \\
\text { category }\end{array}$} & \multirow{2}{*}{$\begin{array}{l}\text { Value } \\
\text { (R) }\end{array}$} & \multirow{2}{*}{$\begin{array}{l}\text { Standard } \\
\text { error } \\
\text { (SE) }\end{array}$} & \multicolumn{2}{|c|}{ Number of cases } & \multirow{2}{*}{$\begin{array}{l}\text { Design } \\
\text { effect } \\
\text { (DEFT) }\end{array}$} & \multirow{2}{*}{$\begin{array}{l}\text { Relative } \\
\text { standard } \\
\text { error } \\
(\mathrm{SE} / \mathrm{R})\end{array}$} & \multicolumn{2}{|c|}{ 95\% Confidence limits } \\
\hline & & & $\begin{array}{c}\text { Unweighted } \\
\text { (N) }\end{array}$ & $\begin{array}{l}\text { Weighted } \\
\text { (WN) }\end{array}$ & & & Lower & Upper \\
\hline \multicolumn{9}{|c|}{ Discussed friendships with father (young men and women whose father was alive at the time of interview) } \\
\hline \multicolumn{9}{|c|}{ Combined } \\
\hline M (15-24) & 0.4938 & 0.0177 & 2,115 & 2,146 & 1.6241 & 0.0358 & 0.4590 & 0.5285 \\
\hline W (15-24) & 0.5170 & 0.0124 & 4,096 & 4,088 & 1.5828 & 0.0239 & 0.4927 & 0.5414 \\
\hline MM (15-29) & 0.3620 & 0.0209 & 1,045 & 1,060 & 1.4019 & 0.0576 & 0.3209 & 0.4030 \\
\hline MW (15-24) & 0.4612 & 0.0159 & 1,885 & 1,902 & 1.3814 & 0.0344 & 0.4300 & 0.4924 \\
\hline UM (15-24) & 0.5307 & 0.0179 & 1,787 & 1,789 & 1.5148 & 0.0337 & 0.4955 & 0.5660 \\
\hline UW (15-24) & 0.5832 & 0.0138 & 2,211 & 2,213 & 1.3161 & 0.0237 & 0.5561 & 0.6104 \\
\hline \multicolumn{9}{|c|}{ Urban } \\
\hline M (15-24) & 0.5613 & 0.0250 & 1,090 & 595 & 1.6604 & 0.0445 & 0.5122 & 0.6105 \\
\hline W (15-24) & 0.5916 & 0.0190 & 1,777 & 1,204 & 1.6290 & 0.0321 & 0.5542 & 0.6290 \\
\hline MM (15-29) & 0.4190 & 0.0276 & 464 & 220 & 1.2048 & 0.0659 & 0.3647 & 0.4734 \\
\hline MW (15-24) & 0.5226 & 0.0208 & 822 & 416 & 1.1936 & 0.0398 & 0.4816 & 0.5635 \\
\hline UM (15-24) & 0.5850 & 0.0248 & 972 & 541 & 1.5690 & 0.0424 & 0.5361 & 0.6338 \\
\hline UW (15-24) & 0.6421 & 0.0223 & 955 & 797 & 1.4361 & 0.0347 & 0.5982 & 0.6860 \\
\hline \multicolumn{9}{|c|}{ Rural } \\
\hline$M(15-24)$ & 0.4678 & 0.0224 & 1,025 & 1,551 & 1.4372 & 0.0479 & 0.4237 & 0.5119 \\
\hline W (15-24) & 0.4859 & 0.0151 & 2,319 & 2,884 & 1.4564 & 0.0311 & 0.4562 & 0.5157 \\
\hline MM (15-29) & 0.3471 & 0.0253 & 581 & 840 & 1.2787 & 0.0728 & 0.2973 & 0.3968 \\
\hline MW (15-24) & 0.4440 & 0.0193 & 1,063 & 1,486 & 1.2647 & 0.0434 & 0.4061 & 0.4820 \\
\hline UM (15-24) & 0.5073 & 0.0232 & 815 & 1,249 & 1.3236 & 0.0457 & 0.4616 & 0.5529 \\
\hline UW (15-24) & 0.5501 & 0.0168 & 1,256 & 1,416 & 1.1990 & 0.0306 & 0.5169 & 0.5832 \\
\hline \multicolumn{9}{|c|}{ Discussed friendships with mother (young men and women whose mother was alive at the time of interview) } \\
\hline \multicolumn{9}{|c|}{ Combined } \\
\hline M (15-24) & 0.5653 & 0.0151 & 2,389 & 2,418 & 1.4888 & 0.0267 & 0.5356 & 0.5951 \\
\hline W (15-24) & 0.8414 & 0.0108 & 4,575 & 4,550 & 1.9932 & 0.0128 & 0.8202 & 0.8626 \\
\hline MM (15-29) & 0.4336 & 0.0205 & 1,274 & 1,286 & 1.4726 & 0.0472 & 0.3933 & 0.4738 \\
\hline MW (15-24) & 0.7957 & 0.0158 & 2,147 & 2,143 & 1.8199 & 0.0199 & 0.7645 & 0.8269 \\
\hline UM (15-24) & 0.5925 & 0.0161 & 2,012 & 2,013 & 1.4712 & 0.0272 & 0.5608 & 0.6242 \\
\hline UW (15-24) & 0.8976 & 0.0066 & 2,428 & 2,424 & 1.0735 & 0.0074 & 0.8846 & 0.9106 \\
\hline \multicolumn{9}{|c|}{ Urban } \\
\hline M (15-24) & 0.6032 & 0.0210 & 1,236 & 675 & 1.5058 & 0.0348 & 0.5619 & 0.6444 \\
\hline W (15-24) & 0.8988 & 0.0106 & 2,026 & 1,370 & 1.5832 & 0.0118 & 0.8779 & 0.9197 \\
\hline MM (15-29) & 0.5058 & 0.0249 & 575 & 272 & 1.1911 & 0.0491 & 0.4569 & 0.5547 \\
\hline MW (15-24) & 0.8615 & 0.0166 & 960 & 486 & 1.4859 & 0.0192 & 0.8289 & 0.8942 \\
\hline UM (15-24) & 0.6275 & 0.0212 & 1,095 & 609 & 1.4474 & 0.0337 & 0.5859 & 0.6691 \\
\hline UW (15-24) & 0.9274 & 0.0086 & 1,066 & 890 & 1.0801 & 0.0093 & 0.9105 & 0.9443 \\
\hline \multicolumn{9}{|c|}{ Rural } \\
\hline M (15-24) & 0.5507 & 0.0192 & 1,153 & 1,743 & 1.3108 & 0.0349 & 0.5129 & 0.5885 \\
\hline W (15-24) & 0.8167 & 0.0145 & 2,549 & 3,180 & 1.8861 & 0.0177 & 0.7883 & 0.8452 \\
\hline MM (15-29) & 0.4142 & 0.0249 & 699 & 1,014 & 1.3333 & 0.0600 & 0.3653 & 0.4631 \\
\hline MW (15-24) & 0.7764 & 0.0197 & 1,187 & 1,657 & 1.6252 & 0.0253 & 0.7377 & 0.8151 \\
\hline UM (15-24) & 0.5773 & 0.0211 & 917 & 1,404 & 1.2897 & 0.0365 & 0.5359 & 0.6187 \\
\hline UW (15-24) & 0.8803 & 0.0091 & 1,362 & 1,534 & 1.0342 & 0.0103 & 0.8624 & 0.8982 \\
\hline
\end{tabular}


Table B.2: (Cont'd)

\begin{tabular}{|c|c|c|c|c|c|c|c|c|}
\hline \multirow{2}{*}{$\begin{array}{l}\text { Variable/ } \\
\text { respondent } \\
\text { category }\end{array}$} & \multirow{2}{*}{$\begin{array}{l}\text { Value } \\
\text { (R) }\end{array}$} & \multirow{2}{*}{$\begin{array}{l}\text { Standard } \\
\text { error } \\
\text { (SE) }\end{array}$} & \multicolumn{2}{|c|}{ Number of cases } & \multirow{2}{*}{$\begin{array}{l}\text { Design } \\
\text { effect } \\
\text { (DEFT) }\end{array}$} & \multirow{2}{*}{$\begin{array}{c}\text { Relative } \\
\text { standard } \\
\text { error } \\
(\mathrm{SE} / \mathrm{R})\end{array}$} & \multicolumn{2}{|c|}{ 95\% Confidence limits } \\
\hline & & & \begin{tabular}{|} 
Unweighted \\
$(\mathbf{N})$
\end{tabular} & $\begin{array}{c}\text { Weighted } \\
\text { (WN) }\end{array}$ & & & Lower & $\overline{\text { Upper }}$ \\
\hline \multicolumn{9}{|c|}{ Independently makes decisions about choice of friends, spending money and buying clothes for oneself (young men and women) } \\
\hline \multicolumn{9}{|c|}{ Combined } \\
\hline M (15-24) & 0.5483 & 0.0157 & 2,479 & 2,504 & 1.5681 & 0.0286 & 0.5174 & 0.5791 \\
\hline W (15-24) & 0.2630 & 0.0130 & 4,848 & 4,848 & 2.0610 & 0.0496 & 0.2373 & 0.2887 \\
\hline MM (15-29) & 0.7211 & 0.0185 & 1,405 & 1,405 & 1.5436 & 0.0256 & 0.6847 & 0.7575 \\
\hline MW (15-24) & 0.2583 & 0.0158 & 2,330 & 2,330 & 1.7419 & 0.0612 & 0.2272 & 0.2894 \\
\hline UM (15-24) & 0.5243 & 0.0161 & 2,077 & 2,077 & 1.4670 & 0.0307 & 0.4926 & 0.5559 \\
\hline UW (15-24) & 0.2662 & 0.0144 & 2,518 & 2,518 & 1.6391 & 0.0542 & 0.2378 & 0.2946 \\
\hline \multicolumn{9}{|c|}{ Urban } \\
\hline M (15-24) & 0.5900 & 0.0225 & 1,289 & 706 & 1.6434 & 0.0382 & 0.5457 & 0.6344 \\
\hline W (15-24) & 0.3306 & 0.0175 & 2,126 & 1,434 & 1.7127 & 0.0529 & 0.2962 & 0.3650 \\
\hline MM (15-29) & 0.8014 & 0.0201 & 648 & 306 & 1.2794 & 0.0250 & 0.7619 & 0.8409 \\
\hline MW (15-24) & 0.3109 & 0.0211 & 1,037 & 525 & 1.4652 & 0.0678 & 0.2694 & 0.3523 \\
\hline UM (15-24) & 0.5664 & 0.0232 & 1,132 & 631 & 1.5717 & 0.0409 & 0.5208 & 0.6120 \\
\hline UW (15-24) & 0.3467 & 0.0186 & 1,089 & 909 & 1.2919 & 0.0538 & 0.3100 & 0.3834 \\
\hline \multicolumn{9}{|c|}{ Rural } \\
\hline M (15-24) & 0.5319 & 0.0199 & 1,190 & 1,799 & 1.3747 & 0.0374 & 0.4927 & 0.5710 \\
\hline $\mathrm{W}(15-24)$ & 0.2346 & 0.0167 & 2,722 & 3,414 & 2.0504 & 0.0710 & 0.2018 & 0.2674 \\
\hline MM (15-29) & 0.6987 & 0.0228 & 757 & 1,099 & 1.3674 & 0.0327 & 0.6538 & 0.7436 \\
\hline MW (15-24) & 0.2430 & 0.0193 & 1,293 & 1,805 & 1.6167 & 0.0794 & 0.2051 & 0.2810 \\
\hline UM (15-24) & 0.5059 & 0.0206 & 945 & 1,446 & 1.2679 & 0.0408 & 0.4653 & 0.5465 \\
\hline UW (15-24) & 0.2208 & 0.0194 & 1,429 & 1,609 & 1.7652 & 0.0878 & 0.1826 & 0.2589 \\
\hline \multicolumn{9}{|c|}{ Can visit any place outside village or neighbourhood unescorted (young men and women) } \\
\hline \multicolumn{9}{|c|}{ Combined } \\
\hline $\mathrm{W}(15-24)$ & 0.2323 & 0.0098 & 4,848 & 4,848 & 1.6180 & 0.0422 & 0.2130 & 0.2516 \\
\hline MW (15-24) & 0.2363 & 0.0124 & 2,330 & 2,330 & 1.4073 & 0.0524 & 0.2119 & 0.2607 \\
\hline UM (15-24) & 0.7624 & 0.0178 & 2,077 & 2,077 & 1.9021 & 0.0233 & 0.7275 & 0.7974 \\
\hline UW (15-24) & 0.2275 & 0.0111 & 2,518 & 2,518 & 1.3295 & 0.0488 & 0.2057 & 0.2494 \\
\hline \multicolumn{9}{|c|}{ Urban } \\
\hline W (15-24) & 0.2168 & 0.0128 & 2,126 & 1,434 & 1.4306 & 0.0590 & 0.1917 & 0.2420 \\
\hline MW (15-24) & 0.2110 & 0.0173 & 1,037 & 525 & 1.3609 & 0.0818 & 0.1770 & 0.2449 \\
\hline UM (15-24) & 0.7769 & 0.0210 & 1,132 & 631 & 1.6998 & 0.0271 & 0.7355 & 0.8183 \\
\hline UW (15-24) & 0.2216 & 0.0145 & 1,089 & 909 & 1.1546 & 0.0656 & 0.1930 & 0.2502 \\
\hline \multicolumn{9}{|c|}{ Rural } \\
\hline W (15-24) & 0.2388 & 0.0128 & 2,722 & 3,414 & 1.5670 & 0.0536 & 0.2136 & 0.2640 \\
\hline MW (15-24) & 0.2436 & 0.0152 & 1,293 & 1,805 & 1.2692 & 0.0622 & 0.2138 & 0.2735 \\
\hline UM (15-24) & 0.7561 & 0.0238 & 945 & 1,446 & 1.7026 & 0.0315 & 0.7093 & 0.8030 \\
\hline UW (15-24) & 0.2309 & 0.0153 & 1,429 & 1,609 & 1.3705 & 0.0662 & 0.2008 & 0.2610 \\
\hline
\end{tabular}

Cont'd on next page... 
Table B.2: (Cont'd)

\begin{tabular}{|c|c|c|c|c|c|c|c|c|}
\hline \multirow{2}{*}{$\begin{array}{l}\text { Variable/ } \\
\text { respondent } \\
\text { category }\end{array}$} & \multirow{2}{*}{$\begin{array}{l}\text { Value } \\
\text { (R) }\end{array}$} & \multirow{2}{*}{$\begin{array}{l}\text { Standard } \\
\text { error } \\
\text { (SE) }\end{array}$} & \multicolumn{2}{|c|}{ Number of cases } & \multirow{2}{*}{$\begin{array}{l}\text { Design } \\
\text { effect } \\
\text { (DEFT) }\end{array}$} & \multirow{2}{*}{$\begin{array}{c}\text { Relative } \\
\text { standard } \\
\text { error } \\
(\mathrm{SE} / \mathrm{R})\end{array}$} & \multicolumn{2}{|c|}{ 95\% Confidence limits } \\
\hline & & & $\begin{array}{c}\text { Unweighted } \\
\text { (N) }\end{array}$ & $\begin{array}{c}\text { Weighted } \\
\text { (WN) }\end{array}$ & & & Lower & Upper \\
\hline \multicolumn{9}{|c|}{ Has savings of any amount (young men and women) } \\
\hline \multicolumn{9}{|c|}{ Combined } \\
\hline$M(15-24)$ & 0.2420 & 0.0149 & 2,479 & 2,504 & 1.7296 & 0.0615 & 0.2127 & 0.2713 \\
\hline $\mathrm{W}(15-24)$ & 0.2521 & 0.0122 & 4,848 & 4,848 & 1.9500 & 0.0482 & 0.2281 & 0.2760 \\
\hline MM (15-29) & 0.3712 & 0.0245 & 1,405 & 1,405 & 1.8965 & 0.0659 & 0.3230 & 0.4193 \\
\hline MW (15-24) & 0.2400 & 0.0155 & 2,330 & 2,330 & 1.7483 & 0.0645 & 0.2096 & 0.2705 \\
\hline UM (15-24) & 0.2266 & 0.0143 & 2,077 & 2,077 & 1.5578 & 0.0632 & 0.1984 & 0.2548 \\
\hline UW (15-24) & 0.2658 & 0.0134 & 2,518 & 2,518 & 1.5212 & 0.0504 & 0.2394 & 0.2922 \\
\hline \multicolumn{9}{|c|}{ Urban } \\
\hline M (15-24) & 0.2871 & 0.0191 & 1,289 & 706 & 1.5170 & 0.0666 & 0.2494 & 0.3247 \\
\hline $\mathrm{W}(15-24)$ & 0.2987 & 0.0170 & 2,126 & 1,434 & 1.7160 & 0.0570 & 0.2652 & 0.3322 \\
\hline MM (15-29) & 0.4512 & 0.0334 & 648 & 306 & 1.7074 & 0.0740 & 0.3855 & 0.5170 \\
\hline MW (15-24) & 0.2510 & 0.0166 & 1,037 & 525 & 1.2331 & 0.0662 & 0.2183 & 0.2837 \\
\hline UM (15-24) & 0.2731 & 0.0199 & 1,132 & 631 & 1.5053 & 0.0730 & 0.2338 & 0.3123 \\
\hline UW (15-24) & 0.3374 & 0.0217 & 1,089 & 909 & 1.5162 & 0.0644 & 0.2946 & 0.3801 \\
\hline \multicolumn{9}{|c|}{ Rural } \\
\hline M (15-24) & 0.2244 & 0.0193 & 1,190 & 1,799 & 1.5913 & 0.0858 & 0.1865 & 0.2623 \\
\hline $\mathrm{W}(15-24)$ & 0.2325 & 0.0157 & 2,722 & 3,414 & 1.9395 & 0.0676 & 0.2016 & 0.2634 \\
\hline MM (15-29) & 0.3489 & 0.0298 & 757 & 1,099 & 1.7175 & 0.0853 & 0.2902 & 0.4075 \\
\hline MW (15-24) & 0.2368 & 0.0194 & 1,293 & 1,805 & 1.6390 & 0.0819 & 0.1987 & 0.2750 \\
\hline UM (15-24) & 0.2063 & 0.0185 & 945 & 1,446 & 1.4040 & 0.0896 & 0.1699 & 0.2427 \\
\hline UW (15-24) & 0.2254 & 0.0164 & 1,429 & 1,609 & 1.4855 & 0.0729 & 0.1931 & 0.2577 \\
\hline \multicolumn{9}{|c|}{ Justified wife beating in at least one situation (young men and women) } \\
\hline \multicolumn{9}{|c|}{ Combined } \\
\hline M (15-24) & 0.6325 & 0.0207 & 2,479 & 2,504 & 2.1371 & 0.0327 & 0.5918 & 0.6733 \\
\hline W (15-24) & 0.8754 & 0.0062 & 4,848 & 4,848 & 1.3159 & 0.0071 & 0.8631 & 0.8877 \\
\hline MM (15-29) & 0.6543 & 0.0206 & 1,405 & 1,405 & 1.6226 & 0.0315 & 0.6138 & 0.6949 \\
\hline MW (15-24) & 0.8985 & 0.0073 & 2,330 & 2,330 & 1.1664 & 0.0081 & 0.8842 & 0.9129 \\
\hline UM (15-24) & 0.6148 & 0.0217 & 2,077 & 2,077 & 2.0338 & 0.0353 & 0.5720 & 0.6575 \\
\hline UW (15-24) & 0.8473 & 0.0083 & 2,518 & 2,518 & 1.1626 & 0.0098 & 0.8309 & 0.8637 \\
\hline \multicolumn{9}{|c|}{ Urban } \\
\hline M (15-24) & 0.5527 & 0.0273 & 1,289 & 706 & 1.9692 & 0.0494 & 0.4990 & 0.6064 \\
\hline $\mathrm{W}(15-24)$ & 0.8121 & 0.0141 & 2,126 & 1,434 & 1.6643 & 0.0174 & 0.7844 & 0.8399 \\
\hline MM (15-29) & 0.5428 & 0.0337 & 648 & 306 & 1.7188 & 0.0620 & 0.4765 & 0.6090 \\
\hline MW (15-24) & 0.8452 & 0.0167 & 1,037 & 525 & 1.4864 & 0.0198 & 0.8124 & 0.8781 \\
\hline UM (15-24) & 0.5458 & 0.0276 & 1,132 & 631 & 1.8647 & 0.0506 & 0.4915 & 0.6002 \\
\hline UW (15-24) & 0.7853 & 0.0157 & 1,089 & 909 & 1.2616 & 0.0200 & 0.7544 & 0.8162 \\
\hline \multicolumn{9}{|c|}{ Rural } \\
\hline M (15-24) & 0.6639 & 0.0267 & 1,190 & 1,799 & 1.9484 & 0.0402 & 0.6113 & 0.7164 \\
\hline W (15-24) & 0.9020 & 0.0058 & 2,722 & 3,414 & 1.0212 & 0.0065 & 0.8905 & 0.9134 \\
\hline MM (15-29) & 0.6855 & 0.0241 & 757 & 1,099 & 1.4270 & 0.0352 & 0.6380 & 0.7329 \\
\hline MW (15-24) & 0.9140 & 0.0078 & 1,293 & 1,805 & 0.9954 & 0.0085 & 0.8987 & 0.9293 \\
\hline UM (15-24) & 0.6448 & 0.0289 & 945 & 1,446 & 1.8568 & 0.0449 & 0.5879 & 0.7018 \\
\hline UW (15-24) & 0.8823 & 0.0082 & 1,429 & 1,609 & 0.9634 & 0.0093 & 0.8661 & 0.8984 \\
\hline
\end{tabular}


Table B.2: (Cont'd)

\begin{tabular}{|c|c|c|c|c|c|c|c|c|}
\hline \multirow{2}{*}{$\begin{array}{l}\text { Variable/ } \\
\text { respondent } \\
\text { category }\end{array}$} & \multirow{2}{*}{$\begin{array}{l}\text { Value } \\
\text { (R) }\end{array}$} & \multirow{2}{*}{$\begin{array}{c}\text { Standard } \\
\text { error } \\
\text { (SE) }\end{array}$} & \multicolumn{2}{|c|}{ Number of cases } & \multirow{2}{*}{$\begin{array}{c}\text { Design } \\
\text { effect } \\
\text { (DEFT) }\end{array}$} & \multirow{2}{*}{$\begin{array}{c}\text { Relative } \\
\text { standard } \\
\text { error } \\
\text { (SE/R) }\end{array}$} & \multicolumn{2}{|c|}{ 95\% Confidence limits } \\
\hline & & & $\begin{array}{c}\text { Unweighted } \\
(\mathbf{N})\end{array}$ & $\begin{array}{l}\text { Weighted } \\
\text { (WN) }\end{array}$ & & & Lower & Upper \\
\hline \multicolumn{9}{|c|}{ Awareness of sex- and pregnancy-related matters (young men and women) } \\
\hline \multicolumn{9}{|c|}{ Combined } \\
\hline M (15-24) & 0.1113 & 0.0113 & 2,479 & 2,504 & 1.7818 & 0.1011 & 0.0892 & 0.1335 \\
\hline $\mathrm{W}(15-24)$ & 0.0620 & 0.0056 & 4,848 & 4,848 & 1.6166 & 0.0903 & 0.0509 & 0.0730 \\
\hline MM (15-29) & 0.1660 & 0.0157 & 1,405 & 1,405 & 1.5775 & 0.0944 & 0.1351 & 0.1968 \\
\hline MW (15-24) & 0.0879 & 0.0092 & 2,330 & 2,330 & 1.5730 & 0.1050 & 0.0697 & 0.1061 \\
\hline UM (15-24) & 0.1066 & 0.0112 & 2,077 & 2,077 & 1.6546 & 0.1051 & 0.0846 & 0.1287 \\
\hline UW (15-24) & 0.0275 & 0.0037 & 2,518 & 2,518 & 1.1321 & 0.1343 & 0.0202 & 0.0347 \\
\hline \multicolumn{9}{|c|}{ Urban } \\
\hline M (15-24) & 0.1207 & 0.0134 & 1,289 & 706 & 1.4784 & 0.1112 & 0.0943 & 0.1471 \\
\hline $\mathrm{W}(15-24)$ & 0.0600 & 0.0070 & 2,126 & 1,434 & 1.3666 & 0.1174 & 0.0461 & 0.0738 \\
\hline MM (15-29) & 0.1792 & 0.0184 & 648 & 306 & 1.2204 & 0.1027 & 0.1430 & 0.2154 \\
\hline MW (15-24) & 0.0986 & 0.0125 & 1,037 & 525 & 1.3480 & 0.1266 & 0.0740 & 0.1232 \\
\hline UM (15-24) & 0.1212 & 0.0144 & 1,132 & 631 & 1.4886 & 0.1192 & 0.0928 & 0.1497 \\
\hline UW (15-24) & 0.0286 & 0.0051 & 1,089 & 909 & 1.0159 & 0.1794 & 0.0185 & 0.0388 \\
\hline \multicolumn{9}{|c|}{ Rural } \\
\hline M (15-24) & 0.1077 & 0.0148 & 1,190 & 1,799 & 1.6439 & 0.1372 & 0.0786 & 0.1368 \\
\hline W (15-24) & 0.0628 & 0.0074 & 2,722 & 3,414 & 1.5861 & 0.1175 & 0.0483 & 0.0773 \\
\hline MM (15-29) & 0.1623 & 0.0193 & 757 & 1,099 & 1.4427 & 0.1192 & 0.1242 & 0.2004 \\
\hline MW (15-24) & 0.0848 & 0.0113 & 1,293 & 1,805 & 1.4631 & 0.1337 & 0.0625 & 0.1071 \\
\hline UM (15-24) & 0.1003 & 0.0149 & 945 & 1,446 & 1.5193 & 0.1481 & 0.0710 & 0.1295 \\
\hline UW (15-24) & 0.0268 & 0.0050 & 1,429 & 1,609 & 1.1681 & 0.1862 & 0.0170 & 0.0366 \\
\hline \multicolumn{9}{|c|}{ Correct specific knowledge of at least one contraceptive method (young men and women) } \\
\hline \multicolumn{9}{|c|}{ Combined } \\
\hline$M(15-24)$ & 0.8130 & 0.0139 & 2,479 & 2,504 & 1.7717 & 0.0171 & 0.7857 & 0.8403 \\
\hline W (15-24) & 0.3018 & 0.0154 & 4,848 & 4,848 & 2.3336 & 0.0510 & 0.2715 & 0.3321 \\
\hline MM (15-29) & 0.8437 & 0.0160 & 1,405 & 1,405 & 1.6507 & 0.0190 & 0.8122 & 0.8752 \\
\hline MW (15-24) & 0.3367 & 0.0197 & 2,330 & 2,330 & 2.0086 & 0.0584 & 0.2980 & 0.3754 \\
\hline UM (15-24) & 0.8098 & 0.0154 & 2,077 & 2,077 & 1.7867 & 0.0190 & 0.7796 & 0.8401 \\
\hline UW (15-24) & 0.2527 & 0.0160 & 2,518 & 2,518 & 1.8428 & 0.0632 & 0.2212 & 0.2841 \\
\hline \multicolumn{9}{|c|}{ Urban } \\
\hline M (15-24) & 0.8168 & 0.0167 & 1,289 & 706 & 1.5525 & 0.0205 & 0.7838 & 0.8497 \\
\hline W (15-24) & 0.3710 & 0.0195 & 2,126 & 1,434 & 1.8603 & 0.0525 & 0.3326 & 0.4094 \\
\hline MM (15-29) & 0.8953 & 0.0170 & 648 & 306 & 1.4162 & 0.0190 & 0.8618 & 0.9289 \\
\hline MW (15-24) & 0.4435 & 0.0245 & 1,037 & 525 & 1.5842 & 0.0551 & 0.3954 & 0.4916 \\
\hline UM (15-24) & 0.8130 & 0.0172 & 1,132 & 631 & 1.4810 & 0.0211 & 0.7792 & 0.8468 \\
\hline UW (15-24) & 0.3123 & 0.0228 & 1,089 & 909 & 1.6198 & 0.0729 & 0.2675 & 0.3571 \\
\hline \multicolumn{9}{|c|}{ Rural } \\
\hline$M(15-24)$ & 0.8115 & 0.0182 & 1,190 & 1,799 & 1.6013 & 0.0224 & 0.7758 & 0.8473 \\
\hline W (15-24) & 0.2728 & 0.0201 & 2,722 & 3,414 & 2.3498 & 0.0736 & 0.2333 & 0.3122 \\
\hline MM (15-29) & 0.8293 & 0.0198 & 757 & 1,099 & 1.4460 & 0.0239 & 0.7904 & 0.8683 \\
\hline MW (15-24) & 0.3056 & 0.0241 & 1,293 & 1,805 & 1.8802 & 0.0788 & 0.2582 & 0.3531 \\
\hline UM (15-24) & 0.8085 & 0.0208 & 945 & 1,446 & 1.6227 & 0.0257 & 0.7676 & 0.8494 \\
\hline UW (15-24) & 0.2190 & 0.0210 & 1,429 & 1,609 & 1.9201 & 0.0960 & 0.1776 & 0.2603 \\
\hline
\end{tabular}


Table B.2: (Cont'd)

\begin{tabular}{|c|c|c|c|c|c|c|c|c|}
\hline \multirow{2}{*}{$\begin{array}{l}\text { Variable/ } \\
\text { respondent } \\
\text { category }\end{array}$} & \multirow{2}{*}{$\begin{array}{l}\text { Value } \\
\text { (R) }\end{array}$} & \multirow{2}{*}{$\begin{array}{c}\text { Standard } \\
\text { error } \\
\text { (SE) }\end{array}$} & \multicolumn{2}{|c|}{ Number of cases } & \multirow{2}{*}{$\begin{array}{c}\text { Design } \\
\text { effect } \\
\text { (DEFT) }\end{array}$} & \multirow{2}{*}{$\begin{array}{c}\text { Relative } \\
\text { standard } \\
\text { error } \\
(\mathrm{SE} / \mathrm{R})\end{array}$} & \multicolumn{2}{|c|}{ 95\% Confidence limits } \\
\hline & & & $\begin{array}{c}\text { Unweighted } \\
\text { (N) }\end{array}$ & $\begin{array}{c}\text { Weighted } \\
\text { (WN) }\end{array}$ & & & Lower & Upper \\
\hline \multicolumn{9}{|c|}{ Correct specific knowledge of condoms (young men and women) } \\
\hline \multicolumn{9}{|c|}{ Combined } \\
\hline$M(15-24)$ & 0.8027 & 0.0143 & 2,479 & 2,504 & 1.7863 & 0.0178 & 0.7746 & 0.8308 \\
\hline $\mathrm{W}(15-24)$ & 0.2050 & 0.0146 & 4,848 & 4,848 & 2.5200 & 0.0713 & 0.1763 & 0.2338 \\
\hline MM (15-29) & 0.8199 & 0.0177 & 1,405 & 1,405 & 1.7284 & 0.0216 & 0.7850 & 0.8548 \\
\hline MW (15-24) & 0.2111 & 0.0175 & 2,330 & 2,330 & 2.0657 & 0.0827 & 0.1767 & 0.2455 \\
\hline UM (15-24) & 0.8015 & 0.0156 & 2,077 & 2,077 & 1.7808 & 0.0194 & 0.7709 & 0.8322 \\
\hline UW (15-24) & 0.1949 & 0.0153 & 2,518 & 2,518 & 1.9342 & 0.0784 & 0.1648 & 0.2249 \\
\hline \multicolumn{9}{|c|}{ Urban } \\
\hline M (15-24) & 0.8079 & 0.0179 & 1,289 & 706 & 1.6268 & 0.0221 & 0.7728 & 0.8431 \\
\hline $\mathrm{W}(15-24)$ & 0.2594 & 0.0190 & 2,126 & 1,434 & 1.9970 & 0.0732 & 0.2220 & 0.2968 \\
\hline MM (15-29) & 0.8788 & 0.0184 & 648 & 306 & 1.4348 & 0.0209 & 0.8426 & 0.9151 \\
\hline MW (15-24) & 0.2945 & 0.0220 & 1,037 & 525 & 1.5535 & 0.0747 & 0.2512 & 0.3378 \\
\hline UM (15-24) & 0.8056 & 0.0181 & 1,132 & 631 & 1.5393 & 0.0225 & 0.7699 & 0.8413 \\
\hline UW (15-24) & 0.2310 & 0.0221 & 1,089 & 909 & 1.7316 & 0.0958 & 0.1874 & 0.2746 \\
\hline \multicolumn{9}{|c|}{ Rural } \\
\hline$M(15-24)$ & 0.8006 & 0.0186 & 1,190 & 1,799 & 1.6048 & 0.0232 & 0.7640 & 0.8372 \\
\hline $\mathrm{W}(15-24)$ & 0.1822 & 0.0191 & 2,722 & 3,414 & 2.5778 & 0.1047 & 0.1446 & 0.2197 \\
\hline MM (15-29) & 0.8034 & 0.0219 & 757 & 1,099 & 1.5174 & 0.0273 & 0.7603 & 0.8466 \\
\hline MW (15-24) & 0.1869 & 0.0215 & 1,293 & 1,805 & 1.9851 & 0.1152 & 0.1445 & 0.2293 \\
\hline UM (15-24) & 0.7998 & 0.0209 & 945 & 1,446 & 1.6075 & 0.0262 & 0.7586 & 0.8410 \\
\hline UW (15-24) & 0.1745 & 0.0202 & 1,429 & 1,609 & 2.0160 & 0.1160 & 0.1346 & 0.2143 \\
\hline \multicolumn{9}{|c|}{ Ever heard of HIV/AIDS (young men and women) } \\
\hline \multicolumn{9}{|c|}{ Combined } \\
\hline M (15-24) & 0.9646 & 0.0069 & 2,479 & 2,504 & 1.8632 & 0.0072 & 0.9510 & 0.9783 \\
\hline W (15-24) & 0.9456 & 0.0073 & 4,848 & 4,848 & 2.2398 & 0.0077 & 0.9313 & 0.9600 \\
\hline MM (15-29) & 0.9477 & 0.0098 & 1,405 & 1,405 & 1.6533 & 0.0104 & 0.9284 & 0.9671 \\
\hline MW (15-24) & 0.9302 & 0.0110 & 2,330 & 2,330 & 2.0863 & 0.0118 & 0.9085 & 0.9519 \\
\hline UM (15-24) & 0.9680 & 0.0065 & 2,077 & 2,077 & 1.6769 & 0.0067 & 0.9553 & 0.9808 \\
\hline UW (15-24) & 0.9650 & 0.0048 & 2,518 & 2,518 & 1.3218 & 0.0050 & 0.9555 & 0.9745 \\
\hline \multicolumn{9}{|c|}{ Urban } \\
\hline M (15-24) & 0.9766 & 0.0056 & 1,289 & 706 & 1.3349 & 0.0058 & 0.9655 & 0.9876 \\
\hline W (15-24) & 0.9790 & 0.0041 & 2,126 & 1,434 & 1.3304 & 0.0042 & 0.9709 & 0.9872 \\
\hline MM (15-29) & 0.9637 & 0.0108 & 648 & 306 & 1.4706 & 0.0112 & 0.9424 & 0.9850 \\
\hline MW (15-24) & 0.9787 & 0.0059 & 1,037 & 525 & 1.3191 & 0.0060 & 0.9671 & 0.9904 \\
\hline UM (15-24) & 0.9801 & 0.0056 & 1,132 & 631 & 1.3508 & 0.0057 & 0.9690 & 0.9911 \\
\hline UW (15-24) & 0.9793 & 0.0053 & 1,089 & 909 & 1.2208 & 0.0054 & 0.9689 & 0.9897 \\
\hline \multicolumn{9}{|c|}{ Rural } \\
\hline$M(15-24)$ & 0.9600 & 0.0093 & 1,190 & 1,799 & 1.6389 & 0.0097 & 0.9416 & 0.9783 \\
\hline W (15-24) & 0.9316 & 0.0101 & 2,722 & 3,414 & 2.0802 & 0.0108 & 0.9118 & 0.9514 \\
\hline MM (15-29) & 0.9433 & 0.0122 & 757 & 1,099 & 1.4479 & 0.0129 & 0.9193 & 0.9673 \\
\hline MW (15-24) & 0.9161 & 0.0139 & 1,293 & 1,805 & 1.8056 & 0.0152 & 0.8887 & 0.9435 \\
\hline UM (15-24) & 0.9628 & 0.0089 & 945 & 1,446 & 1.4474 & 0.0093 & 0.9452 & 0.9803 \\
\hline UW (15-24) & 0.9569 & 0.0069 & 1,429 & 1,609 & 1.2842 & 0.0072 & 0.9434 & 0.9705 \\
\hline
\end{tabular}

Cont'd on next page... 
Table B.2: (Cont'd)

\begin{tabular}{|c|c|c|c|c|c|c|c|c|}
\hline \multirow{2}{*}{$\begin{array}{l}\text { Variable/ } \\
\text { respondent } \\
\text { category }\end{array}$} & \multirow{2}{*}{$\begin{array}{l}\text { Value } \\
\text { (R) }\end{array}$} & \multirow{2}{*}{$\begin{array}{l}\text { Standard } \\
\text { error } \\
\text { (SE) }\end{array}$} & \multicolumn{2}{|c|}{ Number of cases } & \multirow{2}{*}{$\begin{array}{l}\text { Design } \\
\text { effect } \\
\text { (DEFT) }\end{array}$} & \multirow{2}{*}{$\begin{array}{c}\text { Relative } \\
\text { standard } \\
\text { error } \\
\text { (SE/R) }\end{array}$} & \multicolumn{2}{|c|}{ 95\% Confidence limits } \\
\hline & & & \begin{tabular}{|c|} 
Unweighted \\
$(\mathbf{N})$
\end{tabular} & $\begin{array}{l}\text { Weighted } \\
(\mathrm{WN})\end{array}$ & & & Lower & Upper \\
\hline \multicolumn{9}{|c|}{ Comprehensive knowledge of HIV/AIDS (young men and women) } \\
\hline \multicolumn{9}{|c|}{ Combined } \\
\hline$M(15-24)$ & 0.6179 & 0.0169 & 2,479 & 2,504 & 1.7354 & 0.0274 & 0.5845 & 0.6512 \\
\hline $\mathrm{W}(15-24)$ & 0.3302 & 0.0132 & 4,848 & 4,848 & 1.9478 & 0.0398 & 0.3043 & 0.3561 \\
\hline MM (15-29) & 0.5519 & 0.0192 & 1,405 & 1,405 & 1.4491 & 0.0348 & 0.5141 & 0.5898 \\
\hline MW (15-24) & 0.2660 & 0.0159 & 2,330 & 2,330 & 1.7331 & 0.0597 & 0.2348 & 0.2973 \\
\hline UM (15-24) & 0.6439 & 0.0169 & 2,077 & 2,077 & 1.6064 & 0.0262 & 0.6107 & 0.6771 \\
\hline UW (15-24) & 0.4106 & 0.0137 & 2,518 & 2,518 & 1.3932 & 0.0333 & 0.3837 & 0.4375 \\
\hline \multicolumn{9}{|c|}{ Urban } \\
\hline M (15-24) & 0.6610 & 0.0203 & 1,289 & 706 & 1.5391 & 0.0307 & 0.6210 & 0.7010 \\
\hline $\mathrm{W}(15-24)$ & 0.4608 & 0.0216 & 2,126 & 1,434 & 1.9941 & 0.0468 & 0.4183 & 0.5032 \\
\hline MM (15-29) & 0.6303 & 0.0292 & 648 & 306 & 1.5371 & 0.0463 & 0.5729 & 0.6877 \\
\hline MW (15-24) & 0.4061 & 0.0245 & 1,037 & 525 & 1.6027 & 0.0602 & 0.3579 & 0.4542 \\
\hline UM (15-24) & 0.6786 & 0.0203 & 1,132 & 631 & 1.4616 & 0.0299 & 0.6386 & 0.7185 \\
\hline UW (15-24) & 0.5051 & 0.0235 & 1,089 & 909 & 1.5489 & 0.0465 & 0.4589 & 0.5513 \\
\hline \multicolumn{9}{|c|}{ Rural } \\
\hline M (15-24) & 0.6010 & 0.0223 & 1,190 & 1,799 & 1.5692 & 0.0371 & 0.5571 & 0.6448 \\
\hline $\mathrm{W}(15-24)$ & 0.2753 & 0.0154 & 2,722 & 3,414 & 1.7995 & 0.0560 & 0.2450 & 0.3057 \\
\hline MM (15-29) & 0.5301 & 0.0231 & 757 & 1,099 & 1.2726 & 0.0436 & 0.4846 & 0.5755 \\
\hline MW (15-24) & 0.2253 & 0.0185 & 1,293 & 1,805 & 1.5926 & 0.0822 & 0.1889 & 0.2617 \\
\hline UM (15-24) & 0.6288 & 0.0228 & 945 & 1,446 & 1.4475 & 0.0362 & 0.5840 & 0.6736 \\
\hline UW (15-24) & 0.3572 & 0.0158 & 1,429 & 1,609 & 1.2496 & 0.0444 & 0.3260 & 0.3884 \\
\hline \multicolumn{9}{|c|}{ Ever heard of STIs other than HIV (young men and women) } \\
\hline \multicolumn{9}{|c|}{ Combined } \\
\hline$M(15-24)$ & 0.2957 & 0.0177 & 2,479 & 2,504 & 1.9349 & 0.0600 & 0.2608 & 0.3306 \\
\hline W (15-24) & 0.1559 & 0.0096 & 4,848 & 4,848 & 1.8504 & 0.0619 & 0.1369 & 0.1748 \\
\hline MM (15-29) & 0.3434 & 0.0240 & 1,405 & 1,405 & 1.8925 & 0.0698 & 0.2962 & 0.3907 \\
\hline MW (15-24) & 0.1574 & 0.0126 & 2,330 & 2,330 & 1.6659 & 0.0799 & 0.1327 & 0.1822 \\
\hline UM (15-24) & 0.3019 & 0.0177 & 2,077 & 2,077 & 1.7550 & 0.0586 & 0.2671 & 0.3367 \\
\hline UW (15-24) & 0.1535 & 0.0091 & 2,518 & 2,518 & 1.2671 & 0.0593 & 0.1356 & 0.1714 \\
\hline \multicolumn{9}{|c|}{ Urban } \\
\hline M (15-24) & 0.3376 & 0.0227 & 1,289 & 706 & 1.7256 & 0.0674 & 0.2928 & 0.3823 \\
\hline W (15-24) & 0.1657 & 0.0119 & 2,126 & 1,434 & 1.4775 & 0.0719 & 0.1422 & 0.1892 \\
\hline MM (15-29) & 0.4119 & 0.0288 & 648 & 306 & 1.4880 & 0.0699 & 0.3552 & 0.4686 \\
\hline MW (15-24) & 0.1801 & 0.0153 & 1,037 & 525 & 1.2781 & 0.0847 & 0.1500 & 0.2101 \\
\hline UM (15-24) & 0.3360 & 0.0237 & 1,132 & 631 & 1.6871 & 0.0705 & 0.2893 & 0.3826 \\
\hline UW (15-24) & 0.1541 & 0.0133 & 1,089 & 909 & 1.2192 & 0.0866 & 0.1278 & 0.1803 \\
\hline \multicolumn{9}{|c|}{ Rural } \\
\hline$M(15-24)$ & 0.2793 & 0.0230 & 1,190 & 1,799 & 1.7661 & 0.0823 & 0.2341 & 0.3245 \\
\hline W (15-24) & 0.1517 & 0.0128 & 2,722 & 3,414 & 1.8568 & 0.0842 & 0.1266 & 0.1769 \\
\hline MM (15-29) & 0.3243 & 0.0296 & 757 & 1,099 & 1.7379 & 0.0912 & 0.2661 & 0.3826 \\
\hline MW (15-24) & 0.1508 & 0.0156 & 1,293 & 1,805 & 1.5707 & 0.1037 & 0.1201 & 0.1816 \\
\hline UM (15-24) & 0.2870 & 0.0232 & 945 & 1,446 & 1.5783 & 0.0810 & 0.2413 & 0.3328 \\
\hline UW (15-24) & 0.1532 & 0.0121 & 1,429 & 1,609 & 1.2686 & 0.0789 & 0.1294 & 0.1770 \\
\hline
\end{tabular}


Table B.2: (Cont'd)

\begin{tabular}{|c|c|c|c|c|c|c|c|c|}
\hline \multirow{2}{*}{$\begin{array}{l}\text { Variable/ } \\
\text { respondent } \\
\text { category }\end{array}$} & \multirow{2}{*}{$\begin{array}{l}\text { Value } \\
\text { (R) }\end{array}$} & \multirow{2}{*}{$\begin{array}{c}\text { Standard } \\
\text { error } \\
\text { (SE) }\end{array}$} & \multicolumn{2}{|c|}{ Number of cases } & \multirow{2}{*}{$\begin{array}{c}\text { Design } \\
\text { effect } \\
\text { (DEFT) }\end{array}$} & \multirow{2}{*}{$\begin{array}{c}\text { Relative } \\
\text { standard } \\
\text { error } \\
(\mathrm{SE} / \mathrm{R})\end{array}$} & \multicolumn{2}{|c|}{ 95\% Confidence limits } \\
\hline & & & $\begin{array}{c}\text { Unweighted } \\
\text { (N) }\end{array}$ & $\begin{array}{c}\text { Weighted } \\
\text { (WN) }\end{array}$ & & & Lower & Upper \\
\hline \multicolumn{9}{|c|}{ Correct knowledge of the conditions under which abortion is legal (young men and women) } \\
\hline \multicolumn{9}{|c|}{ Combined } \\
\hline M (15-24) & 0.0396 & 0.0055 & 2,479 & 2,504 & 1.3911 & 0.1376 & 0.0289 & 0.0504 \\
\hline $\mathrm{W}(15-24)$ & 0.0761 & 0.0081 & 4,848 & 4,848 & 2.1141 & 0.1058 & 0.0603 & 0.0920 \\
\hline MM (15-29) & 0.0337 & 0.0063 & 1,405 & 1,405 & 1.3031 & 0.1862 & 0.0214 & 0.0461 \\
\hline MW (15-24) & 0.0761 & 0.0096 & 2,330 & 2,330 & 1.7388 & 0.1255 & 0.0573 & 0.0949 \\
\hline UM (15-24) & 0.0387 & 0.0059 & 2,077 & 2,077 & 1.3827 & 0.1513 & 0.0272 & 0.0502 \\
\hline UW (15-24) & 0.0755 & 0.0088 & 2,518 & 2,518 & 1.6627 & 0.1160 & 0.0582 & 0.0927 \\
\hline \multicolumn{9}{|c|}{ Urban } \\
\hline M (15-24) & 0.0467 & 0.0074 & 1,289 & 706 & 1.2544 & 0.1580 & 0.0322 & 0.0612 \\
\hline $\mathrm{W}(15-24)$ & 0.0907 & 0.0132 & 2,126 & 1,434 & 2.1240 & 0.1459 & 0.0647 & 0.1168 \\
\hline MM (15-29) & 0.0499 & 0.0093 & 648 & 306 & 1.0814 & 0.1855 & 0.0317 & 0.0681 \\
\hline MW (15-24) & 0.0892 & 0.0165 & 1,037 & 525 & 1.8644 & 0.1850 & 0.0567 & 0.1217 \\
\hline UM (15-24) & 0.0489 & 0.0080 & 1,132 & 631 & 1.2440 & 0.1631 & 0.0332 & 0.0646 \\
\hline UW (15-24) & 0.0919 & 0.0130 & 1,089 & 909 & 1.4856 & 0.1416 & 0.0663 & 0.1175 \\
\hline \multicolumn{9}{|c|}{ Rural } \\
\hline$M(15-24)$ & 0.0369 & 0.0070 & 1,190 & 1,799 & 1.2767 & 0.1892 & 0.0231 & 0.0506 \\
\hline $\mathrm{W}(15-24)$ & 0.0700 & 0.0100 & 2,722 & 3,414 & 2.0357 & 0.1423 & 0.0504 & 0.0896 \\
\hline MM (15-29) & 0.0292 & 0.0076 & 757 & 1,099 & 1.2346 & 0.2589 & 0.0143 & 0.0441 \\
\hline MW (15-24) & 0.0723 & 0.0114 & 1,293 & 1,805 & 1.5774 & 0.1572 & 0.0499 & 0.0947 \\
\hline UM (15-24) & 0.0342 & 0.0076 & 945 & 1,446 & 1.2768 & 0.2208 & 0.0193 & 0.0491 \\
\hline UW (15-24) & 0.0662 & 0.0115 & 1,429 & 1,609 & 1.7430 & 0.1733 & 0.0436 & 0.0887 \\
\hline \multicolumn{9}{|c|}{ Ever received family life or sex education (young men and women) } \\
\hline \multicolumn{9}{|c|}{ Combined } \\
\hline M (15-24) & 0.3341 & 0.0152 & 2,479 & 2,504 & 1.6061 & 0.0455 & 0.3041 & 0.3641 \\
\hline W (15-24) & 0.2353 & 0.0120 & 4,848 & 4,848 & 1.9708 & 0.0510 & 0.2116 & 0.2589 \\
\hline MM (15-29) & 0.1748 & 0.0124 & 1,405 & 1,405 & 1.2258 & 0.0711 & 0.1504 & 0.1993 \\
\hline MW (15-24) & 0.1299 & 0.0133 & 2,330 & 2,330 & 1.9072 & 0.1023 & 0.1038 & 0.1561 \\
\hline UM (15-24) & 0.3695 & 0.0165 & 2,077 & 2,077 & 1.5595 & 0.0447 & 0.3369 & 0.4020 \\
\hline UW (15-24) & 0.3738 & 0.0144 & 2,518 & 2,518 & 1.4916 & 0.0385 & 0.3455 & 0.4021 \\
\hline \multicolumn{9}{|c|}{ Urban } \\
\hline M (15-24) & 0.3083 & 0.0176 & 1,289 & 706 & 1.3645 & 0.0569 & 0.2737 & 0.3429 \\
\hline W (15-24) & 0.2882 & 0.0205 & 2,126 & 1,434 & 2.0884 & 0.0712 & 0.2478 & 0.3286 \\
\hline MM (15-29) & 0.1861 & 0.0180 & 648 & 306 & 1.1746 & 0.0966 & 0.1507 & 0.2215 \\
\hline MW (15-24) & 0.1613 & 0.0179 & 1,037 & 525 & 1.5643 & 0.1108 & 0.1261 & 0.1965 \\
\hline UM (15-24) & 0.3326 & 0.0188 & 1,132 & 631 & 1.3447 & 0.0566 & 0.2955 & 0.3697 \\
\hline UW (15-24) & 0.3910 & 0.0251 & 1,089 & 909 & 1.7000 & 0.0643 & 0.3415 & 0.4405 \\
\hline \multicolumn{9}{|c|}{ Rural } \\
\hline$M(15-24)$ & 0.3442 & 0.0200 & 1,190 & 1,799 & 1.4512 & 0.0581 & 0.3049 & 0.3836 \\
\hline W (15-24) & 0.2130 & 0.0147 & 2,722 & 3,414 & 1.8668 & 0.0688 & 0.1842 & 0.2419 \\
\hline MM (15-29) & 0.1717 & 0.0151 & 757 & 1,099 & 1.0992 & 0.0878 & 0.1420 & 0.2014 \\
\hline MW (15-24) & 0.1208 & 0.0163 & 1,293 & 1,805 & 1.8000 & 0.1351 & 0.0887 & 0.1529 \\
\hline UM (15-24) & 0.3855 & 0.0222 & 945 & 1,446 & 1.3997 & 0.0575 & 0.3419 & 0.4292 \\
\hline UW (15-24) & 0.3640 & 0.0175 & 1,429 & 1,609 & 1.3748 & 0.0481 & 0.3296 & 0.3985 \\
\hline
\end{tabular}

Cont'd on next page... 
Table B.2: (Cont'd)

\begin{tabular}{|c|c|c|c|c|c|c|c|c|}
\hline \multirow{2}{*}{$\begin{array}{l}\text { Variable/ } \\
\text { respondent } \\
\text { category }\end{array}$} & \multirow{2}{*}{$\begin{array}{l}\text { Value } \\
\text { (R) }\end{array}$} & \multirow{2}{*}{$\begin{array}{l}\text { Standard } \\
\text { error } \\
\text { (SE) }\end{array}$} & \multicolumn{2}{|c|}{ Number of cases } & \multirow{2}{*}{$\begin{array}{c}\text { Design } \\
\text { effect } \\
\text { (DEFT) }\end{array}$} & \multirow{2}{*}{$\begin{array}{c}\text { Relative } \\
\text { standard } \\
\text { error } \\
\text { (SE/R) }\end{array}$} & \multicolumn{2}{|c|}{ 95\% Confidence limits } \\
\hline & & & $\begin{array}{c}\text { Unweighted } \\
(\mathbf{N})\end{array}$ & $\begin{array}{c}\text { Weighted } \\
\text { (WN) }\end{array}$ & & & Lower & Upper \\
\hline \multicolumn{9}{|c|}{ Ever had an opposite-sex romantic partner (young men and women) } \\
\hline \multicolumn{9}{|c|}{ Combined } \\
\hline$M(15-24)$ & 0.1738 & 0.0119 & 2,479 & 2,504 & 1.5672 & 0.0687 & 0.1503 & 0.1972 \\
\hline $\mathrm{W}(15-24)$ & 0.1047 & 0.0069 & 4,848 & 4,848 & 1.5616 & 0.0656 & 0.0912 & 0.1182 \\
\hline MM (15-29) & 0.2051 & 0.0147 & 1,405 & 1,405 & 1.3661 & 0.0718 & 0.1761 & 0.2341 \\
\hline MW (15-24) & 0.1073 & 0.0093 & 2,330 & 2,330 & 1.4565 & 0.0870 & 0.0889 & 0.1257 \\
\hline UM (15-24) & 0.1629 & 0.0116 & 2,077 & 2,077 & 1.4320 & 0.0713 & 0.1400 & 0.1857 \\
\hline UW (15-24) & 0.1007 & 0.0068 & 2,518 & 2,518 & 1.1335 & 0.0675 & 0.0873 & 0.1141 \\
\hline \multicolumn{9}{|c|}{ Urban } \\
\hline M (15-24) & 0.1703 & 0.0115 & 1,289 & 706 & 1.0998 & 0.0676 & 0.1476 & 0.1930 \\
\hline W (15-24) & 0.1245 & 0.0102 & 2,126 & 1,434 & 1.4293 & 0.0822 & 0.1044 & 0.1447 \\
\hline MM (15-29) & 0.2311 & 0.0214 & 648 & 306 & 1.2934 & 0.0927 & 0.1889 & 0.2733 \\
\hline MW (15-24) & 0.1505 & 0.0154 & 1,037 & 525 & 1.3820 & 0.1020 & 0.1203 & 0.1808 \\
\hline UM (15-24) & 0.1504 & 0.0104 & 1,132 & 631 & 0.9746 & 0.0689 & 0.1300 & 0.1708 \\
\hline UW (15-24) & 0.1034 & 0.0096 & 1,089 & 909 & 1.0347 & 0.0924 & 0.0846 & 0.1222 \\
\hline \multicolumn{9}{|c|}{ Rural } \\
\hline M (15-24) & 0.1751 & 0.0160 & 1,190 & 1,799 & 1.4499 & 0.0913 & 0.1436 & 0.2066 \\
\hline W (15-24) & 0.0964 & 0.0087 & 2,722 & 3,414 & 1.5392 & 0.0903 & 0.0793 & 0.1136 \\
\hline MM (15-29) & 0.1979 & 0.0178 & 757 & 1,099 & 1.2313 & 0.0902 & 0.1627 & 0.2330 \\
\hline MW (15-24) & 0.0947 & 0.0110 & 1,293 & 1,805 & 1.3557 & 0.1166 & 0.0730 & 0.1165 \\
\hline UM (15-24) & 0.1683 & 0.0160 & 945 & 1,446 & 1.3174 & 0.0953 & 0.1367 & 0.1999 \\
\hline UW (15-24) & 0.0991 & 0.0092 & 1,429 & 1,609 & 1.1591 & 0.0925 & 0.0811 & 0.1172 \\
\hline \multicolumn{9}{|c|}{ Ever had sex with an opposite-sex romantic partner (young men and women) } \\
\hline \multicolumn{9}{|c|}{ Combined } \\
\hline$M(15-24)$ & 0.0797 & 0.0083 & 2,479 & 2,504 & 1.5247 & 0.1041 & 0.0634 & 0.0960 \\
\hline W (15-24) & 0.0470 & 0.0040 & 4,848 & 4,848 & 1.3161 & 0.0851 & 0.0391 & 0.0549 \\
\hline MM (15-29) & 0.1293 & 0.0123 & 1,405 & 1,405 & 1.3722 & 0.0950 & 0.1051 & 0.1535 \\
\hline MW (15-24) & 0.0517 & 0.0056 & 2,330 & 2,330 & 1.2105 & 0.1074 & 0.0408 & 0.0626 \\
\hline UM (15-24) & 0.0672 & 0.0073 & 2,077 & 2,077 & 1.3311 & 0.1088 & 0.0528 & 0.0816 \\
\hline UW (15-24) & 0.0409 & 0.0043 & 2,518 & 2,518 & 1.0833 & 0.1046 & 0.0325 & 0.0493 \\
\hline \multicolumn{9}{|c|}{ Urban } \\
\hline M (15-24) & 0.0572 & 0.0082 & 1,289 & 706 & 1.2689 & 0.1435 & 0.0411 & 0.0734 \\
\hline W (15-24) & 0.0474 & 0.0056 & 2,126 & 1,434 & 1.2148 & 0.1181 & 0.0364 & 0.0584 \\
\hline MM (15-29) & 0.0993 & 0.0164 & 648 & 306 & 1.3957 & 0.1652 & 0.0670 & 0.1317 \\
\hline MW (15-24) & 0.0636 & 0.0093 & 1,037 & 525 & 1.2240 & 0.1459 & 0.0454 & 0.0819 \\
\hline UM (15-24) & 0.0468 & 0.0063 & 1,132 & 631 & 1.0099 & 0.1355 & 0.0343 & 0.0593 \\
\hline UW (15-24) & 0.0343 & 0.0057 & 1,089 & 909 & 1.0273 & 0.1653 & 0.0231 & 0.0454 \\
\hline \multicolumn{9}{|c|}{ Rural } \\
\hline$M(15-24)$ & 0.0885 & 0.0111 & 1,190 & 1,799 & 1.3446 & 0.1251 & 0.0667 & 0.1103 \\
\hline W (15-24) & 0.0468 & 0.0052 & 2,722 & 3,414 & 1.2767 & 0.1104 & 0.0367 & 0.0570 \\
\hline MM (15-29) & 0.1377 & 0.0150 & 757 & 1,099 & 1.1969 & 0.1089 & 0.1082 & 0.1672 \\
\hline MW (15-24) & 0.0482 & 0.0066 & 1,293 & 1,805 & 1.1080 & 0.1369 & 0.0352 & 0.0612 \\
\hline UM (15-24) & 0.0761 & 0.0102 & 945 & 1,446 & 1.1792 & 0.1337 & 0.0560 & 0.0961 \\
\hline UW (15-24) & 0.0446 & 0.0059 & 1,429 & 1,609 & 1.0719 & 0.1313 & 0.0331 & 0.0561 \\
\hline
\end{tabular}


Table B.2: (Cont'd)

\begin{tabular}{|c|c|c|c|c|c|c|c|c|}
\hline \multirow{2}{*}{$\begin{array}{l}\text { Variable/ } \\
\text { respondent } \\
\text { category }\end{array}$} & \multirow{2}{*}{$\begin{array}{l}\text { Value } \\
(\mathbf{R})\end{array}$} & \multirow{2}{*}{$\begin{array}{c}\text { Standard } \\
\text { error } \\
\text { (SE) }\end{array}$} & \multicolumn{2}{|c|}{ Number of cases } & \multirow{2}{*}{$\begin{array}{c}\text { Design } \\
\text { effect } \\
\text { (DEFT) }\end{array}$} & \multirow{2}{*}{$\begin{array}{c}\text { Relative } \\
\text { standard } \\
\text { error } \\
\text { (SE/R) }\end{array}$} & \multicolumn{2}{|c|}{ 95\% Confidence limits } \\
\hline & & & $\begin{array}{l}\text { Unweighted } \\
(\mathbf{N})\end{array}$ & $\begin{array}{l}\text { Weighted } \\
\text { (WN) }\end{array}$ & & & Lower & Upper \\
\hline \multicolumn{9}{|c|}{ Ever had pre-marital sex (young men and women) } \\
\hline \multicolumn{9}{|c|}{ Combined } \\
\hline$M(15-24)$ & 0.1640 & 0.0117 & 2,479 & 2,504 & 1.5697 & 0.0712 & 0.1411 & 0.1870 \\
\hline $\mathrm{W}(15-24)$ & 0.0582 & 0.0046 & 4,848 & 4,848 & 1.3565 & 0.0784 & 0.0492 & 0.0672 \\
\hline MM (15-29) & 0.2660 & 0.0168 & 1,405 & 1,405 & 1.4232 & 0.0631 & 0.2330 & 0.2991 \\
\hline MW (15-24) & 0.0632 & 0.0060 & 2,330 & 2,330 & 1.1883 & 0.0948 & 0.0514 & 0.0750 \\
\hline UM (15-24) & 0.1441 & 0.0115 & 2,077 & 2,077 & 1.4886 & 0.0796 & 0.1215 & 0.1667 \\
\hline UW (15-24) & 0.0516 & 0.0051 & 2,518 & 2,518 & 1.1612 & 0.0992 & 0.0415 & 0.0617 \\
\hline \multicolumn{9}{|c|}{ Urban } \\
\hline M (15-24) & 0.1442 & 0.0119 & 1,289 & 706 & 1.2108 & 0.0822 & 0.1209 & 0.1675 \\
\hline $\mathrm{W}(15-24)$ & 0.0610 & 0.0066 & 2,126 & 1,434 & 1.2693 & 0.1080 & 0.0481 & 0.0740 \\
\hline MM (15-29) & 0.2673 & 0.0212 & 648 & 306 & 1.2180 & 0.0793 & 0.2256 & 0.3090 \\
\hline MW (15-24) & 0.0791 & 0.0099 & 1,037 & 525 & 1.1775 & 0.1248 & 0.0597 & 0.0985 \\
\hline UM (15-24) & 0.1290 & 0.0114 & 1,132 & 631 & 1.1464 & 0.0886 & 0.1065 & 0.1515 \\
\hline UW (15-24) & 0.0464 & 0.0068 & 1,089 & 909 & 1.0622 & 0.1460 & 0.0331 & 0.0597 \\
\hline \multicolumn{9}{|c|}{ Rural } \\
\hline$M(15-24)$ & 0.1718 & 0.0155 & 1,190 & 1,799 & 1.4202 & 0.0904 & 0.1412 & 0.2024 \\
\hline $\mathrm{W}(15-24)$ & 0.0570 & 0.0059 & 2,722 & 3,414 & 1.3178 & 0.1027 & 0.0455 & 0.0686 \\
\hline MM (15-29) & 0.2657 & 0.0206 & 757 & 1,099 & 1.2846 & 0.0777 & 0.2250 & 0.3063 \\
\hline MW (15-24) & 0.0586 & 0.0071 & 1,293 & 1,805 & 1.0928 & 0.1219 & 0.0445 & 0.0726 \\
\hline UM (15-24) & 0.1507 & 0.0157 & 945 & 1,446 & 1.3466 & 0.1040 & 0.1198 & 0.1816 \\
\hline UW (15-24) & 0.0545 & 0.0070 & 1,429 & 1,609 & 1.1685 & 0.1288 & 0.0407 & 0.0683 \\
\hline \multicolumn{9}{|c|}{$\begin{array}{l}\text { Used condoms consistently in pre-marital relations } \\
\text { (young men and women who reported pre-marital sex in face-to-face interview) }\end{array}$} \\
\hline \multicolumn{9}{|c|}{ Combined } \\
\hline M (15-24) & 0.1693 & 0.0233 & 313 & 335 & 1.0971 & 0.1376 & 0.1234 & 0.2152 \\
\hline $\mathrm{W}(15-24)$ & 0.0179 & 0.0075 & 248 & 245 & 0.8834 & 0.4162 & 0.0032 & 0.0326 \\
\hline MM (15-29) & 0.1887 & 0.0313 & 294 & 299 & 1.3699 & 0.1659 & 0.1270 & 0.2505 \\
\hline UM (15-24) & 0.1717 & 0.0270 & 234 & 245 & 1.0942 & 0.1575 & 0.1184 & 0.2249 \\
\hline \multicolumn{9}{|c|}{$\begin{array}{c}\text { Ever communicated with spouse on contraception } \\
\text { (married young men and women who had begun cohabiting) }\end{array}$} \\
\hline \multicolumn{9}{|c|}{ Combined } \\
\hline MM (15-29) & 0.1216 & 0.0133 & 1,404 & 1,404 & 1.5241 & 0.1093 & 0.0954 & 0.1478 \\
\hline MW (15-24) & 0.3401 & 0.0179 & 2,325 & 2,325 & 1.8214 & 0.0526 & 0.3048 & 0.3753 \\
\hline \multicolumn{9}{|c|}{ Urban } \\
\hline MM (15-29) & 0.1982 & 0.0236 & 648 & 306 & 1.5064 & 0.1191 & 0.1517 & 0.2447 \\
\hline MW (15-24) & 0.4169 & 0.0255 & 1,036 & 525 & 1.6662 & 0.0612 & 0.3666 & 0.4671 \\
\hline \multicolumn{9}{|c|}{ Rural } \\
\hline MM (15-29) & 0.1002 & 0.0152 & 756 & 1,097 & 1.3936 & 0.1520 & 0.0703 & 0.1302 \\
\hline MW (15-24) & 0.3177 & 0.0218 & 1,289 & 1,800 & 1.6781 & 0.0685 & 0.2748 & 0.3605 \\
\hline
\end{tabular}

Cont'd on next page... 
Table B.2: (Cont'd)

\begin{tabular}{|c|c|c|c|c|c|c|c|c|}
\hline \multirow{2}{*}{$\begin{array}{l}\text { Variable/ } \\
\text { respondent } \\
\text { category }\end{array}$} & \multirow{2}{*}{$\begin{array}{l}\text { Value } \\
\text { (R) }\end{array}$} & \multirow{2}{*}{$\begin{array}{l}\text { Standard } \\
\text { error } \\
\text { (SE) }\end{array}$} & \multicolumn{2}{|c|}{ Number of cases } & \multirow{2}{*}{$\begin{array}{l}\text { Design } \\
\text { effect } \\
\text { (DEFT) }\end{array}$} & \multirow{2}{*}{$\begin{array}{c}\text { Relative } \\
\text { standard } \\
\text { error } \\
(\mathrm{SE} / \mathrm{R})\end{array}$} & \multicolumn{2}{|c|}{ 95\% Confidence limits } \\
\hline & & & $\begin{array}{l}\text { Unweighted } \\
(\mathbf{N})\end{array}$ & $\begin{array}{l}\text { Weighted } \\
(\text { WN) }\end{array}$ & & & Lower & Upper \\
\hline \multicolumn{9}{|c|}{$\begin{array}{l}\text { Husband ever forced wife to have sex } \\
\text { (married young men and women who had begun cohabiting) }\end{array}$} \\
\hline \multicolumn{9}{|c|}{ Combined } \\
\hline MM (15-29) & 0.1465 & 0.0164 & 1,404 & 1,404 & 1.7410 & 0.1122 & 0.1142 & 0.1789 \\
\hline MW (15-24) & 0.1028 & 0.0116 & 2,325 & 2,325 & 1.8497 & 0.1133 & 0.0798 & 0.1257 \\
\hline \multicolumn{9}{|c|}{ Urban } \\
\hline MM (15-29) & 0.1386 & 0.0244 & 648 & 306 & 1.7934 & 0.1757 & 0.0907 & 0.1866 \\
\hline MW (15-24) & 0.0594 & 0.0112 & 1,036 & 524 & 1.5262 & 0.1888 & 0.0373 & 0.0814 \\
\hline \multicolumn{9}{|c|}{ Rural } \\
\hline MM (15-29) & 0.1488 & 0.0199 & 756 & 1,097 & 1.5370 & 0.1338 & 0.1096 & 0.1879 \\
\hline MW (15-24) & 0.1154 & 0.0145 & 1,289 & 1,800 & 1.6248 & 0.1253 & 0.0869 & 0.1439 \\
\hline \multicolumn{9}{|c|}{$\begin{array}{l}\text { Husband ever perpetrated physical violence on wife } \\
\text { Irried young men and women who had begun cohabiting) }\end{array}$} \\
\hline \multicolumn{9}{|c|}{ Combined } \\
\hline MM (15-29) & 0.1970 & 0.0178 & 1,404 & 1,404 & 1.6790 & 0.0905 & 0.1619 & 0.2321 \\
\hline MW (15-24) & 0.2293 & 0.0120 & 2,325 & 2,324 & 1.3801 & 0.0525 & 0.2056 & 0.2530 \\
\hline \multicolumn{9}{|c|}{ Urban } \\
\hline MM (15-29) & 0.2140 & 0.0240 & 648 & 306 & 1.4874 & 0.1121 & 0.1668 & 0.2613 \\
\hline MW (15-24) & 0.1911 & 0.0177 & 1,036 & 524 & 1.4482 & 0.0926 & 0.1563 & 0.2260 \\
\hline \multicolumn{9}{|c|}{ Rural } \\
\hline MM (15-29) & 0.1923 & 0.0218 & 756 & 1,097 & 1.5197 & 0.1134 & 0.1494 & 0.2352 \\
\hline MW (15-24) & 0.2404 & 0.0146 & 1,289 & 1,800 & 1.2289 & 0.0609 & 0.2116 & 0.2693 \\
\hline \multicolumn{9}{|c|}{$\begin{array}{l}\text { Husband ever perpetrated physical violence on wife in last } 12 \text { months } \\
\text { (married young men and women who had begun cohabiting) }\end{array}$} \\
\hline \multicolumn{9}{|c|}{ Combined } \\
\hline MM (15-29) & 0.1646 & 0.0153 & 1,404 & 1,404 & 1.5472 & 0.0930 & 0.1345 & 0.1948 \\
\hline MW (15-24) & 0.1864 & 0.0118 & 2,325 & 2,324 & 1.4556 & 0.0631 & 0.1633 & 0.2096 \\
\hline \multicolumn{9}{|c|}{ Urban } \\
\hline MM (15-29) & 0.1836 & 0.0197 & 648 & 306 & 1.2925 & 0.1072 & 0.1449 & 0.2223 \\
\hline MW (15-24) & 0.1466 & 0.0147 & 1,036 & 524 & 1.3359 & 0.1002 & 0.1177 & 0.1755 \\
\hline \multicolumn{9}{|c|}{ Rural } \\
\hline MM (15-29) & 0.1593 & 0.0188 & 756 & 1,097 & 1.4123 & 0.1181 & 0.1223 & 0.1964 \\
\hline MW (15-24) & 0.1980 & 0.0145 & 1,289 & 1,800 & 1.3093 & 0.0734 & 0.1694 & 0.2266 \\
\hline \multicolumn{9}{|c|}{$\begin{array}{l}\text { Currently using any modern contraceptive method } \\
\text { (married young men and women who had begun cohabiting) }\end{array}$} \\
\hline \multicolumn{9}{|c|}{ Combined } \\
\hline MM (15-29) & 0.0778 & 0.0137 & 1,404 & 1,404 & 1.9196 & 0.1765 & 0.0507 & 0.1048 \\
\hline MW (15-24) & 0.2125 & 0.0133 & 2,325 & 2,324 & 1.5681 & 0.0626 & 0.1863 & 0.2387 \\
\hline \multicolumn{9}{|c|}{ Urban } \\
\hline MM (15-29) & 0.0867 & 0.0169 & 648 & 306 & 1.5281 & 0.1950 & 0.0534 & 0.1199 \\
\hline MW (15-24) & 0.2308 & 0.0199 & 1,036 & 524 & 1.5179 & 0.0861 & 0.1917 & 0.2700 \\
\hline \multicolumn{9}{|c|}{ Rural } \\
\hline MM (15-29) & 0.0753 & 0.0169 & 756 & 1,097 & 1.7620 & 0.2248 & 0.0420 & 0.1086 \\
\hline MW (15-24) & 0.2072 & 0.0162 & 1,289 & 1,800 & 1.4309 & 0.0780 & 0.1754 & 0.2390 \\
\hline
\end{tabular}

Cont'd on next page... 
Table B.2: (Cont'd)

\begin{tabular}{|c|c|c|c|c|c|c|c|c|}
\hline \multirow{2}{*}{$\begin{array}{l}\text { Variable/ } \\
\text { respondent } \\
\text { category }\end{array}$} & \multirow{2}{*}{$\begin{array}{l}\text { Value } \\
(\mathbf{R})\end{array}$} & \multirow{2}{*}{$\begin{array}{l}\text { Standard } \\
\text { error } \\
\text { (SE) }\end{array}$} & \multicolumn{2}{|c|}{ Number of cases } & \multirow{2}{*}{$\begin{array}{l}\text { Design } \\
\text { effect } \\
\text { (DEFT) }\end{array}$} & \multirow{2}{*}{$\begin{array}{c}\text { Relative } \\
\text { standard } \\
\text { error } \\
\text { (SE/R) }\end{array}$} & \multicolumn{2}{|c|}{ 95\% Confidence limits } \\
\hline & & & $\begin{array}{l}\text { Unweighted } \\
\text { (N) }\end{array}$ & $\begin{array}{l}\text { Weighted } \\
\text { (WN) }\end{array}$ & & & Lower & Upper \\
\hline \multicolumn{9}{|c|}{$\begin{array}{r}\text { First delivery in a healt } \\
\text { (married young men and women whose first pregr }\end{array}$} \\
\hline \multicolumn{9}{|c|}{ Combined } \\
\hline MM (15-29) & 0.7143 & 0.0254 & 919 & 937 & 1.7068 & 0.0356 & 0.6642 & 0.7644 \\
\hline MW (15-24) & 0.7598 & 0.0193 & 1,587 & 1,600 & 1.8015 & 0.0254 & 0.7218 & 0.7978 \\
\hline \multicolumn{9}{|c|}{ Urban } \\
\hline MM (15-29) & 0.8362 & 0.0225 & 402 & 190 & 1.2167 & 0.0269 & 0.7920 & 0.8805 \\
\hline MW (15-24) & 0.8549 & 0.0207 & 696 & 352 & 1.5485 & 0.0242 & 0.8142 & 0.8956 \\
\hline \multicolumn{9}{|c|}{ Rural } \\
\hline MM (15-29) & 0.6834 & 0.0309 & 517 & 748 & 1.5088 & 0.0452 & 0.6225 & 0.7442 \\
\hline MW (15-24) & 0.7330 & 0.0237 & 891 & 1,248 & 1.5982 & 0.0323 & 0.6863 & 0.7796 \\
\hline \multicolumn{9}{|c|}{$\begin{array}{c}\text { Mean number of children ever born } \\
\text { (married young men and women who had begun cohabiting) }\end{array}$} \\
\hline \multicolumn{9}{|c|}{ Combined } \\
\hline MM (15-29) & 1.1626 & 0.0352 & 1,405 & 1,405 & 1.3573 & 0.0303 & 1.0934 & 1.2319 \\
\hline MW (15-24) & 1.2351 & 0.0261 & 2,330 & 2,330 & 1.2910 & 0.0211 & 1.1837 & 1.2865 \\
\hline \multicolumn{9}{|c|}{ Urban } \\
\hline MM (15-29) & 1.0488 & 0.0410 & 648 & 306 & 1.1119 & 0.0391 & 0.9680 & 1.1295 \\
\hline MW (15-24) & 1.1379 & 0.0328 & 1,037 & 525 & 1.1238 & 0.0288 & 1.0734 & 1.2024 \\
\hline \multicolumn{9}{|c|}{ Rural } \\
\hline MM (15-29) & 1.1944 & 0.0430 & 757 & 1,099 & 1.2073 & 0.0360 & 1.1098 & 1.2790 \\
\hline MW (15-24) & 1.2634 & 0.0320 & 1,293 & 1,805 & 1.1695 & 0.0254 & 1.2003 & 1.3265 \\
\hline \multicolumn{9}{|c|}{$\begin{array}{l}\text { Mean number of children surviving } \\
\text { (married young men and women who had begun cohabiting) }\end{array}$} \\
\hline \multicolumn{9}{|c|}{ Combined } \\
\hline MM (15-29) & 1.1221 & 0.0329 & 1,405 & 1,405 & 1.3374 & 0.0293 & 1.0573 & 1.1868 \\
\hline MW (15-24) & 1.1748 & 0.0239 & 2,330 & 2,330 & 1.2277 & 0.0203 & 1.1279 & 1.2218 \\
\hline \multicolumn{9}{|c|}{ Urban } \\
\hline MM (15-29) & 1.0218 & 0.0365 & 648 & 306 & 1.0404 & 0.0357 & 0.9499 & 1.0937 \\
\hline MW (15-24) & 1.1156 & 0.0329 & 1,037 & 525 & 1.1461 & 0.0295 & 1.0508 & 1.1804 \\
\hline \multicolumn{9}{|c|}{ Rural } \\
\hline MM (15-29) & 1.1500 & 0.0403 & 757 & 1,099 & 1.1947 & 0.0350 & 1.0707 & 1.2293 \\
\hline MW (15-24) & 1.1921 & 0.0291 & 1,293 & 1,805 & 1.1127 & 0.0244 & 1.1347 & 1.2494 \\
\hline \multicolumn{9}{|c|}{$\begin{array}{c}\text { Mean ideal number of children } \\
\text { (married young men and women who had begun cohabiting and gave a numeric response) }\end{array}$} \\
\hline \multicolumn{9}{|c|}{ Combined } \\
\hline MM (15-29) & 2.1276 & 0.0202 & 1,311 & 1,293 & 1.3317 & 0.0095 & 2.0879 & 2.1674 \\
\hline MW (15-24) & 2.0720 & 0.0130 & 2,256 & 2,246 & 1.5503 & 0.0063 & 2.0463 & 2.0977 \\
\hline \multicolumn{9}{|c|}{ Urban } \\
\hline MM (15-29) & 2.1060 & 0.0315 & 619 & 292 & 1.5871 & 0.0150 & 2.0440 & 2.1680 \\
\hline MW (15-24) & 2.0385 & 0.0233 & 1,016 & 514 & 1.6435 & 0.0114 & 1.9926 & 2.0844 \\
\hline \multicolumn{9}{|c|}{ Rural } \\
\hline MM (15-29) & 2.1339 & 0.0245 & 692 & 1,001 & 1.1408 & 0.0115 & 2.0858 & 2.1821 \\
\hline MW (15-24) & 2.0820 & 0.0154 & 1,240 & 1,732 & & & 2.0516 & 2.1124 \\
\hline
\end{tabular}

Cont'd on next page... 
Table B.2: (Cont'd)

\begin{tabular}{|c|c|c|c|c|c|c|c|c|}
\hline \multirow{2}{*}{$\begin{array}{l}\text { Variable/ } \\
\text { respondent } \\
\text { category }\end{array}$} & \multirow{2}{*}{$\begin{array}{l}\text { Value } \\
(\mathbf{R})\end{array}$} & \multirow{2}{*}{$\begin{array}{l}\text { Standard } \\
\text { error } \\
\text { (SE) }\end{array}$} & \multicolumn{2}{|c|}{ Number of cases } & \multirow{2}{*}{$\begin{array}{l}\text { Design } \\
\text { effect } \\
\text { (DEFT) }\end{array}$} & \multirow{2}{*}{$\begin{array}{c}\text { Relative } \\
\text { standard } \\
\text { error } \\
\text { (SE/R) }\end{array}$} & \multicolumn{2}{|c|}{ 95\% Confidence limits } \\
\hline & & & $\begin{array}{l}\text { Unweighted } \\
\text { (N) }\end{array}$ & $\begin{array}{l}\text { Weighted } \\
\text { (WN) }\end{array}$ & & & Lower & Upper \\
\hline \multicolumn{9}{|c|}{$\begin{array}{c}\text { Experienced } 3 \text { or more symptoms or behaviours suggestive of mental health disorders in the month } \\
\text { preceding the interview (young men and women) }\end{array}$} \\
\hline \multicolumn{9}{|c|}{ Combined } \\
\hline$M(15-24)$ & 0.1060 & 0.0148 & 2,479 & 2,504 & 2.3935 & 0.1396 & 0.0769 & 0.1352 \\
\hline W (15-24) & 0.1010 & 0.0084 & 4,848 & 4,848 & 1.9463 & 0.0834 & 0.0844 & 0.1176 \\
\hline MM (15-29) & 0.1245 & 0.0150 & 1,405 & 1,405 & 1.7078 & 0.1208 & 0.0949 & 0.1542 \\
\hline MW (15-24) & 0.1106 & 0.0114 & 2,330 & 2,330 & 1.7617 & 0.1035 & 0.0880 & 0.1331 \\
\hline UM (15-24) & 0.0969 & 0.0150 & 2,077 & 2,077 & 2.3053 & 0.1545 & 0.0674 & 0.1263 \\
\hline UW (15-24) & 0.0889 & 0.0075 & 2,518 & 2,518 & 1.3224 & 0.0844 & 0.0741 & 0.1037 \\
\hline \multicolumn{9}{|c|}{ Urban } \\
\hline$M(15-24)$ & 0.0809 & 0.0115 & 1,289 & 706 & 1.5179 & 0.1425 & 0.0582 & 0.1037 \\
\hline W (15-24) & 0.0828 & 0.0083 & 2,126 & 1,434 & 1.3810 & 0.0997 & 0.0665 & 0.0990 \\
\hline MM (15-29) & 0.0712 & 0.0141 & 648 & 306 & 1.3942 & 0.1980 & 0.0434 & 0.0989 \\
\hline MW (15-24) & 0.0831 & 0.0109 & 1,037 & 525 & 1.2723 & 0.1313 & 0.0616 & 0.1045 \\
\hline UM (15-24) & 0.0762 & 0.0113 & 1,132 & 631 & 1.4304 & 0.1481 & 0.0540 & 0.0985 \\
\hline UW (15-24) & 0.0825 & 0.0108 & 1,089 & 909 & 1.2975 & 0.1311 & 0.0612 & 0.1039 \\
\hline \multicolumn{9}{|c|}{ Rural } \\
\hline$M(15-24)$ & 0.1159 & 0.0200 & 1,190 & 1,799 & 2.1565 & 0.1728 & 0.0765 & 0.1553 \\
\hline W (15-24) & 0.1087 & 0.0115 & 2,722 & 3,414 & 1.9200 & 0.1054 & 0.0861 & 0.1312 \\
\hline MM (15-29) & 0.1394 & 0.0186 & 757 & 1,099 & 1.4776 & 0.1335 & 0.1028 & 0.1761 \\
\hline MW (15-24) & 0.1186 & 0.0144 & 1,293 & 1,805 & 1.6040 & 0.1217 & 0.0902 & 0.1470 \\
\hline UM (15-24) & 0.1058 & 0.0209 & 945 & 1,446 & 2.0838 & 0.1971 & 0.0648 & 0.1469 \\
\hline UW (15-24) & 0.0925 & 0.0100 & 1,429 & 1,609 & 1.3106 & 0.1086 & 0.0727 & 0.1123 \\
\hline \multicolumn{9}{|c|}{ Ever consumed alcohol (young men and women) } \\
\hline \multicolumn{9}{|c|}{ Combined } \\
\hline M (15-24) & 0.2733 & 0.0162 & 2,479 & 2,504 & 1.8117 & 0.0593 & 0.2414 & 0.3052 \\
\hline $\mathrm{W}(15-24)$ & 0.0154 & 0.0036 & 4,848 & 4,848 & 2.0384 & 0.2343 & 0.0083 & 0.0225 \\
\hline MM (15-29) & 0.4889 & 0.0248 & 1,405 & 1,405 & 1.8572 & 0.0507 & 0.4401 & 0.5377 \\
\hline MW (15-24) & 0.0227 & 0.0056 & 2,330 & 2,330 & 1.8225 & 0.2479 & 0.0116 & 0.0337 \\
\hline UM (15-24) & 0.2332 & 0.0156 & 2,077 & 2,077 & 1.6773 & 0.0668 & 0.2025 & 0.2638 \\
\hline UW (15-24) & 0.0060 & 0.0016 & 2,518 & 2,518 & 1.0648 & 0.2741 & 0.0027 & 0.0092 \\
\hline \multicolumn{9}{|c|}{ Urban } \\
\hline M (15-24) & 0.3195 & 0.0212 & 1,289 & 706 & 1.6302 & 0.0663 & 0.2779 & 0.3612 \\
\hline $\mathrm{W}(15-24)$ & 0.0062 & 0.0025 & 2,126 & 1,434 & 1.4892 & 0.4097 & 0.0012 & 0.0112 \\
\hline MM (15-29) & 0.6069 & 0.0293 & 648 & 306 & 1.5244 & 0.0482 & 0.5492 & 0.6645 \\
\hline MW (15-24) & 0.0103 & 0.0049 & 1,037 & 525 & 1.5580 & 0.4754 & 0.0007 & 0.0199 \\
\hline UM (15-24) & 0.2819 & 0.0201 & 1,132 & 631 & 1.5032 & 0.0713 & 0.2423 & 0.3215 \\
\hline UW (15-24) & 0.0029 & 0.0016 & 1,089 & 909 & 1.0012 & 0.5654 & 0.0000 & 0.0061 \\
\hline \multicolumn{9}{|c|}{ Rural } \\
\hline M (15-24) & 0.2551 & 0.0208 & 1,190 & 1,799 & 1.6466 & 0.0816 & 0.2142 & 0.2961 \\
\hline $\mathrm{W}(15-24)$ & 0.0192 & 0.0050 & 2,722 & 3,414 & 1.8949 & 0.2594 & 0.0094 & 0.0291 \\
\hline MM (15-29) & 0.4560 & 0.0302 & 757 & 1,099 & 1.6697 & 0.0663 & 0.3965 & 0.5155 \\
\hline MW (15-24) & 0.0263 & 0.0071 & 1,293 & 1,805 & 1.5999 & 0.2709 & 0.0123 & 0.0403 \\
\hline UM (15-24) & 0.2119 & 0.0203 & 945 & 1,446 & 1.5273 & 0.0959 & 0.1719 & 0.2519 \\
\hline UW (15-24) & 0.0077 & 0.0024 & 1,429 & 1,609 & 1.0318 & 0.3099 & 0.0030 & 0.0124 \\
\hline
\end{tabular}


Table B.2: (Cont'd)

\begin{tabular}{|c|c|c|c|c|c|c|c|c|}
\hline \multirow{2}{*}{$\begin{array}{l}\text { Variable/ } \\
\text { respondent } \\
\text { category }\end{array}$} & \multirow{2}{*}{$\begin{array}{l}\text { Value } \\
(\mathbf{R})\end{array}$} & \multirow{2}{*}{$\begin{array}{l}\text { Standard } \\
\text { error } \\
\text { (SE) }\end{array}$} & \multicolumn{2}{|c|}{ Number of cases } & \multirow{2}{*}{$\begin{array}{l}\text { Design } \\
\text { effect } \\
\text { (DEFT) }\end{array}$} & \multirow{2}{*}{$\begin{array}{l}\text { Relative } \\
\text { standard } \\
\text { error } \\
(\mathrm{SE} / \mathrm{R})\end{array}$} & \multicolumn{2}{|c|}{ 95\% Confidence limits } \\
\hline & & & $\begin{array}{l}\text { Unweighted } \\
(\mathbf{N})\end{array}$ & $\begin{array}{l}\text { Weighted } \\
(\text { WN) }\end{array}$ & & & Lower & Upper \\
\hline \multicolumn{9}{|c|}{$\begin{array}{l}\text { Participated in a government- /NGO- sponsored programme in the } 3 \text { years preceding the interview } \\
\text { (young men and women) }\end{array}$} \\
\hline \multicolumn{9}{|c|}{ Combined } \\
\hline M (15-24) & 0.1939 & 0.0132 & 2,479 & 2,504 & 1.6631 & 0.0681 & 0.1679 & 0.2199 \\
\hline W (15-24) & 0.2177 & 0.0099 & 4,848 & 4,848 & 1.6626 & 0.0453 & 0.1983 & 0.2371 \\
\hline MM (15-29) & 0.1828 & 0.0157 & 1,405 & 1,405 & 1.5270 & 0.0862 & 0.1517 & 0.2138 \\
\hline MW (15-24) & 0.3067 & 0.0158 & 2,330 & 2,330 & 1.6500 & 0.0514 & 0.2756 & 0.3377 \\
\hline UM (15-24) & 0.1973 & 0.0137 & 2,077 & 2,077 & 1.5690 & 0.0695 & 0.1703 & 0.2243 \\
\hline UW (15-24) & 0.1021 & 0.0076 & 2,518 & 2,518 & 1.2540 & 0.0741 & 0.0872 & 0.1170 \\
\hline \multicolumn{9}{|c|}{ Urban } \\
\hline M (15-24) & 0.1257 & 0.0152 & 1,289 & 706 & 1.6497 & 0.1212 & 0.0957 & 0.1557 \\
\hline W (15-24) & 0.1343 & 0.0097 & 2,126 & 1,434 & 1.3114 & 0.0722 & 0.1152 & 0.1533 \\
\hline MM (15-29) & 0.0979 & 0.0146 & 648 & 306 & 1.2455 & 0.1486 & 0.0693 & 0.1266 \\
\hline MW (15-24) & 0.2201 & 0.0151 & 1,037 & 525 & 1.1729 & 0.0686 & 0.1904 & 0.2499 \\
\hline UM (15-24) & 0.1271 & 0.0166 & 1,132 & 631 & 1.6781 & 0.1308 & 0.0944 & 0.1599 \\
\hline UW (15-24) & 0.0647 & 0.0082 & 1,089 & 909 & 1.1041 & 0.1273 & 0.0485 & 0.0809 \\
\hline \multicolumn{9}{|c|}{ Rural } \\
\hline M (15-24) & 0.2207 & 0.0173 & 1,190 & 1,799 & 1.4409 & 0.0785 & 0.1866 & 0.2548 \\
\hline $\mathrm{W}(15-24)$ & 0.2527 & 0.0127 & 2,722 & 3,414 & 1.5259 & 0.0503 & 0.2277 & 0.2777 \\
\hline MM (15-29) & 0.2064 & 0.0191 & 757 & 1,099 & 1.2987 & 0.0926 & 0.1688 & 0.2440 \\
\hline MW (15-24) & 0.3318 & 0.0197 & 1,293 & 1,805 & 1.5030 & 0.0593 & 0.2931 & 0.3706 \\
\hline UM (15-24) & 0.2279 & 0.0185 & 945 & 1,446 & 1.3550 & 0.0812 & 0.1915 & 0.2643 \\
\hline UW (15-24) & 0.1233 & 0.0104 & 1,429 & 1,609 & 1.1980 & 0.0845 & 0.1028 & 0.1438 \\
\hline \multicolumn{9}{|c|}{ Voted in last election (young men and women, aged 20 and above) } \\
\hline \multicolumn{9}{|c|}{ Combined } \\
\hline$M(15-24)$ & 0.6327 & 0.0201 & 1,314 & 1,322 & 1.5097 & 0.0317 & 0.5932 & 0.6723 \\
\hline W (15-24) & 0.6174 & 0.0143 & 2,245 & 2,379 & 1.3984 & 0.0232 & 0.5891 & 0.6456 \\
\hline MM (15-29) & 0.8809 & 0.0119 & 1,391 & 1,388 & 1.3748 & 0.0136 & 0.8574 & 0.9044 \\
\hline MW (15-24) & 0.6780 & 0.0151 & 1,669 & 1,617 & 1.3231 & 0.0223 & 0.6482 & 0.7078 \\
\hline UM (15-24) & 0.5606 & 0.0228 & 926 & 887 & 1.3971 & 0.0407 & 0.5157 & 0.6055 \\
\hline UW (15-24) & 0.3774 & 0.0225 & 576 & 548 & 1.1127 & 0.0596 & 0.3331 & 0.4216 \\
\hline \multicolumn{9}{|c|}{ Urban } \\
\hline M (15-24) & 0.4872 & 0.0207 & 706 & 391 & 1.1008 & 0.0425 & 0.4464 & 0.5280 \\
\hline W (15-24) & 0.4179 & 0.0189 & 1,151 & 751 & 1.2983 & 0.0452 & 0.3807 & 0.4551 \\
\hline MM (15-29) & 0.8223 & 0.0158 & 645 & 305 & 1.0457 & 0.0192 & 0.7913 & 0.8533 \\
\hline MW (15-24) & 0.4884 & 0.0209 & 803 & 407 & 1.1857 & 0.0429 & 0.4472 & 0.5296 \\
\hline UM (15-24) & 0.4299 & 0.0225 & 552 & 310 & 1.0673 & 0.0524 & 0.3856 & 0.4742 \\
\hline UW (15-24) & 0.2797 & 0.0296 & 348 & 291 & 1.2269 & 0.1057 & 0.2215 & 0.3379 \\
\hline \multicolumn{9}{|c|}{ Rural } \\
\hline M (15-24) & 0.6938 & 0.0261 & 608 & 931 & 1.3975 & 0.0377 & 0.6423 & 0.7453 \\
\hline $\mathrm{W}(15-24)$ & 0.7094 & 0.0158 & 1,094 & 1,628 & 1.1495 & 0.0223 & 0.6784 & 0.7405 \\
\hline MM (15-29) & 0.8974 & 0.0145 & 746 & 1,083 & 1.3047 & 0.0162 & 0.8688 & 0.9259 \\
\hline MW (15-24) & 0.7417 & 0.0176 & 866 & 1,210 & 1.1856 & 0.0238 & 0.7070 & 0.7764 \\
\hline UM (15-24) & 0.6306 & 0.0326 & 374 & 578 & 1.3048 & 0.0517 & 0.5664 & 0.6948 \\
\hline UW (15-24) & 0.4879 & 0.0325 & 228 & 257 & 0.9811 & 0.0667 & 0.4238 & 0.5520 \\
\hline
\end{tabular}

Note: M: Men, W: Women, MM: Married men, MW: Married women, UM: Unmarried men, UW: Unmarried women. 
Appendix C

\section{Data quality tables}

Table C.1: Household age distribution

Single-year age distribution of the de jure household population by sex (weighted), Andhra Pradesh, 2007-08

\begin{tabular}{|c|c|c|c|c|c|c|c|c|c|}
\hline \multirow{2}{*}{$\begin{array}{l}\text { Age } \\
\text { (year) }\end{array}$} & \multicolumn{2}{|c|}{ Women } & \multicolumn{2}{|c|}{ Men } & \multirow{2}{*}{$\begin{array}{l}\text { Age } \\
\text { (year) }\end{array}$} & \multicolumn{2}{|c|}{ Women } & \multicolumn{2}{|c|}{ Men } \\
\hline & $\begin{array}{l}\text { Unweighted } \\
\text { Number }\end{array}$ & Percent & $\begin{array}{c}\text { Unweighted } \\
\text { Number }\end{array}$ & Percent & & $\begin{array}{l}\text { Unweighted } \\
\text { Number }\end{array}$ & Percent & $\begin{array}{l}\text { Unweighted } \\
\text { Number }\end{array}$ & Percent \\
\hline 0 & 937 & 1.5 & 955 & 1.6 & 36 & 669 & 1.1 & 682 & 1.1 \\
\hline 1 & 808 & 1.2 & 871 & 1.4 & 37 & 468 & 0.7 & 534 & 0.8 \\
\hline 2 & 917 & 1.5 & 896 & 1.4 & 38 & 1,146 & 1.8 & 968 & 1.5 \\
\hline 3 & 1,057 & 1.7 & 1,141 & 1.9 & 39 & 436 & 0.6 & 379 & 0.6 \\
\hline 4 & 1,047 & 1.7 & 1,038 & 1.7 & 40 & 2,260 & 3.7 & 2,472 & 4.0 \\
\hline 5 & 1,127 & 1.8 & 1,298 & 2.1 & 41 & 182 & 0.3 & 241 & 0.4 \\
\hline 6 & 1,234 & 2.0 & 1,179 & 1.9 & 42 & 665 & 1.0 & 747 & 1.1 \\
\hline 7 & 1,178 & 1.9 & 1,249 & 2.0 & 43 & 295 & 0.4 & 309 & 0.4 \\
\hline 8 & 1,259 & 2.0 & 1,329 & 2.1 & 44 & 201 & 0.3 & 222 & 0.3 \\
\hline 9 & 1,159 & 1.8 & 1,121 & 1.8 & 45 & 2,105 & 3.5 & 2,241 & 3.7 \\
\hline 10 & 1,398 & 2.2 & 1,511 & 2.4 & 46 & 345 & 0.5 & 328 & 0.5 \\
\hline 11 & 1,127 & 1.7 & 1,085 & 1.8 & 47 & 289 & 0.4 & 310 & 0.5 \\
\hline 12 & 1,466 & 2.3 & 1,466 & 2.3 & 48 & 643 & 1.0 & 604 & 1.0 \\
\hline 13 & 1,284 & 2.1 & 1,314 & 2.2 & 49 & 259 & 0.4 & 248 & 0.4 \\
\hline 14 & 1,047 & 1.7 & 1,233 & 1.9 & 50 & 1,833 & 3.0 & 1,940 & 3.3 \\
\hline 15 & 965 & 1.6 & 1,083 & 1.6 & 51 & 154 & 0.3 & 171 & 0.2 \\
\hline 16 & 1,078 & 1.8 & 1,115 & 1.7 & 52 & 393 & 0.6 & 387 & 0.6 \\
\hline 17 & 1,015 & 1.6 & 1,060 & 1.6 & 53 & 200 & 0.3 & 207 & 0.3 \\
\hline 18 & 1,537 & 2.5 & 1,582 & 2.5 & 54 & 233 & 0.3 & 201 & 0.3 \\
\hline 19 & 997 & 1.5 & 983 & 1.5 & 55 & 1,478 & 2.4 & 1,126 & 1.9 \\
\hline 20 & 1,792 & 2.9 & 1,748 & 2.7 & 56 & 210 & 0.3 & 234 & 0.3 \\
\hline 21 & 861 & 1.3 & 913 & 1.4 & 57 & 143 & 0.2 & 136 & 0.2 \\
\hline 22 & 1,287 & 2.0 & 1,281 & 1.9 & 58 & 321 & 0.5 & 273 & 0.4 \\
\hline 23 & 1,008 & 1.5 & 983 & 1.5 & 59 & 107 & 0.2 & 122 & 0.2 \\
\hline 24 & 878 & 1.3 & 903 & 1.4 & 60 & 1,765 & 2.9 & 1,663 & 2.8 \\
\hline 25 & 1,418 & 2.6 & 1,681 & 2.6 & 61 & 89 & 0.1 & 95 & 0.2 \\
\hline 26 & 1,169 & 1.8 & 1,014 & 1.6 & 62 & 279 & 0.4 & 256 & 0.4 \\
\hline 27 & 1,019 & 1.5 & 850 & 1.3 & 63 & 118 & 0.2 & 117 & 0.2 \\
\hline 28 & 1,766 & 2.7 & 1,194 & 1.8 & 64 & 104 & 0.2 & 118 & 0.2 \\
\hline 29 & 828 & 1.2 & 667 & 1.0 & 65 & 1,126 & 1.8 & 926 & 1.6 \\
\hline 30 & 2,594 & 4.1 & 1,911 & 2.7 & 66 & 66 & 0.1 & 88 & 0.1 \\
\hline 31 & 394 & 0.6 & 612 & 1.1 & 67 & 71 & 0.1 & 92 & 0.2 \\
\hline 32 & 1,081 & 1.6 & 1,280 & 2.1 & 68 & 183 & 0.3 & 149 & 0.2 \\
\hline 33 & 480 & 0.7 & 613 & 1.0 & 69 & 63 & 0.1 & 58 & 0.1 \\
\hline 34 & 427 & 0.6 & 605 & 1.0 & $70+$ & 1,911 & 3.1 & 1,994 & 3.3 \\
\hline 35 & 2,491 & 4.0 & 2,608 & 4.2 & Total & 62,940 & 100.0 & 63,010 & 100.0 \\
\hline
\end{tabular}

Note: The de jure population includes usual residents of the household. 
Table C.2: Single-year age distribution of eligible, selected and interviewed young men

Number and percentage of eligible, selected and interviewed young men and percentage of selected young men who were interviewed by single-year age (unweighted), Andhra Pradesh, 2007-08

\begin{tabular}{|c|c|c|c|c|c|c|c|}
\hline \multirow[t]{2}{*}{ Age (years) } & \multicolumn{2}{|c|}{ Eligible } & \multicolumn{2}{|c|}{ Selected for interview } & \multicolumn{2}{|c|}{ Interviewed } & \multirow{2}{*}{$\begin{array}{l}\% \text { selected } \\
\text { respondents } \\
\text { interviewed }\end{array}$} \\
\hline & No. & $\%$ & No. & $\%$ & No. & $\%$ & \\
\hline \multicolumn{8}{|c|}{ MM (15-29) } \\
\hline 15 & 0 & 0.0 & 0 & 0.0 & 0 & 0.0 & $\mathrm{NC}$ \\
\hline 16 & 1 & 0.1 & 1 & 0.1 & 1 & 0.1 & 100.0 \\
\hline 17 & 1 & 0.1 & 1 & 0.1 & 1 & 0.1 & 100.0 \\
\hline 18 & 6 & 0.4 & 5 & 0.3 & 4 & 0.3 & 80.0 \\
\hline 19 & 10 & 0.6 & 8 & 0.5 & 8 & 0.6 & 100.0 \\
\hline 20 & 52 & 3.1 & 46 & 2.9 & 37 & 2.6 & 80.4 \\
\hline 21 & 55 & 3.3 & 53 & 3.4 & 47 & 3.3 & 88.7 \\
\hline 22 & 93 & 5.6 & 86 & 5.5 & 72 & 5.1 & 83.7 \\
\hline 23 & 120 & 7.3 & 114 & 7.3 & 105 & 7.5 & 92.1 \\
\hline 24 & 147 & 8.9 & 139 & 8.8 & 127 & 9.0 & 91.4 \\
\hline 25 & 286 & 17.3 & 271 & 17.2 & 234 & 16.7 & 86.3 \\
\hline 26 & 188 & 11.4 & 180 & 11.5 & 167 & 11.9 & 92.8 \\
\hline 27 & 214 & 12.9 & 204 & 13.0 & 186 & 13.2 & 91.2 \\
\hline 28 & 275 & 16.6 & 265 & 16.9 & 236 & 16.8 & 89.1 \\
\hline 29 & 206 & 12.5 & 199 & 12.7 & 180 & 12.8 & 90.5 \\
\hline Total & 1,654 & 100.0 & 1,572 & 100.0 & 1,405 & 100.0 & 89.4 \\
\hline \multicolumn{8}{|c|}{ UM (15-24) } \\
\hline 15 & 244 & 8.1 & 189 & 8.2 & 168 & 8.1 & 88.9 \\
\hline 16 & 320 & 10.7 & 245 & 10.6 & 218 & 10.5 & 89.0 \\
\hline 17 & 290 & 9.7 & 231 & 10.0 & 214 & 10.3 & 92.6 \\
\hline 18 & 430 & 14.3 & 331 & 14.4 & 293 & 14.1 & 88.5 \\
\hline 19 & 330 & 11.0 & 270 & 11.7 & 258 & 12.4 & 95.6 \\
\hline 20 & 422 & 14.1 & 321 & 14.0 & 284 & 13.7 & 88.5 \\
\hline 21 & 288 & 9.6 & 220 & 9.6 & 198 & 9.5 & 90.0 \\
\hline 22 & 294 & 9.8 & 217 & 9.4 & 195 & 9.4 & 89.9 \\
\hline 23 & 204 & 6.8 & 147 & 6.4 & 131 & 6.3 & 89.1 \\
\hline 24 & 177 & 5.9 & 130 & 5.6 & 118 & 5.7 & 90.8 \\
\hline Total & 2,999 & 100.0 & 2,301 & 100.0 & 2,077 & 100.0 & 90.3 \\
\hline
\end{tabular}

Note: NC: Not calculated, as there is no case in the denominator. The difference between the number of respondents eligible for interview and the number who were selected for interview is due to the sampling design adopted in the Youth Study. Please refer to Chapter 1 for details. 
Table C.3: Single-year age distribution of eligible, selected and interviewed young women

Number and percentages of eligible, selected and interviewed female respondents and percentage of selected respondents who were interviewed by single-year age (unweighted), Andhra Pradesh, 2007-08

\begin{tabular}{|c|c|c|c|c|c|c|c|}
\hline \multirow[t]{2}{*}{ Age (years) } & \multicolumn{2}{|c|}{ Eligible } & \multicolumn{2}{|c|}{ Selected for interview } & \multicolumn{2}{|c|}{ Interviewed } & \multirow{2}{*}{$\begin{array}{l}\% \text { selected } \\
\text { respondents } \\
\text { interviewed }\end{array}$} \\
\hline & No. & $\%$ & No. & $\%$ & No. & $\%$ & \\
\hline \multicolumn{8}{|c|}{ MW(15-24) } \\
\hline 15 & 30 & 0.8 & 24 & 0.9 & 24 & 1.0 & 100.0 \\
\hline 16 & 90 & 2.3 & 62 & 2.4 & 53 & 2.3 & 85.5 \\
\hline 17 & 184 & 4.6 & 140 & 5.4 & 125 & 5.4 & 89.3 \\
\hline 18 & 428 & 10.8 & 262 & 10.2 & 226 & 9.7 & 86.3 \\
\hline 19 & 383 & 9.7 & 250 & 9.7 & 233 & 10.0 & 93.2 \\
\hline 20 & 785 & 19.8 & 452 & 17.6 & 390 & 16.7 & 86.3 \\
\hline 21 & 432 & 10.9 & 318 & 12.3 & 293 & 12.6 & 92.1 \\
\hline 22 & 586 & 14.8 & 385 & 15.0 & 351 & 15.1 & 91.2 \\
\hline 23 & 566 & 14.3 & 365 & 14.2 & 335 & 14.4 & 91.8 \\
\hline 24 & 478 & 12.1 & 317 & 12.3 & 300 & 12.9 & 94.6 \\
\hline Total & 3,962 & 100.0 & 2,575 & 100.0 & 2,330 & 100.0 & 90.5 \\
\hline \multicolumn{8}{|c|}{ UW (15-24) } \\
\hline 15 & 534 & 17.1 & 482 & 18.0 & 460 & 18.3 & 95.4 \\
\hline 16 & 556 & 17.8 & 484 & 18.1 & 457 & 18.1 & 94.4 \\
\hline 17 & 477 & 15.3 & 407 & 15.2 & 386 & 15.3 & 94.8 \\
\hline 18 & 496 & 15.9 & 432 & 16.2 & 410 & 16.3 & 94.9 \\
\hline 19 & 292 & 9.4 & 243 & 9.1 & 229 & 9.1 & 94.2 \\
\hline 20 & 289 & 9.3 & 231 & 8.6 & 217 & 8.6 & 93.9 \\
\hline 21 & 164 & 5.3 & 138 & 5.2 & 126 & 5.0 & 91.3 \\
\hline 22 & 148 & 4.7 & 120 & 4.5 & 104 & 4.1 & 86.7 \\
\hline 23 & 100 & 3.2 & 84 & 3.1 & 81 & 3.2 & 96.4 \\
\hline 24 & 61 & 2.0 & 52 & 1.9 & 48 & 1.9 & 92.3 \\
\hline Total & 3,117 & 100.0 & 2,673 & 100.0 & 2,518 & 100.0 & 94.2 \\
\hline
\end{tabular}

Note: The difference between the number of respondents eligible for interview and the number who were selected for interview is due to the sampling design adopted in the Youth Study. Please refer to Chapter 1 for details. 


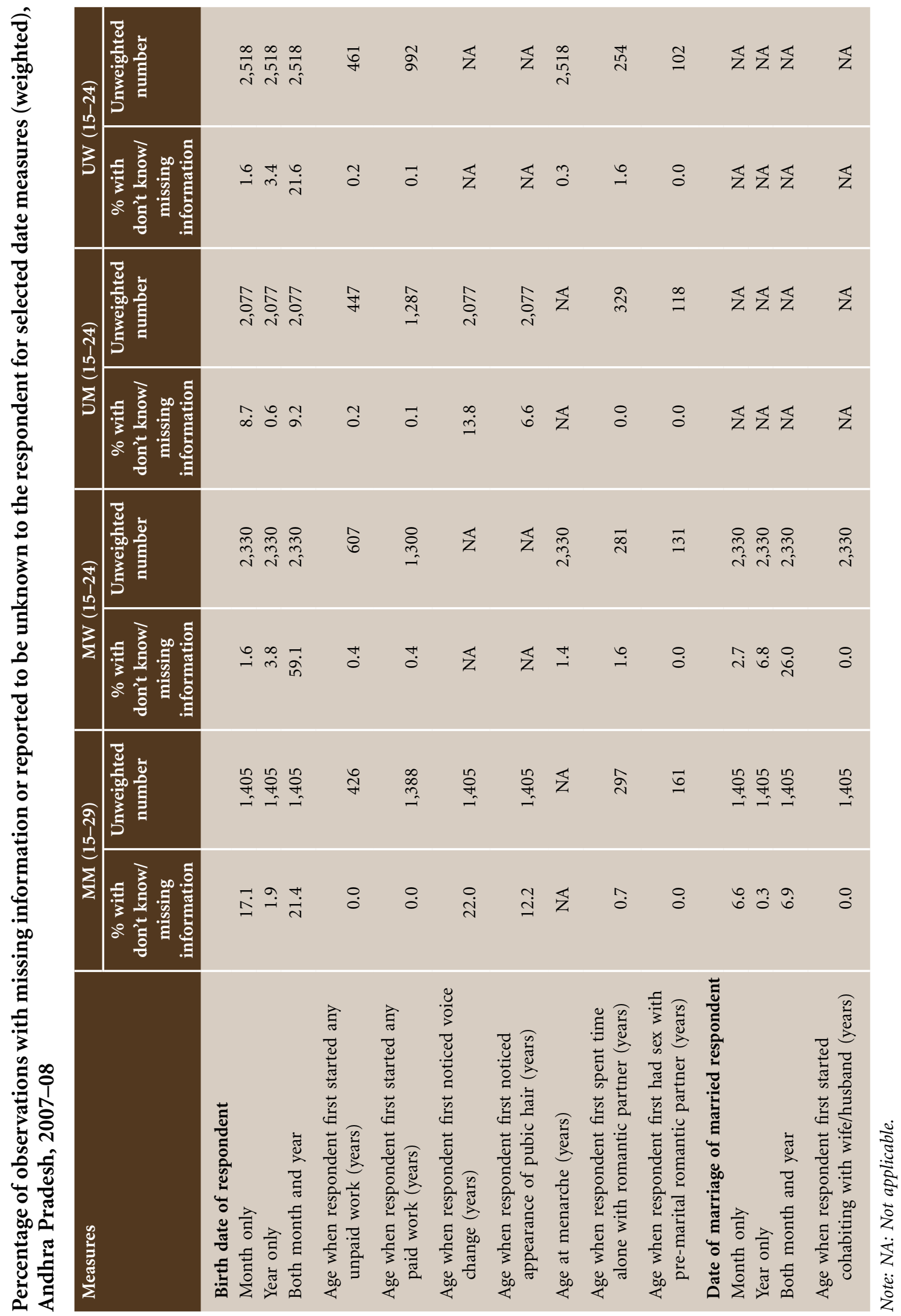




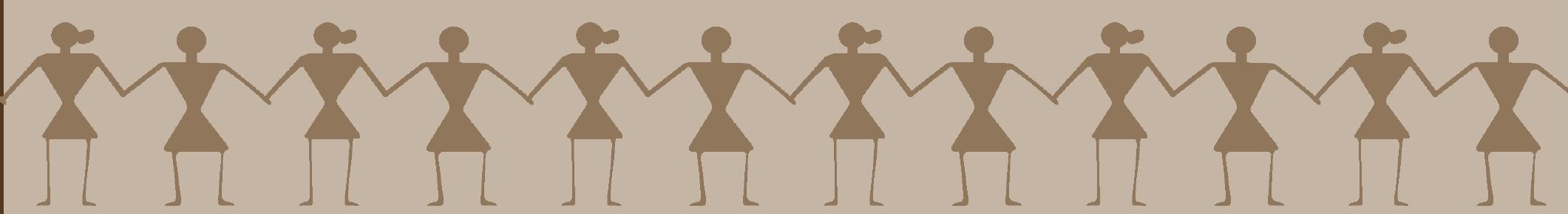


Supported by:

the David

Lucile PaCkard MACARTHUR

FOUNDATION 